Supporting Information

\title{
Pincer Iron Hydride Complexes for Alkene Isomerization: Catalytic Approach to Trisubstituted (Z)-Alkenyl Boronates
}

Songgen $\mathrm{Xu},{ }^{\dagger}$ Peiyu Geng, ${ }^{\dagger}$ Yuling Li, ${ }^{\dagger}$ Guixia Liu, ${ }^{\dagger}, \S$ Lei Zhang, ${ }^{\dagger}$ Yinlong Guo $*, \dagger$ and Zheng Huang*,†,\$,

The State Key Laboratory of Organometallic Chemistry, Shanghai Institute of Organic Chemistry, University of Chinese Academy of Sciences, Chinese Academy of Sciences, 345 Lingling Road, Shanghai 200032, China

* School of Chemistry and Material Sciences, Hangzhou Institute of Advanced Study, University of Chinese Academy of Sciences, 1 Sub-lane Xiangshan, Hangzhou 310024, China ${ }^{\S}$ Chang-Kung Chuang Institute, East China Normal University, Shanghai 200062, China

huangzh@sioc.ac.cn 


\section{Table of content}

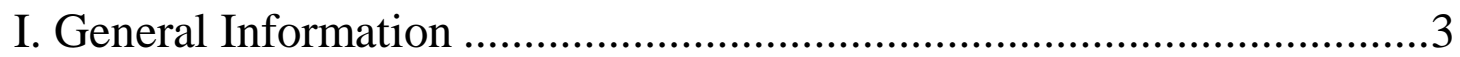

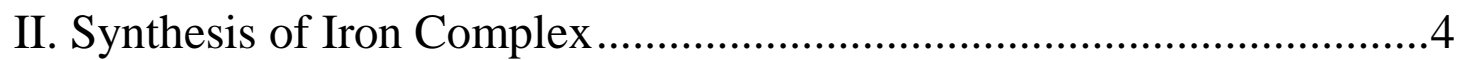

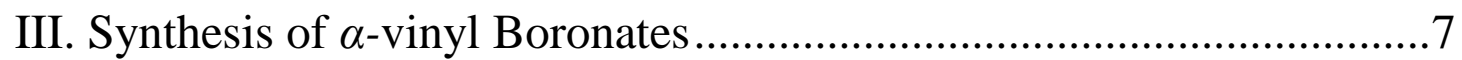

IV. Procedures for Isomerization of 1,1-Disubstituted Vinyl Boronates .21

V. Procedures for Tandem Isomerization and Coupling Reactions ..........37

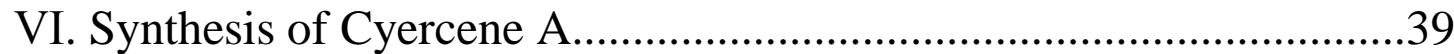

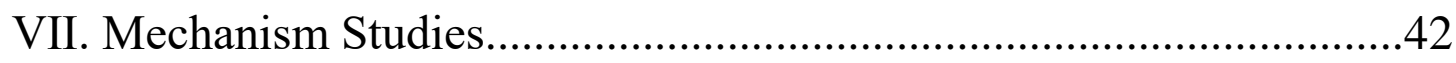

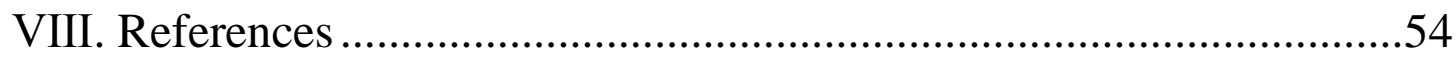

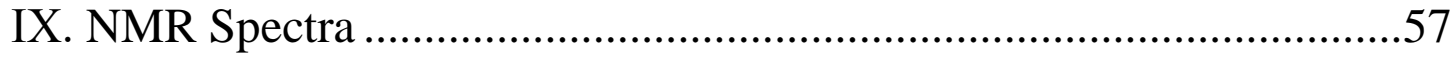




\section{General Information}

Unless otherwise noted, all reagents were purchased from commercial suppliers and used without further purification. All manipulations were carried out using standard Schlenk, high-vacuum and glovebox techniques. Pentane, tetrahydrofuran (THF) and ether were purchased from Sinopharm Group Chemical Co., Ltd. and dried over $\mathrm{LiAlH}_{4}$ overnight under an atmosphere of dry argon, then distilled prior to use and stored in an argon atmosphere glovebox. Toluene was distilled from sodium benzophenone ketyl prior to use. 6-methylpicolinic acid, (S)-tert-leucinol, TBTU and aniline were purchased from TCI chemistry. $t \mathrm{Bu}_{2} \mathrm{PCl}$, ferrous chloride tetrahydrate, $i \mathrm{PrOB}$ in were purchased from Alfa Aesar. $\mathrm{NaHBEt}_{3}$ was purchased from Acros. Complexes $\left(\mathrm{PNN}_{\text {oxa }}\right) \mathrm{FeCl}_{2},{ }^{1}\left(\mathrm{PNN}_{\mathrm{py}}\right) \mathrm{FeCl}_{2},{ }^{2}\left(\mathrm{PNN}_{\mathrm{im}}\right) \mathrm{FeCl}_{2},{ }^{3}(\mathrm{PNP}) \mathrm{FeCl}_{2}{ }^{4}$ were prepared according to previously reported procedures.

NMR spectra were recorded on Agilent $400 \mathrm{MHz}$, Varian Mercury $400 \mathrm{MHz}$ or Bruker $400 \mathrm{MHz} .{ }^{1} \mathrm{H}$ NMR chemical shifts were referenced to residual protio-solvent peaks or tetramethylsilane signal $(0 \mathrm{ppm})$, and ${ }^{13} \mathrm{C}$ NMR chemical shifts were referenced to the solvent resonance. Data for ${ }^{1} \mathrm{H}$ NMR are recorded as follows: chemical shift $(\delta, \mathrm{ppm})$, multiplicity $(\mathrm{s}=$ singlet, $\mathrm{d}=$ doublet, $\mathrm{t}=$ triplet, $\mathrm{q}=$ quartlet quint $=$ quintuplet, sext $=$ sextuplet, $\mathrm{m}=$ multiplet or unresolved, coupling constant $(\mathrm{s})$ in $\mathrm{Hz}$, integration). Data for ${ }^{13} \mathrm{C} \mathrm{NMR}$ are reported in terms of chemical shift $(\delta, \mathrm{ppm})$. Elemental analyses and high resolution mass spectrometer (HR-MS) were carried out by the Analytical Laboratory of Shanghai Institute of Organic Chemistry (CAS) or State Key Laboratory of Organometallic Chemistry of Shanghai Institute of Organic Chemistry (CAS). 


\section{Synthesis of Iron Complex}

Procedure for preparation of complex $\left(\mathrm{PNN}_{\mathrm{imid}}\right) \mathrm{FeCl}_{2}$ :

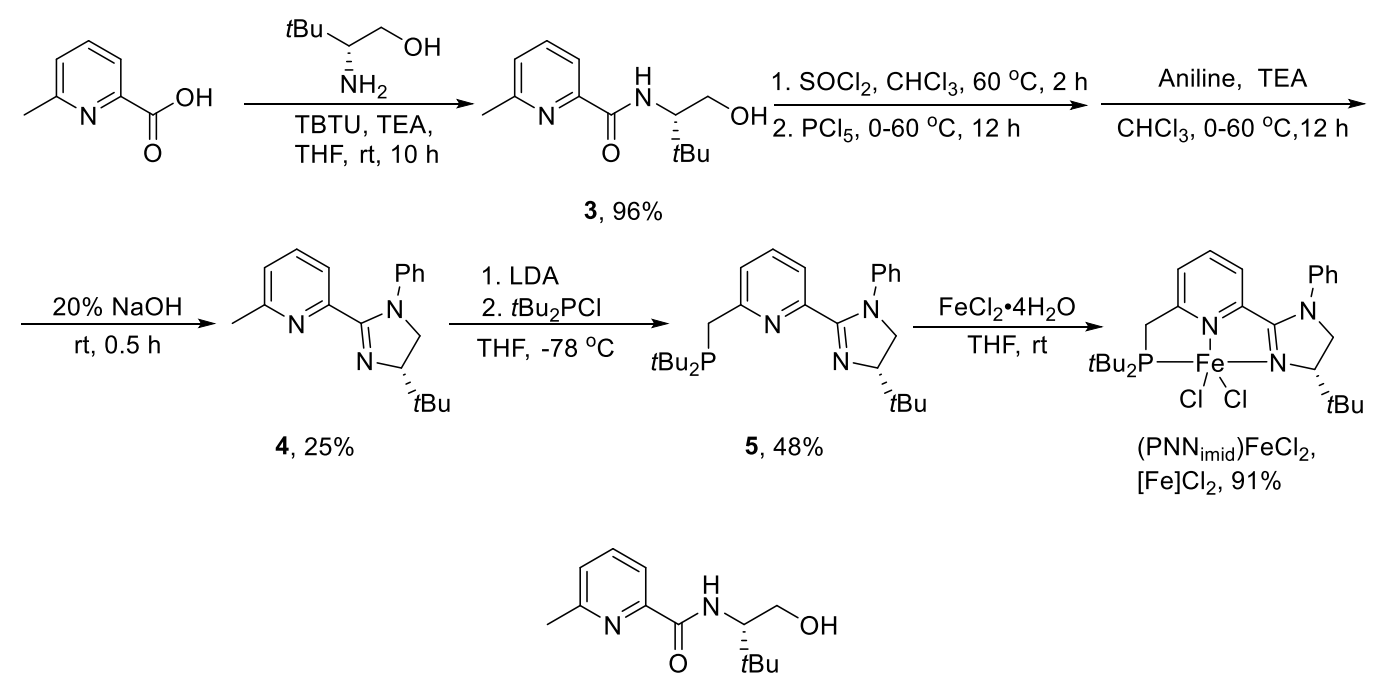

Preparation of (S)-N-(1-hydroxy-3,3-dimethylbutan-2-yl)-6-methylpicolinamide

(3). An oven-dried $250 \mathrm{~mL}$ three-necked flask was charged with 6-methylpicolinic acid (5 g, $36 \mathrm{mmol})$, (S)-tert-leucinol (4.3 g, $36 \mathrm{mmol})$, and TBTU (11.7 g, $36 \mathrm{mmol})$ in 150 $\mathrm{mL}$ THF. $10 \mathrm{~mL}$ triethylamine was added to the mixture and the suspension was stirred vigorous overnight. The solvent was removed under vacuo and the residue was extracted with dichloromethane $(2 \times 50 \mathrm{~mL})$ and washed with brine. The combined organics were dried over $\mathrm{Na}_{2} \mathrm{SO}_{4}$, filtered, and the solvent was removed in vacuo. The residue was purified by flash column chromatography (petroleum ether/EtOAc $=10: 1$ 1:1) to give product $(9.3 \mathrm{~g}, 96 \%)$ as yellow solid. ${ }^{1} \mathrm{H}$ NMR $\left(400 \mathrm{MHz}, \mathrm{CDCl}_{3}\right) \delta 8.41$ (s, 1H), $8.03-7.93(\mathrm{~m}, 1 \mathrm{H}), 7.78-7.67(\mathrm{~m}, 1 \mathrm{H}), 7.32-7.29(\mathrm{~m}, 1 \mathrm{H}), 4.04-3.93(\mathrm{~m}$, 2H), $3.74-3.66(\mathrm{~m}, 1 \mathrm{H}), 2.81(\mathrm{~s}, 1 \mathrm{H}), 2.60(\mathrm{~s}, 3 \mathrm{H}), 1.06(\mathrm{~s}, 9 \mathrm{H}) .{ }^{13} \mathrm{C}$ NMR (101 MHz, $\left.\mathrm{CDCl}_{3}\right) \delta 165.8,157.2,148.9,137.5,126.0,119.4,63.6,60.5,33.7,27.0,24.4$. HRMS$\mathrm{EI}(\mathrm{m} / \mathrm{z})$ : Calc. for $\mathrm{C}_{13} \mathrm{H}_{20} \mathrm{~N}_{2} \mathrm{O}_{2}[\mathrm{M}]^{+}$236.1525, found 236.1521 .<smiles>Cc1cccc(C2=NC(C(C)(C)C)CN2c2ccccc2)n1</smiles>

Preparation of (S)-2-(4-(tert-butyl)-1-phenyl-4,5-dihydro-1H-imidazol-2-yl)-6methylpyridine (4). (S)-N-(1-hydroxy-3,3-dimethylbutan-2-yl)-6-methylpicolinamide 
(3) (3.2 g,13.6 mmol) was added to an oven-dried $100 \mathrm{~mL}$ three-necked flask fitted with a reflux condenser and the system was purged with argon. $5 \mathrm{~mL}$ anhydrous chloroform was added and thionyl chloride $(1.26 \mathrm{~mL}, 15.1 \mathrm{mmol})$ was added dropwise. The solution was stirred at reflux for $2 \mathrm{~h}$. After cooling to room temperature, Phosphorus pentachloride ( $2.86 \mathrm{~g}, 13.7 \mathrm{mmol})$ was added, and the resulting suspension was stirred at reflux for a further $2 \mathrm{~h}$. The mixture was cooled to $0{ }^{\circ} \mathrm{C}$ and a solution of aniline $(1.8$ $\mathrm{g}, 16.9 \mathrm{mmol})$ in triethylamine $(5.7 \mathrm{~mL}, 46 \mathrm{mmol})$ and chloroform $(10 \mathrm{~mL})$ was added via cannula. The solution was stirred at $0{ }^{\circ} \mathrm{C}$ for $30 \mathrm{~min}$ and then at reflux for $12 \mathrm{~h}$. The chloroform was removed in vacuo and $20 \% \mathrm{NaOH}(50 \mathrm{~mL})$ was added to the crude product. The aqueous was extracted with dichloromethane $(2 \times 50 \mathrm{~mL})$, and the combined organics were dried over $\mathrm{Na}_{2} \mathrm{SO}_{4}$, filtered, and the solvent was removed in vacuo. The residue was purified by flash column chromatography (petroleum ether/EtOAc $=10: 1-1: 1)$ to give product $(1.01 \mathrm{~g}, 25 \%)$ as yellow solid. ${ }^{1} \mathrm{H}$ NMR (400 $\left.\mathrm{MHz}, \mathrm{CDCl}_{3}\right) \delta 7.55(\mathrm{t}, J=7.7 \mathrm{~Hz}, 1 \mathrm{H}), 7.47(\mathrm{~d}, J=7.6 \mathrm{~Hz}, 1 \mathrm{H}), 7.09(\mathrm{dt}, J=7.8,4.2$ $\mathrm{Hz}, 3 \mathrm{H}), 6.90$ (t, $J=7.4 \mathrm{~Hz}, 1 \mathrm{H}), 6.67(\mathrm{~d}, J=7.7 \mathrm{~Hz}, 2 \mathrm{H}), 4.15-4.01(\mathrm{~m}, 2 \mathrm{H}), 3.76(\mathrm{t}$, $J=7.2 \mathrm{~Hz}, 1 \mathrm{H}), 2.38(\mathrm{~s}, 3 \mathrm{H}), 0.99(\mathrm{~s}, 9 \mathrm{H}) .{ }^{13} \mathrm{C} \mathrm{NMR}\left(101 \mathrm{MHz}, \mathrm{CDCl}_{3}\right) \delta 160.4,158.2$, $150.3,142.9,136.7,128.4,123.9,122.7,121.7,121.2,74.3,54.7,34.2,26.1,24.4$. HRMS-EI (m/z): Calc. for $\mathrm{C}_{19} \mathrm{H}_{23} \mathrm{~N}_{3}[\mathrm{M}]^{+} 293.1892$, found 293.1891.

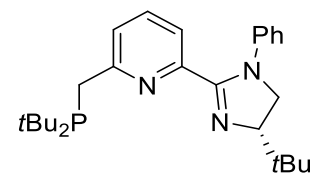

\section{Preparation of (S)-2-(4-(tert-butyl)-1-phenyl-4,5-dihydro-1H-imidazol-2-yl)-6-} ((di-tert-butylphosphaneyl)methyl)pyridine (5). An oven-dried $100 \mathrm{~mL}$ Schlenk flask was charged with (S)-2-(4-(tert-butyl)-1-phenyl-4,5-dihydro-1H-imidazol-2-yl)6-methylpyridine (4) (805 $\mathrm{mg}, 2.75 \mathrm{mmol}$ ) and $30 \mathrm{~mL}$ dry THF. Then the solution was cooled to $-78^{\circ} \mathrm{C}$ and a solution of newly prepared lithium diisopropylamide (LDA, 1.0 M, $3.3 \mathrm{~mL}, 3.3 \mathrm{mmol}$ ) was added dropwise via syringe over $10 \mathrm{~min}$. The resulting brown colored mixture was stirred for $1 \mathrm{~h}$ at $-78{ }^{\circ} \mathrm{C}$ and then a solution of ditertbutylchlorophosphine (584 mg, $3.3 \mathrm{mmol})$ in THF $(10 \mathrm{~mL})$ was added dropwise via syringe over $10 \mathrm{~min}$. The solution was stirred for $1 \mathrm{~h}$, then slowly warm up to room 
temperature and stirred overnight. Then the reaction was quenched by adding degassed water $(20 \mathrm{~mL})$ slowly into the mixture under argon atmosphere. The aqueous phase was extracted with $n$-hexane $(3 \times 20 \mathrm{~mL})$. The combined organic solution was dried over anhydrous $\mathrm{Na}_{2} \mathrm{SO}_{4}$, filtered under argon atmosphere, and the solvent was removed under vacuum. Then the crude solid dissolved in THF and recrystallized at $-27^{\circ} \mathrm{C}$ to give product as white solid (578 mg, 48\%). ${ }^{1} \mathrm{H} \mathrm{NMR}\left(400 \mathrm{MHz}, \mathrm{CDCl}_{3}\right) \delta 7.84(\mathrm{~d}, J=$ $7.5 \mathrm{~Hz}, 1 \mathrm{H}), 7.43(\mathrm{~d}, J=7.9 \mathrm{~Hz}, 1 \mathrm{H}), 7.22(\mathrm{t}, J=7.7 \mathrm{~Hz}, 1 \mathrm{H}), 7.10(\mathrm{t}, J=7.7 \mathrm{~Hz}, 2 \mathrm{H})$, $6.92(\mathrm{t}, J=7.2 \mathrm{~Hz}, 1 \mathrm{H}), 6.84(\mathrm{~d}, J=7.9 \mathrm{~Hz}, 2 \mathrm{H}), 4.04(\mathrm{t}, J=9.7 \mathrm{~Hz}, 1 \mathrm{H}), 3.76(\mathrm{t}, J=$ $10.0 \mathrm{~Hz}, 1 \mathrm{H}), 3.65$ (t, $J=9.1 \mathrm{~Hz}, 1 \mathrm{H}), 2.85$ (d, $J=2.2 \mathrm{~Hz}, 2 \mathrm{H}), 1.11(\mathrm{~s}, 9 \mathrm{H}), 1.06$ (d, $J$ $=5.9 \mathrm{~Hz}, 9 \mathrm{H}), 1.03(\mathrm{~d}, J=5.9 \mathrm{~Hz}, 9 \mathrm{H}) .{ }^{13} \mathrm{C} \mathrm{NMR}\left(101 \mathrm{MHz}, \mathrm{CDCl}_{3}\right) \delta 162.3(\mathrm{~d}, J=$ $15.8 \mathrm{~Hz}), 160.9,151.5,145.0,136.6,129.1,124.8$ (d, $J=10.9 \mathrm{~Hz}), 123.0,122.8,121.7$ $(\mathrm{d}, J=1.6 \mathrm{~Hz}), 75.0,55.8,34.7,32.3(\mathrm{~d}, J=25.5 \mathrm{~Hz}), \delta 32.1(\mathrm{~d}, J=23.3 \mathrm{~Hz}), 30.1(\mathrm{~d}$, $J=13.9 \mathrm{~Hz}), 26.6 .{ }^{31} \mathrm{P}$ NMR $\left(162 \mathrm{MHz}, \mathrm{CDCl}_{3}\right) \delta 34.4$. HRMS-EI $(\mathrm{m} / z)$ : Calc. For $\mathrm{C}_{27} \mathrm{H}_{40} \mathrm{~N}_{3} \mathrm{P}[\mathrm{M}]^{+}$437.2960, found 437.2966.

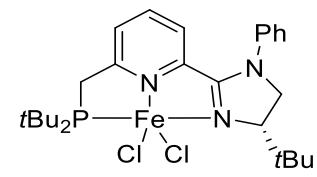

Iron complex $\left(\mathrm{PNN}_{\mathrm{imid}}\right) \mathrm{FeCl}_{2}$ : In a nitrogen filled glove-box, $\mathrm{FeCl}_{2} \cdot 4 \mathrm{H}_{2} \mathrm{O}(128 \mathrm{mg}, 0.64$ mmol, 1 equiv.) was added to THF $(30 \mathrm{~mL})$ and the resulting mixture was stirred vigorously overnight. In another vial, PPI ligand (5) (297 mg, $0.68 \mathrm{mmol}, 1.05$ equiv.) was dissolved in THF (10 mL). The PPI ligand solution was added to the $\mathrm{FeCl}_{2} \cdot 4 \mathrm{H}_{2} \mathrm{O}$ solution slowly. The reaction mixture was stirred at room temperature for $8 \mathrm{~h}$. The solvent was removed under vacuum and the precipitate was washed with pentane. $[\mathrm{Fe}] \mathrm{Cl}_{2}$ was collected by filtration and dried under vacuum as gray solid (331 $\left.\mathrm{mg}, 91 \%\right)$. ${ }^{1} \mathrm{H}$ NMR (400 MHz, $\left.\mathrm{CDCl}_{3}\right) \delta 75.98$ (s, 1H), 64.48 (s, 1H), 44.99 (s, 1H), 34.61 (s, 1H), 17.38 (s, 2H), $14.92(\mathrm{~s}, 2 \mathrm{H}), 9.81-8.09$ (m, 11H), 7.50 (s, 9H), 3.82 (s, 1H), 1.93 (s, $1 \mathrm{H}), 1.33(\mathrm{~s}, 1 \mathrm{H}),-12.21$ (s, 9H). Anal. Calcd. $\left(\mathrm{C}_{27} \mathrm{H}_{40} \mathrm{Cl}_{2} \mathrm{FeN}_{3} \mathrm{P}\right): \mathrm{C}, 57.46 ; \mathrm{H}, 7.14 ; \mathrm{N}$, 7.45. Found: C, 57.04; H, 6.87; N, 7.25. 
Table S1. Selected bond length and angles for complex $\left(\mathrm{PNN}_{\text {oxa }}\right) \mathrm{FeCl}_{2}$ and $\left(\mathrm{PNN}_{\text {imid }}\right) \mathrm{FeCl}_{2}$
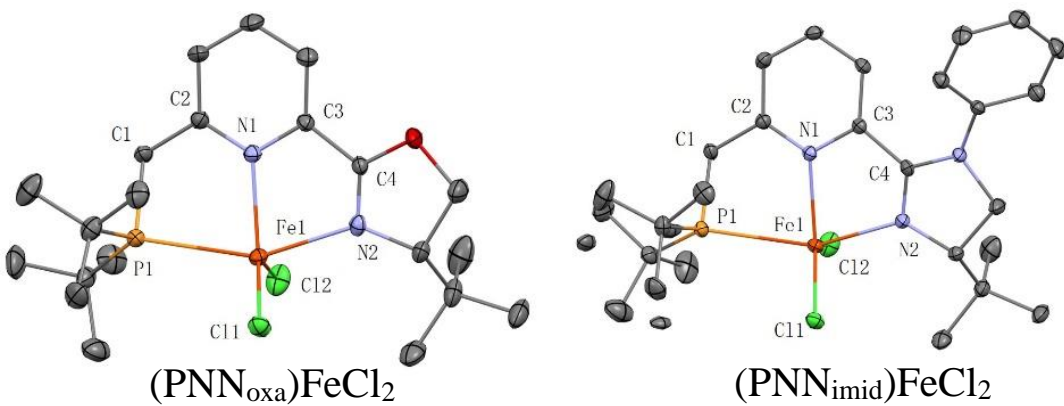

$\left(\mathrm{PNN}_{\text {imid }}\right) \mathrm{FeCl}_{2}$

\begin{tabular}{ccc}
\hline & $\left(\mathrm{PNN}_{\text {oxa }}\right) \mathrm{FeCl}_{2}$ & $\left(\mathrm{PNN}_{\text {imid }}\right) \mathrm{FeCl}_{2}$ \\
\hline Selected Bond Lengths & & \\
$\mathrm{Fe}(1)-\mathrm{Cl}(1)$ & $2.2962(17)$ & Distance $(\AA)$ \\
$\mathrm{Fe}(1)-\mathrm{Cl}(2)$ & $2.3087(15)$ & $2.3222(8)$ \\
$\mathrm{Fe}(1)-\mathrm{P}(1)$ & $2.6040(16)$ & $2.6438(10)$ \\
$\mathrm{Fe}(1)-\mathrm{N}(1)$ & $2.159(4)$ & $2.152(2)$ \\
$\mathrm{Fe}(1)-\mathrm{N}(2)$ & $2.246(5)$ & $2.153(3)$ \\
$\mathrm{C}(1)-\mathrm{C}(2)$ & $1.494(7)$ & $1.505(4)$ \\
$\mathrm{C}(3)-\mathrm{C}(4)$ & $1.471(8)$ & $1.374(8)$ \\
$\mathrm{Selected} \mathrm{Bond} \mathrm{Angles}$ & & $78.81(7)$ \\
$\mathrm{N}(1)-\mathrm{Fe}(1)-\mathrm{P}(1)$ & $78.11(13)$ & $75.72(9)$ \\
$\mathrm{N}(1)-\mathrm{Fe}(1)-\mathrm{N}(2)$ & $75.74(17)$ & $119.1(3)$ \\
$\mathrm{N}(1)-\mathrm{C}(2)-\mathrm{C}(1)$ & $118.8(5)$ & $112.6(4)$ \\
$\mathrm{N}(1)-\mathrm{C}(3)-\mathrm{C}(4)$ & $113.6(4)$ & $152.80(8)$ \\
$\mathrm{N}(2)-\mathrm{Fe}(1)-\mathrm{P}(1)$ & $152.48(13)$ & $75.74(17)$ \\
$\mathrm{N}(1)-\mathrm{Fe}(1)-\mathrm{N}(2)$ & $75.72(9)$ &
\end{tabular}

\section{Synthesis of $\alpha$-vinyl Boronates}

\section{General Procedure A:}

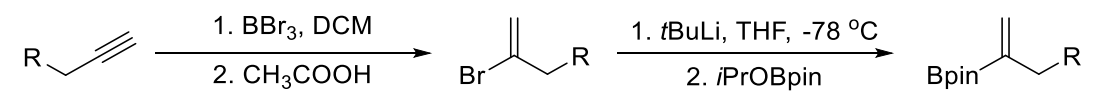

The $\alpha$-vinyl boronates were prepared according to the literature procedure. ${ }^{5}$ An oven-dried Schlenk flask was charged with a magnetic bar then sealed, evacuated and refilled with argon. $\mathrm{BBr}_{3}(0.5$ equiv.) was added to the flask through syringe with $\mathrm{CH}_{2} \mathrm{Cl}_{2}$ (2 M). After cooling to $-78{ }^{\circ} \mathrm{C}$, alkyne (1 equiv.) was added dropwise to the 
solution; then the mixture slowly warm up to room temperature and stirred for $3 \mathrm{~h}$. Acetic acid (6 equiv.) was added to the mixture slowly then stirred for $1 \mathrm{~h}$. This reaction was quenched with saturated sodium bicarbonate and extracted with $\mathrm{CH}_{2} \mathrm{Cl}_{2}$. The combined organics were dried over $\mathrm{Na}_{2} \mathrm{SO}_{4}$, filtered, and the solvent was removed in vacuo. The residue was purified by flash column chromatography with petroleum ether to provide the corresponding vinyl bromide. Next, the vinyl bromide was dissolved in anhydrous THF $(0.75 \mathrm{M})$, which was then added to an oven-dried Schlenk flask in argon atmosphere. After cooling to $-78{ }^{\circ} \mathrm{C}, t \mathrm{BuLi}$ (2 equiv., $1.3 \mathrm{M}$ in pentane) was added dropwise and the mixture stirred for $30 \mathrm{~min}$. 2-Isopropoxy-4,4,5,5-tetramethyl$[1,3,2]$ dioxaborolane (1.5 equiv.) was added dropwise via syringe and the mixture keep at $-78{ }^{\circ} \mathrm{C}$ for $15 \mathrm{~min}$ and then allowed to warm to room temperature over $3 \mathrm{~h}$. The reaction was quenched by water and extracted with $\mathrm{CH}_{2} \mathrm{Cl}_{2}$. The combined organics was dried over $\mathrm{Na}_{2} \mathrm{SO}_{4}$, filtered, and the solvent was removed in vacuo. The residue was purified by flash column chromatography (petroleum ether/ EtOAc $=100: 1-10: 1$ ) provided the $\alpha$-vinyl boronates.

\section{General Procedure B:}

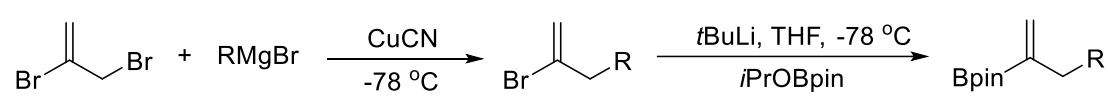

The $\alpha$-vinyl bromides were prepared according to the literature procedure ${ }^{6}$ and the $\alpha$ vinyl boronates were prepared according to the literature procedure. ${ }^{5}$ An oven-dried $100 \mathrm{~mL}$ Schlenk flask was charged with $\mathrm{CuCN}$ (0.1 equiv.) and anhydrous THF (1 M), which was added a solution of newly prepared alkylmagnesium bromide (1 equiv, $1 \mathrm{M}$ in THF) at $-78{ }^{\circ} \mathrm{C}$. The suspension was stirred at $-78{ }^{\circ} \mathrm{C}$ for $15 \mathrm{~min}$ and $2,3-$ dibromopropene (1 equiv.) was added dropwise over $15 \mathrm{~min}$. The mixture was stirred at the same temperature for $2 \mathrm{~h}$. The reaction was quenched by saturated ammonium chloride and extracted with EtOAc. The combined organics were dried over $\mathrm{Na}_{2} \mathrm{SO}_{4}$, filtered, and the solvent was removed in vacuo. The residue was purified by flash column chromatography with petroleum ether to provide the corresponding vinyl bromide. The vinyl bromide was added to an oven-dried Schlenk flask under argon and dissolved in anhydrous THF $(0.75 \mathrm{M})$. After cooling to $-78^{\circ} \mathrm{C}, t \mathrm{BuLi}$ (2 qeuiv., $1.3 \mathrm{M}$ 
in pentane) was added dropwise and the mixture was stirred for $30 \mathrm{~min}$. 2-Isopropoxy4,4,5,5-tetramethyl-[1,3,2] dioxaborolane (1.5 equiv.) was added dropwise via syringe and the mixture keep at $-78{ }^{\circ} \mathrm{C}$ for 15 min then allowed to warm to room temperature over $3 \mathrm{~h}$. The reaction was quenched by water and extracted with $\mathrm{CH}_{2} \mathrm{Cl}_{2}$. The combined organics were dried over $\mathrm{Na}_{2} \mathrm{SO}_{4}$, filtered, and the solvent was removed in vacuo. The residue was purified by flash column chromatography (petroleum ether/ EtOAc $=100: 1)$ to provide the $\alpha$-vinyl boronates.

\section{General Procedure C:}

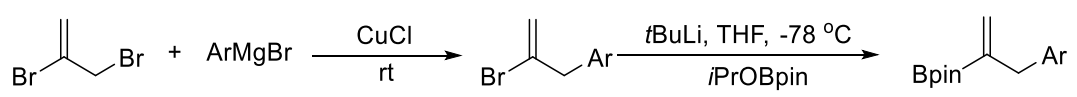

The $\alpha$-vinyl bromides were prepared according to the literature procedure ${ }^{7}$ and the $\alpha$ vinyl boronates were prepared according to the literature procedure. ${ }^{5}$ An oven-dried $100 \mathrm{~mL}$ Schlenk flask charged with magnetic stir bar, $\mathrm{CuCl}(0.1$ equiv.) and 2,3dibromopropene (1 equiv.) and anhydrous THF (1 M) under argon atmosphere. The solution of newly prepared arylmagnesium bromide (1 equiv., $1 \mathrm{M}$ in THF) was added dropwise through syringe at room temperature over $15 \mathrm{~min}$. The suspension was stirred at the same temperature for $4 \mathrm{~h}$. The reaction was quenched by saturated ammonium chloride and extracted with EtOAc. The combined organics were dried over $\mathrm{Na}_{2} \mathrm{SO}_{4}$, filtered, and the solvent was removed in vacuo. The residue was purified by flash column chromatography with petroleum ether to provide the corresponding vinyl bromide. The vinyl bromide was added to an oven-dried Schlenk flask and dissolved in anhydrous THF $(0.75 \mathrm{M})$ under argon. After cooling to $-78^{\circ} \mathrm{C}, t \mathrm{BuLi}(2$ qeuiv., $1.3 \mathrm{M}$ in pentane) was added dropwise and the mixture was stirred for $30 \mathrm{~min}$. 2-Isopropoxy4,4,5,5-tetramethyl-[1,3,2]dioxaborolane (1.5 equiv.) was added dropwise via syringe and the mixture keep at $-78{ }^{\circ} \mathrm{C}$ for 15 min then allowed to warm to room temperature over $3 \mathrm{~h}$. The reaction was quenched by water and extracted with $\mathrm{CH}_{2} \mathrm{Cl}_{2}$. The combined organics were dried over $\mathrm{Na}_{2} \mathrm{SO}_{4}$, filtered, and the solvent was removed in vacuo. The residue was purified by flash column chromatography (petroleum ether/ EtOAc $=100: 1)$ to provide the $\alpha$-vinyl boronates.

In order to achieve high conversion and stereoselectivity in the isomerization reaction, 
it is important to purify the starting materials, 1,1-disubstituted alkenyl boronates, thoroughly. For some substrates, a purification procedure involving distillation following column chromatography is required.

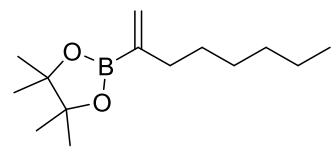

\section{4,4,5,5-Tetramethyl-2-(oct-1-en-2-yl)-1,3,2-dioxaborolane (1a).}

Prepared according to procedure A. Colorless oil, $76 \%$ yield over two steps. ${ }^{1} \mathrm{H}$ NMR $\left(400 \mathrm{MHz}, \mathrm{CDCl}_{3}\right) \delta 5.75(\mathrm{~d}, J=3.5 \mathrm{~Hz}, 1 \mathrm{H}), 5.58(\mathrm{~s}, 1 \mathrm{H}), 2.14(\mathrm{t}, J=7.5 \mathrm{~Hz}, 2 \mathrm{H})$, $1.45-1.36(\mathrm{~m}, 2 \mathrm{H}), 1.34-1.21(\mathrm{~m}, 18 \mathrm{H}), 0.88(\mathrm{t}, J=6.7 \mathrm{~Hz}, 3 \mathrm{H})$. These spectroscopic data correspond to reported data. ${ }^{5}$

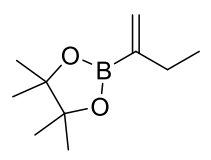

\section{2-(But-1-en-2-yl)-4,4,5,5-tetramethyl-1,3,2-dioxaborolane (1b).}

Prepared according to procedure A. Colorless oil, $29 \%$ yield over two steps. ${ }^{1} \mathrm{H}$ NMR $\left(400 \mathrm{MHz}, \mathrm{CDCl}_{3}\right) \delta 5.74(\mathrm{~d}, J=3.0 \mathrm{~Hz}, 1 \mathrm{H}), 5.60(\mathrm{~s}, 1 \mathrm{H}), 2.16(\mathrm{q}, J=7.4 \mathrm{~Hz}, 2 \mathrm{H})$, $1.27(\mathrm{~s}, 12 \mathrm{H}), 1.01(\mathrm{t}, J=7.4 \mathrm{~Hz}, 3 \mathrm{H})$. These spectroscopic data correspond to reported data. $^{8}$

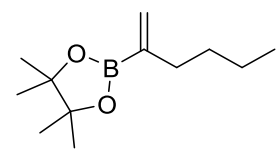

2-(Hex-1-en-2-yl)-4,4,5,5-tetramethyl-1,3,2-dioxaborolane (1c).

Prepared according to procedure A. Colorless oil, $23 \%$ yield over two steps. ${ }^{1} \mathrm{H}$ NMR $\left(400 \mathrm{MHz}, \mathrm{CDCl}_{3}\right) \delta 5.73(\mathrm{~d}, J=3.6 \mathrm{~Hz}, 1 \mathrm{H}), 5.56(\mathrm{~s}, 1 \mathrm{H}), 2.12(\mathrm{t}, J=7.2 \mathrm{~Hz}, 2 \mathrm{H})$, $1.46-1.33(\mathrm{~m}, 2 \mathrm{H}), 1.32-1.27(\mathrm{~m}, 2 \mathrm{H}), 1.24(\mathrm{~s}, 12 \mathrm{H}), 0.87(\mathrm{t}, J=7.1 \mathrm{~Hz}, 3 \mathrm{H})$. These spectroscopic data correspond to reported data. ${ }^{9}$

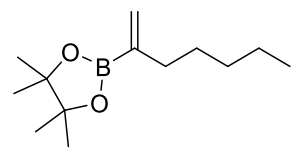

2-(Hept-1-en-2-yl)-4,4,5,5-tetramethyl-1,3,2-dioxaborolane (1d).

Prepared according to procedure A. Colorless oil, $21 \%$ yield over two steps. ${ }^{1}$ H NMR (400 MHz, $\left.\mathrm{CDCl}_{3}\right) \delta 5.74(\mathrm{~s}, 1 \mathrm{H}), 5.58(\mathrm{~s}, 1 \mathrm{H}), 2.13(\mathrm{t}, J=7.5 \mathrm{~Hz}$, 
2H), $1.46-1.36(\mathrm{~m}, 2 \mathrm{H}), 1.27-1.24(\mathrm{~m}, 16 \mathrm{H}), 0.87(\mathrm{t}, J=6.7 \mathrm{~Hz}, 3 \mathrm{H}) . \mathrm{Th}$ ese spectroscopic data correspond to reported data ${ }^{10}$.

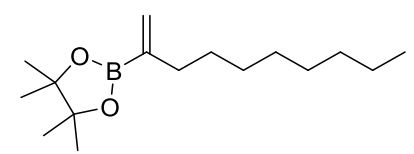

\section{2-(Dec-1-en-2-yl)-4,4,5,5-tetramethyl-1,3,2-dioxaborolane (1e).}

Prepared according to procedure A. Colorless oil, $47 \%$ yield over two steps. ${ }^{1} \mathrm{H}$ NMR $\left(400 \mathrm{MHz}, \mathrm{CDCl}_{3}\right) \delta 5.74(\mathrm{~d}, J=3.5 \mathrm{~Hz}, 1 \mathrm{H}), 5.58(\mathrm{~s}, 1 \mathrm{H}), 2.13(\mathrm{t}, J=7.3 \mathrm{~Hz}, 2 \mathrm{H})$, $1.47-1.35(\mathrm{~m}, 2 \mathrm{H}), 1.34-1.13(\mathrm{~m}, 22 \mathrm{H}), 0.87(\mathrm{t}, J=5.8 \mathrm{~Hz}, 3 \mathrm{H}) .{ }^{13} \mathrm{C}$ NMR $(101$ $\left.\mathrm{MHz}, \mathrm{CDCl}_{3}\right) \delta 128.8,83.4,35.5,32.1,29.7,29.4,29.44,24.9,22.8,14.3$. Carbon attached to boron not observed due to quadrupolar relaxation, two ${ }^{13} \mathrm{C}$ peaks overlap in this NMR instrument. ${ }^{11} \mathrm{~B} \mathrm{NMR}\left(128 \mathrm{MHz}, \mathrm{CDCl}_{3}\right) \delta 30.4$. Calc. for $\mathrm{C}_{16} \mathrm{H}_{31}{ }^{10} \mathrm{BO}_{2}[\mathrm{M}]^{+}$ 265.2450, found 265.2449.

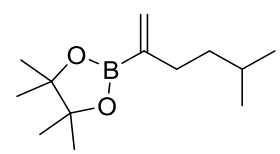

\section{4,4,5,5-tetramethyl-2-(5-methylhex-1-en-2-yl)-1,3,2-dioxaborolane (1f).}

Prepared according to procedure A. Colorless oil, $28 \%$ yield over two steps. ${ }^{1} \mathrm{H}$ NMR $\left(400 \mathrm{MHz}, \mathrm{CDCl}_{3}\right) \delta 5.73(\mathrm{~d}, J=3.2 \mathrm{~Hz}, 1 \mathrm{H}), 5.58(\mathrm{~s}, 1 \mathrm{H}), 2.14(\mathrm{t}, J=8.0 \mathrm{~Hz}, 2 \mathrm{H})$, $1.59-1.46(\mathrm{~m}, 1 \mathrm{H}), 1.27-1.24(\mathrm{~m}, 14 \mathrm{H}), 0.87(\mathrm{~d}, J=6.6 \mathrm{~Hz}, 6 \mathrm{H}) .{ }^{13} \mathrm{C} \mathrm{NMR}(101 \mathrm{MHz}$, $\left.\mathrm{CDCl}_{3}\right) \delta 128.5,83.4,38.5,33.2,27.7,24.9,22.8$. Carbon attached to boron not observed due to quadrupolar relaxation. ${ }^{11} \mathrm{~B} \mathrm{NMR}\left(128 \mathrm{MHz}, \mathrm{CDCl}_{3}\right) \delta 30.2$. These spectroscopic data correspond to reported data. ${ }^{11}$

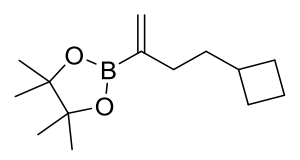

\section{2-(4-Cyclobutylbut-1-en-2-yl)-4,4,5,5-tetramethyl-1,3,2-dioxaborolane (1g).}

Prepared according to procedure B. Colorless oil, 39\% yield over two steps. ${ }^{1} \mathrm{H}$ NMR $\left(400 \mathrm{MHz}, \mathrm{CDCl}_{3}\right) \delta 5.74(\mathrm{~d}, J=3.3 \mathrm{~Hz}, 1 \mathrm{H}), 5.57(\mathrm{~s}, 1 \mathrm{H}), 2.32-2.18(\mathrm{~m}, 1 \mathrm{H}), 2.08-$ $2.00(\mathrm{~m}, 4 \mathrm{H}), 1.87-1.73(\mathrm{~m}, 2 \mathrm{H}), 1.64-1.44(\mathrm{~m}, 4 \mathrm{H}), 1.27(\mathrm{~s}, 12 \mathrm{H}) .{ }^{13} \mathrm{C}$ NMR $(101$ $\left.\mathrm{MHz}, \mathrm{CDCl}_{3}\right) \delta 128.6,83.3,36.7,35.9,33.0,28.5,24.8,18.6$. Carbon attached to boron 
not observed due to quadrupolar relaxation. ${ }^{11} \mathrm{~B}$ NMR $\left(128 \mathrm{MHz}, \mathrm{CDCl}_{3}\right) \delta$ 26.6. HRMS-EI $(m / z)$ : Calc. for $\mathrm{C}_{14} \mathrm{H}_{25}{ }^{10} \mathrm{BO}_{2}[\mathrm{M}]^{+} 235.1978$, found 235.1985.

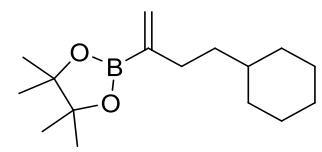

\section{2-(5-Cyclohexylpent-1-en-2-yl)-4,4,5,5-tetramethyl-1,3,2-dioxaborolane (1h).}

Prepared according to procedure A. Colorless oil, $49 \%$ yield over two steps. ${ }^{1} \mathrm{H}$ NMR $\left(400 \mathrm{MHz}, \mathrm{CDCl}_{3}\right) \delta 5.71(\mathrm{~d}, J=3.2 \mathrm{~Hz}, 1 \mathrm{H}), 5.55(\mathrm{~s}, 1 \mathrm{H}), 2.19-2.02(\mathrm{~m}, 2 \mathrm{H}), 1.76-$ $1.53(\mathrm{~m}, 4 \mathrm{H}), 1.34-1.08(\mathrm{~m}, 19 \mathrm{H}), 0.84(\mathrm{q}, J=10.9,10.1 \mathrm{~Hz}, 2 \mathrm{H}) .{ }^{13} \mathrm{C}$ NMR $(101$ $\left.\mathrm{MHz}, \mathrm{CDCl}_{3}\right) \delta 128.5,83.3,37.3,37.0,33.5,32.6,26.8,26.5,24.8$. Carbon attached to boron not observed due to quadrupolar relaxation. ${ }^{11} \mathrm{~B} \mathrm{NMR}\left(128 \mathrm{MHz}, \mathrm{CDCl}_{3}\right) \delta 30.1$. HRMS-EI (m/z): Calc. for $\mathrm{C}_{16} \mathrm{H}_{29}{ }^{10} \mathrm{BO}_{2}[\mathrm{M}]^{+}$263.2291, found 263.2292.

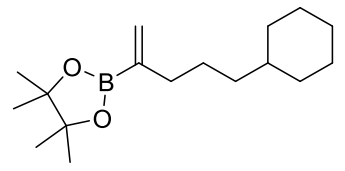

2-(5-Cyclohexylpent-1-en-2-yl)-4,4,5,5-tetramethyl-1,3,2-dioxaborolane (1i).

Prepared according to procedure B. Colorless oil, $49 \%$ yield over two steps. ${ }^{1} \mathrm{H}$ NMR $\left(400 \mathrm{MHz}, \mathrm{CDCl}_{3}\right) \delta 5.72(\mathrm{~d}, J=3.3 \mathrm{~Hz}, 1 \mathrm{H}), 5.55(\mathrm{~s}, 1 \mathrm{H}), 2.09(\mathrm{t}, J=7.5 \mathrm{~Hz}, 2 \mathrm{H})$, $1.73-1.56(\mathrm{~m}, 4 \mathrm{H}), 1.45-1.33(\mathrm{~m}, 2 \mathrm{H}), 1.28-1.07(\mathrm{~m}, 19 \mathrm{H}), 0.89-0.75(\mathrm{~m}, 2 \mathrm{H})$. ${ }^{13} \mathrm{C} \mathrm{NMR}\left(101 \mathrm{MHz}, \mathrm{CDCl}_{3}\right) \delta 128.8,83.3,37.7,37.2,35.7,33.5,26.9,26.6,26.5,24.8$. Carbon attached to boron not observed due to quadrupolar relaxation. ${ }^{11} \mathrm{~B}$ NMR (128 $\left.\mathrm{MHz}, \mathrm{CDCl}_{3}\right) \delta$ 29.8. HRMS-EI $(\mathrm{m} / \mathrm{z})$ : Calc. for $\mathrm{C}_{17} \mathrm{H}_{31}{ }^{10} \mathrm{BO}_{2}[\mathrm{M}]^{+}$277.2448, found 277.2437.

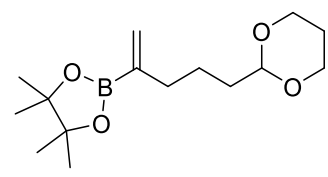

2-(5-(1,3-Dioxan-2-yl)pent-1-en-2-yl)-4,4,5,5-tetramethyl-1,3,2-dioxaborolane (1j). Prepared according to procedure B. Colorless oil, $31 \%$ yield over two steps. ${ }^{1} \mathrm{H}$ NMR $\left(400 \mathrm{MHz}, \mathrm{CDCl}_{3}\right) \delta 5.77(\mathrm{~d}, J=3.4 \mathrm{~Hz}, 1 \mathrm{H}), 5.60(\mathrm{~s}, 1 \mathrm{H}), 4.51(\mathrm{t}, J=5.0 \mathrm{~Hz}, 1 \mathrm{H})$, $4.13-4.05$ (m, 2H), $3.80-3.70(\mathrm{~m}, 2 \mathrm{H}), 2.14(\mathrm{t}, J=7.4 \mathrm{~Hz}, 2 \mathrm{H}), 2.11-1.99(\mathrm{~m}, 1 \mathrm{H})$, $1.63-1.46(\mathrm{~m}, 4 \mathrm{H}), 1.36-1.29(\mathrm{~m}, 1 \mathrm{H}), 1.26(\mathrm{~d}, J=1.0 \mathrm{~Hz}, 12 \mathrm{H}) .{ }^{13} \mathrm{C}$ NMR $(101$ 
$\left.\mathrm{MHz}, \mathrm{CDCl}_{3}\right) \delta 129.1,102.3,83.2,66.8,53.4,34.8,25.9,24.7,23.4$. Carbon attached to boron not observed due to quadrupolar relaxation. ${ }^{11} \mathrm{~B} \mathrm{NMR}\left(128 \mathrm{MHz}, \mathrm{C}_{6} \mathrm{D}_{6}\right) \delta 26.2$. HRMS-EI (m/z): Calc. for $\mathrm{C}_{15} \mathrm{H}_{26}{ }^{10} \mathrm{BO}_{4}[\mathrm{M}-\mathrm{H}]^{+} 280.1955$, found 280.1952.

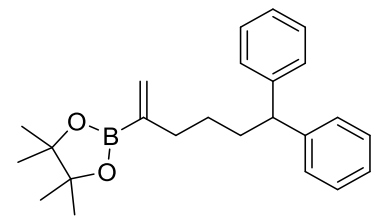

2-(6,6-Diphenylhex-1-en-2-yl)-4,4,5,5-tetramethyl-1,3,2-dioxaborolane (1k).

Prepared according to procedure B. Colorless oil, $37 \%$ yield over two steps. ${ }^{1} \mathrm{H}$ NMR $\left(400 \mathrm{MHz}, \mathrm{CDCl}_{3}\right) \delta 7.32-7.22(\mathrm{~m}, 8 \mathrm{H}), 7.21-7.11(\mathrm{~m}, 2 \mathrm{H}), 5.77(\mathrm{~s}, 1 \mathrm{H}), 5.59(\mathrm{~s}$, 1H), $3.92(\mathrm{t}, J=7.8 \mathrm{~Hz}, 1 \mathrm{H}), 2.22(\mathrm{t}, J=6.8 \mathrm{~Hz}, 2 \mathrm{H}), 2.12-2.01(\mathrm{~m}, 2 \mathrm{H}), 1.23(\mathrm{~s}$, 12H), $0.95-0.85(\mathrm{~m}, 2 \mathrm{H}) .{ }^{13} \mathrm{C} \mathrm{NMR}\left(101 \mathrm{MHz}, \mathrm{CDCl}_{3}\right) \delta 144.5,128.2,127.4,127.0$, $125.0,82.4,50.4,34.5,34.4,26.7,23.8$. Carbon attached to boron not observed due to quadrupolar relaxation. ${ }^{11} \mathrm{~B}$ NMR $\left(128 \mathrm{MHz}, \mathrm{CDCl}_{3}\right) \delta 30.1 . \mathrm{HRMS}-\mathrm{EI}(\mathrm{m} / \mathrm{z})$ : Calc. for $\mathrm{C}_{24} \mathrm{H}_{31}{ }^{10} \mathrm{BO}_{2}[\mathrm{M}]^{+}$361.2448, found 361.2461.<smiles>C=C(CC[Si](C)(C)C)OC1(C)OC(C)(C)C1(C)C</smiles>

Trimethyl(3-(4,4,5,5-tetramethyl-1,3,2-dioxaborolan-2-yl)but-3-en-1-yl)silane (11).

Prepared according to procedure B. Colorless oil, $24 \%$ yield over two steps. ${ }^{1} \mathrm{H}$ NMR $\left(400 \mathrm{MHz}, \mathrm{CDCl}_{3}\right) \delta 5.71(\mathrm{~d}, J=3.0 \mathrm{~Hz}, 1 \mathrm{H}), 5.60(\mathrm{~s}, 1 \mathrm{H}), 2.13(\mathrm{~d}, J=8.7 \mathrm{~Hz}, 2 \mathrm{H})$, $1.26(\mathrm{~s}, 12 \mathrm{H}), 0.66-0.58(\mathrm{~m}, 2 \mathrm{H}),-0.01(\mathrm{~s}, 9 \mathrm{H}) .{ }^{13} \mathrm{C} \mathrm{NMR}\left(101 \mathrm{MHz}, \mathrm{CDCl}_{3}\right) \delta 127.1$, $83.4,29.4,24.9,15.9,-1.4$. Carbon attached to boron not observed due to quadrupolar relaxation. ${ }^{11} \mathrm{~B}$ NMR $\left(128 \mathrm{MHz}, \mathrm{CDCl}_{3}\right) \delta$ 30.3. HRMS-EI $(\mathrm{m} / \mathrm{z})$ : Calc. for $\mathrm{C}_{13} \mathrm{H}_{27}{ }^{10} \mathrm{BO}_{2} \mathrm{Si}[\mathrm{M}]^{+} 253.1904$, found 253.1896.

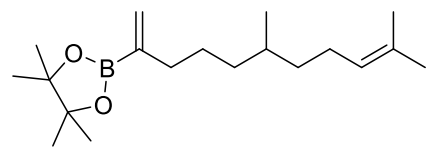

2-(6,10-Dimethylundeca-1,9-dien-2-yl)-4,4,5,5-tetramethyl-1,3,2-dioxaborolane (1m). 
Prepared according to procedure B. Colorless oil, $11 \%$ yield over two steps. ${ }^{1} \mathrm{H}$ NMR $\left(400 \mathrm{MHz}, \mathrm{CDCl}_{3}\right) \delta 5.75(\mathrm{~d}, J=3.4 \mathrm{~Hz}, 1 \mathrm{H}), 5.59(\mathrm{~s}, 1 \mathrm{H}), 5.09(\mathrm{t}, J=7.0 \mathrm{~Hz}, 1 \mathrm{H})$, $2.18-2.05(\mathrm{~m}, 2 \mathrm{H}), 2.02-1.86(\mathrm{~m}, 2 \mathrm{H}), 1.67(\mathrm{~s}, 3 \mathrm{H}), 1.60(\mathrm{~s}, 3 \mathrm{H}), 1.48-1.35(\mathrm{~m}$, $3 \mathrm{H}), 1.34-1.29(\mathrm{~m}, 2 \mathrm{H}), 1.26(\mathrm{~s}, 12 \mathrm{H}), 1.16-1.04(\mathrm{~m}, 2 \mathrm{H}), 0.85$ (d, J = 6.5 Hz, 3H). ${ }^{13} \mathrm{C} \mathrm{NMR}\left(101 \mathrm{MHz}, \mathrm{CDCl}_{3}\right) \delta 131.0,128.9,125.3,83.4,37.3,36.7,35.8,32.5,26.7$, 25.9, 25.7, 24.9, 19.7, 17.8. Carbon attached to boron not observed due to quadrupolar relaxation. ${ }^{11} \mathrm{~B}$ NMR $\left(128 \mathrm{MHz}, \mathrm{CDCl}_{3}\right) \delta$ 30.4. HRMS-EI $(\mathrm{m} / \mathrm{z})$ : Calc. for $\mathrm{C}_{19} \mathrm{H}_{35}{ }^{10} \mathrm{BO}_{2}[\mathrm{M}]^{+}$305.2761, found 305.2757.

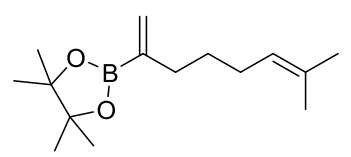

4,4,5,5-Tetramethyl-2-(7-methylocta-1,6-dien-2-yl)-1,3,2-dioxaborolane (1n).

Prepared according to procedure B. Colorless oil, $20 \%$ yield over two steps. ${ }^{1} \mathrm{H}$ NMR $\left(400 \mathrm{MHz}, \mathrm{CDCl}_{3}\right) \delta 5.76(\mathrm{~d}, J=3.3 \mathrm{~Hz}, 1 \mathrm{H}), 5.59(\mathrm{~s}, 1 \mathrm{H}), 5.12(\mathrm{t}, J=7.0 \mathrm{~Hz}, 1 \mathrm{H})$, $2.14(\mathrm{t}, J=7.5 \mathrm{~Hz}, 2 \mathrm{H}), 2.01-1.88(\mathrm{~m}, 2 \mathrm{H}), 1.68(\mathrm{~s}, 3 \mathrm{H}), 1.59$ (s, 3H), $1.49-1.39$ (m, 2H), 1.26 (s, 12H). ${ }^{13} \mathrm{C} \mathrm{NMR}\left(101 \mathrm{MHz}, \mathrm{CDCl}_{3}\right) \delta 131.4,129.0,124.9,83.4,35.2,29.5$, 27.9, 25.9, 24.9, 17.8. Carbon attached to boron not observed due to quadrupolar relaxation. ${ }^{11} \mathrm{~B}$ NMR $\left(128 \mathrm{MHz}, \mathrm{CDCl}_{3}\right) \delta$ 30.5. HRMS-EI $(\mathrm{m} / z)$ : Calc. for $\mathrm{C}_{15} \mathrm{H}_{27}{ }^{10} \mathrm{BO}_{2}[\mathrm{M}]^{+}$249.2135, found 249.2132.

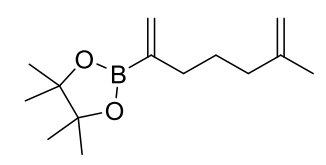

\section{4,4,5,5-tetramethyl-2-(6-methylhepta-1,6-dien-2-yl)-1,3,2-dioxaborolane (10).}

Prepared according to procedure B. Colorless oil, $31 \%$ yield over two steps. ${ }^{1} \mathrm{H}$ NMR $\left(400 \mathrm{MHz} \mathrm{CDCl}_{3}\right) \delta 5.76(\mathrm{~d}, J=3.0 \mathrm{~Hz}, 1 \mathrm{H}), 5.59(\mathrm{~s}, 1 \mathrm{H}), 4.68(\mathrm{~s}, 1 \mathrm{H}), 4.67(\mathrm{~s}, 1 \mathrm{H})$, $2.13(\mathrm{t}, J=7.5 \mathrm{~Hz}, 2 \mathrm{H}), 2.00(\mathrm{t}, J=7.7 \mathrm{~Hz}, 2 \mathrm{H}), 1.70(\mathrm{~s}, 3 \mathrm{H}), 1.62-1.50(\mathrm{~m}, 2 \mathrm{H}), 1.26$ (s, 12H). ${ }^{13} \mathrm{C}$ NMR $\left(101 \mathrm{MHz}, \mathrm{CDCl}_{3}\right) \delta 146.3,146.2,129.2,109.8,83.4,37.5,35.2$, 27.3, 24.9, 22.5. Carbon attached to boron not observed due to quadrupolar relaxation. ${ }^{11}$ B NMR $\left(128 \mathrm{MHz}, \mathrm{CDCl}_{3}\right) \delta$ 30.3. HRMS-EI $(\mathrm{m} / z)$ : Calc. for $\mathrm{C}_{15} \mathrm{H}_{27}{ }^{11} \mathrm{BO}_{2}[\mathrm{M}]^{+}$ 236.1942 , found 236.1943 . 


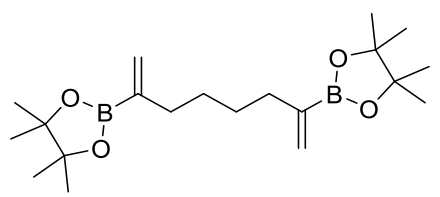

2,2'-(Octa-1,7-diene-2,7-diyl)bis(4,4,5,5-tetramethyl-1,3,2-dioxaborolane) (1p).

Prepared according to procedure $\mathrm{A}, \mathrm{BBr}_{3}$ (1.0 equiv.). White solid, $40 \%$ yield over two steps. ${ }^{1} \mathrm{H}$ NMR (400 MHz, $\left.\mathrm{CDCl}_{3}\right) \delta 5.73(\mathrm{~s}, 2 \mathrm{H}), 5.58(\mathrm{~s}, 2 \mathrm{H}), 2.12(\mathrm{t}, J=6.3 \mathrm{~Hz}, 4 \mathrm{H})$, $1.44-1.35(\mathrm{~m}, 4 \mathrm{H}), 1.25(\mathrm{~s}, 24 \mathrm{H}) .{ }^{13} \mathrm{C} \mathrm{NMR}\left(101 \mathrm{MHz}, \mathrm{CDCl}_{3}\right) \delta$ 128.8, 83.4, 35.3, 29.0, 24.9. Carbon attached to boron not observed due to quadrupolar relaxation. ${ }^{11} \mathrm{~B}$ NMR (128 MHz, $\left.\mathrm{CDCl}_{3}\right) \delta$ 30.2. HRMS-EI $(\mathrm{m} / z)$ : Calc. for $\mathrm{C}_{20} \mathrm{H}_{36}{ }^{11} \mathrm{~B}_{2} \mathrm{O}_{4}[\mathrm{M}]^{+}$ 362.2794, found 362.2802 .

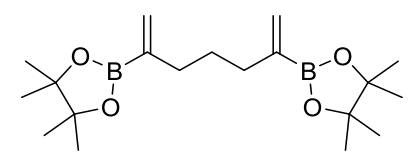

2,2' -(Hepta-1,6-diene-2,6-diyl)bis(4,4,5,5-tetramethyl-1,3,2-dioxaborolane) (1q).

Prepared according to procedure $\mathrm{A} . \mathrm{BBr}_{3}$ (1.0 equiv.). White solid, $11 \%$ yield over two steps. ${ }^{1} \mathrm{H}$ NMR $\left(400 \mathrm{MHz}, \mathrm{CDCl}_{3}\right) \delta 5.75(\mathrm{~d}, J=3.4 \mathrm{~Hz}, 2 \mathrm{H}), 5.60(\mathrm{~s}, 2 \mathrm{H}), 2.15(\mathrm{t}, J=$ $7.6 \mathrm{~Hz}, 4 \mathrm{H}), 1.54$ (p, $J=7.8 \mathrm{~Hz}, 2 \mathrm{H}), 1.26(\mathrm{~s}, 24 \mathrm{H}) .{ }^{13} \mathrm{C} \mathrm{NMR}\left(101 \mathrm{MHz}, \mathrm{CDCl}_{3}\right) \delta$ 129.0, 83.4, 35.2, 28.8, 24.9. Carbon attached to boron not observed due to quadrupolar relaxation. ${ }^{11} \mathrm{~B}$ NMR $\left(128 \mathrm{MHz}, \mathrm{CDCl}_{3}\right) \delta$ 30.0. HRMS-EI $(\mathrm{m} / \mathrm{z})$ : Calc. for $\mathrm{C}_{19} \mathrm{H}_{34}{ }^{11} \mathrm{~B}_{2} \mathrm{O}_{4}[\mathrm{M}]^{+}$348.2638, found 338.2637.

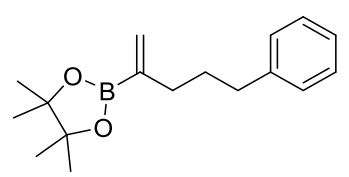

\section{4,4,5,5-tetramethyl-2-(5-phenylpent-1-en-2-yl)-1,3,2-dioxaborolane (1r).}

Prepared according to procedure A. Colorless oil, $22 \%$ yield over two steps. ${ }^{1} \mathrm{H}$ NMR $\left(400 \mathrm{MHz}, \mathrm{CDCl}_{3}\right) \delta 7.34-7.26(\mathrm{~m}, 2 \mathrm{H}), 7.25-7.16(\mathrm{~m}, 3 \mathrm{H}), 5.82(\mathrm{~d}, J=3.3 \mathrm{~Hz}, 1 \mathrm{H})$, $5.65(\mathrm{~s}, 1 \mathrm{H}), 2.64(\mathrm{t}, J=8.6 \mathrm{~Hz}, 2 \mathrm{H}), 2.24(\mathrm{t}, J=7.5 \mathrm{~Hz}, 2 \mathrm{H}), 1.79(\mathrm{tt}, J=8.6,7.5 \mathrm{~Hz}$, $2 \mathrm{H}), 1.30(\mathrm{~s}, 12 \mathrm{H})$. These spectroscopic data correspond to reported data. ${ }^{12}$<smiles>C=C(CCc1ccccc1)OB1OC(C)(C)C(C)(C)O1</smiles>

4,4,5,5-tetramethyl-2-(4-phenylbut-1-en-2-yl)-1,3,2-dioxaborolane (1s). 
Prepared according to procedure A. Colorless oil, $42 \%$ yield over two steps. ${ }^{1} \mathrm{H}$ NMR $\left(400 \mathrm{MHz}, \mathrm{CDCl}_{3}\right) \delta 7.35-7.26(\mathrm{~m}, 2 \mathrm{H}), 7.25-7.16(\mathrm{~m}, 3 \mathrm{H}), 5.82(\mathrm{~d}, J=3.2 \mathrm{~Hz}, 1 \mathrm{H})$, $5.64(\mathrm{~s}, 1 \mathrm{H}), 2.78(\mathrm{t}, J=8.3 \mathrm{~Hz}, 2 \mathrm{H}), 2.51(\mathrm{t}, J=8.3 \mathrm{~Hz}, 2 \mathrm{H}), 1.30(\mathrm{~s}, 12 \mathrm{H})$. These spectroscopic data correspond to reported data. ${ }^{9}$

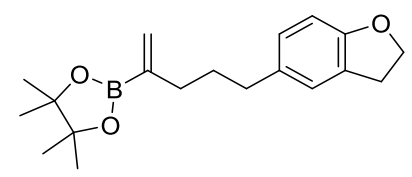

\section{2-(5-(2,3-dihydrobenzofuran-5-yl)pent-1-en-2-yl)-4,4,5,5-tetramethyl-1,3,2-} dioxaborolane (1t).

Prepared according to procedure B. Colorless oil, $38 \%$ yield over two steps. ${ }^{1} \mathrm{H}$ NMR $\left(400 \mathrm{MHz}, \mathrm{CDCl}_{3}\right) \delta 7.02(\mathrm{~s}, 1 \mathrm{H}), 6.92(\mathrm{~d}, J=8.1 \mathrm{~Hz}, 1 \mathrm{H}), 6.69(\mathrm{~d}, J=8.1 \mathrm{~Hz}, 1 \mathrm{H})$, $5.79(\mathrm{~d}, J=3.3 \mathrm{~Hz}, 1 \mathrm{H}), 5.62(\mathrm{~s}, 1 \mathrm{H}), 4.54(\mathrm{t}, J=8.7 \mathrm{~Hz}, 2 \mathrm{H}), 3.18(\mathrm{t}, J=8.7 \mathrm{~Hz}, 2 \mathrm{H})$, $2.54(\mathrm{t}, J=8.0 \mathrm{~Hz}, 2 \mathrm{H}), 2.20(\mathrm{t}, J=7.4 \mathrm{~Hz}, 2 \mathrm{H}), 1.72(\mathrm{tt}, J=8.0,7.4 \mathrm{~Hz}, 2 \mathrm{H}), 1.28(\mathrm{~s}$, 12H). ${ }^{13} \mathrm{C} \mathrm{NMR}\left(101 \mathrm{MHz}, \mathrm{CDCl}_{3}\right) \delta 158.2,135.0,129.3,127.9,126.9,125.0,108.9$, 83.5, 71.2, 35.1, 35.1, 31.6, 30.0, 24.9. Carbon attached to boron not observed due to quadrupolar relaxation. ${ }^{11} \mathrm{~B} \mathrm{NMR}\left(128 \mathrm{MHz}, \mathrm{CDCl}_{3}\right) \delta 26.5$. HRMS-EI $(\mathrm{m} / z)$ : Calc. for $\mathrm{C}_{19} \mathrm{H}_{27}{ }^{10} \mathrm{BO}_{3}[\mathrm{M}]^{+}$313.2084, found 313.2082.

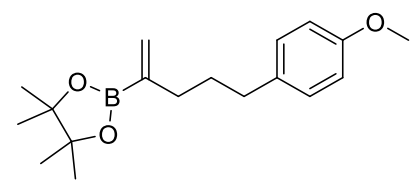

\section{2-(5-(4-Methoxyphenyl)pent-1-en-2-yl)-4,4,5,5-tetramethyl-1,3,2-dioxaborolane} (1u).

Prepared according to procedure B. Colorless oil, $20 \%$ yield over two steps. ${ }^{1} \mathrm{H}$ NMR $\left(400 \mathrm{MHz}, \mathrm{CDCl}_{3}\right) \delta 7.10(\mathrm{~d}, J=8.6 \mathrm{~Hz}, 2 \mathrm{H}), 6.82(\mathrm{~d}, J=8.6 \mathrm{~Hz}, 2 \mathrm{H}), 5.79(\mathrm{~d}, J=3.1$ $\mathrm{Hz}, 1 \mathrm{H}), 5.61(\mathrm{~s}, 1 \mathrm{H}), 3.79(\mathrm{~s}, 3 \mathrm{H}), 2.55(\mathrm{~d}, J=8.1 \mathrm{~Hz}, 2 \mathrm{H}), 2.20(\mathrm{t}, J=7.5 \mathrm{~Hz}, 2 \mathrm{H})$, $1.77-1.63(\mathrm{~m}, 2 \mathrm{H}), 1.27$ (s, 12H). ${ }^{13} \mathrm{C} \mathrm{NMR}\left(101 \mathrm{MHz}, \mathrm{CDCl}_{3}\right) \delta$ 159.7, 137.0, 131.4, 131.3, 115.7, 85.4, 57.3, 37.2, 36.7, 33.3, 26.9. Carbon attached to boron not observed due to quadrupolar relaxation. ${ }^{11} \mathrm{~B}$ NMR $\left(128 \mathrm{MHz}, \mathrm{CDCl}_{3}\right) \delta 30.3$. HRMS-EI $(\mathrm{m} / \mathrm{z})$ : Calc. for $\mathrm{C}_{18} \mathrm{H}_{27}{ }^{11} \mathrm{BO}_{3}[\mathrm{M}]^{+}$302.2048, found 302.2050. 


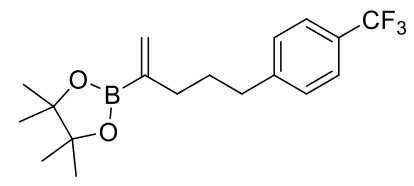

4,4,5,5-Tetramethyl-2-(5-(4-(trifluoromethyl)phenyl)pent-1-en-2-yl)-1,3,2dioxaborolane (1v).

Prepared according to procedure B. Colorless oil, 9\% yield over two steps. ${ }^{1} \mathrm{H}$ NMR $\left(400 \mathrm{MHz}, \mathrm{CDCl}_{3}\right) \delta 7.52(\mathrm{~d}, J=7.9 \mathrm{~Hz}, 2 \mathrm{H}), 7.29(\mathrm{~d}, J=7.9 \mathrm{~Hz}, 2 \mathrm{H}), 5.83(\mathrm{~d}, J=3.2$ $\mathrm{Hz}, 1 \mathrm{H}), 5.63(\mathrm{~s}, 1 \mathrm{H}), 2.66$ (t, $J=8.3 \mathrm{~Hz}, 2 \mathrm{H}), 2.22$ (t, $J=7.4 \mathrm{~Hz}, 2 \mathrm{H}), 1.83-1.73$ (m, 2H), $1.27(\mathrm{~s}, 12 \mathrm{H}) .{ }^{13} \mathrm{C} \mathrm{NMR}\left(101 \mathrm{MHz}, \mathrm{CDCl}_{3}\right) \delta 147.0,129.7,128.8, \delta 128.1(\mathrm{q}, J=$ $32.3 \mathrm{~Hz}), 125.3(\mathrm{q}, J=3.8 \mathrm{~Hz}), 124.6(\mathrm{q}, J=271.8 \mathrm{~Hz}), 83.5,35.4,35.1,30.7,24.9$. Carbon attached to boron not observed due to quadrupolar relaxation. ${ }^{19} \mathrm{~F}$ NMR (377 $\left.\mathrm{MHz}, \mathrm{CDCl}_{3}\right) \delta-62.3 .{ }^{11} \mathrm{~B}$ NMR $\left(128 \mathrm{MHz}, \mathrm{CDCl}_{3}\right) \delta 30.1$. HRMS-EI $(\mathrm{m} / z)$ : Calc. for $\mathrm{C}_{18} \mathrm{H}_{24}{ }^{11} \mathrm{BF}_{3} \mathrm{O}_{2}[\mathrm{M}]^{+}$340.1816, found 340.1817.<smiles>C=C(CC(C)C)B1OC(C)(C)C(C)(C)O1</smiles>

4,4,5,5-tetramethyl-2-(4-methylpent-1-en-2-yl)-1,3,2-dioxaborolane (1w).

Prepared according to procedure A. Colorless oil, $37 \%$ yield over two steps. ${ }^{1} \mathrm{H}$ NMR $\left(400 \mathrm{MHz}, \mathrm{CDCl}_{3}\right) \delta 5.78(\mathrm{~d}, J=3.3 \mathrm{~Hz}, 1 \mathrm{H}), 5.55(\mathrm{~s}, 1 \mathrm{H}), 2.02(\mathrm{~d}, J=6.9 \mathrm{~Hz}, 2 \mathrm{H})$, $1.80-1.64(\mathrm{~m}, 1 \mathrm{H}), 1.25(\mathrm{~s}, 12 \mathrm{H}), 0.85(\mathrm{~d}, J=6.6 \mathrm{~Hz}, 6 \mathrm{H})$. These spectroscopic data correspond to reported data. ${ }^{13}$

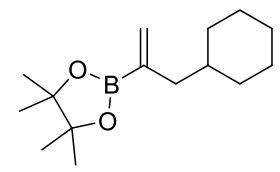

2-(3-cyclohexylprop-1-en-2-yl)-4,4,5,5-tetramethyl-1,3,2-dioxaborolane (1x).

Prepared according to procedure C. Colorless oil, $27 \%$ yield over two steps. ${ }^{1} \mathrm{H}$ NMR $\left(400 \mathrm{MHz}, \mathrm{CDCl}_{3}\right) \delta 5.77(\mathrm{~d}, J=3.3 \mathrm{~Hz}, 1 \mathrm{H}), 5.54(\mathrm{~s}, 1 \mathrm{H}), 2.11-1.95(\mathrm{~m}, 2 \mathrm{H}), 1.74-$ $1.56(\mathrm{~m}, 4 \mathrm{H}), 1.26(\mathrm{~s}, 17 \mathrm{H}), 0.93-0.77(\mathrm{~m}, 2 \mathrm{H})$. These spectroscopic data correspond to reported data. ${ }^{10}$

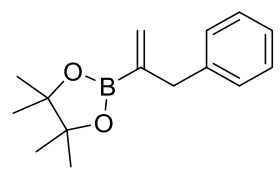




\section{4,4,5,5-Tetramethyl-2-(3-phenylprop-1-en-2-yl)-1,3,2-dioxaborolane (1y).}

Prepared according to procedure C. Colorless oil, $11 \%$ yield over two steps. ${ }^{1} \mathrm{H}$ NMR $\left(400 \mathrm{MHz}, \mathrm{CDCl}_{3}\right) \delta 7.29-7.23(\mathrm{~m}, 2 \mathrm{H}), 7.22-7.13(\mathrm{~m}, 3 \mathrm{H}), 5.83(\mathrm{~d}, J=2.5 \mathrm{~Hz}, 1 \mathrm{H})$, $5.52(\mathrm{~s}, 1 \mathrm{H}), 3.48(\mathrm{~s}, 2 \mathrm{H}), 1.21(\mathrm{~s}, 12 \mathrm{H})$. These spectroscopic data correspond to reported data. ${ }^{9}$

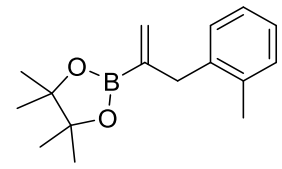

4,4,5,5-Tetramethyl-2-(3-(o-tolyl)prop-1-en-2-yl)-1,3,2-dioxaborolane (1z).

Prepared according to procedure C. Colorless oil, $23 \%$ yield over two steps. ${ }^{1} \mathrm{H}$ NMR $\left(400 \mathrm{MHz}, \mathrm{CDCl}_{3}\right) \delta 7.20-7.06(\mathrm{~m}, 4 \mathrm{H}), 5.85(\mathrm{~s}, 1 \mathrm{H}), 5.33(\mathrm{~s}, 1 \mathrm{H}), 3.48(\mathrm{~s}, 2 \mathrm{H}), 2.27$ $(\mathrm{s}, 3 \mathrm{H}), 1.27(\mathrm{~s}, 12 \mathrm{H})$. These spectroscopic data correspond to reported data. ${ }^{14}$

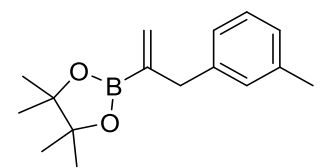

\section{4,4,5,5-tetramethyl-2-(3-(m-tolyl)prop-1-en-2-yl)-1,3,2-dioxaborolane (1aa).}

Prepared according to procedure C, purified by distillation after column chromatography. Colorless oil, $19 \%$ yield over two steps. ${ }^{1} \mathrm{H}$ NMR (400 MHz, $\left.\mathrm{CDCl}_{3}\right)$ $\delta 7.17-7.12(\mathrm{~m}, 1 \mathrm{H}), 7.02(\mathrm{~s}, 1 \mathrm{H}), 7.01-6.96(\mathrm{~m}, 2 \mathrm{H}), 5.82(\mathrm{~d}, J=3.1 \mathrm{~Hz}, 1 \mathrm{H}), 5.53$ $(\mathrm{s}, 1 \mathrm{H}), 3.45(\mathrm{~s}, 2 \mathrm{H}), 2.31(\mathrm{~s}, 3 \mathrm{H}), 1.22(\mathrm{~s}, 12 \mathrm{H}) .{ }^{13} \mathrm{C} \mathrm{NMR}\left(101 \mathrm{MHz}, \mathrm{CDCl}_{3}\right) \delta$ 140.7, $137.7,130.0,129.9,128.1,126.5,126.3,83.6,41.4,24.8,21.5$. Carbon attached to boron not observed due to quadrupolar relaxation. ${ }^{11} \mathrm{~B} \mathrm{NMR}\left(128 \mathrm{MHz}, \mathrm{CDCl}_{3}\right) \delta 30.4$. HRMS-EI $(m / z)$ : Calc. for $\mathrm{C}_{16} \mathrm{H}_{23}{ }^{11} \mathrm{BO}_{2}[\mathrm{M}]^{+} 258.1786$, found 258.1787.

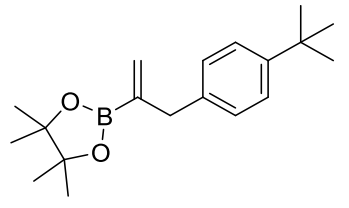

\section{2-(3-(4-(Tert-butyl)phenyl)prop-1-en-2-yl)-4,4,5,5-tetramethyl-1,3,2-} dioxaborolane (1ab).

Prepared according to Procedure C. Colorless oil, $30 \%$ yield over two steps ${ }^{1} \mathrm{H}$ NMR $\left(400 \mathrm{MHz}, \mathrm{CDCl}_{3}\right) \delta 7.28(\mathrm{~d}, J=8.1 \mathrm{~Hz}, 2 \mathrm{H}), 7.12(\mathrm{~d}, J=8.1 \mathrm{~Hz}, 2 \mathrm{H}), 5.81(\mathrm{~d}, J=3.2$ 
$\mathrm{Hz}, 1 \mathrm{H}), 5.52$ (s, 1H), 3.45 (s, 2H), 1.30 (s, 9H), 1.21 (s, 12H). ${ }^{13} \mathrm{C}$ NMR (101 MHz, $\left.\mathrm{CDCl}_{3}\right) \delta 147.5,136.7,128.8,127.9,124.1,82.6,39.9,33.4,30.6,23.8$. Carbon attached to boron not observed due to quadrupolar relaxation. ${ }^{11} \mathrm{~B}$ NMR (128 MHz, $\left.\mathrm{CDCl}_{3}\right) \delta$ 26.5. HRMS-EI (m/z): Calc. for $\mathrm{C}_{19} \mathrm{H}_{29}{ }^{11} \mathrm{BO}_{2}[\mathrm{M}]^{+} 300.2255$, found 300.2259.

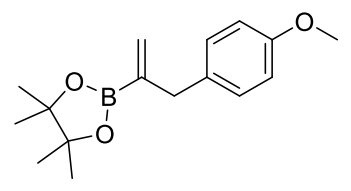

2-(3-(4-Methoxyphenyl)prop-1-en-2-yl)-4,4,5,5-tetramethyl-1,3,2-dioxaborolane (1ac).

Prepared according to procedure C, purified by distillation after column chromatography. Colorless oil, $19 \%$ yield over two steps. ${ }^{1} \mathrm{H}$ NMR (400 MHz, $\mathrm{CDCl}_{3}$ ) $\delta 7.11(\mathrm{~d}, J=8.6 \mathrm{~Hz}, 2 \mathrm{H}), 6.81(\mathrm{~d}, J=8.6 \mathrm{~Hz}, 2 \mathrm{H}), 5.81(\mathrm{~d}, J=3.1 \mathrm{~Hz}, 1 \mathrm{H}), 5.51(\mathrm{~s}$, $1 \mathrm{H}), 3.78(\mathrm{~s}, 3 \mathrm{H}), 3.42(\mathrm{~s}, 2 \mathrm{H}), 1.22(\mathrm{~s}, 12 \mathrm{H})$. These spectroscopic data correspond to reported data. ${ }^{15}$

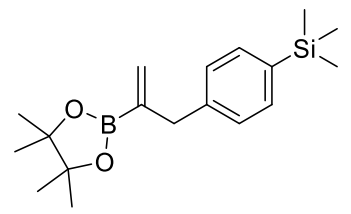

\section{Trimethyl(4-(2-(4,4,5,5-tetramethyl-1,3,2-dioxaborolan-2-yl)allyl)phenyl)silane} (1ad).

Prepared according to procedure C, purified by distillation after column chromatography. Colorless oil, $20 \%$ yield over two steps. ${ }^{1} \mathrm{H}$ NMR (400 MHz, $\mathrm{CDCl}_{3}$ ) $\delta 7.45(\mathrm{~d}, J=8.0 \mathrm{~Hz}, 2 \mathrm{H}), 7.22(\mathrm{~d}, J=8.0 \mathrm{~Hz}, 2 \mathrm{H}), 5.87(\mathrm{~d}, J=3.2 \mathrm{~Hz}, 1 \mathrm{H}), 5.56(\mathrm{~s}$, $1 \mathrm{H}), 3.50(\mathrm{~s}, 2 \mathrm{H}), 1.25(\mathrm{~s}, 12 \mathrm{H}), 0.28(\mathrm{~s}, 9 \mathrm{H}) .{ }^{13} \mathrm{C} \mathrm{NMR}\left(101 \mathrm{MHz}, \mathrm{CDCl}_{3}\right) \delta 141.5$, 137.3, 133.3, 130.1, 128.7, 83.6, 41.3, 24.8, -0.9. Carbon attached to boron not observed due to quadrupolar relaxation. ${ }^{11} \mathrm{~B}$ NMR $\left(128 \mathrm{MHz}, \mathrm{CDCl}_{3}\right) \delta 29.9 . \mathrm{HRMS}-\mathrm{EI}(\mathrm{m} / \mathrm{z})$ : Calc. for $\mathrm{C}_{18} \mathrm{H}_{29}{ }^{11} \mathrm{BO}_{2} \mathrm{Si}[\mathrm{M}]^{+} 316.2024$, found 316.2028.

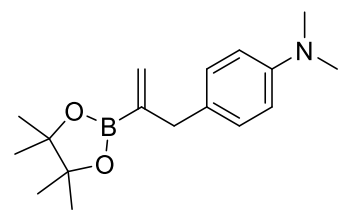




\section{N,N-dimethyl-4-(2-(4,4,5,5-tetramethyl-1,3,2-dioxaborolan-2-yl)allyl)aniline}

(1ae).

Prepared according to procedure C. Colorless oil, $20 \%$ yield over two steps. ${ }^{1} \mathrm{H}$ NMR $\left(400 \mathrm{MHz}, \mathrm{CDCl}_{3}\right) \delta 7.07(\mathrm{~d}, J=8.5 \mathrm{~Hz}, 2 \mathrm{H}), 6.69(\mathrm{~d}, J=8.5 \mathrm{~Hz}, 2 \mathrm{H}), 5.80(\mathrm{~d}, J=2.3$ $\mathrm{Hz}, 1 \mathrm{H}), 5.50$ (s, 1H), 3.39 (s, 2H), 2.91 (s, 6H), 1.23 (s, 12H). ${ }^{13} \mathrm{C}$ NMR (101 MHz, $\left.\mathrm{CDCl}_{3}\right) \delta 149.1,129.9,129.3,129.0,113.1,83.5,41.1,40.4,24.9$. Carbon attached to boron not observed due to quadrupolar relaxation. ${ }^{11} \mathrm{~B} \mathrm{NMR}\left(128 \mathrm{MHz}, \mathrm{CDCl}_{3}\right) \delta 30.4$. HRMS-EI $(m / z)$ : Calc. for $\mathrm{C}_{17} \mathrm{H}_{26}{ }^{11} \mathrm{BNO}_{2}[\mathrm{M}]^{+}$287.2051, found 287.2052.

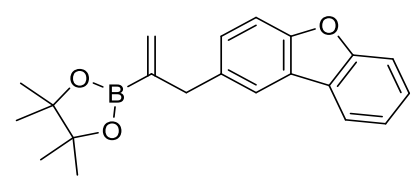

\section{2-(3-(Dibenzo[b,d]furan-2-yl)prop-1-en-2-yl)-4,4,5,5-tetramethyl-1,3,2- dioxaborolane (1af).}

Prepared according to procedure C. White solid, $17 \%$ yield over two steps. ${ }^{1} \mathrm{H}$ NMR $\left(400 \mathrm{MHz}, \mathrm{CDCl}_{3}\right) \delta 7.82(\mathrm{~d}, J=7.6 \mathrm{~Hz}, 1 \mathrm{H}), 7.70(\mathrm{~s}, 1 \mathrm{H}), 7.45(\mathrm{~d}, J=7.6 \mathrm{~Hz}, 1 \mathrm{H})$, $7.40-7.29(\mathrm{~m}, 2 \mathrm{H}), 7.27-7.18(\mathrm{~m}, 2 \mathrm{H}), 5.79(\mathrm{~d}, J=3.0 \mathrm{~Hz}, 1 \mathrm{H}), 5.52(\mathrm{~s}, 1 \mathrm{H}), 3.56$ (s, 2H), $1.11(\mathrm{~s}, 12 \mathrm{H}) .{ }^{13} \mathrm{C} \mathrm{NMR}\left(101 \mathrm{MHz}, \mathrm{CDCl}_{3}\right) \delta 156.6,154.9,135.3,129.9,128.6$, 126.9, 124.5, 124.2, 122.6, 121.0, 120.7, 111.7, 111.2, 83.7, 41.6, 24.8. Carbon attached to boron not observed due to quadrupolar relaxation. ${ }^{11} \mathrm{~B}$ NMR $(128 \mathrm{MHz}$, $\left.\mathrm{CDCl}_{3}\right) \delta$ 30.4. HRMS-EI $(\mathrm{m} / \mathrm{z})$ : Calc. for $\mathrm{C}_{21} \mathrm{H}_{23} \mathrm{BO}_{3}[\mathrm{M}]^{+}$334.1735, found 334.1743.<smiles></smiles>

tert-butyl 4-(4,4,5,5-tetramethyl-1,3,2-dioxaborolan-2-yl)pent-4-enoate (1ag).

Prepared according to according to previously reported procedure ${ }^{5}$. Colorless oil. ${ }^{1} \mathrm{H}$ NMR (400 MHz, $\left.\mathrm{CDCl}_{3}\right) \delta 5.78(\mathrm{~d}, J=3.0 \mathrm{~Hz}, 1 \mathrm{H}), 5.62(\mathrm{~s}, 1 \mathrm{H}), 2.45-2.39(\mathrm{~m}, 2 \mathrm{H})$, $2.38-2.32(\mathrm{~m}, 2 \mathrm{H}), 1.42(\mathrm{~s}, 9 \mathrm{H}), 1.25(\mathrm{~s}, 12 \mathrm{H})$.<smiles>C=C(CCCCl)OC1(C)OC(C)(C)C1(C)C</smiles>

2-(5-chloropent-1-en-2-yl)-4,4,5,5-tetramethyl-1,3,2-dioxaborolane (1ah). 
Prepared according to according to previously reported procedure. ${ }^{16}$ Colorless oil. ${ }^{1} \mathrm{H}$ NMR (400 MHz, $\left.\mathrm{CDCl}_{3}\right) \delta 5.82(\mathrm{~d}, J=3.0 \mathrm{~Hz}, 1 \mathrm{H}), 5.65(\mathrm{~s}, 1 \mathrm{H}), 3.51(\mathrm{t}, J=6.8 \mathrm{~Hz}$, 2H), $2.28(\mathrm{t}, J=7.3 \mathrm{~Hz}, 2 \mathrm{H}), 1.97-1.78(\mathrm{~m}, 2 \mathrm{H}), 1.26(\mathrm{~s}, 12 \mathrm{H})$.

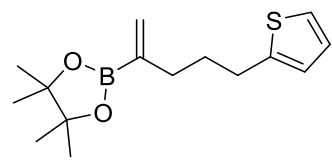

4,4,5,5-tetramethyl-2-(5-(thiophen-2-yl)pent-1-en-2-yl)-1,3,2-dioxaborolane (1ai). Prepared according to according procedure B. Colorless oil, 17\% yield over two steps. ${ }^{1} \mathrm{H}$ NMR $\left(400 \mathrm{MHz}, \mathrm{CDCl}_{3}\right) \delta 7.10(\mathrm{dd}, J=5.2,0.8 \mathrm{~Hz}, 1 \mathrm{H}), 6.91(\mathrm{dd}, J=5.1,3.4 \mathrm{~Hz}$, $1 \mathrm{H}), 6.79(\mathrm{~d}, J=2.9 \mathrm{~Hz}, 1 \mathrm{H}), 5.82(\mathrm{~d}, J=3.2 \mathrm{~Hz}, 1 \mathrm{H}), 5.64(\mathrm{~s}, 1 \mathrm{H}), 2.82(\mathrm{~d}, J=7.7 \mathrm{~Hz}$, 2H), $2.24(\mathrm{t}, J=7.4 \mathrm{~Hz}, 2 \mathrm{H}), 1.89-1.79(\mathrm{~m}, 2 \mathrm{H}), 1.28(\mathrm{~s}, 12 \mathrm{H}) .{ }^{13} \mathrm{C}$ NMR $(101 \mathrm{MHz}$, $\left.\mathrm{CDCl}_{3}\right) \delta 145.9,129.7,126.7,124.0,122.8,83.5,77.5,77.2,76.8,35.0,31.3,29.6,24.9$. ${ }^{11} \mathrm{~B}$ NMR $\left(128 \mathrm{MHz}, \mathrm{CDCl}_{3}\right) \delta 30.2$.

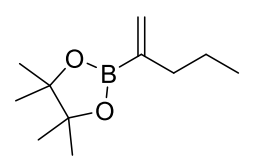

\section{4,4,5,5-tetramethyl-2-(pent-1-en-2-yl)-1,3,2-dioxaborolane (1al).}

Prepared according to according procedure A and purified by distillation after column chromatography. Colorless oil, $10 \%$ yield over two steps. ${ }^{1} \mathrm{H}$ NMR (400 $\mathrm{MHz}, \mathrm{CDCl}_{3}$ ) $\delta 5.76(\mathrm{~d}, J=3.4 \mathrm{~Hz}, 1 \mathrm{H}), 5.59(\mathrm{~s}, 1 \mathrm{H}), 2.12(\mathrm{t}, J=7.5 \mathrm{~Hz}, 2 \mathrm{H}), 1.49-1.37(\mathrm{~m}, 2 \mathrm{H})$, $1.26(\mathrm{~s}, 12 \mathrm{H}), 0.89(\mathrm{t}, J=7.3 \mathrm{~Hz}, 3 \mathrm{H})$. These spectroscopic data correspond to reported data. ${ }^{17}$

\section{Procedures for Isomerization of 1,1-Disubstituted Vinyl Boronates}

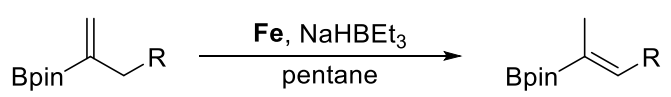

Procedure for evaluating iron complexes (table 1): In a nitrogen-filled glove-box, an oven-dried $10 \mathrm{~mL}$ thin-wall glass tube was charged with an iron complex (10 $\mu \mathrm{mol})$, solvent $(2 \mathrm{~mL}), \mathbf{1 a}(0.2 \mathrm{mmol})$. Afterwards, $\mathrm{NaHBEt}_{3}(1 \mathrm{M}$ in THF) was added to the glass tube. The tube was sealed with a Teflon plug, and the reaction mixture was stirred at room temperature for $2 \mathrm{~h}$. The reaction was quenched by exposing the solution to air. 
Mesitylene was added as the internal standard. An aliquot was removed from the flask and analyzed by GC.

Condition A for isomerization of 1,1-Disubstituted Vinyl Boronates. In a nitrogenfilled glove-box, an oven-dried $10 \mathrm{~mL}$ thin-wall glass tube was charged with $[\mathrm{Fe}] \mathrm{Cl}_{2}$ (10 $\mu \mathrm{mol}, 5.6 \mathrm{mg})$, pentane $(2 \mathrm{~mL}), \alpha$-vinyl boronates $(0.2 \mathrm{mmol})$, then $\mathrm{NaHBEt}_{3}(15$ $\mu$ mol, $15 \mu \mathrm{L}, 1 \mathrm{M}$ in THF) was added to the glass tube. The tube was sealed with a Teflon plug, and the solution was stirred at room temperature for $2 \mathrm{~h}$. The resulting mixture was concentrated under vacuum and the residue was purified by flash silica gel column chromatography to give the product. The conversions and $Z / E$ ratios were confirmed by ${ }^{1} \mathrm{H}$ NMR analysis of isolated products. The yields are referring to those for the isolated compounds that contain $Z, E$ isomers and the starting compound, as the starting compounds cannot be separated from the products. The amounts of the remaining starting compound for each substrate are shown, along with the $Z / E$ ratios.

Condition B for isomerization of 1,1-Disubstituted Vinyl Boronates. In a nitrogenfilled glove-box, an oven-dried $10 \mathrm{~mL}$ thin-wall glass tube was charged with $\left[\mathrm{Fe}^{-} \mathrm{Cl}_{2}\right.$ (10 $\mu \mathrm{mol}, 5.6 \mathrm{mg})$, pentane $(2 \mathrm{~mL}), \alpha$-vinyl boronates $(0.1 \mathrm{mmol})$, then $\mathrm{NaHBEt}_{3}(30$ $\mu \mathrm{mol}, 30 \mu \mathrm{L}, 1 \mathrm{M}$ in THF) was added to the glass tube. The tube was sealed with a Teflon plug, and the solution was stirred at room temperature for $8 \mathrm{~h}$. The reaction was quenched by exposing the solution to air. The resulting mixture was concentrated under vacuum and the residue was purified by flash silica gel column chromatography to give the product. The conversions and Z/E ratios were confirmed by ${ }^{1} \mathrm{H}$ NMR analysis of isolated products. The yields are referring to those for the isolated compounds that contain $Z, E$ isomers and the starting compound, as the starting compounds cannot be separated from the products. The amounts of the remaining starting compound for each substrate are shown, along with the $Z / E$ ratios.

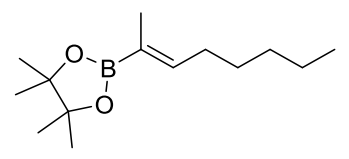

\section{(Z)-4,4,5,5-tetramethyl-2-(oct-2-en-2-yl)-1,3,2-dioxaborolane (2a).}

The reaction was performed according to condition A. Colorless oil (98\% covn., $45 \mathrm{mg}$, 
93\% yield, $Z / E>20: 1,2 \%$ 1a). ${ }^{1} \mathrm{H}$ NMR $\left(400 \mathrm{MHz}, \mathrm{CDCl}_{3}\right) \delta 6.31(\mathrm{t}, J=6.4 \mathrm{~Hz}, 1 \mathrm{H})$, $2.17-2.04(\mathrm{~m}, 2 \mathrm{H}), 1.67(\mathrm{~s}, 3 \mathrm{H}), 1.44-1.35(\mathrm{~m}, 2 \mathrm{H}), 1.34-1.27(\mathrm{~m}, 4 \mathrm{H}), 1.26(\mathrm{~s}$, $12 \mathrm{H}), 0.88(\mathrm{t}, J=6.6 \mathrm{~Hz}, 3 \mathrm{H}) .{ }^{13} \mathrm{C} \mathrm{NMR}\left(101 \mathrm{MHz}, \mathrm{CDCl}_{3}\right) \delta 146.9,83.2,31.9,28.8$, 28.7, 24.9, 22.7, 14.2, 14.0. Carbon attached to boron not observed due to quadrupolar relaxation. ${ }^{11}$ B NMR $\left(128 \mathrm{MHz}, \mathrm{CDCl}_{3}\right) \delta 30.5$. HRMS-EI $(\mathrm{m} / z)$ : Calc. for $\mathrm{C}_{14} \mathrm{H}_{27}{ }^{11} \mathrm{BO}_{2}$ $[\mathrm{M}]^{+} 238.2099$, found 238.2102.

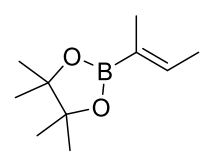

\section{(Z)-2-(But-2-en-2-yl)-4,4,5,5-tetramethyl-1,3,2-dioxaborolane (2b).}

The reaction was performed according to condition A. Colorless oil (98\% covn., $33 \mathrm{mg}$, $91 \%$ yield, $Z / E>20: 1,2 \%$ 1b). ${ }^{1} \mathrm{H}$ NMR $\left(400 \mathrm{MHz}, \mathrm{CDCl}_{3}\right) \delta 6.42(\mathrm{qd}, J=6.6,1.5$ $\mathrm{Hz}, 1 \mathrm{H}), 1.70(\mathrm{~d}, J=6.6 \mathrm{~Hz}, 3 \mathrm{H}), 1.67(\mathrm{~s}, 3 \mathrm{H}), 1.25$ (s, 12H). ${ }^{13} \mathrm{C}$ NMR $(101 \mathrm{MHz}$, $\left.\mathrm{CDCl}_{3}\right) \delta 140.9,83.2,24.9,14.4,13.6$. Carbon attached to boron not observed due to quadrupolar relaxation. These spectroscopic data correspond to reported data. ${ }^{18}$

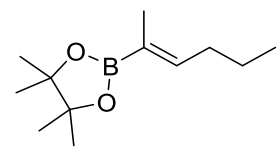

(Z)-2-(Hex-2-en-2-yl)-4,4,5,5-tetramethyl-1,3,2-dioxaborolane (2c).

The reaction was performed according to condition A. Colorless oil (98\% covn., $36 \mathrm{mg}$, $86 \%$ yield, $Z / E>20: 1,3 \%$ 1c). ${ }^{1} \mathrm{H} \mathrm{NMR}\left(400 \mathrm{MHz}, \mathrm{CDCl}_{3}\right) \delta 6.31(\mathrm{td}, J=6.9,1.5 \mathrm{~Hz}$, $1 \mathrm{H}), 2.15-2.06(\mathrm{~m}, 2 \mathrm{H}), 1.67(\mathrm{~s}, 3 \mathrm{H}), 1.46-1.37(\mathrm{~m}, 2 \mathrm{H}), 1.26(\mathrm{~s}, 12 \mathrm{H}), 0.91(\mathrm{t}, J=$ $7.3 \mathrm{~Hz}, 3 \mathrm{H}) .{ }^{13} \mathrm{C} \mathrm{NMR}\left(101 \mathrm{MHz}, \mathrm{CDCl}_{3}\right) \delta 146.5,83.1,30.8,26.9,24.8,22.1,14.1$. Carbon attached to boron not observed due to quadrupolar relaxation. These spectroscopic data correspond to reported data. ${ }^{19}$

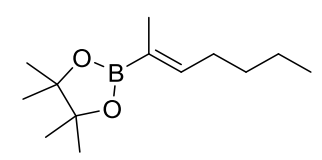

\section{(Z)-2-(Hept-2-en-2-yl)-4,4,5,5-tetramethyl-1,3,2-dioxaborolane (2d).}

The reaction was performed according to condition A. Colorless oil (97\% covn., $41 \mathrm{mg}$, $92 \%$ yield, $Z / E>20: 1,3 \%$ 1d). ${ }^{1} \mathrm{H} \mathrm{NMR}\left(400 \mathrm{MHz}, \mathrm{CDCl}_{3}\right) \delta 6.31(\mathrm{td}, J=7.1,1.6 \mathrm{~Hz}$, $1 \mathrm{H}), 2.15-2.03(\mathrm{~m}, 2 \mathrm{H}), 1.67(\mathrm{~s}, 3 \mathrm{H}), 1.43-1.29(\mathrm{~m}, 4 \mathrm{H}), 1.25(\mathrm{~s}, 12 \mathrm{H}), 0.89(\mathrm{t}, J=$ 
7.0 Hz, 3H). $\left.{ }^{13} \mathrm{C} \mathrm{NMR} \mathrm{(101} \mathrm{MHz,} \mathrm{CDCl}_{3}\right) \delta 146.7,83.0,31.0,28.4,24.8,22.6,14.0$, 13.9. Carbon attached to boron not observed due to quadrupolar relaxation. These spectroscopic data correspond to reported data. ${ }^{13}$

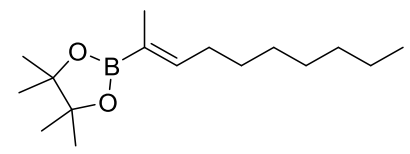

(Z)-2-(Dec-2-en-2-yl)-4,4,5,5-tetramethyl-1,3,2-dioxaborolane (2e).

The reaction was performed according to condition A. Colorless oil (97\% covn., $48 \mathrm{mg}$, $91 \%$ yield, $Z / E>20: 1,3 \%$ 1e). ${ }^{1} \mathrm{H} \mathrm{NMR}\left(400 \mathrm{MHz}, \mathrm{CDCl}_{3}\right) \delta 6.31(\mathrm{t}, J=6.5 \mathrm{~Hz}, 1 \mathrm{H})$, $2.15-2.06(\mathrm{~m}, 2 \mathrm{H}), 1.66(\mathrm{~s}, 3 \mathrm{H}), 1.44-1.27(\mathrm{~m}, 10 \mathrm{H}), 1.25(\mathrm{~s}, 12 \mathrm{H}), 0.87(\mathrm{t}, J=6.6$ $\mathrm{Hz}, 3 \mathrm{H}) .{ }^{13} \mathrm{C} \mathrm{NMR}\left(101 \mathrm{MHz}, \mathrm{CDCl}_{3}\right) \delta 146.9,83.2,32.0,29.6,29.4,29.0,28.8,24.9$, 22.8, 14.3, 14.0. Carbon attached to boron not observed due to quadrupolar relaxation. ${ }^{11} \mathrm{~B}$ NMR $\left(128 \mathrm{MHz}, \mathrm{CDCl}_{3}\right) \delta$ 30.5. HRMS-FI $(\mathrm{m} / \mathrm{z})$ : Calc. for $\mathrm{C}_{16} \mathrm{H}_{31}{ }^{10} \mathrm{BO}_{2}[\mathrm{M}]^{+}$ 265.2448, found 265.2445.

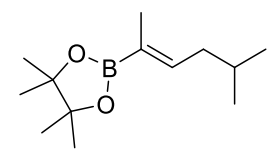

(Z)-4,4,5,5-tetramethyl-2-(5-methylhex-2-en-2-yl)-1,3,2-dioxaborolane (2f).

The reaction was performed according to condition A. Colorless oil (> 98\% covn., 43 mg, $96 \%$ yield, $Z / E>20: 1,1 \%$ 1f). ${ }^{1} \mathrm{H}$ NMR $\left(400 \mathrm{MHz}, \mathrm{CDCl}_{3}\right) \delta 6.34(\mathrm{td}, J=7.0,1.0$ $\mathrm{Hz}, 1 \mathrm{H}), 2.03-1.97(\mathrm{~m}, 2 \mathrm{H}), 1.75-1.64(\mathrm{~m}, 4 \mathrm{H}), 1.26(\mathrm{~s}, 12 \mathrm{H}), 0.90$ (d, J = 6.6 Hz, $6 \mathrm{H}) .{ }^{13} \mathrm{C} \mathrm{NMR}\left(101 \mathrm{MHz}, \mathrm{CDCl}_{3}\right) \delta 145.7,83.2,38.0,28.4,24.9,22.8,14.2$. Carbon attached to boron not observed due to quadrupolar relaxation. ${ }^{11} \mathrm{~B}$ NMR $(128 \mathrm{MHz}$, $\left.\mathrm{CDCl}_{3}\right) \delta$ 30.6. ${ }^{11} \mathrm{~B}$ NMR $\left(128 \mathrm{MHz}, \mathrm{CDCl}_{3}\right) \delta$ 30.6. HRMS-EI $(\mathrm{m} / \mathrm{z})$ : Calc. for $\mathrm{C}_{13} \mathrm{H}_{25}{ }^{11} \mathrm{BO}_{2}[\mathrm{M}]^{+}$224.1942, found 224.1945.

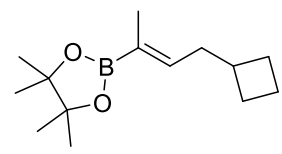

\section{(Z)-2-(4-Cyclobutylbut-2-en-2-yl)-4,4,5,5-tetramethyl-1,3,2-dioxaborolane (2g).}

The reaction was performed according to condition A. Colorless oil (> 98\% covn., 44 mg, $93 \%$ yield, $Z / E>20: 1,1 \%$ 1g). ${ }^{1} \mathrm{H}$ NMR $\left(400 \mathrm{MHz}, \mathrm{CDCl}_{3}\right) \delta 6.21(\mathrm{td}, J=7.0,1.4$ $\mathrm{Hz}, 1 \mathrm{H}), 2.41-2.26(\mathrm{~m}, 1 \mathrm{H}), 2.23-2.15(\mathrm{~m}, 2 \mathrm{H}), 2.08-1.98(\mathrm{~m}, 2 \mathrm{H}), 1.85-1.75(\mathrm{~m}$, 
2H), $1.65(\mathrm{~s}, 3 \mathrm{H}), 1.64-1.55(\mathrm{~m}, 2 \mathrm{H}), 1.23(\mathrm{~s}, 12 \mathrm{H}) .{ }^{13} \mathrm{C} \mathrm{NMR}\left(101 \mathrm{MHz}, \mathrm{CDCl}_{3}\right) \delta$ 144.6, 83.2, 36.0, 35.5, 28.3, 24.9, 18.6, 14.2. Carbon attached to boron not observed due to quadrupolar relaxation. ${ }^{11} \mathrm{~B}$ NMR $\left(128 \mathrm{MHz}, \mathrm{CDCl}_{3}\right) \delta 30.6$. HRMS-EI $(\mathrm{m} / \mathrm{z})$ : Calc. for $\mathrm{C}_{14} \mathrm{H}_{25}{ }^{10} \mathrm{BO}_{2}[\mathrm{M}]^{+} 235.1978$, found 235.1979.

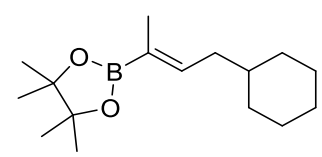

(Z)-2-(4-Cyclohexylbut-2-en-2-yl)-4,4,5,5-tetramethyl-1,3,2-dioxaborolane (2h).

The reaction was performed according to condition A. Colorless oil (98\% covn., $46 \mathrm{mg}$, $87 \%$ yield, $Z / E>20: 1,2 \%$ 1h). ${ }^{1} \mathrm{H} \mathrm{NMR}\left(400 \mathrm{MHz} \mathrm{CDCl}_{3}\right) \delta 6.36(\mathrm{td}, J=7.1,1.6 \mathrm{~Hz}$, 1H), $2.06-1.99(\mathrm{~m}, 2 \mathrm{H}), 1.78-1.60(\mathrm{~m}, 7 \mathrm{H}), 1.41-1.33(\mathrm{~m}, 1 \mathrm{H}), 1.28(\mathrm{~s}, 12 \mathrm{H}), 1.25$ - $1.11(\mathrm{~m}, 4 \mathrm{H}), 0.99-0.85(\mathrm{~m}, 2 \mathrm{H}) .{ }^{13} \mathrm{C} \mathrm{NMR}\left(101 \mathrm{MHz}, \mathrm{CDCl}_{3}\right) \delta$ 145.5, 83.1, 37.9, $36.7,33.5,26.7,26.5,24.9,14.2$. Carbon attached to boron not observed due to quadrupolar relaxation. ${ }^{11} \mathrm{~B}$ NMR $\left(128 \mathrm{MHz}, \mathrm{CDCl}_{3}\right) \delta$ 30.6. HRMS-EI $(\mathrm{m} / \mathrm{z})$ : Calc. for $\mathrm{C}_{16} \mathrm{H}_{29}{ }^{10} \mathrm{BO}_{2}[\mathrm{M}]^{+}$263.2291, found 263.2292.

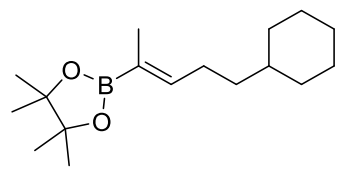

(Z)-2-(5-Cyclohexylpent-2-en-2-yl)-4,4,5,5-tetramethyl-1,3,2-dioxaborolane (2i).

The reaction was performed according to condition A. Colorless oil (96\% covn., $52 \mathrm{mg}$, 95\% yield, $Z / E>20: 1,4 \%$ 1i). ${ }^{1} \mathrm{H}$ NMR $\left(400 \mathrm{MHz}, \mathrm{CDCl}_{3}\right) \delta 6.30(\mathrm{td}, J=7.1,1.2 \mathrm{~Hz}$, 1H), $2.16-2.07(\mathrm{~m}, 1 \mathrm{H}), 1.78-1.60(\mathrm{~m}, 8 \mathrm{H}), 1.35-1.08(\mathrm{~m}, 19 \mathrm{H}), 0.95-0.82(\mathrm{~m}$, 2H). ${ }^{13} \mathrm{C} \mathrm{NMR}\left(101 \mathrm{MHz}, \mathrm{CDCl}_{3}\right) \delta 147.1,83.2,37.5,36.7,33.4,26.9,26.5,26.2,24.9$, 13.9. Carbon attached to boron not observed due to quadrupolar relaxation. ${ }^{11} \mathrm{~B}$ NMR $\left(128 \mathrm{MHz}, \mathrm{CDCl}_{3}\right) \delta$ 30.7. HRMS-EI $(\mathrm{m} / \mathrm{z})$ : Calc. for $\mathrm{C}_{17} \mathrm{H}_{31}{ }^{10} \mathrm{BO}_{2}[\mathrm{M}]^{+}$277.2448, found 277.2452 .

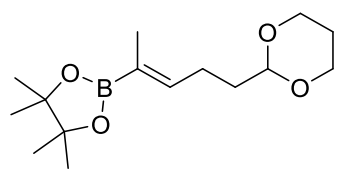

(Z)-2-(5-(1,3-Dioxan-2-yl)pent-2-en-2-yl)-4,4,5,5-tetramethyl-1,3,2-dioxaborolane $(\mathbf{2 j})$. 
The reaction was performed according to condition A. Colorless oil (97\% covn., $47 \mathrm{mg}$, $84 \%$ yield, $Z / E>20: 1,3 \%$ 1j). ${ }^{1} \mathrm{H}$ NMR $\left(400 \mathrm{MHz}, \mathrm{CDCl}_{3}\right) \delta 6.27(\mathrm{td}, J=7.1,1.5 \mathrm{~Hz}$, $1 \mathrm{H}), 4.48(\mathrm{t}, J=5.2 \mathrm{~Hz}, 1 \mathrm{H}), 4.11-4.04(\mathrm{~m}, 2 \mathrm{H}), 3.78-3.69(\mathrm{~m}, 2 \mathrm{H}), 2.25-2.15(\mathrm{~m}$, 2H), $2.12-1.98(\mathrm{~m}, 1 \mathrm{H}), 1.70-1.62(\mathrm{~m}, 5 \mathrm{H}), 1.36-1.29(\mathrm{~m}, 1 \mathrm{H}), 1.24(\mathrm{~s}, 12 \mathrm{H}) .{ }^{13} \mathrm{C}$ NMR (101 MHz, $\left.\mathrm{CDCl}_{3}\right) \delta 145.2,101.9,83.2,67.0,34.3,26.0,24.9,23.3,14.0$. Carbon attached to boron not observed due to quadrupolar relaxation. ${ }^{11} \mathrm{~B}$ NMR (128 MHz, $\left.\mathrm{CDCl}_{3}\right) \delta$ 30.4. HRMS-EI $(\mathrm{m} / \mathrm{z})$ : Calc. for $\mathrm{C}_{15} \mathrm{H}_{26}{ }^{10} \mathrm{BO}_{4}[\mathrm{M}-\mathrm{H}]^{+} 280.1950$, found 280.1961 .

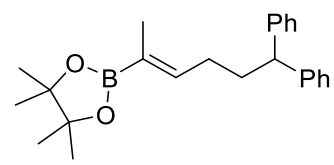

(Z)-2-(6,6-Diphenylhex-2-en-2-yl)-4,4,5,5-tetramethyl-1,3,2-dioxaborolane (2k).

The reaction was performed according to condition A. Colorless oil (97\% covn., $45 \mathrm{mg}$, 94\% yield, $Z / E>20: 1,3 \%$ 1k). ${ }^{1} \mathrm{H}$ NMR $\left(400 \mathrm{MHz} \mathrm{CDCl}_{3}\right) \delta 7.36-7.25(\mathrm{~m}, 8 \mathrm{H})$, $7.24-7.17(\mathrm{~m}, 2 \mathrm{H}), 6.41(\mathrm{t}, J=6.1 \mathrm{~Hz}, 1 \mathrm{H}), 3.97(\mathrm{t}, J=7.5 \mathrm{~Hz}, 1 \mathrm{H}), 2.28-2.19(\mathrm{~m}$, 2H), $2.19-2.10(\mathrm{~m}, 2 \mathrm{H}), 1.63(\mathrm{~s}, 3 \mathrm{H}), 1.32(\mathrm{~s}, 12 \mathrm{H}) .{ }^{13} \mathrm{C} \mathrm{NMR}\left(101 \mathrm{MHz}, \mathrm{CDCl}_{3}\right) \delta$ $145.8,145.1,128.5,128.0,126.2,83.2,51.0,34.8,27.2,24.9,14.0$. Carbon attached to boron not observed due to quadrupolar relaxation. ${ }^{11} \mathrm{~B} \mathrm{NMR}\left(128 \mathrm{MHz}, \mathrm{CDCl}_{3}\right) \delta 30.4$. HRMS-EI (m/z): Calc. for $\mathrm{C}_{24} \mathrm{H}_{31}{ }^{10} \mathrm{BO}_{2}[\mathrm{M}]^{+} 361.2448$, found 361.2456.

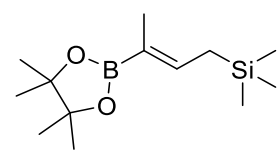

(Z)-Trimethyl(3-(4,4,5,5-tetramethyl-1,3,2-dioxaborolan-2-yl)but-2-en-1-yl)silane (2l).

The reaction was performed according to condition A. Colorless oil (96\% covn., $48 \mathrm{mg}$, $99 \%$ yield, $Z / E=19: 1,4 \%$ 1l). ${ }^{1} \mathrm{H}$ NMR $\left(400 \mathrm{MHz}, \mathrm{CDCl}_{3}\right) \delta 6.43(\mathrm{td}, J=8.7,1.4 \mathrm{~Hz}$, $1 \mathrm{H}), 1.65(\mathrm{~d}, J=8.7 \mathrm{~Hz}, 2 \mathrm{H}), 1.61(\mathrm{~s}, 3 \mathrm{H}), 1.24(\mathrm{~s}, 12 \mathrm{H}), 0.01(\mathrm{~s}, 9 \mathrm{H}) .{ }^{13} \mathrm{C}$ NMR $(101$ $\left.\mathrm{MHz}, \mathrm{CDCl}_{3}\right) \delta 143.6,82.9,24.9,21.5,13.8,-1.3$. Carbon attached to boron not observed due to quadrupolar relaxation. ${ }^{11} \mathrm{~B} \mathrm{NMR}\left(128 \mathrm{MHz}, \mathrm{CDCl}_{3}\right) \delta 30.0$. HRMS$\mathrm{EI}(\mathrm{m} / \mathrm{z})$ : Calc. for $\mathrm{C}_{13} \mathrm{H}_{27}{ }^{10} \mathrm{BO}_{2} \mathrm{Si}[\mathrm{M}]^{+} 253.1904$, found 253.1902 . 


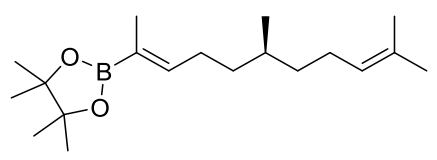

(R,Z)-2-(6,10-Dimethylundeca-2,9-dien-2-yl)-4,4,5,5-tetramethyl-1,3,2-

dioxaborolane $(\mathbf{2 m})$.

The reaction was performed according to condition A. Colorless oil (97\% covn., $59 \mathrm{mg}$, $97 \%$ yield, $Z / E>20: 1,3 \%$ 1m). ${ }^{1} \mathrm{H}$ NMR $\left(400 \mathrm{MHz}, \mathrm{CDCl}_{3}\right) \delta 6.29(\mathrm{td}, J=6.9,1.6$ $\mathrm{Hz}, 1 \mathrm{H}), 5.07(\mathrm{t}, J=7.1 \mathrm{~Hz}, 1 \mathrm{H}), 2.18-2.02(\mathrm{~m}, 2 \mathrm{H}), 2.01-1.85(\mathrm{~m}, 2 \mathrm{H}), 1.66(\mathrm{~s}$, $6 \mathrm{H}), 1.57$ (s, 3H), $1.45-1.35(\mathrm{~m}, 2 \mathrm{H}), 1.29-1.22(\mathrm{~m}, 13 \mathrm{H}), 1.19-1.05(\mathrm{~m}, 2 \mathrm{H}), 0.85$ $(\mathrm{d}, J=6.4 \mathrm{~Hz}, 3 \mathrm{H}) .{ }^{13} \mathrm{C} \mathrm{NMR}\left(101 \mathrm{MHz}, \mathrm{CDCl}_{3}\right) \delta 147.0,131.1,125.1,83.2,37.1$, 36.1, 32.4, 26.3, 25.9, 25.6, 24.9, 19.6, 17.8, 14.0. Carbon attached to boron not observed due to quadrupolar relaxation. ${ }^{11} \mathrm{~B} \mathrm{NMR}\left(128 \mathrm{MHz}, \mathrm{CDCl}_{3}\right) \delta 30.7$. HRMSEI $(m / z)$ : Calc. for $\mathrm{C}_{19} \mathrm{H}_{35}{ }^{10} \mathrm{BO}_{2}[\mathrm{M}]^{+} 305.2761$, found 305.2766.

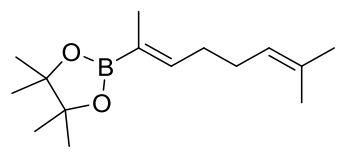

(Z)-4,4,5,5-Tetramethyl-2-(7-methylocta-2,6-dien-2-yl)-1,3,2-dioxaborolane (2n).

The reaction was performed according to condition A, 8 h. Colorless oil (96\% covn., $46 \mathrm{mg}, 91 \%$ yield, $Z / E>20: 1,3 \%$ 1n). ${ }^{1} \mathrm{H}$ NMR $\left(400 \mathrm{MHz}, \mathrm{CDCl}_{3}\right) \delta 6.32(\mathrm{dt}, J=6.7$, $3.3 \mathrm{~Hz}, 1 \mathrm{H}), 5.14(\mathrm{t}, J=6.9 \mathrm{~Hz}, 1 \mathrm{H}), 2.19-2.10(\mathrm{~m}, 2 \mathrm{H}), 2.10-2.01(\mathrm{~m}, 2 \mathrm{H}), 1.67(\mathrm{~s}$, 6H), 1.59 (s, 3H), 1.26 (s, 12H). $\left.{ }^{13} \mathrm{C} \mathrm{NMR} \mathrm{(101} \mathrm{MHz,} \mathrm{CDCl}_{3}\right) \delta 146.3,131.9,124.3$, 83.2, 29.2, 27.6, 25.8, 24.9, 17.8, 14.0. Carbon attached to boron not observed due to quadrupolar relaxation. HRMS-EI $(\mathrm{m} / z)$ : Calc. for $\mathrm{C}_{15} \mathrm{H}_{27}{ }^{10} \mathrm{BO}_{2}[\mathrm{M}]^{+} 249.2135$, found 249.2134 .

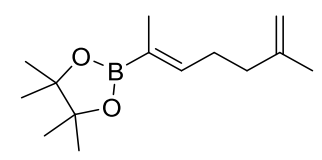

(Z)-4,4,5,5-tetramethyl-2-(6-methylhepta-2,6-dien-2-yl)-1,3,2-dioxaborolane (2o).

The reaction was performed according to condition A ( $0.1 \mathrm{mmol}$ scale). Colorless oil (95\% covn., $20 \mathrm{mg}, 83 \%$ yield, $Z / E>20: 1,4 \%$ 1o). ${ }^{1} \mathrm{H} \mathrm{NMR}\left(400 \mathrm{MHz}, \mathrm{CDCl}_{3}\right) \delta 6.30$ (td, $J=6.8,1.3 \mathrm{~Hz}, 1 \mathrm{H}), 4.71(\mathrm{~s}, 1 \mathrm{H}), 4.69(\mathrm{~s}, 1 \mathrm{H}), 2.31-2.22(\mathrm{~m}, 2 \mathrm{H}), 2.09$ (t, $J=8.6$ $\mathrm{Hz}, 2 \mathrm{H}), 1.72$ (s, 3H), 1.69 (s, 3H), 1.25 (s, 12H). ${ }^{13} \mathrm{C} \mathrm{NMR}\left(101 \mathrm{MHz}, \mathrm{CDCl}_{3}\right) \delta 145.9$, 
$145.8,110.0,83.2,36.9,27.2,24.9,22.7,14.0$. Carbon attached to boron not observed due to quadrupolar relaxation. ${ }^{11} \mathrm{~B}$ NMR $\left(128 \mathrm{MHz}, \mathrm{CDCl}_{3}\right) \delta 30.2$. HRMS-EI $(\mathrm{m} / \mathrm{z})$ : Calc. for $\mathrm{C}_{15} \mathrm{H}_{27}{ }^{11} \mathrm{BO}_{2}[\mathrm{M}]^{+}$236.1942, found 236.1941.

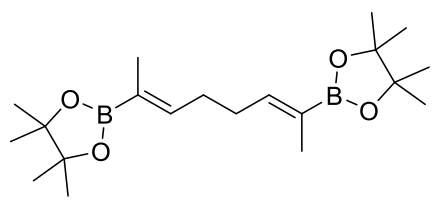

2,2'-((2Z,6Z)-Octa-2,6-diene-2,7-diyl)bis(4,4,5,5-tetramethyl-1,3,2-dioxaborolane) $(\mathbf{2 p})$.

The reaction was performed according to condition A. Colorless oil (97\% covn., $71 \mathrm{mg}$, $99 \%$ yield, $Z / E=17: 1,3 \%$ 1p). ${ }^{1} \mathrm{H}$ NMR $\left(400 \mathrm{MHz}, \mathrm{CDCl}_{3}\right) \delta 6.35(\mathrm{t}, J=5.1 \mathrm{~Hz}, 2 \mathrm{H})$, $2.28-2.16(\mathrm{~m}, 4 \mathrm{H}), 1.69$ (s, 6H), 1.28 (s, 24H). $\left.{ }^{13} \mathrm{C} \mathrm{NMR} \mathrm{(101} \mathrm{MHz,} \mathrm{CDCl}_{3}\right) \delta 145.8$, $83.2,28.2,24.9,14.0$. Carbon attached to boron not observed due to quadrupolar relaxation. ${ }^{11} \mathrm{~B}$ NMR $\left(128 \mathrm{MHz}, \mathrm{CDCl}_{3}\right) \delta$ 30.2. HRMS-FI $(\mathrm{m} / \mathrm{z})$ : Calc. for $\mathrm{C}_{20} \mathrm{H}_{36}{ }^{10} \mathrm{~B}_{2} \mathrm{O}_{4}[\mathrm{M}]^{+} 360.2867$, found 360.2874.

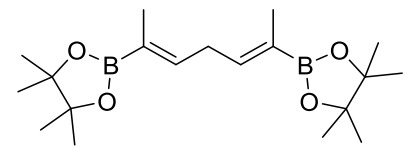

\section{2,2'-((2Z,5Z)-Hepta-2,5-diene-2,6-diyl)bis(4,4,5,5-tetramethyl-1,3,2-di}

oxaborolane) (2q).

The reaction was performed according to condition A $(0.1 \mathrm{mmol}$ scale $)$. Colorless oil (91\% covn., $32 \mathrm{mg}, 91 \%$ yield, $Z / E>20: 1,8 \%$ 1q). ${ }^{1} \mathrm{H} \mathrm{NMR}\left(400 \mathrm{MHz}, \mathrm{CDCl}_{3}\right) \delta 6.34$ $(\mathrm{td}, J=7.0,1.1 \mathrm{~Hz}, 2 \mathrm{H}), 2.99(\mathrm{t}, J=7.0 \mathrm{~Hz}, 2 \mathrm{H}), 1.72(\mathrm{~s}, 6 \mathrm{H}), 1.27(\mathrm{~s}, 24 \mathrm{H}) .{ }^{13} \mathrm{C} \mathrm{NMR}$ $\left(101 \mathrm{MHz}, \mathrm{CDCl}_{3}\right) \delta 143.2,83.3,28.7,24.9,14.1$. Carbon attached to boron not observed due to quadrupolar relaxation. ${ }^{11} \mathrm{~B}$ NMR $\left(128 \mathrm{MHz}, \mathrm{CDCl}_{3}\right) \delta 30.4$. HRMSFI $(m / z)$ : Calc. for $\mathrm{C}_{19} \mathrm{H}_{34}{ }^{10} \mathrm{~B}_{2} \mathrm{O}_{4}[\mathrm{M}]^{+} 346.2710$, found 346.2714 .

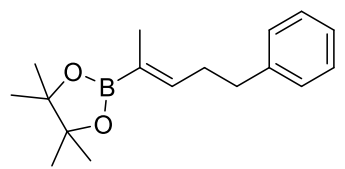

\section{(Z)-4,4,5,5-Tetramethyl-2-(5-phenylpent-2-en-2-yl)-1,3,2-dioxaborolane (2r).}

The reaction was performed according to condition A (reaction time: $8 \mathrm{~h}$ ). Colorless oil (97\% covn., $53 \mathrm{mg}, 99 \%$ yield, Z/E > 20:1, 3\% 1r). ${ }^{1} \mathrm{H} \mathrm{NMR}\left(400 \mathrm{MHz}, \mathrm{CDCl}_{3}\right) \delta 7.30$ 
$-7.22(\mathrm{~m}, 2 \mathrm{H}), 7.22-7.14(\mathrm{~m}, 3 \mathrm{H}), 6.39(\mathrm{t}, J=6.3 \mathrm{~Hz}, 1 \mathrm{H}), 2.70(\mathrm{t}, J=8.3 \mathrm{~Hz}, 2 \mathrm{H})$, $2.49-2.37$ (m, 2H), 1.66 (s, 3H), 1.26 (s, 12H). $\left.{ }^{13} \mathrm{C} \mathrm{NMR} \mathrm{(101} \mathrm{MHz,} \mathrm{CDCl}_{3}\right) \delta 145.4$, $142.3,128.4,125.9,83.3,35.3,30.9,25.0,24.9,14.0$. Carbon attached to boron not observed due to quadrupolar relaxation. These spectroscopic data correspond to reported data. ${ }^{20}$

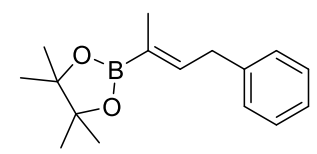

(Z)-4,4,5,5-Tetramethyl-2-(4-phenylbut-2-en-2-yl)-1,3,2-dioxaborolane (2s).

The reaction was performed according to condition A (reaction time: $8 \mathrm{~h}$ ). Colorless oil (92\% covn., $48 \mathrm{mg}, 95 \%$ yield, $Z / E>20: 1,7 \%$ 1s). ${ }^{1} \mathrm{H} \mathrm{NMR}\left(400 \mathrm{MHz}, \mathrm{CDCl}_{3}\right) \delta 7.36$ - $7.27(\mathrm{~m}, 2 \mathrm{H}), 7.27-7.17(\mathrm{~m}, 3 \mathrm{H}), 6.52$ (t, $J=6.9 \mathrm{~Hz}, 1 \mathrm{H}), 3.52(\mathrm{~d}, J=6.9 \mathrm{~Hz}, 2 \mathrm{H})$, 1.85 (s, 3H), 1.29 (s, 12H). ${ }^{13} \mathrm{C}$ NMR (101 MHz, $\left.\mathrm{CDCl}_{3}\right) \delta 144.4,140.6,128.8,128.5$, $126.0,83.3,35.2,24.9,14.2$. Carbon attached to boron not observed due to quadrupolar relaxation. These spectroscopic data correspond to reported data. ${ }^{13}$

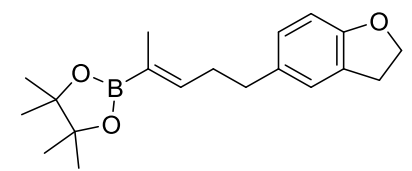

\section{(Z)-2-(5-(2,3-Dihydrobenzofuran-6-yl)pent-2-en-2-yl)-4,4,5,5-tetramethyl-1,3,2-}

dioxaborolane $(2 t)$.

The reaction was performed according to condition A (reaction time: $8 \mathrm{~h}$ ). Colorless oil (96\% covn., $49 \mathrm{mg}, 79 \%$ yield, $Z / E>20: 1,3 \%$ 1s). ${ }^{1} \mathrm{H} \mathrm{NMR}\left(400 \mathrm{MHz}, \mathrm{CDCl}_{3}\right) \delta 7.04$ (s, 1H), $6.93(\mathrm{~d}, J=8.1 \mathrm{~Hz}, 1 \mathrm{H}), 6.70(\mathrm{~d}, J=8.1 \mathrm{~Hz}, 1 \mathrm{H}), 6.39(\mathrm{td}, J=7.0,1.6 \mathrm{~Hz}, 1 \mathrm{H})$, $4.54(\mathrm{t}, J=8.7 \mathrm{~Hz}, 2 \mathrm{H}), 3.18(\mathrm{t}, J=8.7 \mathrm{~Hz}, 2 \mathrm{H}), 2.64(\mathrm{t}, J=8.1 \mathrm{~Hz}, 2 \mathrm{H}), 2.43-2.36$ (m, 2H), 1.67 (s, 3H), 1.27 (s, 12H). $\left.{ }^{13} \mathrm{C} \mathrm{NMR} \mathrm{(101} \mathrm{MHz,} \mathrm{CDCl}_{3}\right) \delta$ 158.3, 145.5, 134.3, $127.8,127.0,124.9,109.0,83.2,71.2,34.7,31.5,29.9,24.9,14.0$. Carbon attached to boron not observed due to quadrupolar relaxation. ${ }^{11} \mathrm{~B} \mathrm{NMR}\left(128 \mathrm{MHz}, \mathrm{CDCl}_{3}\right) \delta 30.4$. HRMS-EI (m/z): Calc. for $\mathrm{C}_{19} \mathrm{H}_{27}{ }^{10} \mathrm{BO}_{3}[\mathrm{M}]^{+} 313.2084$, found 313.2080.

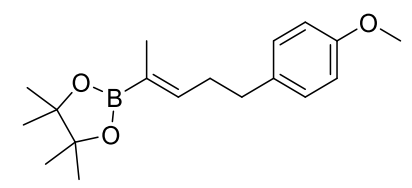




\section{(Z)-2-(5-(4-Methoxyphenyl)pent-2-en-2-yl)-4,4,5,5-tetramethyl-1,3,2-}

dioxaborolane $(2 \mathrm{u})$.

The reaction was performed according to condition A (reaction time: $8 \mathrm{~h}$ ). Colorless oil (97\% covn., $58 \mathrm{mg}, 96 \%$ yield, $Z / E>20: 1,3 \%$ 1t). ${ }^{1} \mathrm{H}$ NMR $\left(400 \mathrm{MHz}, \mathrm{CDCl}_{3}\right) \delta 7.12$ $(\mathrm{d}, J=8.4 \mathrm{~Hz}, 2 \mathrm{H}), 6.83(\mathrm{~d}, J=8.4 \mathrm{~Hz}, 2 \mathrm{H}), 6.39(\mathrm{td}, J=6.7,1.6 \mathrm{~Hz}, 1 \mathrm{H}), 3.79(\mathrm{~s}, 3 \mathrm{H})$, $2.66(\mathrm{t}, J=8.4 \mathrm{~Hz}, 2 \mathrm{H}), 2.46-2.37(\mathrm{~m}, 2 \mathrm{H}), 1.66(\mathrm{~s}, 3 \mathrm{H}), 1.27$ (s, 12H). Carbon attached to boron not observed due to quadrupolar relaxation. ${ }^{13} \mathrm{C}$ NMR $(101 \mathrm{MHz}$, $\left.\mathrm{CDCl}_{3}\right) \delta 157.9,145.5,134.4,129.3,113.8,83.3,55.4,34.3,31.2,24.9,14.0 .{ }^{11} \mathrm{~B}$ NMR $\left(128 \mathrm{MHz}, \mathrm{CDCl}_{3}\right) \delta$ 30.3. HRMS-EI $(\mathrm{m} / \mathrm{z})$ : Calc. for $\mathrm{C}_{18} \mathrm{H}_{27}{ }^{11} \mathrm{BO}_{2}[\mathrm{M}]^{+}$302.2048, found 302.2051.

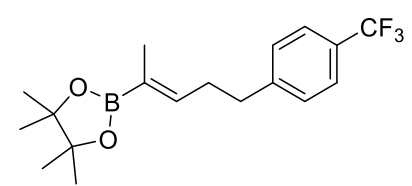

(Z)-4,4,5,5-Tetramethyl-2-(5-(4-(trifluoromethyl)phenyl)pent-2-en-2-yl)-1,3,2dioxaborolane $(2 \mathrm{v})$.

The reaction was performed according to condition A (reaction time: $8 \mathrm{~h}, 0.1 \mathrm{mmol}$ scale). Colorless oil (95\% covn., $32 \mathrm{mg}, 94 \%$ yield, $Z / E>20: 1,5 \%$ 1u). ${ }^{1} \mathrm{H}$ NMR (400 $\left.\mathrm{MHz}, \mathrm{CDCl}_{3}\right) \delta 7.55(\mathrm{~d}, J=8.0 \mathrm{~Hz}, 2 \mathrm{H}), 7.32(\mathrm{~d}, J=7.9 \mathrm{~Hz}, 2 \mathrm{H}), 6.38(\mathrm{td}, J=6.8,1.2$ $\mathrm{Hz}, 1 \mathrm{H}), 2.79(\mathrm{t}, J=7.7 \mathrm{~Hz}, 2 \mathrm{H}), 2.52-2.43(\mathrm{~m}, 2 \mathrm{H}), 1.68(\mathrm{~s}, 3 \mathrm{H}), 1.29(\mathrm{~s}, 12 \mathrm{H}) .{ }^{13} \mathrm{C}$ NMR (101 MHz, $\left.\mathrm{CDCl}_{3}\right) \delta 146.2,144.3,128.6,128.2(\mathrm{q}, J=32.6 \mathrm{~Hz}), 125.2(\mathrm{q}, J=$ $3.7 \mathrm{~Hz}), 124.3(\mathrm{q}, J=270.1 \mathrm{~Hz}), 83.2,34.9,30.3,24.8,13.9$. Carbon attached to boron not observed due to quadrupolar relaxation. ${ }^{11} \mathrm{~B}$ NMR $\left(128 \mathrm{MHz}, \mathrm{CDCl}_{3}\right) \delta 30.4 .{ }^{19} \mathrm{~F}$ NMR $\left(377 \mathrm{MHz}, \mathrm{CDCl}_{3}\right) \delta$-62.3. HRMS-FI $(\mathrm{m} / \mathrm{z})$ : Calc. for $\mathrm{C}_{18} \mathrm{H}_{24}{ }^{10} \mathrm{BF}_{3} \mathrm{O}_{2}[\mathrm{M}]^{+}$ 339.1852 , found 339.1847 .

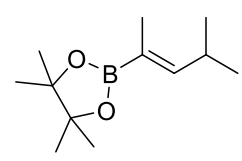

(Z)-4,4,5,5-tetramethyl-2-(4-methylpent-2-en-2-yl)-1,3,2-dioxaborolane (2w).

The reaction was performed according to condition B. Colorless oil (92\% covn., $18 \mathrm{mg}$, $86 \%$ yield, $Z / E=16: 1,7 \%$ 1v). ${ }^{1} \mathrm{H}$ NMR $\left(400 \mathrm{MHz}, \mathrm{CDCl}_{3}\right) \delta 6.12(\mathrm{dd}, J=9.1,1.6 \mathrm{~Hz}$, $1 \mathrm{H}), 2.75-2.61(\mathrm{~m}, 1 \mathrm{H}), 1.68(\mathrm{~s}, 3 \mathrm{H}), 1.26(\mathrm{~s}, 12 \mathrm{H}), 0.96(\mathrm{~d}, J=6.7 \mathrm{~Hz}, 6 \mathrm{H}) .{ }^{13} \mathrm{C} \mathrm{NMR}$ 
$\left(101 \mathrm{MHz}, \mathrm{CDCl}_{3}\right) \delta 153.5,83.2,27.6,25.0,22.4,13.9$. Carbon attached to boron not observed due to quadrupolar relaxation. ${ }^{11} \mathrm{~B} \mathrm{NMR}\left(128 \mathrm{MHz}, \mathrm{CDCl}_{3}\right) \delta 30.3$. These spectroscopic data correspond to reported data. ${ }^{13}$

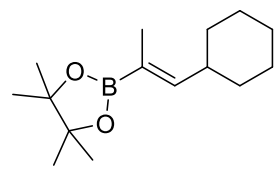

\section{(Z)-2-(1-cyclohexylprop-1-en-2-yl)-4,4,5,5-tetramethyl-1,3,2-dioxaborolane (2x).}

The reaction was performed according to condition B. Colorless oil (92\% covn., $22 \mathrm{mg}$, $89 \%$ yield, $Z / E=15: 1,7 \%$ 1w). ${ }^{1} \mathrm{H}$ NMR $\left(400 \mathrm{MHz}, \mathrm{CDCl}_{3}\right) \delta 6.12(\mathrm{dd}, J=8.9,1.5$ $\mathrm{Hz}, 1 \mathrm{H}), 2.41-2.29(\mathrm{~m}, 1 \mathrm{H}), 1.76-1.56(\mathrm{~m}, 7 \mathrm{H}), 1.25(\mathrm{~m}, 18 \mathrm{H}) .{ }^{13} \mathrm{C}$ NMR $(101 \mathrm{MHz}$, $\left.\mathrm{CDCl}_{3}\right) \delta 151.9,83.2,37.7,32.4,26.3,26.1,25.0,14.0$. Carbon attached to boron not observed due to quadrupolar relaxation. ${ }^{11} \mathrm{~B} \mathrm{NMR}\left(128 \mathrm{MHz}, \mathrm{CDCl}_{3}\right) \delta 30.4$. These spectroscopic data correspond to reported data. ${ }^{21}$

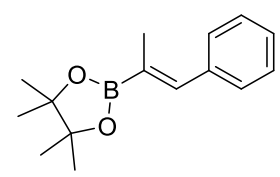

(Z)-4,4,5,5-Tetramethyl-2-(1-phenylprop-1-en-2-yl)-1,3,2-dioxaborolane (2y).

The reaction was performed according to condition B. Colorless oil (97\% covn., $22 \mathrm{mg}$, 91\% yield, $Z / E>20: 1,3 \%$ 1x). ${ }^{1} \mathrm{H}$ NMR $\left(400 \mathrm{MHz}, \mathrm{CDCl}_{3}\right) \delta 7.44-7.30(\mathrm{~m}, 4 \mathrm{H})$, $7.25-7.18(\mathrm{~m}, 2 \mathrm{H}), 2.00(\mathrm{~d}, J=1.4 \mathrm{~Hz}, 3 \mathrm{H}), 1.32(\mathrm{~s}, 12 \mathrm{H}) .{ }^{13} \mathrm{C}$ NMR (101 MHz, $\left.\mathrm{CDCl}_{3}\right) \delta 142.5,138.1,129.5,128.2,127.2,83.6,25.0,16.0$. Carbon attached to boron not observed due to quadrupolar relaxation. These spectroscopic data correspond to reported data. ${ }^{21}$

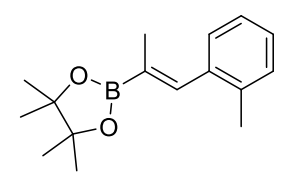

(Z)-4,4,5,5-Tetramethyl-2-(1-(o-tolyl)prop-1-en-2-yl)-1,3,2-dioxaborolane (2z).

The reaction was performed according to condition B. Colorless oil ( >98\% covn., 24 mg, 93\% yield, $Z / E>20: 1,1 \%$ 1y). ${ }^{1} \mathrm{H}$ NMR (400 MHz, $\left.\mathrm{CDCl}_{3}\right) \delta$ 7.29-7.27 (m, 1H), $7.22-7.13(\mathrm{~m}, 4 \mathrm{H}), 2.28(\mathrm{~s}, 3 \mathrm{H}), 1.80(\mathrm{~d}, J=1.8 \mathrm{~Hz}, 3 \mathrm{H}), 1.32(\mathrm{~s}, 12 \mathrm{H}) .{ }^{13} \mathrm{C}$ NMR 
$\left(101 \mathrm{MHz}, \mathrm{CDCl}_{3}\right) \delta 142.0,137.3,136.4,129.9,129.1,127.2,125.2,83.6,25.0,20.0$, 15.8. Carbon attached to boron not observed due to quadrupolar relaxation. These spectroscopic data correspond to reported data. ${ }^{21}$

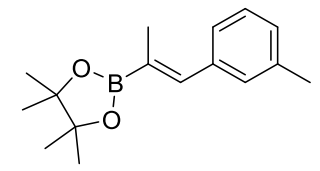

(Z)-4,4,5,5-tetramethyl-2-(1-(m-tolyl)prop-1-en-2-yl)-1,3,2-dioxaborolane (2aa).

The reaction was performed according to condition B. Colorless oil (97\% covn., $24 \mathrm{mg}$, $94 \%$ yield, $Z / E=19: 1,3 \%$ 1aa). ${ }^{1} \mathrm{H}$ NMR $\left(400 \mathrm{MHz}, \mathrm{CDCl}_{3}\right) \delta 7.25-7.18(\mathrm{~m}, 4 \mathrm{H})$, $7.06(\mathrm{~d}, J=7.2 \mathrm{~Hz}, 1 \mathrm{H}), 2.36(\mathrm{~s}, 3 \mathrm{H}), 1.99(\mathrm{~d}, J=1.8 \mathrm{~Hz}, 3 \mathrm{H}), 1.32(\mathrm{~s}, 12 \mathrm{H}) .{ }^{13} \mathrm{C}$ NMR $\left(101 \mathrm{MHz}, \mathrm{CDCl}_{3}\right) \delta 142.7,138.0,137.6,130.3,128.1,128.0,126.6,83.6,25.0,21.6$, 16.1. ${ }^{11} \mathrm{~B}$ NMR $\left(128 \mathrm{MHz}, \mathrm{CDCl}_{3}\right) \delta 30.8$. Carbon attached to boron not observed due to quadrupolar relaxation. These spectroscopic data correspond to reported data. ${ }^{22}$

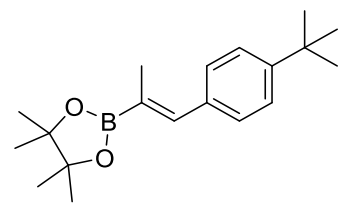

(Z)-2-(1-(4-(Tert-butyl)phenyl)prop-1-en-2-yl)-4,4,5,5-tetramethyl-1,3,2-

dioxaborolane (2ab).

The reaction was performed according to condition B. Colorless oil (98\% covn., $27 \mathrm{mg}$, 91\% yield, $Z / E>20: 1,2 \%$ 1ab). ${ }^{1} \mathrm{H}$ NMR $\left(400 \mathrm{MHz}, \mathrm{CDCl}_{3}\right) \delta 7.41-7.32(\mathrm{~m}, 4 \mathrm{H})$, $7.21(\mathrm{~s}, 1 \mathrm{H}), 2.01(\mathrm{~d}, J=1.6 \mathrm{~Hz}, 3 \mathrm{H}), 1.33(\mathrm{~s}, 9 \mathrm{H}), 1.32(\mathrm{~s}, 12 \mathrm{H}) .{ }^{13} \mathrm{C} \mathrm{NMR}(101 \mathrm{MHz}$, $\left.\mathrm{CDCl}_{3}\right) \delta 150.2,142.4,135.3,129.4,125.1,83.6,34.7,31.6,25.0,16.1$. Carbon attached to boron not observed due to quadrupolar relaxation. ${ }^{11} \mathrm{~B}$ NMR (128 MHz, $\left.\mathrm{CDCl}_{3}\right) \delta$ 31.1. HRMS-FI $(\mathrm{m} / \mathrm{z})$ : Calc. for $\mathrm{C}_{19} \mathrm{H}_{29}{ }^{10} \mathrm{BO}_{2}[\mathrm{M}]^{+} 299.2291$, found 299.2287.

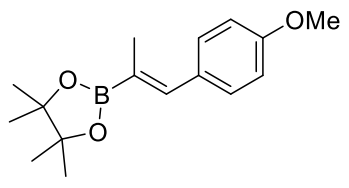

(Z)-2-(1-(4-Methoxyphenyl)prop-1-en-2-yl)-4,4,5,5-tetramethyl-1,3,2-

dioxaborolane (2ac). 
The reaction was performed according to condition B. Colorless oil (97\% covn., $25 \mathrm{mg}$, $93 \%$ yield, $Z / E>20: 1,3 \%$ 1ac).

${ }^{1} \mathrm{H}$ NMR $\left(400 \mathrm{MHz}, \mathrm{CDCl}_{3}\right) \delta 7.36(\mathrm{~d}, J=7.6 \mathrm{~Hz}, 2 \mathrm{H}), 7.18(\mathrm{~s}, 1 \mathrm{H}), 6.88(\mathrm{~d}, J=7.6$ $\mathrm{Hz}, 2 \mathrm{H}), 3.82$ (s, 3H), 1.99 (s, 3H), 1.31 (s, 12H). ${ }^{13} \mathrm{C} \mathrm{NMR}\left(101 \mathrm{MHz}, \mathrm{CDCl}_{3}\right) \delta 158.8$, 142.1, 131.1, 130.9, 113.6, 83.6, 55.4, 25.0, 16.1. Carbon attached to boron not observed due to quadrupolar relaxation. These spectroscopic data correspond to reported data. ${ }^{22}$

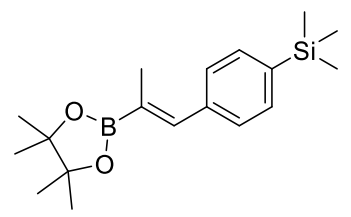

(Z)-trimethyl(4-(2-(4,4,5,5-tetramethyl-1,3,2-dioxaborolan-2-yl)prop-1-en-1yl)phenyl)silane (2ad).

The reaction was performed according to condition B. Colorless oil (97\% covn., $28 \mathrm{mg}$, $89 \%$ yield, $Z / E=19: 1,3 \%$ 1ad). ${ }^{1} \mathrm{H}$ NMR $\left(400 \mathrm{MHz}, \mathrm{CDCl}_{3}\right) \delta 7.51(\mathrm{~d}, J=8.0 \mathrm{~Hz}$, 2H), $7.38(\mathrm{~d}, J=8.0 \mathrm{~Hz}, 2 \mathrm{H}), 7.23(\mathrm{~s}, 1 \mathrm{H}), 2.00(\mathrm{~d}, J=1.7 \mathrm{~Hz}, 3 \mathrm{H}), 1.32(\mathrm{~s}, 12 \mathrm{H}), 0.27$ (s, 9H). ${ }^{13} \mathrm{C}$ NMR $\left(101 \mathrm{MHz}, \mathrm{CDCl}_{3}\right) \delta 142.5,139.5,138.4,133.2,128.8,83.7,25.0$, 16.1, -1.0. Carbon attached to boron not observed due to quadrupolar relaxation. ${ }^{11} \mathrm{~B}$ NMR $\left(128 \mathrm{MHz}, \mathrm{CDCl}_{3}\right) \delta$ 31.0. HRMS-EI (m/z): Calc. for $\mathrm{C}_{18} \mathrm{H}_{29}{ }^{11} \mathrm{BO}_{2}[\mathrm{M}]^{+}$ 316.2024, found 316.2032.

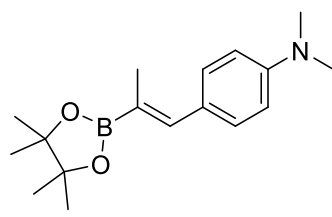

(Z)-N, $N$-dimethyl-4-(2-(4,4,5,5-tetramethyl-1,3,2-dioxaborolan-2-yl)prop-1-en-1yl)aniline (2ae).

The reaction was performed according to condition B. Colorless oil (98\% covn., $25 \mathrm{mg}$, $89 \%$ yield, $Z / E>20: 1,2 \%$ 1ae). ${ }^{1} \mathrm{H} \mathrm{NMR}\left(400 \mathrm{MHz}, \mathrm{CDCl}_{3}\right) \delta 7.38$ (d, $J=8.8 \mathrm{~Hz}$, 2H), $7.16(\mathrm{~s}, 1 \mathrm{H}), 6.71(\mathrm{~d}, J=8.8 \mathrm{~Hz}, 2 \mathrm{H}), 2.98(\mathrm{~s}, 6 \mathrm{H}), 2.04(\mathrm{~s}, 3 \mathrm{H}), 1.32(\mathrm{~s}, 12 \mathrm{H}) .{ }^{13} \mathrm{C}$ NMR (101 MHz, $\left.\mathrm{CDCl}_{3}\right) \delta 149.6,142.8,131.1,126.7,111.8,83.4,40.5,25.0,16.2$. Carbon attached to boron not observed due to quadrupolar relaxation. ${ }^{11} \mathrm{~B}$ NMR (128 $\left.\mathrm{MHz}, \mathrm{CDCl}_{3}\right) \delta$ 31.3. HRMS-EI $(\mathrm{m} / \mathrm{z})$ : Calc. for $\mathrm{C}_{17} \mathrm{H}_{26}{ }^{11} \mathrm{BNO}_{2}[\mathrm{M}]^{+} 287.2051$, found 
287.2052.

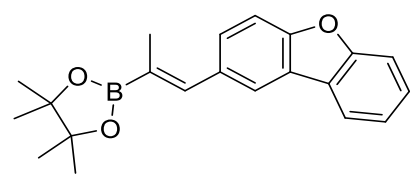

\section{(Z)-2-(1-(Tibenzo[b,d]furan-2-yl)prop-1-en-2-yl)-4,4,5,5-tetramethyl-1,3,2-}

\section{dioxaborolane (2af).}

The reaction was performed according to condition B. Colorless oil (98\% covn., $31 \mathrm{mg}$, 93\% yield, $Z / E>20: 1,2 \%$ 1af). ${ }^{1} \mathrm{H}$ NMR (400 $\left.\mathrm{MHz}, \mathrm{CDCl}_{3}\right) \delta 7.98(\mathrm{~s}, 1 \mathrm{H}), 7.97-$ $7.92(\mathrm{~m}, 1 \mathrm{H}), 7.60-7.48(\mathrm{~m}, 3 \mathrm{H}), 7.48-7.43(\mathrm{~m}, 1 \mathrm{H}), 7.41(\mathrm{~s}, 1 \mathrm{H}), 7.38-7.30(\mathrm{~m}$, $1 \mathrm{H}), 2.08(\mathrm{~d}, J=1.8 \mathrm{~Hz}, 3 \mathrm{H}), 1.34(\mathrm{~s}, 12 \mathrm{H}) .{ }^{13} \mathrm{C} \mathrm{NMR}\left(101 \mathrm{MHz}, \mathrm{CDCl}_{3}\right) \delta 156.7$, 155.4, 142.5, 133.0, 129.1, 127.3, 124.4, 124.2, 122.9, 121.5, 120.8, 111.8, 111.3, 83.7, 25.0, 16.1. Carbon attached to boron not observed due to quadrupolar relaxation. ${ }^{11} \mathrm{~B}$ NMR (128 MHz, $\left.\mathrm{CDCl}_{3}\right) \delta 30.7$. HRMS-EI $(m / z)$ : Calc. for $\mathrm{C}_{21} \mathrm{H}_{23}{ }^{11} \mathrm{BO}_{3}[\mathrm{M}]^{+} 334.1735$, found 334.1748 .

\section{Isomerization of $\omega$-alkenylboronates}

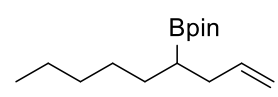

\section{4,4,5,5-tetramethyl-2-(non-1-en-4-yl)-1,3,2-dioxaborolane (1aj).}

Prepared according to according to previously reported procedure. ${ }^{23}{ }^{1} \mathrm{H}$ NMR (400 $\left.\mathrm{MHz}, \mathrm{CDCl}_{3}\right) \delta 5.83(\mathrm{ddt}, J=17.0,10.2,6.9 \mathrm{~Hz}, 1 \mathrm{H}), 5.11-4.88(\mathrm{~m}, 2 \mathrm{H}), 2.26-2.08$ $(\mathrm{m}, 2 \mathrm{H}), 1.50-1.28(\mathrm{~m}, 8 \mathrm{H}), 1.26(\mathrm{~s}, 12 \mathrm{H}), 1.13-1.04(\mathrm{~m}, 1 \mathrm{H}), 0.89(\mathrm{t}, J=6.8 \mathrm{~Hz}$, $3 \mathrm{H})$. These spectroscopic data correspond to reported data. ${ }^{24}$

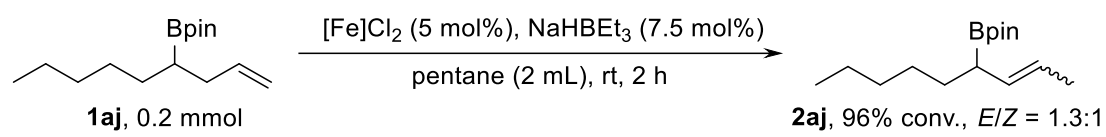

\section{4,4,5,5-tetramethyl-2-(non-2-en-4-yl)-1,3,2-dioxaborolane (2aj).}

In a nitrogen-filled glove-box, an oven-dried $10 \mathrm{~mL}$ thin-wall glass tube was charged with $[\mathrm{Fe}] \mathrm{Cl}_{2}(10 \mu \mathrm{mol}, 5.6 \mathrm{mg})$, pentane $(2 \mathrm{~mL})$, 1aj $(0.2 \mathrm{mmol}, 50 \mathrm{mg})$, then $\mathrm{NaHBEt}_{3}$ (15 $\mu \mathrm{mol}, 15 \mu \mathrm{L}, 1 \mathrm{M}$ in THF) was added to the glass tube. The tube was sealed with a Teflon plug, and the solution was stirred at room temperature for $2 \mathrm{~h}$. The resulting mixture was concentrated under vacuum and the residue was purified by flash silica gel 
column chromatography to give the product as colorless oil. (96\% conv., $44 \mathrm{mg}, 88 \%$ yield, $Z / E=1.3: 1,3 \%$ 1aj). $(\boldsymbol{E})-\mathbf{2 a j}:{ }^{1} \mathrm{H} \mathrm{NMR}\left(400 \mathrm{MHz}, \mathrm{CDCl}_{3}\right) \delta 5.48-5.26(\mathrm{~m}, 2 \mathrm{H})$, $1.77-1.67(\mathrm{~m}, 1 \mathrm{H}), 1.65-1.61(\mathrm{~m}, 3 \mathrm{H}), 1.56-1.25(\mathrm{~m}, 8 \mathrm{H}), 1.22(\mathrm{~s}, 12 \mathrm{H}), 0.86(\mathrm{t}, J$ $=6.7 \mathrm{~Hz}, 3 \mathrm{H}) .{ }^{13} \mathrm{C} \mathrm{NMR}\left(101 \mathrm{MHz}, \mathrm{CDCl}_{3}\right) \delta 131.9,123.9,82.9,31.9,31.0,28.7,24.7$, 24.6, 22.6, 14.0. ${ }^{11} \mathrm{~B}$ NMR (128 MHz, $\left.\mathrm{CDCl}_{3}\right) \delta$ 33.4. HRMS-EI $(\mathrm{m} / \mathrm{z})$ : Calc. for $\mathrm{C}_{21} \mathrm{H}_{23}{ }^{11} \mathrm{BO}_{3}[\mathrm{M}]^{+}$252.2255, found 252.2257. (Z)-2aj: ${ }^{1} \mathrm{H}$ NMR (400 MHz, $\left.\mathrm{CDCl}_{3}\right) \delta$ $5.48-5.26(\mathrm{~m}, 2 \mathrm{H}), 2.15-2.00(\mathrm{~m}, 1 \mathrm{H}), 1.59(\mathrm{dd}, J=6.7,1.7 \mathrm{~Hz}, 3 \mathrm{H}), 1.56-1.25$ $(\mathrm{m}, 8 \mathrm{H}), 1.22(\mathrm{~s}, 12 \mathrm{H}), 0.86(\mathrm{t}, J=6.7 \mathrm{~Hz}, 3 \mathrm{H}) .{ }^{13} \mathrm{C} \mathrm{NMR}\left(101 \mathrm{MHz}, \mathrm{CDCl}_{3}\right) \delta 131.8$, 122.8, 82.9, 31.2, 28.8, 24.8, 24.7, 24.5, 18.1, 13.1. ${ }^{11} \mathrm{~B} \mathrm{NMR}\left(128 \mathrm{MHz}, \mathrm{CDCl}_{3}\right) \delta 33.4$. HRMS-EI $(m / z)$ : Calc. for $\mathrm{C}_{21} \mathrm{H}_{23}{ }^{11} \mathrm{BO}_{3}[\mathrm{M}]^{+}$252.2255, found 252.2257.

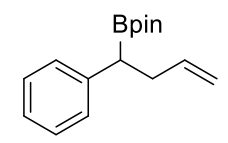

\section{4,4,5,5-tetramethyl-2-(1-phenylbut-3-en-1-yl)-1,3,2-dioxaborolane (1ak).}

Prepared according to according to previously reported procedure. ${ }^{25}{ }^{1} \mathrm{H}$ NMR (400 $\left.\mathrm{MHz}, \mathrm{CDCl}_{3}\right) \delta 7.29-7.19(\mathrm{~m}, 5 \mathrm{H}), 7.16-7.10(\mathrm{~m}, 1 \mathrm{H}), 5.82(\mathrm{ddt}, J=16.6,9.9,6.3$ $\mathrm{Hz}, 1 \mathrm{H}), 5.09-4.85(\mathrm{~m}, 2 \mathrm{H}), 2.66-2.52(\mathrm{~m}, 1 \mathrm{H}), 2.47-2.34(\mathrm{~m}, 2 \mathrm{H}), 1.20(\mathrm{~s}, 6 \mathrm{H})$, $1.18(\mathrm{~s}, 6 \mathrm{H})$. These spectroscopic data correspond to reported data. ${ }^{25}$

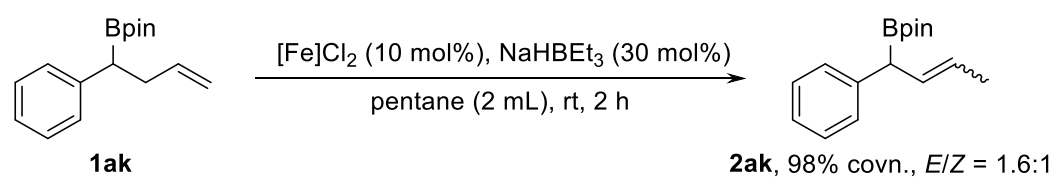

\section{4,4,5,5-tetramethyl-2-(1-phenylbut-3-en-1-yl)-1,3,2-dioxaborolane (2ak).}

In a nitrogen-filled glove-box, an oven-dried $10 \mathrm{~mL}$ thin-wall glass tube was charged with $[\mathrm{Fe}] \mathrm{Cl}_{2}(10 \mu \mathrm{mol}, 5.6 \mathrm{mg})$, pentane $(2 \mathrm{~mL})$, 1ak $(0.1 \mathrm{mmol}, 26 \mathrm{mg})$, then $\mathrm{NaHBEt}_{3}$ (30 $\mu \mathrm{mol}, 30 \mu \mathrm{L}, 1 \mathrm{M}$ in THF) was added to the glass tube. The tube was sealed with a Teflon plug, and the solution was stirred at room temperature for $2 \mathrm{~h}$. The resulting mixture was concentrated under vacuum and the residue was purified by flash silica gel column chromatography to give the product as colorless oil. (98\% conv., $24 \mathrm{mg}, 92 \%$ yield, $Z / E=1.7: 1,2 \%$ 1ak). $(\boldsymbol{E})-2$ ak: ${ }^{1} \mathrm{H}$ NMR $\left(400 \mathrm{MHz}, \mathrm{CDCl}_{3}\right) \delta 7.30-7.19(\mathrm{~m}$, 4H), $7.16-7.11(\mathrm{~m}, 1 \mathrm{H}), 5.78-5.66(\mathrm{~m}, 1 \mathrm{H}), 5.60-5.39(\mathrm{~m}, 1 \mathrm{H}), 3.17(\mathrm{~d}, J=8.2 \mathrm{~Hz}$, $1 \mathrm{H}), 1.70-1.64(\mathrm{~m}, 3 \mathrm{H}), 1.21(\mathrm{~s}, 12 \mathrm{H}) .{ }^{13} \mathrm{C} \mathrm{NMR}\left(101 \mathrm{MHz}, \mathrm{CDCl}_{3}\right) \delta$ 142.3, 130.7, 
128.6, 128.4, 125.4, 83.6, 24.7, 24.7, 13.2. (Z)-2ak: ${ }^{1} \mathrm{H} \mathrm{NMR}\left(400 \mathrm{MHz}, \mathrm{CDCl}_{3}\right) \delta 7.30$ $-7.19(\mathrm{~m}, 4 \mathrm{H}), 7.16-7.11(\mathrm{~m}, 1 \mathrm{H}), 5.78-5.66(\mathrm{~m}, 1 \mathrm{H}), 5.60-5.39(\mathrm{~m}, 1 \mathrm{H}), 3.51(\mathrm{~d}$, $J=9.6 \mathrm{~Hz}, 0 \mathrm{H}), 1.70-1.64(\mathrm{~m}, 3 \mathrm{H}), 1.21(\mathrm{~s}, 8 \mathrm{H}) .{ }^{13} \mathrm{C} \mathrm{NMR}\left(101 \mathrm{MHz}, \mathrm{CDCl}_{3}\right) \delta$ $142.2,130.7,128.5,128.1,125.5,83.6,24.7,18.3,15.0$. These spectroscopic data correspond to reported data. ${ }^{26}$

\section{Isomerization of Bdan-containing substrate}

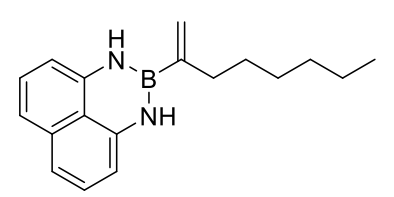

\section{2-(oct-1-en-2-yl)-2,3-dihydro-1H-naphtho[1,8-de][1,3,2]diazaborinine (1am).}

Prepared according to according to previously reported procedure ${ }^{27}{ }^{1} \mathrm{H}$ NMR $(400 \mathrm{MHz}$, $\left.\mathrm{CDCl}_{3}\right) \delta 7.11(\mathrm{t}, J=8.4 \mathrm{~Hz}, 2 \mathrm{H}), 7.02(\mathrm{~d}, J=8.2 \mathrm{~Hz}, 2 \mathrm{H}), 6.34(\mathrm{dd}, J=7.3,0.8 \mathrm{~Hz}$, 2H), $5.75(\mathrm{~s}, 2 \mathrm{H}), 5.51(\mathrm{~s}, 2 \mathrm{H}), 2.21(\mathrm{t}, J=7.4 \mathrm{~Hz}, 2 \mathrm{H}), 1.50-1.41(\mathrm{~m}, 2 \mathrm{H}), 1.39-$ $1.22(\mathrm{~m}, 6 \mathrm{H}), 0.90(\mathrm{~d}, J=6.8 \mathrm{~Hz}, 3 \mathrm{H})$.

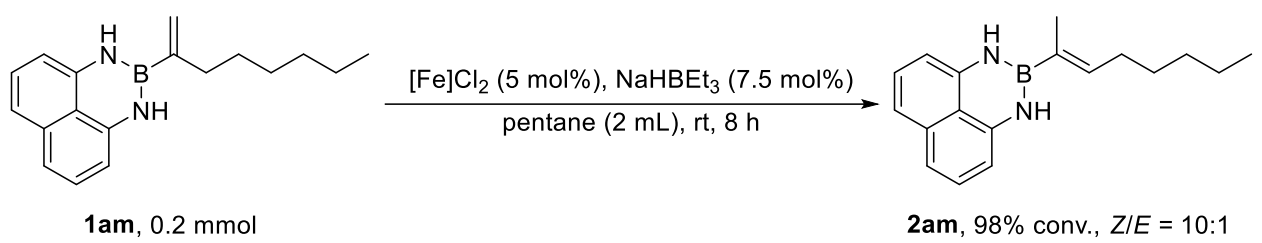

(Z)-2-(oct-2-en-2-yl)-2,3-dihydro-1H-naphtho[1,8-de][1,3,2]diazaborinine (2am).

In a nitrogen-filled glove-box, an oven-dried $10 \mathrm{~mL}$ thin-wall glass tube was charged with $\left[\mathrm{Fe}_{\mathrm{Cl}}(10 \mu \mathrm{mol}, 5.6 \mathrm{mg})\right.$, pentane $(2 \mathrm{~mL})$, $\mathbf{1 a m}(0.2 \mathrm{mmol}, 55 \mathrm{mg})$, then $\mathrm{NaHBEt}_{3}(15 \mu \mathrm{mol}, 15 \mu \mathrm{L}, 1 \mathrm{M}$ in THF) was added to the glass tube. The tube was sealed with a Teflon plug, and the solution was stirred at room temperature for $8 \mathrm{~h}$. The resulting mixture was concentrated under vacuum and the residue was purified by flash silica gel column chromatography to give the product as colorless oil. (98\% conv., 51 mg, 93\% yield, $Z / E=10: 1,2 \%$ 1am). ${ }^{1} \mathrm{H}$ NMR $\left(400 \mathrm{MHz}, \mathrm{CDCl}_{3}\right) \delta 7.12(\mathrm{td}, J=7.7$ $\mathrm{Hz}, 2 \mathrm{H}), 7.02(\mathrm{~d}, J=8.1 \mathrm{~Hz}, 2 \mathrm{H}), 6.35(\mathrm{~d}, J=7.3 \mathrm{~Hz}, 2 \mathrm{H}), 6.05(\mathrm{td}, J=6.9,1.5 \mathrm{~Hz}$, 1H), $5.75(\mathrm{~s}, 2 \mathrm{H}), 2.30-2.11(\mathrm{~m}, 2 \mathrm{H}), 1.78(\mathrm{~s}, 3 \mathrm{H}), 1.52-1.42(\mathrm{~m}, 2 \mathrm{H}), 1.41-1.30$ $(\mathrm{m}, 4 \mathrm{H}), 0.94(\mathrm{t}, J=6.9 \mathrm{~Hz}, 3 \mathrm{H}) .{ }^{13} \mathrm{C} \mathrm{NMR}\left(101 \mathrm{MHz}, \mathrm{CDCl}_{3}\right) \delta 141.5,140.5,136.4$, 
127.7, 119.7, 117.4, 105.7, 31.8, 29.0, 28.7, 22.7, 14.2, 14.1. ${ }^{11} \mathrm{~B}$ NMR (128 MHz, $\left.\mathrm{CDCl}_{3}\right) \delta$ 28.5. HRMS-ESI $(\mathrm{m} / \mathrm{z})$ : Calc. for $\mathrm{C}_{18} \mathrm{H}_{24}{ }^{10} \mathrm{BO}_{3}[\mathrm{M}+\mathrm{H}]^{+} 279.2027$, found 279.2037 .

\section{Procedures for Tandem Isomerization and Coupling Reactions}

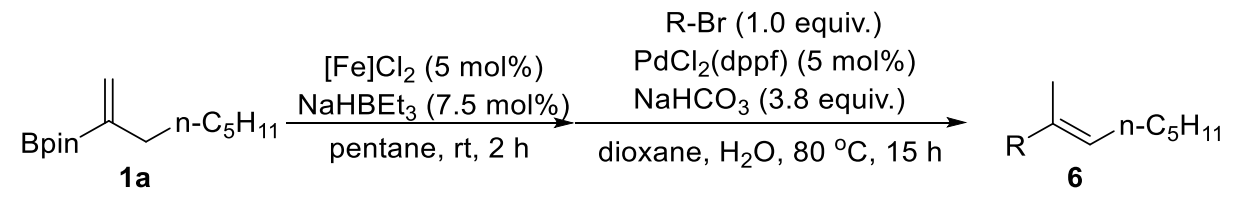

General procedure for tandem isomerization and coupling reactions. In a nitrogenfilled glove-box, an oven-dried $10 \mathrm{~mL}$ thin-wall glass tube was charged with $[\mathrm{Fe}] \mathrm{Cl}_{2}$ (10 $\mu \mathrm{mol}, 5.6 \mathrm{mg})$, pentane $(2 \mathrm{~mL}), \alpha$-vinyl boronates (1a) $(0.2 \mathrm{mmol}) \mathrm{NaHBEt}_{3}(15$ $\mu \mathrm{mol}, 15 \mu \mathrm{L}, 1 \mathrm{M}$ in THF) was then added to the glass tube. The tube was sealed with a Teflon plug, and the solution was stirred at room temperature for $2 \mathrm{~h}$. The reaction was quenched by exposing the solution to air. The solution was evaporated under vacuum. Under argon, the residue was dissolved in $0.8 \mathrm{~mL}$ water and $2 \mathrm{~mL}$ dioxane, followed by adding $\mathrm{R}-\mathrm{Br}(0.2 \mathrm{mmol}), \mathrm{PdCl}_{2}$ (dppf) (7.3 mg, $\left.0.01 \mathrm{mmol}\right), \mathrm{NaHCO}_{3}$ (64 $\mathrm{mg}, 0.76 \mathrm{mmol}$ ). The tube was sealed with a Teflon plug, and the reaction mixture was stirred at $80{ }^{\circ} \mathrm{C}$ temperature for $15 \mathrm{~h}$. The reaction was cooled to room temperature and extracted with ethyl acetate $(5 \mathrm{~mL} \times 3)$. The organic phase was dried over $\mathrm{Na}_{2} \mathrm{SO}_{4}$, filtered and concentrated under vacuum. The residue was purified by flash silica gel column chromatography to give the product.

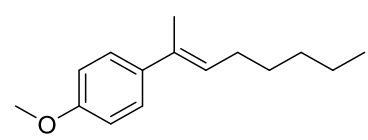

(E)-1-methoxy-4-(oct-2-en-2-yl)benzene (6a). (21 mg, 49\% yield, $E / Z>20: 1) .{ }^{1} \mathrm{H}$ $\operatorname{NMR}\left(400 \mathrm{MHz}, \mathrm{CDCl}_{3}\right) \delta 7.33(\mathrm{~d}, J=8.8 \mathrm{~Hz}, 2 \mathrm{H}), 6.85(\mathrm{~d}, J=8.8 \mathrm{~Hz}, 2 \mathrm{H}), 5.72(\mathrm{t}, J$ $=7.6 \mathrm{~Hz}, 1 \mathrm{H}), 3.81(\mathrm{~s}, 3 \mathrm{H}), 2.24-2.12(\mathrm{~m}, 2 \mathrm{H}), 2.01(\mathrm{~s}, 3 \mathrm{H}), 1.51-1.40(\mathrm{~m}, 2 \mathrm{H}), 1.39$ $-1.25(\mathrm{~m}, 4 \mathrm{H}), 0.91(\mathrm{t}, J=6.7 \mathrm{~Hz}, 3 \mathrm{H}) .{ }^{13} \mathrm{C} \mathrm{NMR}\left(101 \mathrm{MHz}, \mathrm{CDCl}_{3}\right) \delta 158.5,136.8$, 133.9, 127.4, 126.7, 113.6, 55.4, 31.8, 29.6, 28.9, 22.8, 15.9, 14.2. HRMS-EI $(m / z)$ : Calc. for $\mathrm{C}_{15} \mathrm{H}_{22} \mathrm{O}[\mathrm{M}]^{+} 218.1665$, found 218.1664. 


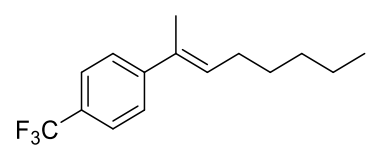

(E)-1-(oct-2-en-2-yl)-4-(trifluoromethyl)benzene (6b). (39 mg, 76\% yield, E/Z > 20:1). ${ }^{1} \mathrm{H}$ NMR (400 MHz, $\left.\mathrm{CDCl}_{3}\right) \delta 7.55(\mathrm{~d}, J=8.2 \mathrm{~Hz}, 2 \mathrm{H}), 7.47(\mathrm{~d}, J=8.2 \mathrm{~Hz}, 2 \mathrm{H})$, $5.87(\mathrm{t}, J=7.2 \mathrm{~Hz}, 1 \mathrm{H}), 2.26-2.17(\mathrm{~m}, 2 \mathrm{H}), 2.04(\mathrm{~s}, 3 \mathrm{H}), 1.52-1.42(\mathrm{~m}, 2 \mathrm{H}), 1.41-$ $1.29(\mathrm{~m}, 4 \mathrm{H}), 0.92(\mathrm{t}, J=6.5 \mathrm{~Hz}, 3 \mathrm{H}) .{ }^{13} \mathrm{C} \mathrm{NMR}\left(101 \mathrm{MHz}, \mathrm{CDCl}_{3}\right) \delta$ 147.7, 133.7, $131.2,128.5$ (q, $J=32.4 \mathrm{~Hz}), 125.9,125.2(\mathrm{q}, J=3.8 \mathrm{~Hz}), 124.27(\mathrm{q}, J=218.7 \mathrm{~Hz})$, 31.8, 29.3, 29.0, 22.8, 15.8, 14.2. ${ }^{19} \mathrm{~F}$ NMR $\left(376 \mathrm{MHz}, \mathrm{CDCl}_{3}\right) \delta-62.4$. HRMS-EI $(\mathrm{m} / z)$ : Calc. for $\mathrm{C}_{15} \mathrm{H}_{19} \mathrm{~F}_{3}[\mathrm{M}]^{+}$236.1607, found 236.1407.

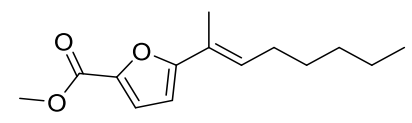

Methyl-(E)-5-(oct-2-en-2-yl)furan-2-carboxylate $(\mathbf{6 c}) .(43 \mathrm{mg}, 91 \%$ yield, $E / Z>$ 20:1). ${ }^{1} \mathrm{H}$ NMR $\left(400 \mathrm{MHz}, \mathrm{CDCl}_{3}\right) \delta 7.13(\mathrm{~d}, J=3.6 \mathrm{~Hz}, 1 \mathrm{H}), 6.33(\mathrm{t}, J=8.1 \mathrm{~Hz}, 1 \mathrm{H})$, $6.26(\mathrm{~d}, J=3.6 \mathrm{~Hz}, 1 \mathrm{H}), 3.86(\mathrm{~s}, 3 \mathrm{H}), 2.24-2.14(\mathrm{~m}, 2 \mathrm{H}), 1.92(\mathrm{~s}, 3 \mathrm{H}), 1.49-1.39(\mathrm{~m}$, $2 \mathrm{H}), 1.38-1.23(\mathrm{~m}, 4 \mathrm{H}), 0.89(\mathrm{t}, J=6.7 \mathrm{~Hz}, 3 \mathrm{H}) .{ }^{13} \mathrm{C} \mathrm{NMR}\left(101 \mathrm{MHz}, \mathrm{CDCl}_{3}\right) \delta 160.1$, 159.4, 142.6, 130.6, 124.0, 119.9, 106.3, 51.8, 31.7, 29.1, 28.3, 22.7, 14.1, 13.2. HRMSEI $(m / z)$ : Calc. for $\mathrm{C}_{14} \mathrm{H}_{20} \mathrm{O}_{3}[\mathrm{M}]^{+} 236.1407$, found 236.1407.

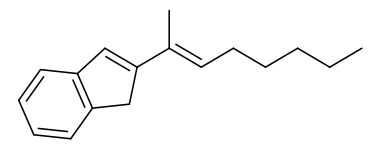

(E)-2-(oct-2-en-2-yl)-1H-indene (6d). (33 mg, 72\% yield, E/Z > 20:1). ${ }^{1} \mathrm{H}$ NMR (400 $\left.\mathrm{MHz}, \mathrm{CDCl}_{3}\right) \delta 7.38(\mathrm{~d}, J=7.3 \mathrm{~Hz}, 1 \mathrm{H}), 7.30(\mathrm{~d}, J=7.4 \mathrm{~Hz}, 1 \mathrm{H}), 7.22(\mathrm{t}, J=7.4 \mathrm{~Hz}$, 1H), $7.12(\mathrm{td}, J=7.4,1.1 \mathrm{~Hz}, 1 \mathrm{H}), 6.69(\mathrm{~s}, 1 \mathrm{H}), 5.84(\mathrm{t}, J=7.3 \mathrm{~Hz}, 1 \mathrm{H}), 3.58(\mathrm{~s}, 2 \mathrm{H})$, $2.28-2.13(\mathrm{~m}, 2 \mathrm{H}), 1.96(\mathrm{~s}, 3 \mathrm{H}), 1.52-1.42(\mathrm{~m}, 3 \mathrm{H}), 1.41-1.28(\mathrm{~m}, 4 \mathrm{H}), 0.93(\mathrm{t}, J$ $=6.9 \mathrm{~Hz}, 3 \mathrm{H}) .{ }^{13} \mathrm{C} \mathrm{NMR}\left(101 \mathrm{MHz}, \mathrm{CDCl}_{3}\right) \delta 150.2,145.7,142.8,131.3,129.4,126.5$, 125.5, 124.4, 123.5, 120.6, 38.3, 31.8, 29.5, 28.6, 22.8, 14.2, 14.1. HRMS-EI $(\mathrm{m} / \mathrm{z})$ : Calc. for $\mathrm{C}_{17} \mathrm{H}_{22}[\mathrm{M}]^{+} 226.1716$, found 226.1714 .

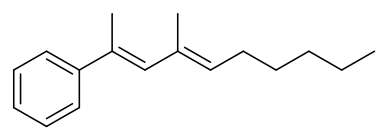


((2E,4E)-4-Methyldeca-2,4-dien-2-yl)benzene (6e). (41 mg, 89\% yield, $E / Z=11: 1$; $E / Z>20: 1) .{ }^{1} \mathrm{H}$ NMR $\left(400 \mathrm{MHz}, \mathrm{CDCl}_{3}\right) \delta 7.51-7.44(\mathrm{~m}, 2 \mathrm{H}), 7.40-7.32(\mathrm{~m}, 2 \mathrm{H})$, $7.31-7.21(\mathrm{~m}, 1 \mathrm{H}), 6.27(\mathrm{~s}, 1 \mathrm{H}), 5.49(\mathrm{t}, J=7.3 \mathrm{~Hz}, 1 \mathrm{H}), 2.24(\mathrm{~d}, J=1.2 \mathrm{~Hz}, 2 \mathrm{H})$, $2.22-2.15(\mathrm{~m}, 1 \mathrm{H}), 1.87(\mathrm{~s}, 3 \mathrm{H}), 1.53-1.43(\mathrm{~m}, 2 \mathrm{H}), 1.43-1.34(\mathrm{~m}, 4 \mathrm{H}), 0.96(\mathrm{t}, J$ $=6.9 \mathrm{~Hz}, 3 \mathrm{H}) .{ }^{13} \mathrm{C} \mathrm{NMR}\left(101 \mathrm{MHz}, \mathrm{CDCl}_{3}\right) \delta 144.7,134.1,132.9,132.1,131.3,128.3$, 126.7, 126.0, 31.8, 29.5, 28.4, 22.8, 17.6, 17.2, 14.3. HRMS-EI $(m / z)$ : Calc. for $\mathrm{C}_{17} \mathrm{H}_{24}$ $[\mathrm{M}]^{+} 228.1873$, found 228.1871 .

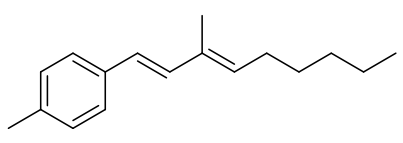

1-methyl-4-((1E,3E)-3-methylnona-1,3-dien-1-yl)benzene (6f). (29 mg, 48\% yield, $E / Z=12 / 1 ; E / Z>20: 1) .{ }^{1} \mathrm{H}$ NMR $\left(400 \mathrm{MHz}, \mathrm{CDCl}_{3}\right) \delta 7.37(\mathrm{~d}, J=8.1 \mathrm{~Hz}, 1 \mathrm{H}), 7.18$ $(\mathrm{d}, J=8.1 \mathrm{~Hz}, 1 \mathrm{H}), 6.83(\mathrm{~d}, J=16.1 \mathrm{~Hz}, 1 \mathrm{H}), 6.49(\mathrm{~d}, J=16.1 \mathrm{~Hz}, 1 \mathrm{H}), 5.69(\mathrm{t}, J=7.3$ $\mathrm{Hz}, 1 \mathrm{H}), 2.40$ (s, 3H), 2.23 - 2.14 (m, 2H), 1.92 (s, 3H), $1.47-1.37$ (m, 2H), 1.42 $1.35(\mathrm{~m}, 4 \mathrm{H}), 0.97(\mathrm{t}, J=6.9 \mathrm{~Hz}, 3 \mathrm{H}) .{ }^{13} \mathrm{C} \mathrm{NMR}\left(101 \mathrm{MHz}, \mathrm{CDCl}_{3}\right) \delta$ 136.7, 135.4, 134.1, 133.9, 133.3, 129.4, 126.2, 125.5, 31.7, 29.5, 28.6, 22.8, 21.3, 14.2, 12.5. HRMS$\mathrm{EI}(\mathrm{m} / \mathrm{z})$ : Calc. for $\mathrm{C}_{17} \mathrm{H}_{24}[\mathrm{M}]^{+} 228.1873$, found 228.1873 .

\section{Synthesis of Cyercene A}

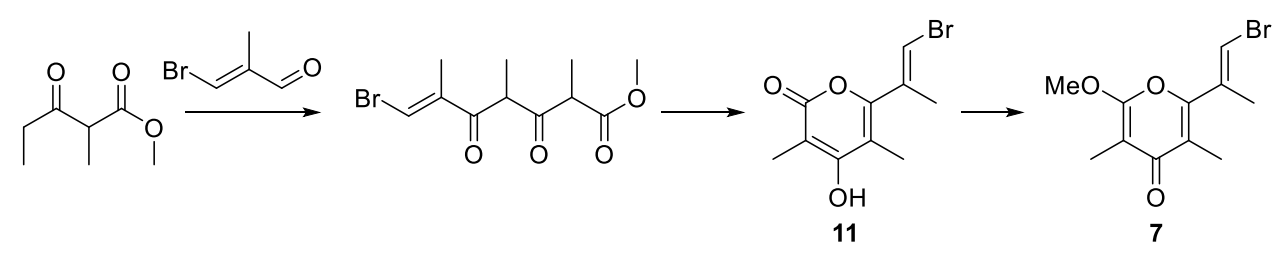

Compound 7 was prepared according to reported literature procedure. ${ }^{28}$

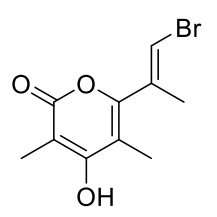

(E)-6-(1-bromoprop-1-en-2-yl)-4-hydroxy-3,5-dimethyl-2H-pyran-2-one (11). ${ }^{1} \mathrm{H}$ NMR (400 MHz, DMSO-d6) $\delta 10.72(\mathrm{~s}, 1 \mathrm{H}), 6.89$ (s, 1H), 1.97 (s, 3H), 1.89 (s, 3H), $1.85(\mathrm{~s}, 3 \mathrm{H}) .{ }^{13} \mathrm{C}$ NMR $\left(101 \mathrm{MHz}, \mathrm{DMSO}-d_{6}\right) \delta 164.4,163.6,153.4,133.5,114.3,108.5$, 
98.9, 18.2, 11.6, 9.4. HRMS-EI $(m / z)$ : Calc. for $\mathrm{C}_{10} \mathrm{H}_{11} \mathrm{BrO}_{3}[\mathrm{M}]^{+}$257.9886, found 257.9887.<smiles>COc1oc(/C=C/Br)c(C)c(=O)c1C</smiles>

(E)-2-(1-bromoprop-1-en-2-yl)-6-methoxy-3,5-dimethyl-4H-pyran-4-one $(7) .{ }^{1} \mathrm{H}$ NMR (400 MHz, $\left.\mathrm{CDCl}_{3}\right) \delta 6.57(\mathrm{~s}, 1 \mathrm{H}), 3.95$ (s, 3H), 2.09 (s, 3H), 1.97 (s, 3H), 1.84 (s, 3H). ${ }^{13} \mathrm{C}$ NMR $\left(101 \mathrm{MHz}, \mathrm{CDCl}_{3}\right) \delta 180.9,162.0,154.6,133.7,119.4,114.6,99.9$, 55.5, 18.2, 11.8, 7.0. HRMS-EI $(\mathrm{m} / \mathrm{z})$ : Calc. for $\mathrm{C}_{11} \mathrm{H}_{13} \mathrm{BrO}_{3}[\mathrm{M}]^{+}$272.0073, found 272.0039 .

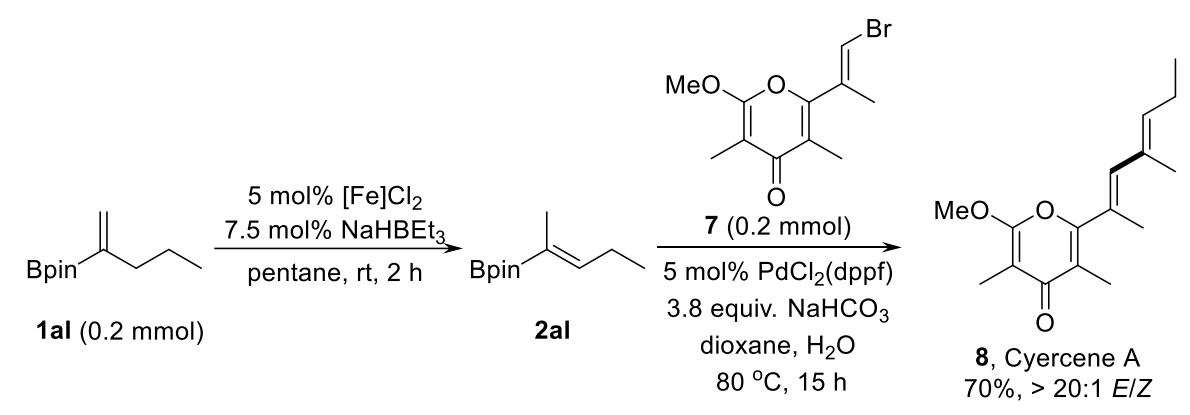

In a nitrogen-filled glove-box, an oven-dried $10 \mathrm{~mL}$ thin-wall glass tube was charged with $[\mathrm{Fe}] \mathrm{Cl}_{2}(10 \mu \mathrm{mol}, 5.6 \mathrm{mg})$, pentane $(2 \mathrm{~mL})$, 1al $(39 \mathrm{mg}, 0.2 \mathrm{mmol})$, then $\mathrm{NaHBEt}_{3}$ (15 $\mu \mathrm{mol}, 15 \mu \mathrm{L}, 1 \mathrm{M}$ in THF) was added to the glass tube. The tube was sealed with a Teflon plug, and the solution was stirred at room temperature for $2 \mathrm{~h}$. The reaction was quenched by exposing the solution to air. The solution was evaporated under vacuum. Under argon, the residue was dissolved in $0.8 \mathrm{~mL}$ water and $2 \mathrm{~mL}$ dioxane, followed by adding 7 (54 mg, $0.2 \mathrm{mmol}), \mathrm{PdCl}_{2}(\mathrm{dppf})(7.3 \mathrm{mg}, 0.01 \mathrm{mmol}), \mathrm{NaHCO}_{3}(64 \mathrm{mg}$, $0.76 \mathrm{mmol})$. The tube was sealed with a Teflon plug, and the reaction mixture was stirred at $80^{\circ} \mathrm{C}$ temperature for $15 \mathrm{~h}$. The reaction was cooled to room temperature and extracted with ethyl acetate $(5 \mathrm{~mL} \times 3)$. The organic phase was dried over $\mathrm{Na}_{2} \mathrm{SO}_{4}$, filtered and concentrated under vacuum. The residue was purified by flash silica gel column chromatography to give the product as white solid (36.2 mg, 70\% yield, > 20:1 $E / Z)$. 


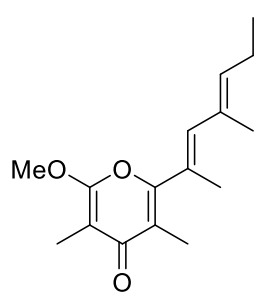

Cyercene A (8). ${ }^{1} \mathrm{H}$ NMR $\left(400 \mathrm{MHz}, \mathrm{CDCl}_{3}\right) \delta 6.09(\mathrm{~s}, 1 \mathrm{H}), 5.51(\mathrm{t}, J=7.2 \mathrm{~Hz}, 1 \mathrm{H})$, $3.94(\mathrm{~s}, 3 \mathrm{H}), 2.21-2.09(\mathrm{~m}, 2 \mathrm{H}), 2.05$ (s, 3H), 1.99 (s, 3H), 1.85 (s, 3H), 1.83 (s, 3H), $1.02(\mathrm{t}, J=7.5 \mathrm{~Hz}, 3 \mathrm{H}) .{ }^{13} \mathrm{C} \mathrm{NMR}\left(101 \mathrm{MHz}, \mathrm{CDCl}_{3}\right) \delta 181.7,162.0,159.3,139.6$, $136.1,131.1,125.5,117.8,99.3,55.3,21.7,16.5,16.2,14.0,12.0,7.0$. These spectroscopic data correspond to reported data. ${ }^{28}$ 


\section{Mechanism Studies}

Time-course of the isomerization under the system of " $1 \mathrm{Fe}+1.5 \mathrm{NaHBEt3}$ " and "1 Fe + 3 NaHBEt3"

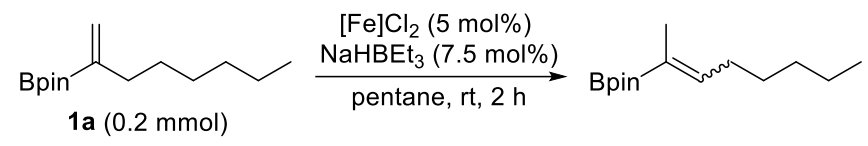

In a nitrogen-filled glove-box, an oven-dried $10 \mathrm{~mL}$ thin-wall glass tube was charged with $[\mathrm{Fe}] \mathrm{Cl}_{2}(10 \mu \mathrm{mol}, 5.6 \mathrm{mg})$, pentane $(2 \mathrm{~mL}), \mathbf{1 a}(0.2 \mathrm{mmol}, 48 \mathrm{mg})$, then $\mathrm{NaHBEt}_{3}$ (15 $\mu \mathrm{mol}, 15 \mu \mathrm{L}, 1 \mathrm{M}$ in THF) was added to the glass tube. The tube was sealed with a Teflon plug, and the solution was stirred at room temperature. Samples $(50 \mu \mathrm{L})$ were recorded periodically and quenched by exposing the solution to air for GC analysis.

Table S2. Time-course of the isomerization under the system of "1 Fe + 1.5 NaHBEt 3 "

\begin{tabular}{|c|c|c|c|c|}
\hline & \multicolumn{2}{|c|}{$[\mathrm{Fe}] \mathrm{Cl}_{2}(5 \mathrm{~mol} \%)$} & \multicolumn{2}{|c|}{ Bpin } \\
\hline 1a & & & & $E-2 \mathbf{a}$ \\
\hline time $(\min )$ & $1 \mathrm{a}(\%)$ & $Z-2 a \quad(\%)$ & $E-2 \mathbf{a}(\%)$ & Z/E \\
\hline 0 & 100.0 & 0 & 0 & -- \\
\hline 5 & 73.9 & 24.1 & 2.0 & $11.9: 1$ \\
\hline 10 & 61.1 & 36.7 & 2.2 & $16.6: 1$ \\
\hline 15 & 51.9 & 45.6 & 2.5 & $18.2: 1$ \\
\hline 20 & 43.7 & 53.9 & 2.5 & $21.7: 1$ \\
\hline 30 & 30.3 & 67.1 & 2.6 & $25.7: 1$ \\
\hline 40 & 20.6 & 76.7 & 2.7 & $28.0: 1$ \\
\hline 50 & 13.7 & 83.5 & 2.8 & $29.5: 1$ \\
\hline 60 & 9.0 & 88.0 & 3.0 & $29.8: 1$ \\
\hline 70 & 6.0 & 91.0 & 3.0 & $30.9: 1$ \\
\hline 80 & 4.2 & 92.7 & 3.1 & $30.3: 1$ \\
\hline 100 & 3.1 & 93.8 & 3.1 & $30: 1$ \\
\hline 120 & 2.1 & 94.7 & 3.2 & $29.5: 1$ \\
\hline 150 & 2.2 & 94.7 & 3.1 & $30.5: 1$ \\
\hline
\end{tabular}




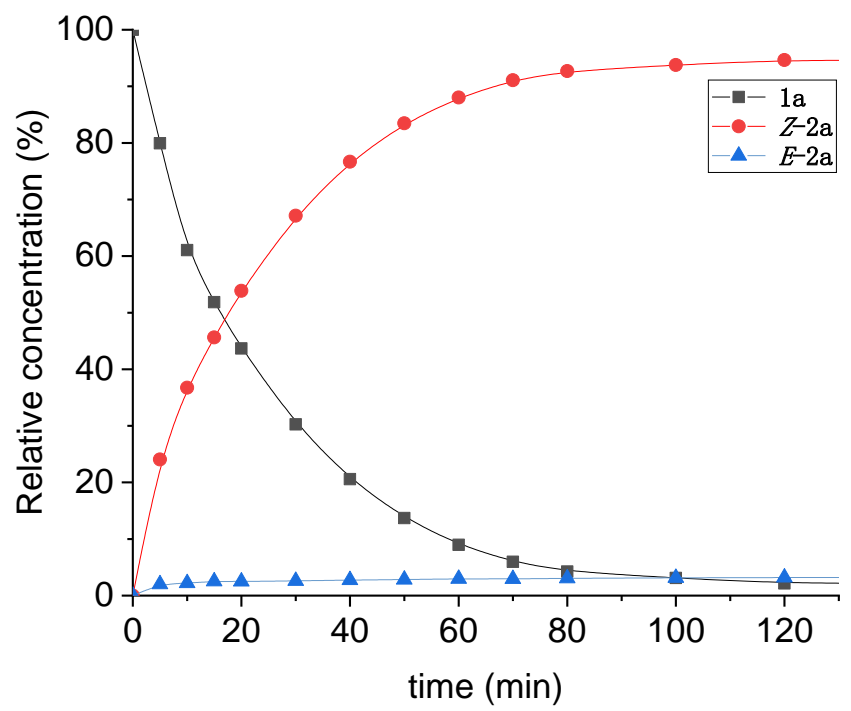

Figure S1-a. Time-course of the isomerization under the system of "1 Fe + 1.5 $\mathrm{NaHBEt}_{3}$ "

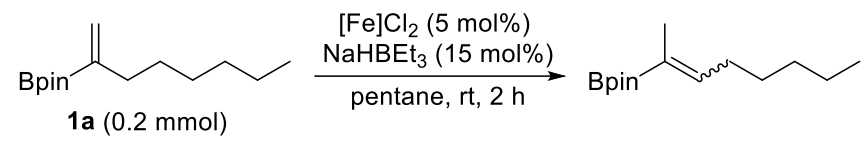

In a nitrogen-filled glove-box, an oven-dried $10 \mathrm{~mL}$ thin-wall glass tube was charged with $[\mathrm{Fe}] \mathrm{Cl}_{2}(10 \mu \mathrm{mol}, 5.6 \mathrm{mg})$, pentane $(2 \mathrm{~mL}), \mathbf{1 a}(0.2 \mathrm{mmol}, 48 \mathrm{mg})$; then $\mathrm{NaHBEt}_{3}$ (30 $\mu \mathrm{mol}, 30 \mu \mathrm{L}, 1 \mathrm{M}$ in THF) was added to the glass tube. The tube was sealed with a Teflon plug, and the solution was stirred at room temperature. Samples $(50 \mu \mathrm{L})$ were recorded periodically and quenched by exposing the solution to air for GC analysis.

Table S3. Time-course of the isomerization under the system of " $1 \mathrm{Fe}+3 \mathrm{NaHBEt}$ "

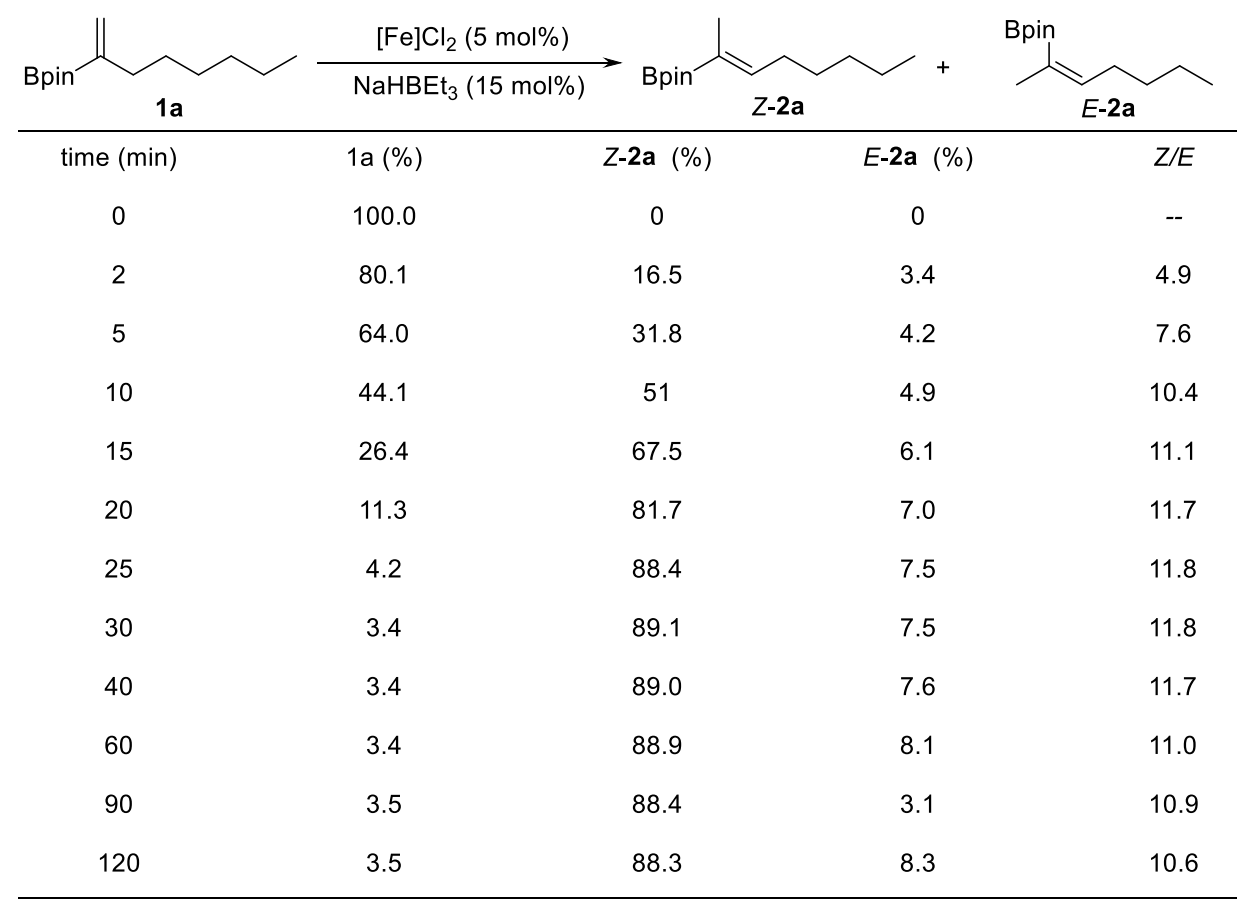




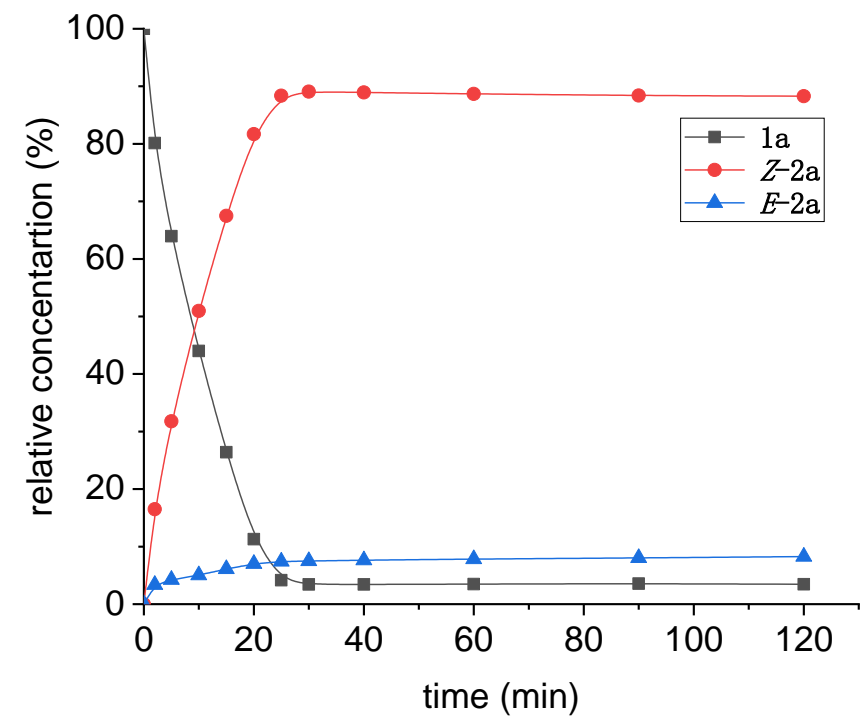

Figure S1-b. Time-course of the isomerization under the system of "1 $\mathrm{Fe}+3 \mathrm{NaHBEt} 3$ "

\section{Successive addition experiment}

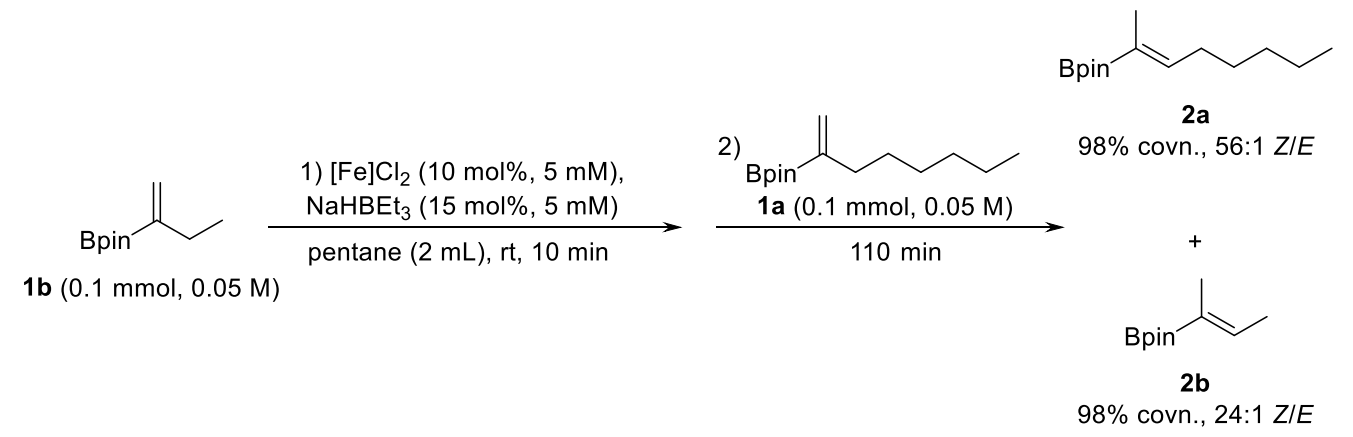

In a nitrogen-filled glove-box, an oven-dried $10 \mathrm{~mL}$ thin-wall glass tube was charged with $[\mathrm{Fe}] \mathrm{Cl}_{2}(10 \mu \mathrm{mol}, 5.6 \mathrm{mg})$, pentane $(2 \mathrm{~mL}), \mathbf{1 b}(18 \mathrm{mg}, 0.1 \mathrm{mmol}) . \mathrm{NaHBEt}_{3}(15$ $\mu$ mol, $15 \mu \mathrm{L}, 1 \mathrm{M}$ in THF) was then added to the glass tube. The tube was sealed with a Teflon plug, and the solution was stirred at room temperature for $10 \mathrm{~min}$. $1 \mathbf{a}(24 \mathrm{mg}$, $0.1 \mathrm{mmol}$ ) was injected into the glass tube and the solution was stirred at room temperature for an additional period of $110 \mathrm{~min}$. Sample $(50 \mu \mathrm{L})$ were quenched by exposing the solution to air for GC analysis.

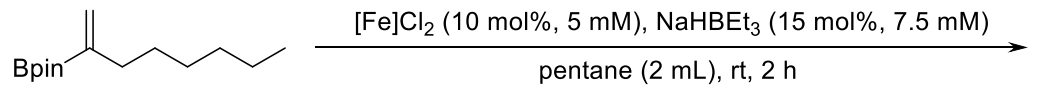

1a, $0.1 \mathrm{mmol}, 0.05 \mathrm{M}$

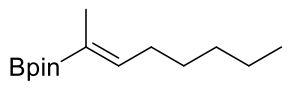

2a, $98 \%$ conv., $Z / E=19: 1$

In a nitrogen-filled glove-box, an oven-dried $10 \mathrm{~mL}$ thin-wall glass tube was charged with $[\mathrm{Fe}] \mathrm{Cl}_{2}(10 \mu \mathrm{mol}, 5.6 \mathrm{mg})$, pentane $(2 \mathrm{~mL}), \mathbf{1 a}(24 \mathrm{mg}, 0.1 \mathrm{mmol})$, then $\mathrm{NaHBEt}_{3}$ 
(15 $\mu$ mol, $15 \mu \mathrm{L}, 1 \mathrm{M}$ in THF) was added to the glass tube. The tube was sealed with a Teflon plug, and the solution was stirred at room temperature for $2 \mathrm{~h}$. Sample (50 $\mu \mathrm{L})$ were quenched by exposing the solution to air for GC analysis.

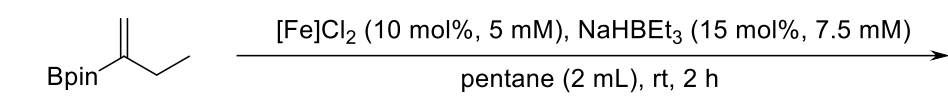

1b, $0.1 \mathrm{mmol}, 0.05 \mathrm{M}$

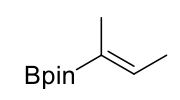

2b, $98 \%$ conv., $Z / E=34: 1$

In a nitrogen-filled glove-box, an oven-dried $10 \mathrm{~mL}$ thin-wall glass tube was charged with $[\mathrm{Fe}] \mathrm{Cl}_{2}(10 \mu \mathrm{mol}, 5.6 \mathrm{mg})$, pentane $(2 \mathrm{~mL}), \mathbf{1 b}(18 \mathrm{mg}, 0.1 \mathrm{mmol})$, then $\mathrm{NaHBEt}_{3}$ (15 $\mu \mathrm{mol}, 15 \mu \mathrm{L}, 1 \mathrm{M}$ in THF) was added to the glass tube. The tube was sealed with a Teflon plug, and the solution was stirred at room temperature for $2 \mathrm{~h}$. Samples (50 $\mu \mathrm{L})$ were quenched by exposing the solution to air for GC analysis.

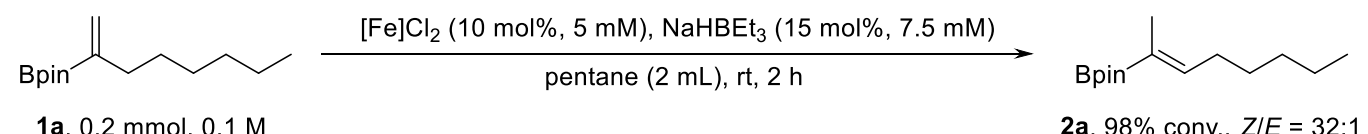

In a nitrogen-filled glove-box, an oven-dried $10 \mathrm{~mL}$ thin-wall glass tube was charged with $[\mathrm{Fe}] \mathrm{Cl}_{2}(10 \mu \mathrm{mol}, 5.6 \mathrm{mg})$, pentane $(2 \mathrm{~mL}), \mathbf{1 a}(48 \mathrm{mg}, 0.2 \mathrm{mmol})$, then $\mathrm{NaHBEt}_{3}$ (15 $\mu \mathrm{mol}, 15 \mu \mathrm{L}, 1 \mathrm{M}$ in THF) was added to the glass tube. The tube was sealed with a Teflon plug, and the solution was stirred at room temperature for $2 \mathrm{~h}$. Sample (50 $\mu \mathrm{L})$ were quenched by exposing the solution to air for GC analysis.

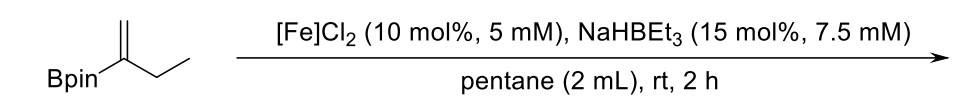

1b, $0.2 \mathrm{mmol}, 0.1 \mathrm{M}$

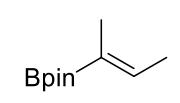

2b, $98 \%$ conv., $Z / E=34: 1$

In a nitrogen-filled glove-box, an oven-dried $10 \mathrm{~mL}$ thin-wall glass tube was charged with $[\mathrm{Fe}] \mathrm{Cl}_{2}(10 \mu \mathrm{mol}, 5.6 \mathrm{mg})$, pentane $(2 \mathrm{~mL}), \mathbf{1 b}(36 \mathrm{mg}, 0.1 \mathrm{mmol})$, then $\mathrm{NaHBEt}_{3}$ (15 $\mu \mathrm{mol}, 15 \mu \mathrm{L}, 1 \mathrm{M}$ in THF) was added to the glass tube. The tube was sealed with a Teflon plug, and the solution was stirred at room temperature for $2 \mathrm{~h}$. Samples (50 $\mu \mathrm{L})$ were quenched by exposing the solution to air for GC analysis. 


\section{SAESI-MS experiment}

SAESI-MS conditions: SAESI-MS spectra were recorded on a Thermo TSQ Quantum Access triple-quadrupole mass spectrometer (Thermo Fisher Scientific, Waltham, MA) equipped with a home-made SAESI ion source in positive mode. The basic SAESI conditions were: vacuum, $3.2 \times 10^{-6}$ torr; spray voltage, $4000 \mathrm{~V}$; capillary temperature, $275^{\circ} \mathrm{C}$; sheath gas pressure of two sprayers, 3 arb. units; the collision energy of CID, 40 $\mathrm{eV}$. Data acquisition and analysis were done with the Xcalibur (version 2.0, Thermo Fisher Scientific) software package. In solvent-assisted electrospray ionization mass spectrometric experiment, the angle ( $\alpha$ ) between the two sprayers is $45^{\circ}$ and the distance (b) between the tip of sprayers and the inlet to the mass is $6 \mathrm{~mm}$. The chemical solutions were injected by a $500-\mu \mathrm{L}$ air-tight syringe with a speed at $5 \mu \mathrm{L} / \mathrm{min}$ to SAESI-MS. The assisted solvent of methanol was injected by another $500-\mu \mathrm{L}$ air-tight syringe with a speed at $5 \mu \mathrm{L} / \mathrm{min}$.

\section{SAESI-MS device:}

(a)

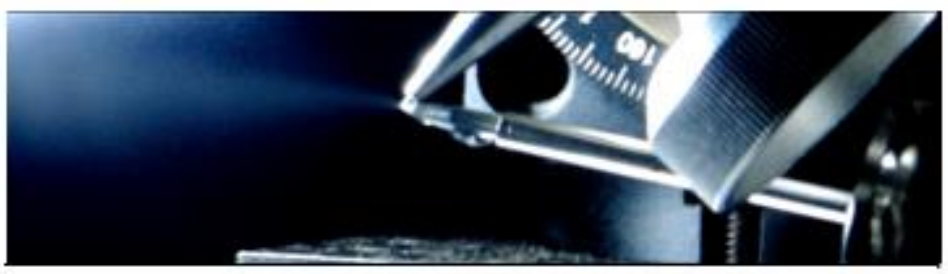

(b)

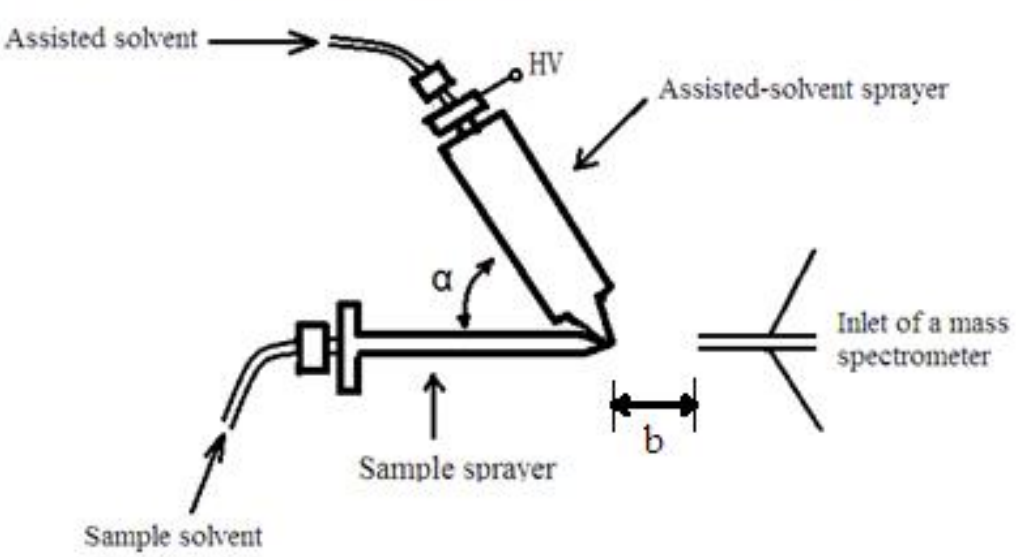

Figure S2 . (a) Photographic image of SAESI apparatus. (b) Schematic representation of the SAESI. The angle $(\alpha)$ between the two sprayers is $45^{\circ}$ andthe distance (b) between the tip of sprayers and the inlet to the mass is $6 \mathrm{~mm}$. 


\section{Mass spectrometric experiment results:}

(a)

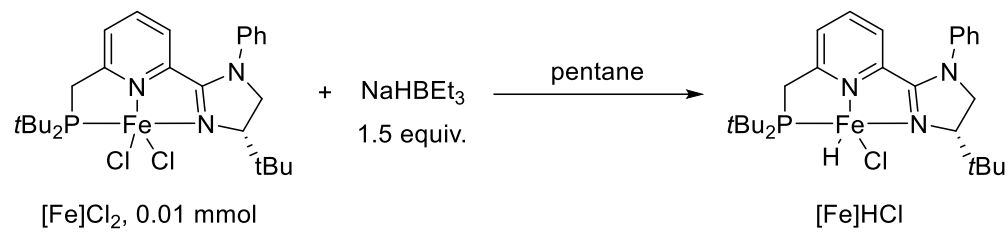

In a nitrogen-filled glove-box, an oven-dried $10 \mathrm{~mL}$ glass tube was charged with $[\mathrm{Fe}] \mathrm{Cl}_{2}(10 \mu \mathrm{mol}, 5.6 \mathrm{mg})$ and pentane $(2 \mathrm{~mL})$. The suspension was sealed and moved out from the glove-box, followed by the injection of $\mathrm{NaHBEt}_{3}(15 \mu \mathrm{mol}, 15 \mu \mathrm{L}, 1 \mathrm{M}$ in THF). The solution was stirred at room temperature for 5 minutes and sent to SAESIMS analysis.

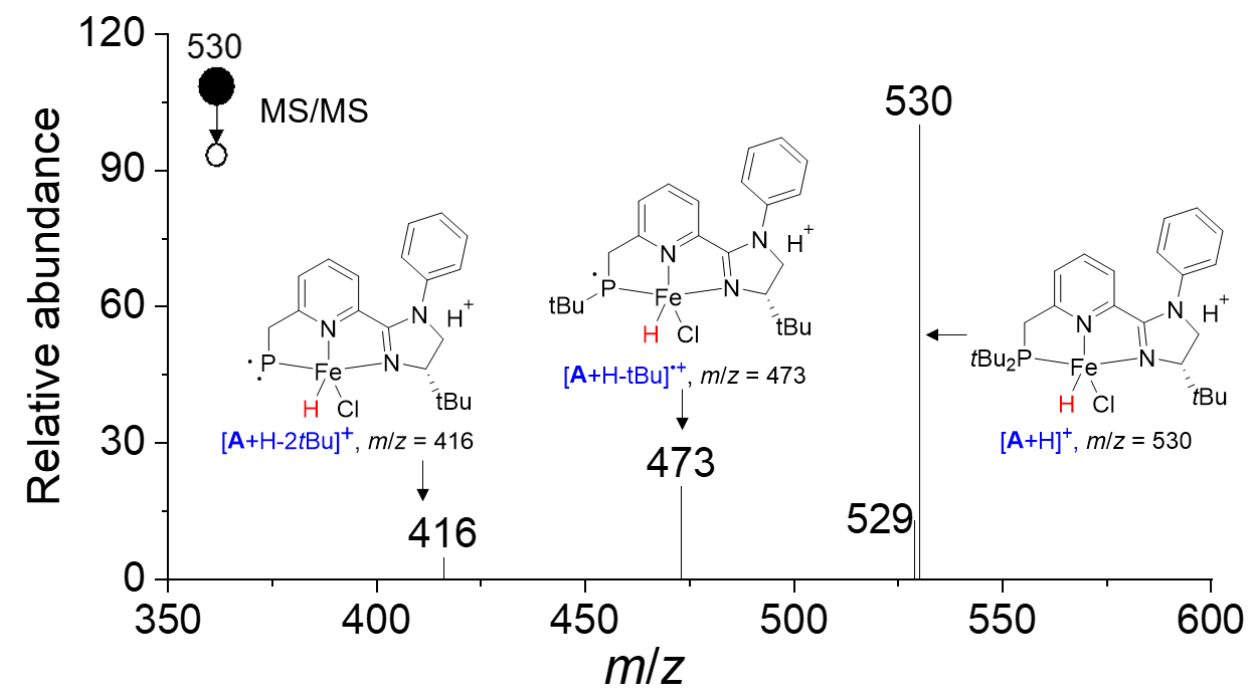

Figure S3-a. SAESI-MS/MS spectrum of complex $[\mathrm{Fe}] \mathrm{HCl}$

(b)

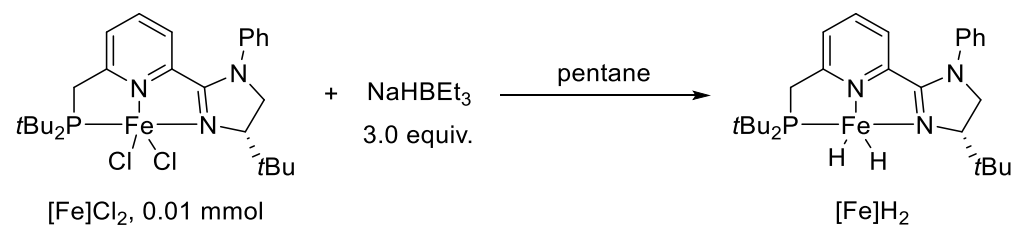

In a nitrogen-filled glove-box, an oven-dried $10 \mathrm{~mL}$ glass tube was charged with $\left[\mathrm{Fe}_{\mathrm{Cl}}(10 \mu \mathrm{mol}, 5.6 \mathrm{mg})\right.$ and pentane $(2 \mathrm{~mL})$. The suspension was sealed and moved out from the glove-box, followed by the injection of $\mathrm{NaHBEt}_{3}(30 \mu \mathrm{mol}, 30 \mu \mathrm{L}, 1 \mathrm{M}$ in THF). The solution was stirred at room temperature for 5 minutes and sent to SAESIMS analysis. 


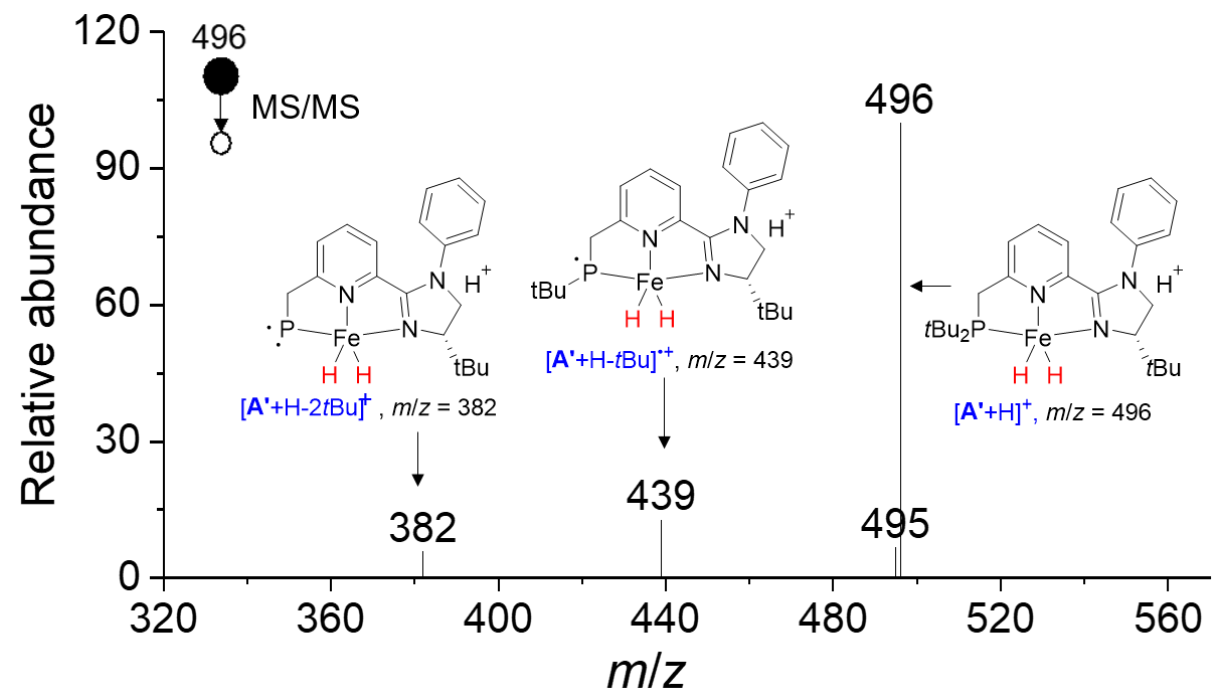

Figure S3-b. SAESI-MS/MS spectrum of complex $[\mathrm{Fe}] \mathrm{H}_{2}$

(c)

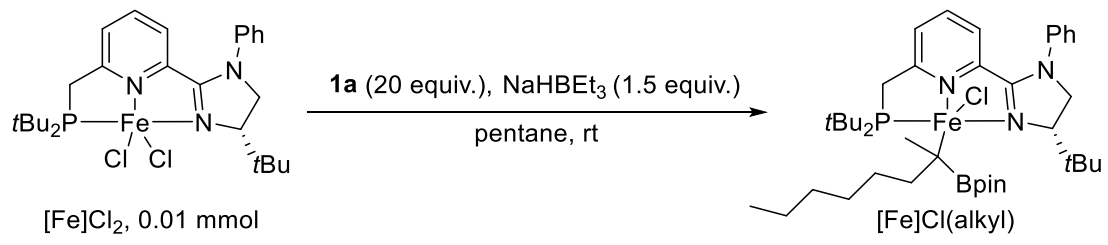

In a nitrogen-filled glove-box, an oven-dried $10 \mathrm{~mL}$ glass tube was charged with $\left[\mathrm{Fe}_{\mathrm{Cl}}(10 \mu \mathrm{mol}, 5.6 \mathrm{mg})\right.$ and pentane $(2 \mathrm{~mL}), \mathbf{1 a}(0.2 \mathrm{mmol})$. The suspension was sealed and moved out from the glove-box, followed by the injection of $\mathrm{NaHBEt}_{3}$ (15 $\mu \mathrm{mol}, 15 \mu \mathrm{L}, 1 \mathrm{M}$ in THF). The solution was stirred at room temperature for 5 minutes and sent to SAESI-MS analysis.

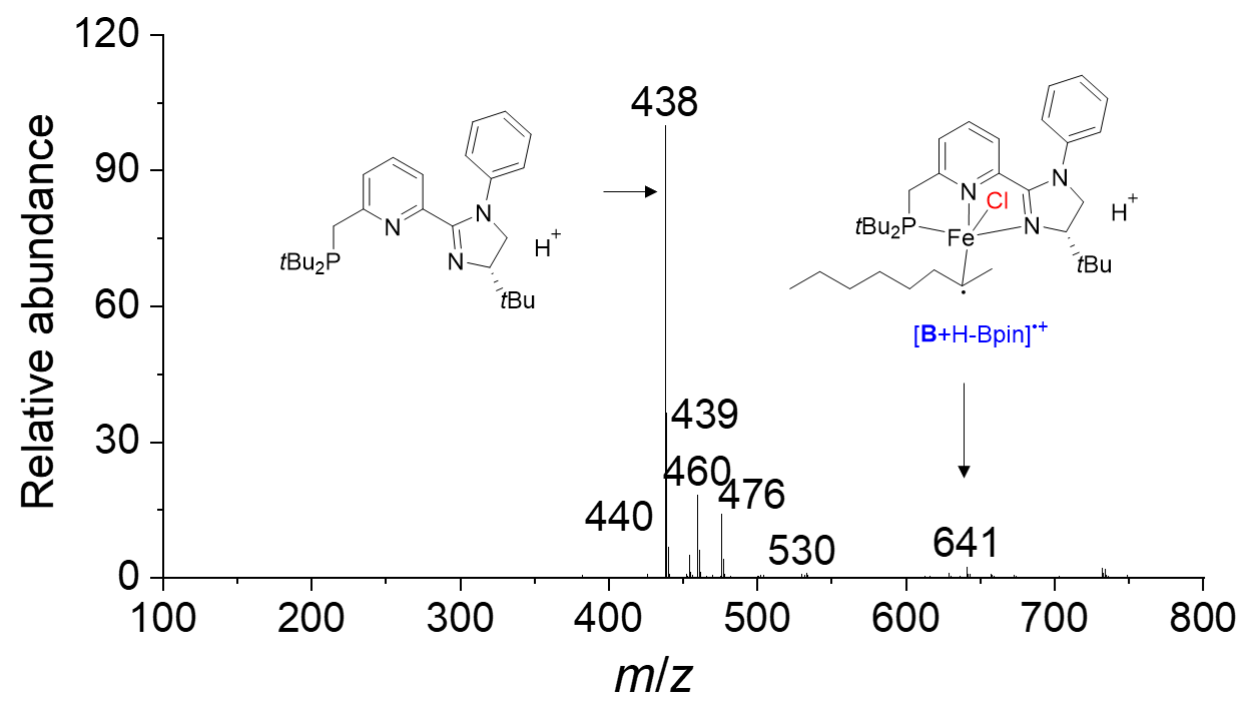

Figure S3-c-1. SAESI-MS spectrum of the reaction solution 


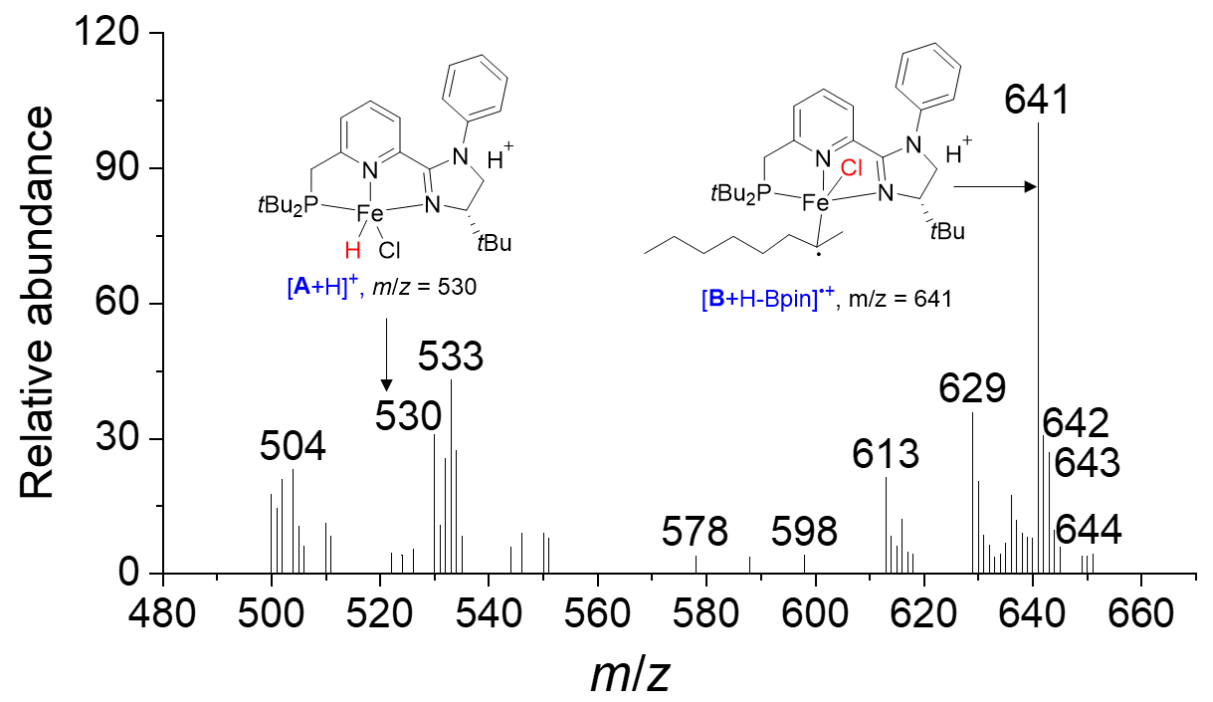

Figure S3-c-2. Expanded SAESI-MS spectrum of the reaction solution

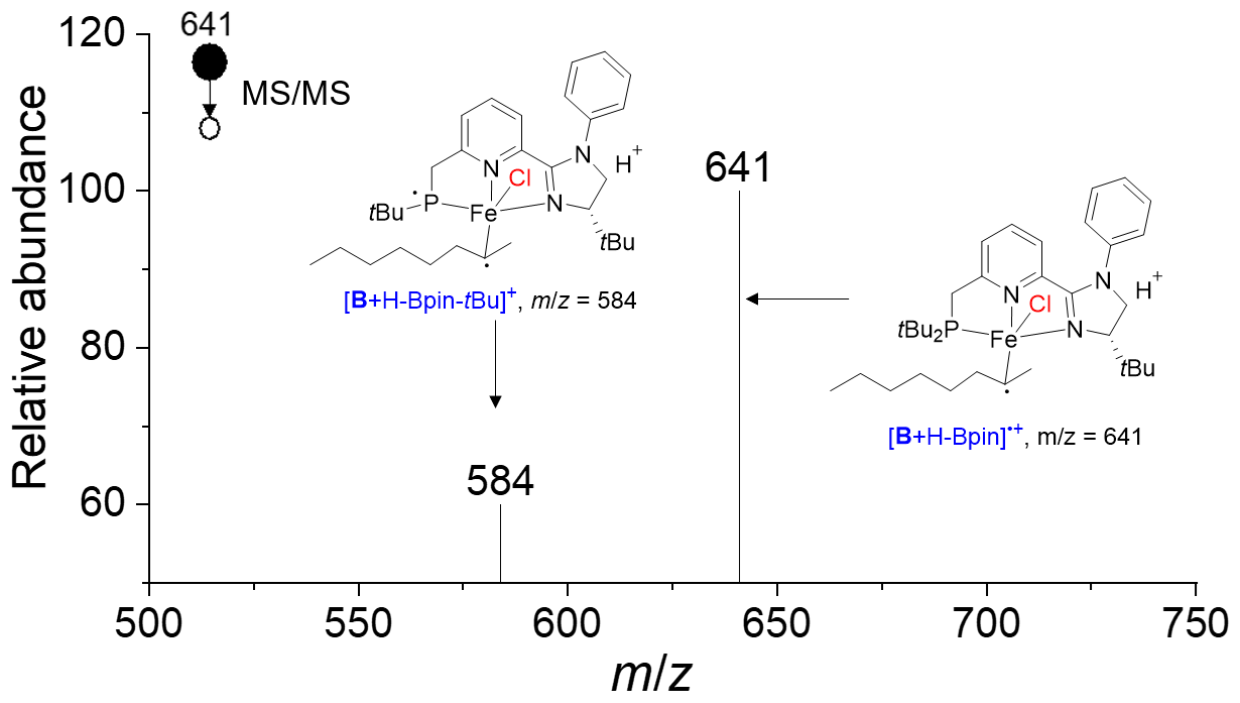

Figure S3-c-3. SAESI-MS/MS spectrum of complex [Fe]Cl(alkyl)

(d)

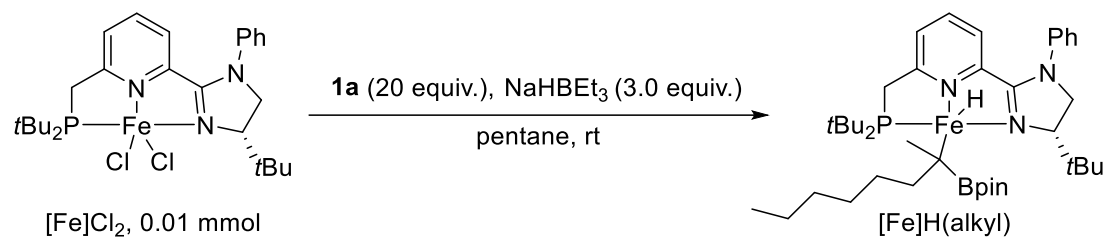

In a nitrogen-filled glove-box, an oven-dried $10 \mathrm{~mL}$ glass tube was charged with $[\mathrm{Fe}] \mathrm{Cl}_{2}(10 \mu \mathrm{mol}, 5.6 \mathrm{mg})$ and pentane $(2 \mathrm{~mL}), \alpha$-vinyl boronates $(0.2 \mathrm{mmol})$. The suspension was sealed and moved out from the glove-box, followed by the injection of $\mathrm{NaHBEt}_{3}(30 \mu \mathrm{mol}, 30 \mu \mathrm{L}, 1 \mathrm{M}$ in THF). The solution was stirred at room temperature for 5 minutes and sent to SAESI-MS analysis. 


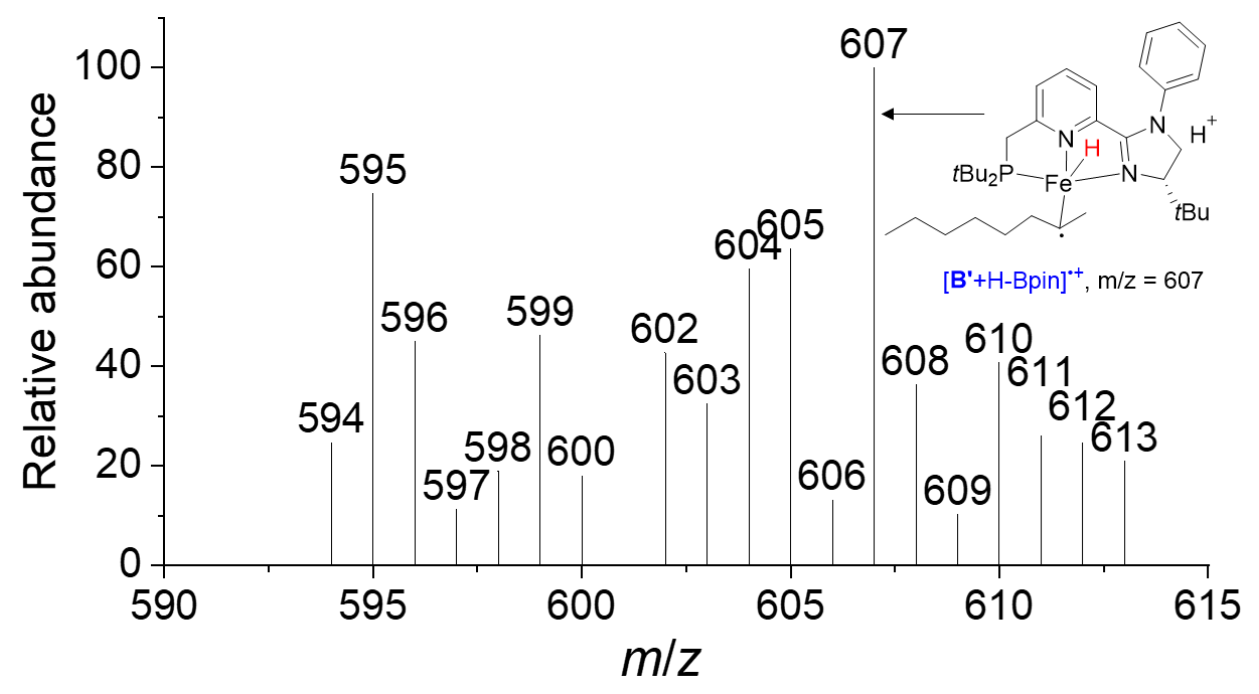

Figure S3-d-1. SAESI-MS spectrum of the reaction solution

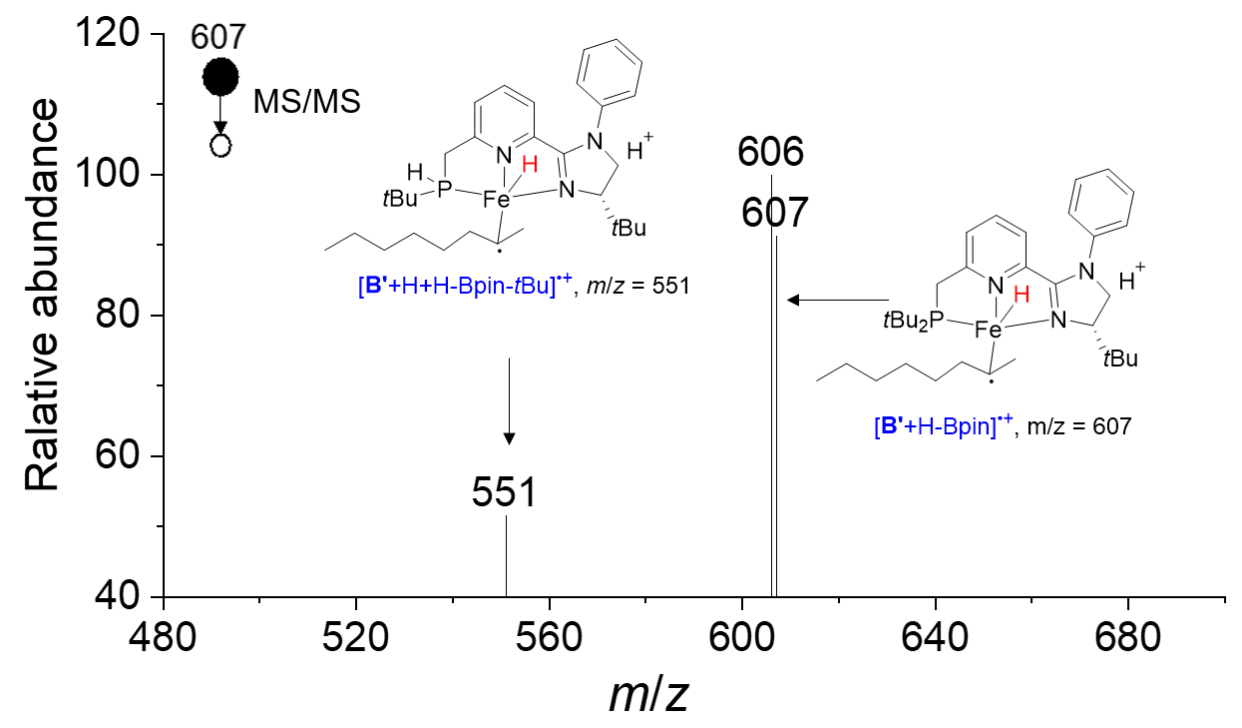

Figure S3-d-2. SAESI-MS/MS spectrum of complex [Fe]H(alkyl)

\section{Deuterium-Labeling}
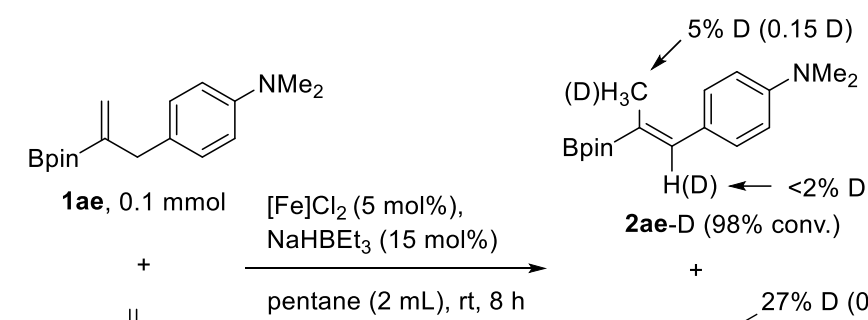

2ae-D (98\% conv.)

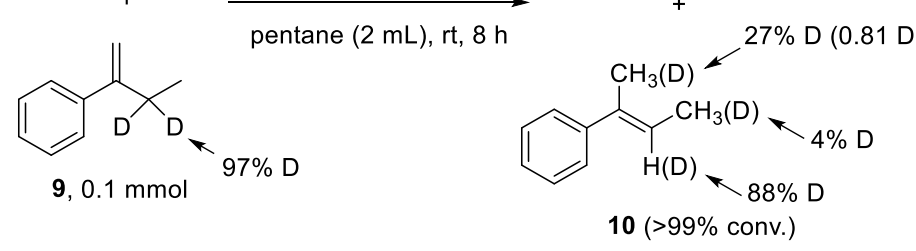

In an nitrogen-filled glove-box, an oven-dried $10 \mathrm{~mL}$ thin-wall glass tube was charged with $[\mathrm{Fe}] \mathrm{Cl}_{2}(10 \mu \mathrm{mol}, 5.6 \mathrm{mg})$, pentane $(2 \mathrm{~mL}), \mathbf{1 a e}(28.7 \mathrm{mg}, 0.1 \mathrm{mmol}), \mathbf{9}(13.4 \mathrm{mg}$, 
$0.1 \mathrm{mml}$ ), then $\mathrm{NaHBEt}_{3}(30 \mu \mathrm{mol}, 30 \mu \mathrm{L}, 1 \mathrm{M}$ in THF) was added to the glass tube. The tube was sealed with a Teflon plug, and the reaction was stirred at room temperature for $2 \mathrm{~h}$. Afterwards, the reaction was quenched by exposing the solution to air. The resulting solution was concentrated in vacuum and the residue was purified by flash silica gel column chromatography to give the product 2 ae-D (98\% conv., $E / Z>$ 20:1), 10 (> 99\% conv., $Z / E>20: 1)$.

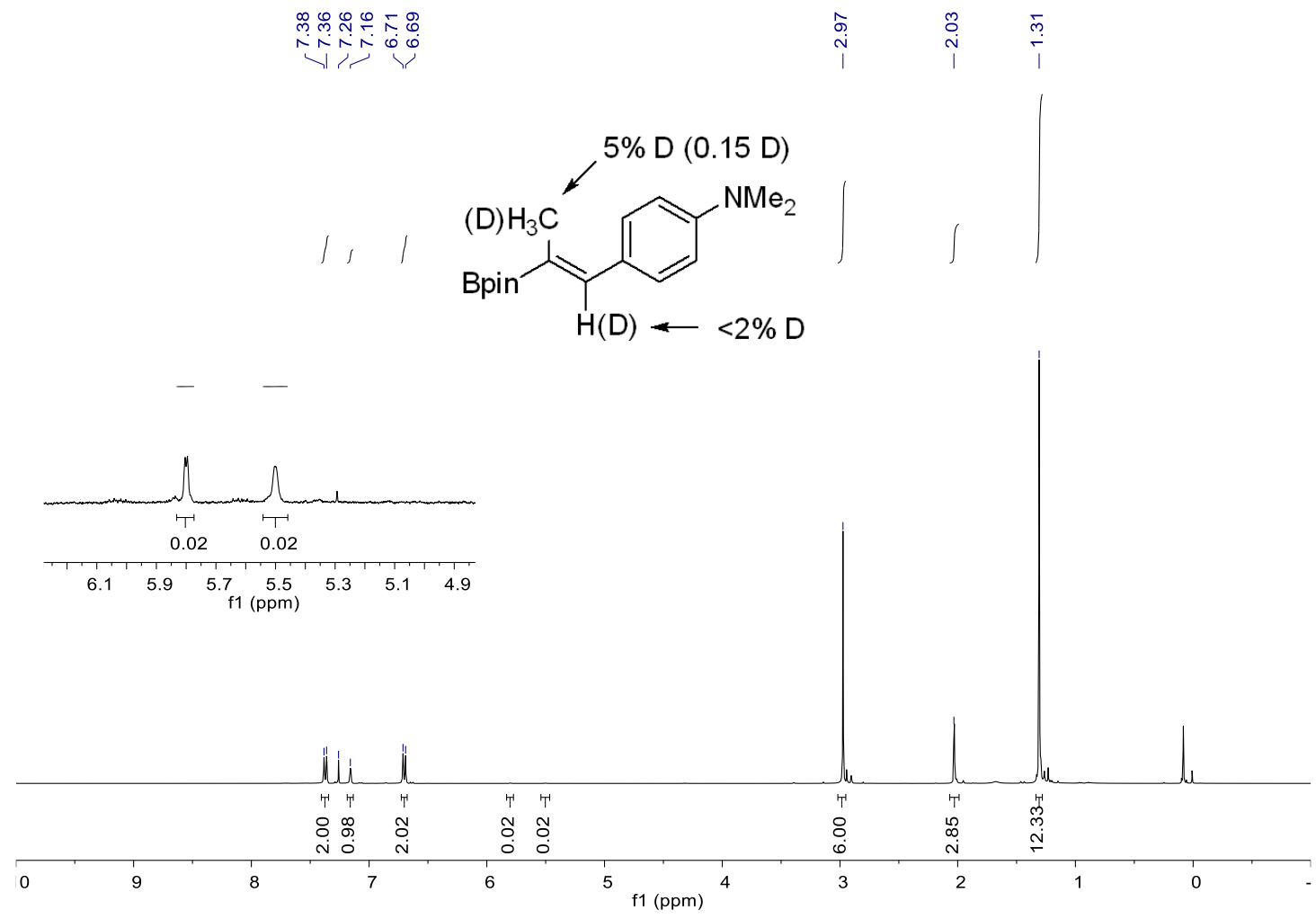

${ }^{1} \mathrm{H}$ NMR (400M, $\mathrm{CDCl}_{3}$ ) spectrum of 2ae-D 

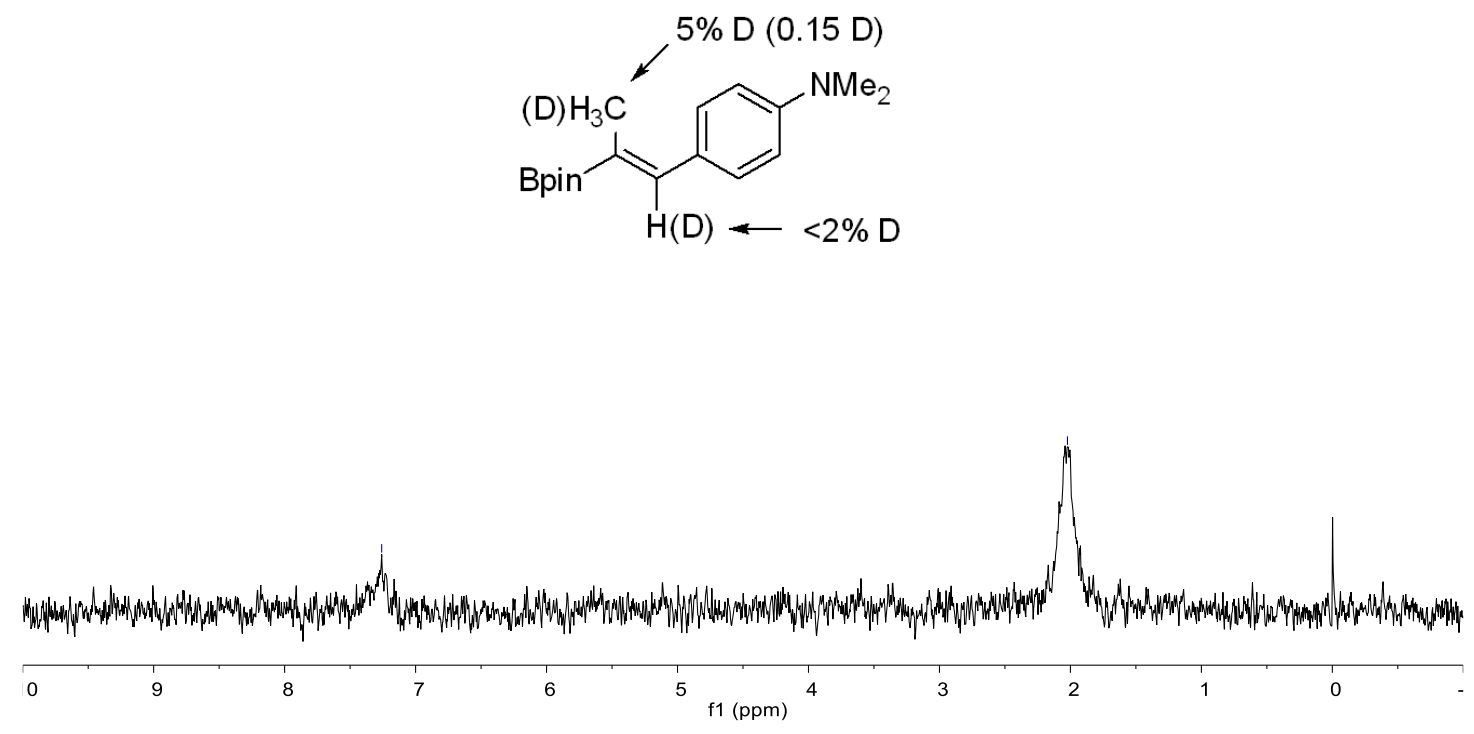

${ }^{2} \mathrm{H}$ NMR (400M, $\mathrm{CHCl}_{3}$ ) spectrum of 2ae-D

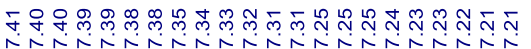

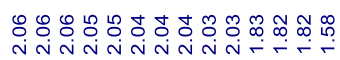

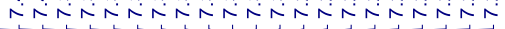

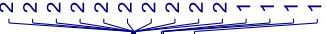
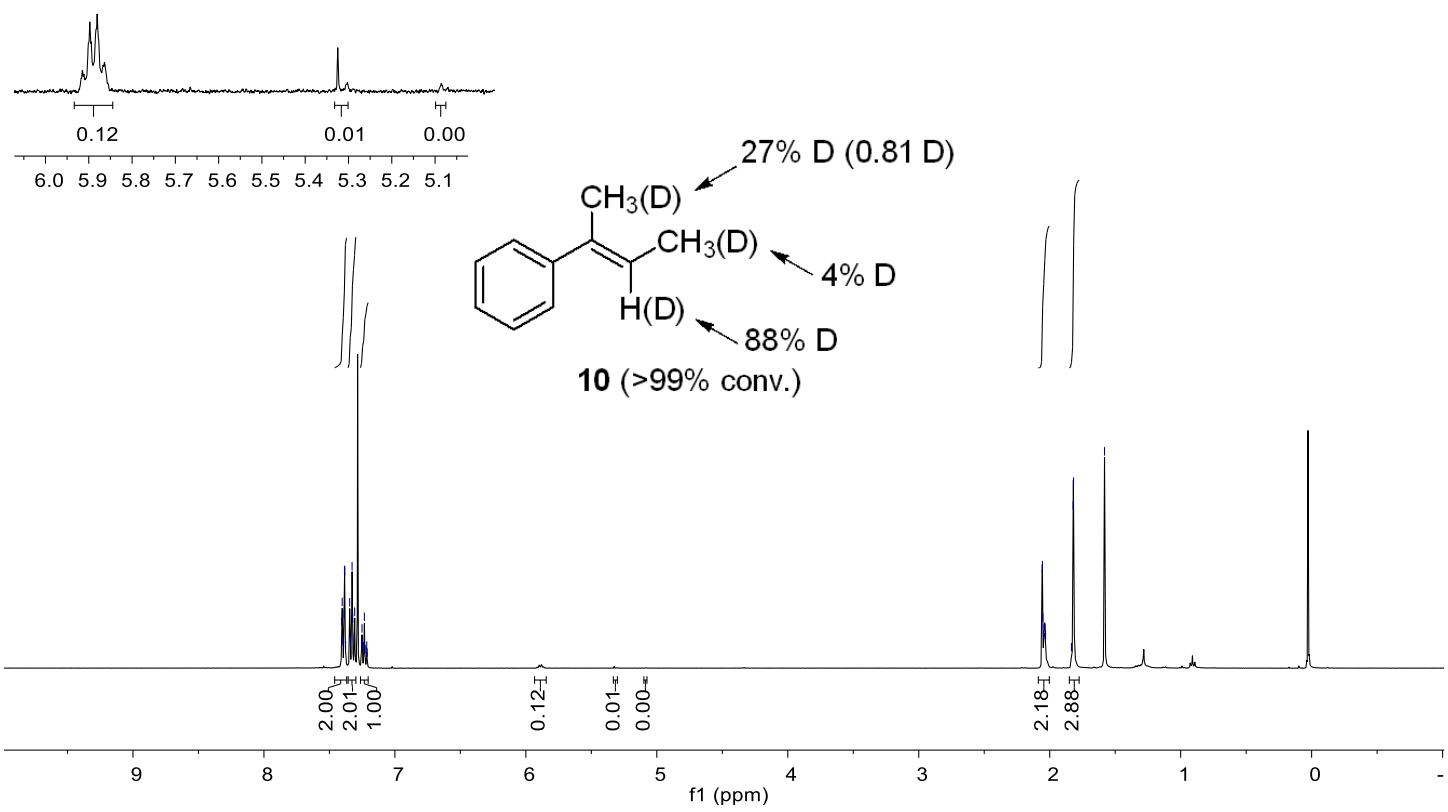

${ }^{1} \mathrm{H} \mathrm{NMR}\left(400 \mathrm{M}, \mathrm{CDCl}_{3}\right.$ ) spectrum of D-2ae 
Studies of reactivity difference between the monohydride A and the dihydride $A^{\text {' }}$

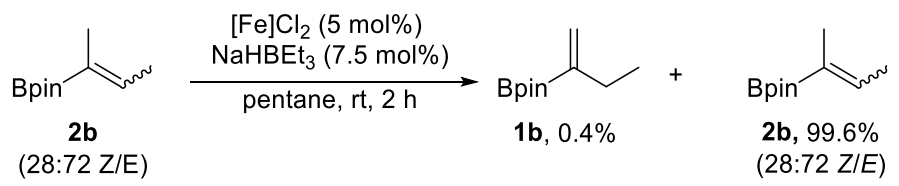

In a nitrogen-filled glove-box, an oven-dried $10 \mathrm{~mL}$ thin-wall glass tube was charged with an $\left[\mathrm{Fe}_{\mathrm{Cl}}(5.6 \mathrm{mg}, 10 \mu \mathrm{mol})\right.$, pentane $(2 \mathrm{~mL}), \mathbf{2 b}(0.2 \mathrm{mmol})$. Afterwards, $\mathrm{NaHBEt}_{3}(15 \mu \mathrm{mol}, 15 \mu \mathrm{L}, 1 \mathrm{M}$ in THF) was added to the glass tube. The tube was sealed with a Teflon plug, and the reaction mixture was stirred at room temperature for $24 \mathrm{~h}$. The reaction was quenched by exposing the solution to air. An aliquot was removed from the flask and analyzed by GC.

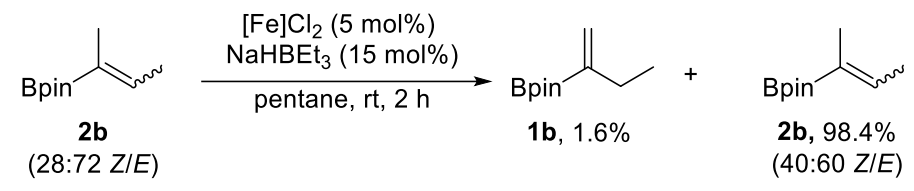

In a nitrogen-filled glove-box, an oven-dried $10 \mathrm{~mL}$ thin-wall glass tube was charged with $[\mathrm{Fe}] \mathrm{Cl}_{2}(5.6 \mathrm{mg}, 10 \mu \mathrm{mol})$, pentane $(2 \mathrm{~mL}), \mathbf{2 b}(0.5 \mathrm{mmol})$. Afterwards, $\mathrm{NaHBEt}_{3}$ (30 $\mu \mathrm{mol}, 30 \mu \mathrm{L}, 1 \mathrm{M}$ in THF) was added to the glass tube. The tube was sealed with a Teflon plug, and the reaction mixture was stirred at room temperature for $24 \mathrm{~h}$. The reaction was quenched by exposing the solution to air. An aliquot was removed from the flask and analyzed by GC.

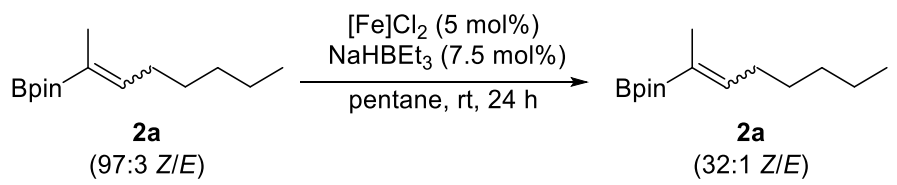

In a nitrogen-filled glove-box, an oven-dried $10 \mathrm{~mL}$ thin-wall glass tube was charged with an $[\mathrm{Fe}] \mathrm{Cl}_{2}(5.6 \mathrm{mg}, 10 \mu \mathrm{mol})$, pentane $(2 \mathrm{~mL}), \mathbf{2 a}(48 \mathrm{mg}, 0.2 \mathrm{mmol})$. Afterwards, $\mathrm{NaHBEt}_{3}(15 \mu \mathrm{mol}, 15 \mu \mathrm{L}, 1 \mathrm{M}$ in THF) was added to the glass tube. The tube was sealed with a Teflon plug, and the reaction mixture was stirred at room temperature for $24 \mathrm{~h}$. The reaction was quenched by exposing the solution to air. An aliquot was removed from the flask and analyzed by GC. 


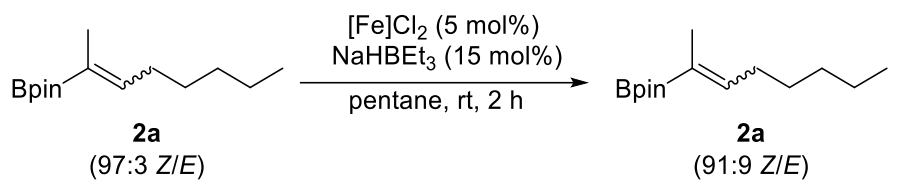

In a nitrogen-filled glove-box, an oven-dried $10 \mathrm{~mL}$ thin-wall glass tube was charged with $[\mathrm{Fe}] \mathrm{Cl}_{2}(5.6 \mathrm{mg}, 10 \mu \mathrm{mol})$, pentane $(2 \mathrm{~mL}), \mathbf{2 a}(48 \mathrm{mg}, 0.2 \mathrm{mmol})$. Afterwards, $\mathrm{NaHBEt}_{3}(30 \mu \mathrm{mol}, 30 \mu \mathrm{L}, 1 \mathrm{M}$ in THF) was added to the glass tube. The tube was sealed with a Teflon plug, and the reaction mixture was stirred at room temperature for $2 \mathrm{~h}$. The reaction was quenched by exposing the solution to air. An aliquot was removed from the flask and analyzed by GC.

\section{References}

(1) Xu, S.; Liu, G.; Huang, Z. Iron Catalyzed Isomerization of $\alpha$-Alkyl Styrenes to Access Trisubstituted Alkenes. Chin. J. Chem . 2021, 39, 585-589.

(2) Zhang, L.; Peng, D.; Leng, X.; Huang, Z. Iron-catalyzed, Atom-economical, Chemo- and Regioselective Alkene Hydroboration with Pinacolborane. Angew. Chem. Int. Ed. 2013, 52, 3676-80.

(3) Zuo, Z.; Zhang, L.; Leng, X.; Huang, Z. Iron-catalyzed Asymmetric Hydrosilylation of Ketones. Chem. Commun. 2015, 51, 5073-6.

(4) Peng, D.; Zhang, Y.; Du, X.; Zhang, L.; Leng, X.; Walter, M. D.; Huang, Z. Phosphinite-iminopyridine Iron Catalysts for Chemoselective Alkene Hydrosilylation. J. Am. Chem. Soc. 2013, 135, 19154-66.

(5) Moran, W. J.; Morken, J. P. Rh-Catalyzed Enantioselective Hydrogenation of Vinyl Boronates for the Construction of Secondary Boronic Esters. Org. Lett. 2006, 8, 2413-2415.

(6) Mushti, C. S.; Kim, J.-H.; Corey, E. J. Total Synthesis of Antheliolide A. J. Am. Chem. Soc. 2006, 128, 14050-14052.

(7) Bigot, A.; Breuninger, D.; Breit, B. One-Pot Desymmetrizing Hydroformylation/Carbonyl Ene Cyclization Process: Straightforward Access to Highly Functionalized Cyclohexanols. Org. Lett. 2008, 10, 5321-5324.

(8) Myhill, J. A.; Zhang, L.; Lovinger, G. J.; Morken, J. P. Enantioselective Construction of Tertiary Boronic Esters by Conjunctive Cross-Coupling. Angew. Chem. Int. Ed. 2018, 57, 12799-12803.

(9) Ganić, A.; Pfaltz, A. Iridium-Catalyzed Enantioselective Hydrogenation of Alkenylboronic Esters. Chem. Eu. J. 2012, 18, 6724-6728.

(10) Coombs, J. R.; Zhang, L.; Morken, J. P. Synthesis of Vinyl Boronates from Aldehydes by a Practical Boron-Wittig Reaction. Org. Lett. 2015, 17, 1708-1711. 
(11) Yao, Z.-J.; Hong, S.; Zhang, W.; Liu, M.; Deng, W. Copper-catalyzed regioselective hydroboration of terminal alkynes in aqueous medium. Tetrahedron Lett. 2016, 57, 910-913.

(12) Aparece, M. D.; Gao, C.; Lovinger, G. J.; Morken, J. P. Vinylidenation of Organoboronic Esters Enabled by a Pd-Catalyzed Metallate Shift. Angew. Chem. Int. Ed. 2019, 58, 592-595.

(13)Guan, W.; Michael, A. K.; McIntosh, M. L.; Koren-Selfridge, L.; Scott, J. P.; Clark, T. B. Stereoselective Formation of Trisubstituted Vinyl Boronate Esters by the Acid-Mediated Elimination of $\alpha$-Hydroxyboronate Esters. J. Org. Chem. 2014, 79, 7199-7204.

(14) Meng, F.; Jang, H.; Jung, B.; Hoveyda, A. H. Cu-catalyzed chemoselective preparation of 2-(pinacolato)boron-substituted allylcopper complexes and their in situ site-, diastereo-, and enantioselective additions to aldehydes and ketones. Angew. Chem. Int. Ed. 2013, 52, 5046-51.

(15) Mao, L.; Bertermann, R.; Emmert, K.; Szabó, K. J.; Marder, T. B. Synthesis of Vinyl-, Allyl-, and 2-Boryl Allylboronates via a Highly Selective CopperCatalyzed Borylation of Propargylic Alcohols. Org. Lett. 2017, 19, 6586-6589.

(16) Takagi, J.; Takahashi, K.; Ishiyama, T.; Miyaura, N. Palladium-Catalyzed CrossCoupling Reaction of Bis(pinacolato)diboron with 1-Alkenyl Halides or Triflates: Convenient Synthesis of Unsymmetrical 1,3-Dienes via the Borylation-Coupling Sequence. J. Am. Chem. Soc. 2002, 124, 8001-8006.

(17) Ojha, D. P.; Prabhu, K. R. Pd-Catalyzed Hydroborylation of Alkynes: A Ligand Controlled Regioselectivity Switch for the Synthesis of $\alpha$ - or $\beta$-Vinylboronates. Org. Lett. 2016, 18, 432-435.

(18) Lawson, J. R.; Wilkins, L. C.; Melen, R. L. Tris(2,4,6-trifluorophenyl)borane: An Efficient Hydroboration Catalyst. Chem. Eu. J. 2017, 23, 10997-11000.

(19) Magre, M.; Maity, B.; Falconnet, A.; Cavallo, L.; Rueping, M. MagnesiumCatalyzed Hydroboration of Terminal and Internal Alkynes. Angew. Chem. Int. Ed. 2019, 58, 7025-7029.

(20) Armstrong, R. J.; Sandford, C.; García-Ruiz, C.; Aggarwal, V. K. Conjunctive Functionalization of Vinyl Boronate Complexes with Electrophiles: A Diastereoselective Three-component Coupling. Chem. Commun. 2017, 53, 49224925.

(21) Meng, F.; Jung, B.; Haeffner, F.; Hoveyda, A. H. NHC-Cu-Catalyzed Protoboration of Monosubstituted Allenes. Ligand-Controlled Site Selectivity, Application to Synthesis and Mechanism. Org. Lett. 2013, 15, 1414-1417.

(22) Yuan, W.; Ma, S. Ligand Controlled Highly Selective Copper-Catalyzed Borylcuprations of Allenes with Bis(pinacolato)diboron. Adv. Synth. Catal. 2012, 354, 1867-1872.

(23) Hong, K.; Liu, X.; Morken, J. P. Simple Access to Elusive $\alpha$-Boryl Carbanions and Their Alkylation: An Umpolung Construction for Organic Synthesis. J. Am. Chem. Soc. 2014, 136, 10581-10584.

(24) Sun, S.-Z.; Talavera, L.; Spieß, P.; Day, C. S.; Martin, R. sp3 Bis-Organometallic Reagents via Catalytic 1,1-Difunctionalization of Unactivated Olefins. Angew. 
Chem. Int. Ed. 2021, 60, 11740-11744.

(25) Weber, F.; Ballmann, M.; Kohlmeyer, C.; Hilt, G. Nickel-Catalyzed Double Bond Transposition of Alkenyl Boronates for in Situ syn-Selective Allylboration Reactions. Org. Lett. 2016, 18, 548-551.

(26) Chausset-Boissarie, L.; Ghozati, K.; LaBine, E.; Chen, J. L. Y.; Aggarwal, V. K.; Crudden, C. M. Enantiospecific, Regioselective Cross-Coupling Reactions of Secondary Allylic Boronic Esters. Chem. Eu. J. 2013, 19, 17698-17701.

(27) Yoshida, H.; Takemoto, Y.; Takaki, K. A Masked Diboron in Cu-catalysed Borylation Reaction: Highly Regioselective Formal Hydroboration of Alkynes for Synthesis of Branched Alkenylborons. Chem. Commun. 2014, 50, 8299-302.

(28) Liang, G.; Miller, A. K.; Trauner, D. Stereoselective Synthesis of Cyercene A and the Placidenes. Org. Lett. 2005, 7, 819-821. 


\section{NMR Spectra}

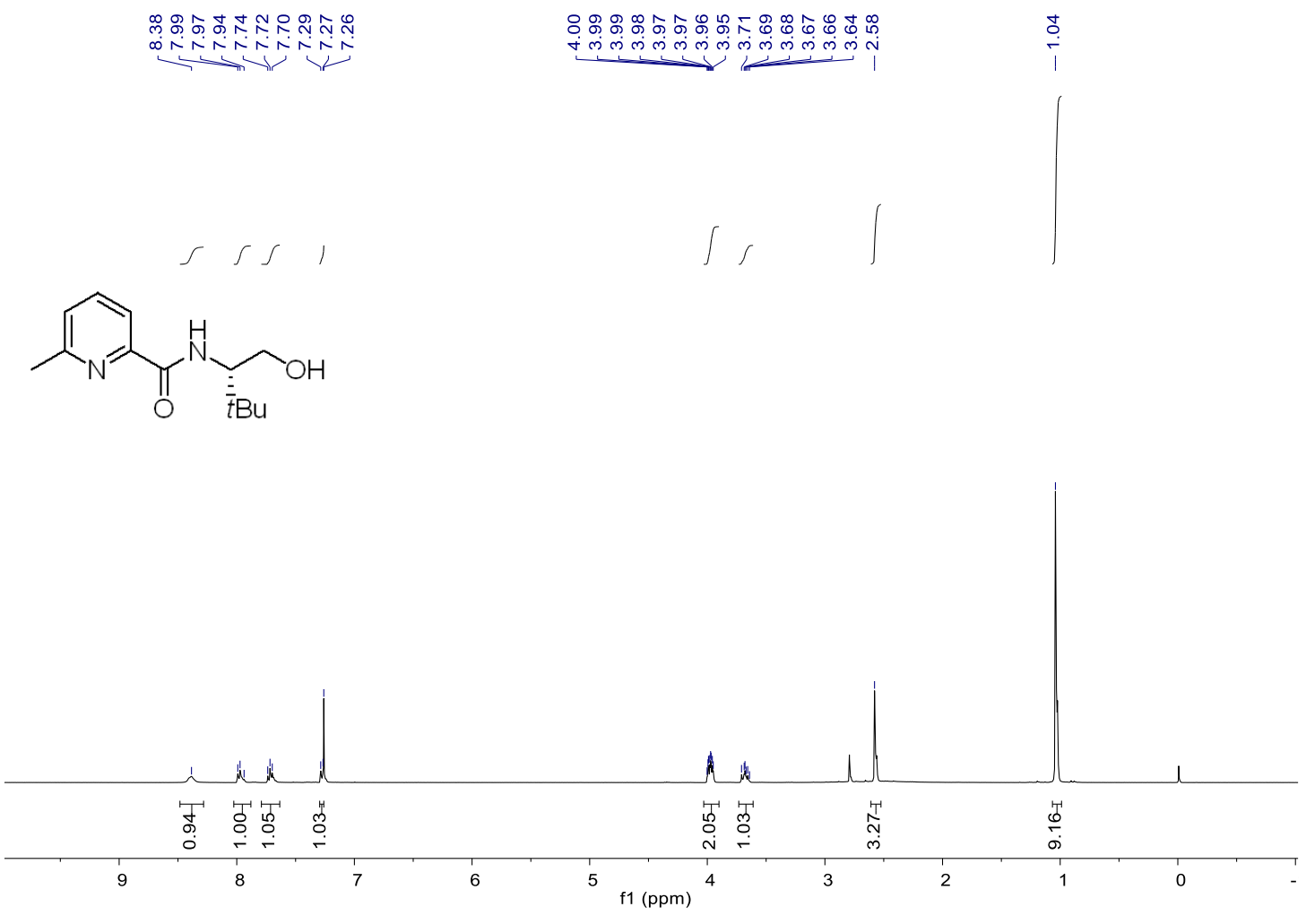

${ }^{1} \mathrm{H}$ NMR (400M, $\mathrm{CDCl}_{3}$ ) spectrum of $\mathbf{3}$

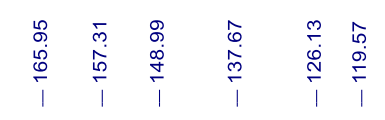

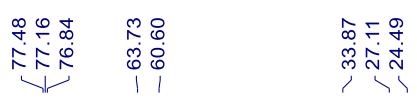

$\overbrace{\mathrm{O}} \overbrace{\overline{\bar{t}}_{\mathrm{Bu}}}^{\mathrm{N}} \mathrm{OH}$

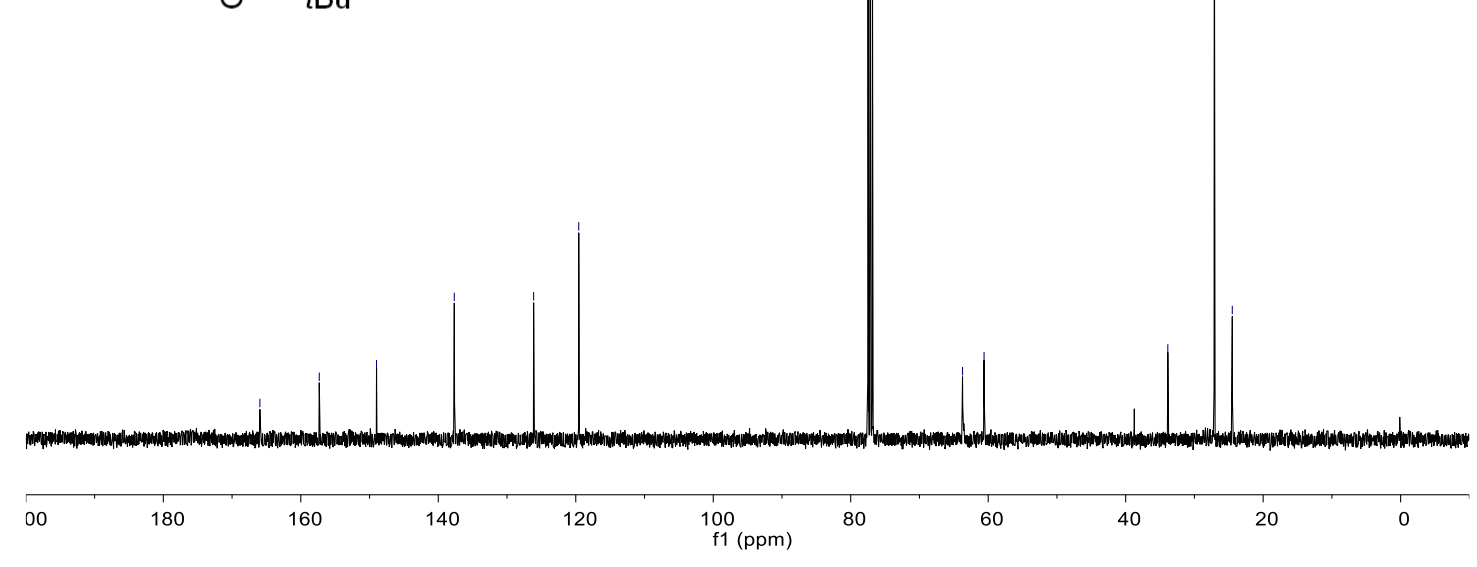

${ }^{13} \mathrm{C}$ NMR $\left(101 \mathrm{M}, \mathrm{CDCl}_{3}\right)$ spectrum of $\mathbf{3}$ 


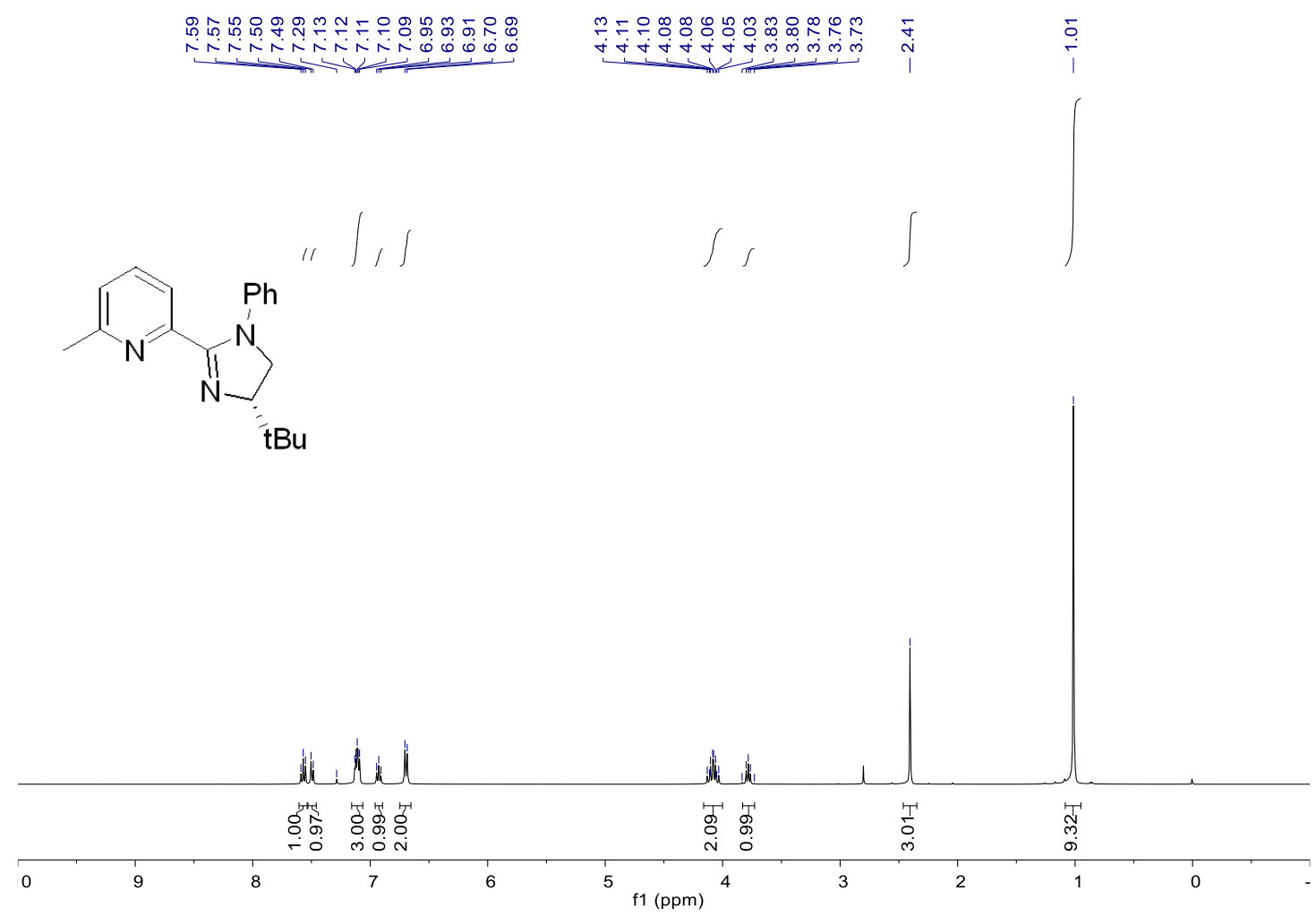

${ }^{1} \mathrm{H}$ NMR (400M, $\mathrm{CDCl}_{3}$ ) spectrum of $\mathbf{4}$

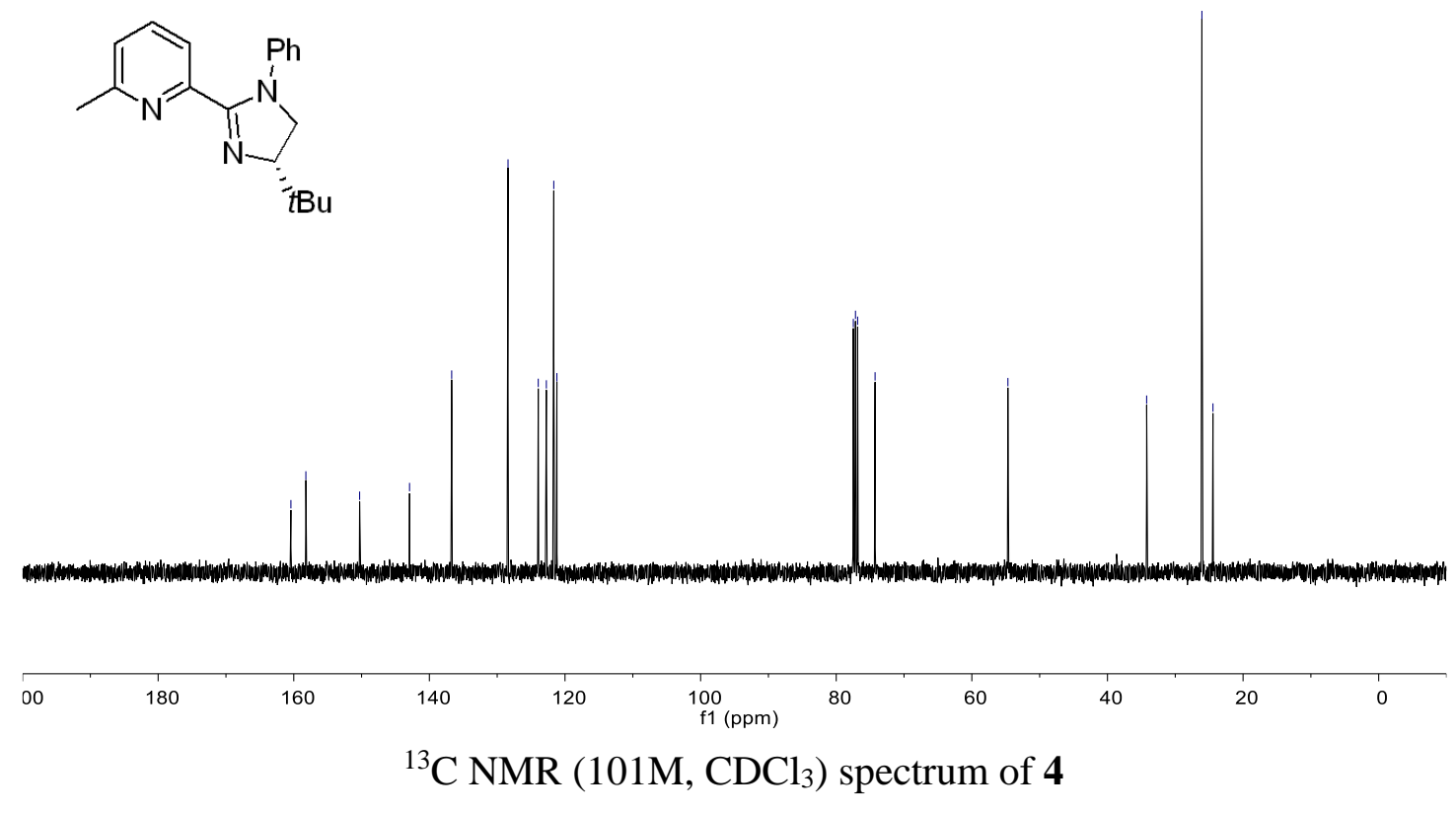




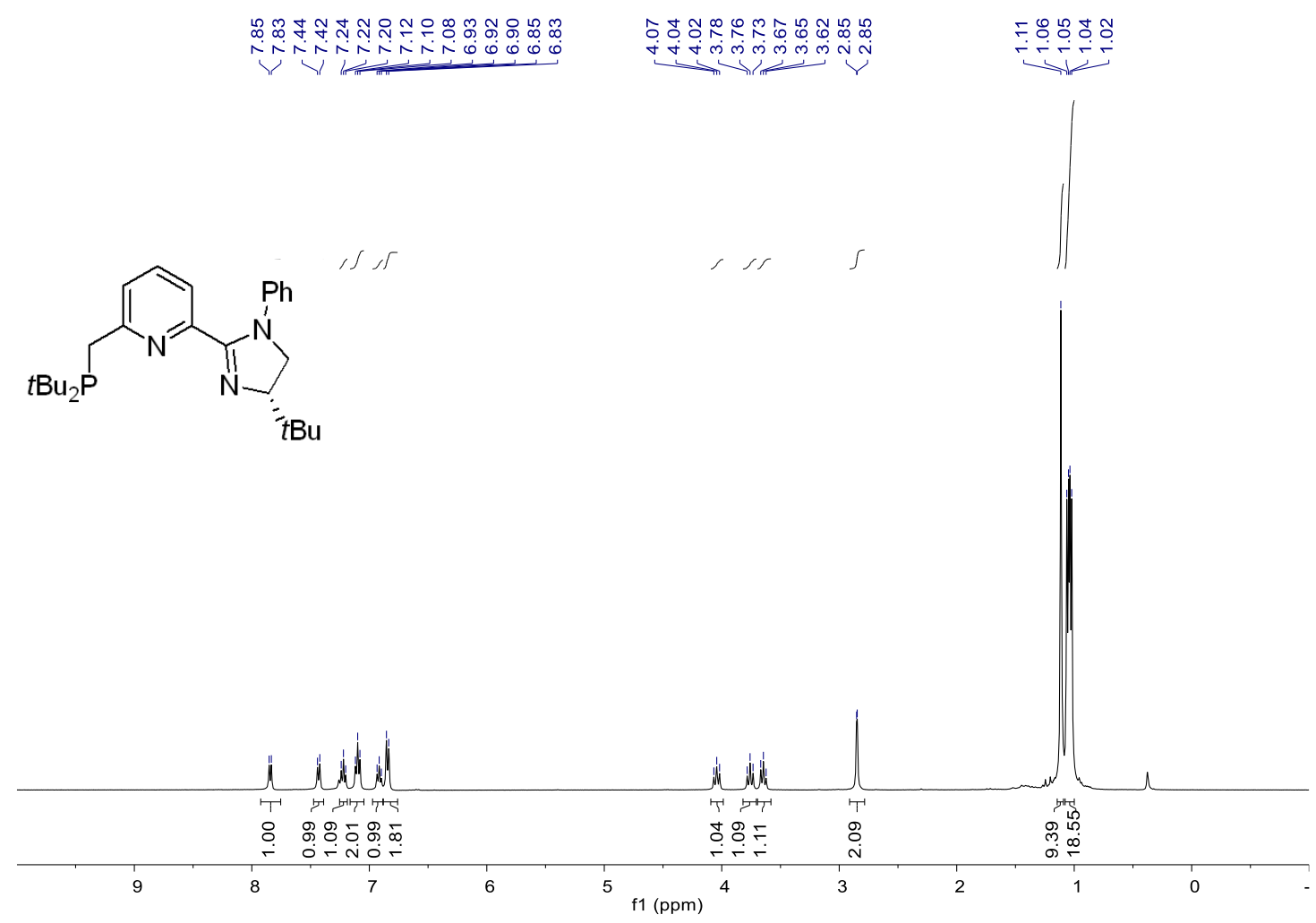

${ }^{1} \mathrm{H}$ NMR (400M, $\mathrm{CDCl}_{3}$ ) spectrum of 5

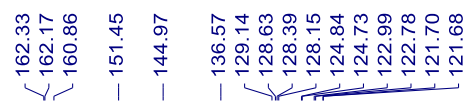

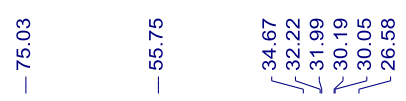

(

180

160

140

$\begin{array}{ll}100 & 80 \\ \mathrm{f} 1(\mathrm{ppm}) & \end{array}$

60

40

20

${ }^{13} \mathrm{C}$ NMR (101M, $\left.\mathrm{C}_{6} \mathrm{D}_{6}\right)$ spectrum of 5 


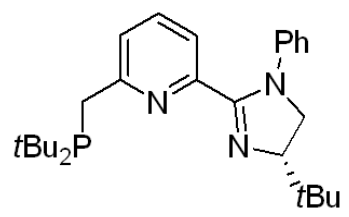
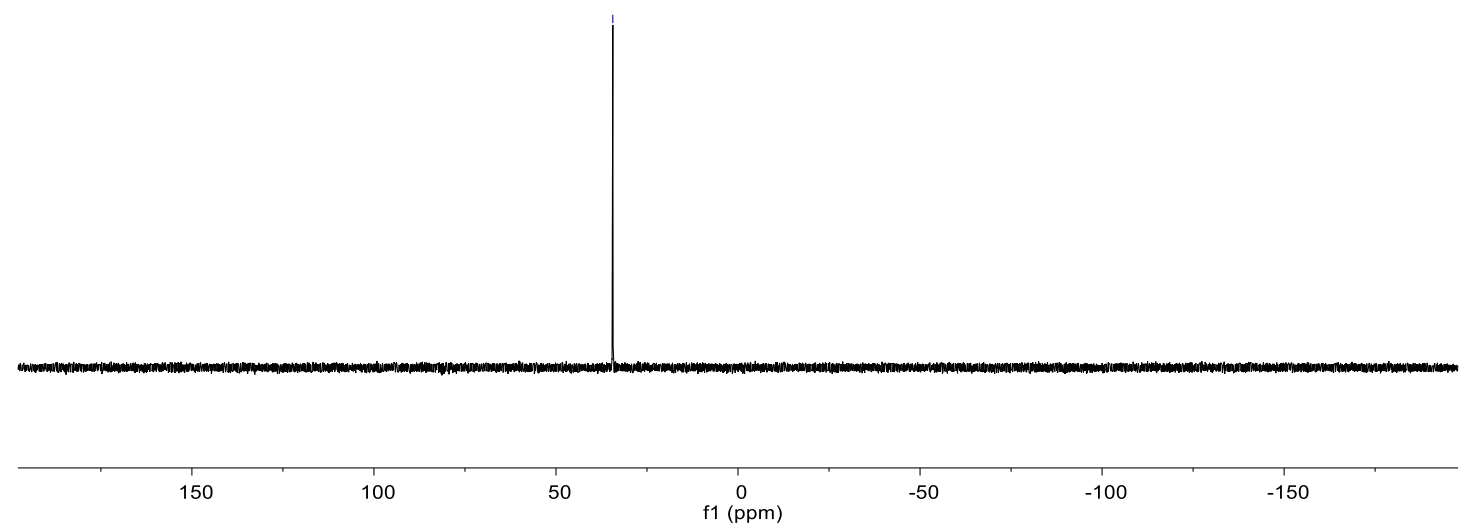

${ }^{31} \mathrm{P}$ NMR (162 MHz, $\left.\mathrm{CDCl}_{3}\right)$ spectrum of 5

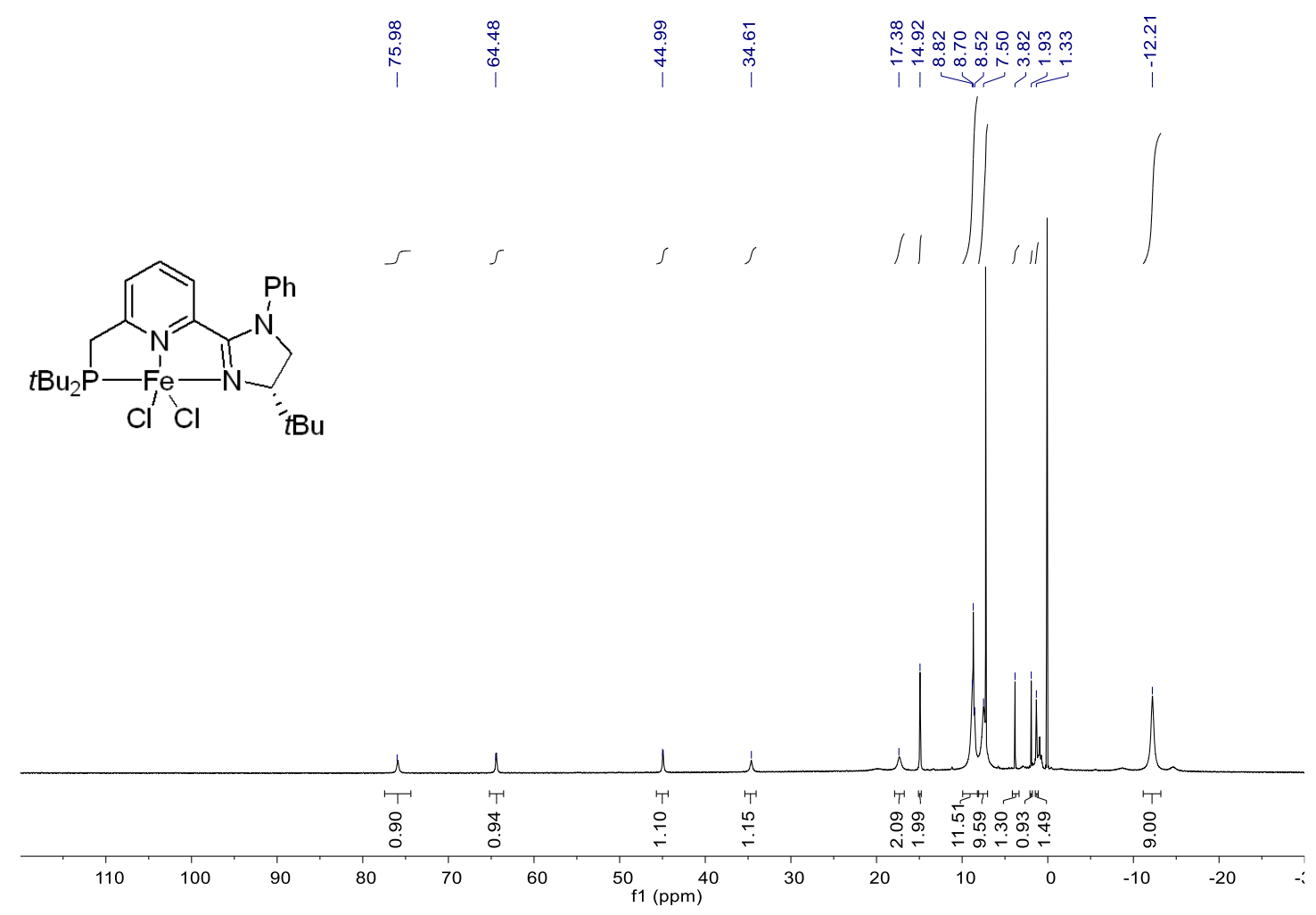

${ }^{1} \mathrm{H}$ NMR (400M, $\mathrm{CDCl}_{3}$ ) spectrum of $[\mathrm{Fe}] \mathrm{Cl}_{2}$ 


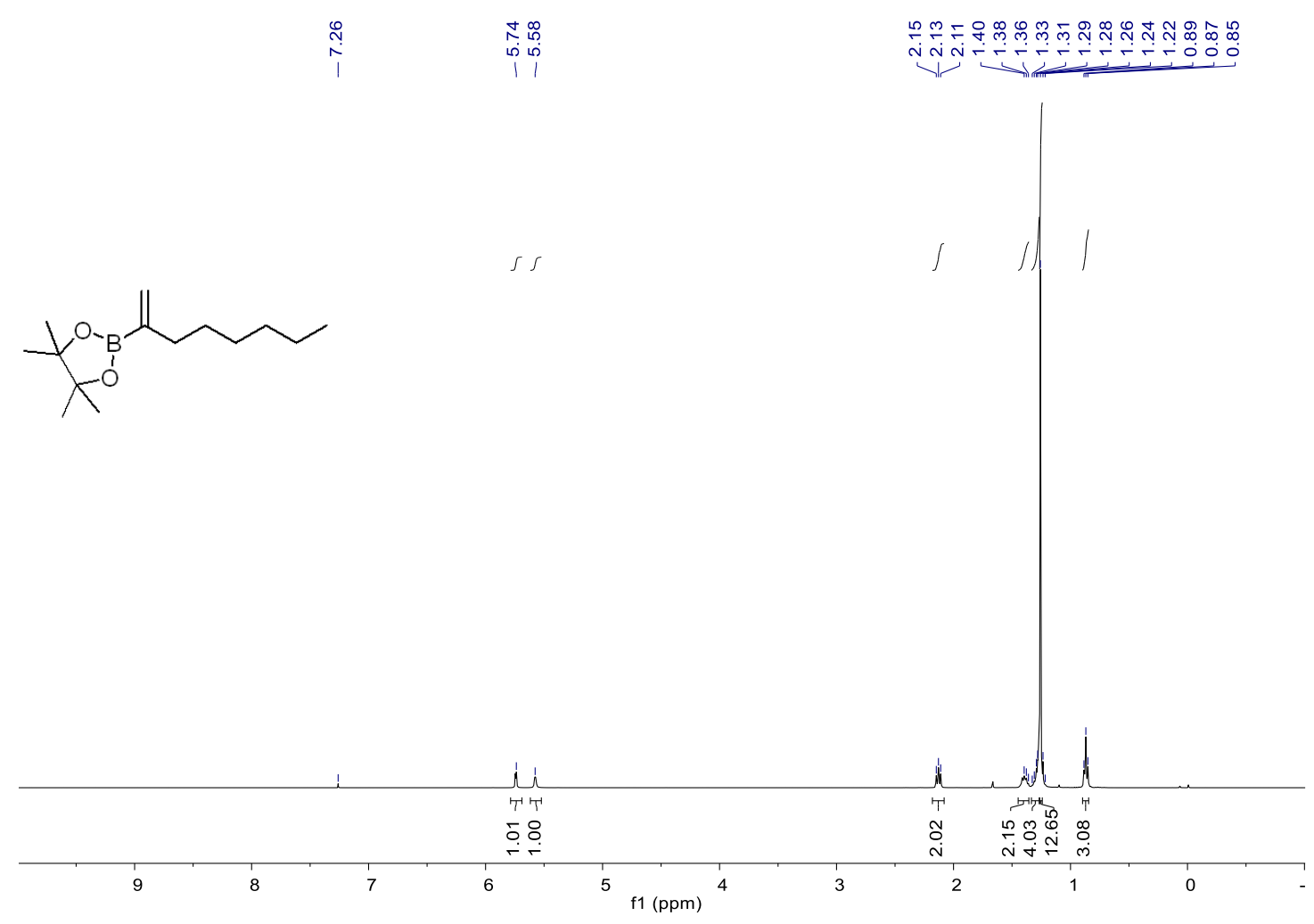

${ }^{1} \mathrm{H}$ NMR (400M, $\mathrm{CDCl}_{3}$ ) spectrum of $\mathbf{1 a}$

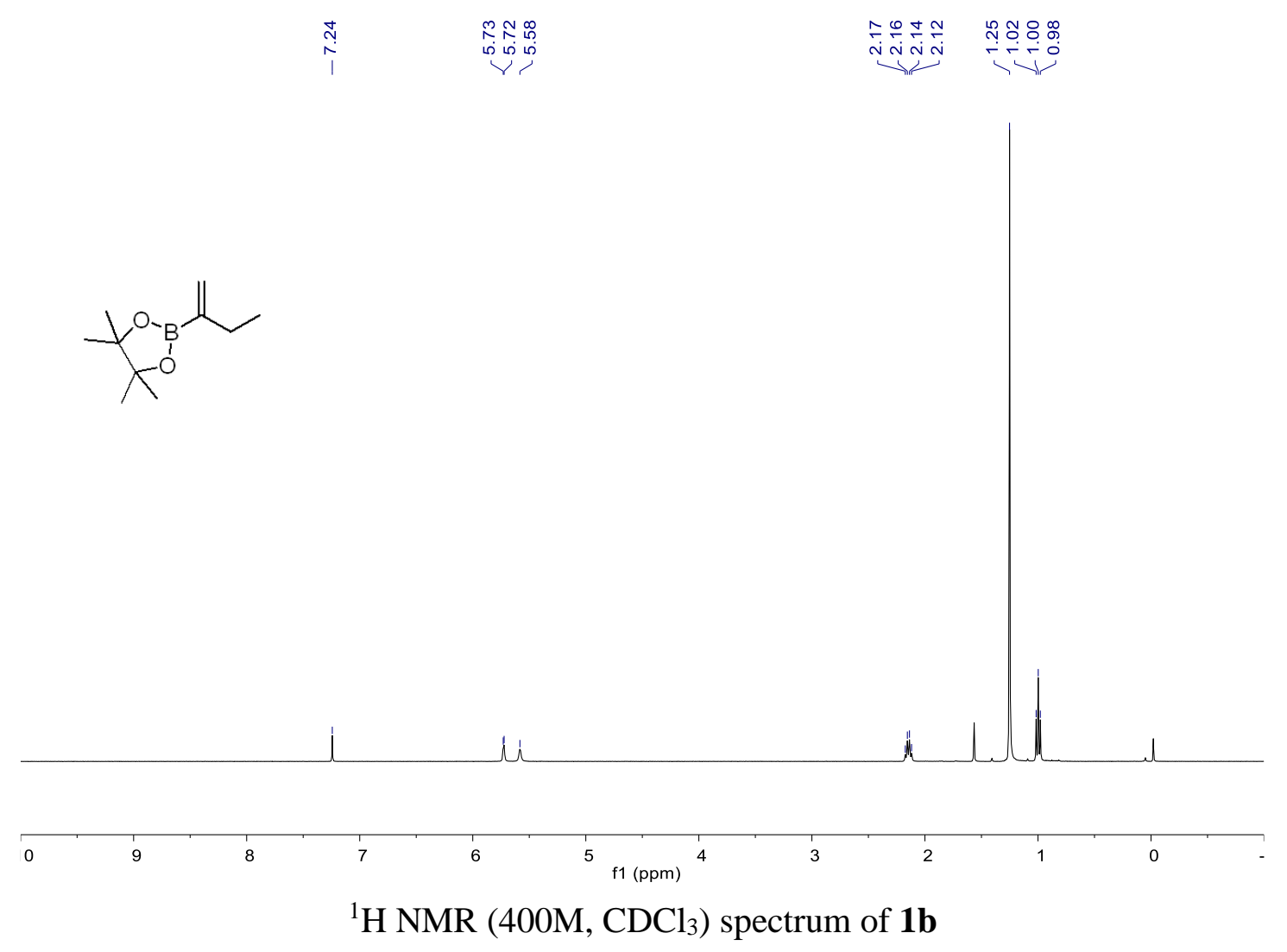




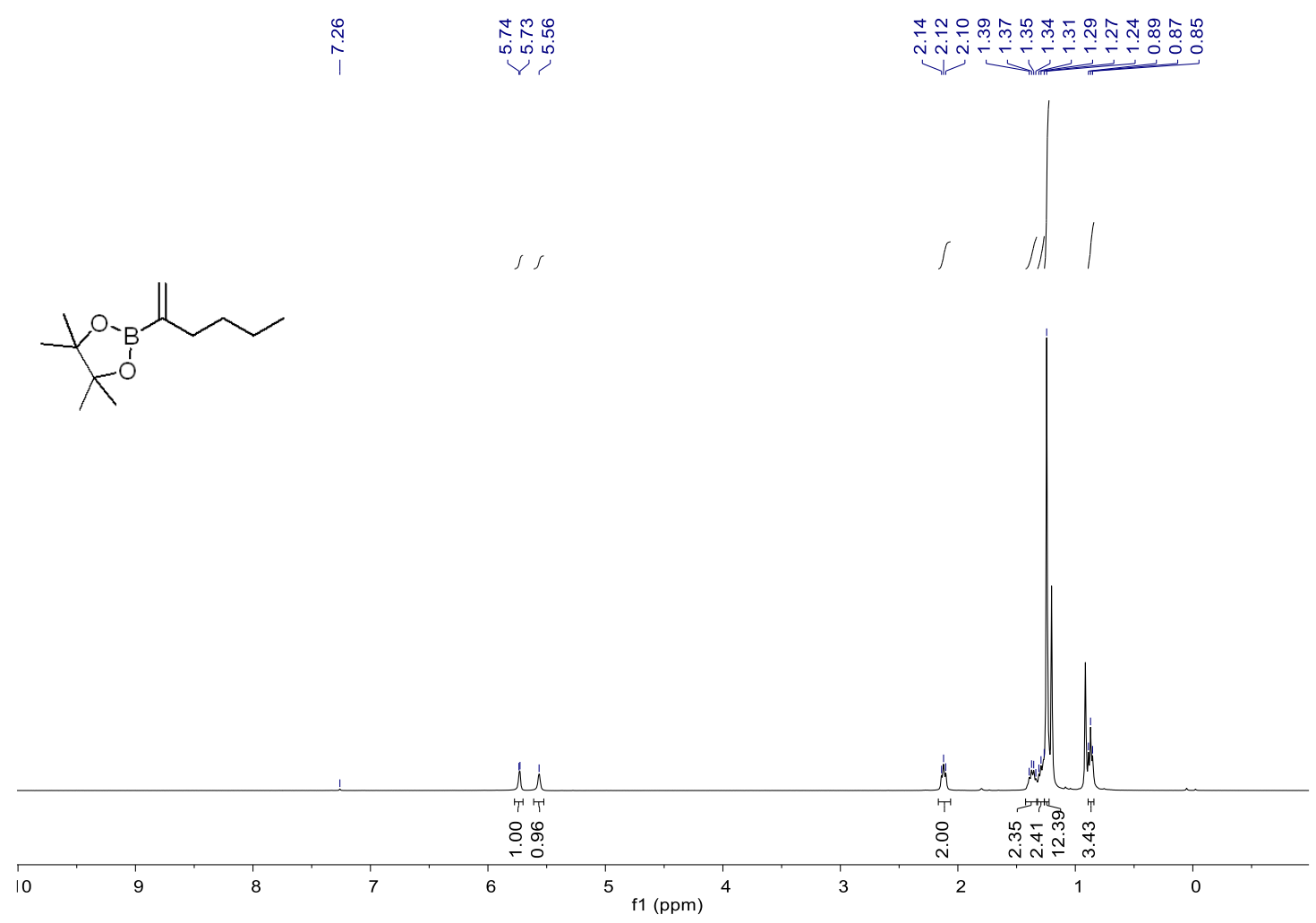

${ }^{1} \mathrm{H}$ NMR (400M, $\mathrm{CDCl}_{3}$ ) spectrum of $\mathbf{1 c}$

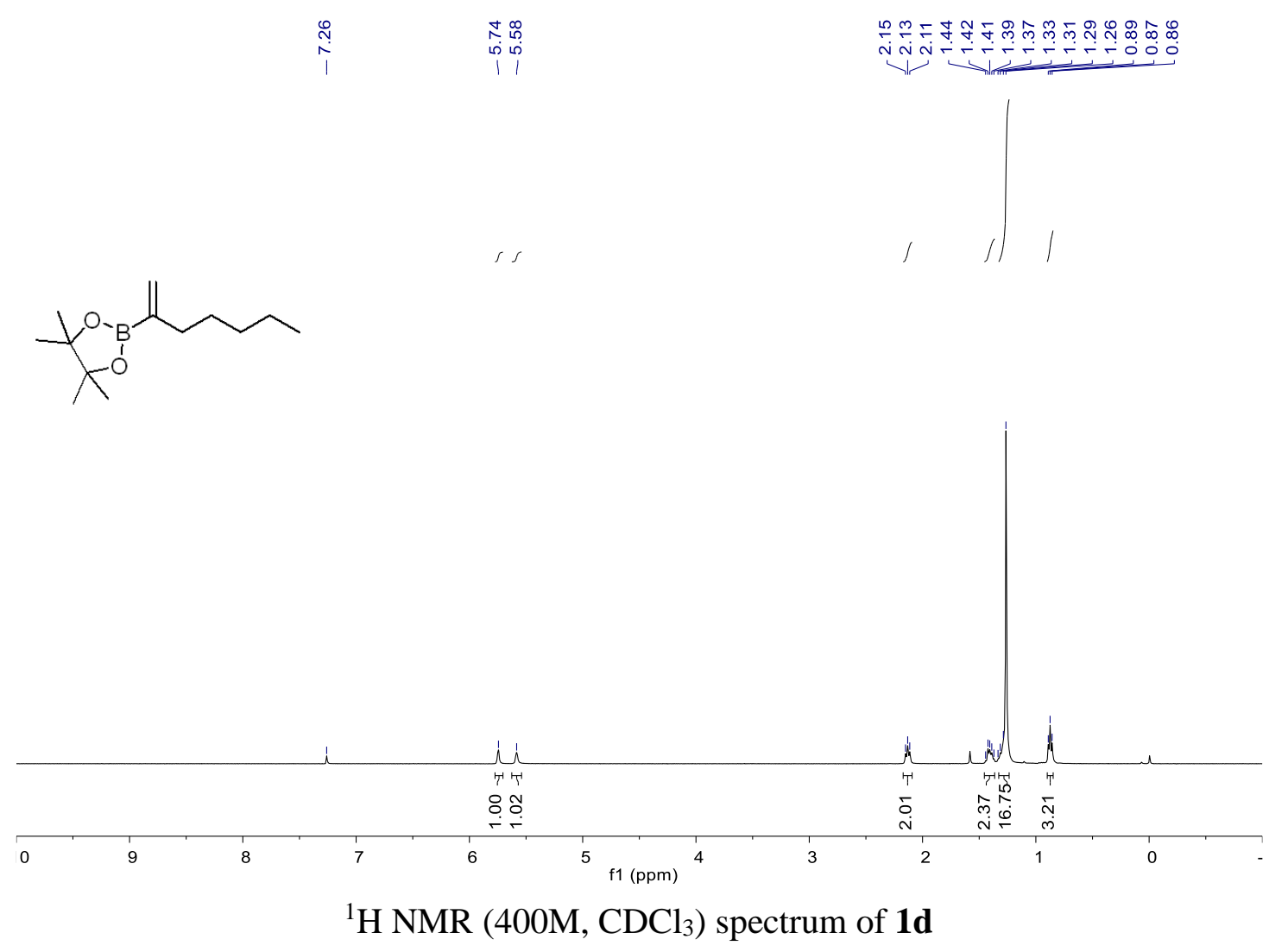




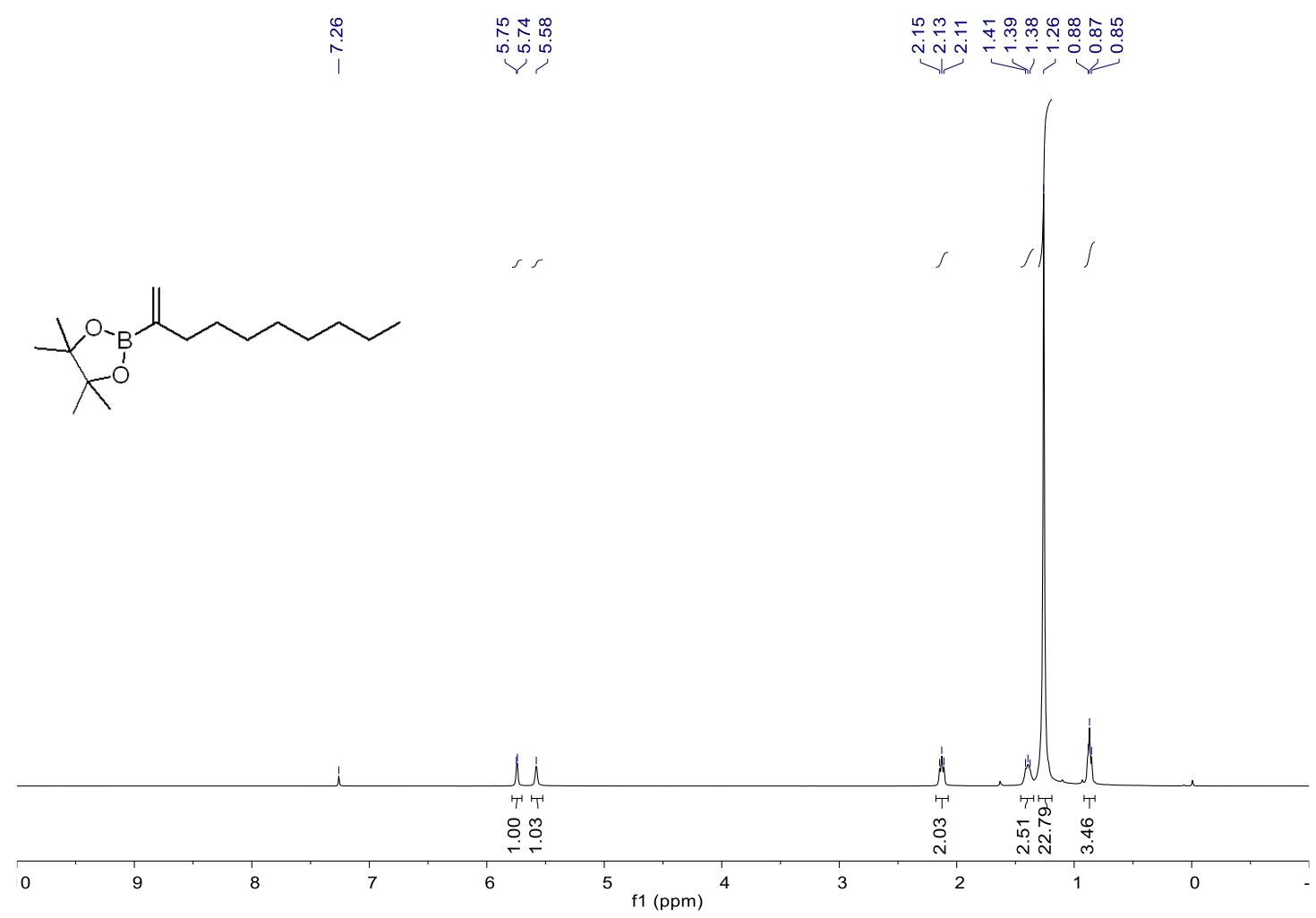

${ }^{1} \mathrm{H}$ NMR (400M, $\mathrm{CDCl}_{3}$ ) spectrum of $\mathbf{1 e}$
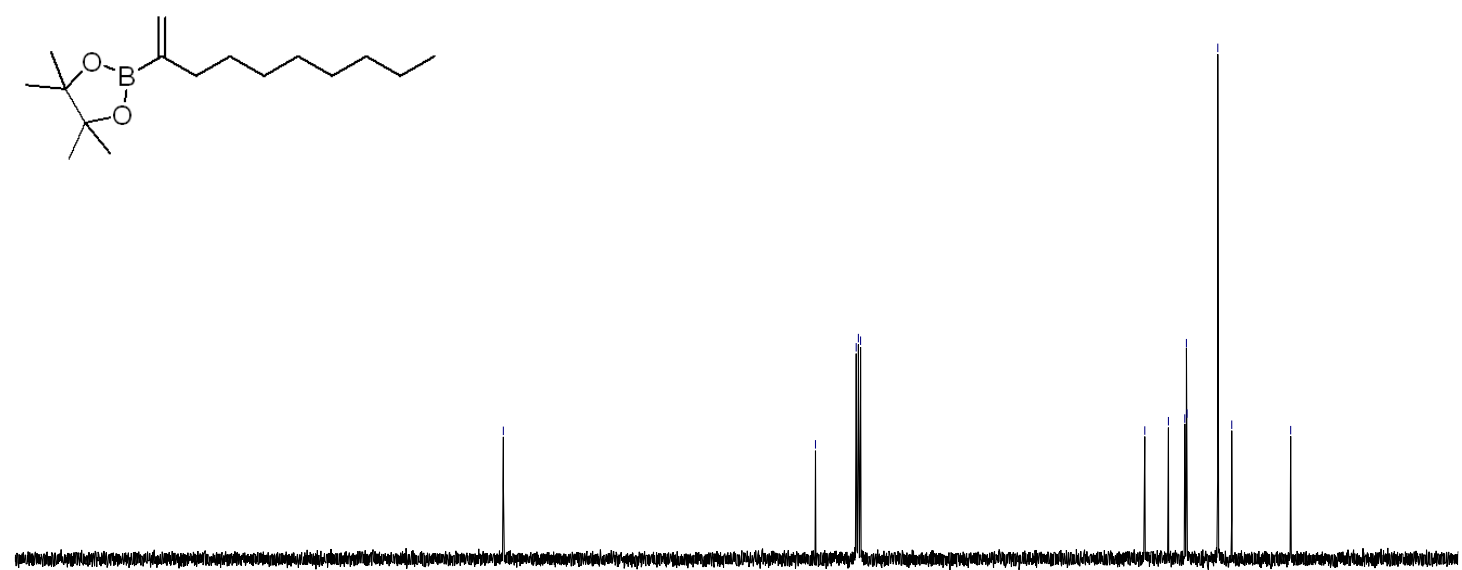

${ }^{13} \mathrm{C} \mathrm{NMR}\left(101 \mathrm{M}, \mathrm{CDCl}_{3}\right)$ spectrum of $\mathbf{1 e}$ 

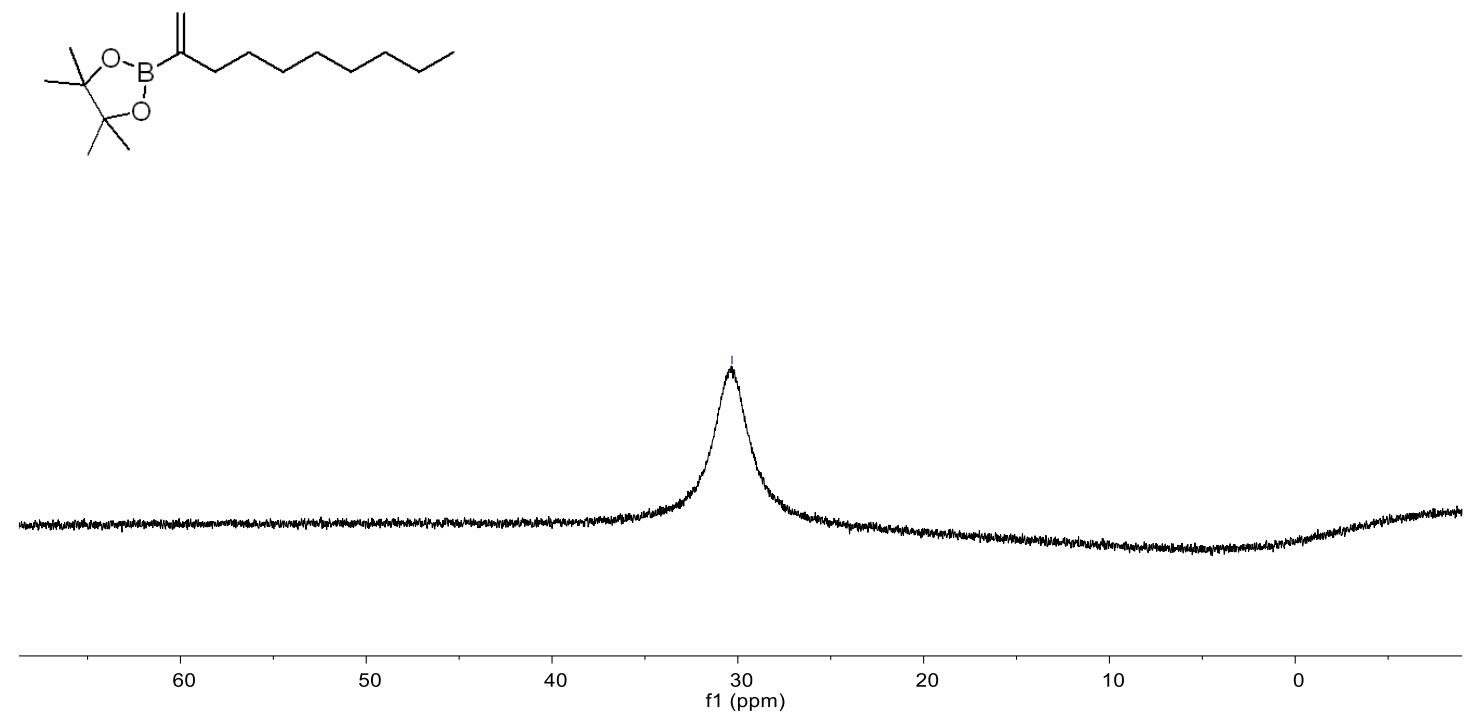

${ }^{11} \mathrm{~B}$ NMR (128M, $\left.\mathrm{CDCl}_{3}\right)$ spectrum of $\mathbf{1 e}$

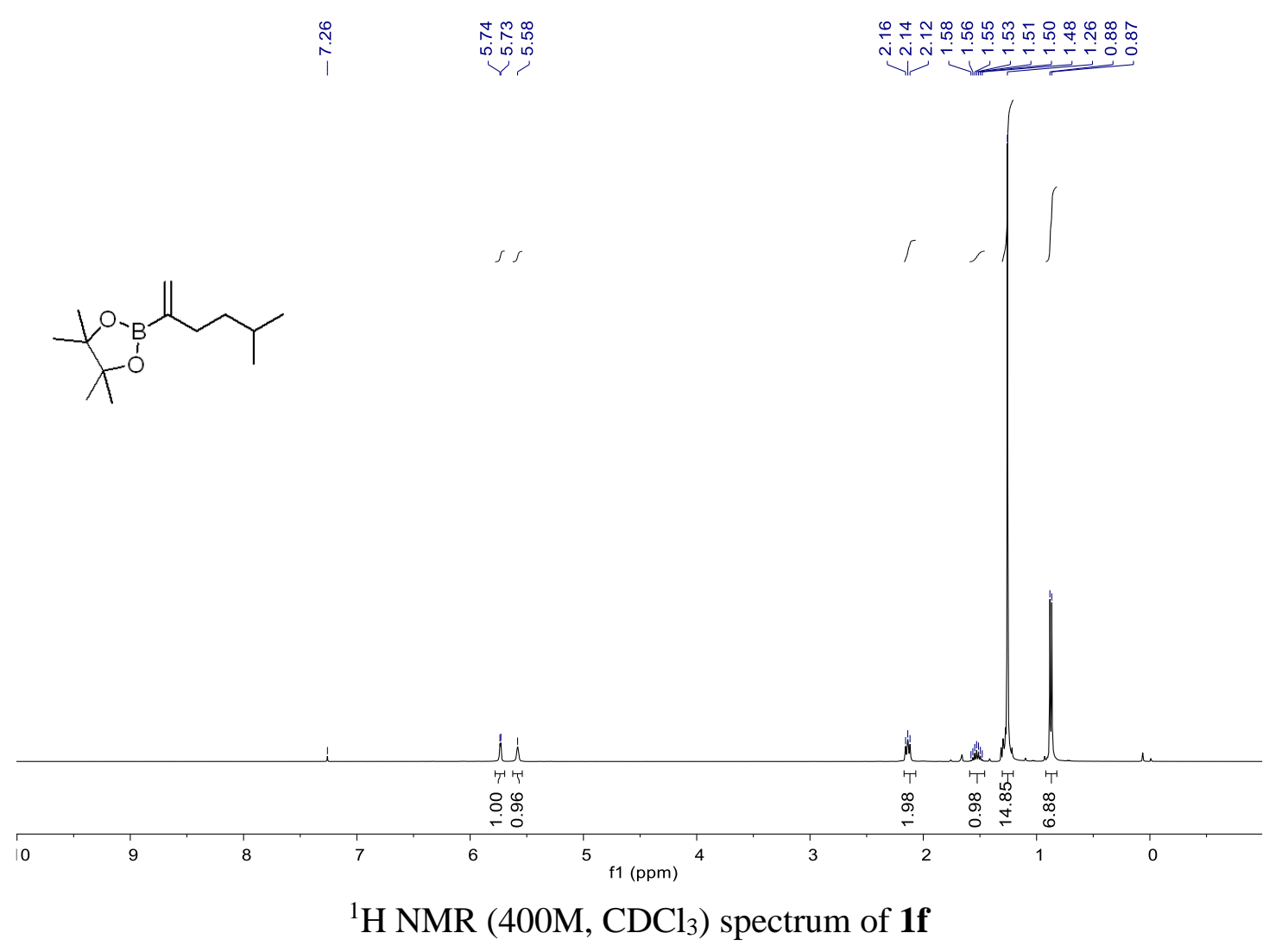



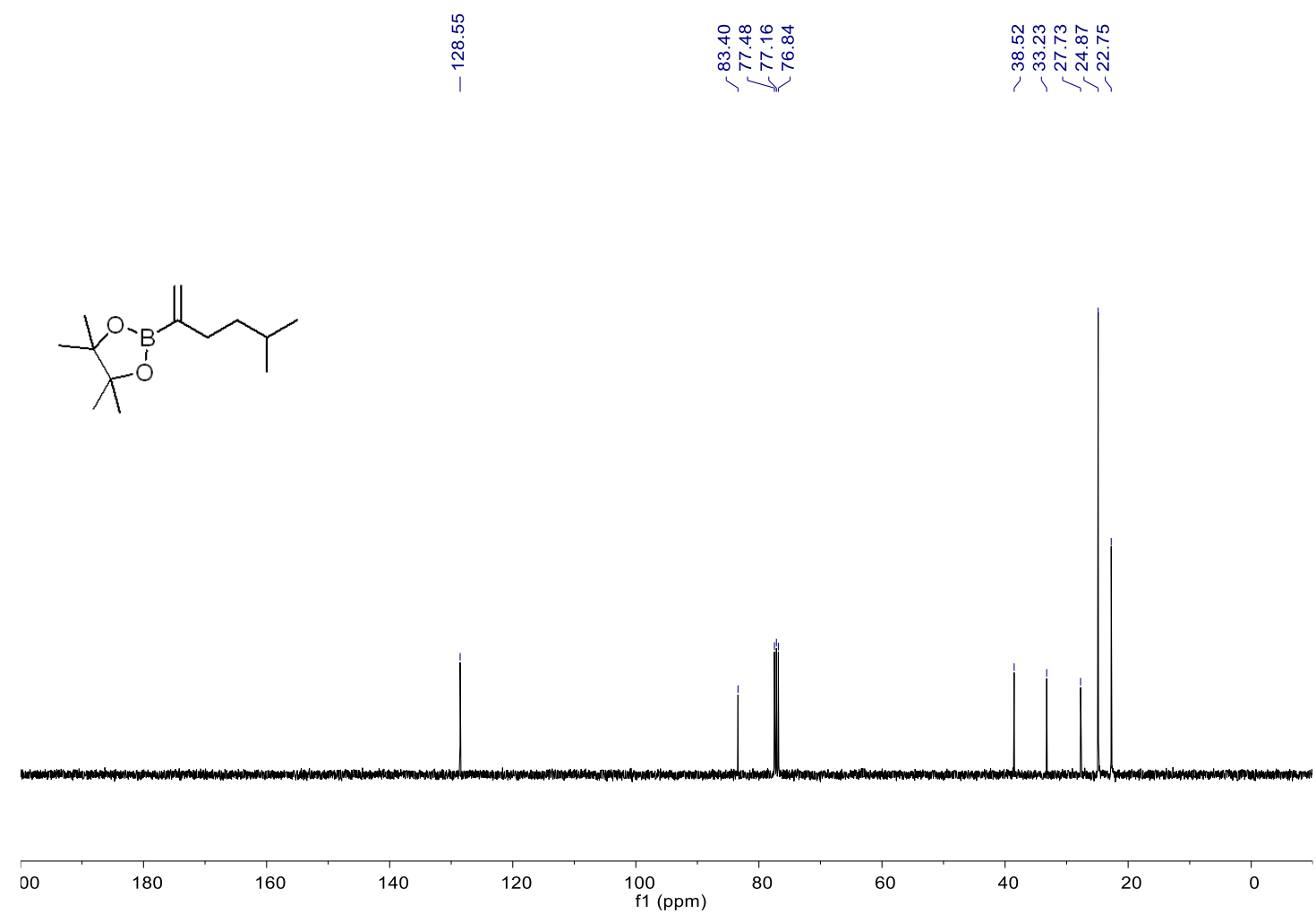

${ }^{13} \mathrm{C} \mathrm{NMR}\left(101 \mathrm{M}, \mathrm{CDCl}_{3}\right)$ spectrum of $\mathbf{1 f}$<smiles>CC(C)CC(C)CC(=O)OC(C)(C)C</smiles>

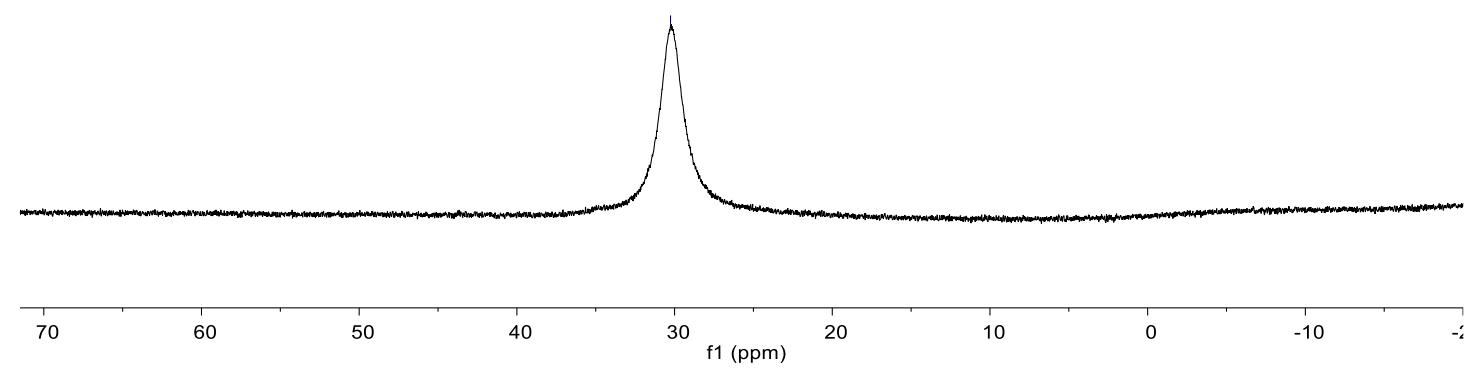

${ }^{11} \mathrm{~B}$ NMR $\left(128 \mathrm{M}, \mathrm{CDCl}_{3}\right)$ spectrum of $\mathbf{1 f}$ 


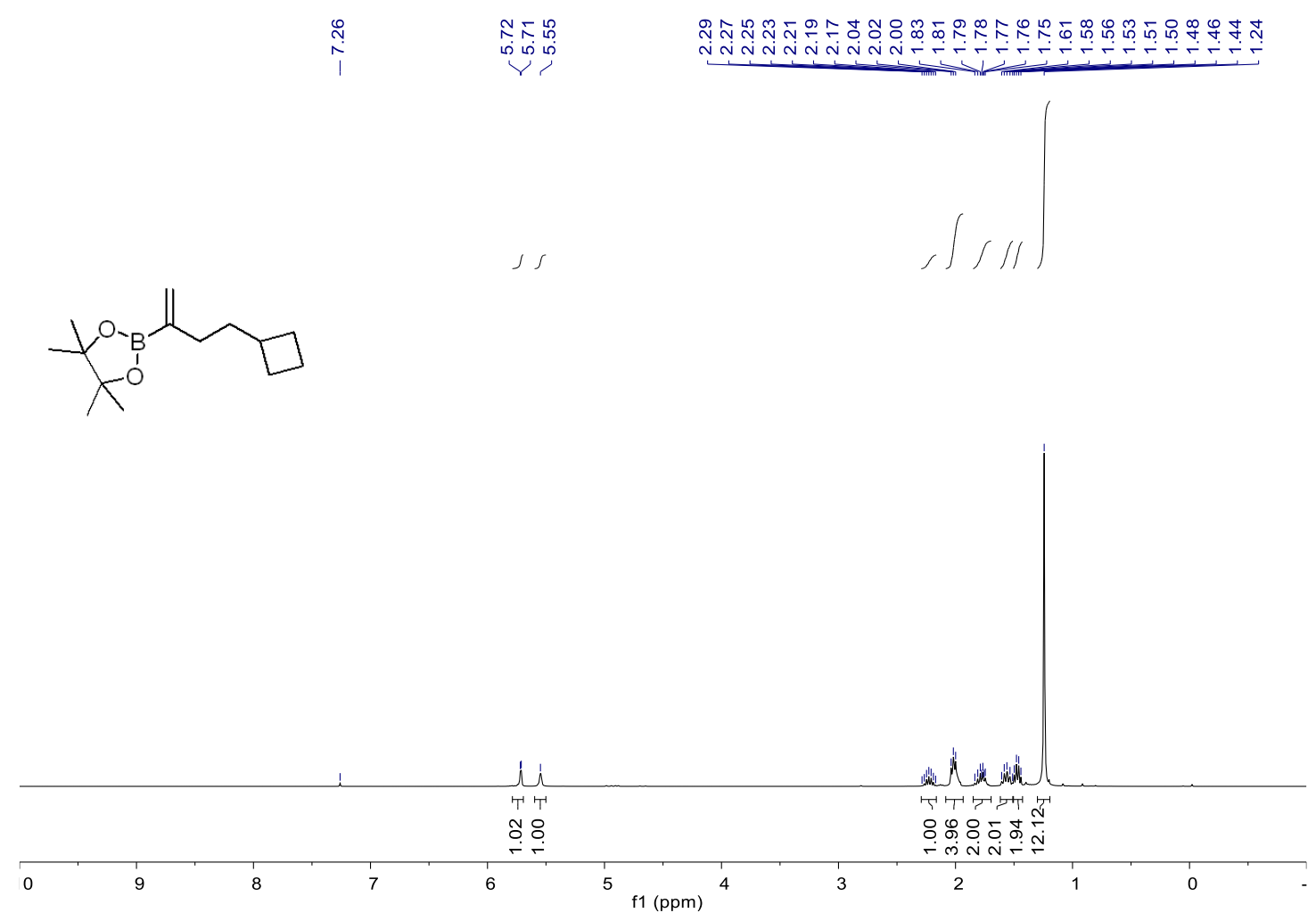

${ }^{1} \mathrm{H}$ NMR $\left(400 \mathrm{M}, \mathrm{CDCl}_{3}\right)$ spectrum of $\mathbf{1 g}$

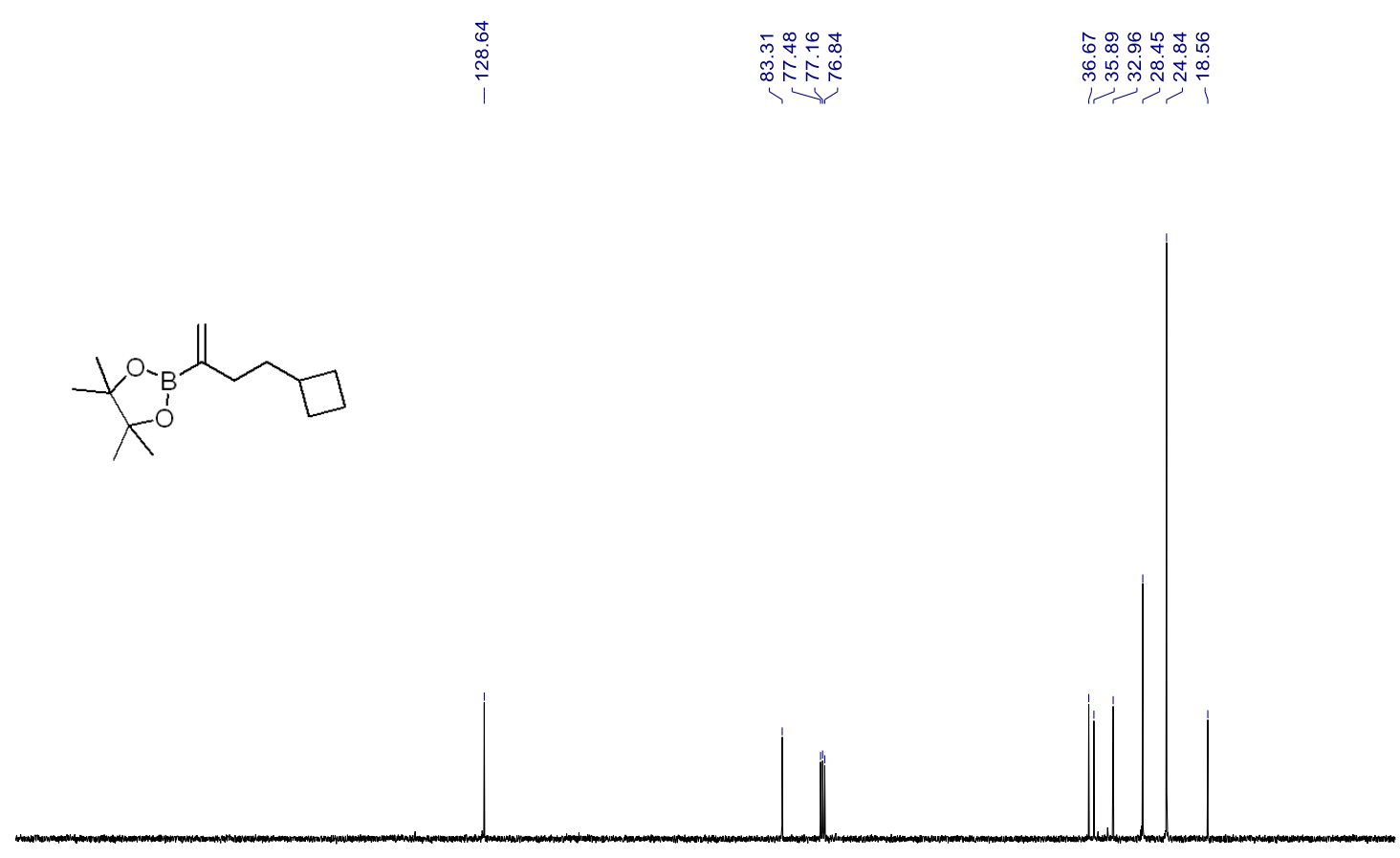

\begin{tabular}{rllllll|lll}
1 & 160 & 140 & 120 & 100 & 1 & 1 & 1 & 1 \\
$\mathrm{f} 1(\mathrm{ppm})$ & 80 & 60 & 40 & 20 & 0
\end{tabular}

${ }^{13} \mathrm{C} \mathrm{NMR}\left(101 \mathrm{M}, \mathrm{CDCl}_{3}\right)$ spectrum of $\mathbf{1 g}$ 

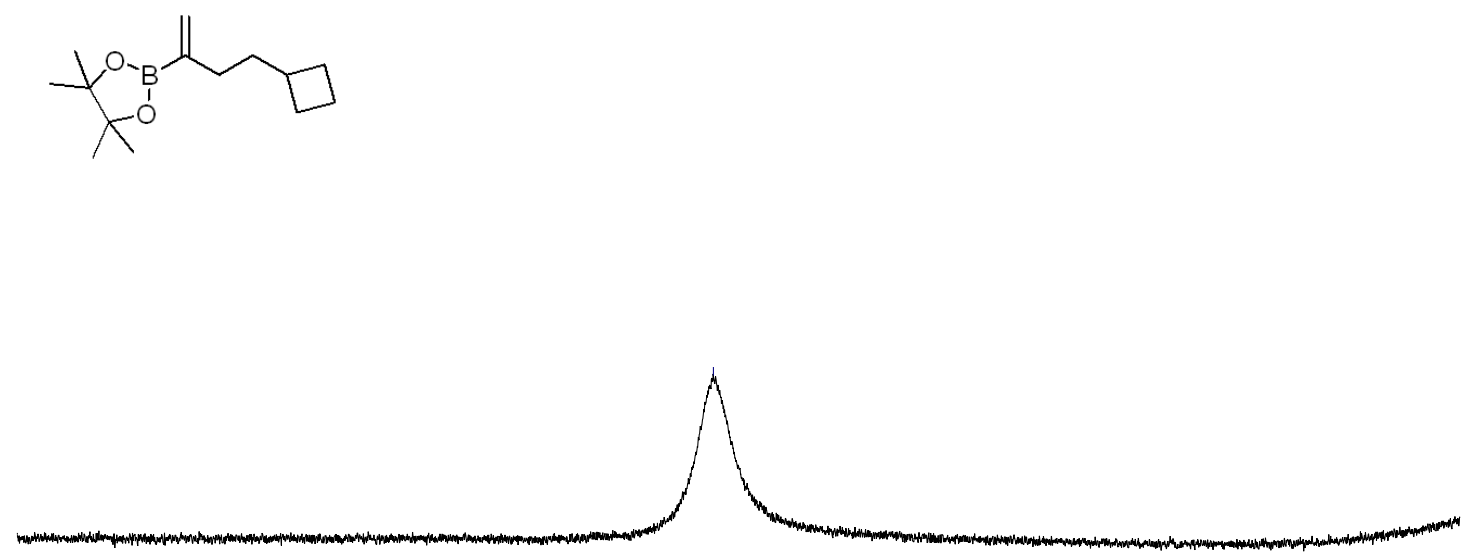

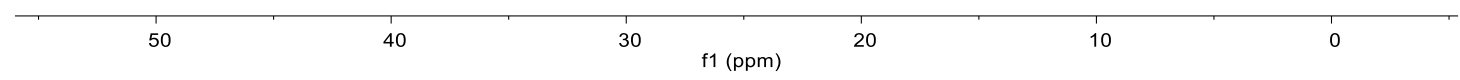

${ }^{11} \mathrm{~B}$ NMR $\left(128 \mathrm{M}, \mathrm{CDCl}_{3}\right)$ spectrum of $\mathbf{1 g}$

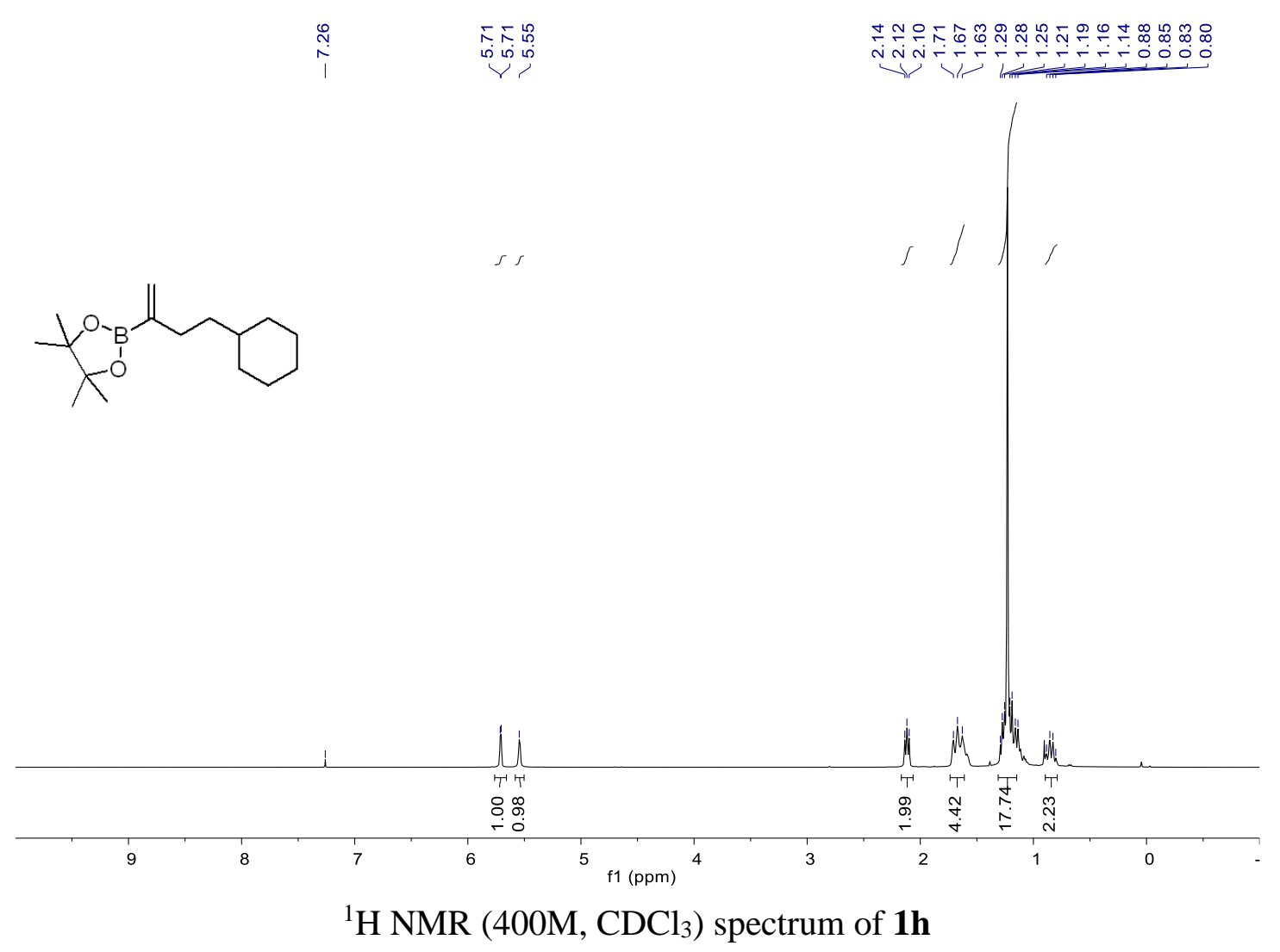



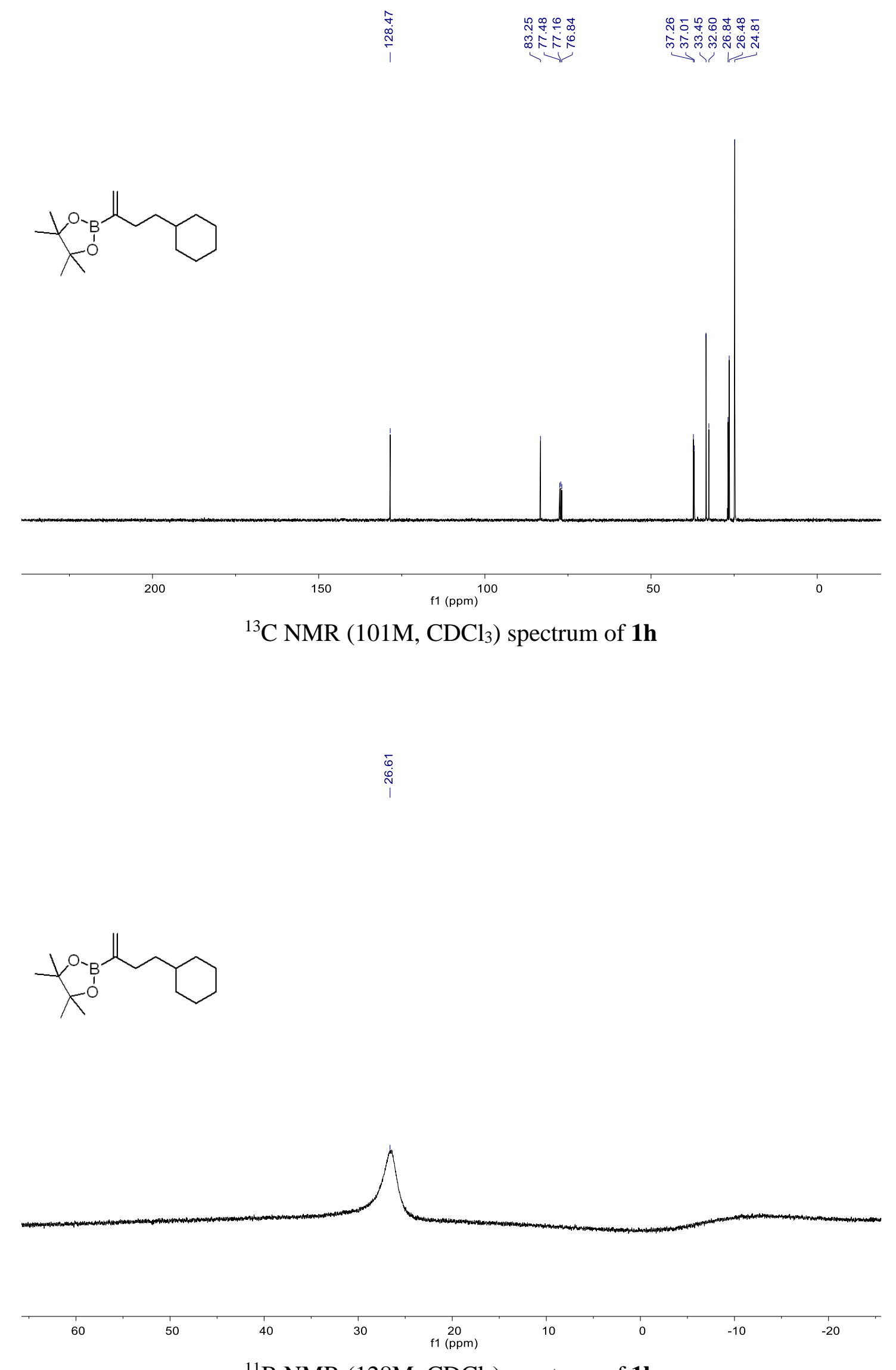

${ }^{11} \mathrm{~B}$ NMR (128M, $\left.\mathrm{CDCl}_{3}\right)$ spectrum of $\mathbf{1 h}$ 


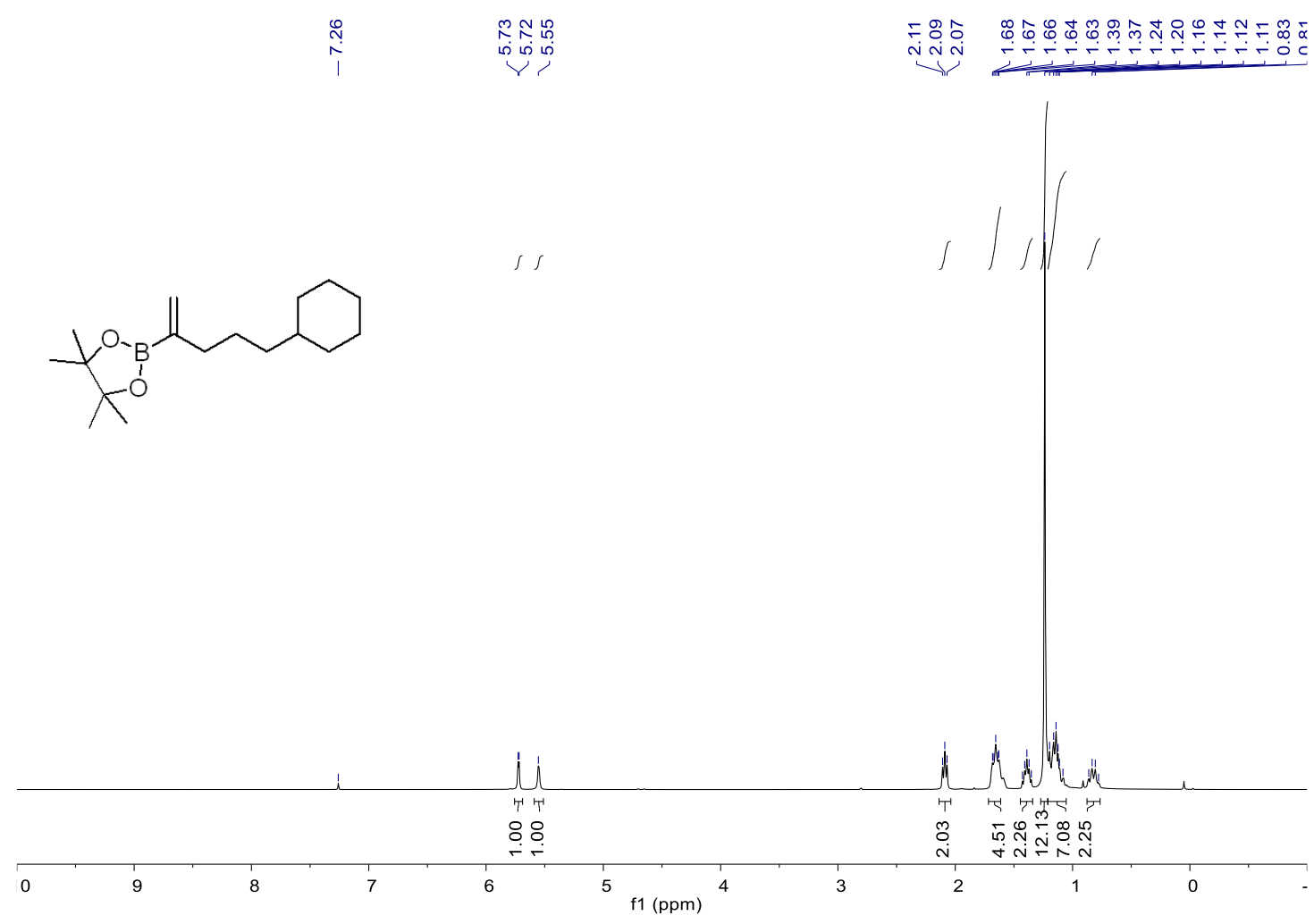

${ }^{1} \mathrm{H}$ NMR (400M, $\mathrm{CDCl}_{3}$ ) spectrum of $\mathbf{1 i}$

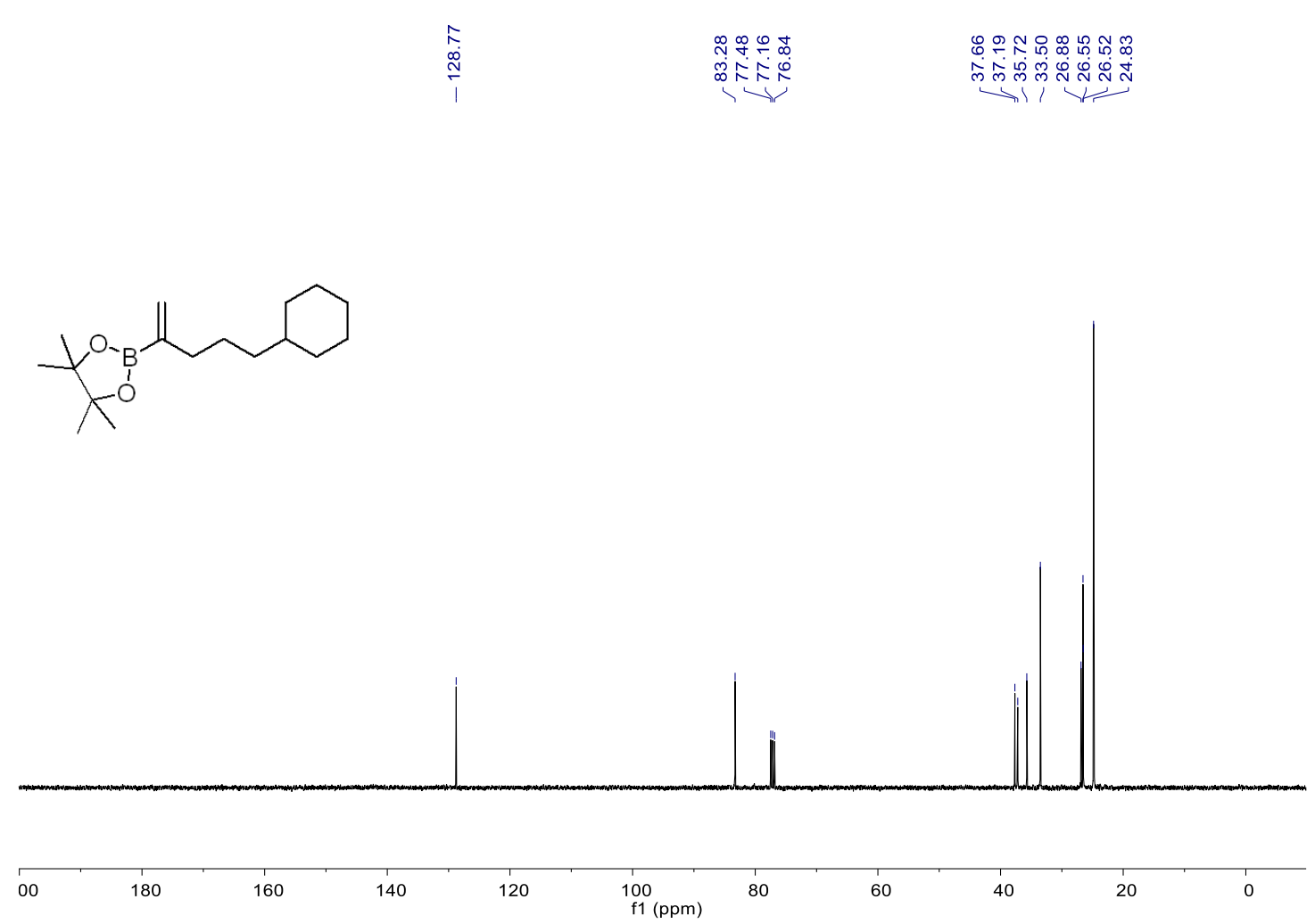

${ }^{13} \mathrm{C}$ NMR $\left(101 \mathrm{M}, \mathrm{CDCl}_{3}\right)$ spectrum of $\mathbf{1 i}$ 
<smiles>C=C(CCCC1CCCCC1)B1OC(C)(C)C(C)(C)O1</smiles>

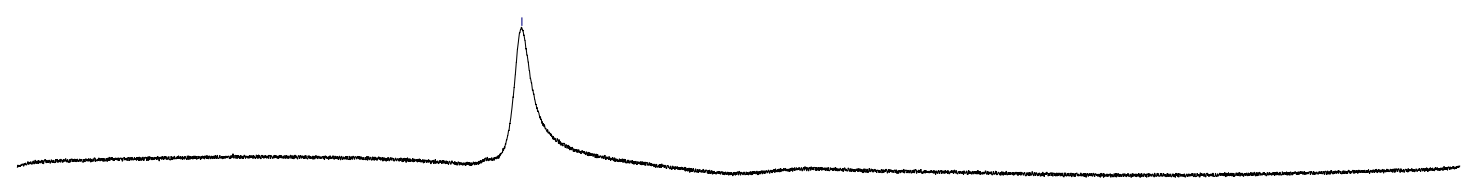

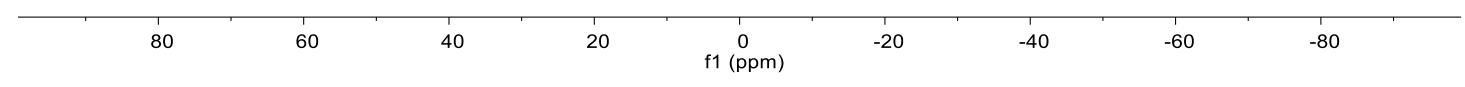

${ }^{11} \mathrm{~B}$ NMR $\left(128 \mathrm{M}, \mathrm{CDCl}_{3}\right)$ spectrum of $\mathbf{1 i}$

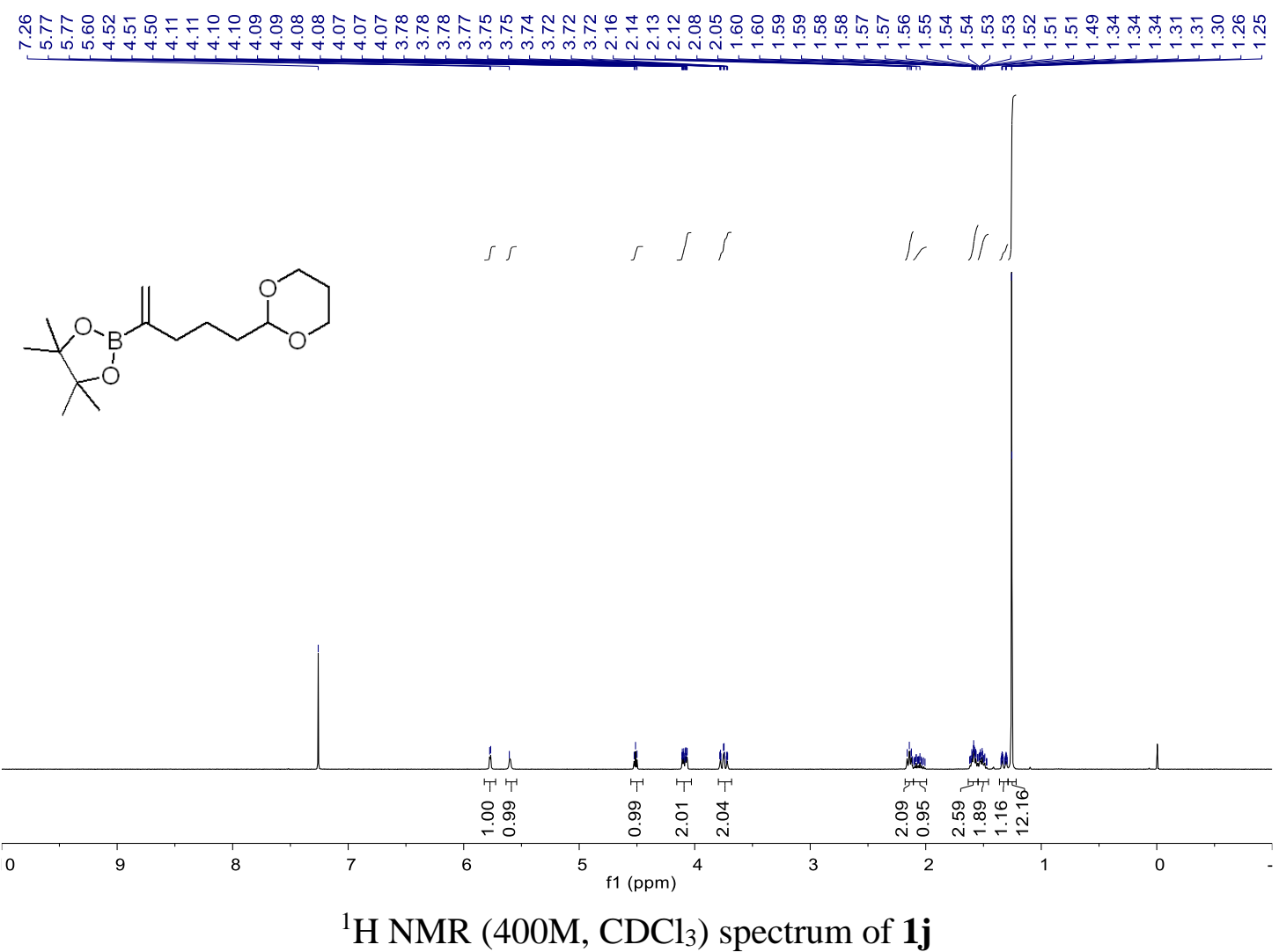



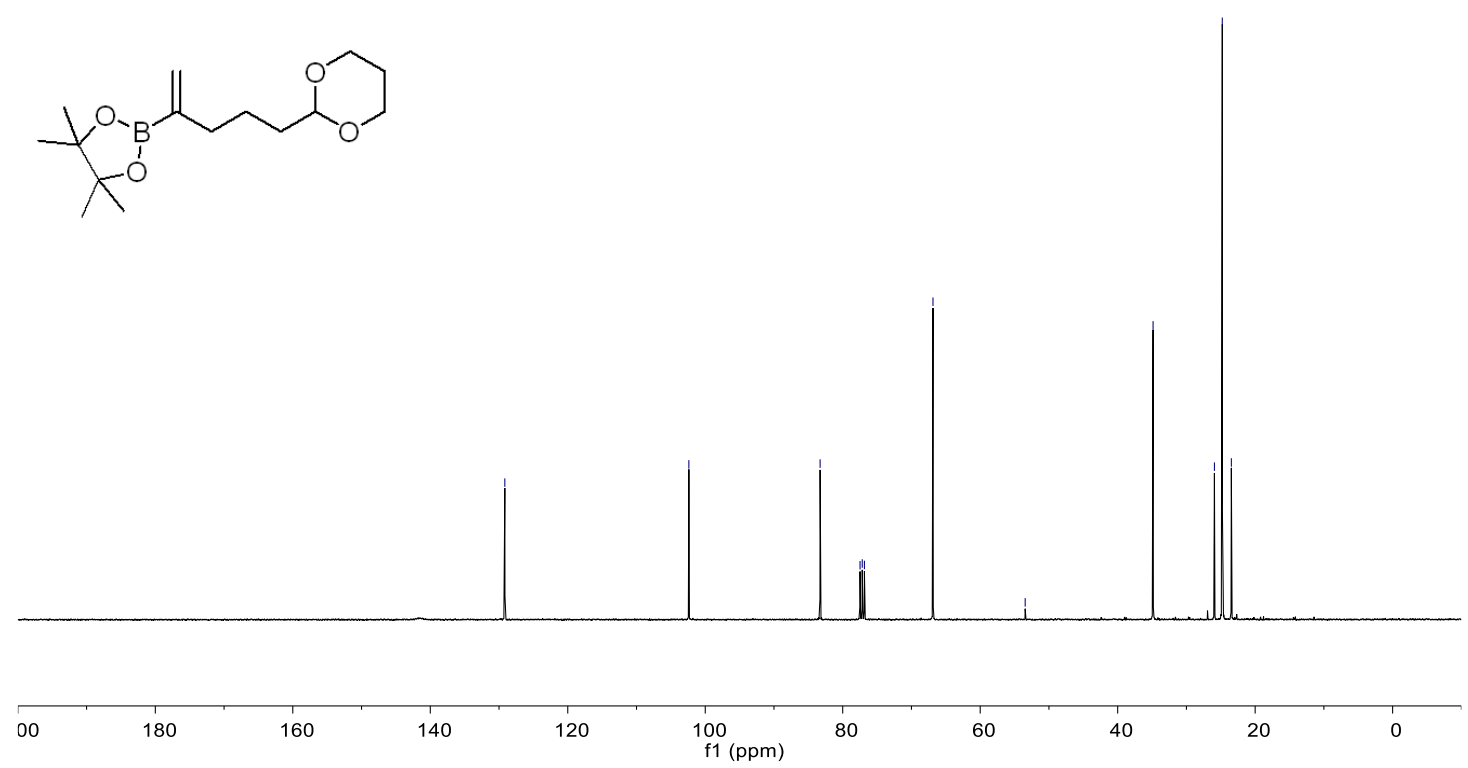

${ }^{13} \mathrm{C} \mathrm{NMR}\left(101 \mathrm{M}, \mathrm{CDCl}_{3}\right)$ spectrum of $\mathbf{1} \mathbf{j}$

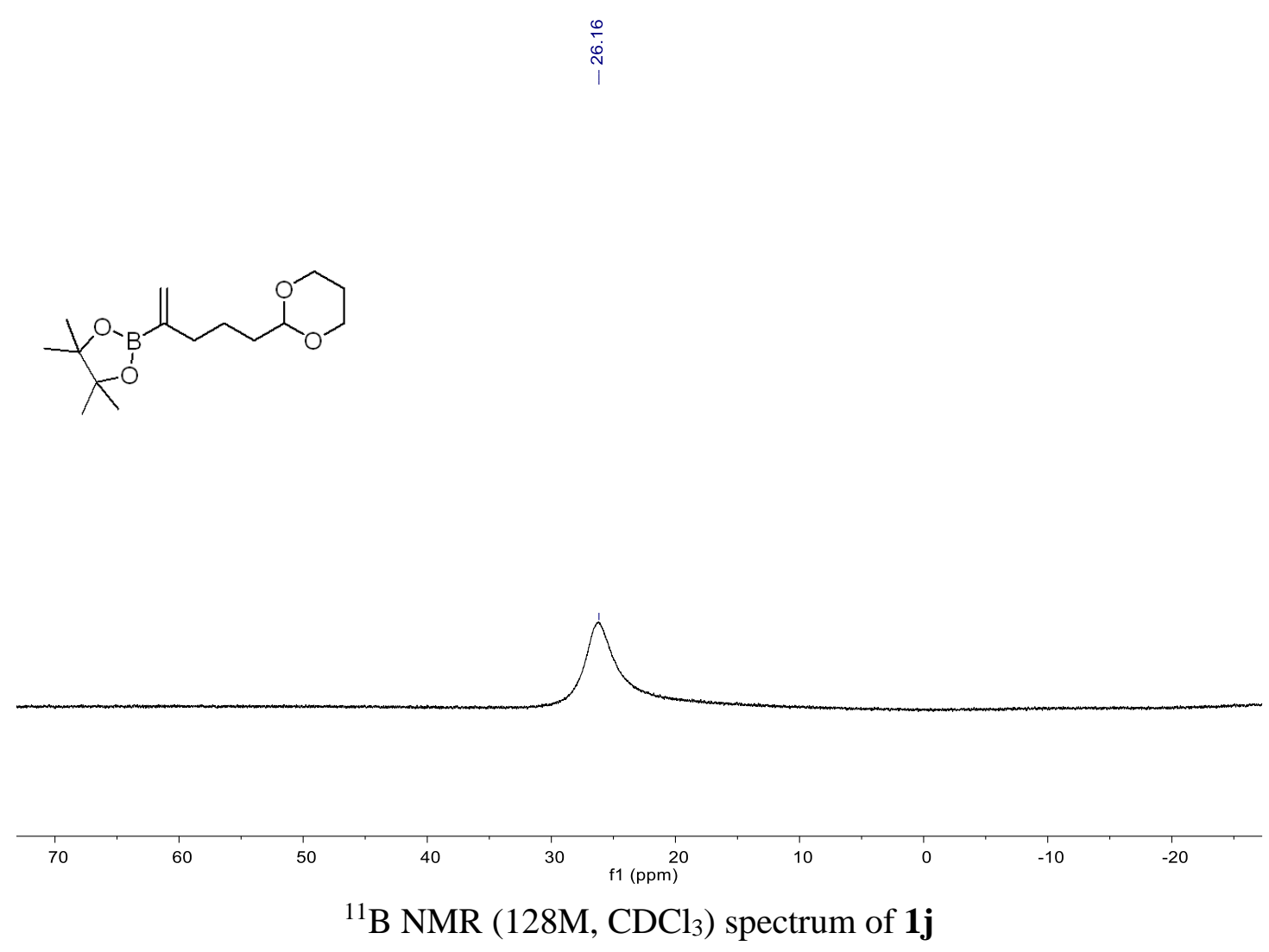




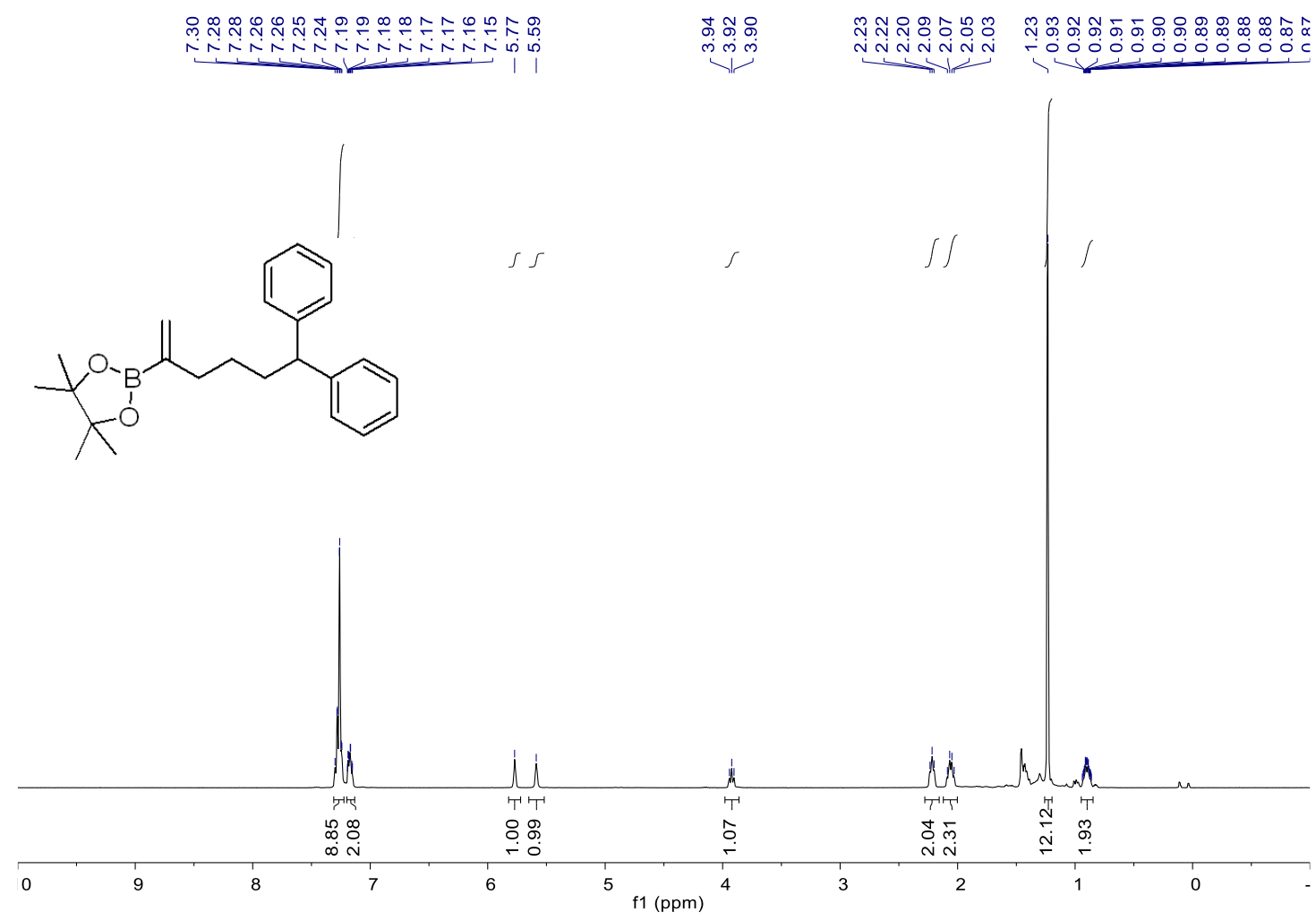

${ }^{1} \mathrm{H}$ NMR (400M, $\mathrm{CDCl}_{3}$ ) spectrum of $\mathbf{1 k}$<smiles>C=C(CCCC(c1ccccc1)c1ccccc1)B1OC(C)(C)C(C)(C)O1</smiles>

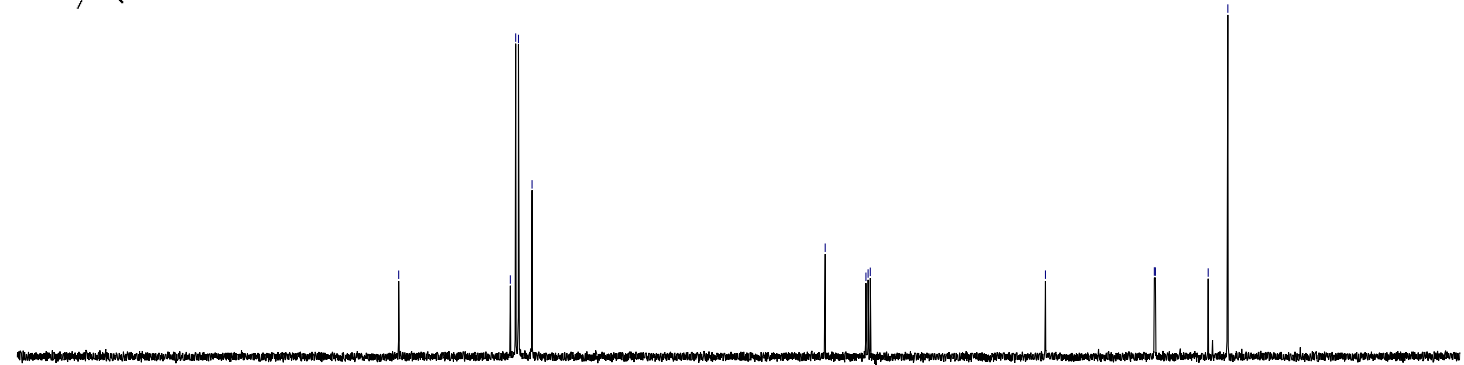
160 $100 \quad 80$ 60 40 20 0

${ }^{13} \mathrm{C}$ NMR $\left(101 \mathrm{M}, \mathrm{CDCl}_{3}\right)$ spectrum of $\mathbf{1 k}$ 


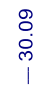
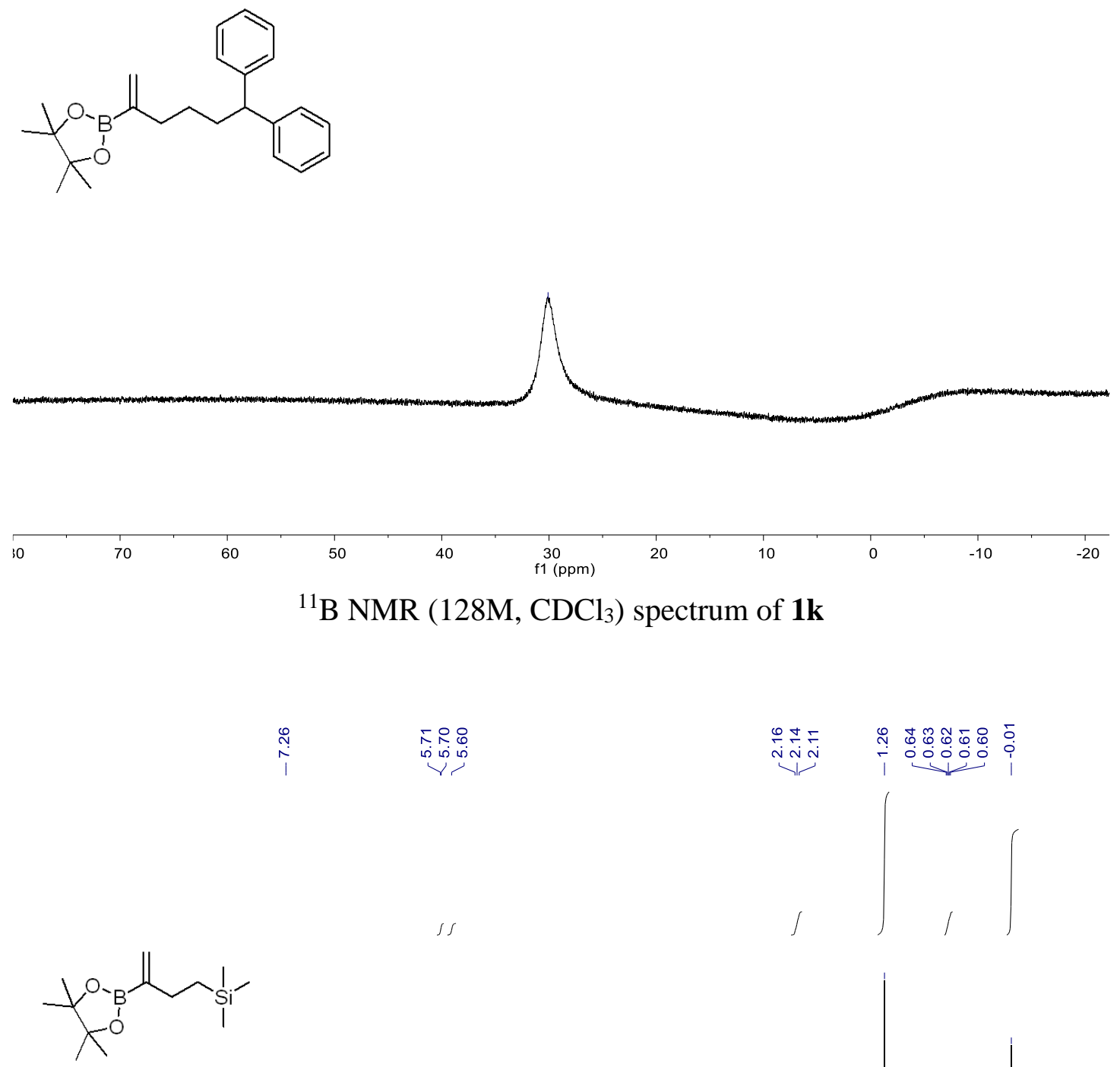

$\iint$

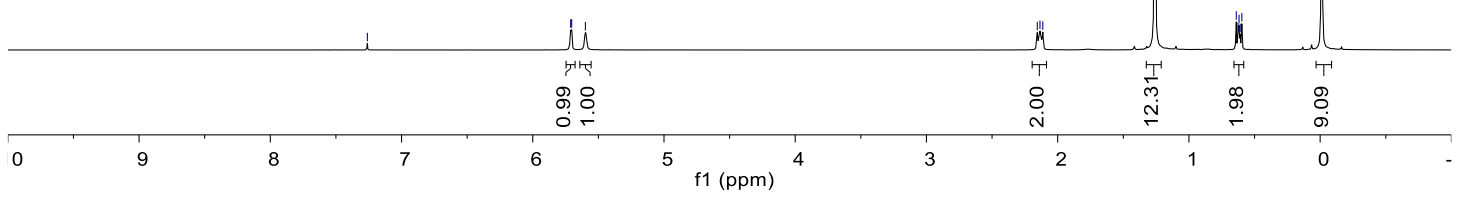

${ }^{1} \mathrm{H}$ NMR (400M, $\mathrm{CDCl}_{3}$ ) spectrum of $\mathbf{1 l}$

S73 

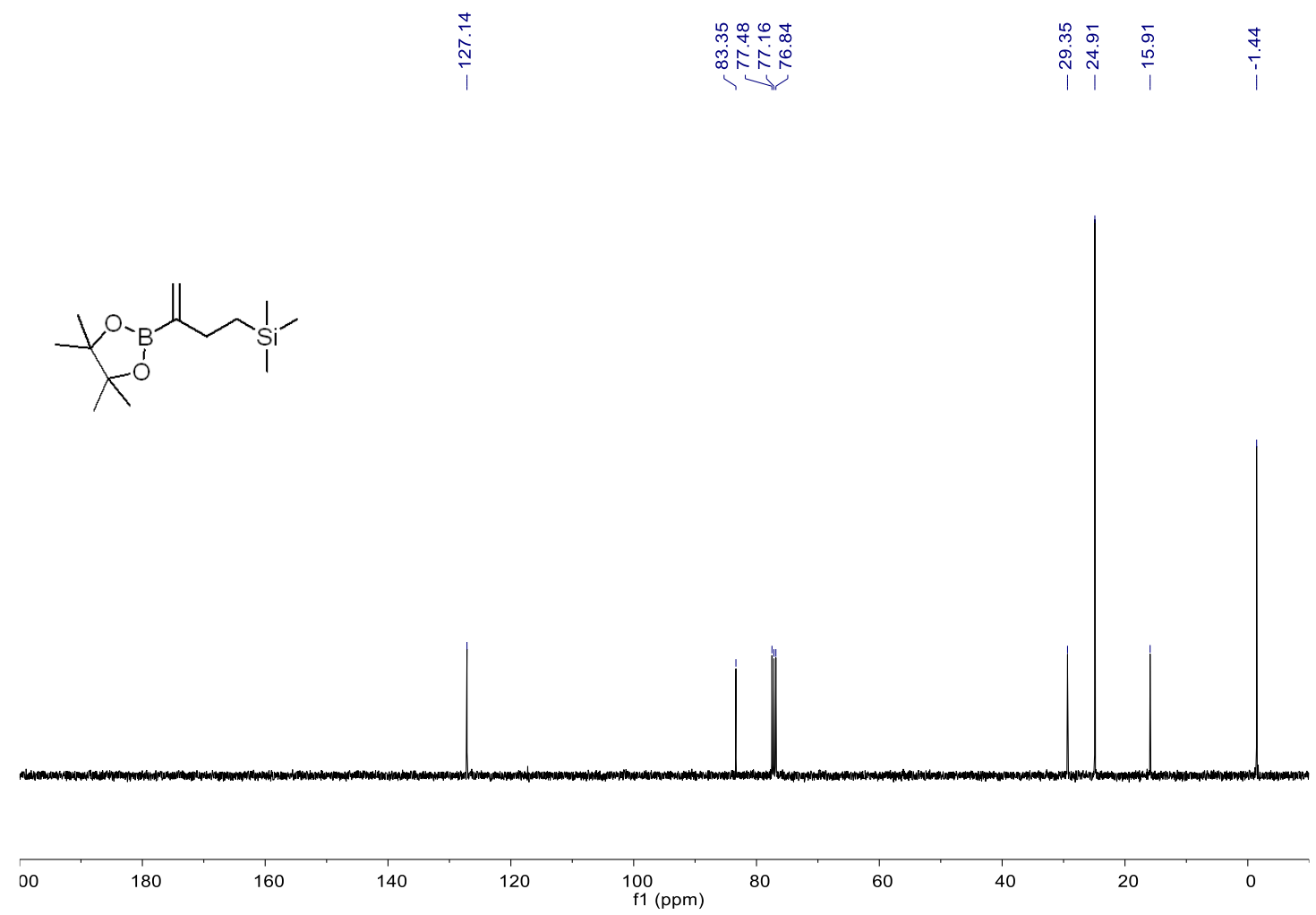

${ }^{13} \mathrm{C} \mathrm{NMR}\left(101 \mathrm{M}, \mathrm{CDCl}_{3}\right)$ spectrum of $\mathbf{1 l}$<smiles>C=C(CC[Si](C)(C)C)OP(OC(C)(C)C)C(C)(C)C</smiles>

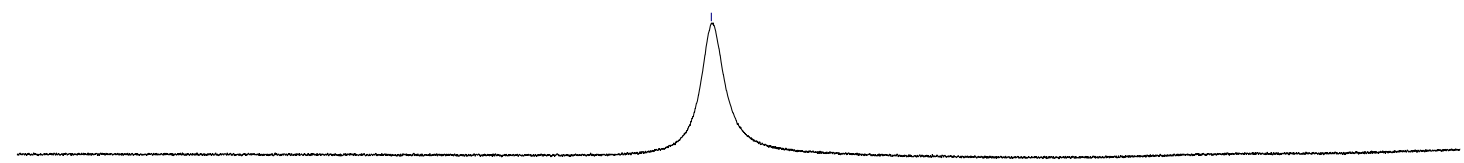
60

50

$\begin{array}{ll}30 & 1 \\ \mathrm{f} 1(\mathrm{ppm}) & \end{array}$

$20 \quad 10$

10

$-10 \quad-20$

${ }^{11} \mathrm{~B}$ NMR (128M, $\left.\mathrm{CDCl}_{3}\right)$ spectrum of $\mathbf{1 l}$ 


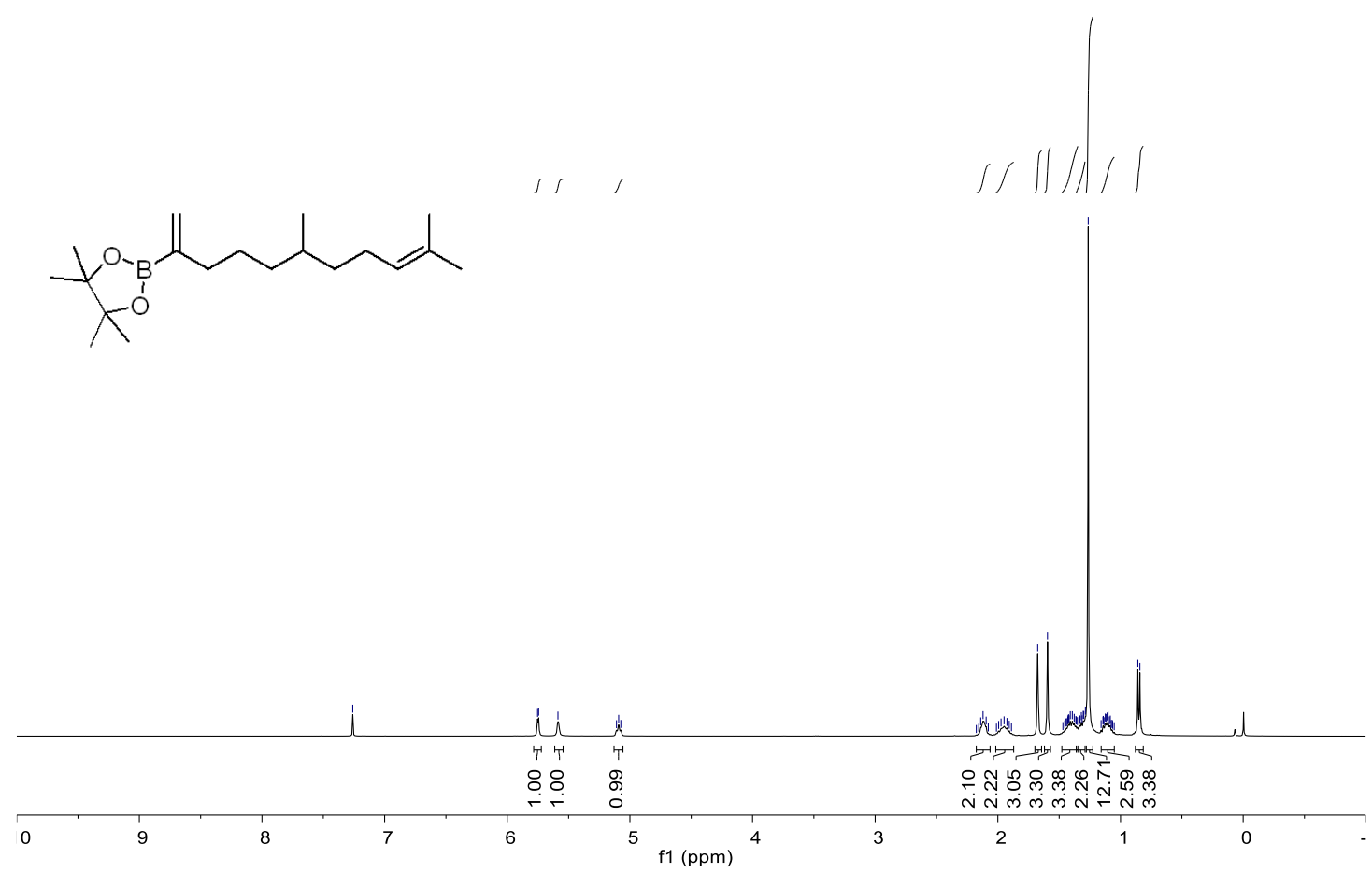

${ }^{1} \mathrm{H}$ NMR (400M, $\mathrm{CDCl}_{3}$ ) spectrum of $\mathbf{1 m}$

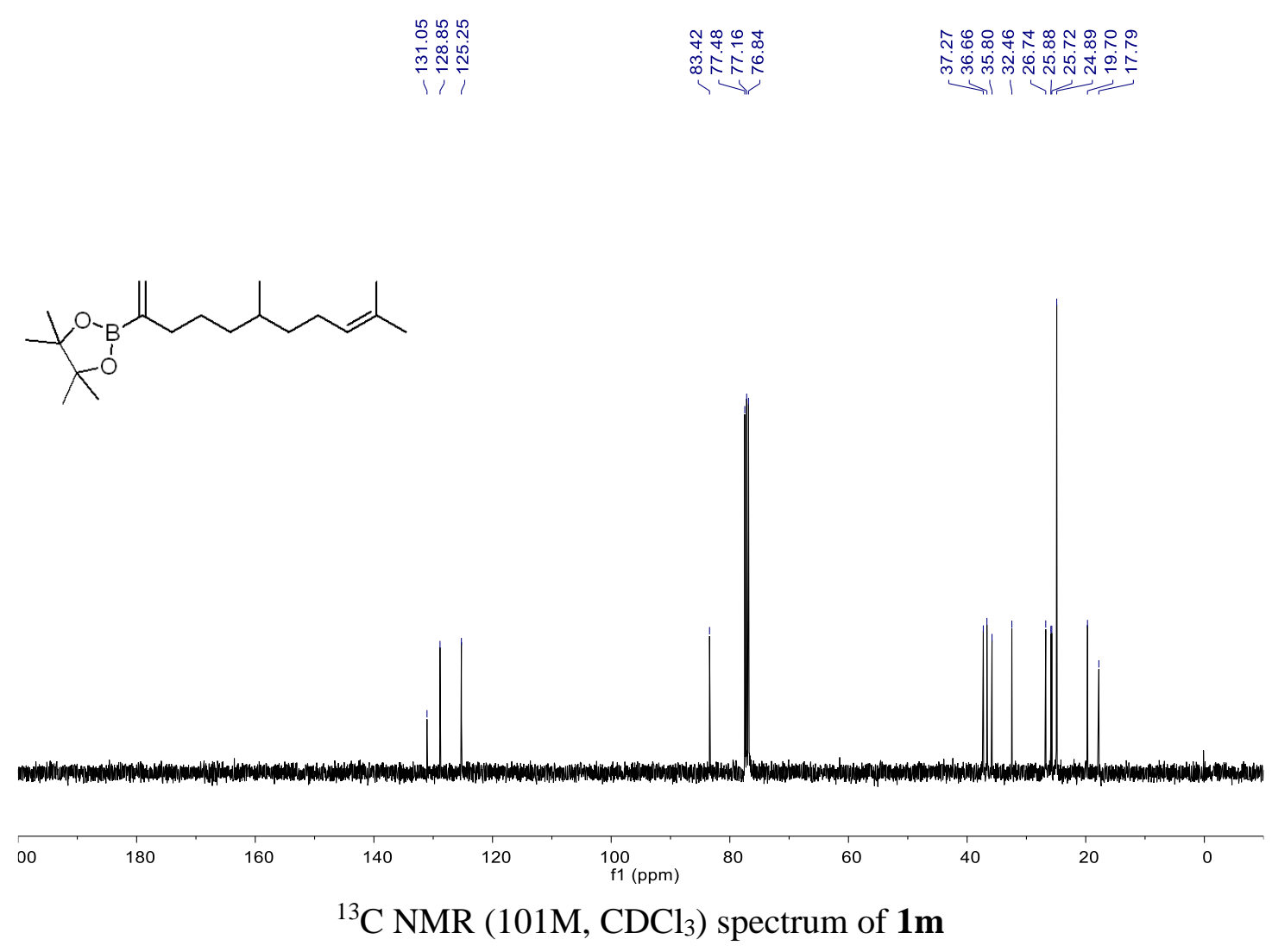


<smiles>CCCCCCCCC(C)C(C)(C)C</smiles>

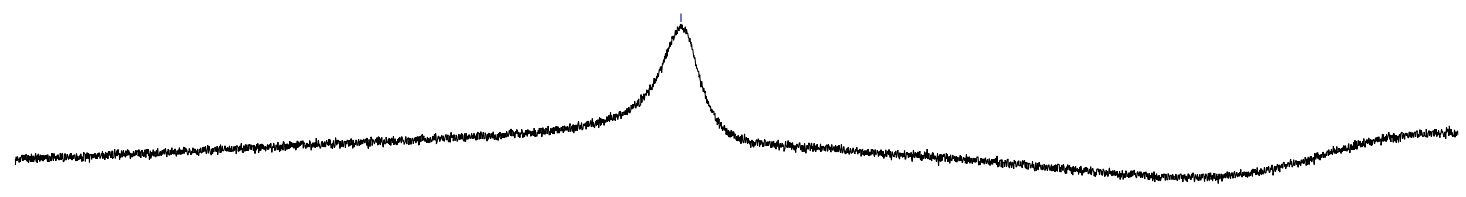

\begin{tabular}{llcccc}
\hline 60 & 50 & 30 & 10 & 10 & 0
\end{tabular}

${ }^{11} \mathrm{~B}$ NMR (128M, $\mathrm{CDCl}_{3}$ ) spectrum of $\mathbf{1 m}$

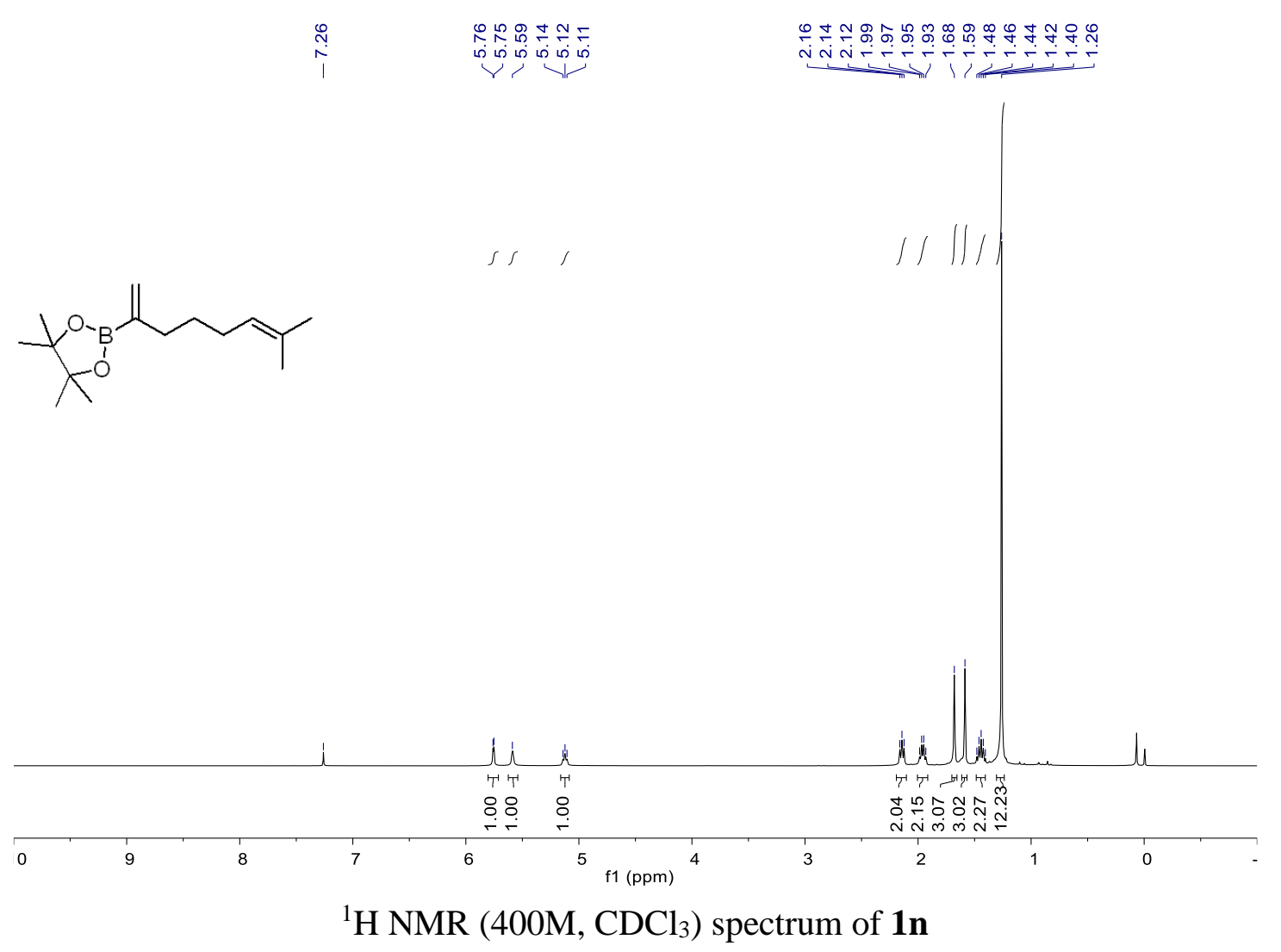



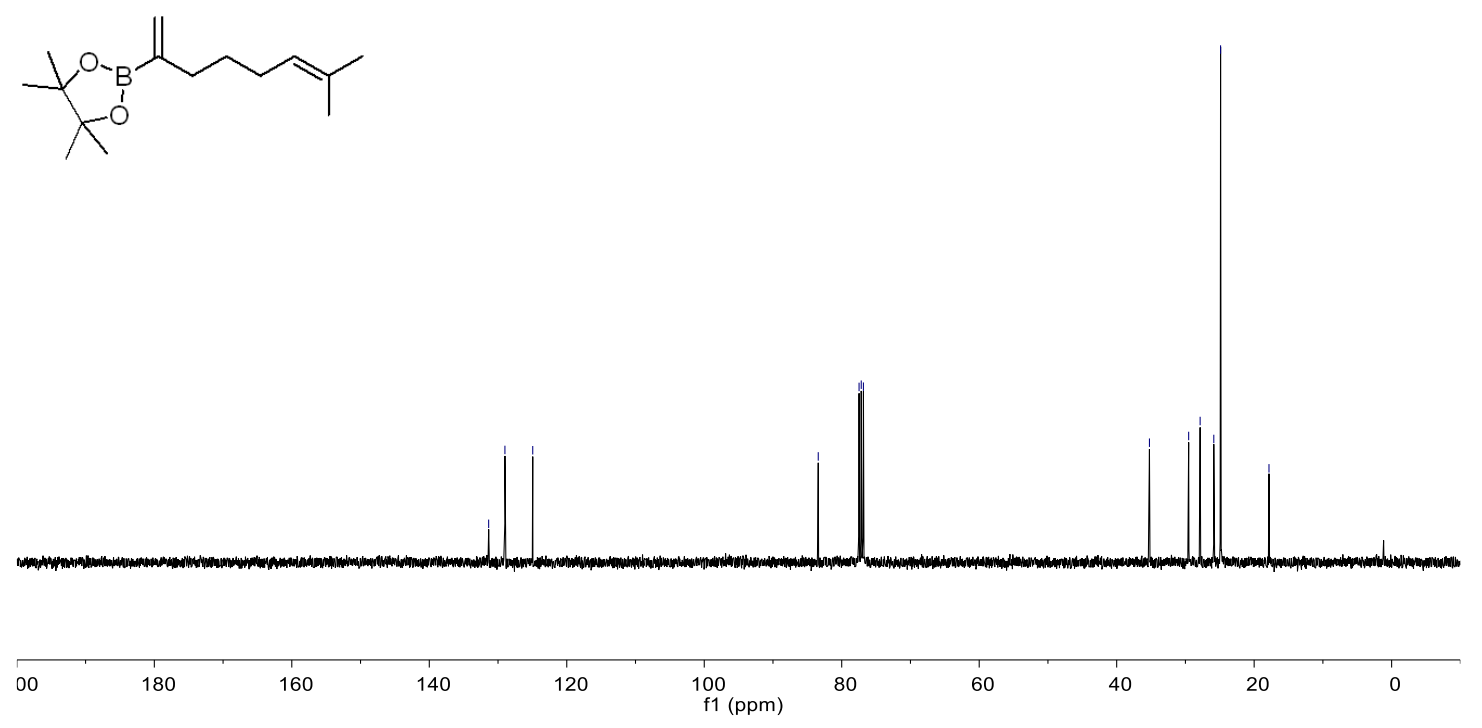

${ }^{13} \mathrm{C} \mathrm{NMR}\left(101 \mathrm{M}, \mathrm{CDCl}_{3}\right)$ spectrum of $\mathbf{1 n}$<smiles>C=C(CCCC=C(C)C)OC(C)(C)C(C)(C)C</smiles>

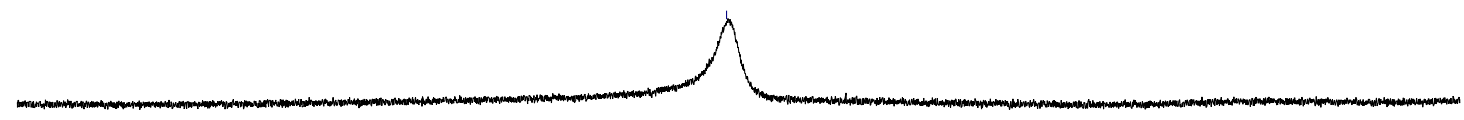

70

60

40

30
30

20

10

$-2$

${ }^{11} \mathrm{~B}$ NMR (128M, $\mathrm{CDCl}_{3}$ ) spectrum of $\mathbf{1 n}$ 


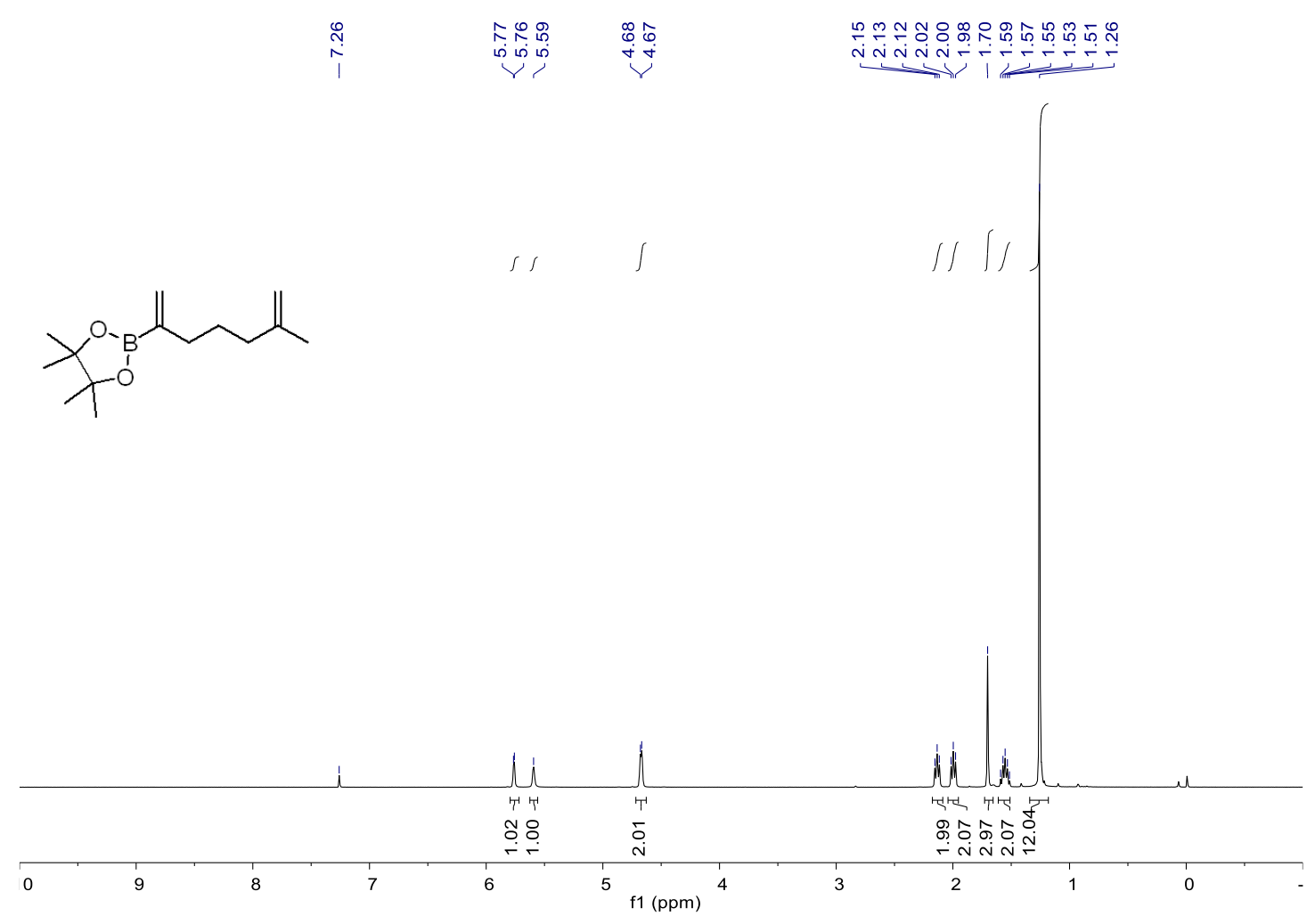

${ }^{1} \mathrm{H}$ NMR (400M, $\mathrm{CDCl}_{3}$ ) spectrum of $\mathbf{1 0}$

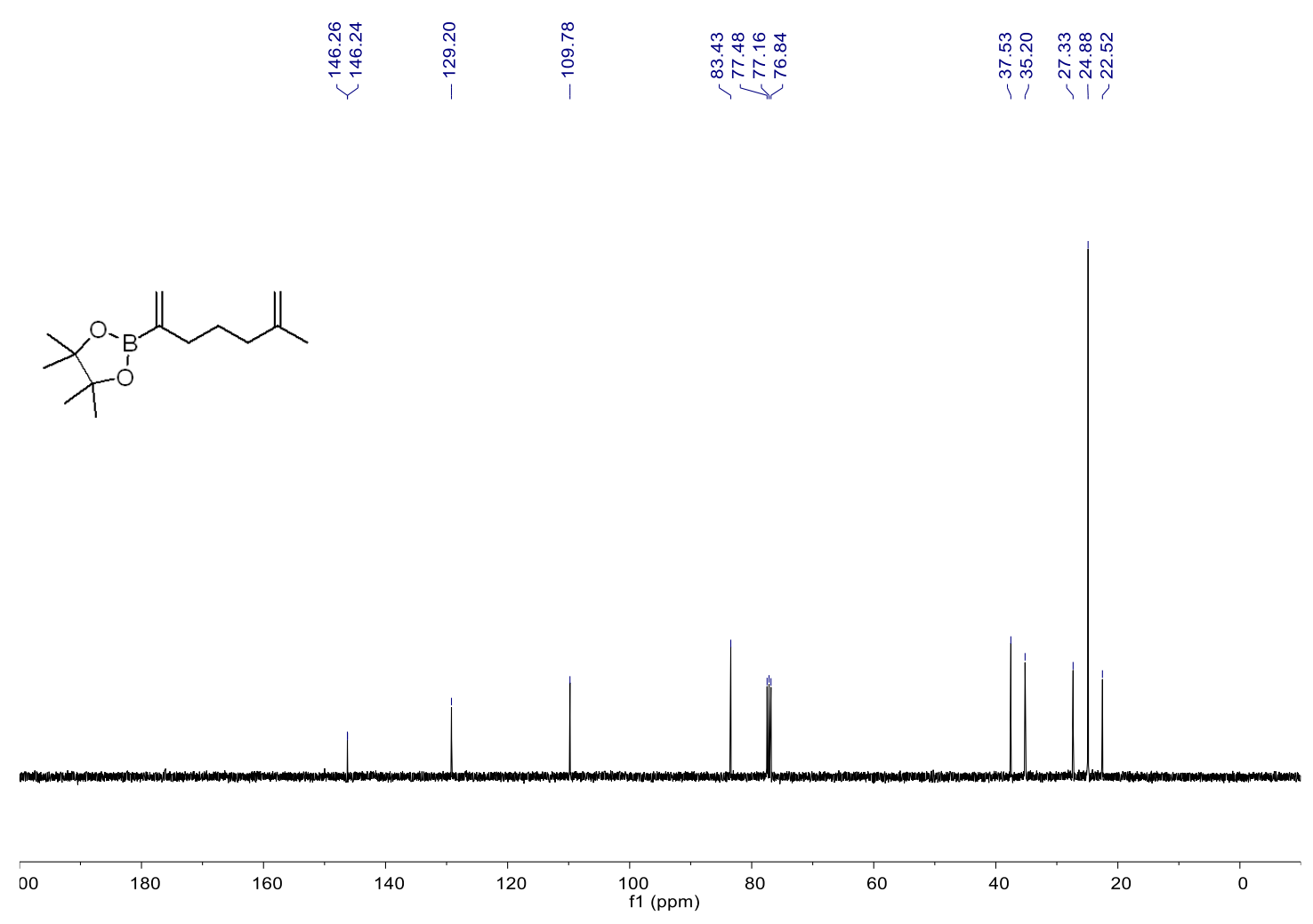

${ }^{13} \mathrm{C}$ NMR $\left(101 \mathrm{M}, \mathrm{CDCl}_{3}\right)$ spectrum of $\mathbf{1 o}$ 
<smiles>C=C(C)CCCC(=C)B1OC(C)(C)C(C)(C)O1</smiles>

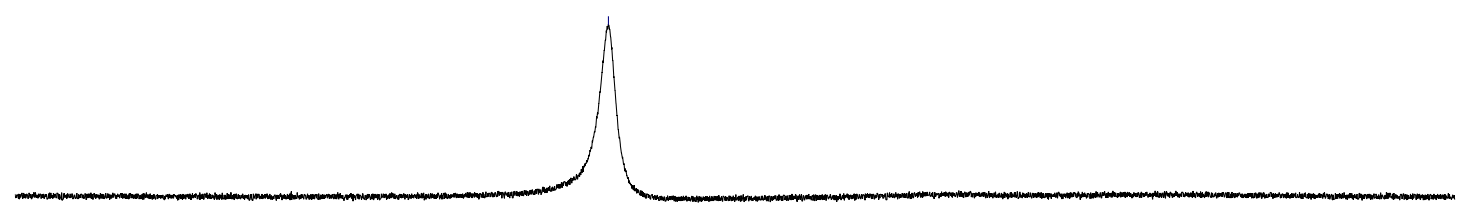

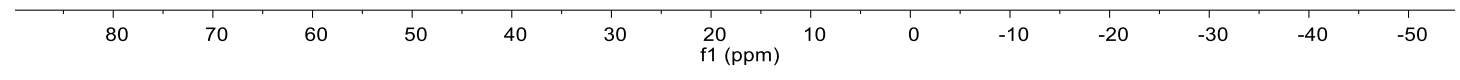

${ }^{11} \mathrm{~B}$ NMR $\left(128 \mathrm{M}, \mathrm{CDCl}_{3}\right)$ spectrum of $\mathbf{1 o}$

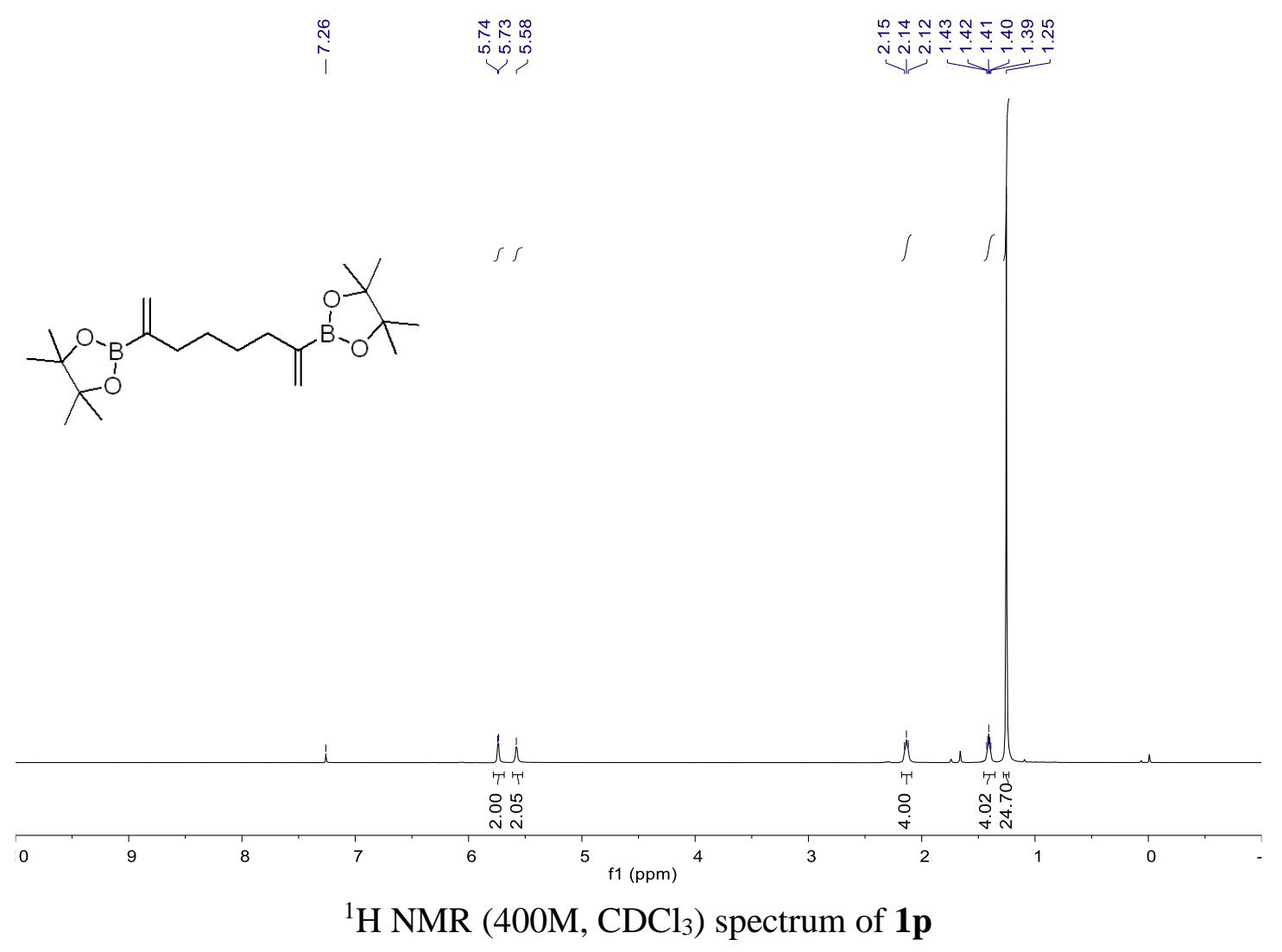




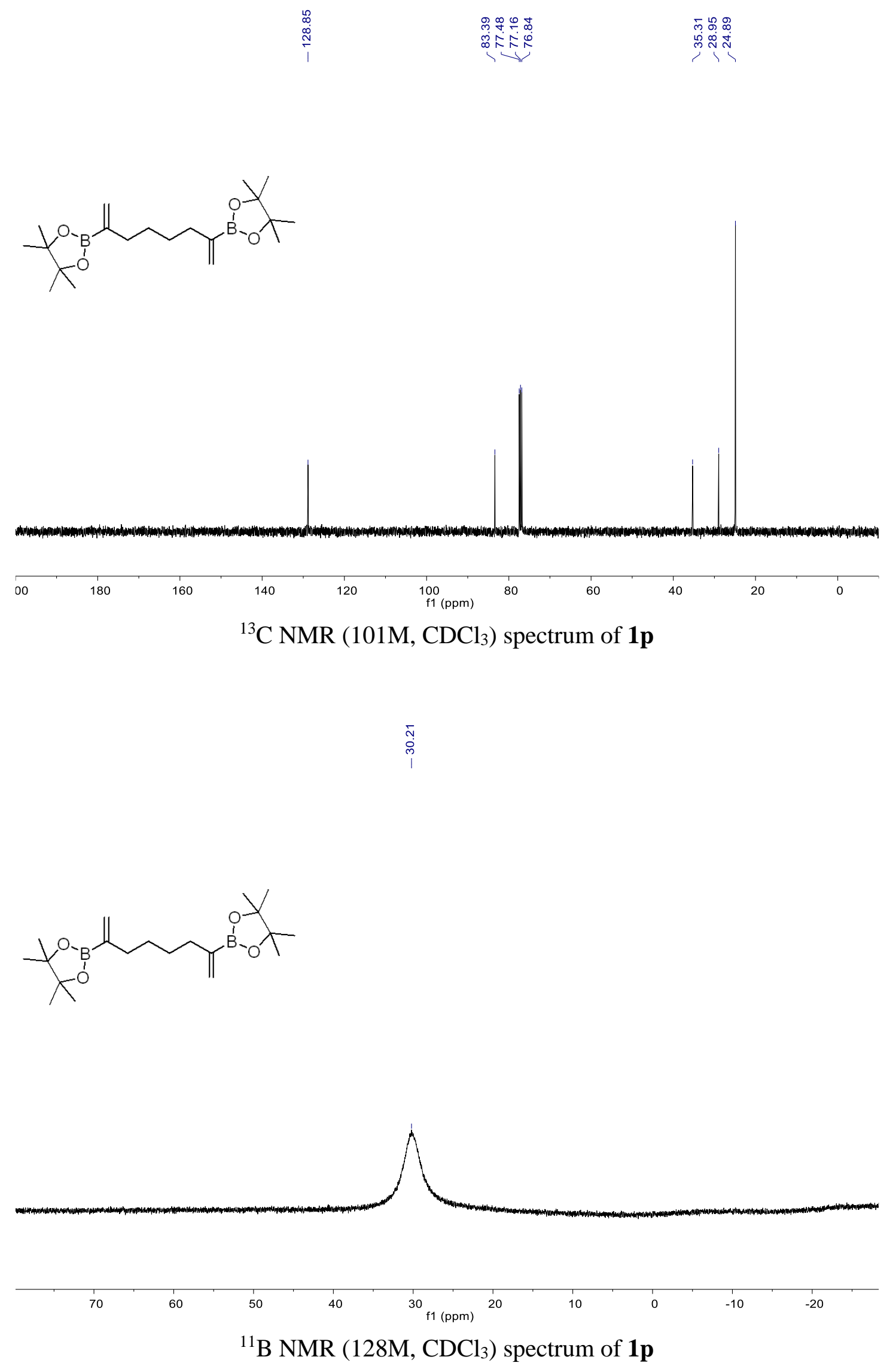




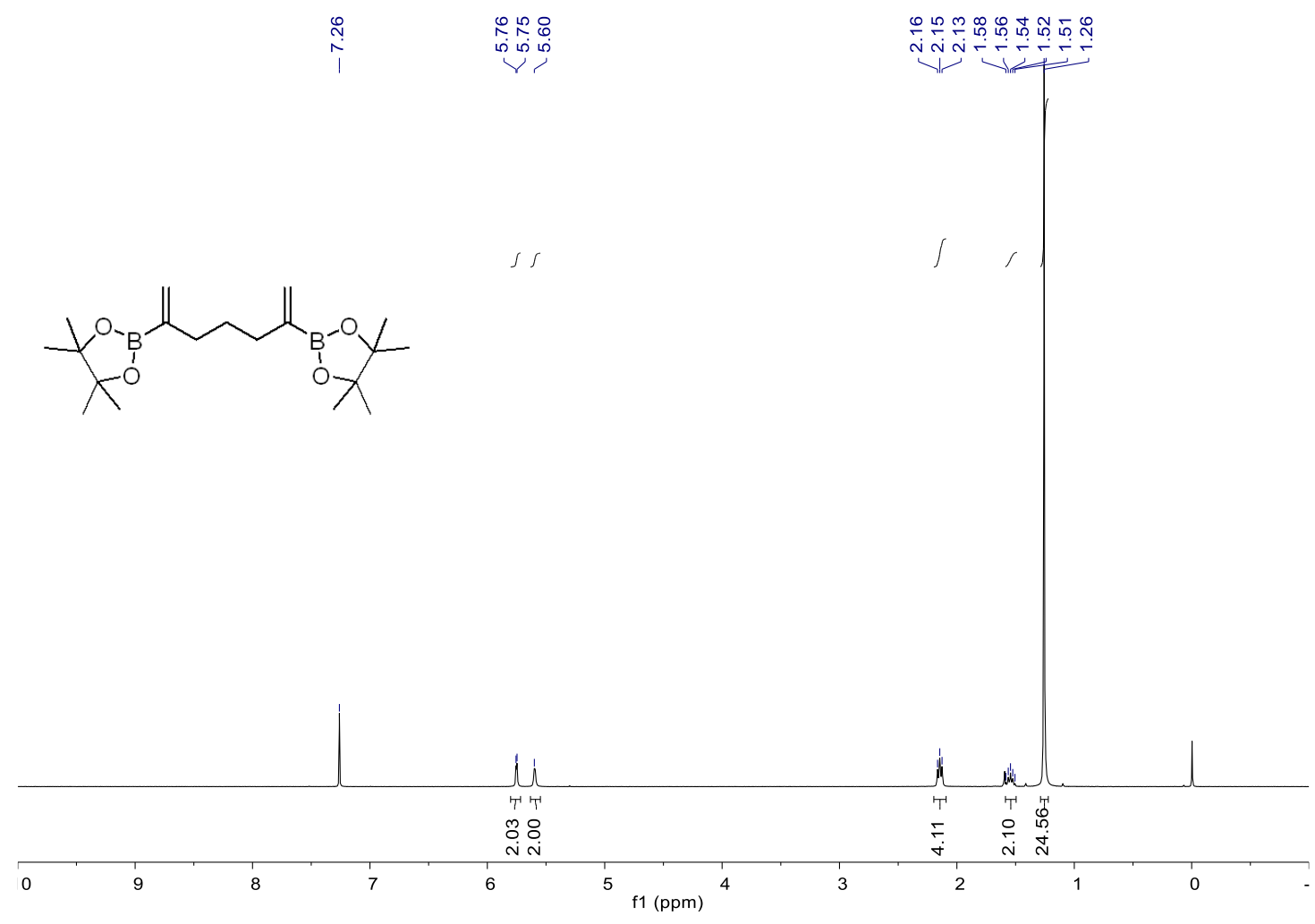

${ }^{1} \mathrm{H}$ NMR (400M, $\mathrm{CDCl}_{3}$ ) spectrum of $\mathbf{1 q}$

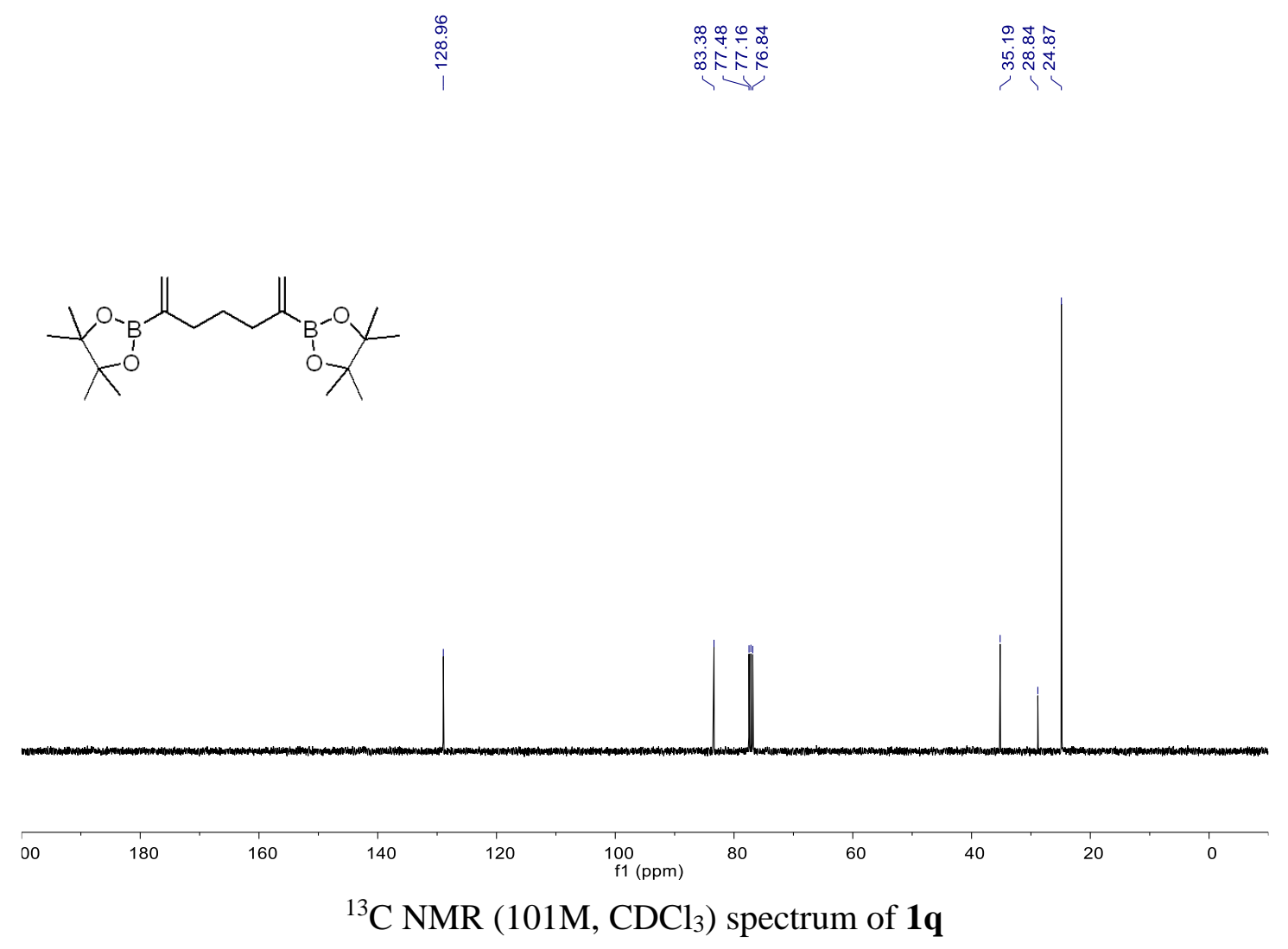



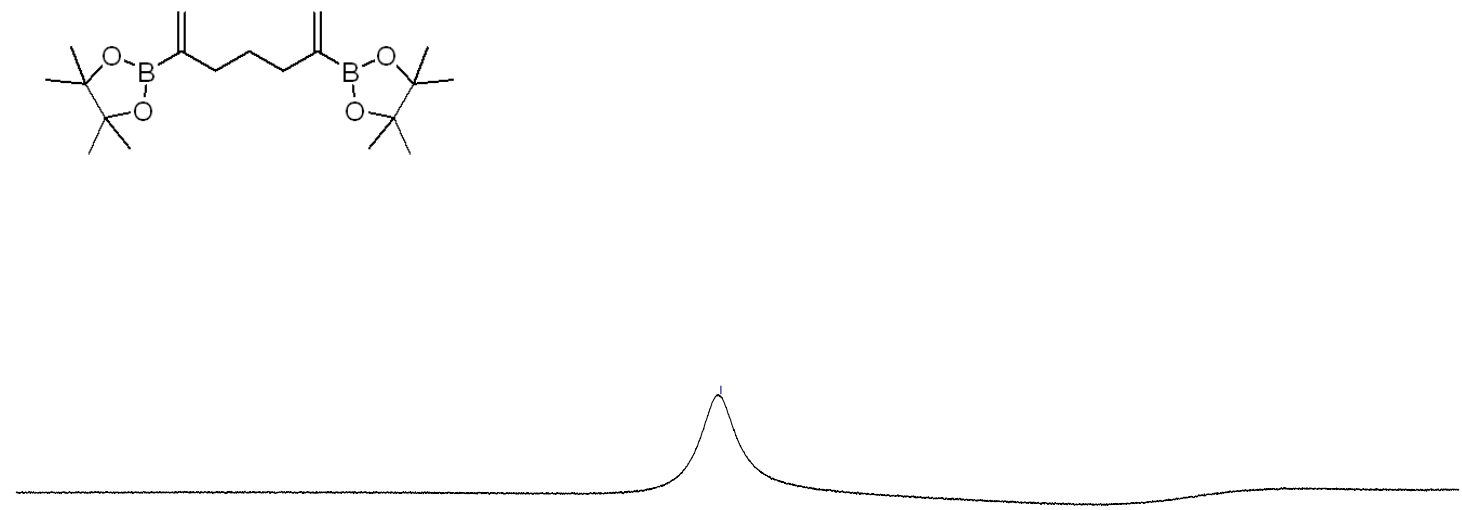

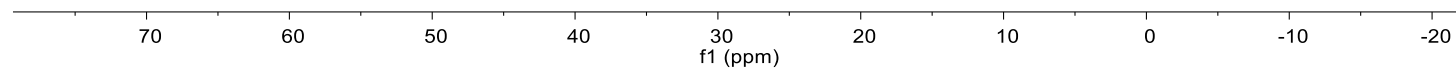

${ }^{11} \mathrm{~B}$ NMR $\left(128 \mathrm{M}, \mathrm{CDCl}_{3}\right)$ spectrum of $\mathbf{1 q}$

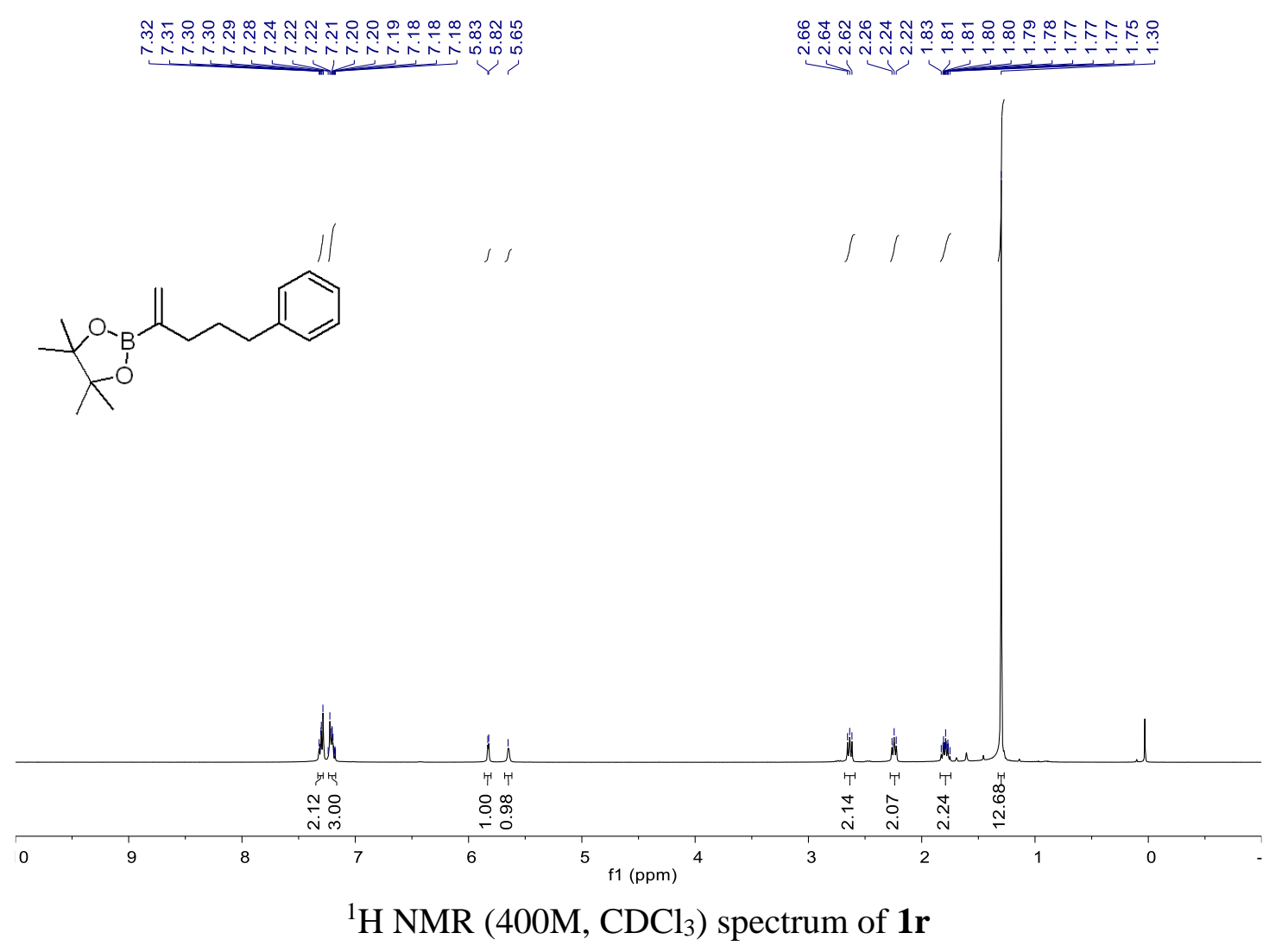



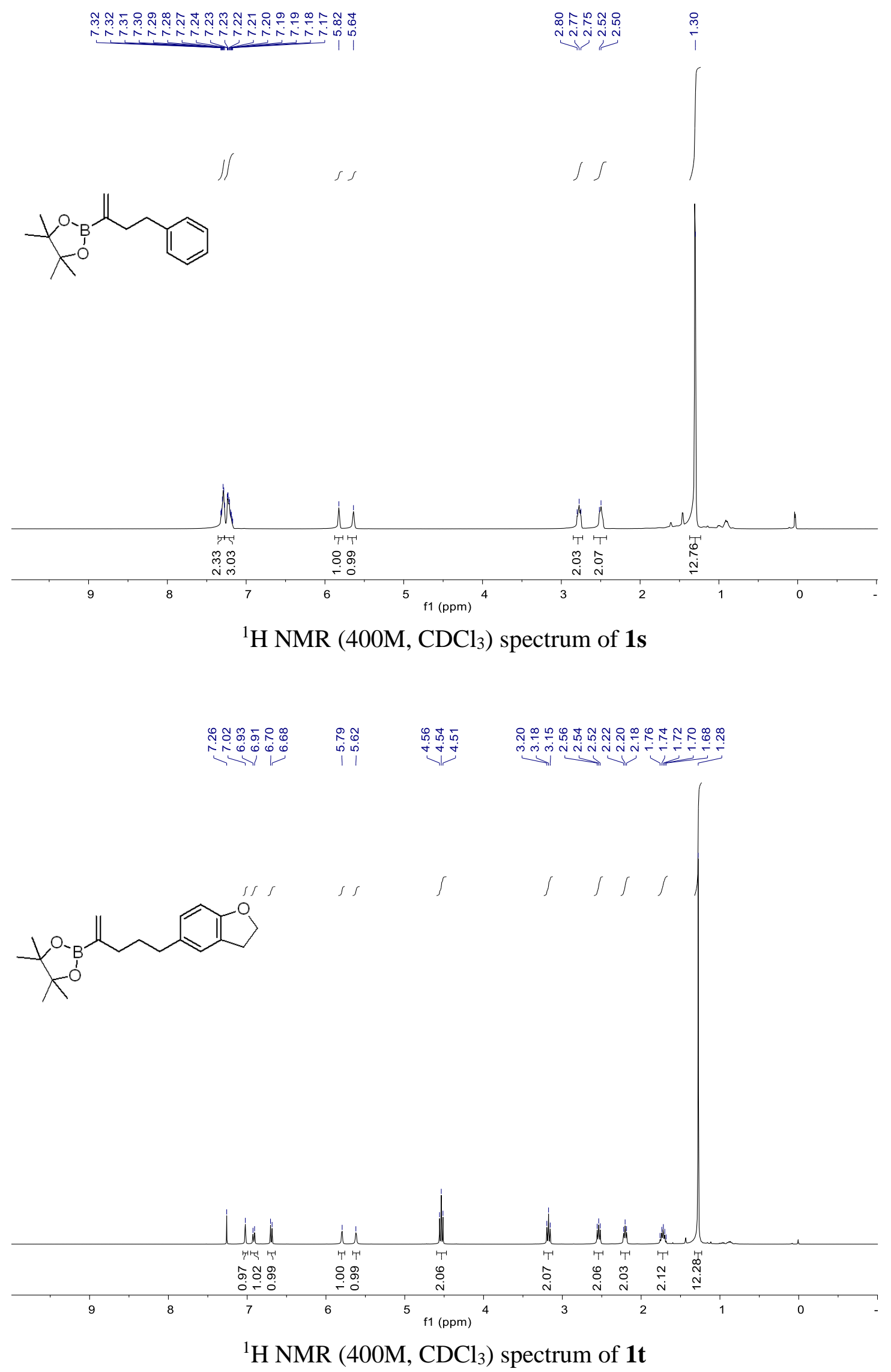

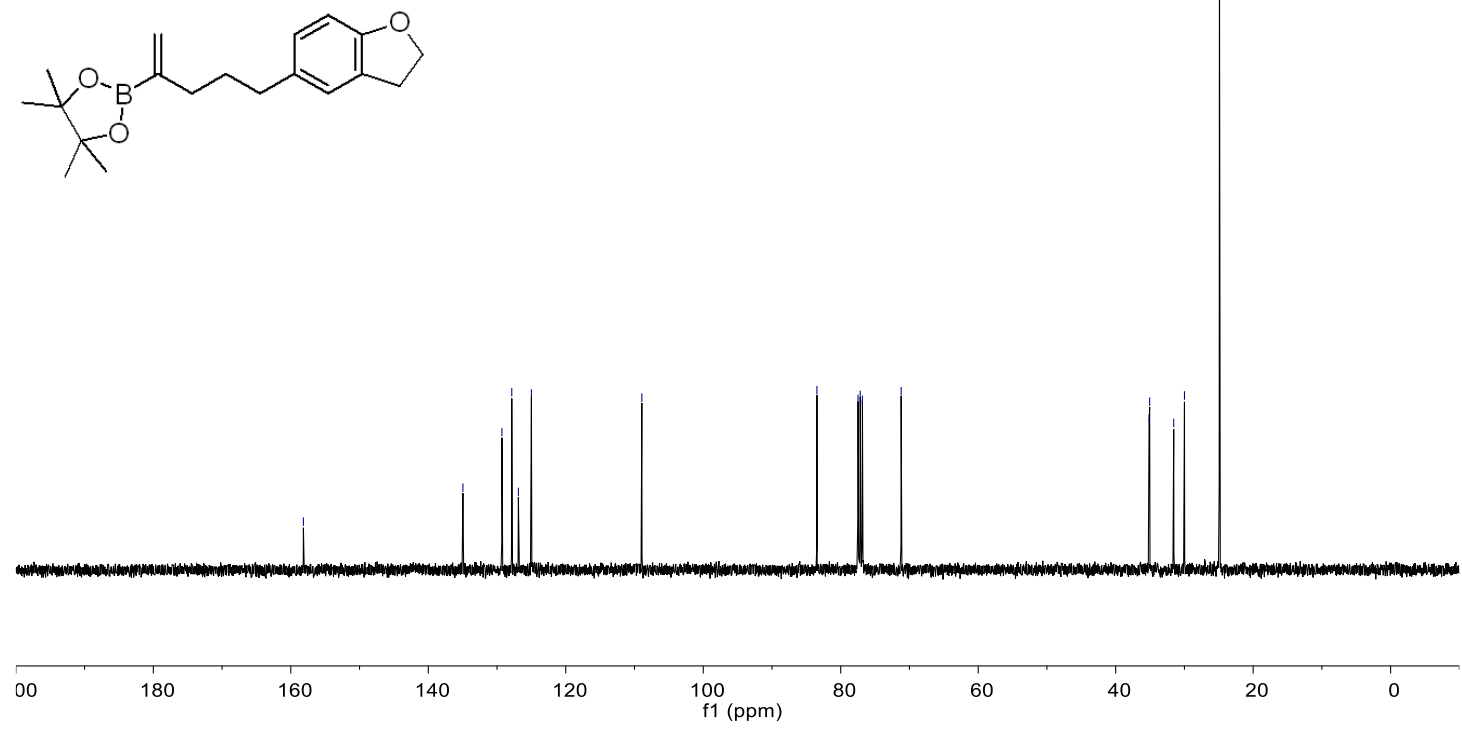

${ }^{13} \mathrm{C}$ NMR $\left(101 \mathrm{M}, \mathrm{CDCl}_{3}\right)$ spectrum of $\mathbf{1 t}$
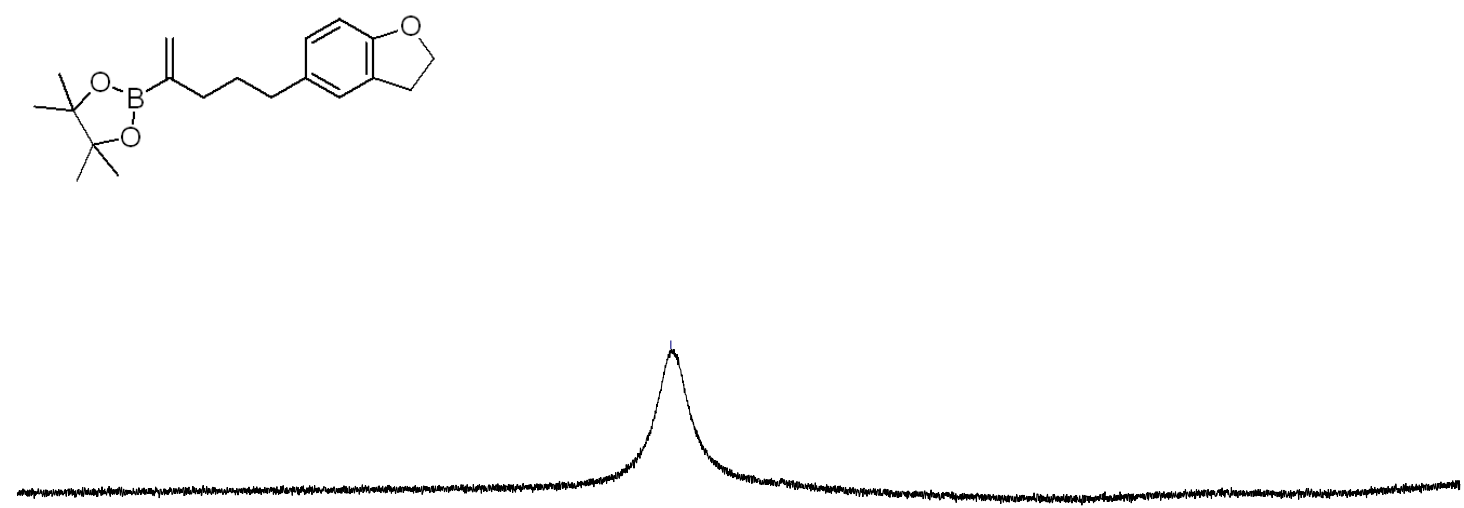

\begin{tabular}{|c|c|c|c|c|c|c|c|c|c|}
\hline 70 & 60 & 50 & 40 & 30 & $\begin{array}{r}1 \\
20 \\
\mathrm{f} 1(\mathrm{ppm})\end{array}$ & 10 & 0 & -10 & -20 \\
\hline
\end{tabular}

${ }^{11} \mathrm{~B}$ NMR (128M, $\mathrm{CDCl}_{3}$ ) spectrum of $\mathbf{1 t}$ 


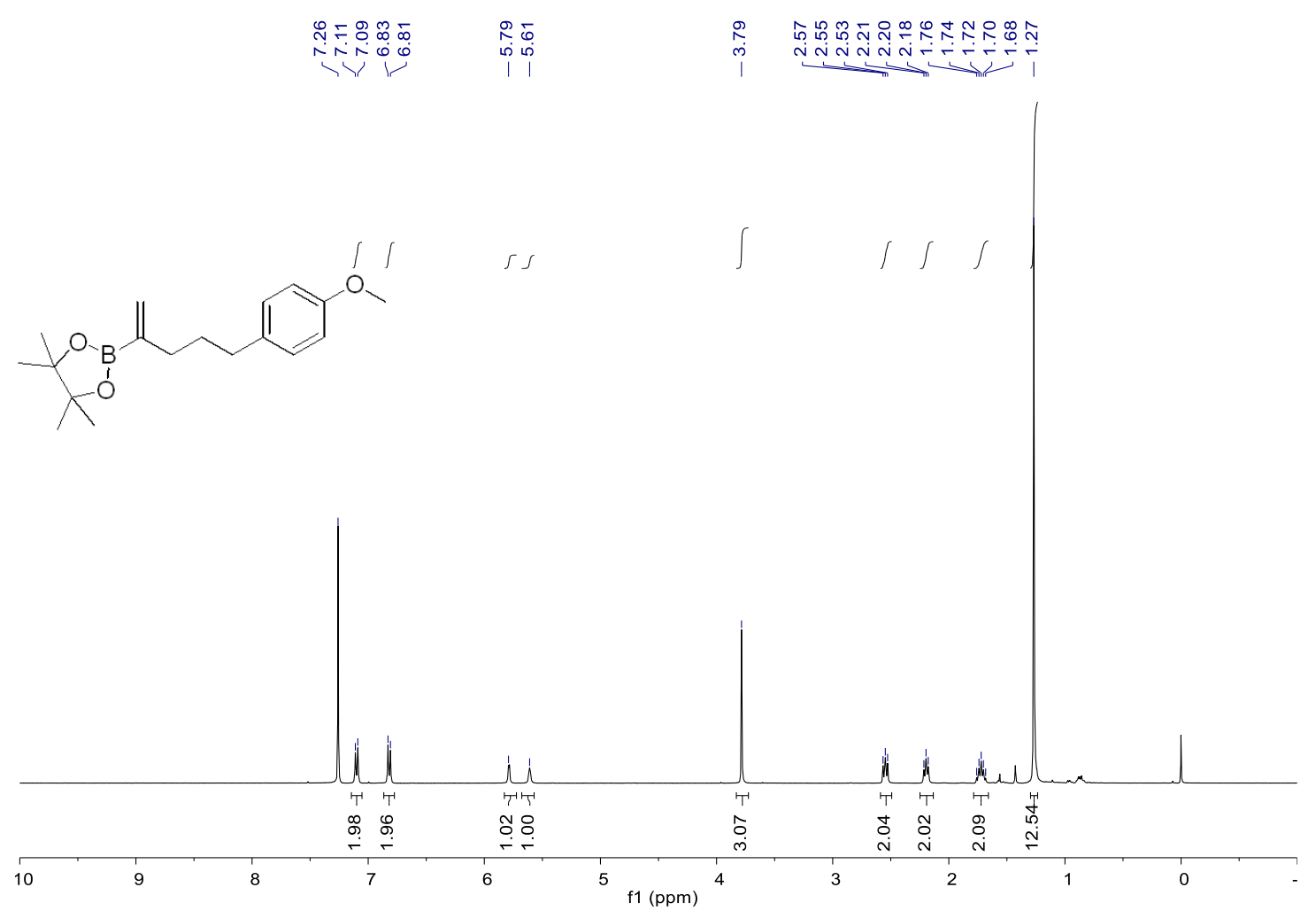

${ }^{1} \mathrm{H}$ NMR (400M, $\mathrm{CDCl}_{3}$ ) spectrum of $\mathbf{1 u}$

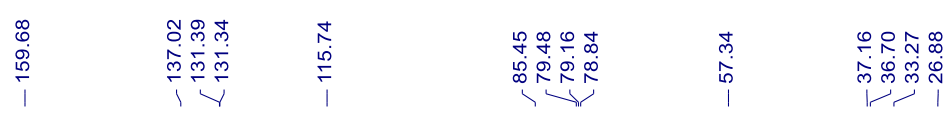<smiles>C=C(CCCc1ccc(OC)cc1)OC(C)(C)C(C)(C)C</smiles>
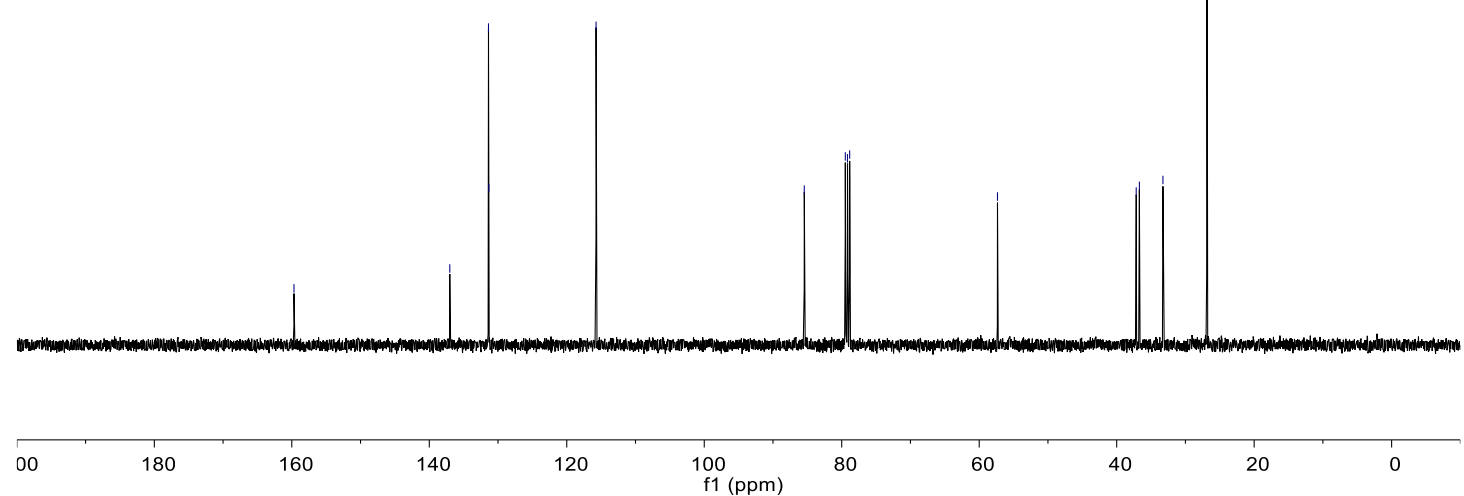

${ }^{13} \mathrm{C}$ NMR (101M, $\left.\mathrm{CDCl}_{3}\right)$ spectrum of $\mathbf{1 u}$ 

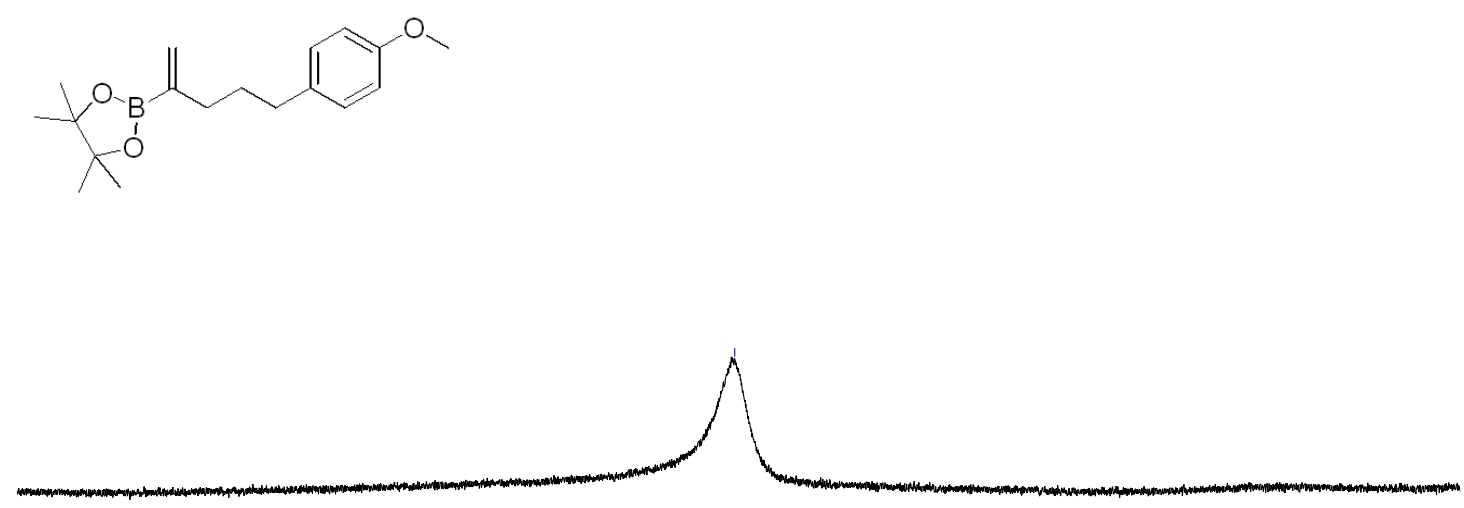

\begin{tabular}{|c|c|c|c|c|c|c|c|c|}
\hline 70 & 60 & 50 & 40 & $\begin{array}{c}30 \\
\text { f1 }(\mathrm{ppm})\end{array}$ & 20 & 10 & 0 & -10 \\
\hline
\end{tabular}

${ }^{11} \mathrm{~B}$ NMR (128M, $\left.\mathrm{CDCl}_{3}\right)$ spectrum of $\mathbf{1 u}$

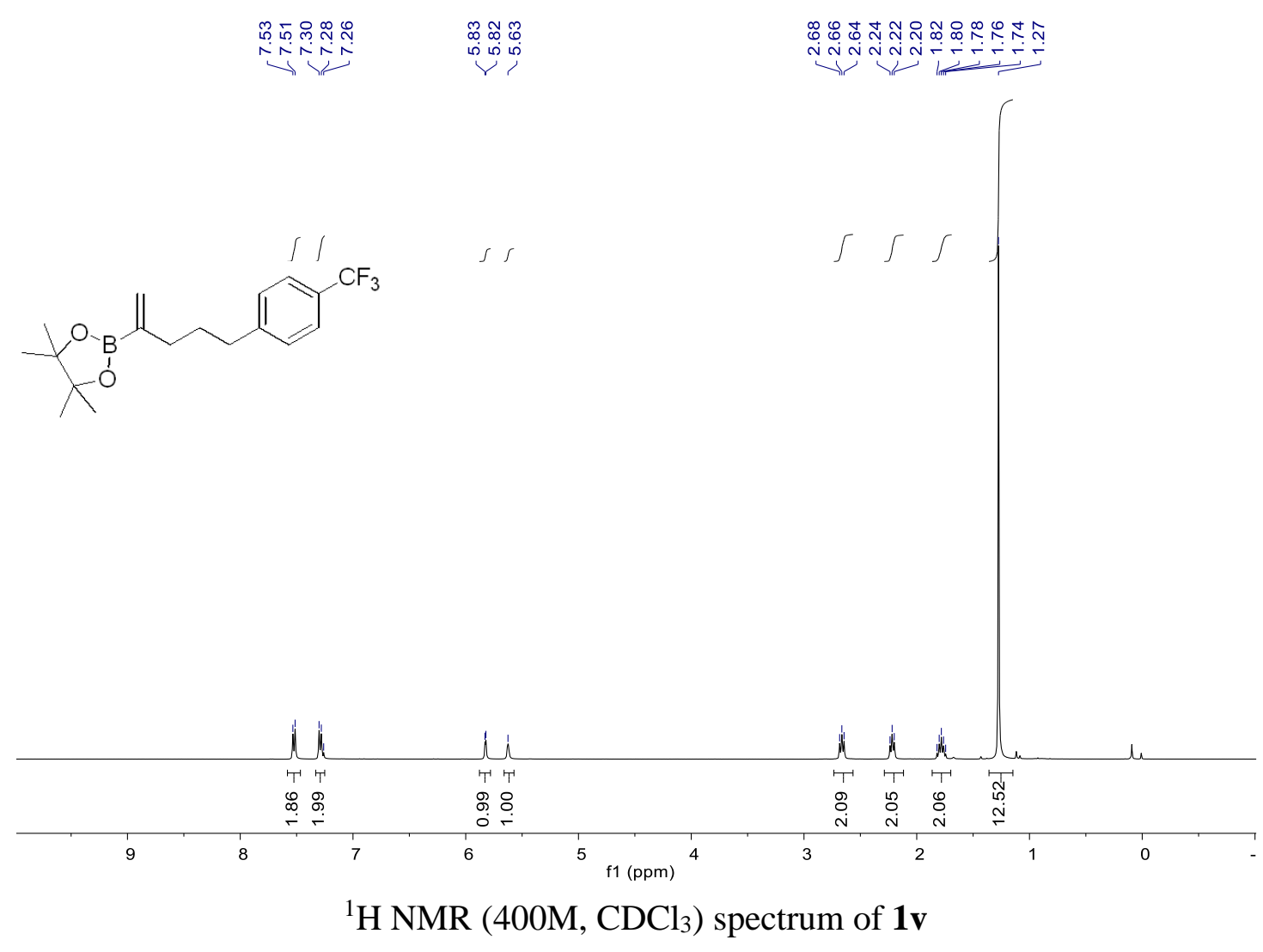



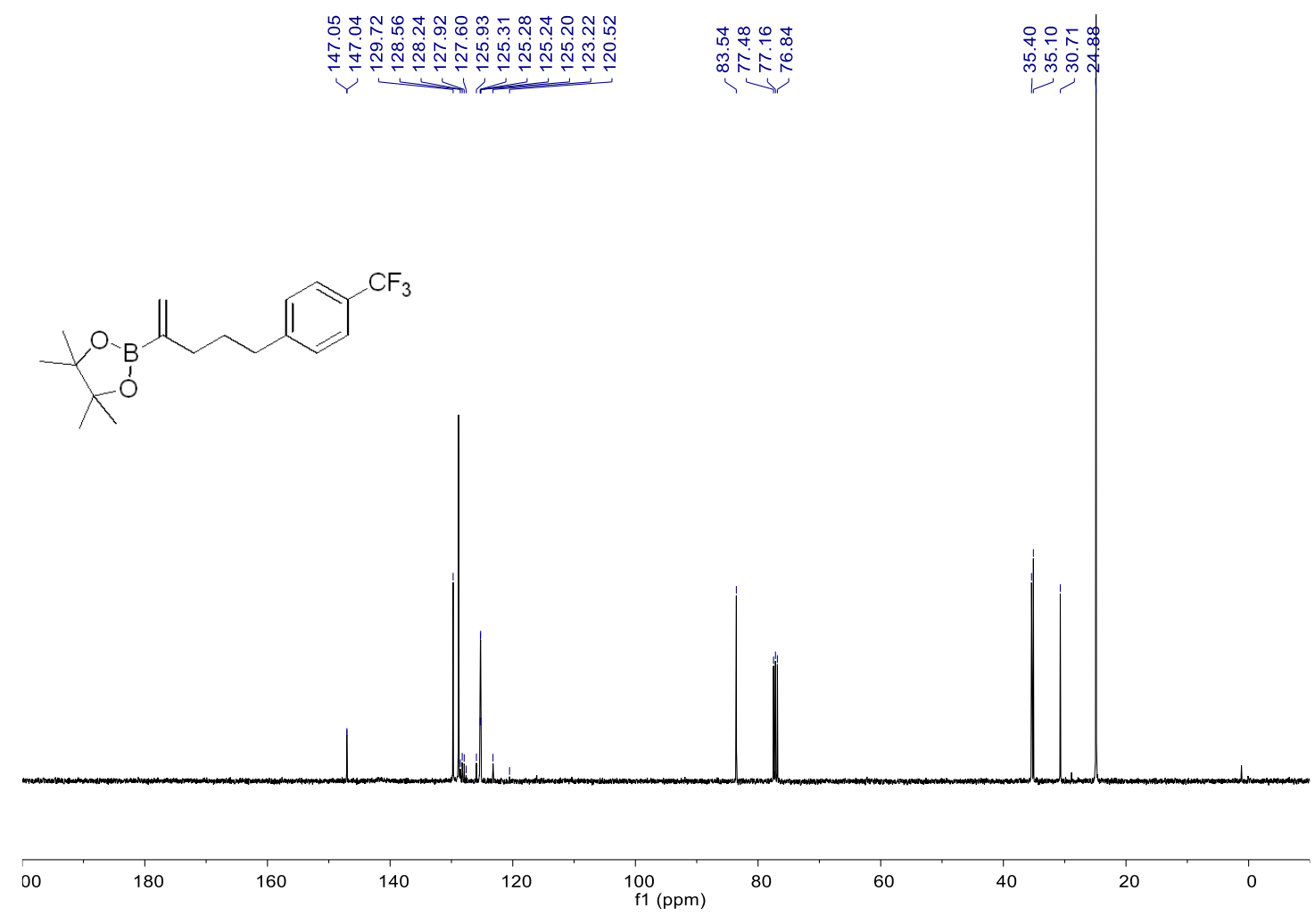

${ }^{13} \mathrm{C} \mathrm{NMR}\left(101 \mathrm{M}, \mathrm{CDCl}_{3}\right)$ spectrum of $\mathbf{1 v}$

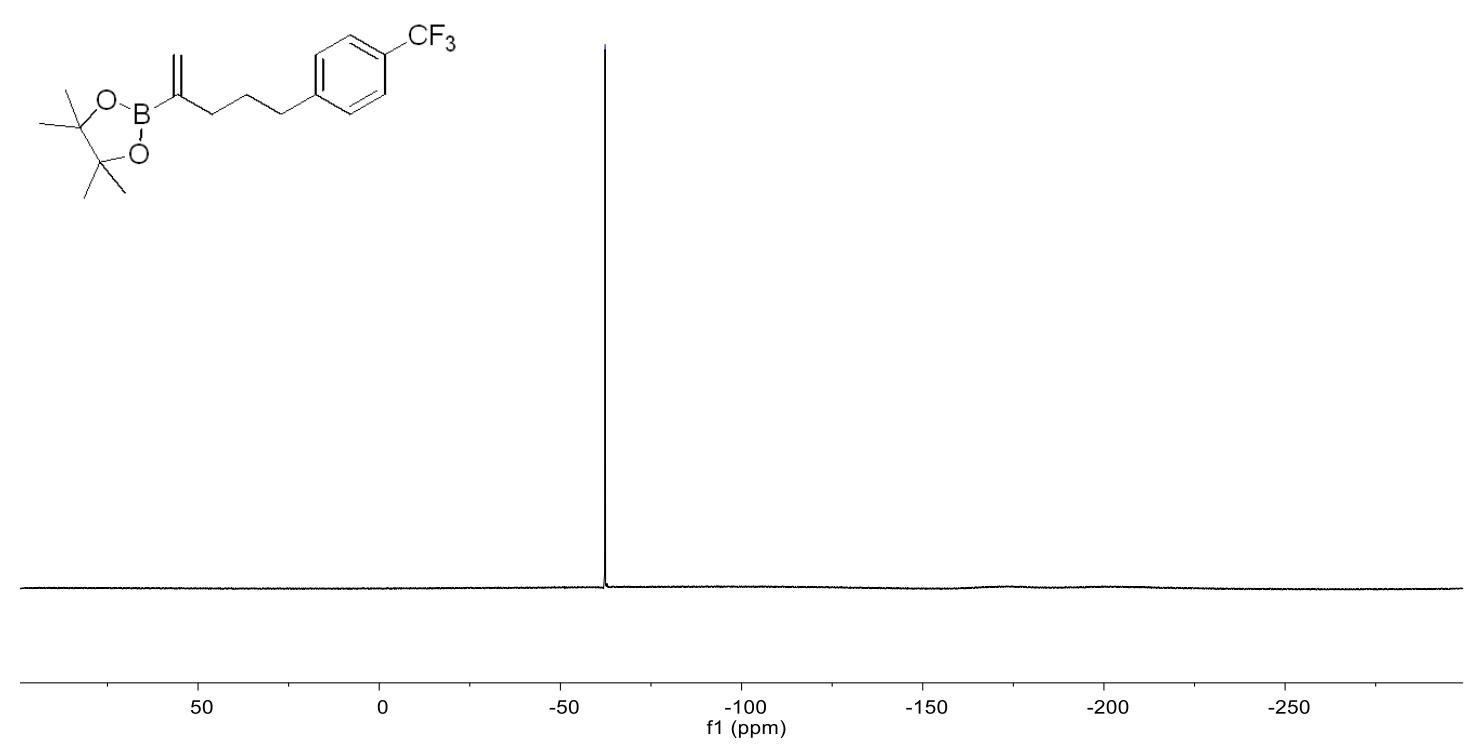

${ }^{19} \mathrm{~F}$ NMR $\left(376 \mathrm{MHz}, \mathrm{CDCl}_{3}\right.$ ) spectrum of $\mathbf{1 v}$ 
<smiles>C=C(CCCc1ccc(C(F)(F)F)cc1)B1OC(C)(C)C(C)(C)O1</smiles>

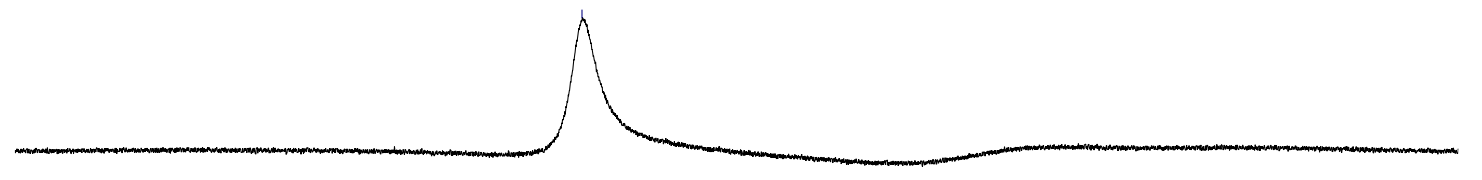

\begin{tabular}{|c|c|c|c|c|c|c|c|c|c|c|c|}
\hline 70 & 60 & 50 & 40 & 30 & $\begin{array}{l}20 \\
\text { f1 (ppm) }\end{array}$ & 10 & 0 & -10 & -20 & -30 & -40 \\
\hline
\end{tabular}

${ }^{11} \mathrm{~B}$ NMR $\left(128 \mathrm{M}, \mathrm{CDCl}_{3}\right)$ spectrum of $\mathbf{1 v}$

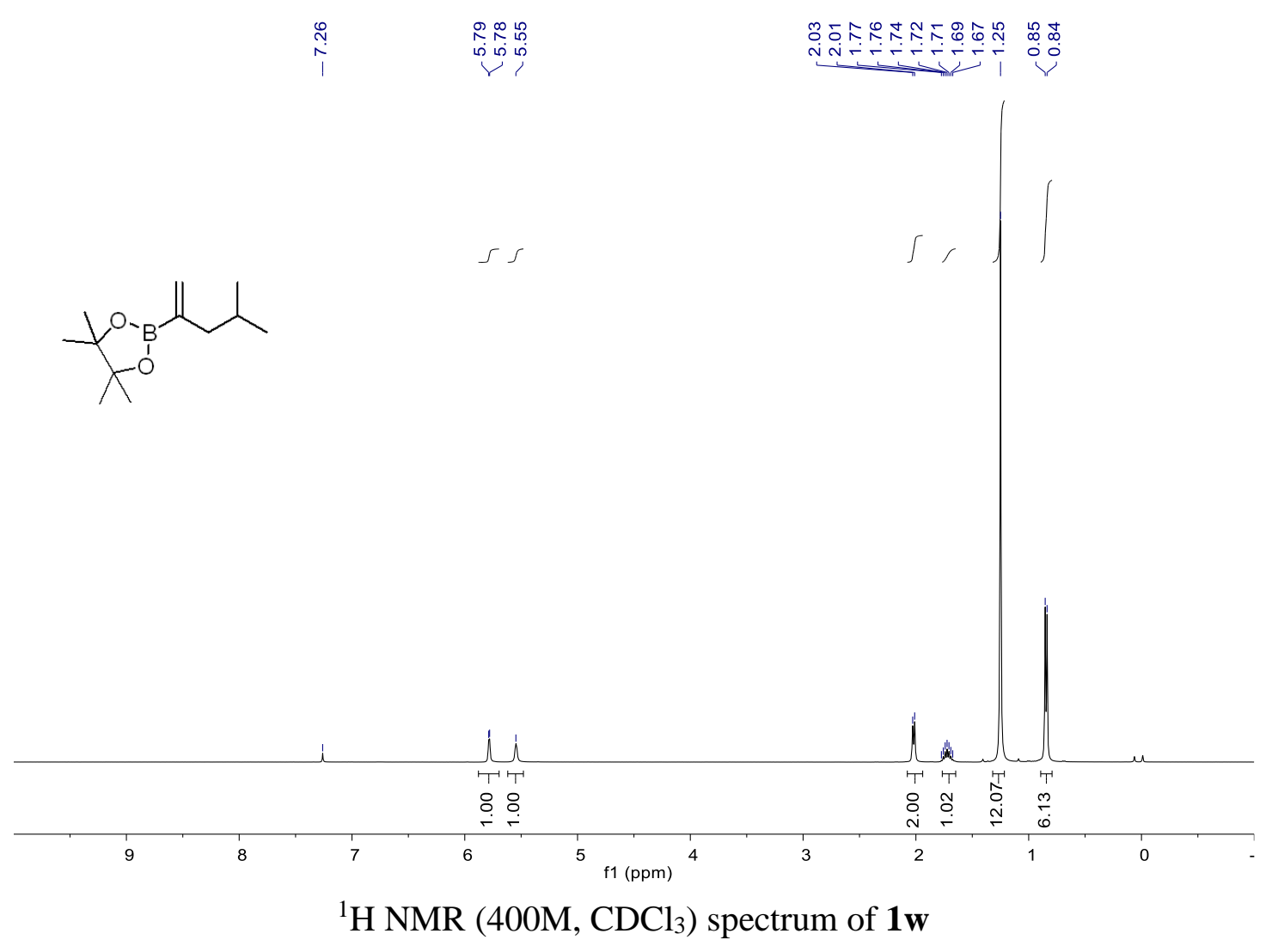




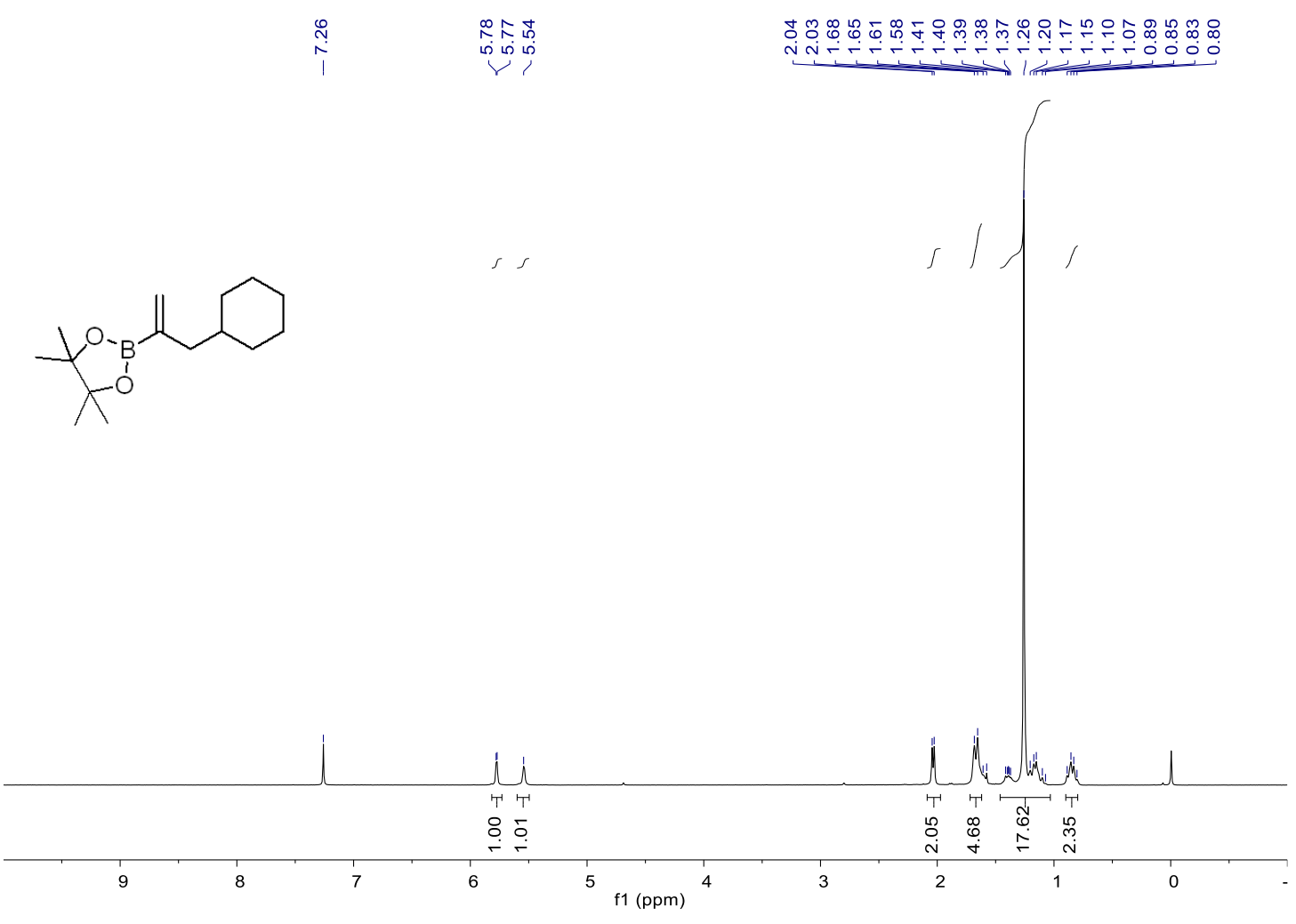

${ }^{1} \mathrm{H}$ NMR (400M, $\mathrm{CDCl}_{3}$ ) spectrum of $\mathbf{1 x}$

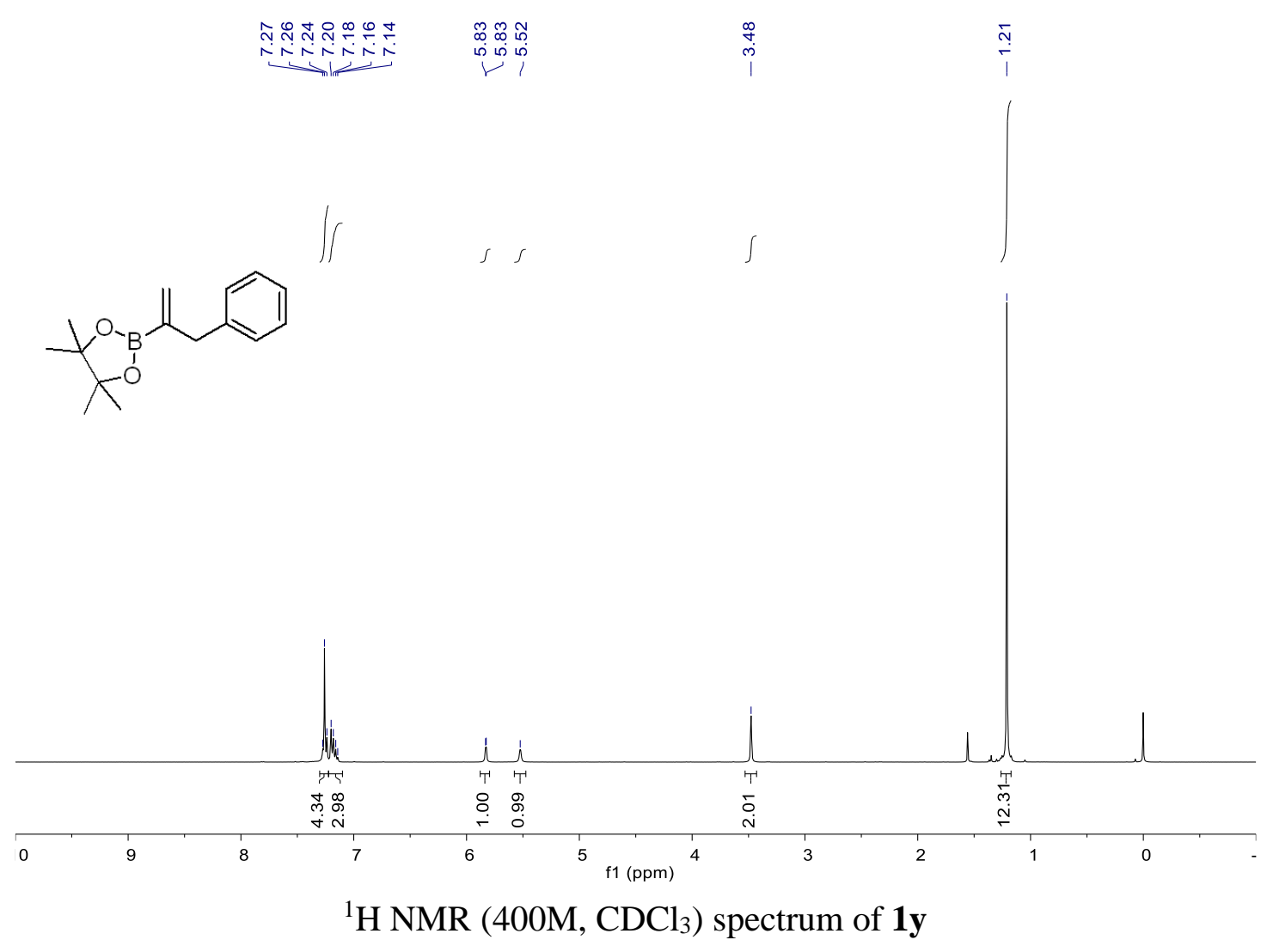




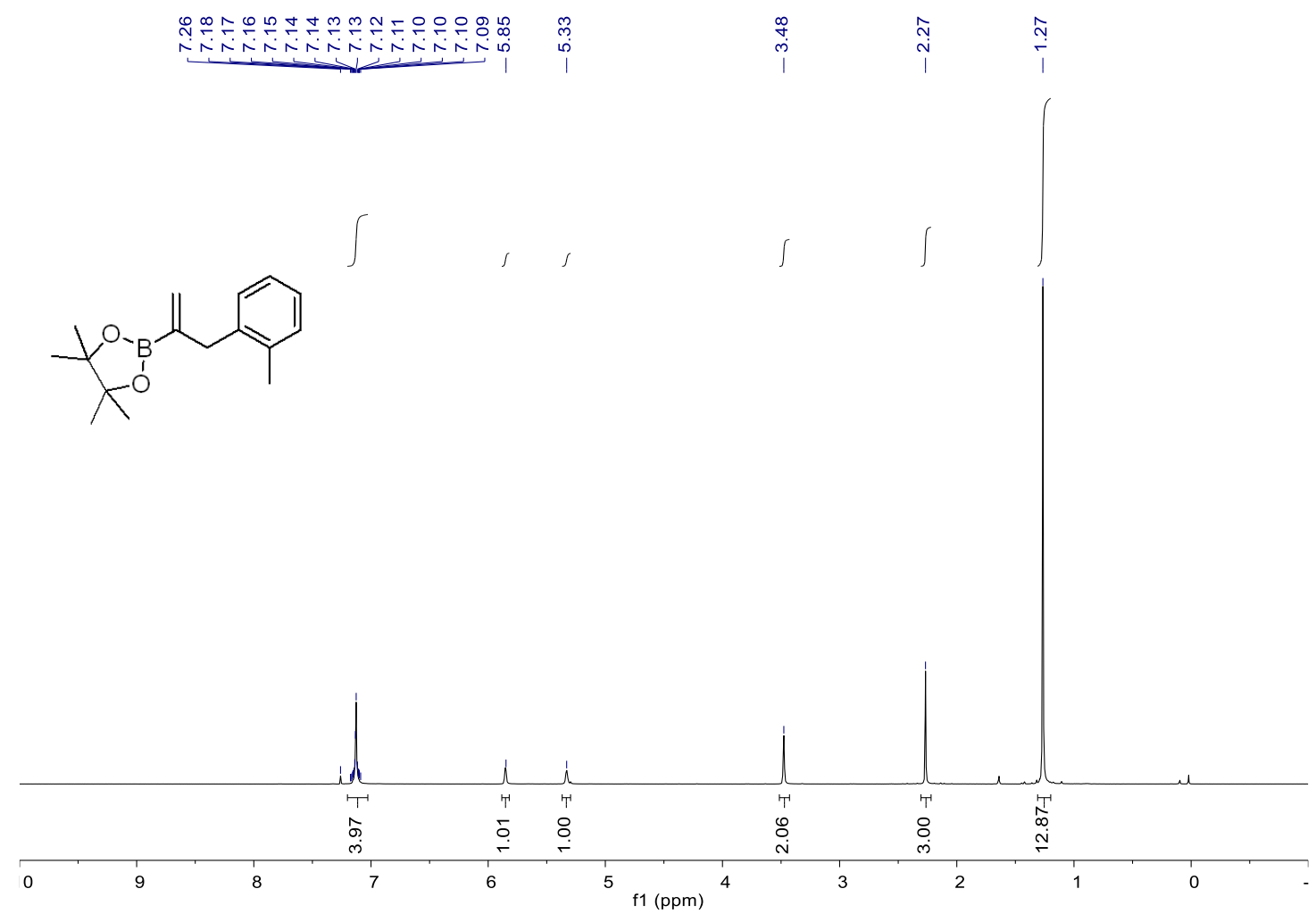

${ }^{1} \mathrm{H}$ NMR (400M, $\mathrm{CDCl}_{3}$ ) spectrum of $\mathbf{1 z}$

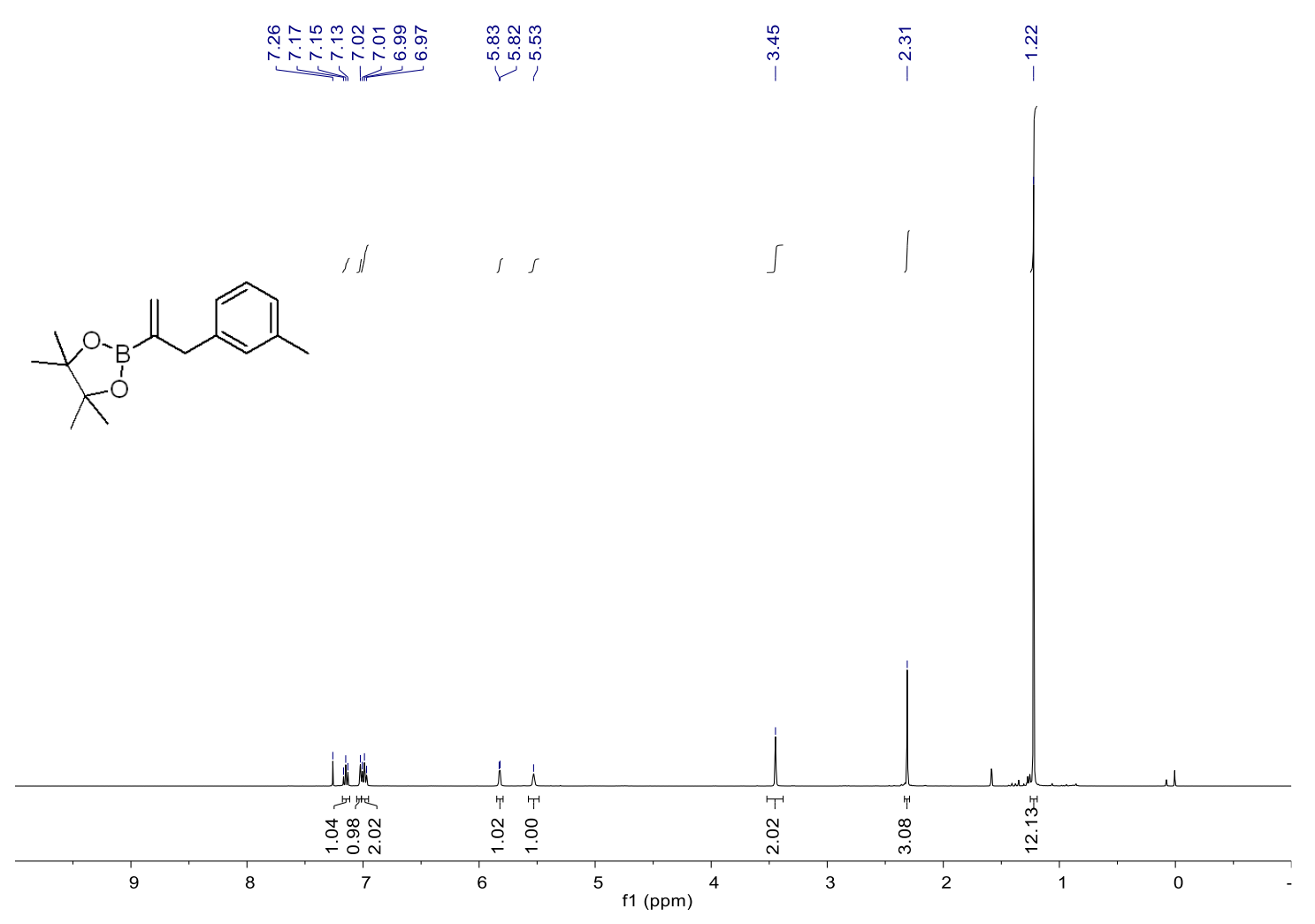

${ }^{1} \mathrm{H}$ NMR (400M, $\mathrm{CDCl}_{3}$ ) spectrum of 1aa 

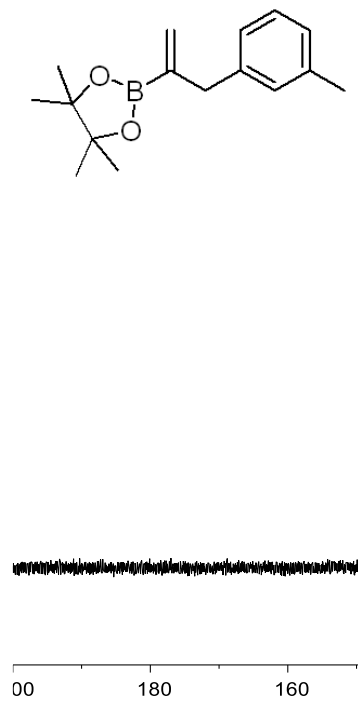

${ }^{13} \mathrm{C} \mathrm{NMR}\left(101 \mathrm{M}, \mathrm{CDCl}_{3}\right)$ spectrum of $1 \mathbf{a a}$<smiles>C=C(Cc1cccc(C)c1)OC(C)(C)C(C)(C)C</smiles>

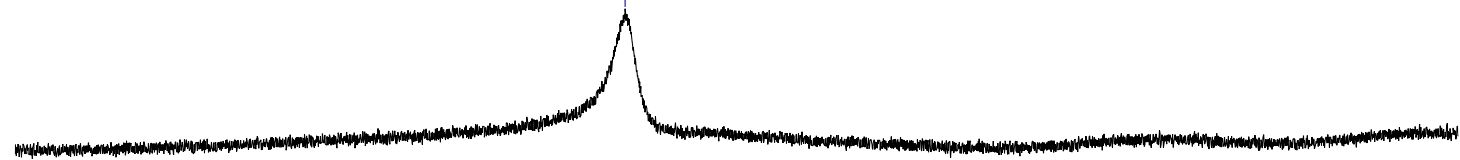

$70 \quad 60$

50

40

$\begin{array}{lr}30 & 20 \\ & \mathrm{f} 1(\mathrm{ppm})\end{array}$

10

0

$-10$

$-20$

${ }^{11} \mathrm{~B}$ NMR (128M, $\left.\mathrm{CDCl}_{3}\right)$ spectrum of $\mathbf{1 a a}$ 


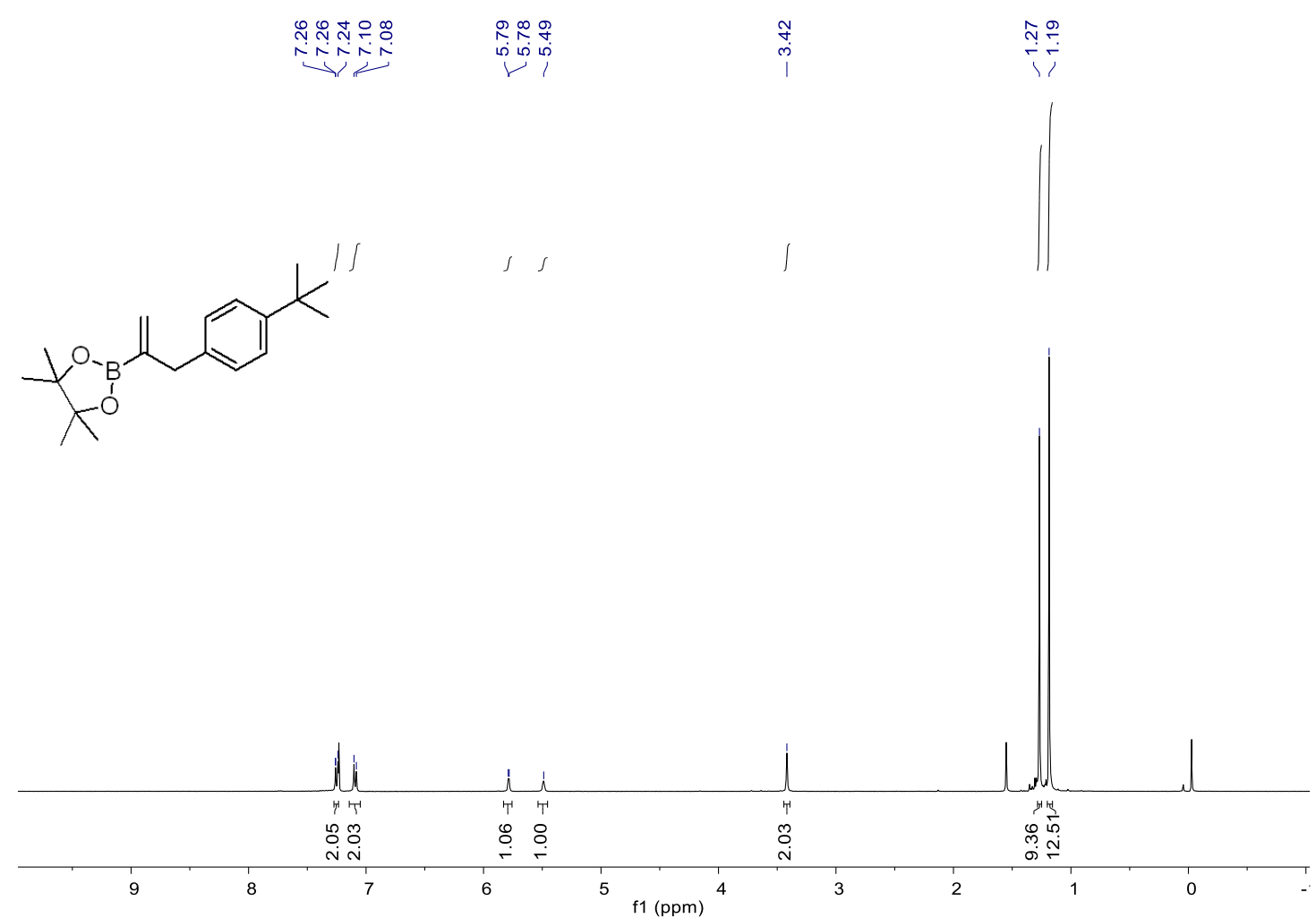

${ }^{1} \mathrm{H}$ NMR (400M, $\mathrm{CDCl}_{3}$ ) spectrum of $\mathbf{1 a b}$
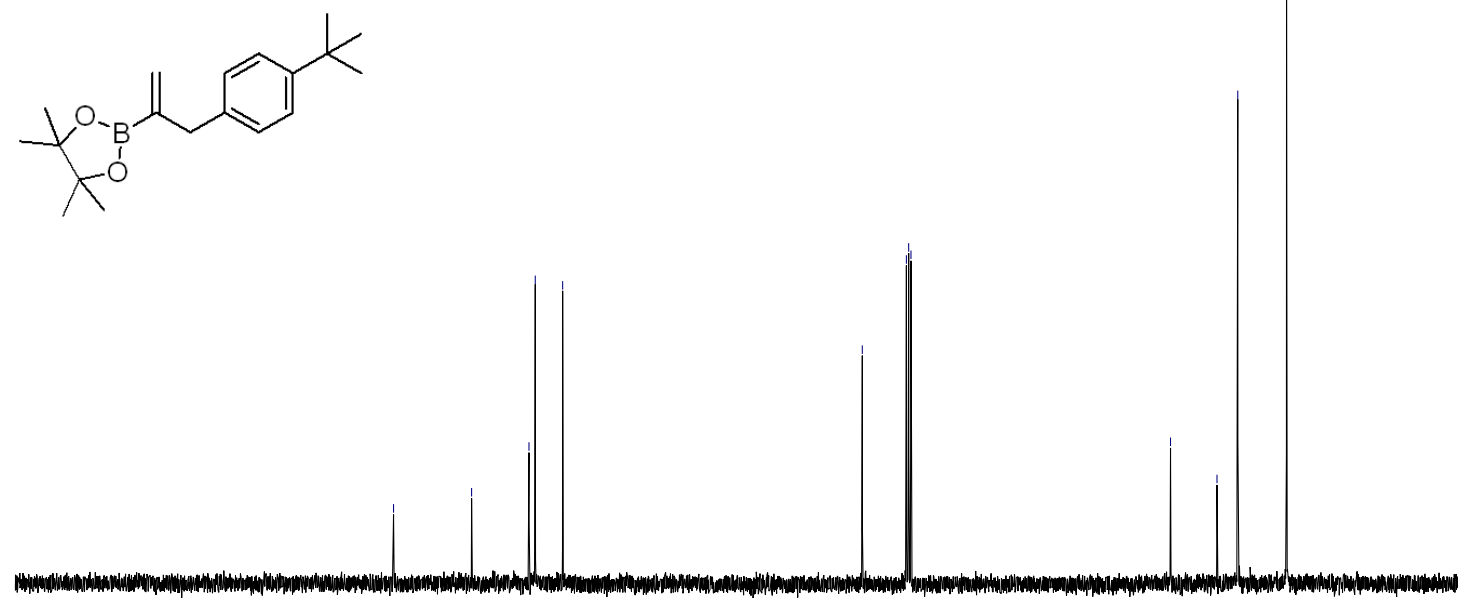

oo

180

160

140

120 100
$\mathrm{f} 1(\mathrm{ppm})$

80

60

40

20

${ }^{13} \mathrm{C}$ NMR $\left(101 \mathrm{M}, \mathrm{CDCl}_{3}\right)$ spectrum of $\mathbf{1 a b}$ 

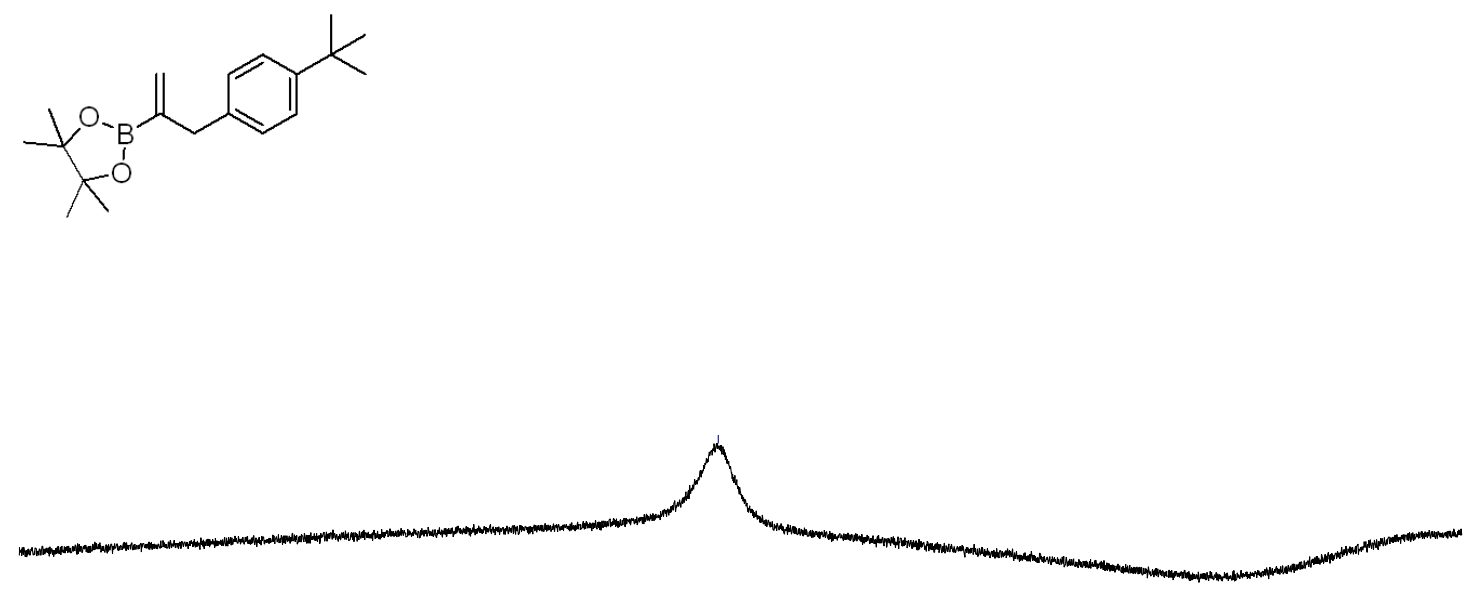

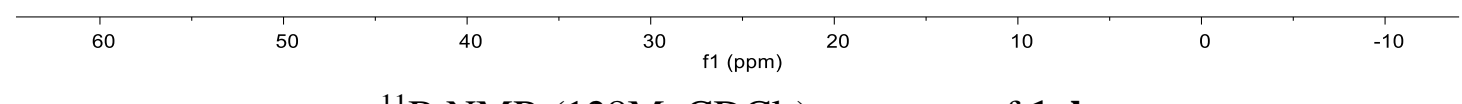

${ }^{11} \mathrm{~B}$ NMR $\left(128 \mathrm{M}, \mathrm{CDCl}_{3}\right)$ spectrum of $\mathbf{1 a b}$

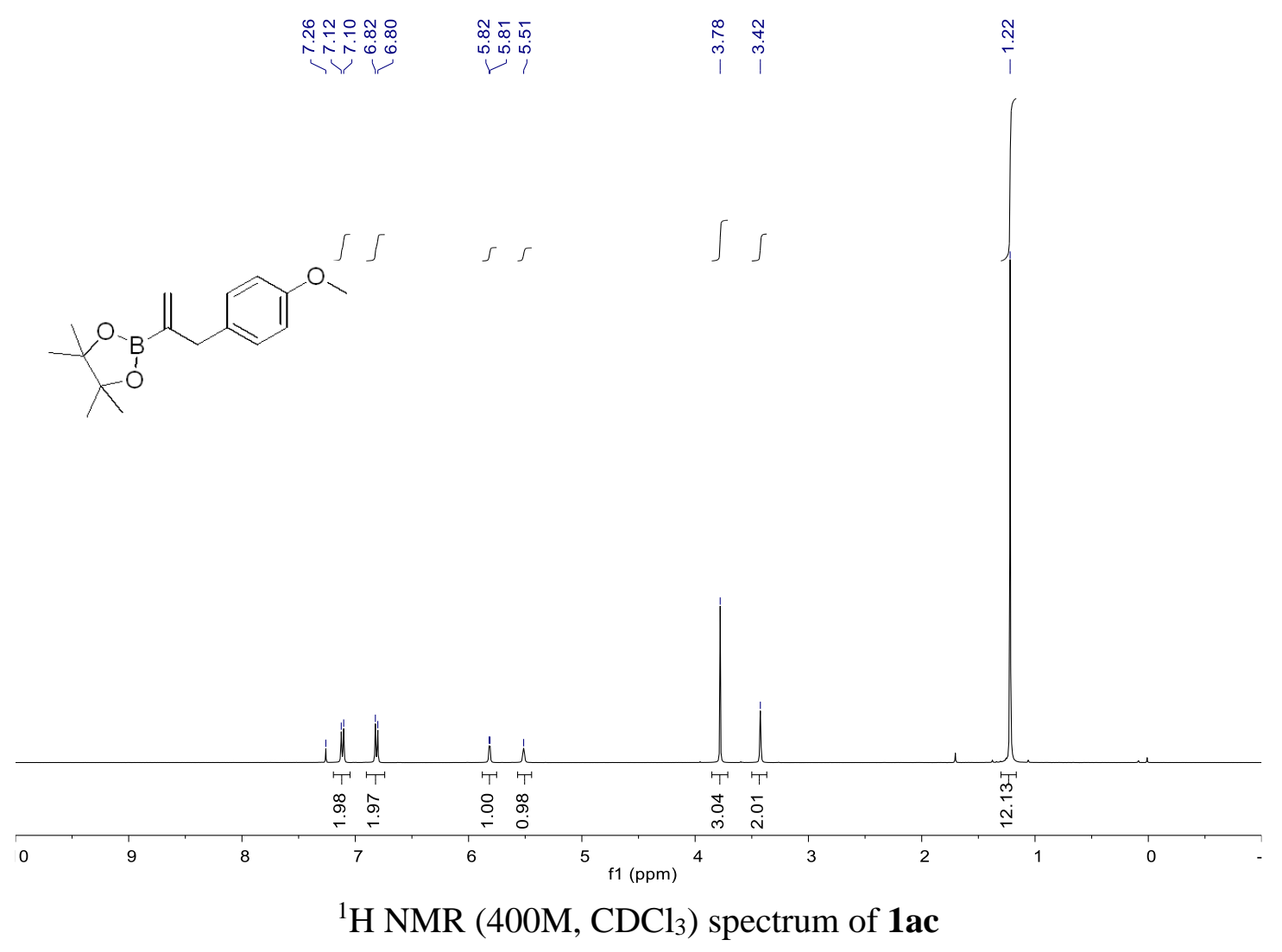




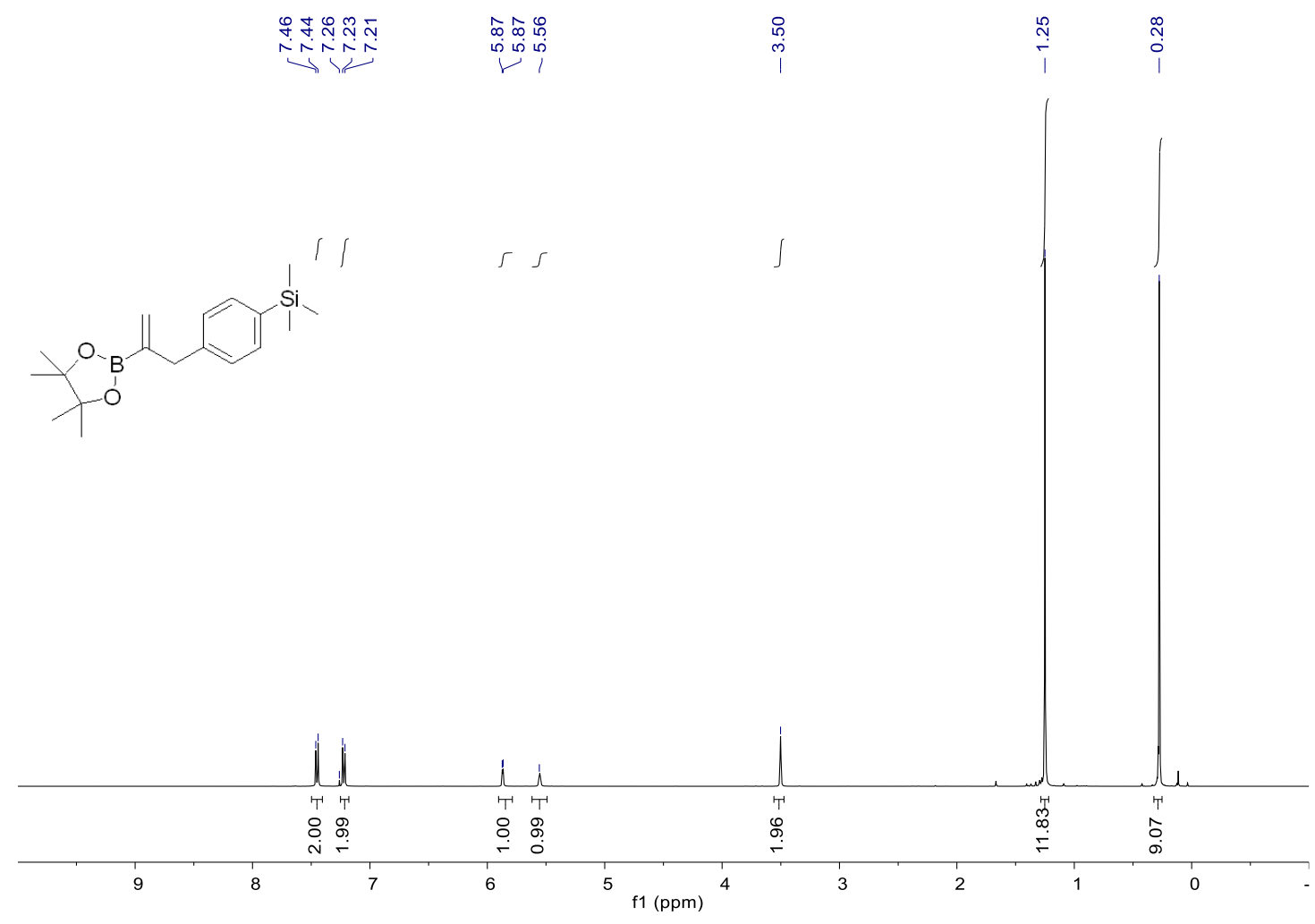

${ }^{1} \mathrm{H}$ NMR (400M, $\mathrm{CDCl}_{3}$ ) spectrum of $\mathbf{1 a d}$

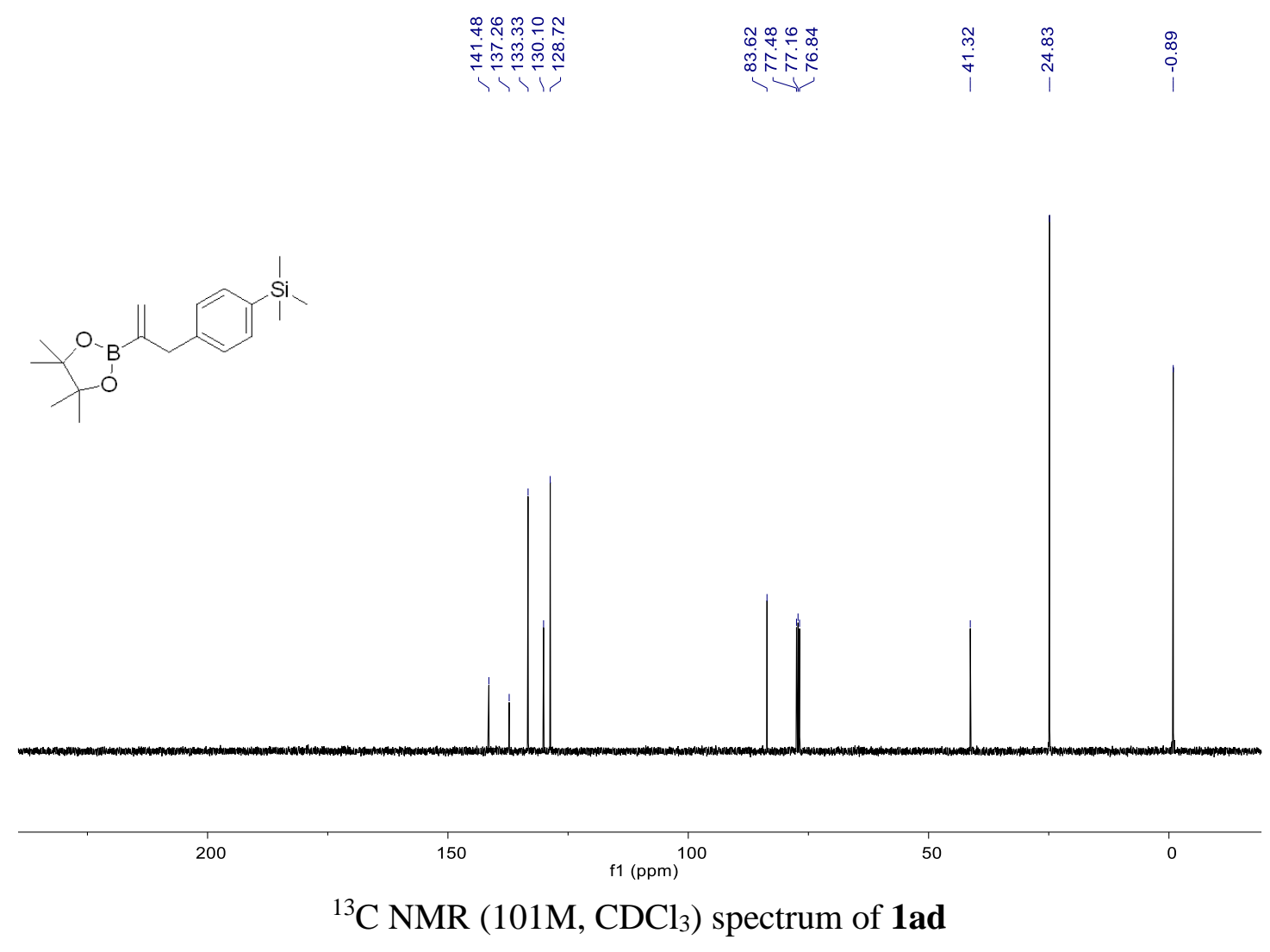



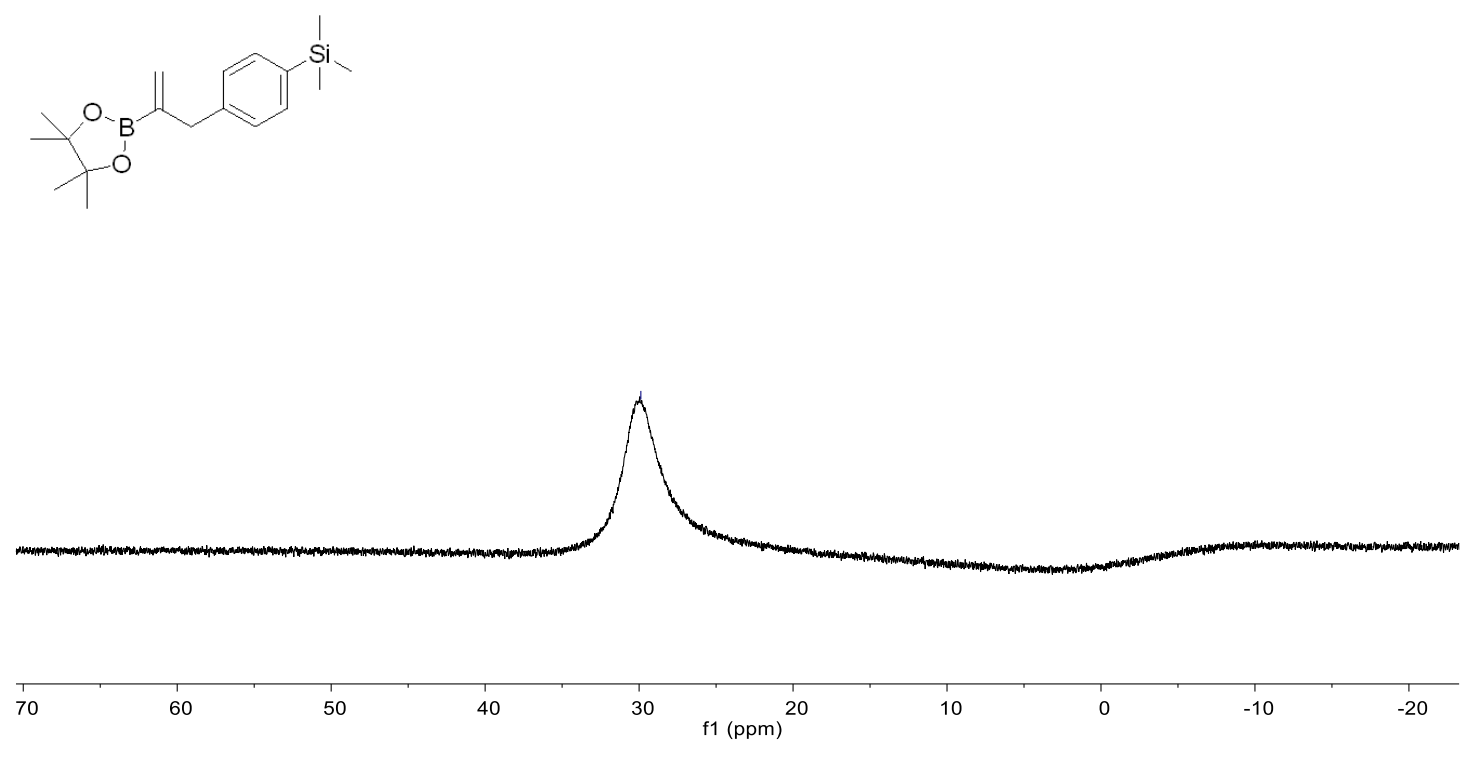

${ }^{11} \mathrm{~B}$ NMR $\left(128 \mathrm{M}, \mathrm{CDCl}_{3}\right)$ spectrum of $\mathbf{1 a d}$

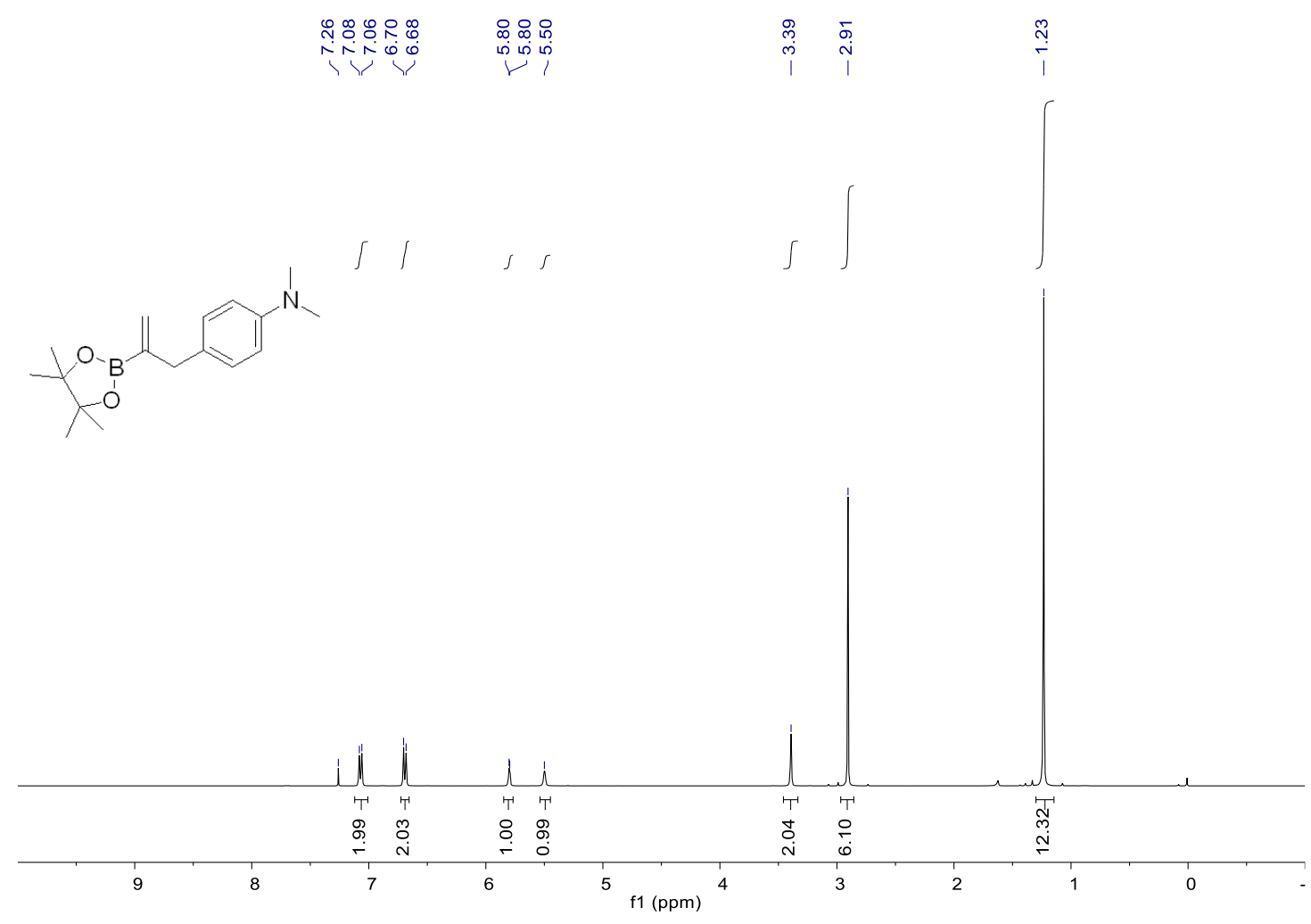

${ }^{1} \mathrm{H}$ NMR (400M, $\mathrm{CDCl}_{3}$ ) spectrum of $\mathbf{2 a e}$ 


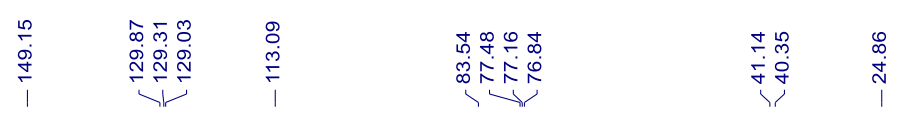
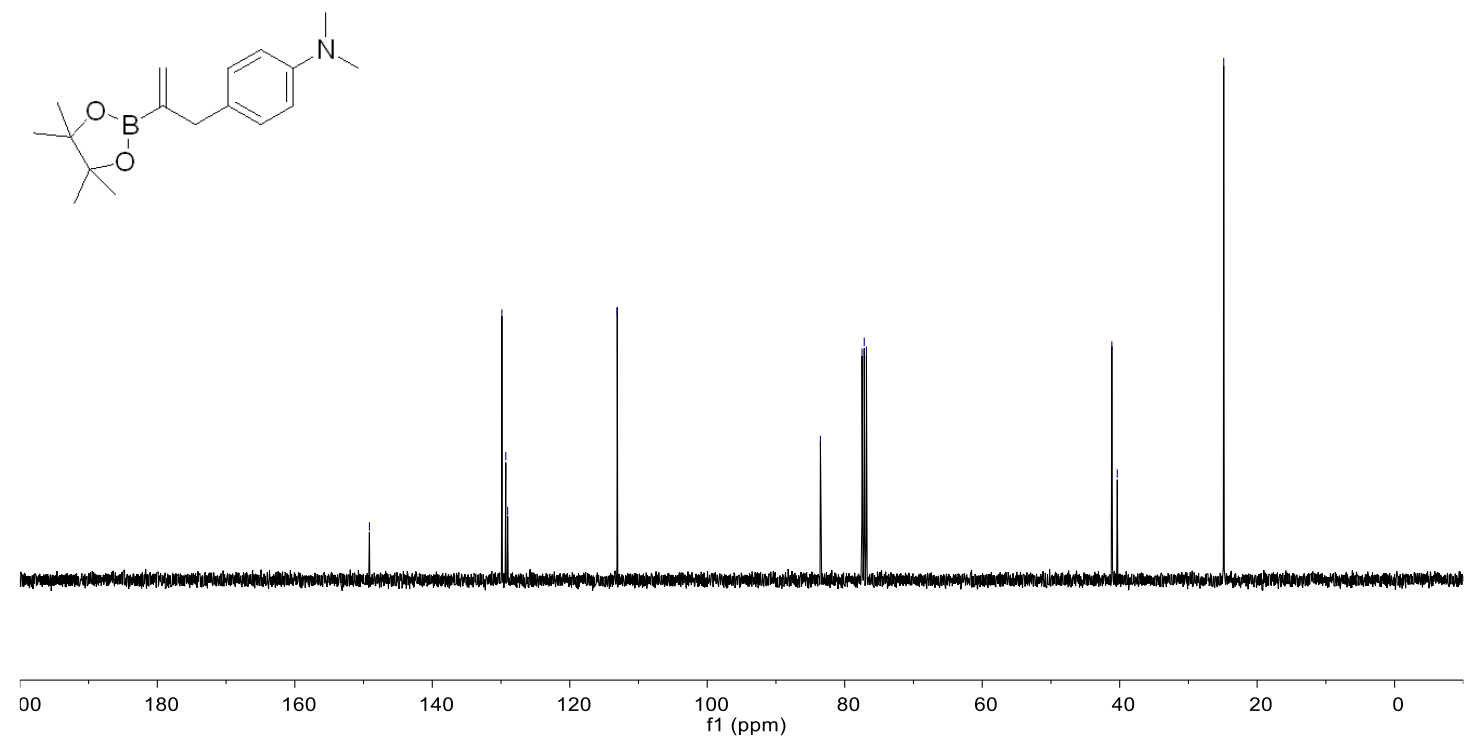

${ }^{13} \mathrm{C} \mathrm{NMR}\left(101 \mathrm{M}, \mathrm{CDCl}_{3}\right)$ spectrum of $\mathbf{1 a e}$
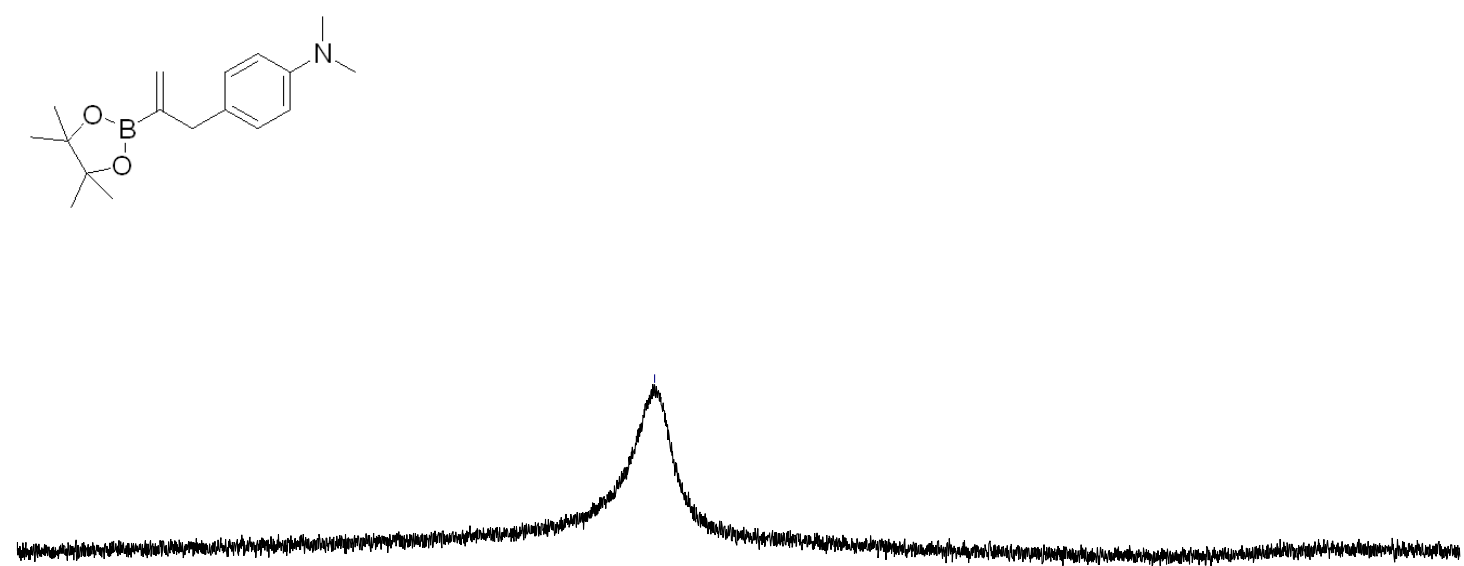

60

50

40 30

20

10

${ }^{11} \mathrm{~B}$ NMR (128M, $\left.\mathrm{CDCl}_{3}\right)$ spectrum of $\mathbf{1 a e}$ 


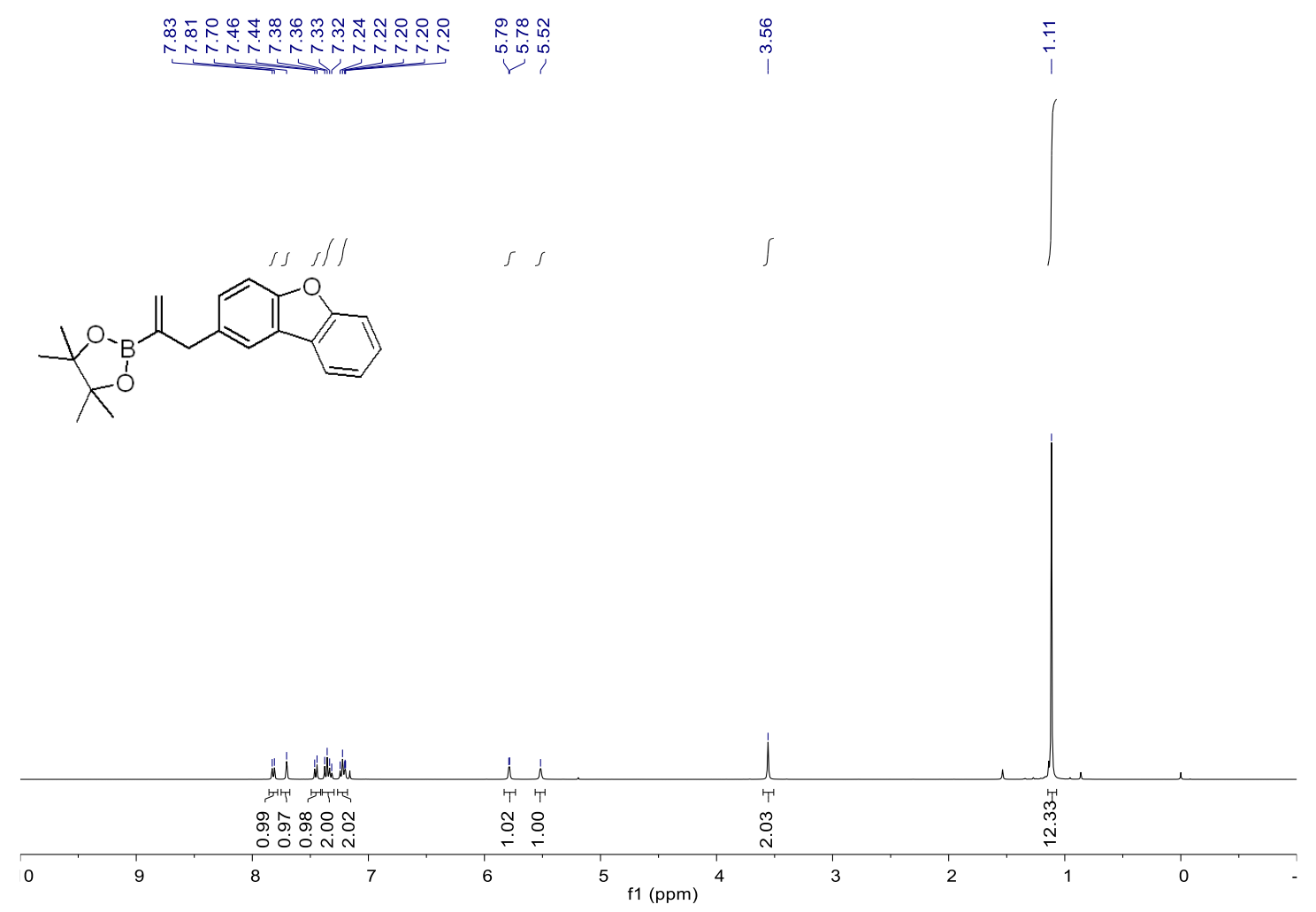

${ }^{1} \mathrm{H}$ NMR (400M, $\mathrm{CDCl}_{3}$ ) spectrum of $\mathbf{1 a f}$
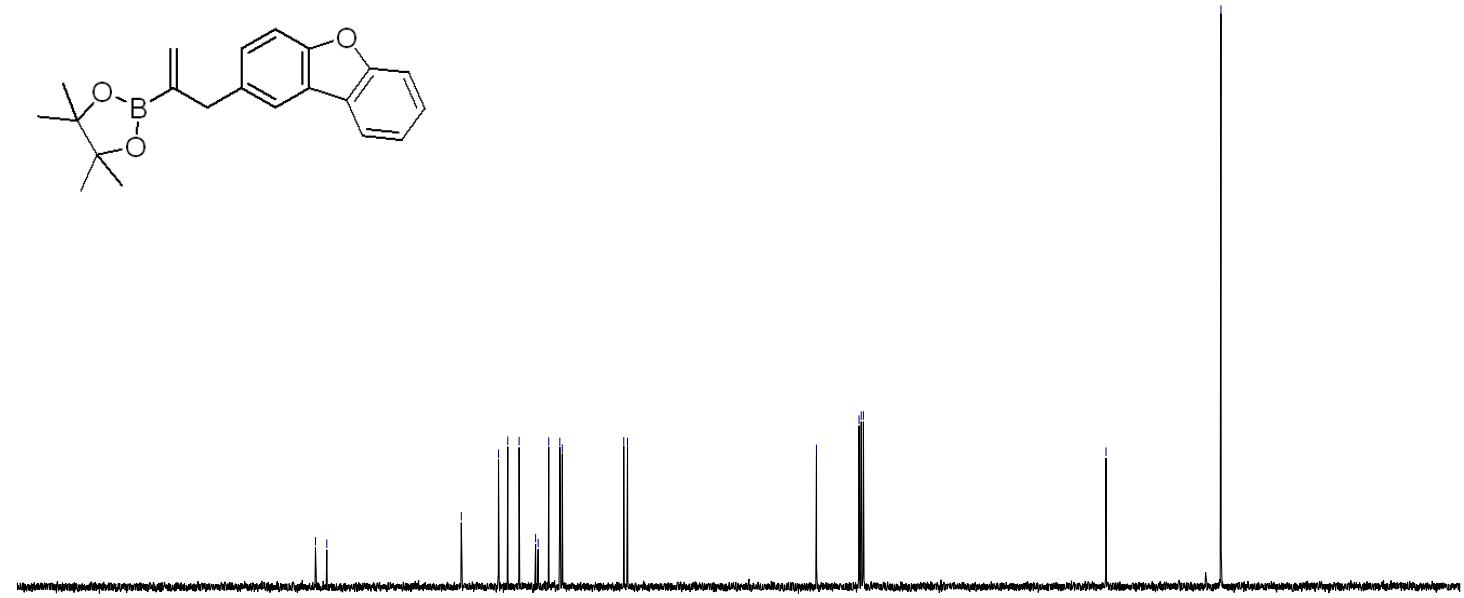

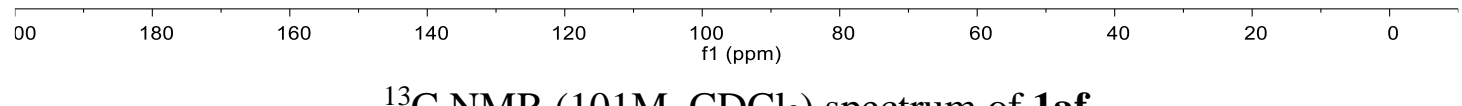

${ }^{13} \mathrm{C}$ NMR $\left(101 \mathrm{M}, \mathrm{CDCl}_{3}\right)$ spectrum of $\mathbf{1 a f}$ 

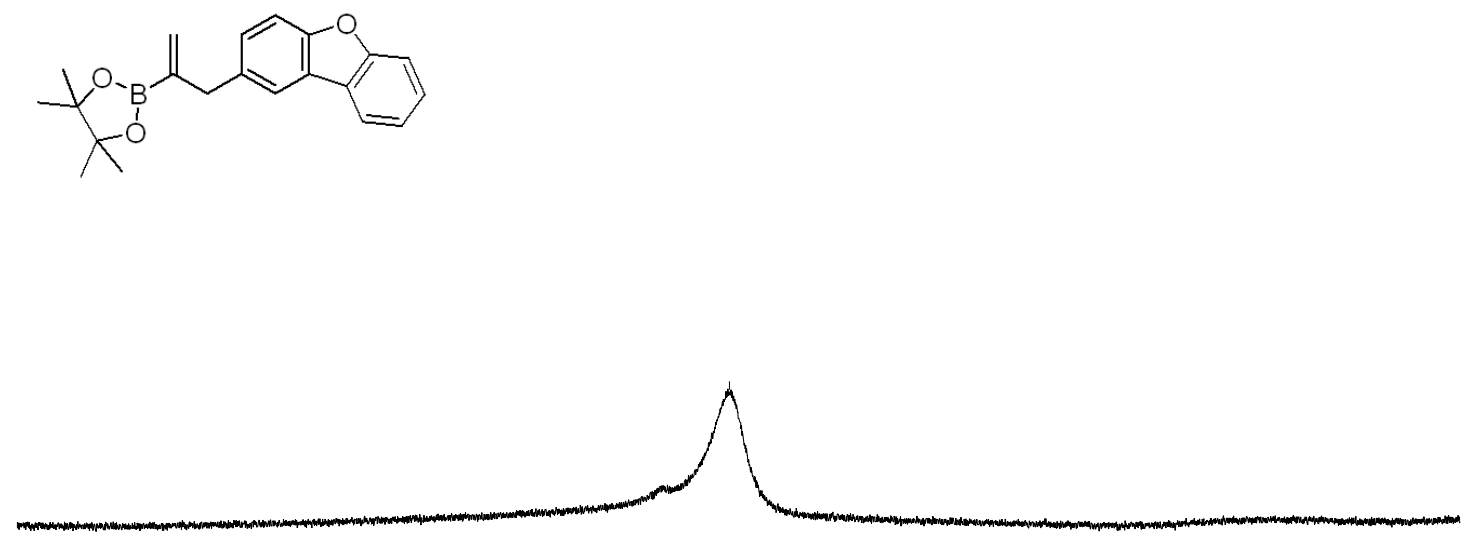

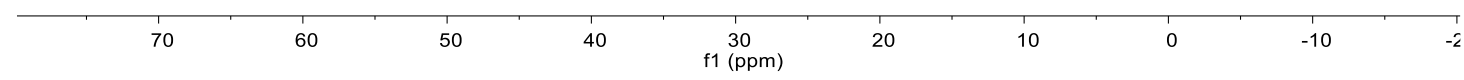

${ }^{11} \mathrm{~B}$ NMR $\left(128 \mathrm{M}, \mathrm{CDCl}_{3}\right)$ spectrum of $\mathbf{1 a f}$

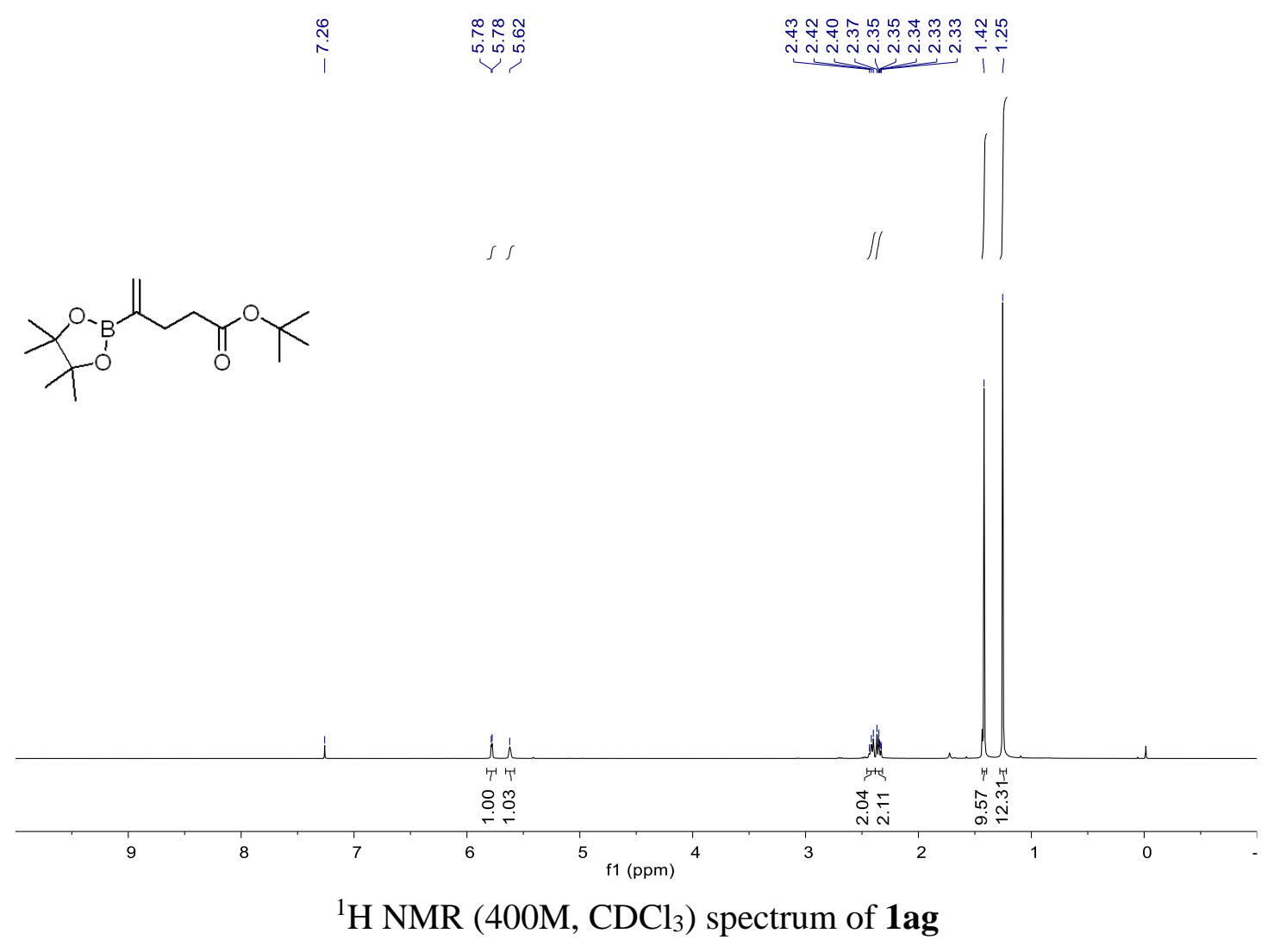




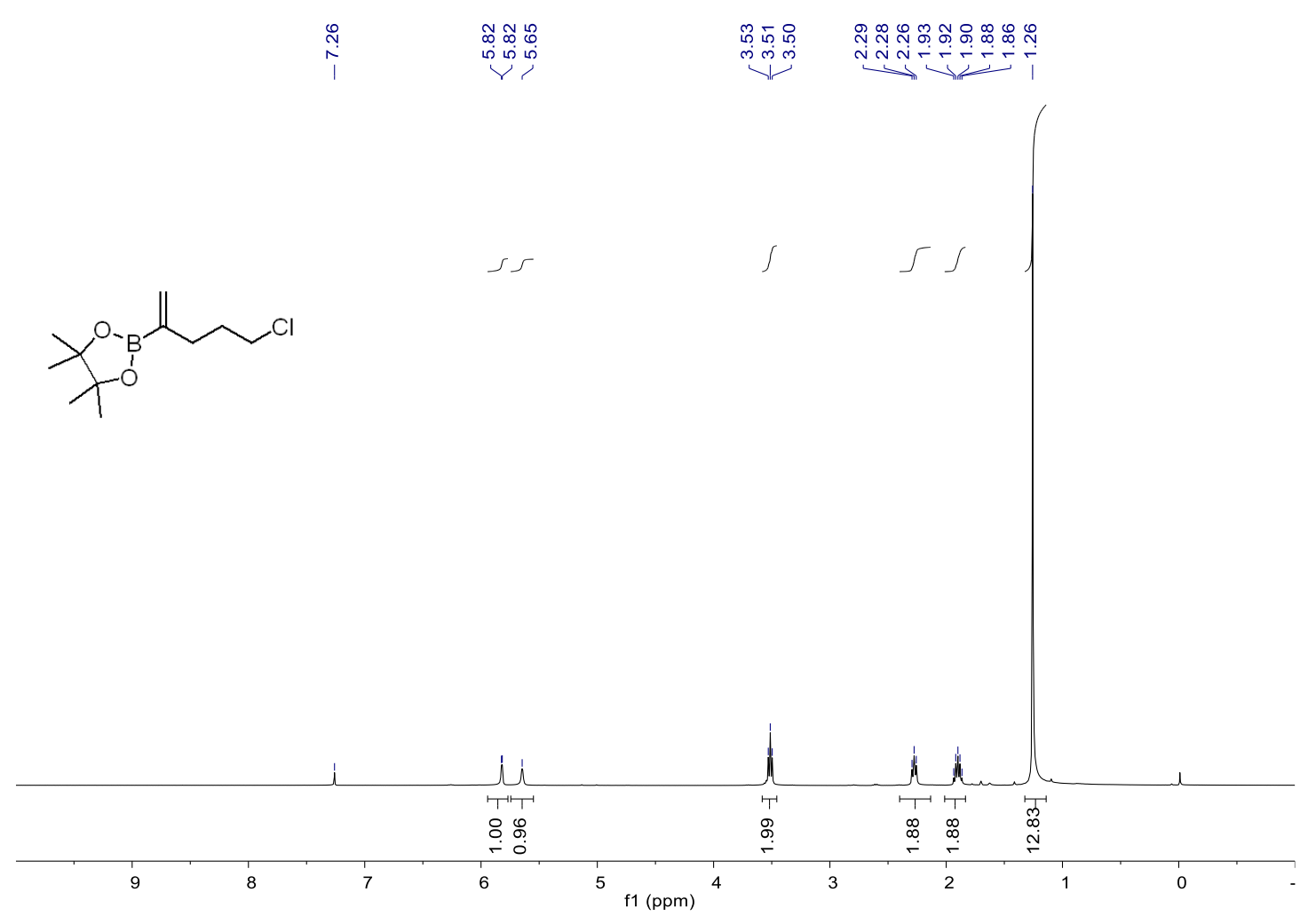

${ }^{1} \mathrm{H}$ NMR (400M, $\mathrm{CDCl}_{3}$ ) spectrum of 1ah

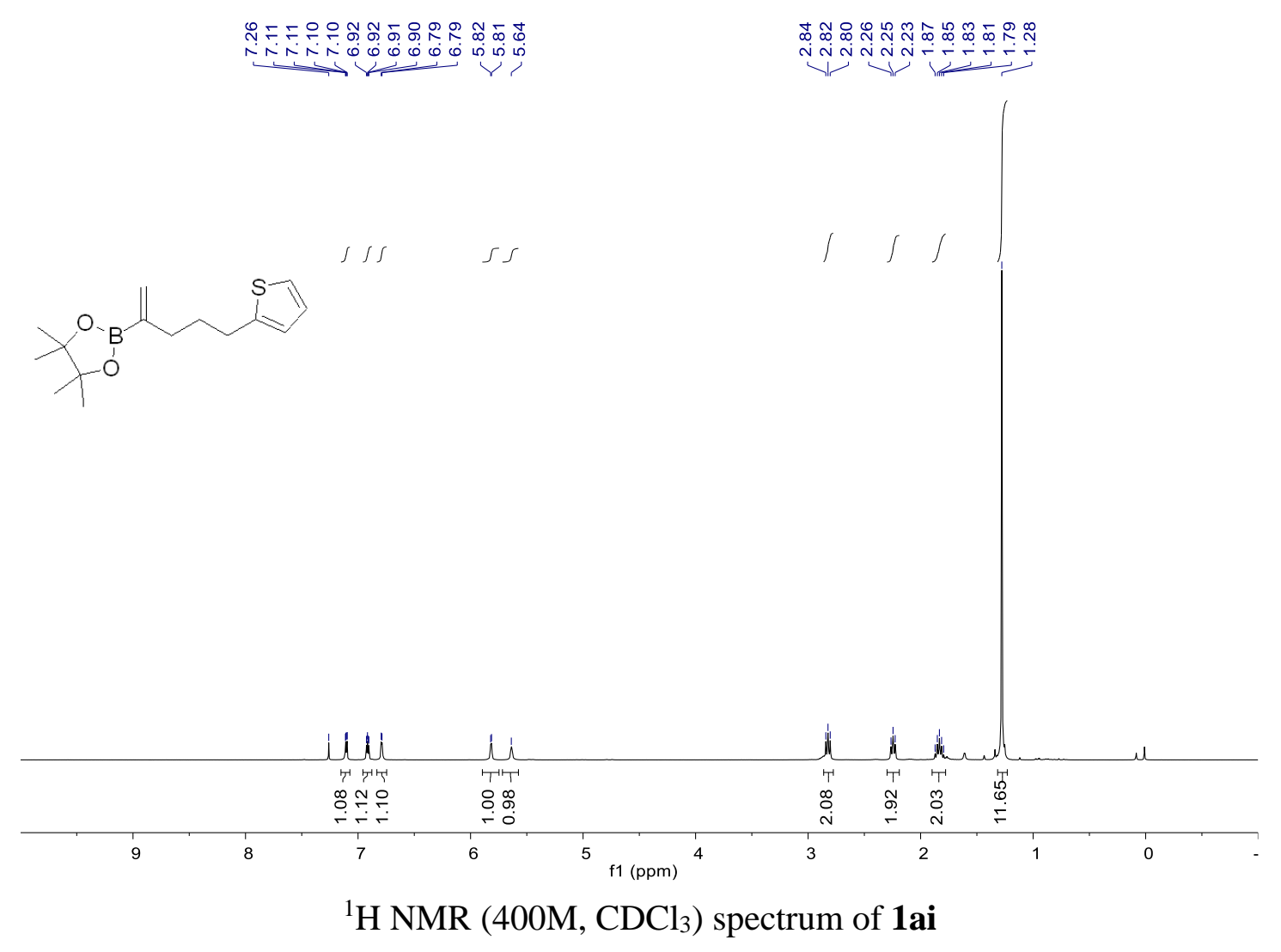



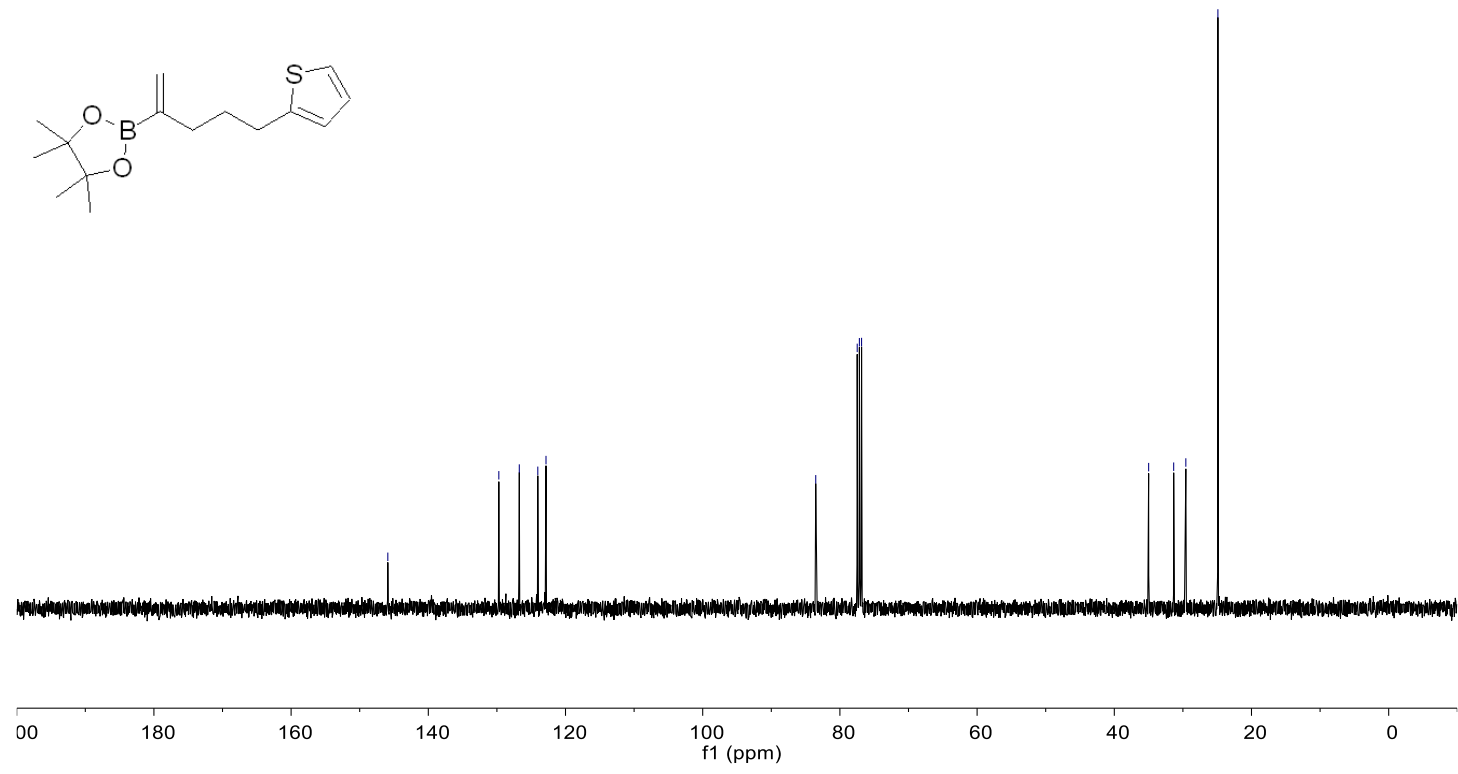

${ }^{13} \mathrm{C} \mathrm{NMR}\left(101 \mathrm{M}, \mathrm{CDCl}_{3}\right)$ spectrum of 1ai
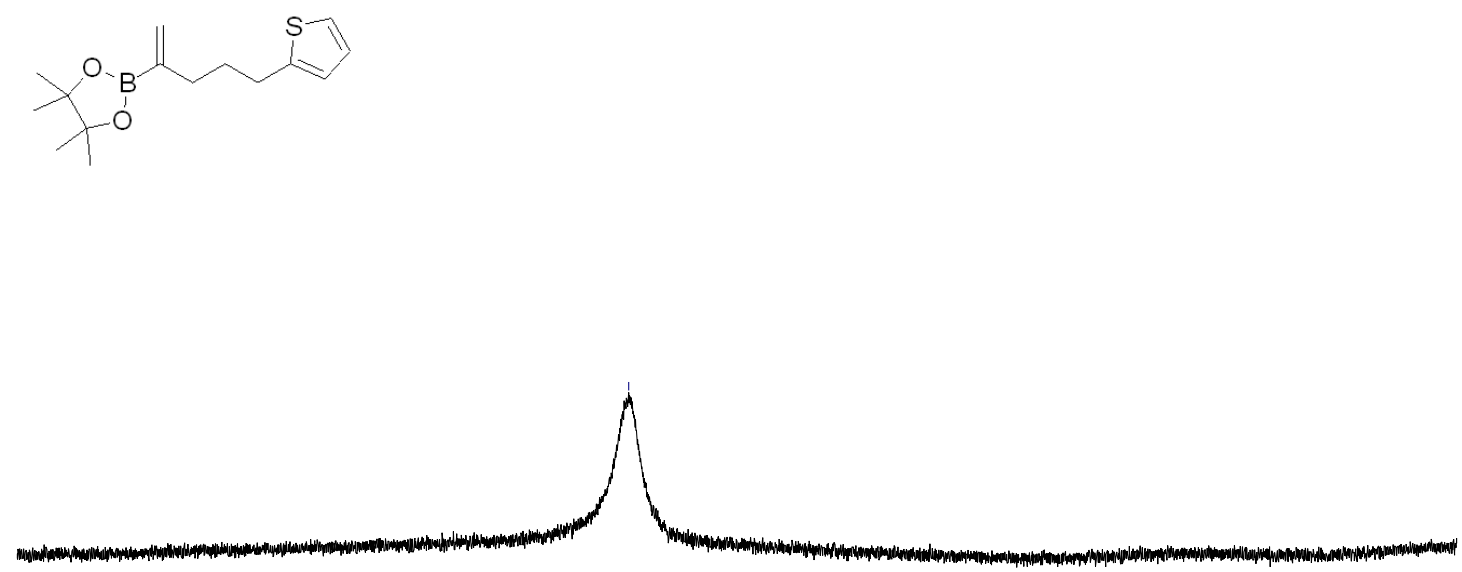

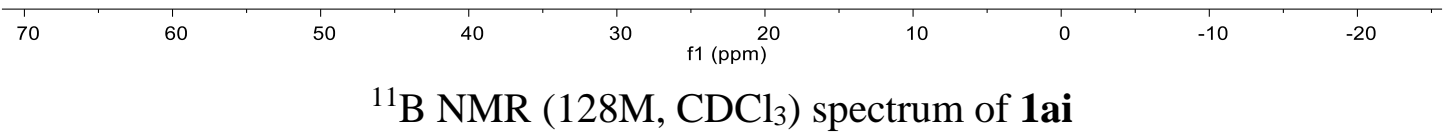




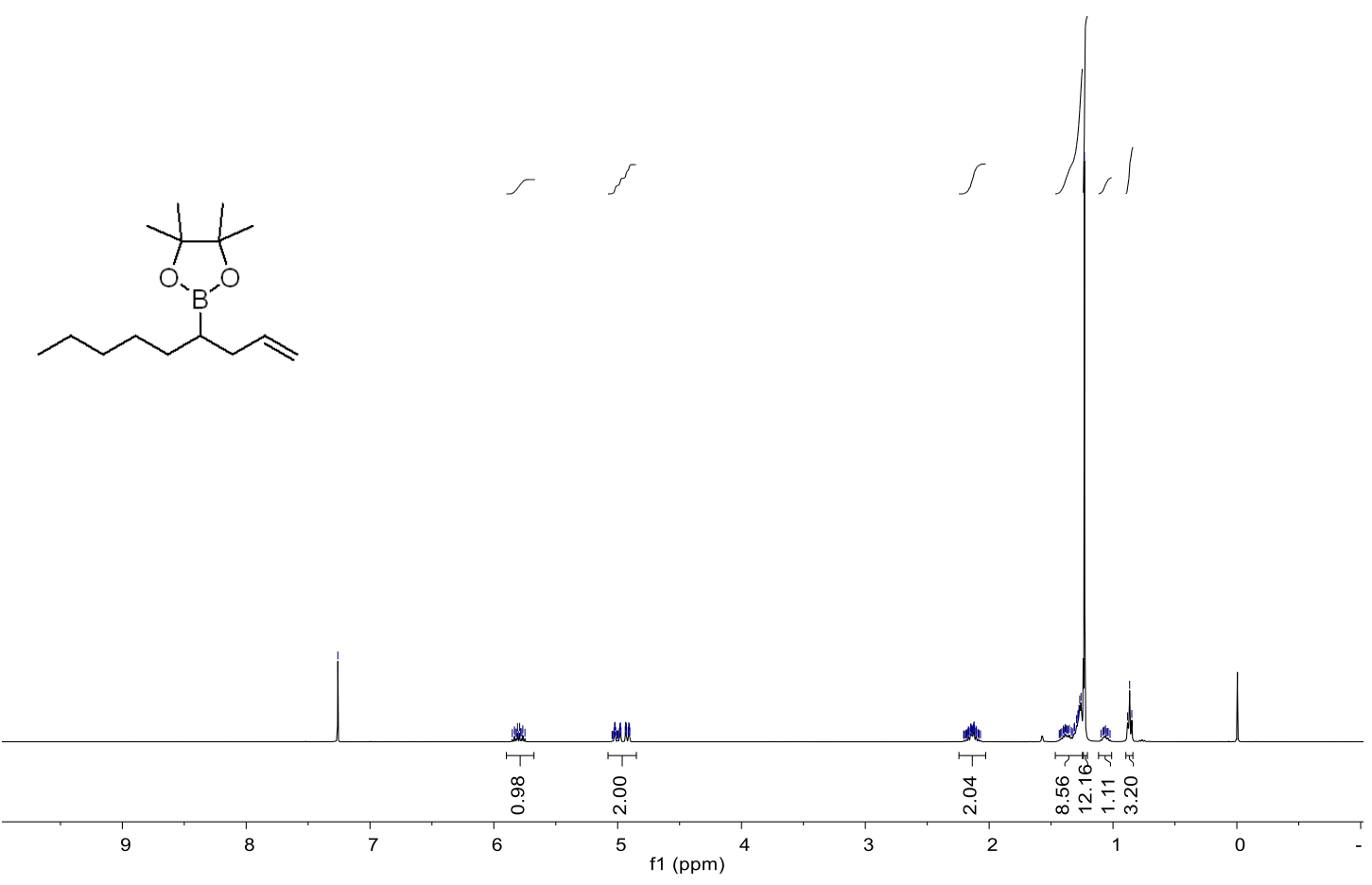

${ }^{1} \mathrm{H}$ NMR (400M, $\left.\mathrm{CDCl}_{3}\right)$ spectrum of 1aj

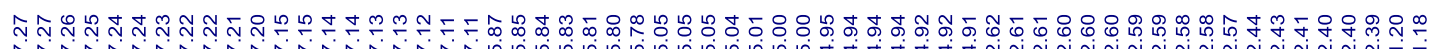

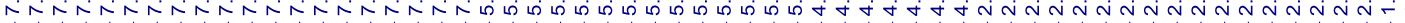
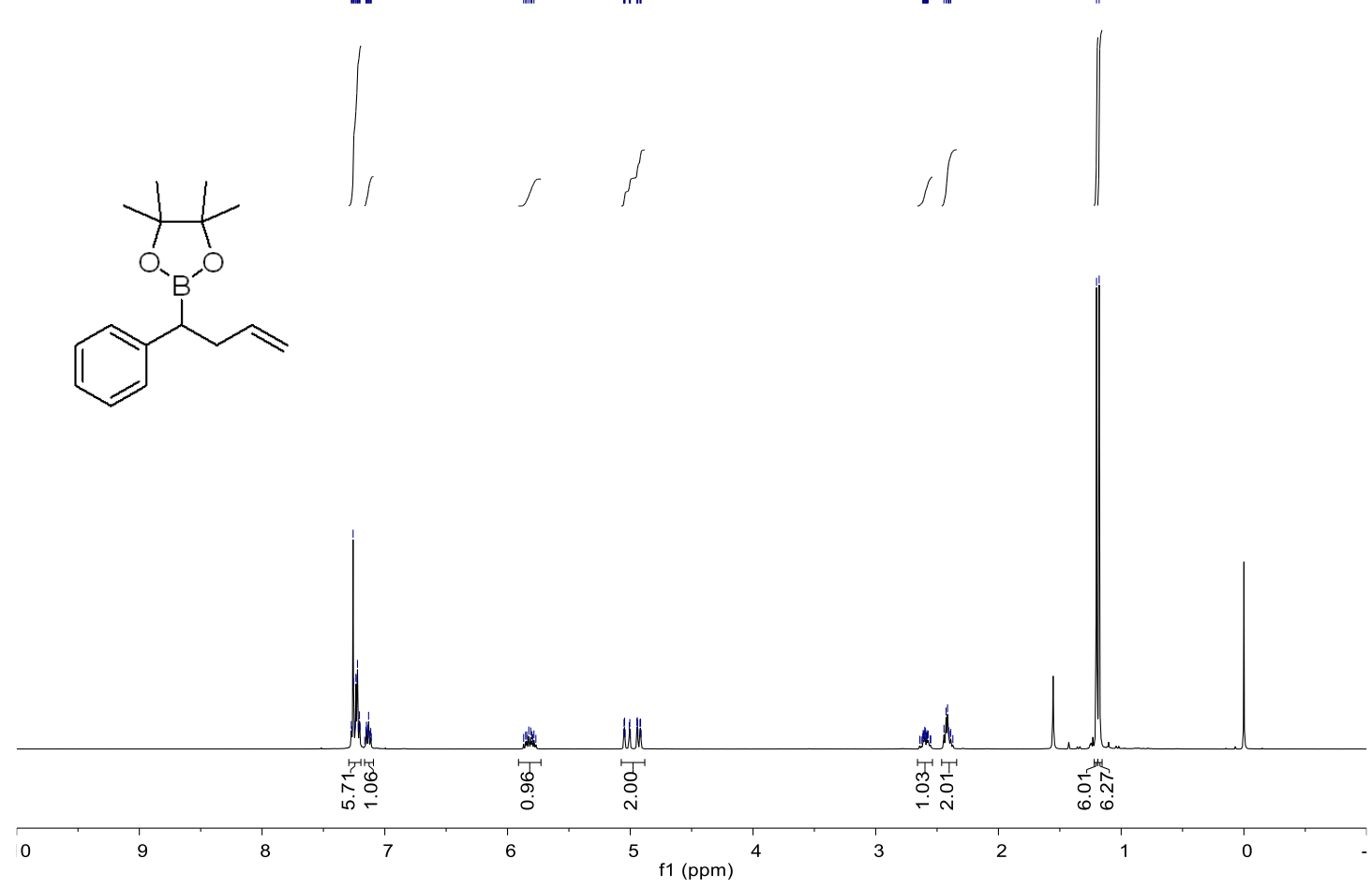

${ }^{1} \mathrm{H}$ NMR $\left(400 \mathrm{M}, \mathrm{CDCl}_{3}\right)$ spectrum of $\mathbf{1 a k}$ 


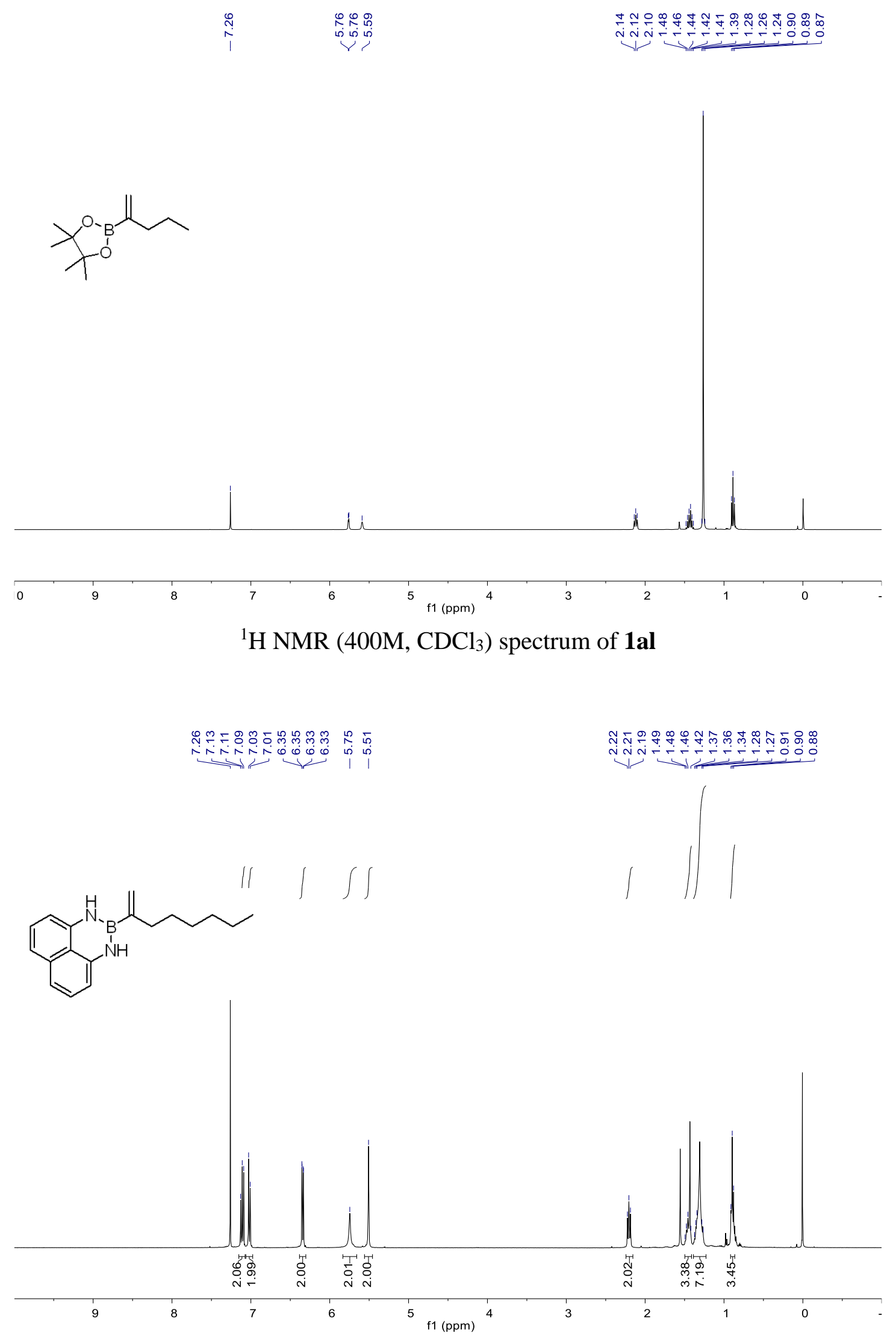

${ }^{1} \mathrm{H}$ NMR (400M, $\mathrm{CDCl}_{3}$ ) spectrum of $\mathbf{1 a m}$ 


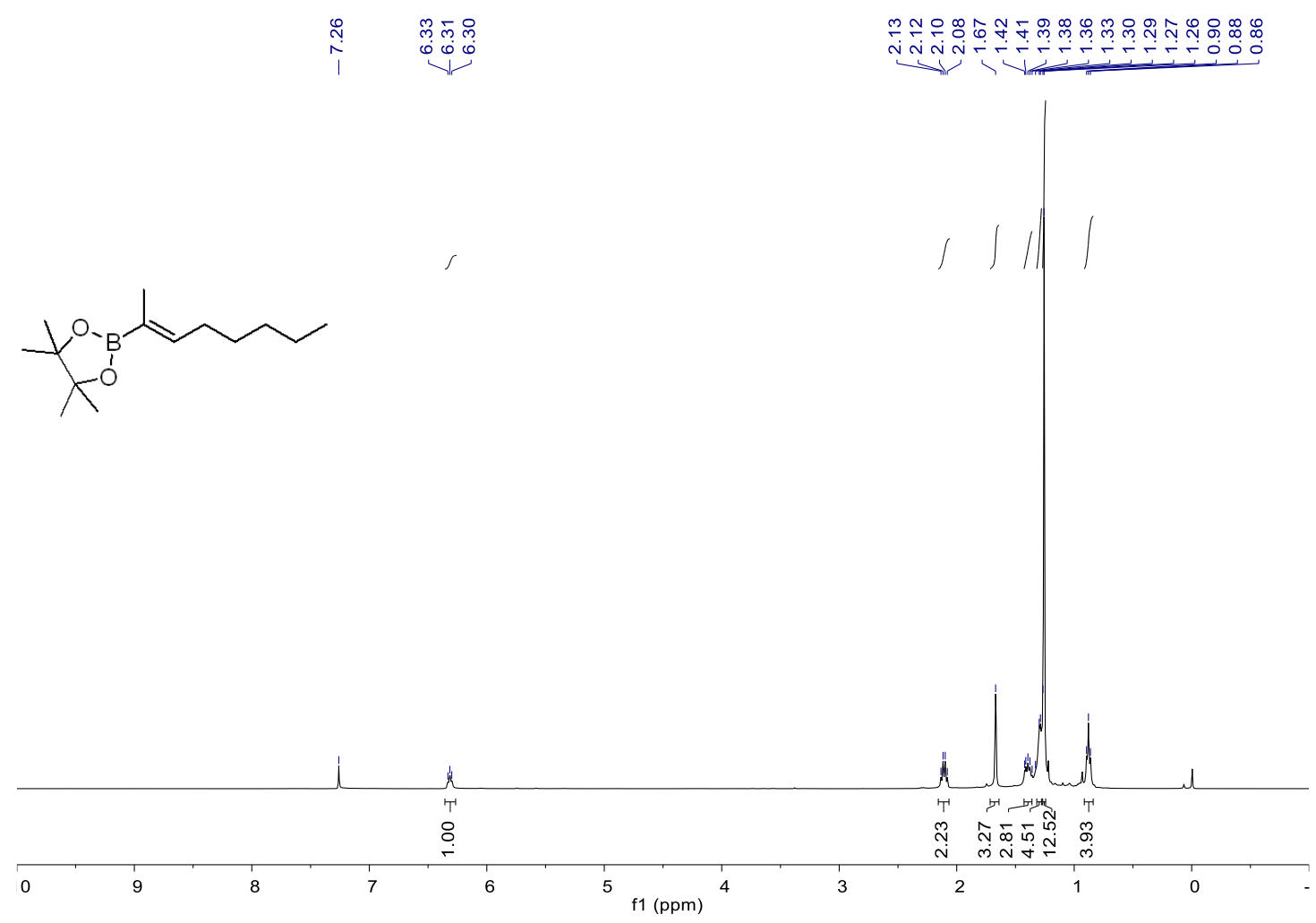

${ }^{1} \mathrm{H} \mathrm{NMR}\left(400 \mathrm{M}, \mathrm{CDCl}_{3}\right)$ spectrum of $\mathbf{2 a}$
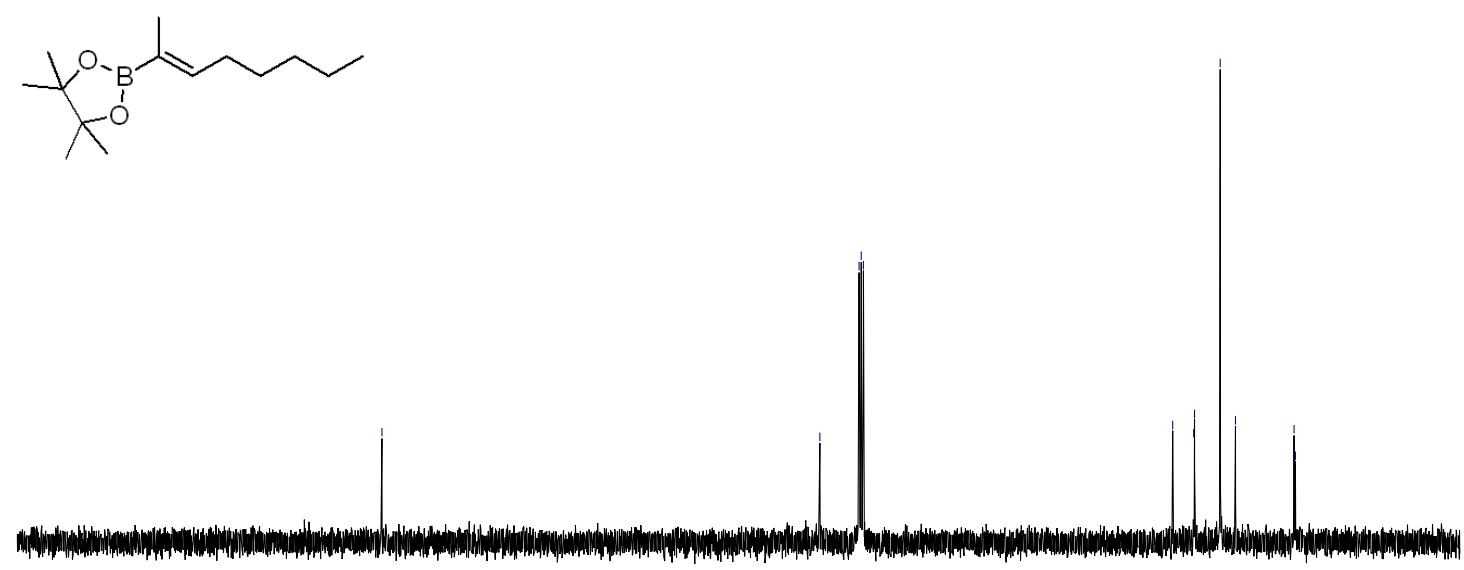

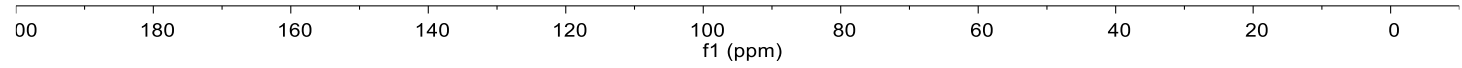

${ }^{13} \mathrm{C}$ NMR $\left(101 \mathrm{M}, \mathrm{CDCl}_{3}\right)$ spectrum of $\mathbf{2 a}$ 


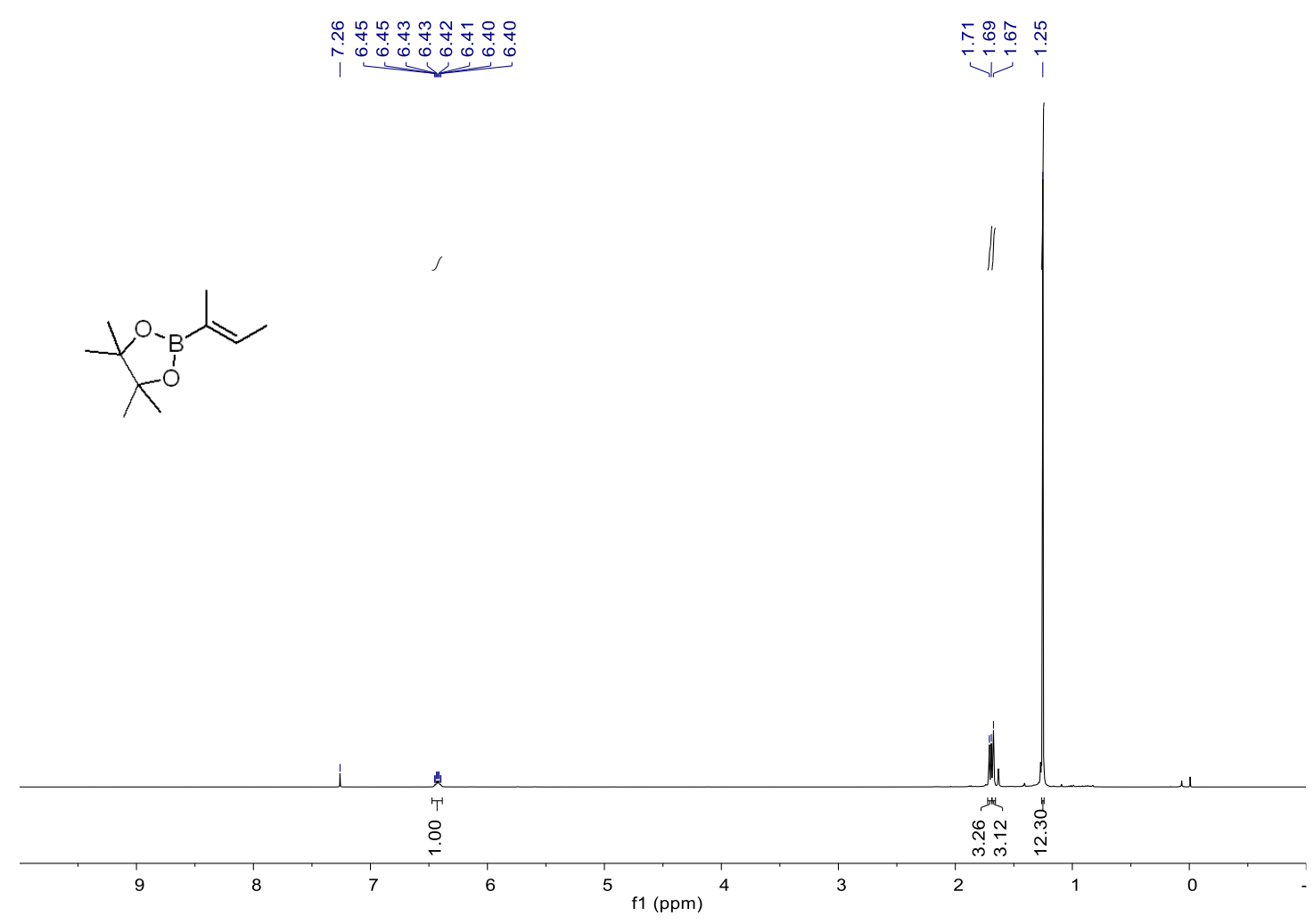

${ }^{1} \mathrm{H}$ NMR (400M, $\mathrm{CDCl}_{3}$ ) spectrum of $\mathbf{2 b}$

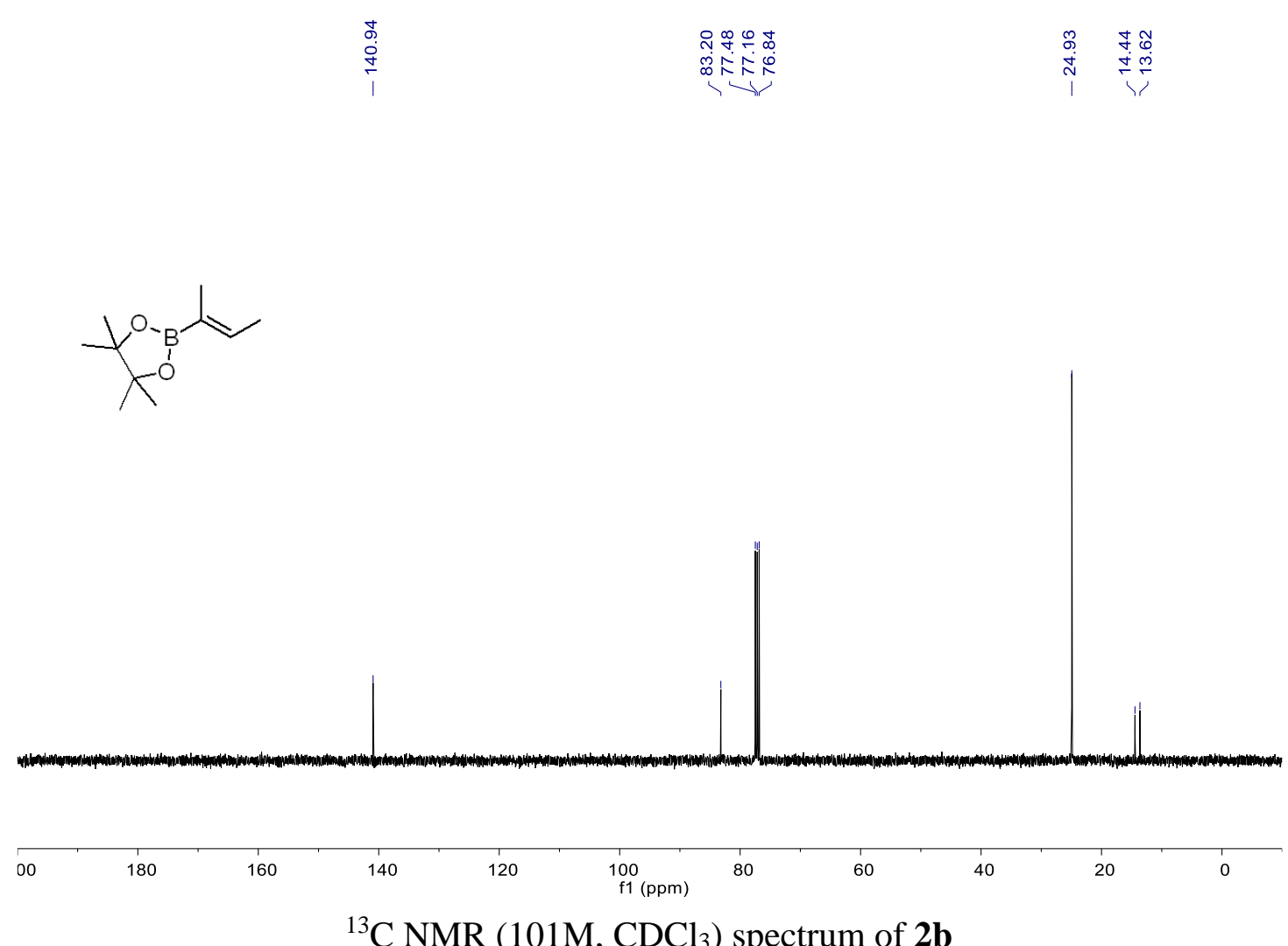

${ }^{13} \mathrm{C} \mathrm{NMR}\left(101 \mathrm{M}, \mathrm{CDCl}_{3}\right)$ spectrum of $\mathbf{2 b}$ 


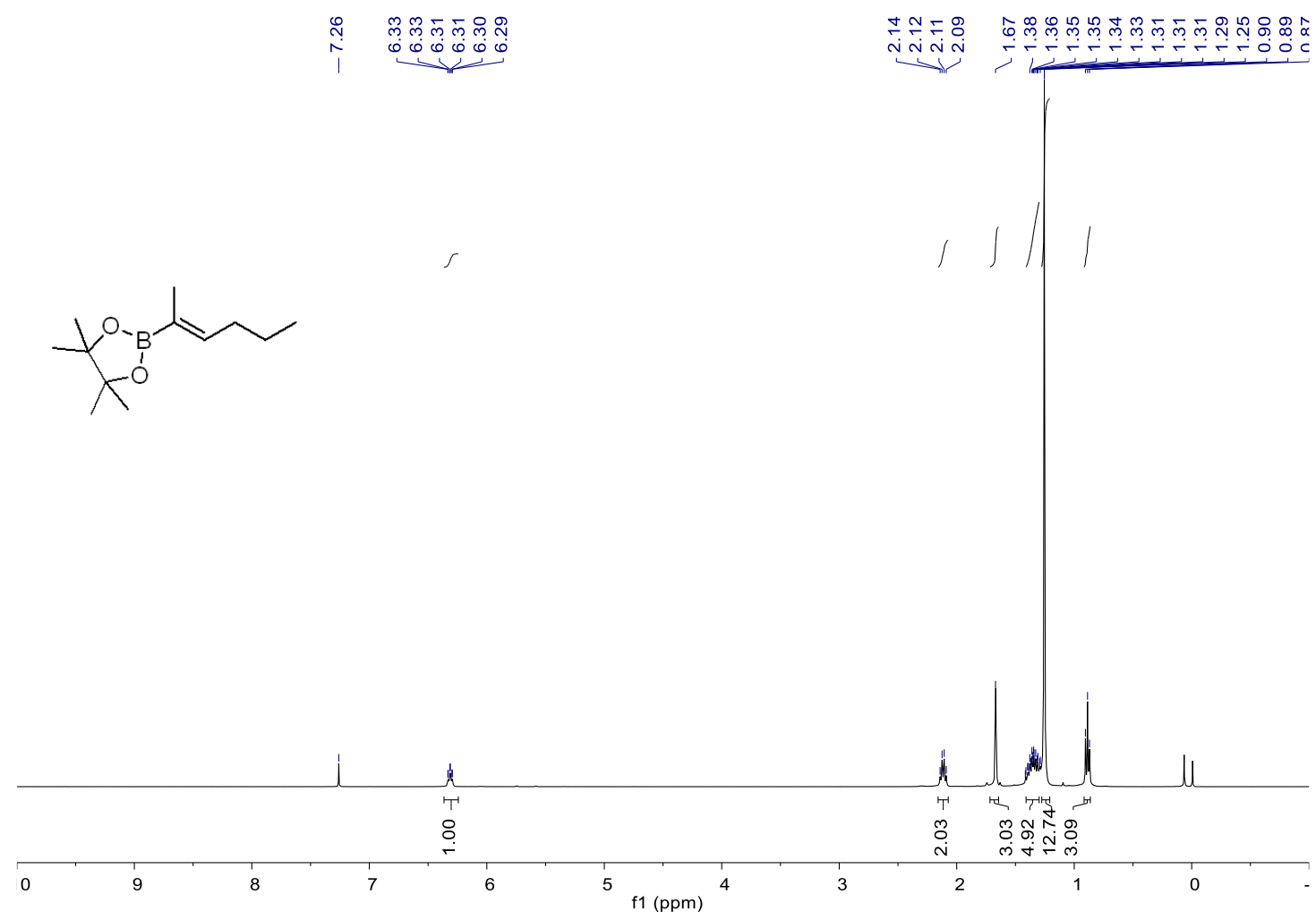

${ }^{1} \mathrm{H} \mathrm{NMR}\left(400 \mathrm{M}, \mathrm{CDCl}_{3}\right)$ spectrum of $\mathbf{2 c}$

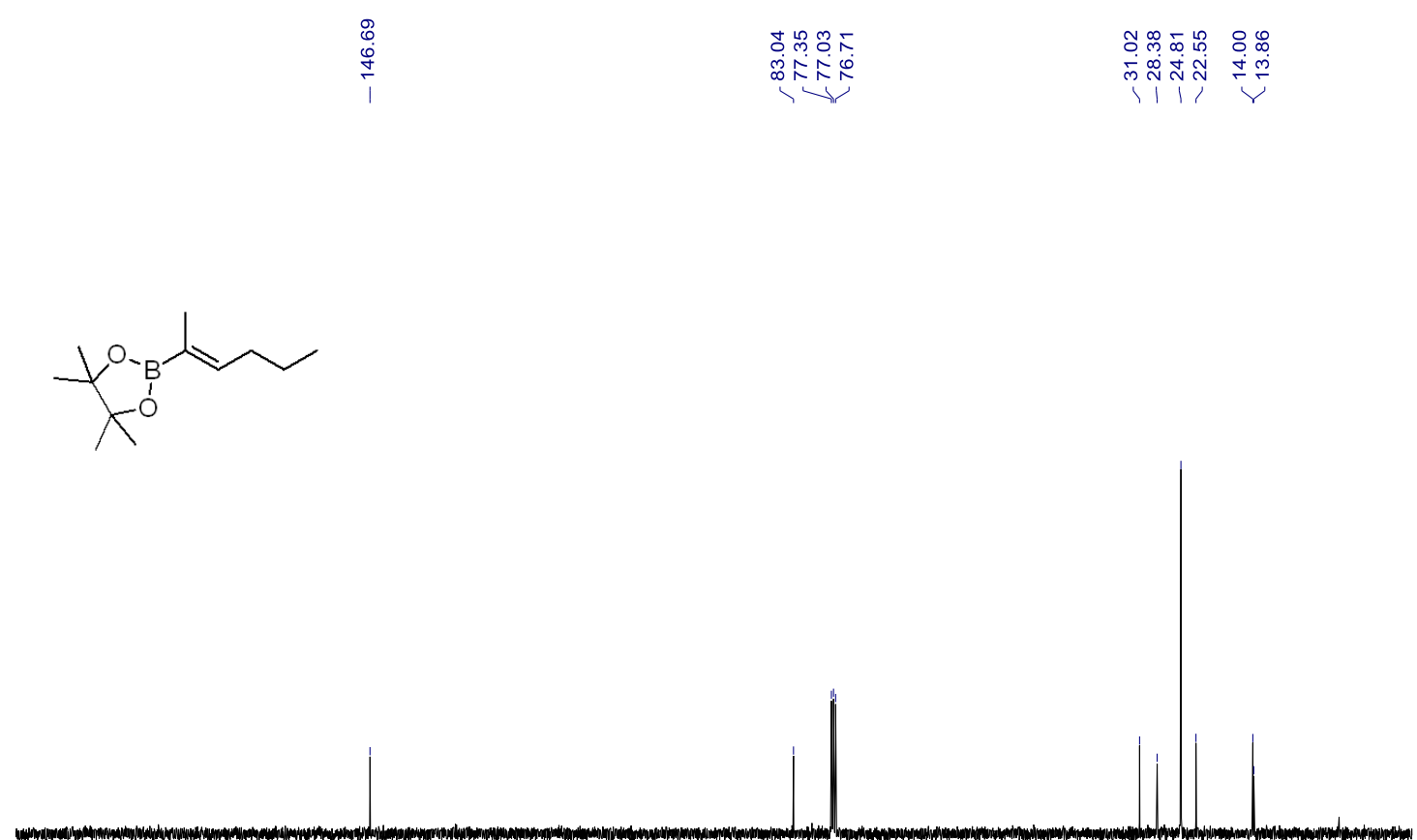

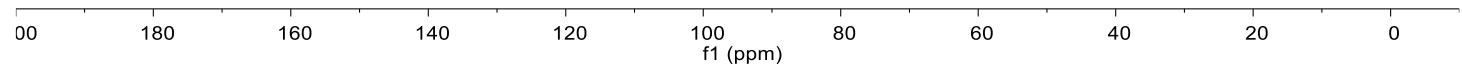

${ }^{13} \mathrm{C} \mathrm{NMR}\left(101 \mathrm{M}, \mathrm{CDCl}_{3}\right)$ spectrum of $\mathbf{2 c}$ 


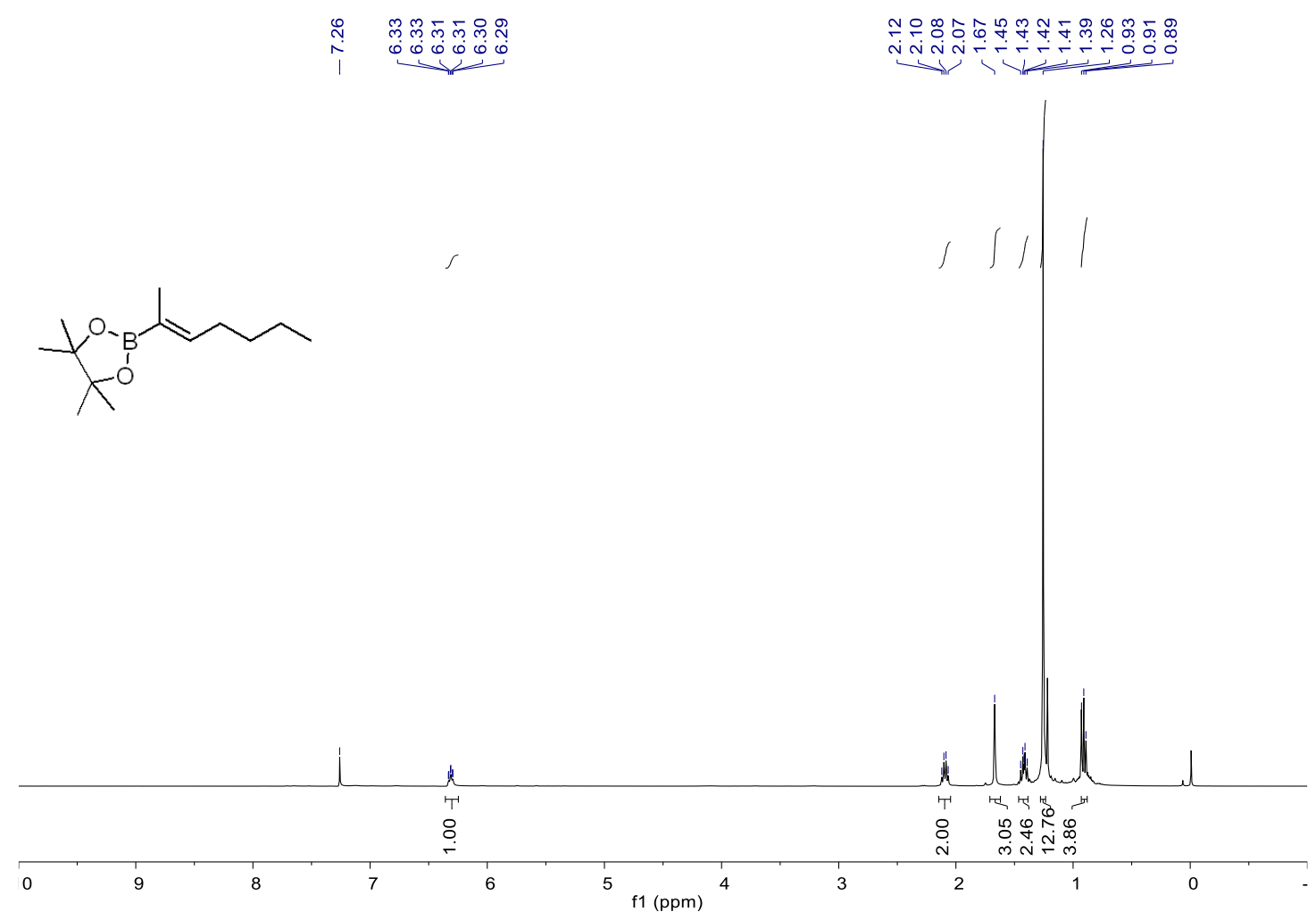

${ }^{1} \mathrm{H}$ NMR (400M, $\left.\mathrm{CDCl}_{3}\right)$ spectrum of $\mathbf{2 d}$
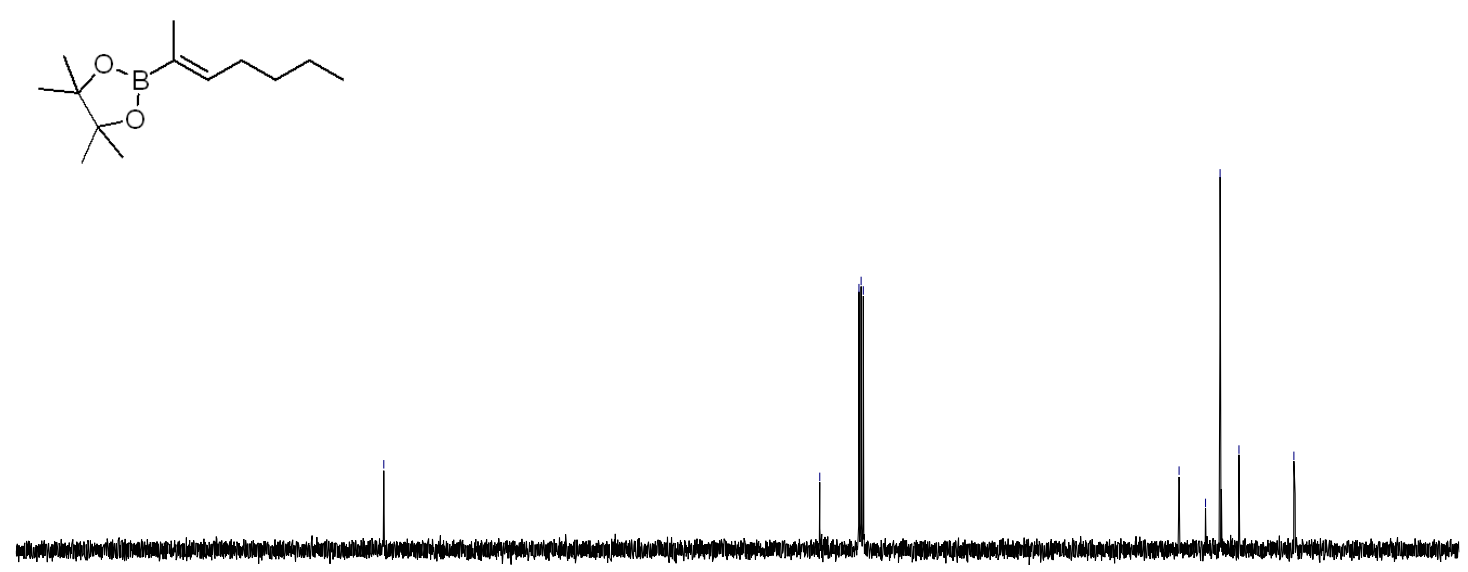

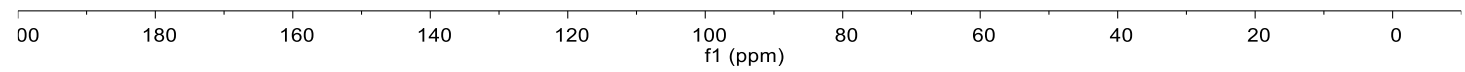

${ }^{13} \mathrm{C} \mathrm{NMR}\left(101 \mathrm{M}, \mathrm{CDCl}_{3}\right)$ spectrum of $\mathbf{2 d}$ 


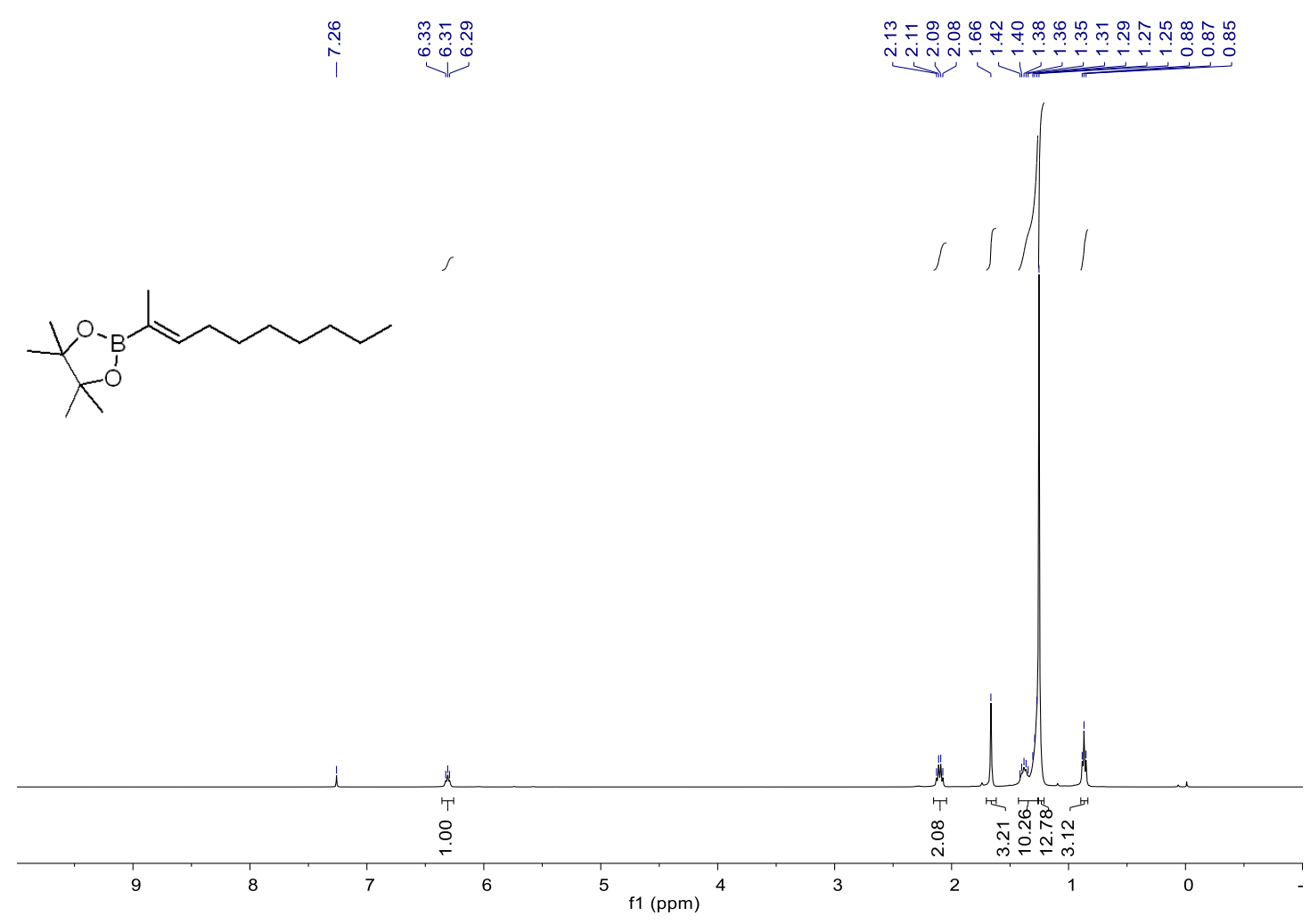

${ }^{1} \mathrm{H}$ NMR (400M, $\left.\mathrm{CDCl}_{3}\right)$ spectrum of $\mathbf{2 e}$

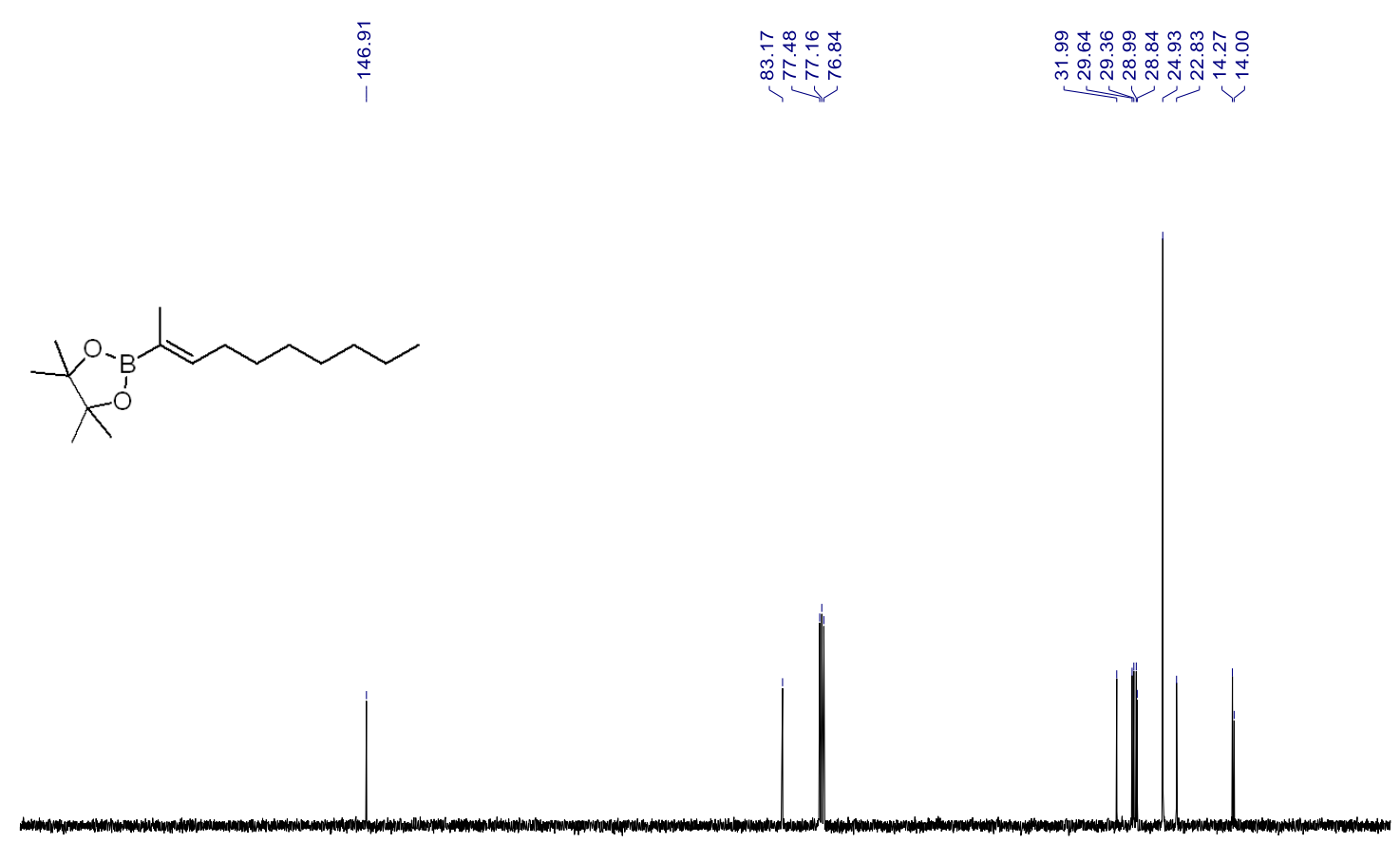

\begin{tabular}{|c|c|c|c|c|c|c|c|c|c|c|}
\hline 00 & 180 & 160 & 140 & 120 & $\begin{array}{l}100 \\
\mathrm{f} 1(\mathrm{ppm})\end{array}$ & 80 & 60 & 40 & 20 & 0 \\
\hline
\end{tabular}

${ }^{13} \mathrm{C} \mathrm{NMR}\left(101 \mathrm{M}, \mathrm{CDCl}_{3}\right)$ spectrum of $\mathbf{2 e}$ 

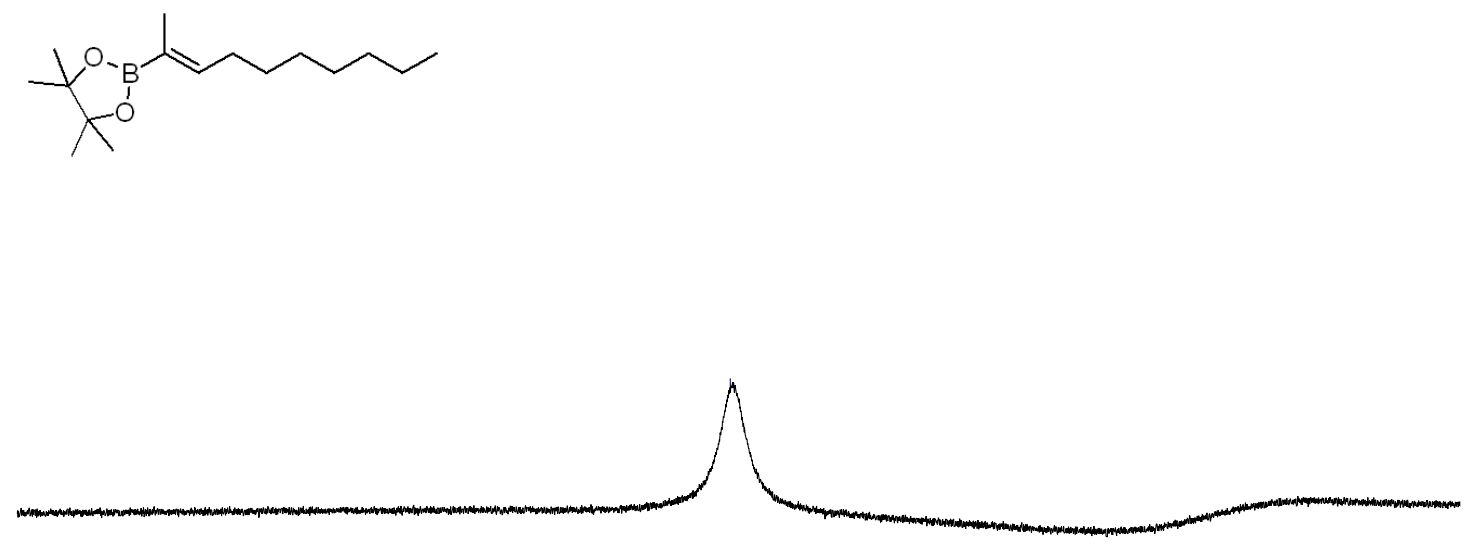

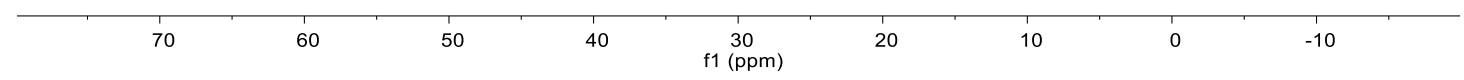

${ }^{11} \mathrm{~B}$ NMR $\left(128 \mathrm{M}, \mathrm{CDCl}_{3}\right)$ spectrum of $\mathbf{2 e}$

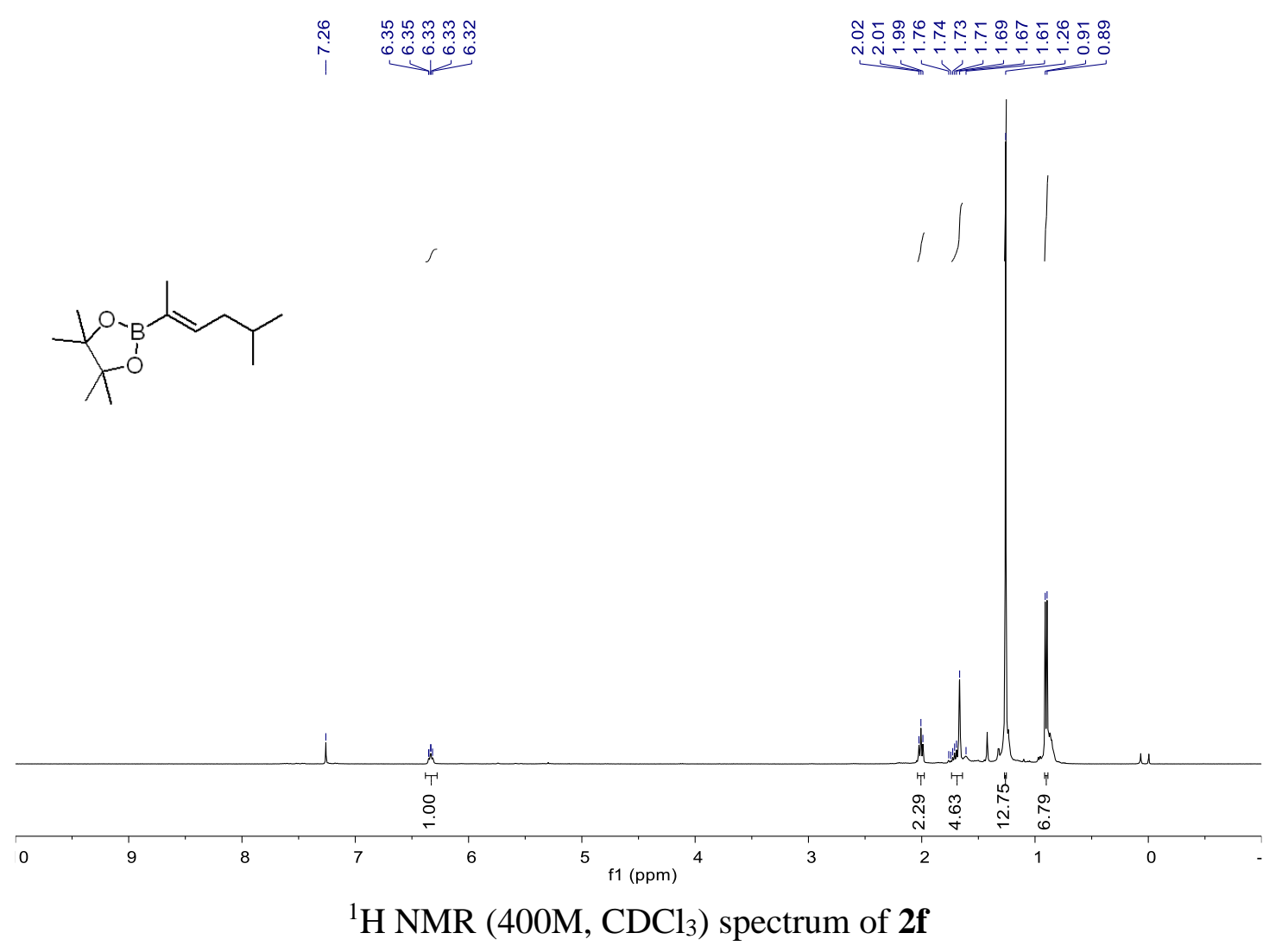



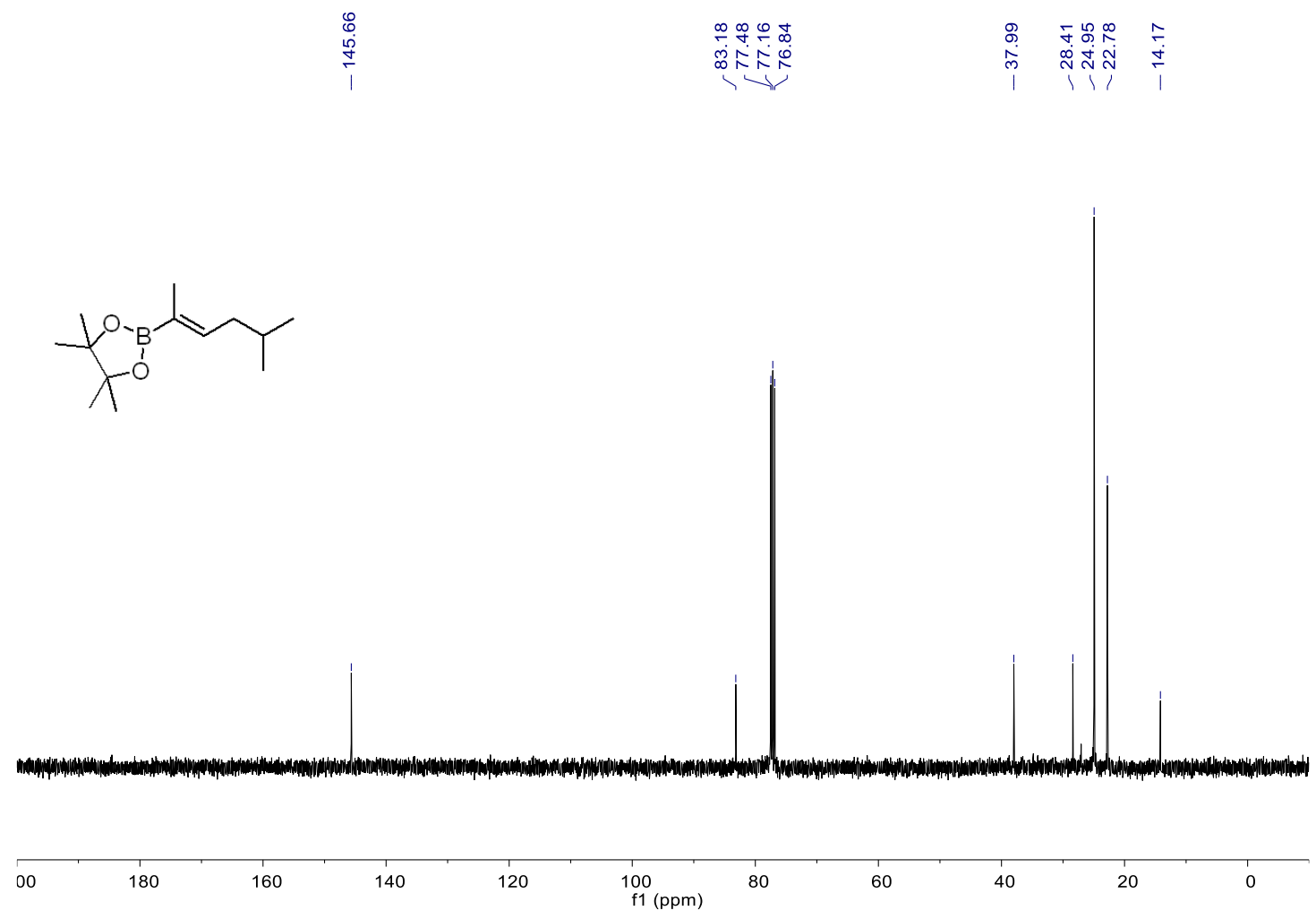

${ }^{13} \mathrm{C} \mathrm{NMR}\left(101 \mathrm{M}, \mathrm{CDCl}_{3}\right)$ spectrum of $\mathbf{2 f}$
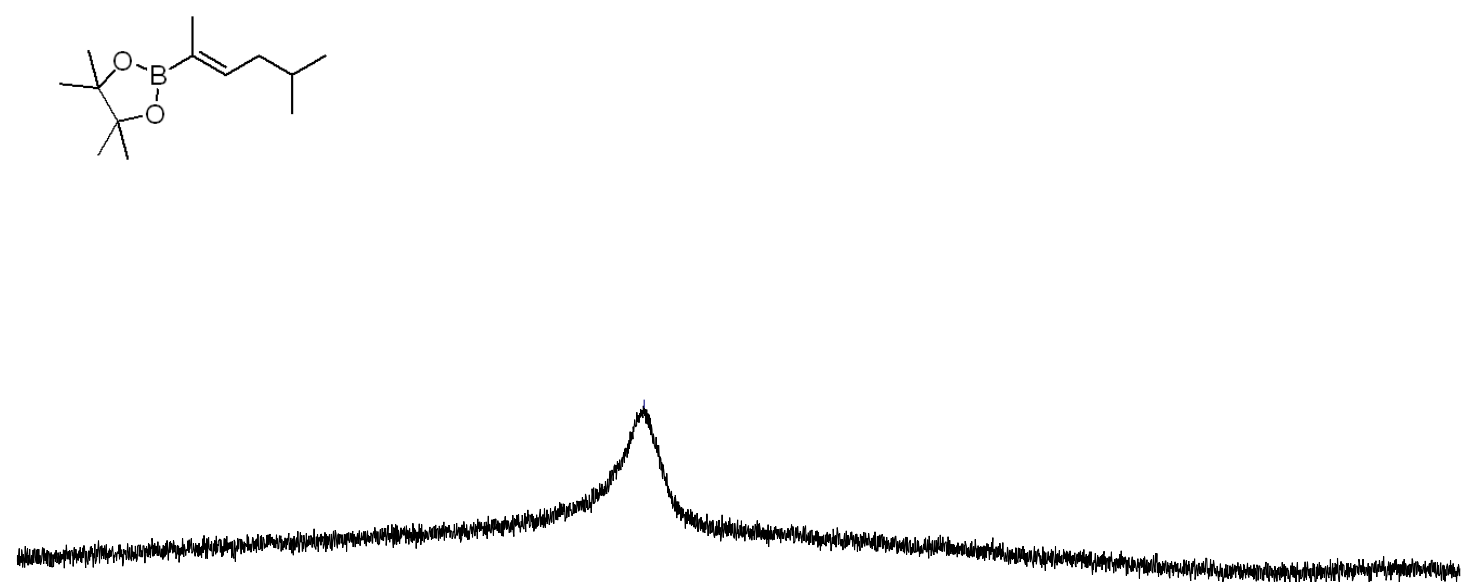

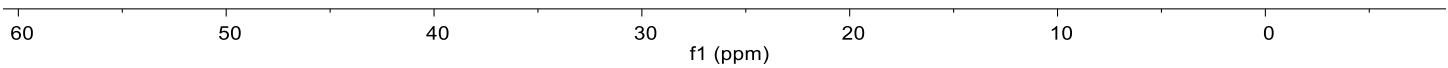

${ }^{11} \mathrm{~B}$ NMR $\left(128 \mathrm{M}, \mathrm{CDCl}_{3}\right)$ spectrum of $\mathbf{2 f}$ 


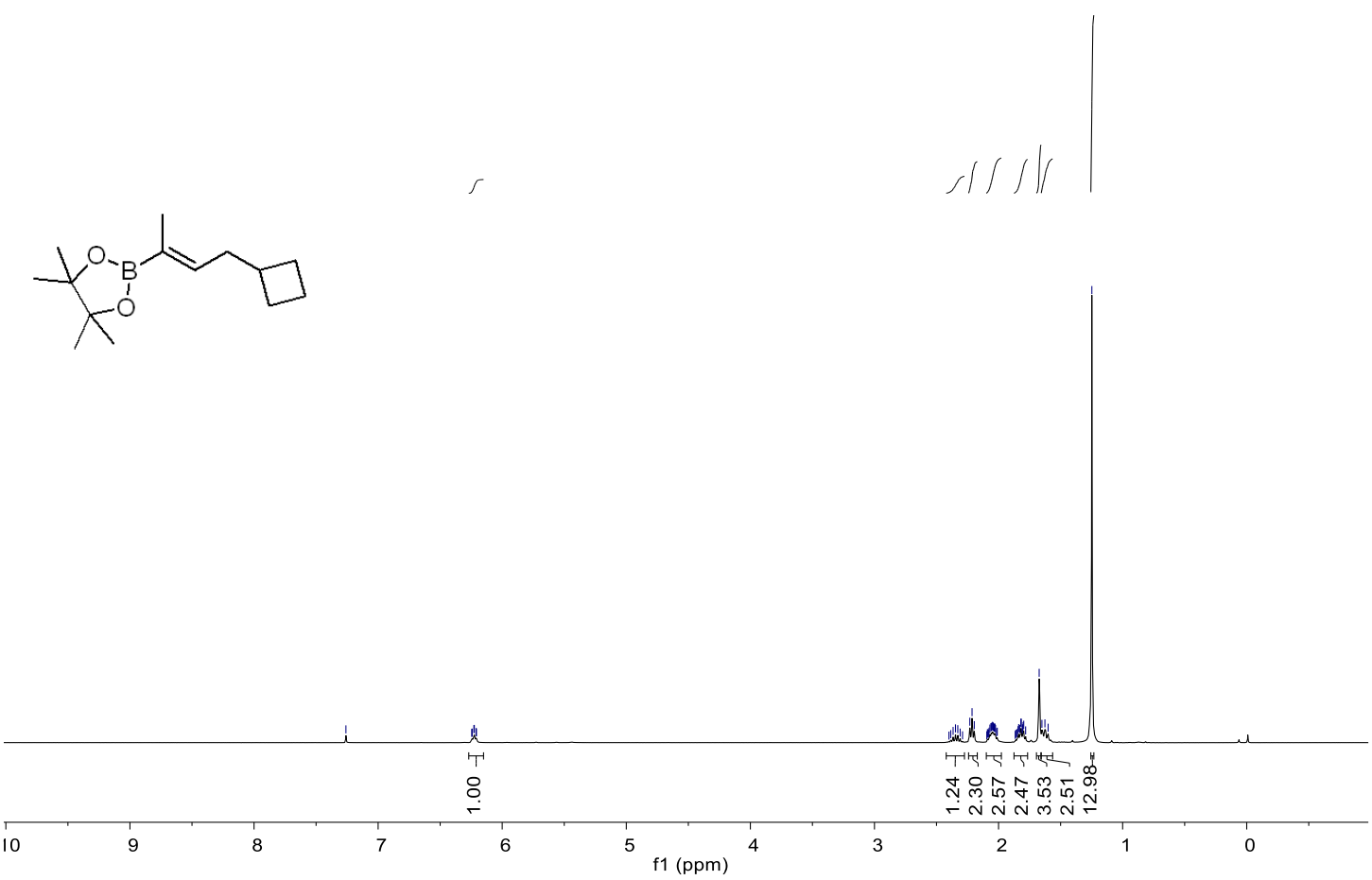

${ }^{1} \mathrm{H}$ NMR (400M, $\mathrm{CDCl}_{3}$ ) spectrum of $\mathbf{2 g}$

$\frac{j}{1}$
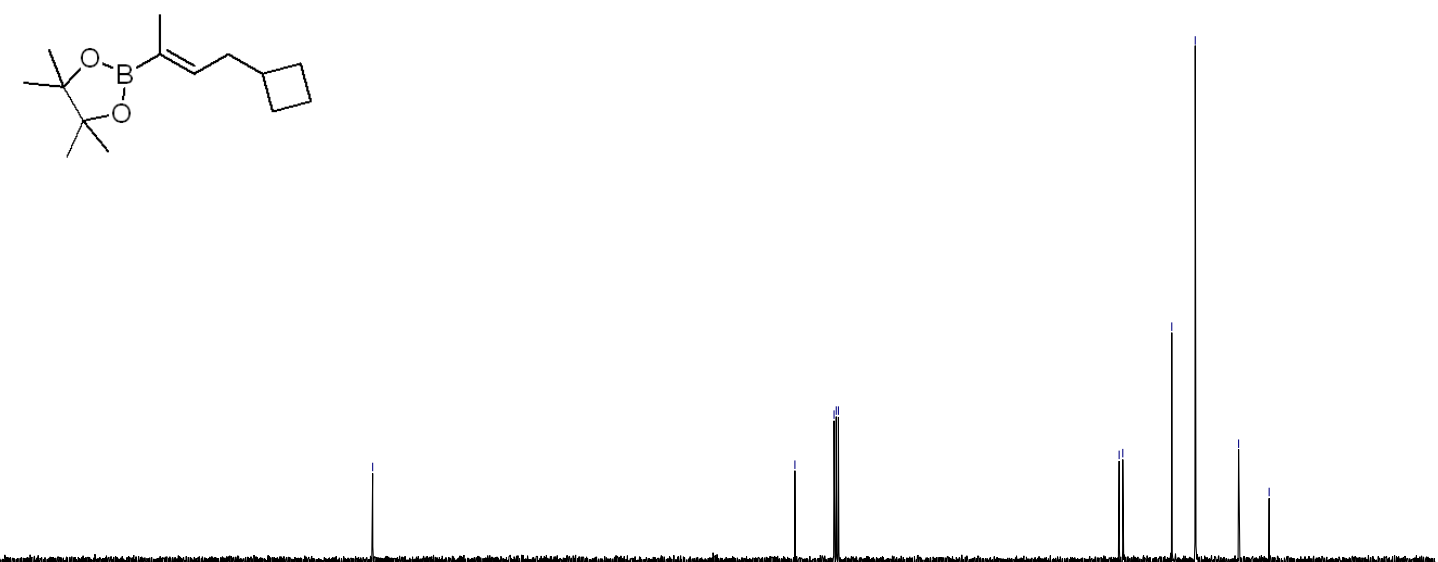
$160 \quad 140$ 120 100
$\mathrm{f} 1(\mathrm{ppm}) \quad 80$ 60 40 20 0

${ }^{13} \mathrm{C}$ NMR (101M, $\mathrm{CDCl}_{3}$ ) spectrum of $\mathbf{2 g}$ 

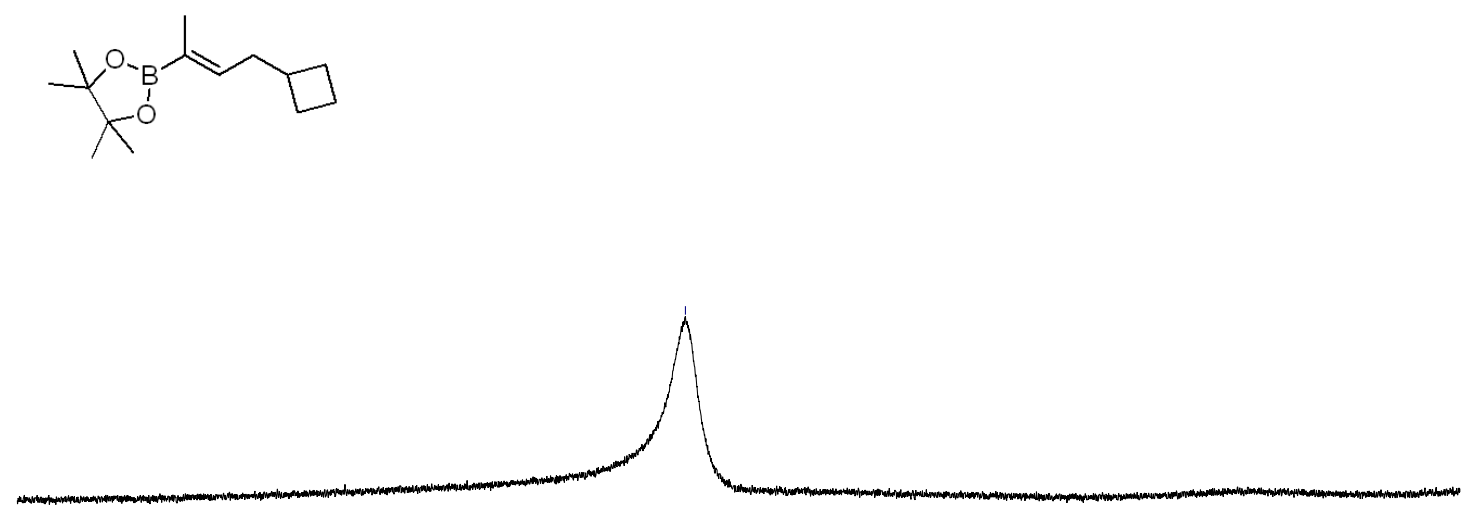

\begin{tabular}{llllllll}
\hline 1 & 1 & 1 & 1 & 1 & 1 & 1 \\
\hline
\end{tabular}

${ }^{11} \mathrm{~B}$ NMR $\left(128 \mathrm{M}, \mathrm{CDCl}_{3}\right)$ spectrum of $\mathbf{2 g}$

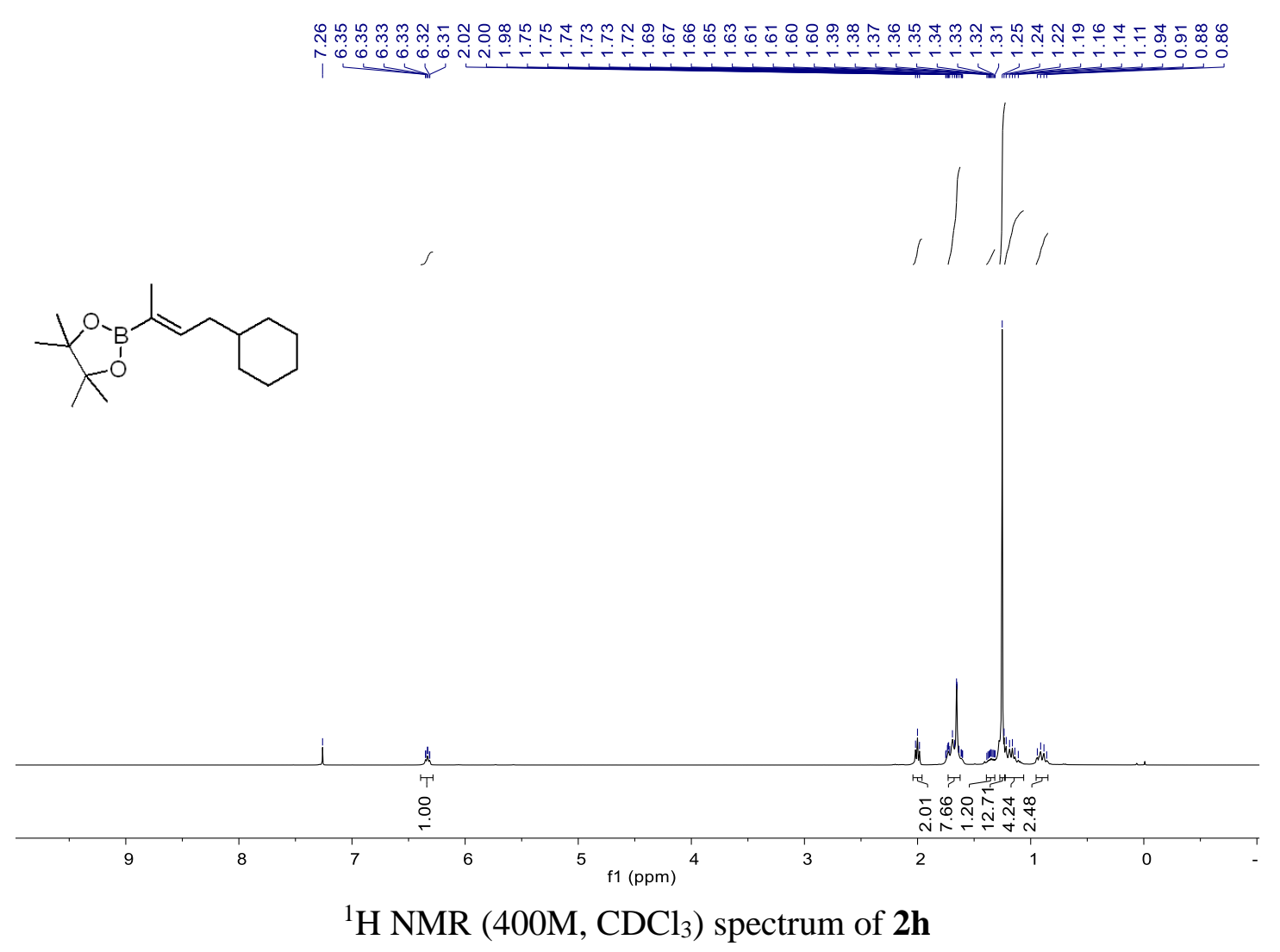



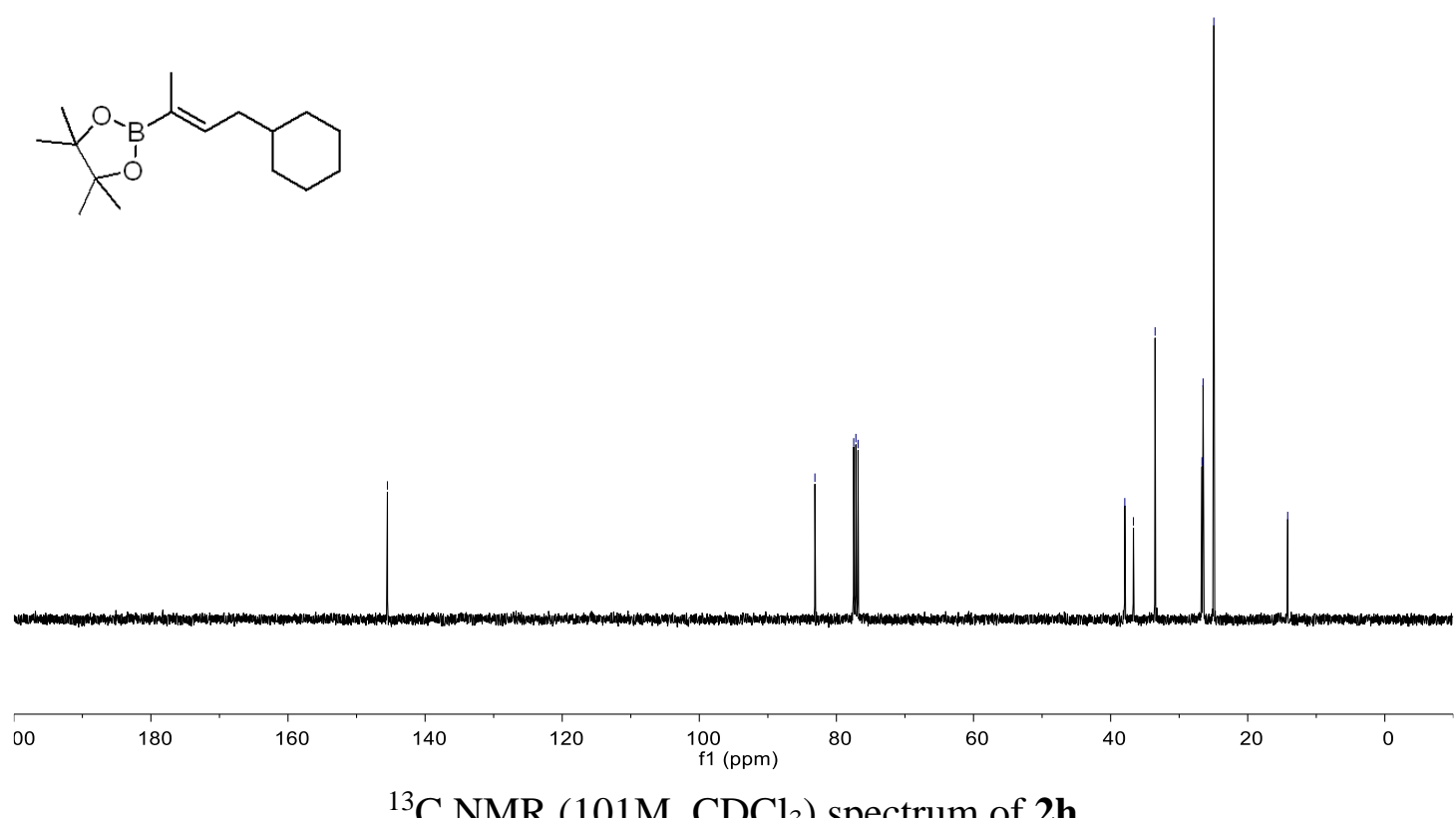

${ }^{13} \mathrm{C}$ NMR (101M, $\mathrm{CDCl}_{3}$ ) spectrum of $\mathbf{2 h}$<smiles>C/C(=C\CC1CCCCC1)OC(C)(C)C(C)(C)C</smiles>

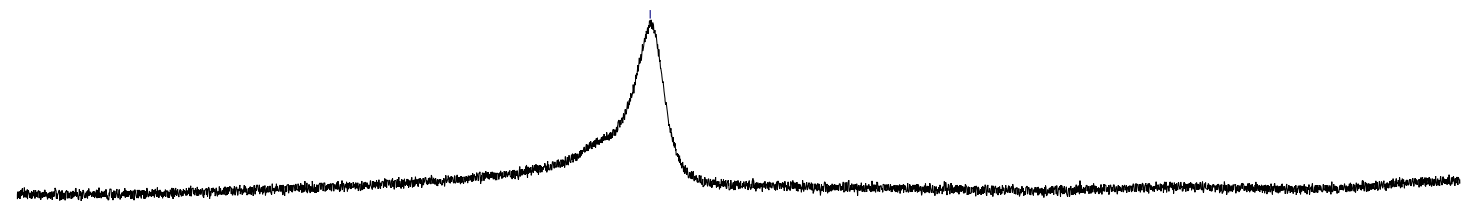

${ }^{11} \mathrm{~B}$ NMR (128M, $\mathrm{CDCl}_{3}$ ) spectrum of $\mathbf{2 h}$ 


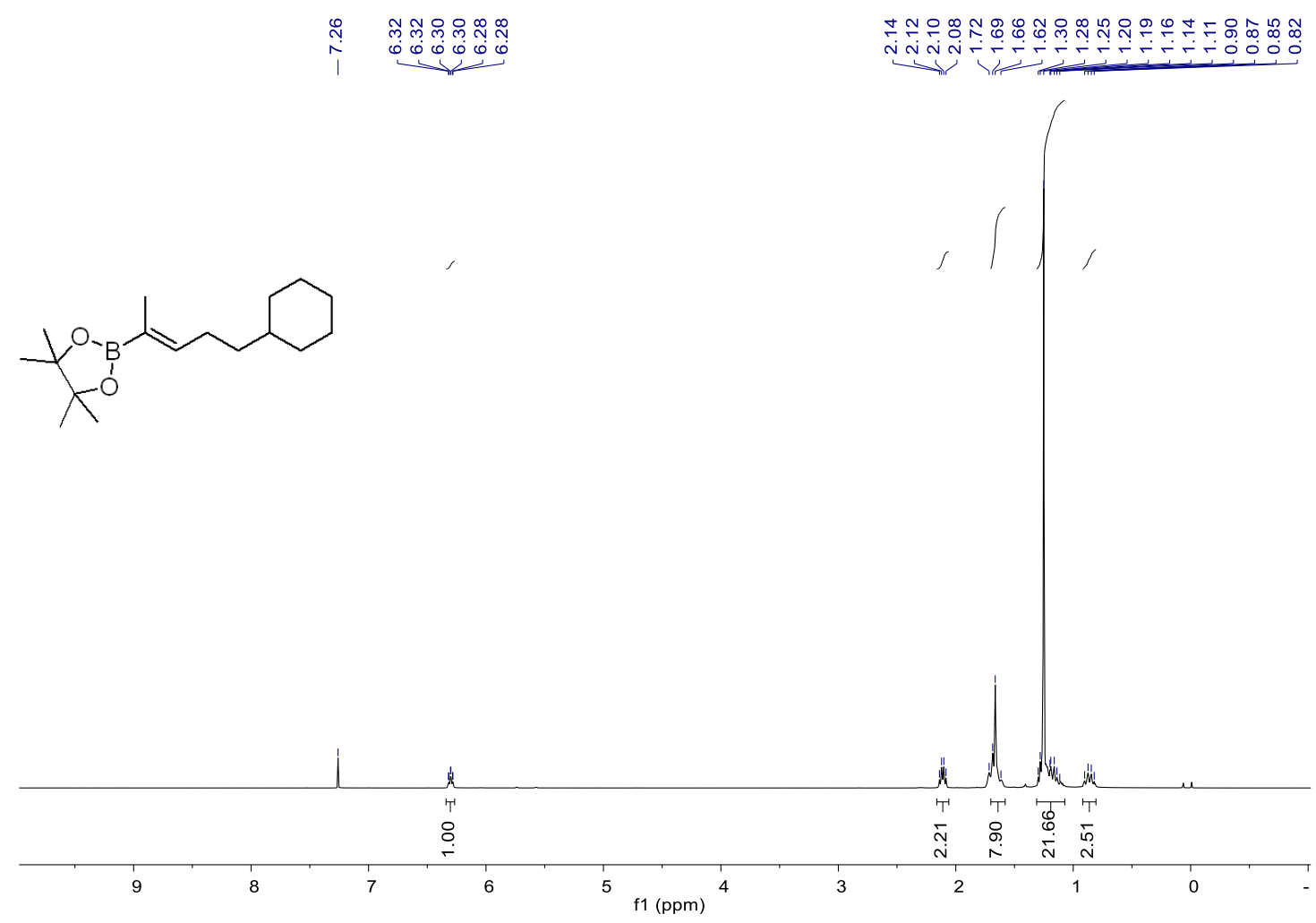

${ }^{1} \mathrm{H}$ NMR (400M, $\left.\mathrm{CDCl}_{3}\right)$ spectrum of $\mathbf{2 i}$
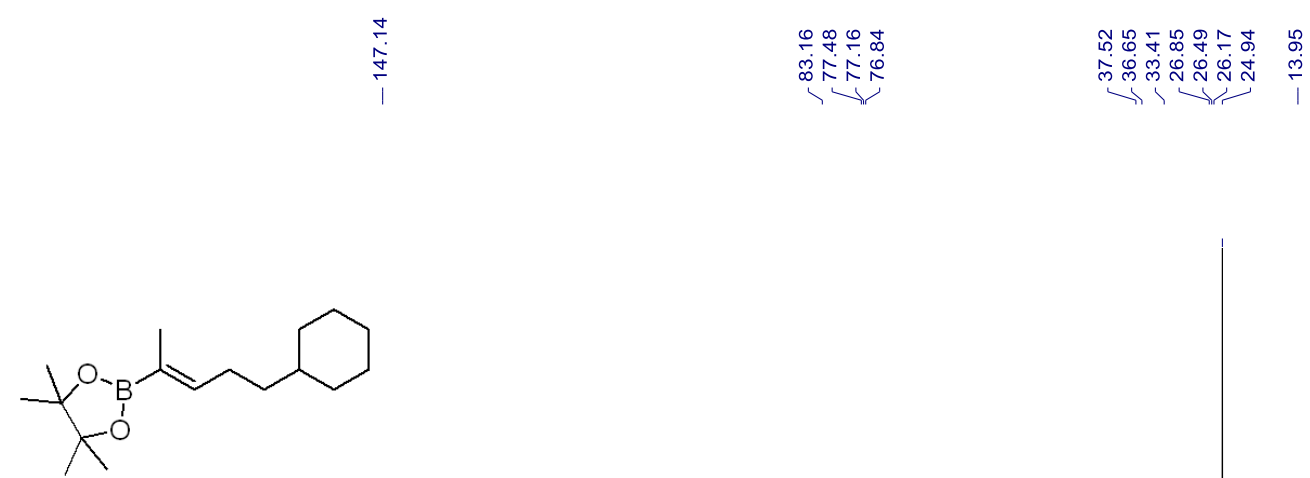

\begin{tabular}{llllcllllll}
\hline 00 & 180 & 160 & 140 & 120 & 100 & 80 & 60 & 40 & 20 & 0 \\
\hline
\end{tabular}

${ }^{13} \mathrm{C} \mathrm{NMR}\left(101 \mathrm{M}, \mathrm{CDCl}_{3}\right)$ spectrum of $\mathbf{2 i}$ 

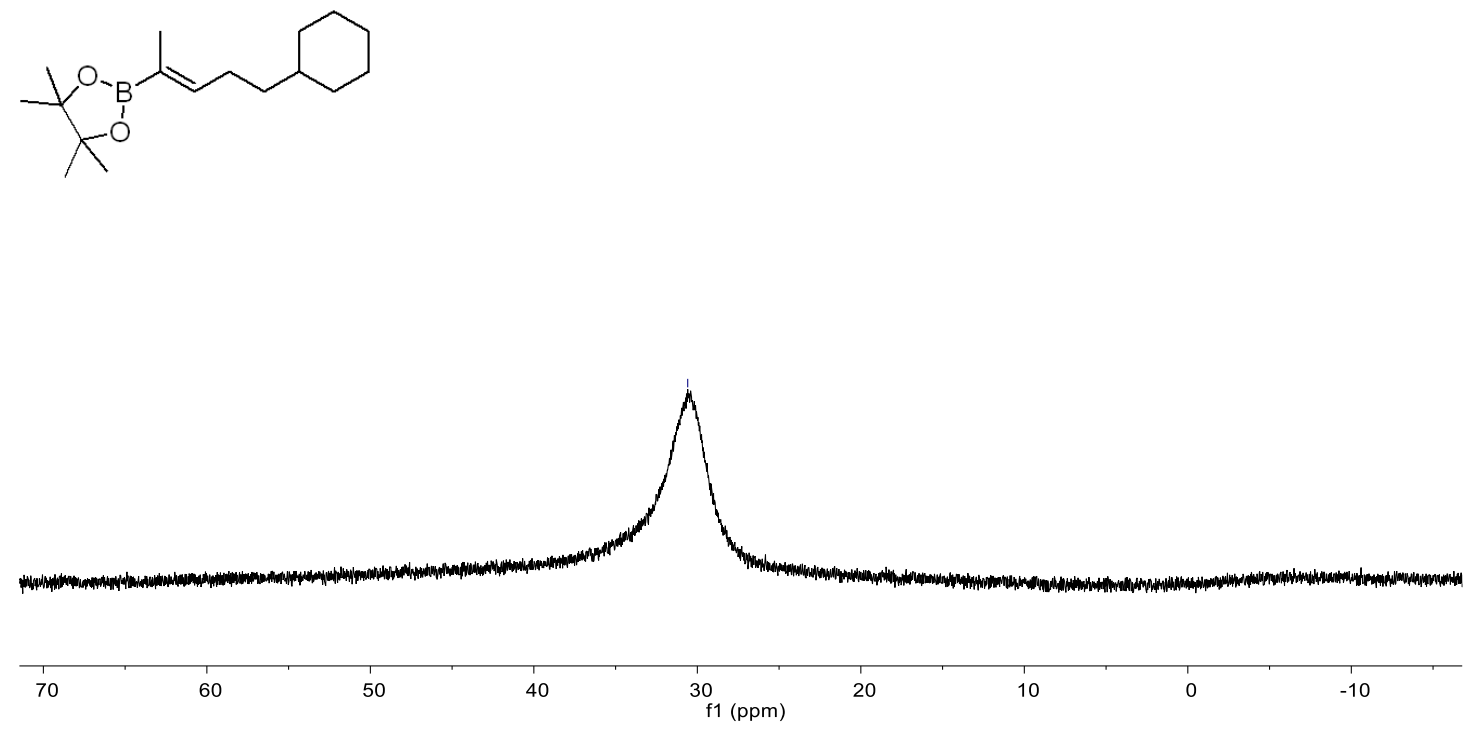

${ }^{11} \mathrm{~B}$ NMR (128M, $\left.\mathrm{CDCl}_{3}\right)$ spectrum of $\mathbf{2 i}$

过
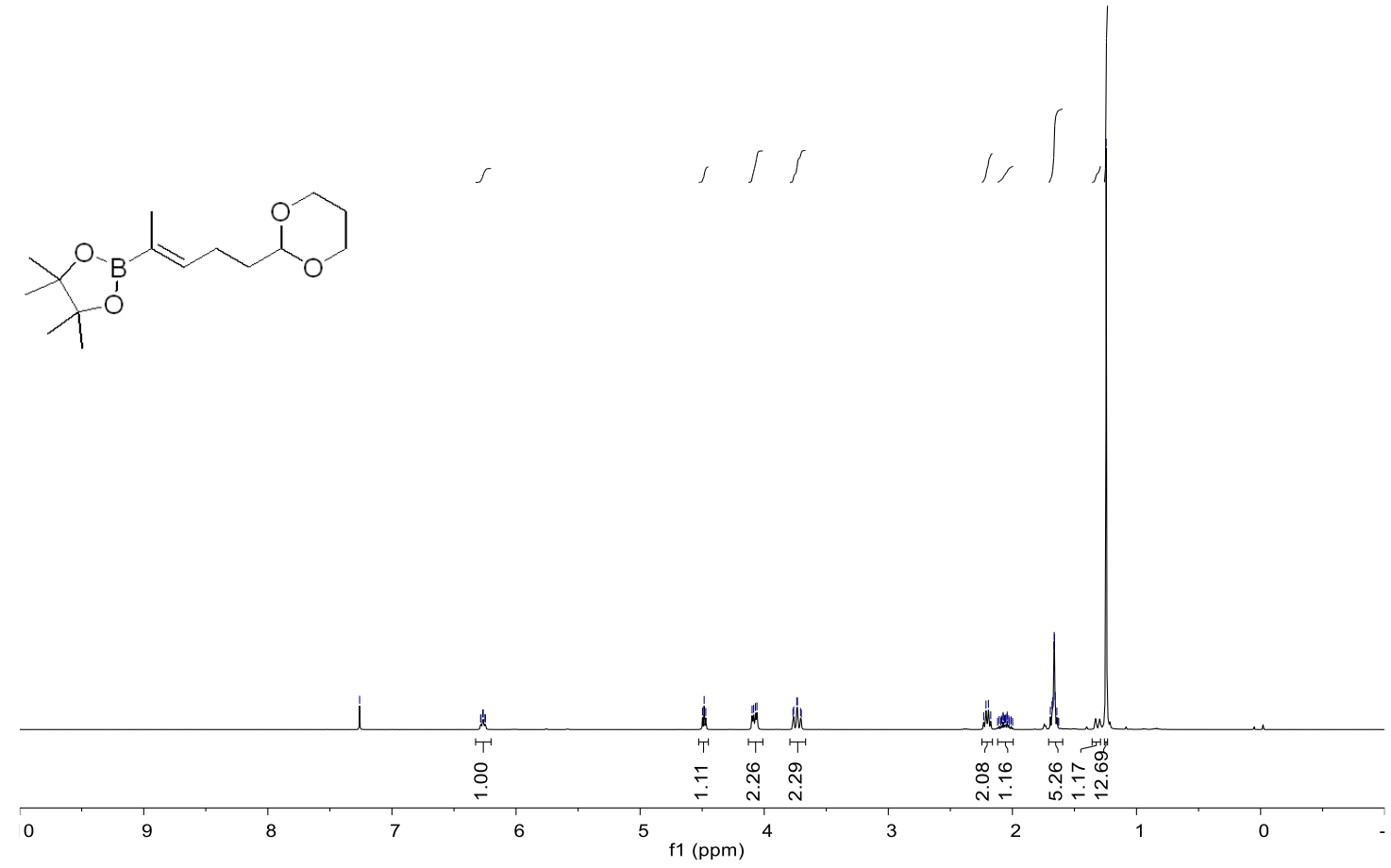

${ }^{1} \mathrm{H}$ NMR (400M, $\left.\mathrm{CDCl}_{3}\right)$ spectrum of $\mathbf{2} \mathbf{j}$ 

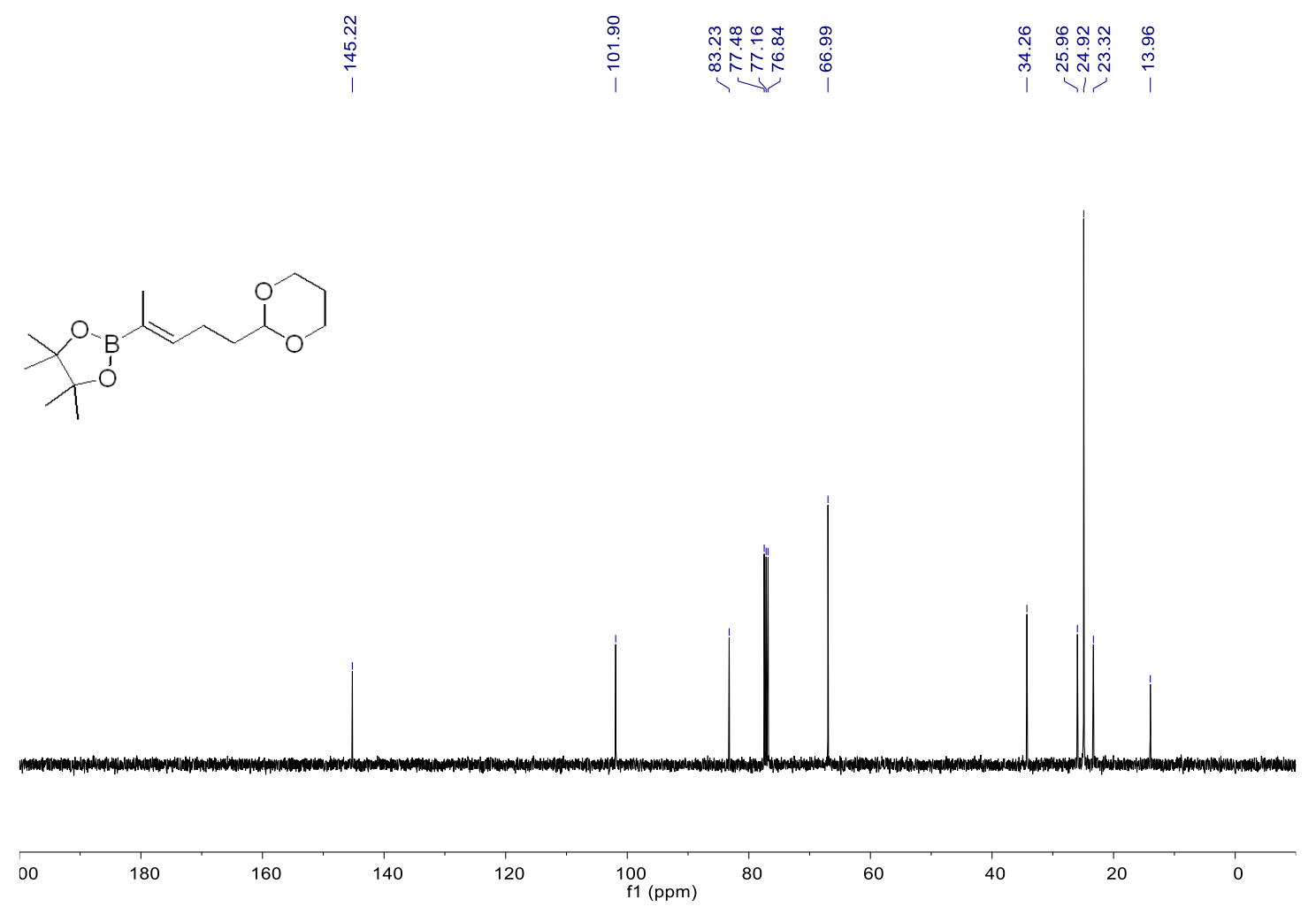

${ }^{13} \mathrm{C} \mathrm{NMR}\left(101 \mathrm{M}, \mathrm{CDCl}_{3}\right)$ spectrum of $\mathbf{2} \mathbf{j}$<smiles>C/C(=C\CCC1OCCCO1)B1OC(C)(C)C(C)(C)O1</smiles>

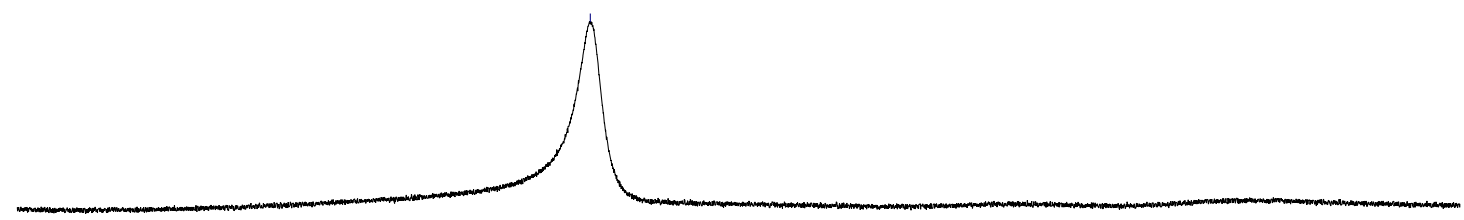

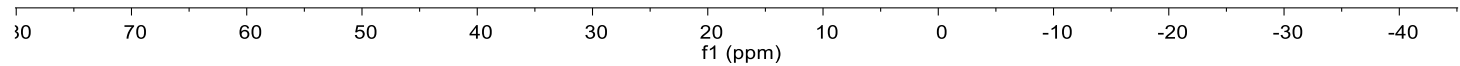

${ }^{11} \mathrm{~B}$ NMR $\left(128 \mathrm{M}, \mathrm{CDCl}_{3}\right)$ spectrum of $\mathbf{2} \mathbf{j}$ 


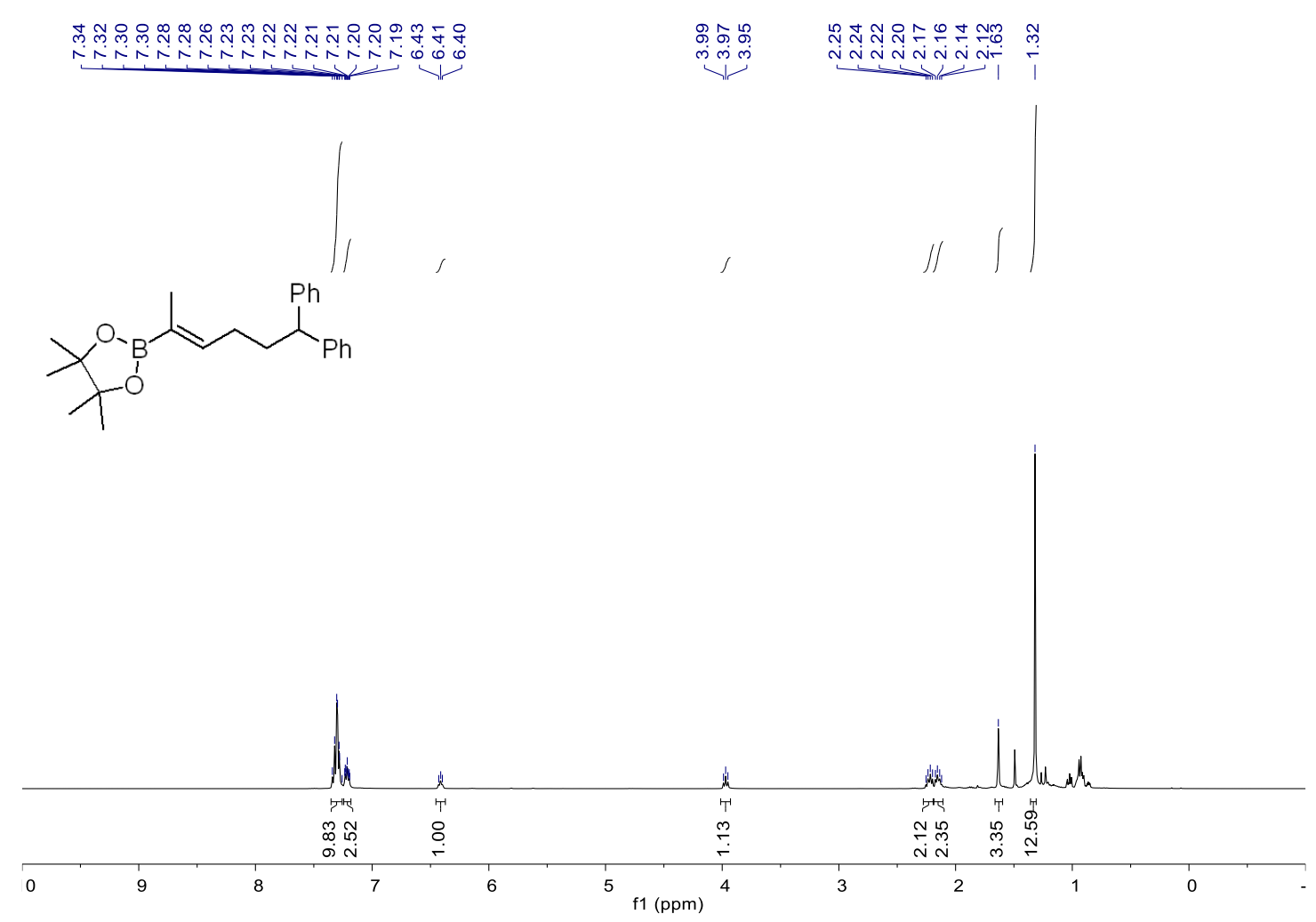

${ }^{1} \mathrm{H}$ NMR (400M, $\mathrm{CDCl}_{3}$ ) spectrum of $\mathbf{2 k}$

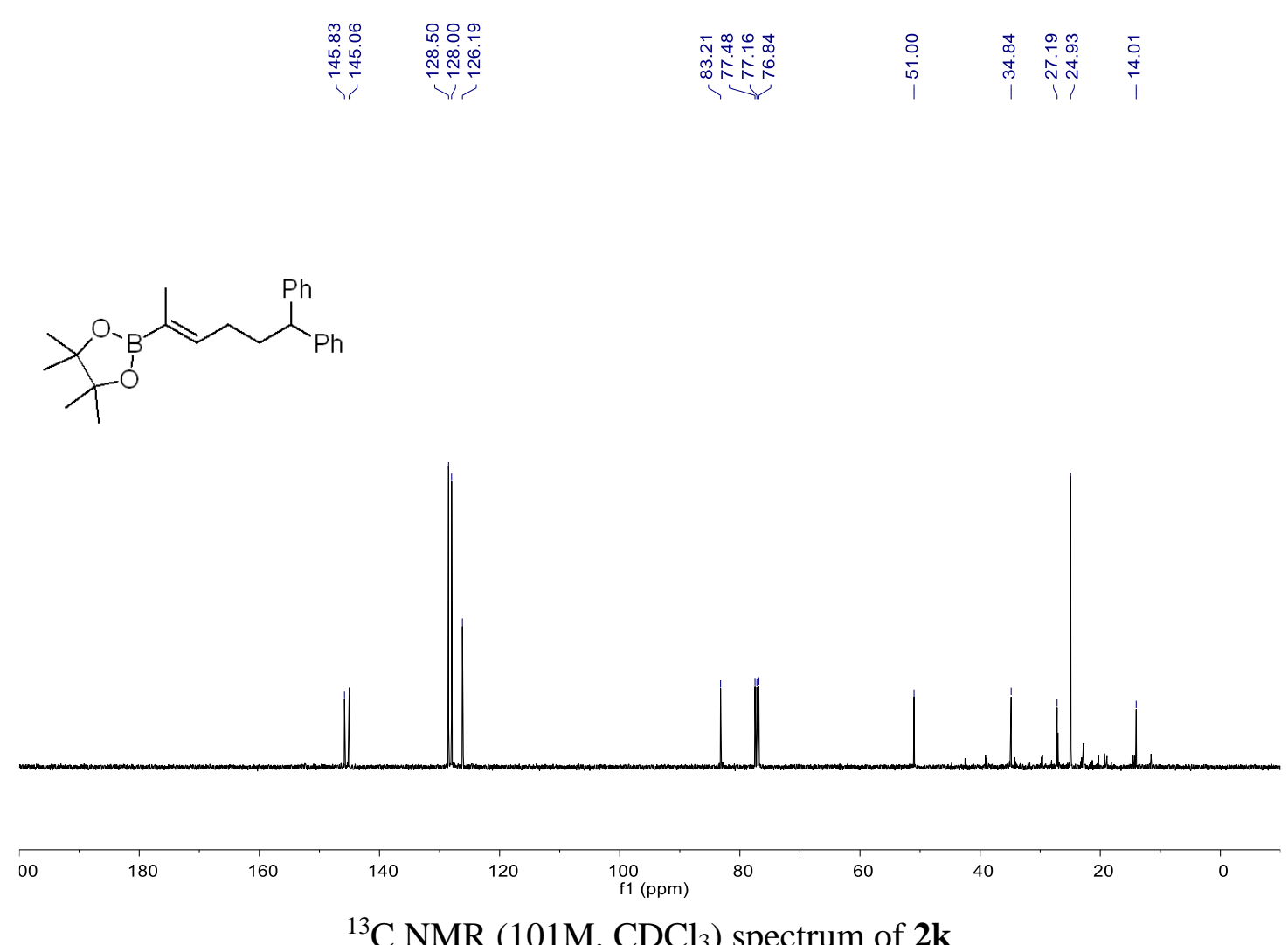

${ }^{13} \mathrm{C}$ NMR (101M, $\left.\mathrm{CDCl}_{3}\right)$ spectrum of $\mathbf{2 k}$ 
<smiles>CC(=CCCC(c1ccccc1)c1ccccc1)B1OC(C)(C)C(C)(C)O1</smiles>

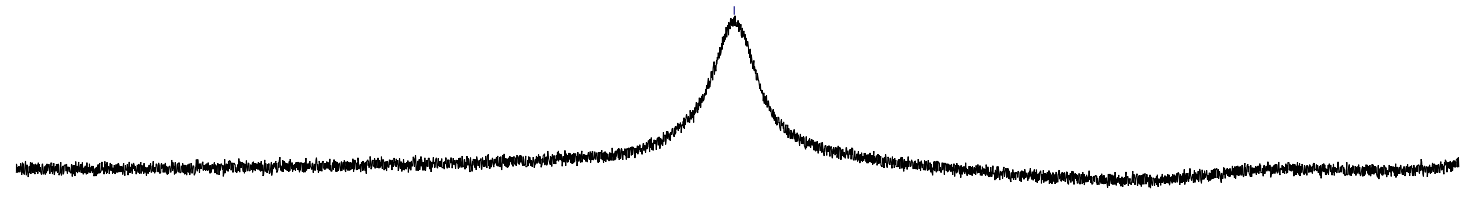

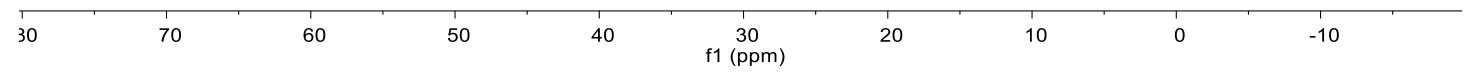

${ }^{11} \mathrm{~B}$ NMR $\left(128 \mathrm{M}, \mathrm{CDCl}_{3}\right)$ spectrum of $\mathbf{2 k}$

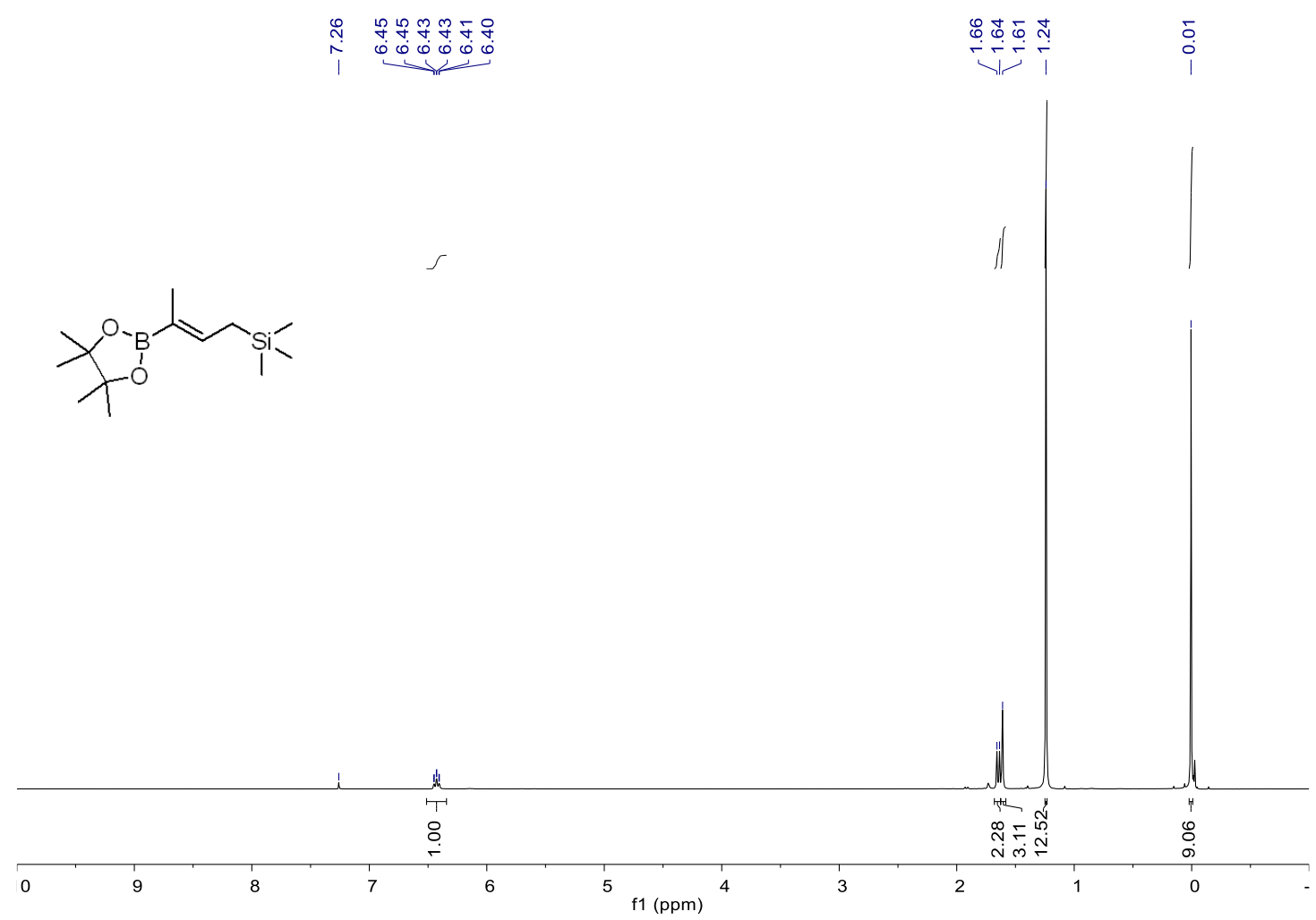

${ }^{1} \mathrm{H}$ NMR (400M, $\left.\mathrm{CDCl}_{3}\right)$ spectrum of $\mathbf{2 l}$ 

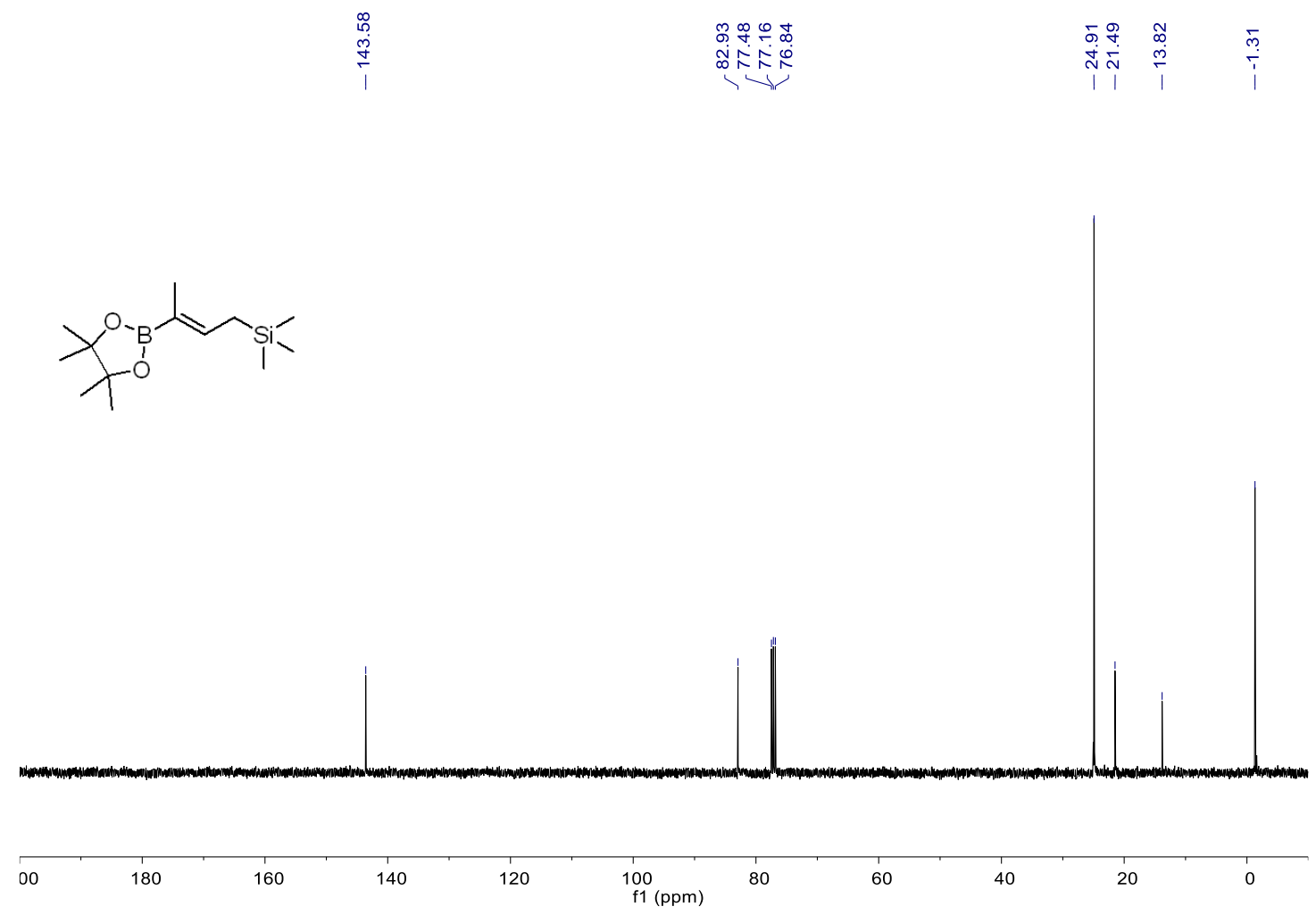

${ }^{13} \mathrm{C}$ NMR $\left(101 \mathrm{M}, \mathrm{CDCl}_{3}\right)$ spectrum of $\mathbf{2 l}$

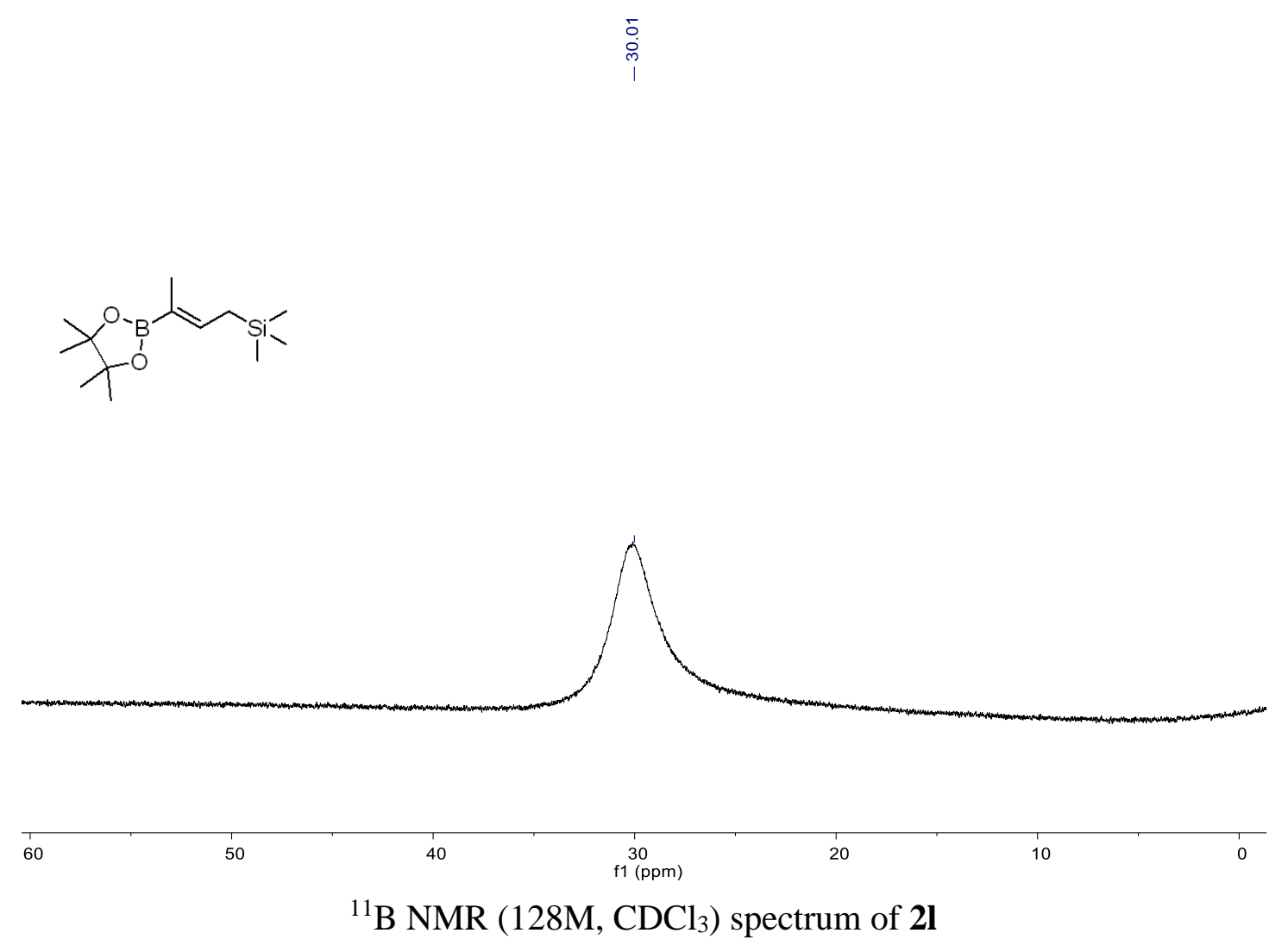




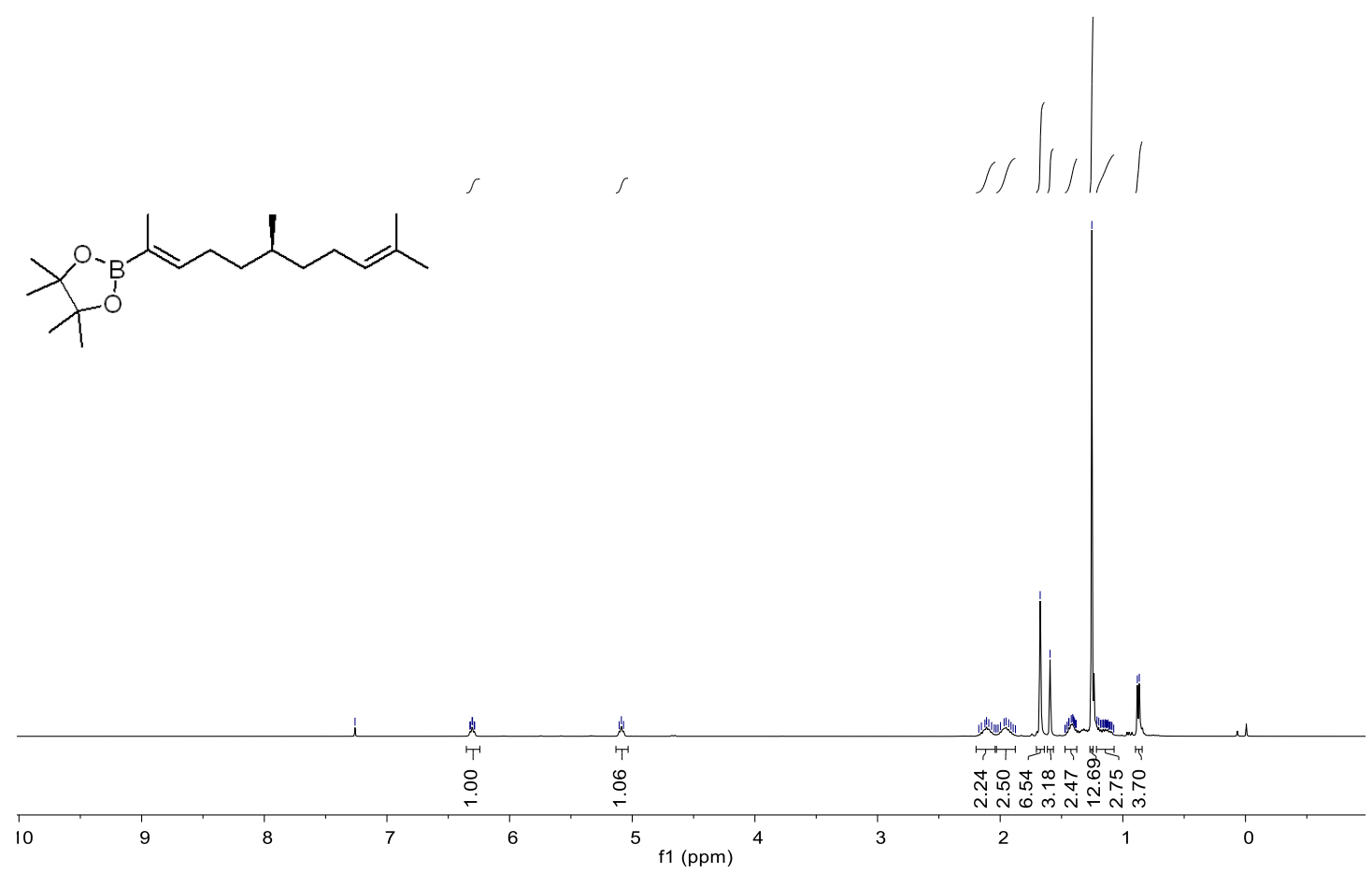

${ }^{1} \mathrm{H}$ NMR (400M, $\left.\mathrm{CDCl}_{3}\right)$ spectrum of $\mathbf{2 m}$

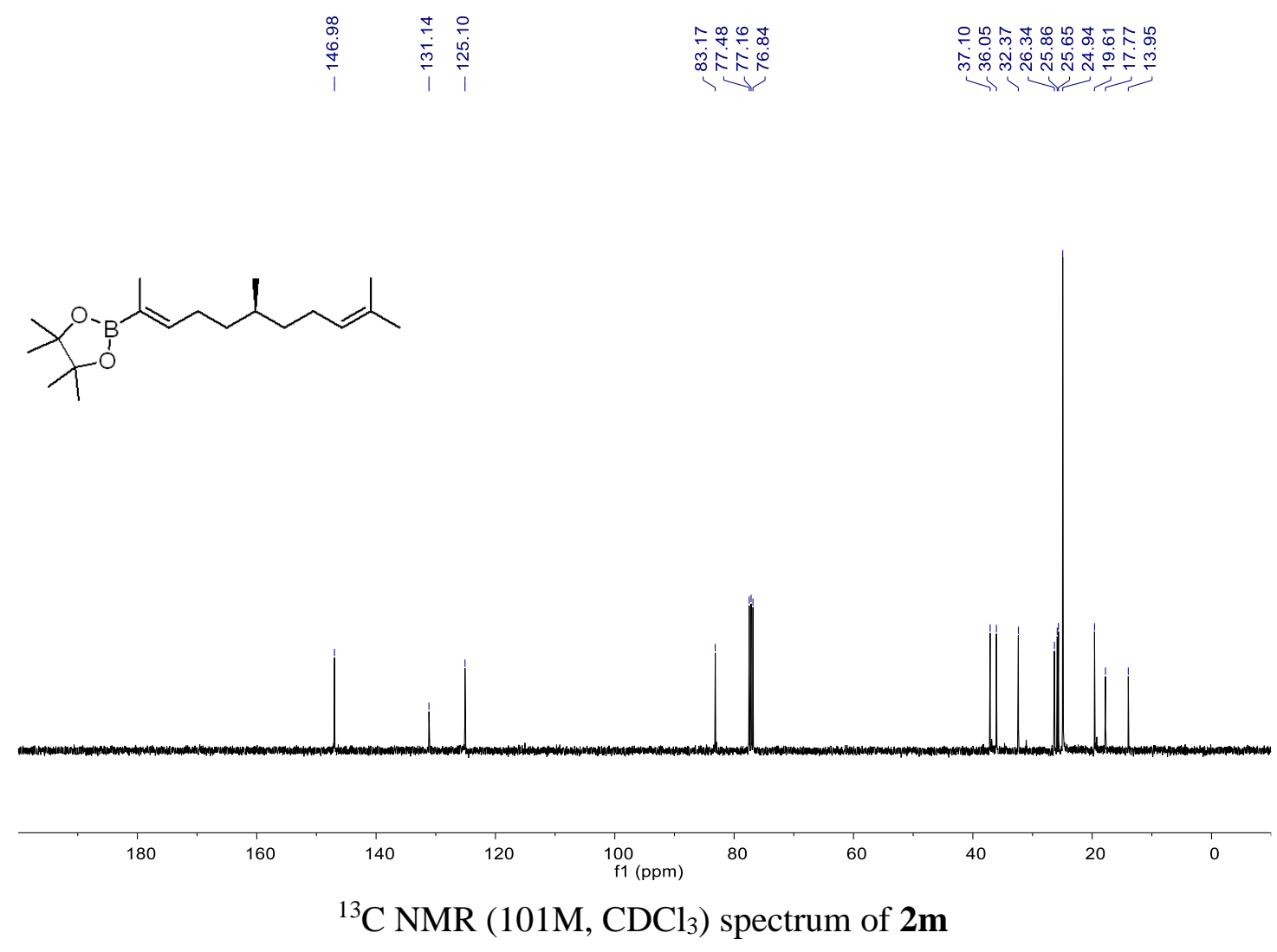


<smiles>CC(C)=CCCC(C)CCC=C(C)OP1OC(C)(C)C(C)(C)O1</smiles>

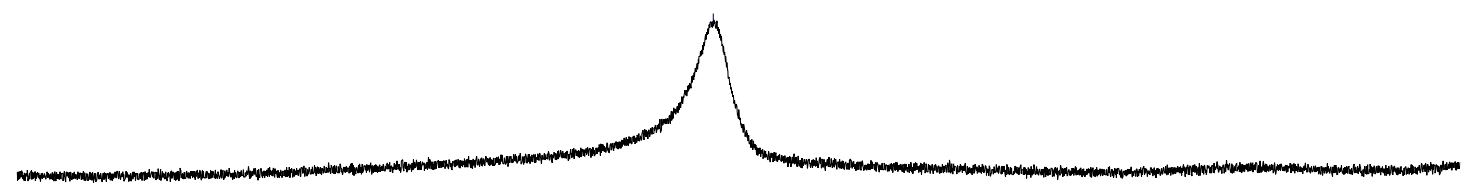

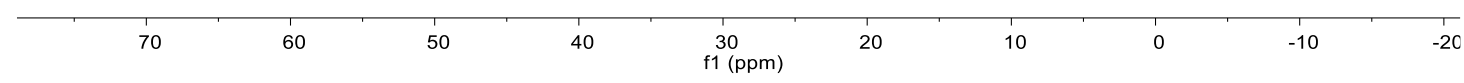

${ }^{11} \mathrm{~B}$ NMR $\left(128 \mathrm{M}, \mathrm{CDCl}_{3}\right)$ spectrum of $\mathbf{2 m}$

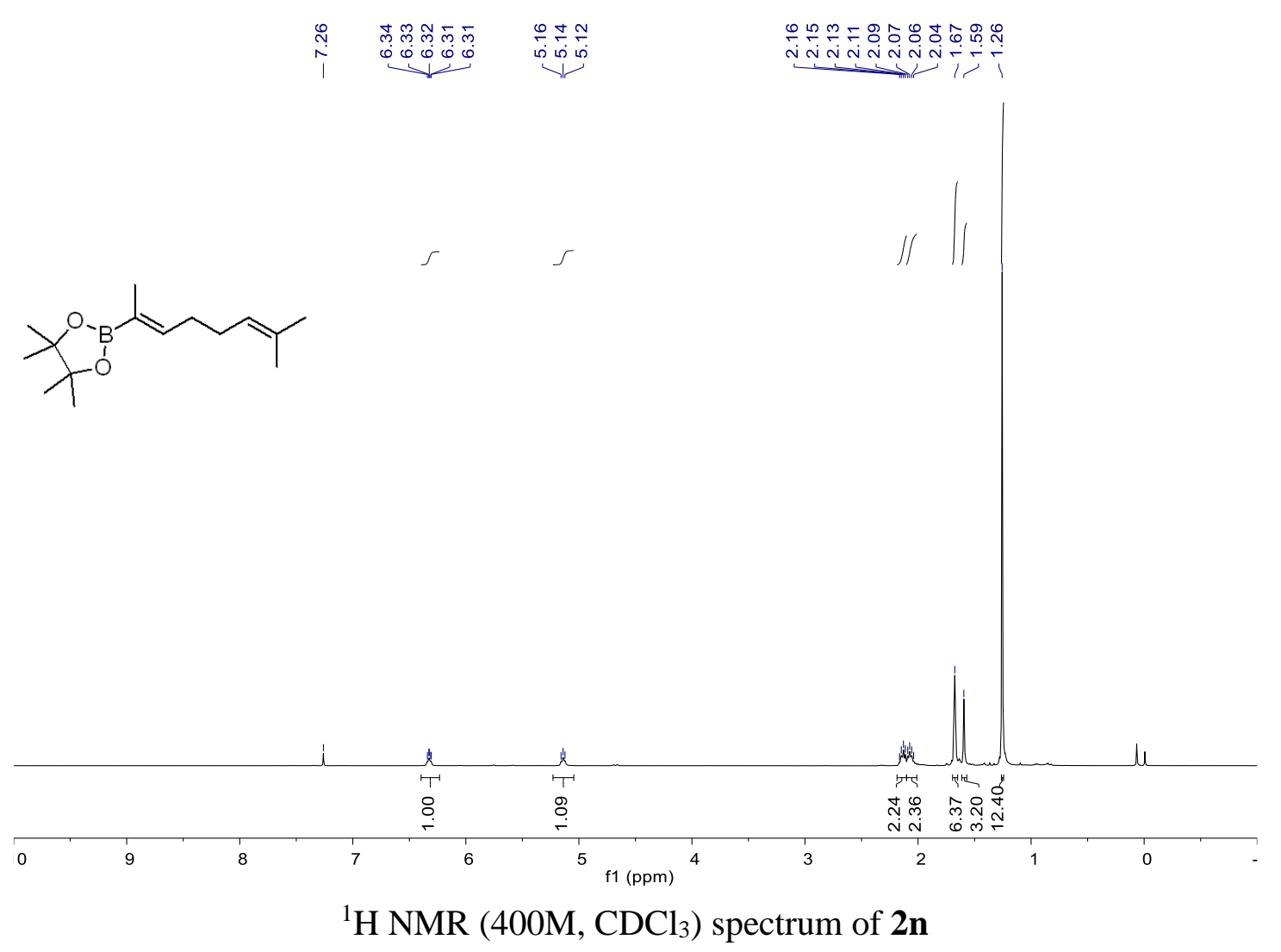



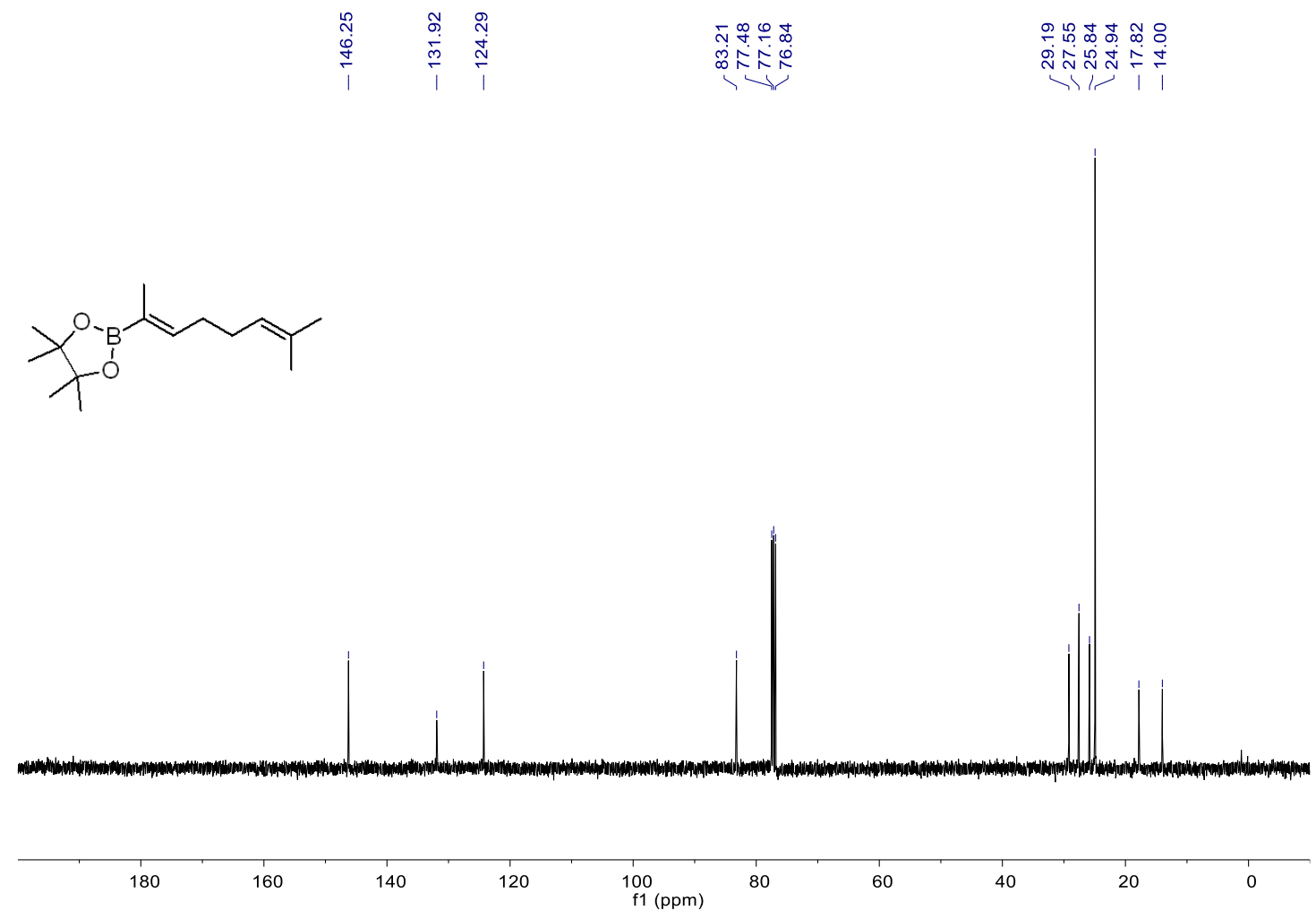

${ }^{13} \mathrm{C} \mathrm{NMR}\left(101 \mathrm{M}, \mathrm{CDCl}_{3}\right)$ spectrum of $\mathbf{2 n}$<smiles>CC(C)=CCCC=C(C)B1OC(C)(C)C(C)(C)O1</smiles>

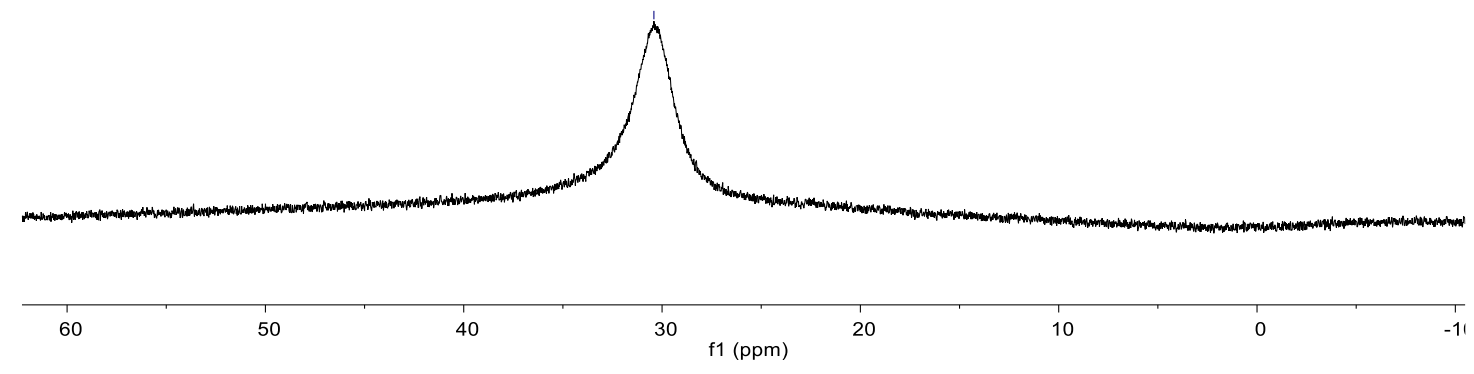

${ }^{11} \mathrm{~B}$ NMR (128M, $\left.\mathrm{CDCl}_{3}\right)$ spectrum of $\mathbf{2 n}$ 


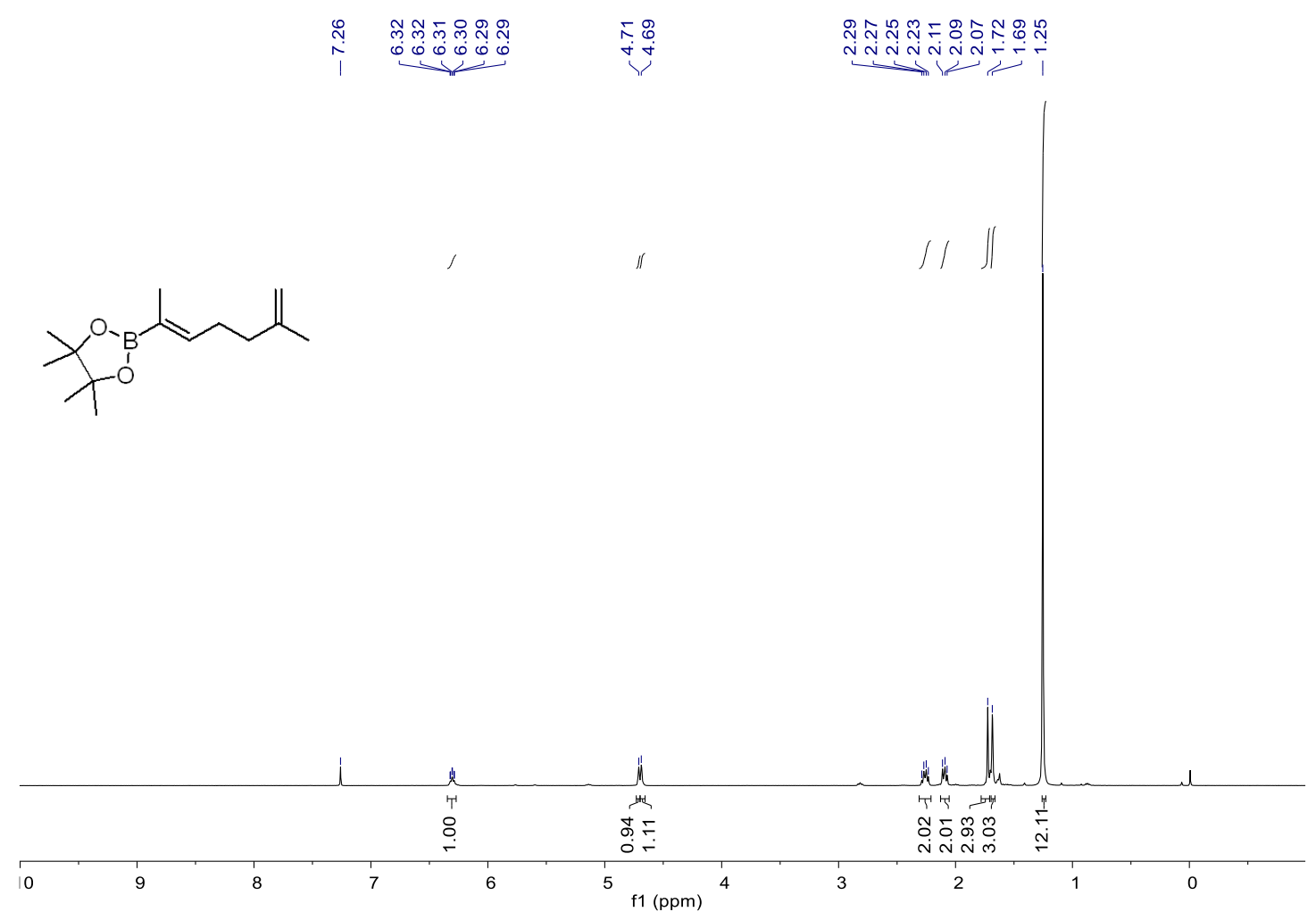

${ }^{1} \mathrm{H}$ NMR (400M, $\mathrm{CDCl}_{3}$ ) spectrum of $\mathbf{2 0}$

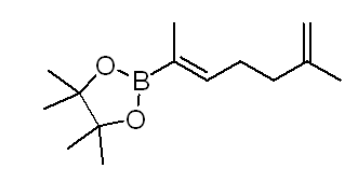

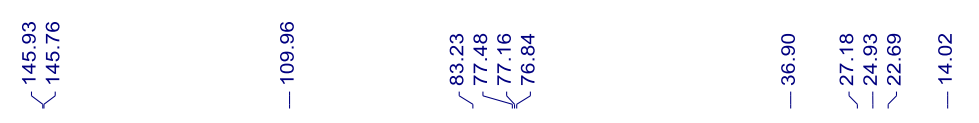

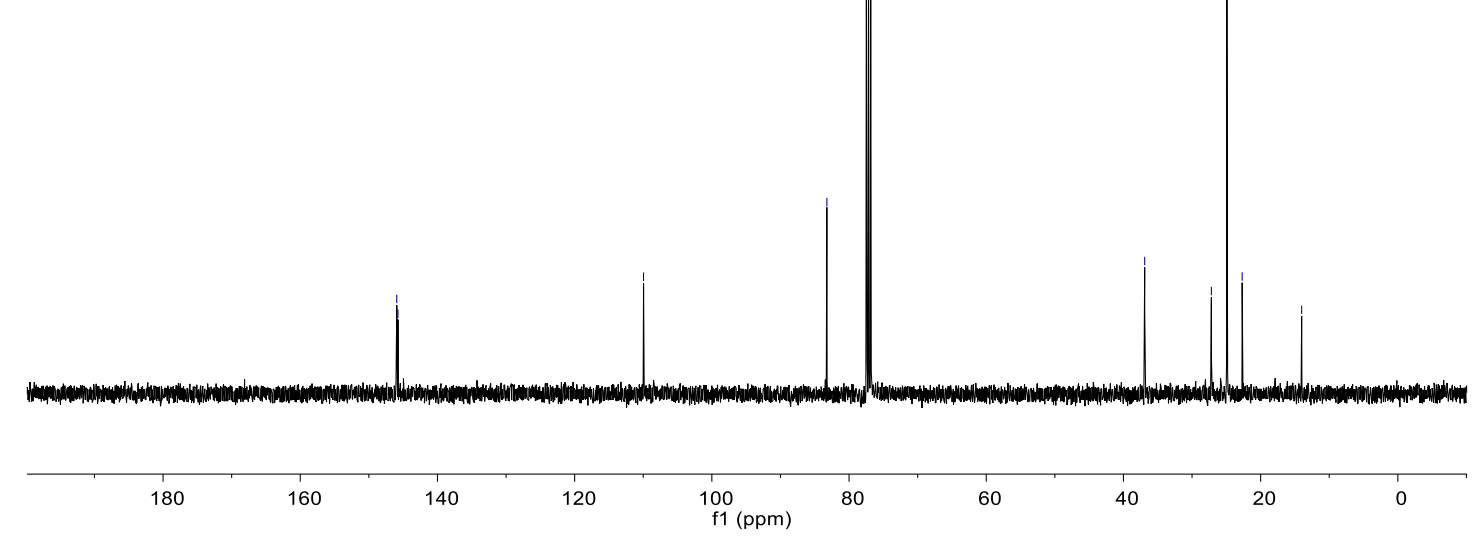

${ }^{13} \mathrm{C}$ NMR $\left(101 \mathrm{M}, \mathrm{CDCl}_{3}\right)$ spectrum of $2 \mathbf{o}$ 
<smiles>C=C(C)CC/C=C(\C)B1OC(C)(C)C(C)(C)O1</smiles>

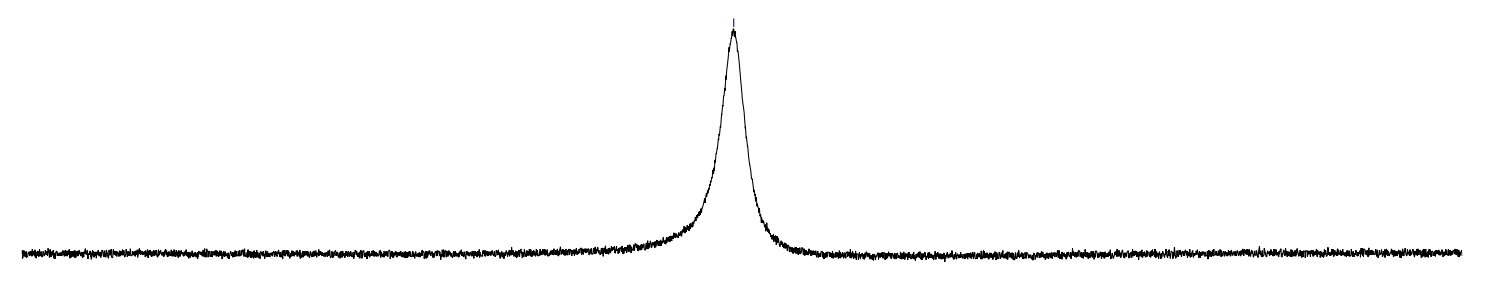

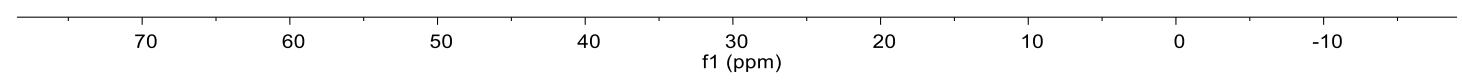

${ }^{11} \mathrm{~B}$ NMR $\left(128 \mathrm{M}, \mathrm{CDCl}_{3}\right)$ spectrum of 20

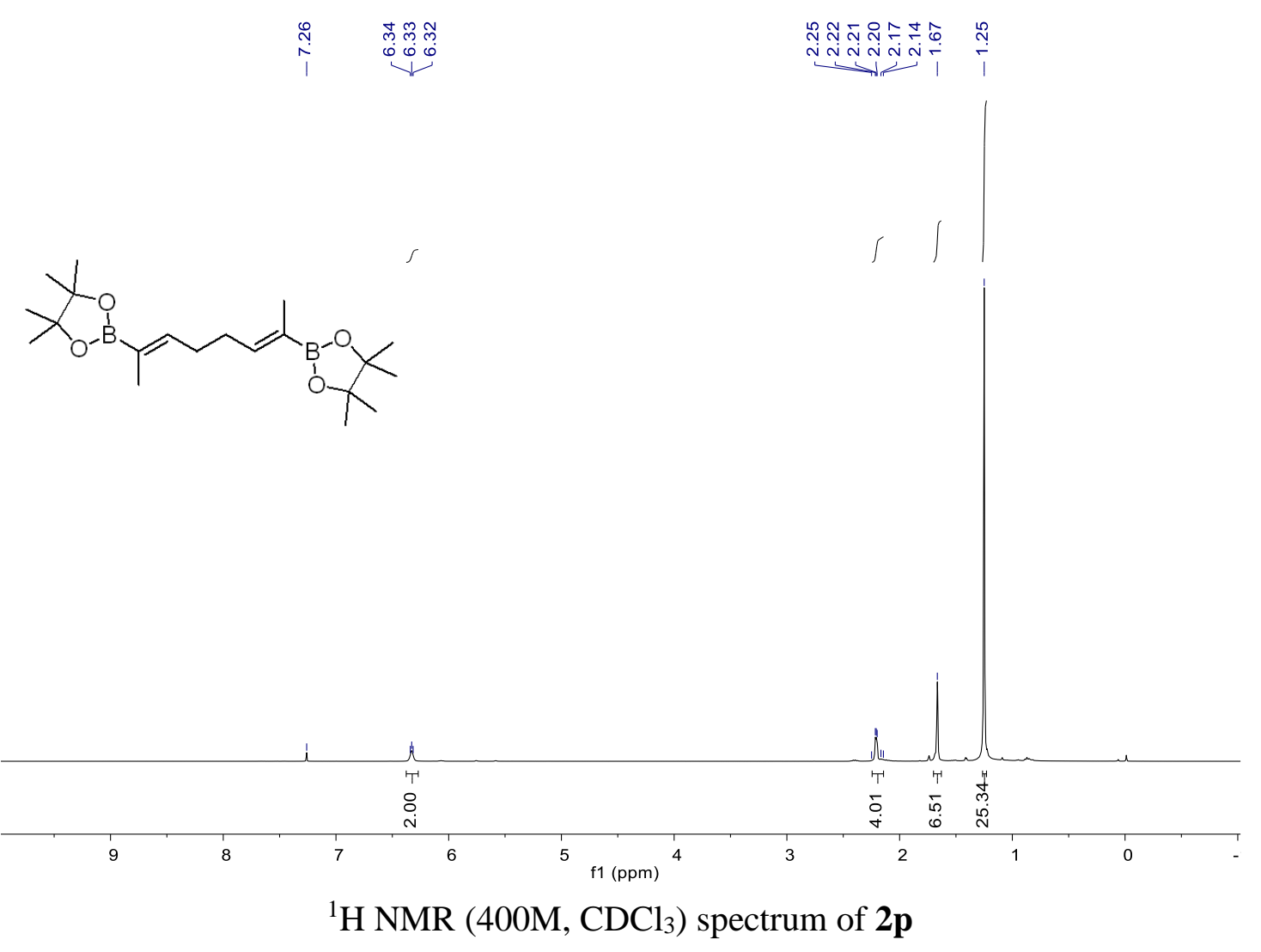




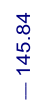

$\sqrt{\infty}$
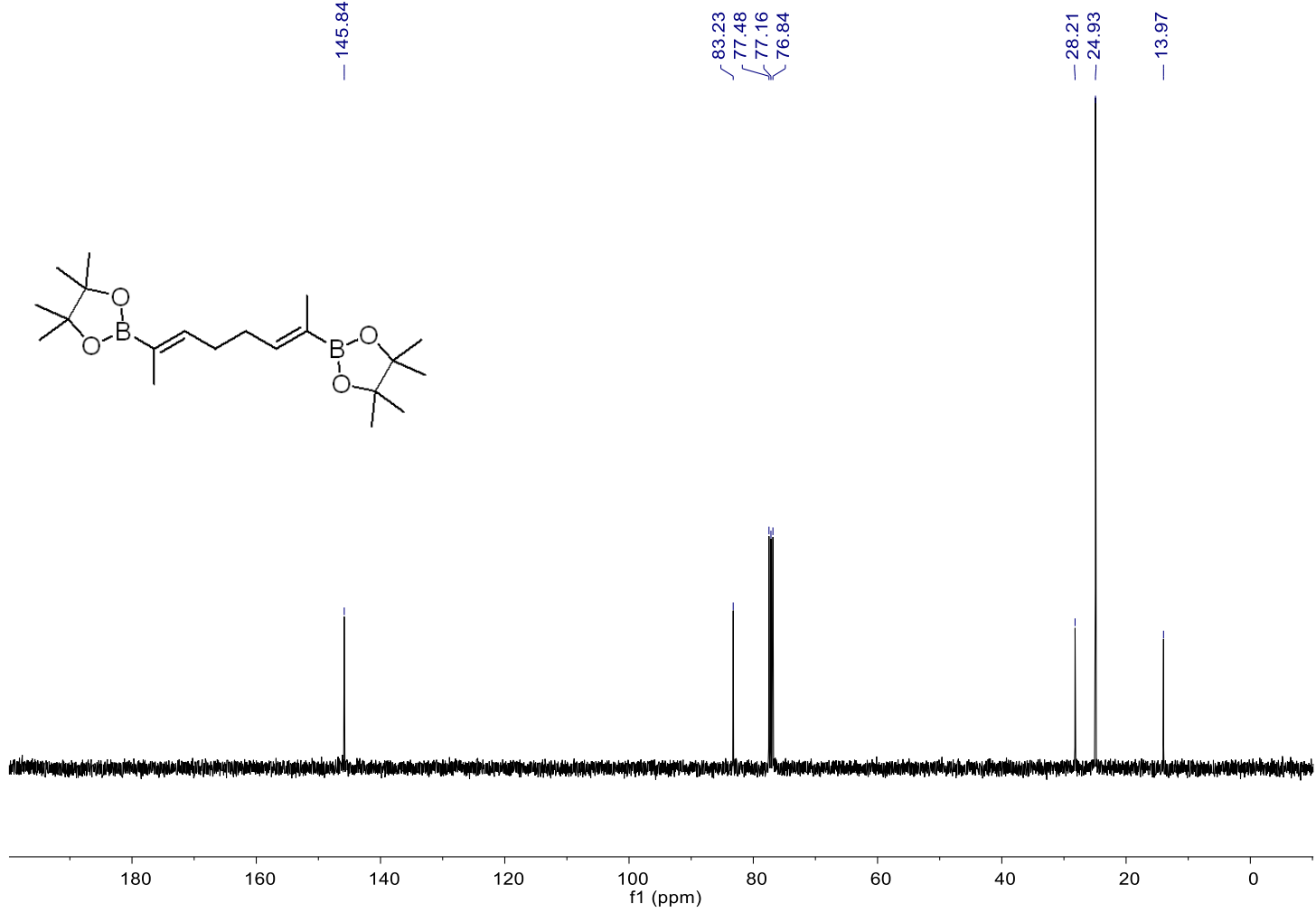

${ }^{13} \mathrm{C}$ NMR (101M, $\left.\mathrm{CDCl}_{3}\right)$ spectrum of $\mathbf{2 p}$

$\stackrel{\hat{O}}{\dot{0}}$
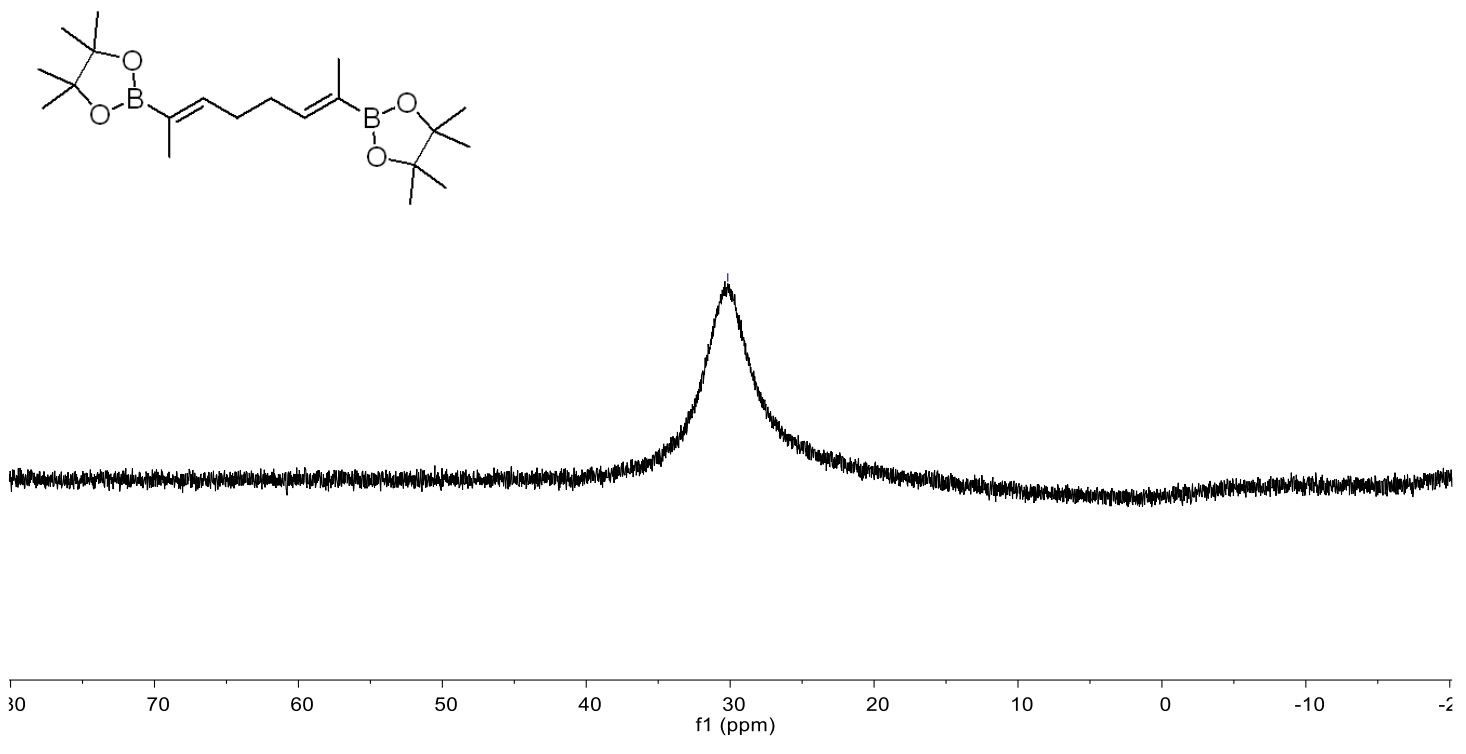

${ }^{11} \mathrm{~B}$ NMR (128M, $\left.\mathrm{CDCl}_{3}\right)$ spectrum of $\mathbf{2 p}$

S124 


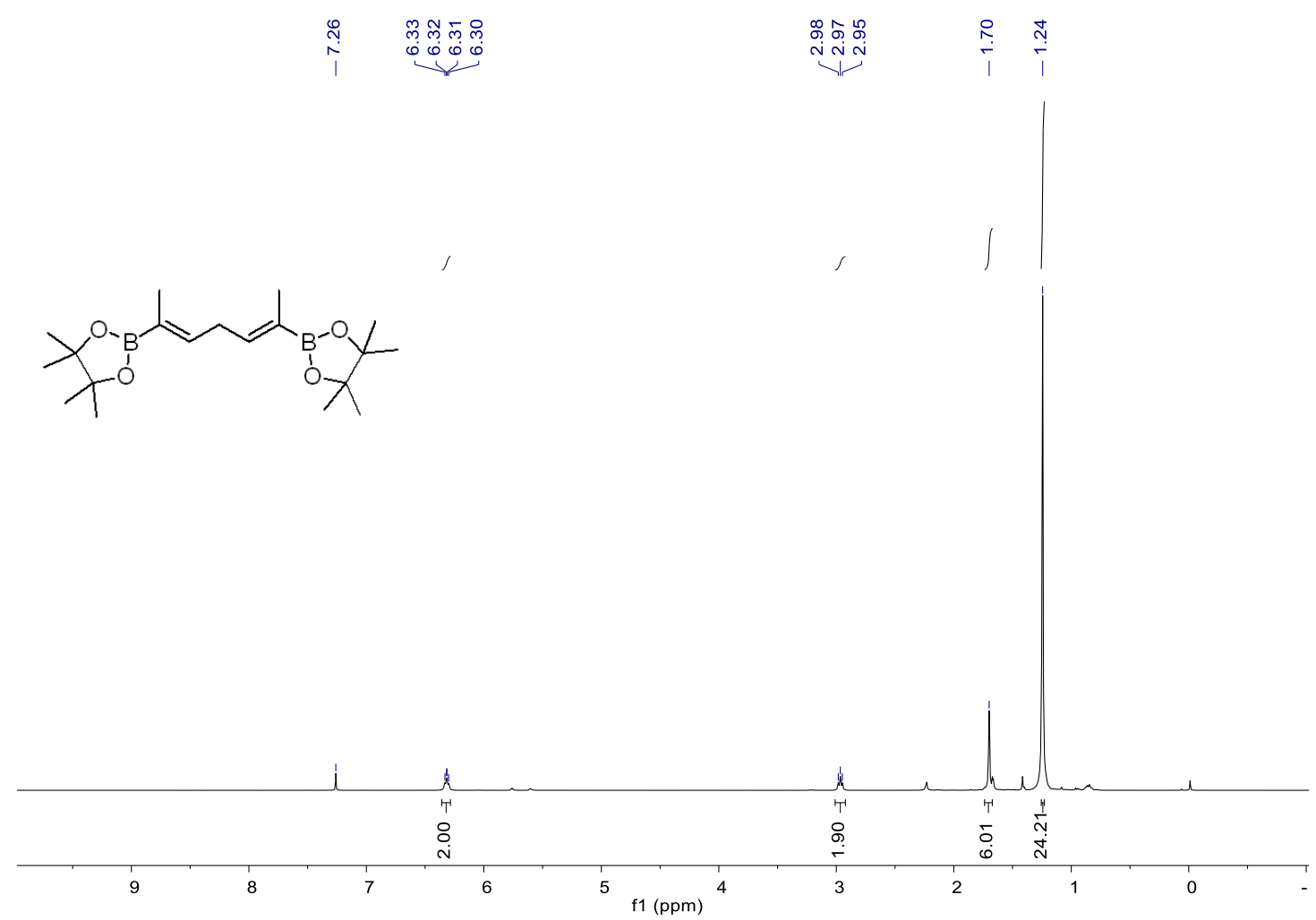

${ }^{1} \mathrm{H}$ NMR (400M, $\mathrm{CDCl}_{3}$ ) spectrum of $\mathbf{2 q}$
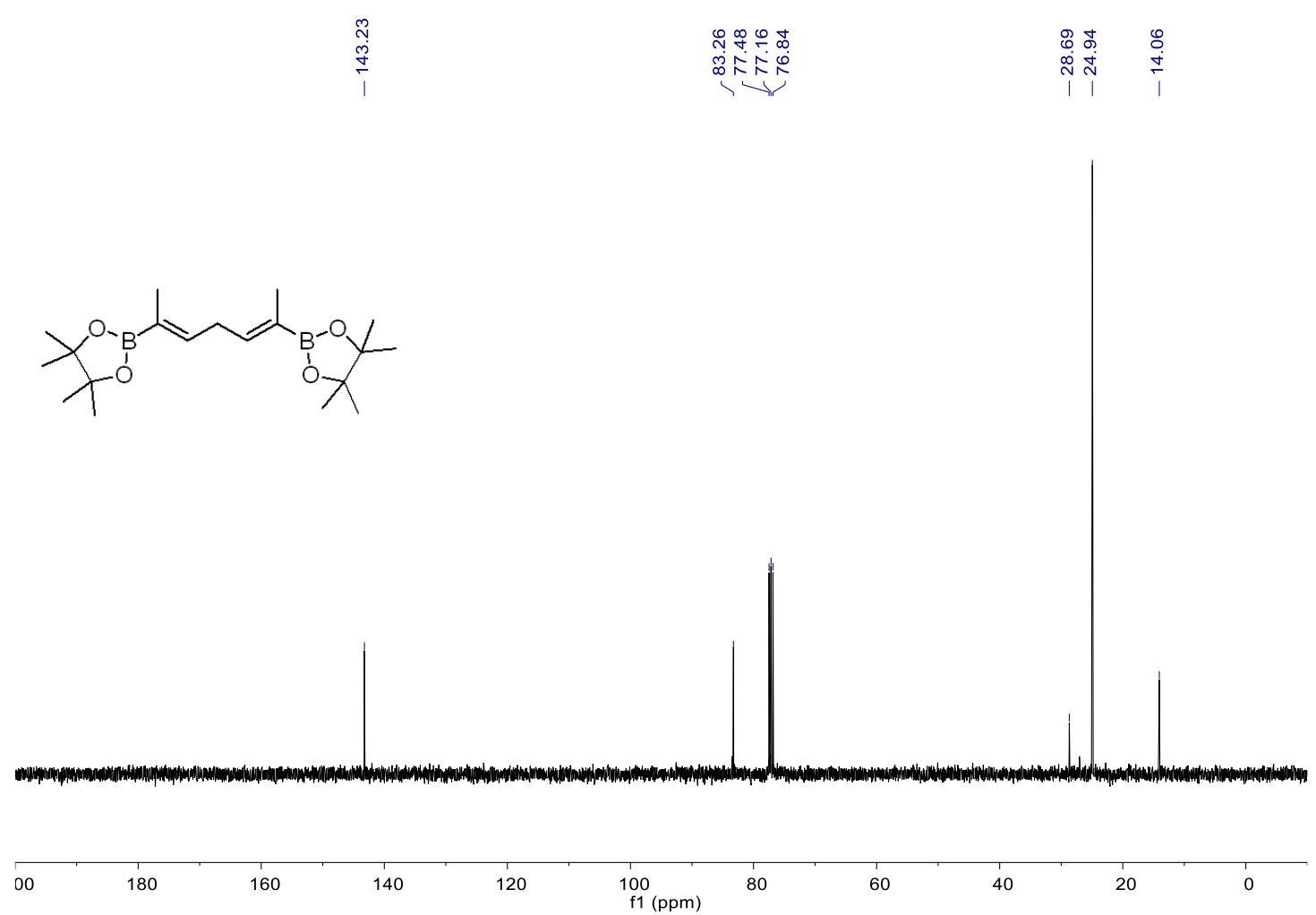

${ }^{13} \mathrm{C} \mathrm{NMR}\left(101 \mathrm{M}, \mathrm{CDCl}_{3}\right)$ spectrum of $\mathbf{2 q}$ 

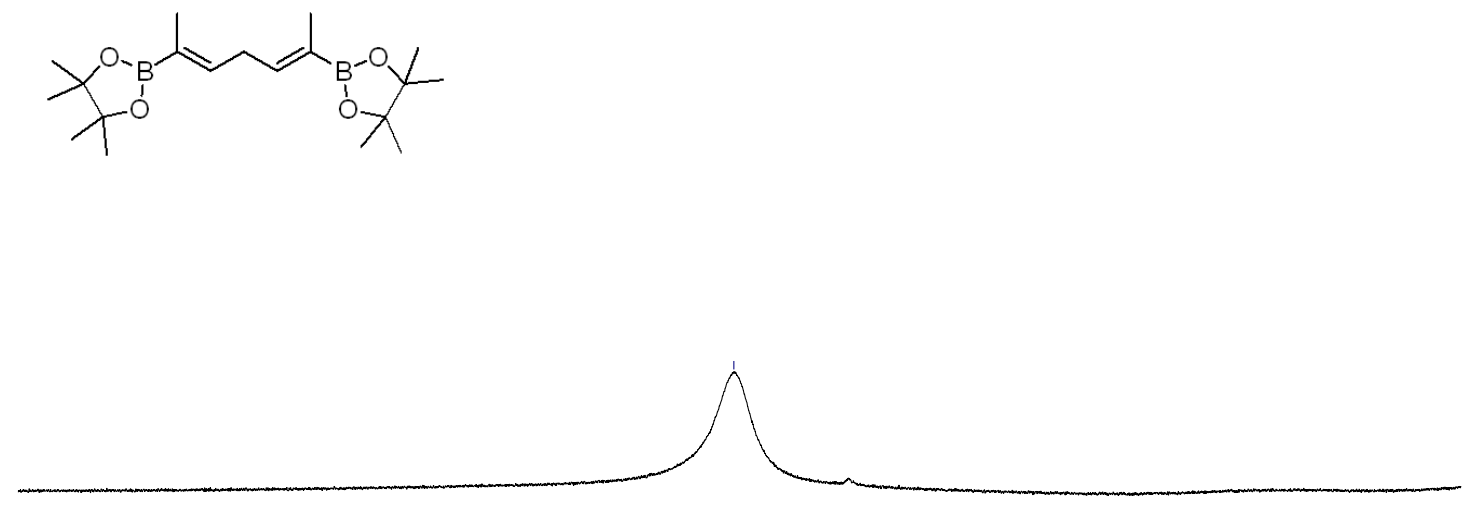

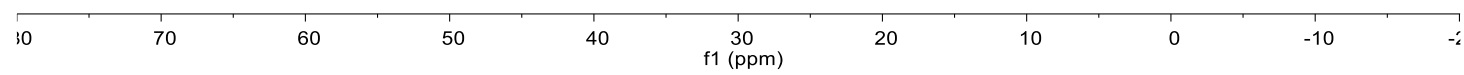

${ }^{11} \mathrm{~B}$ NMR $\left(128 \mathrm{M}, \mathrm{CDCl}_{3}\right)$ spectrum of $\mathbf{2 q}$

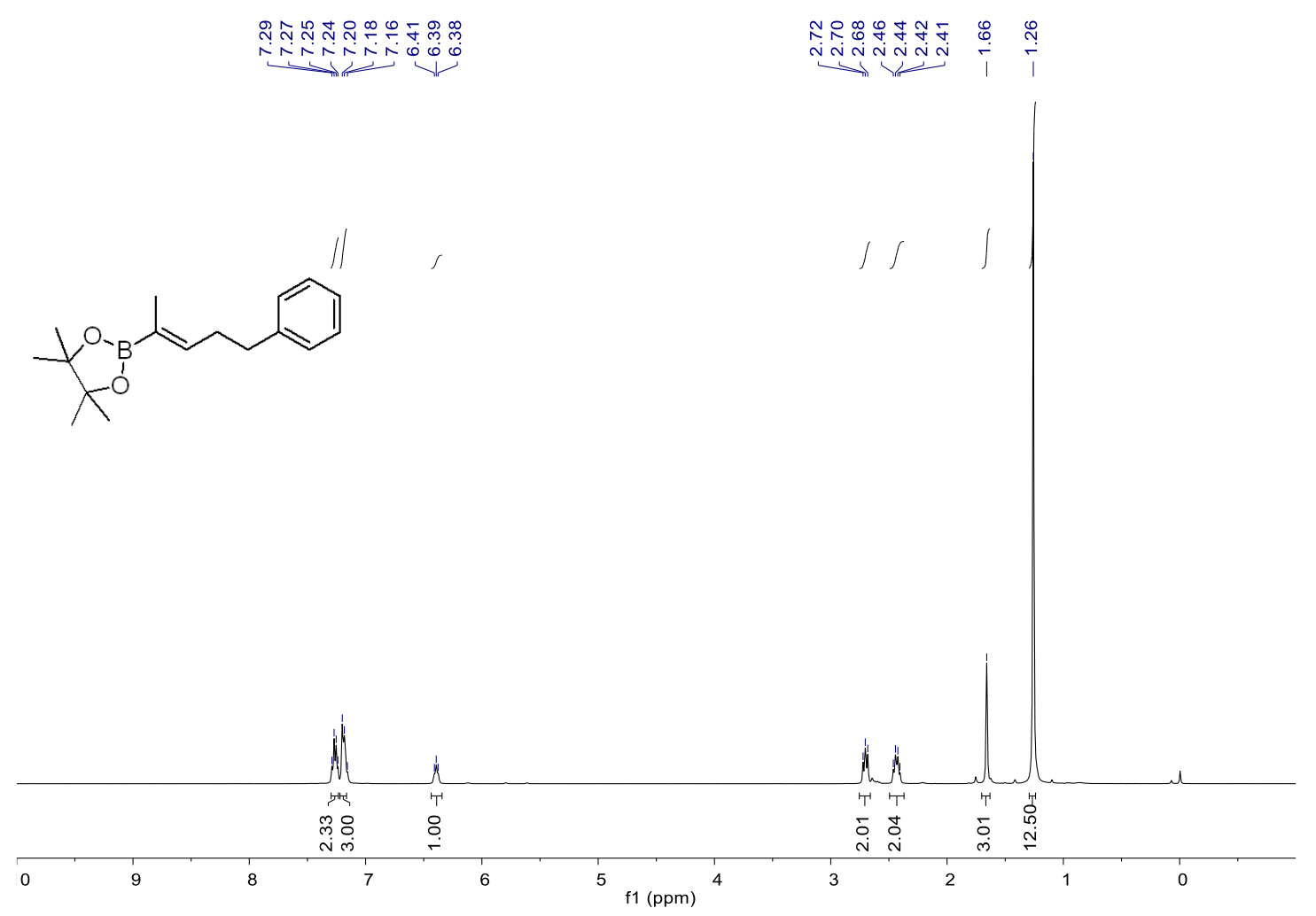

${ }^{1} \mathrm{H}$ NMR $\left(400 \mathrm{M}, \mathrm{CDCl}_{3}\right)$ spectrum of $\mathbf{2 r}$ 

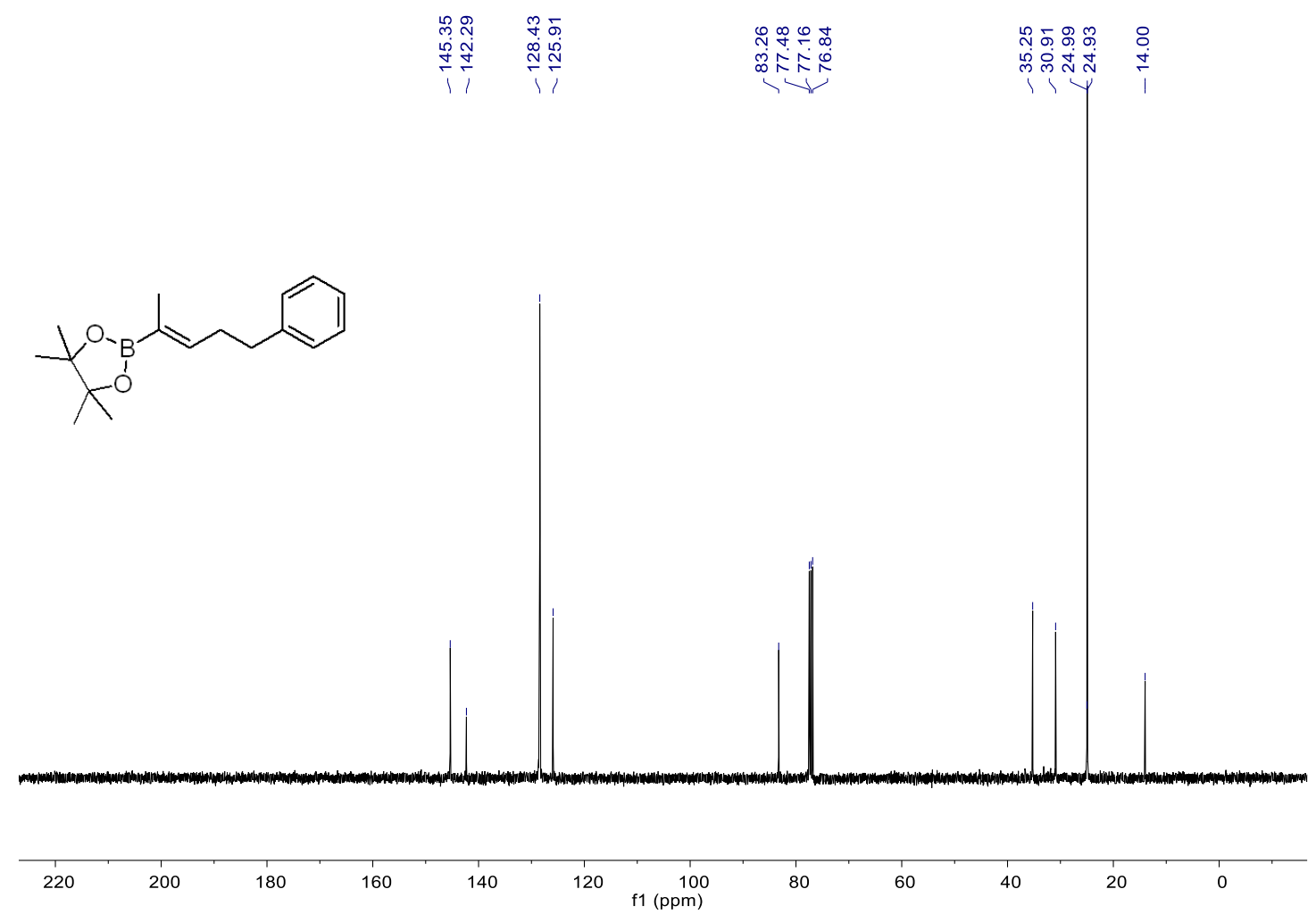

${ }^{13} \mathrm{C}$ NMR (101M, $\left.\mathrm{CDCl}_{3}\right)$ spectrum of $\mathbf{2 r}$

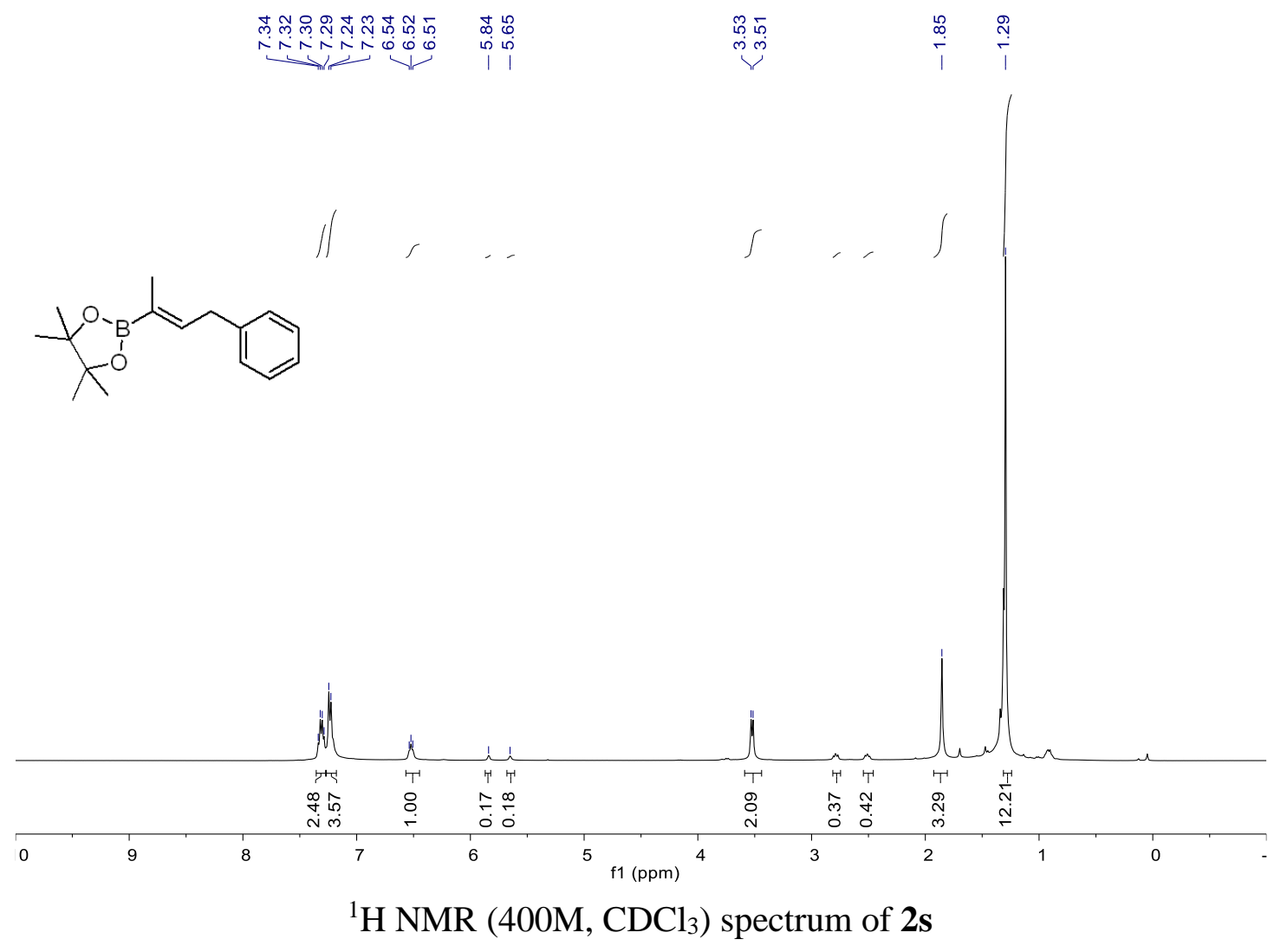




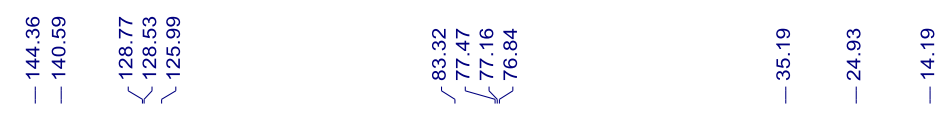<smiles>C/C(=C\Cc1ccccc1)B1OC(C)(C)C(C)(C)O1</smiles>

\begin{tabular}{rlrlllllll}
\hline 180 & 160 & 140 & 120 & $\substack{100 \\
\mathrm{f} 1(\mathrm{ppm})}$ & 80 & 60 & 40 & 20 & 0
\end{tabular}

${ }^{13} \mathrm{C}$ NMR (101M, $\mathrm{CDCl}_{3}$ ) spectrum of $2 \mathrm{~s}$

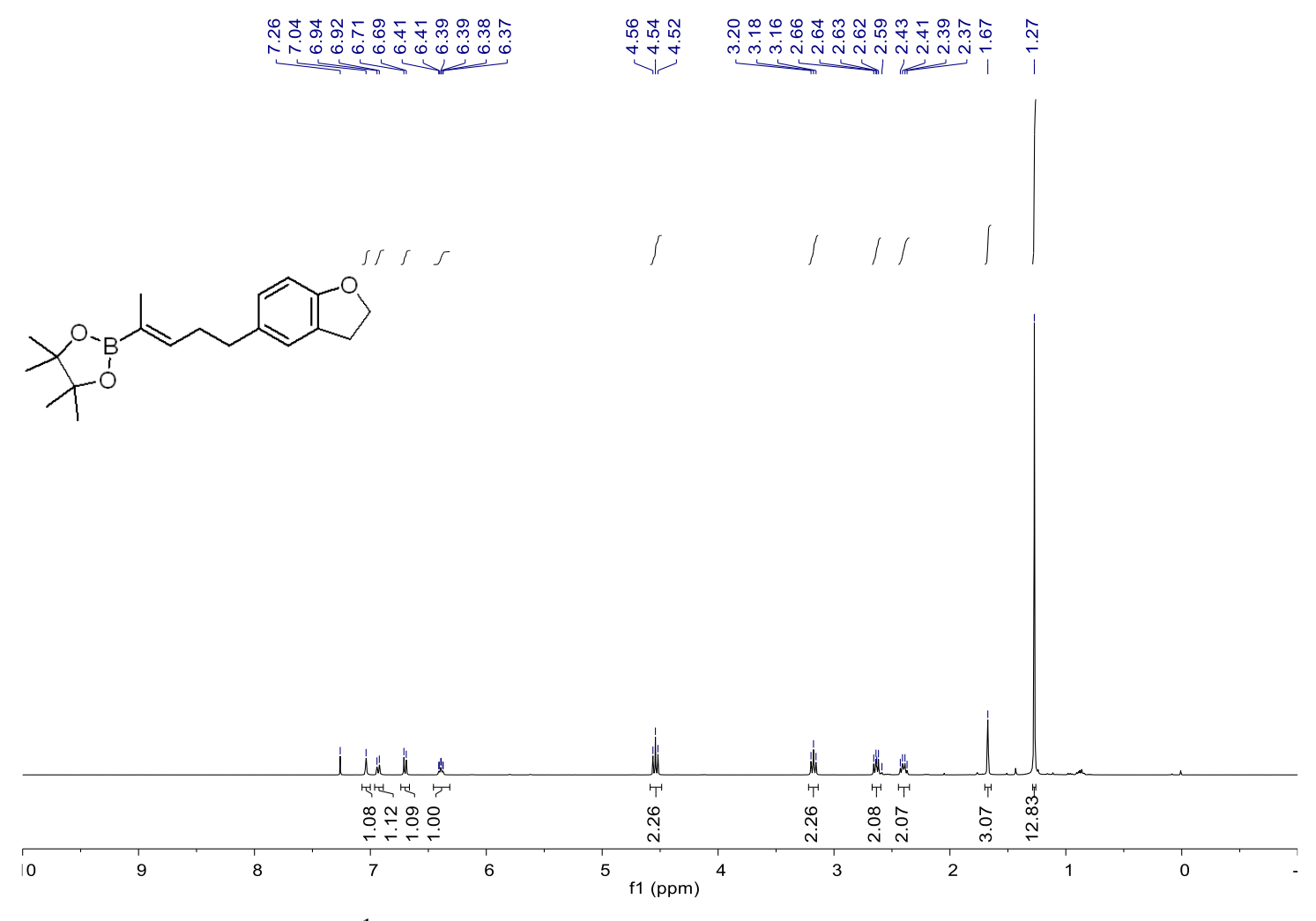

${ }^{1} \mathrm{H}$ NMR (400M, $\mathrm{CDCl}_{3}$ ) spectrum of $\mathbf{2 t}$ 

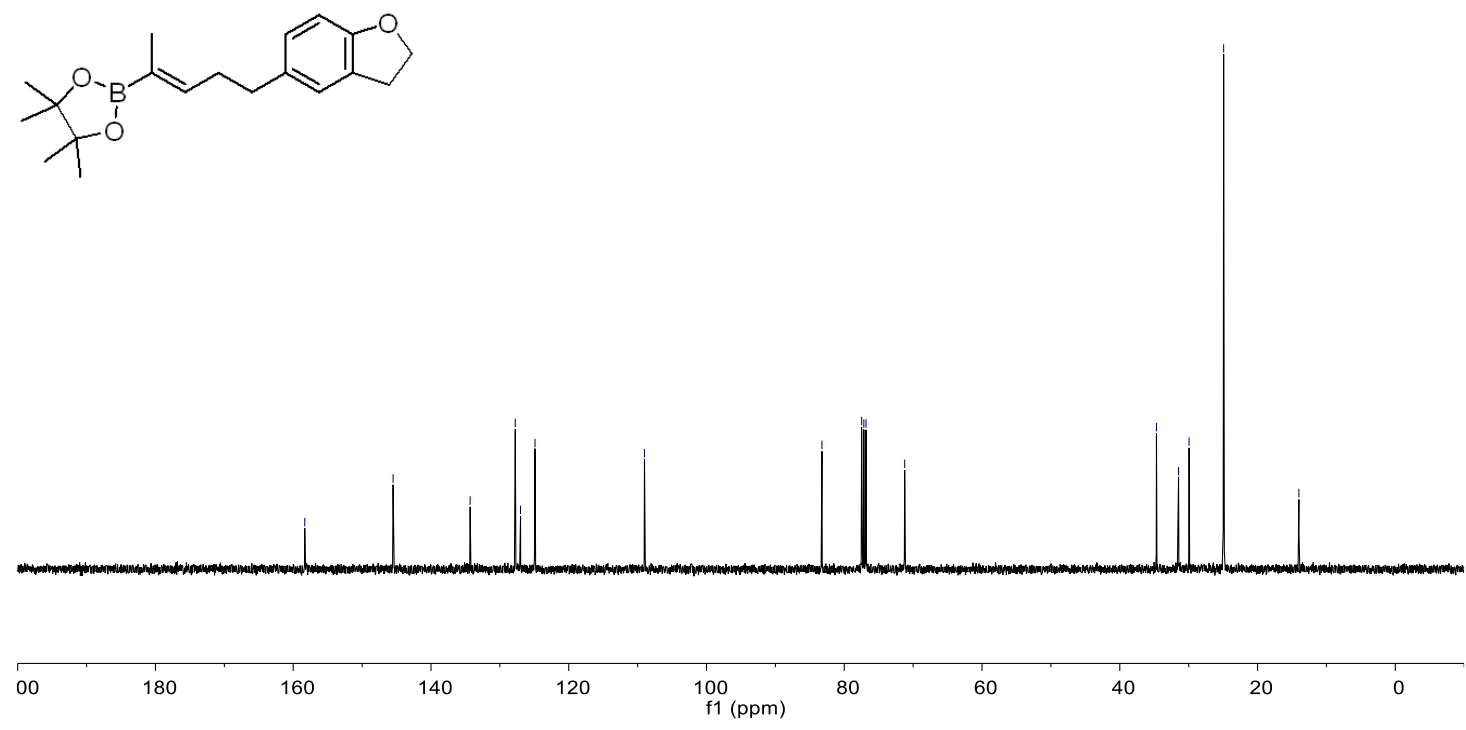

${ }^{13} \mathrm{C}$ NMR (101M, $\left.\mathrm{CDCl}_{3}\right)$ spectrum of $\mathbf{2 t}$<smiles>C/C(=C\CCc1ccc2c(c1)CCO2)OB1OC(C)(C)C(C)(C)O1</smiles>

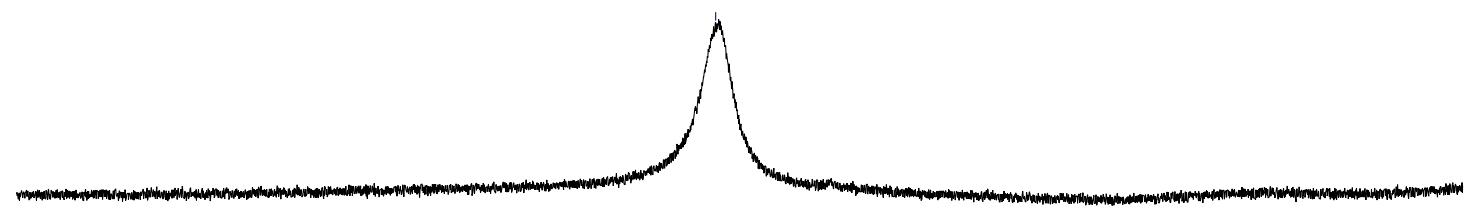

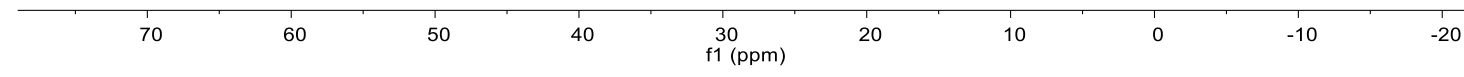

${ }^{11} \mathrm{~B}$ NMR (128M, $\left.\mathrm{CDCl}_{3}\right)$ spectrum of $\mathbf{2 t}$ 


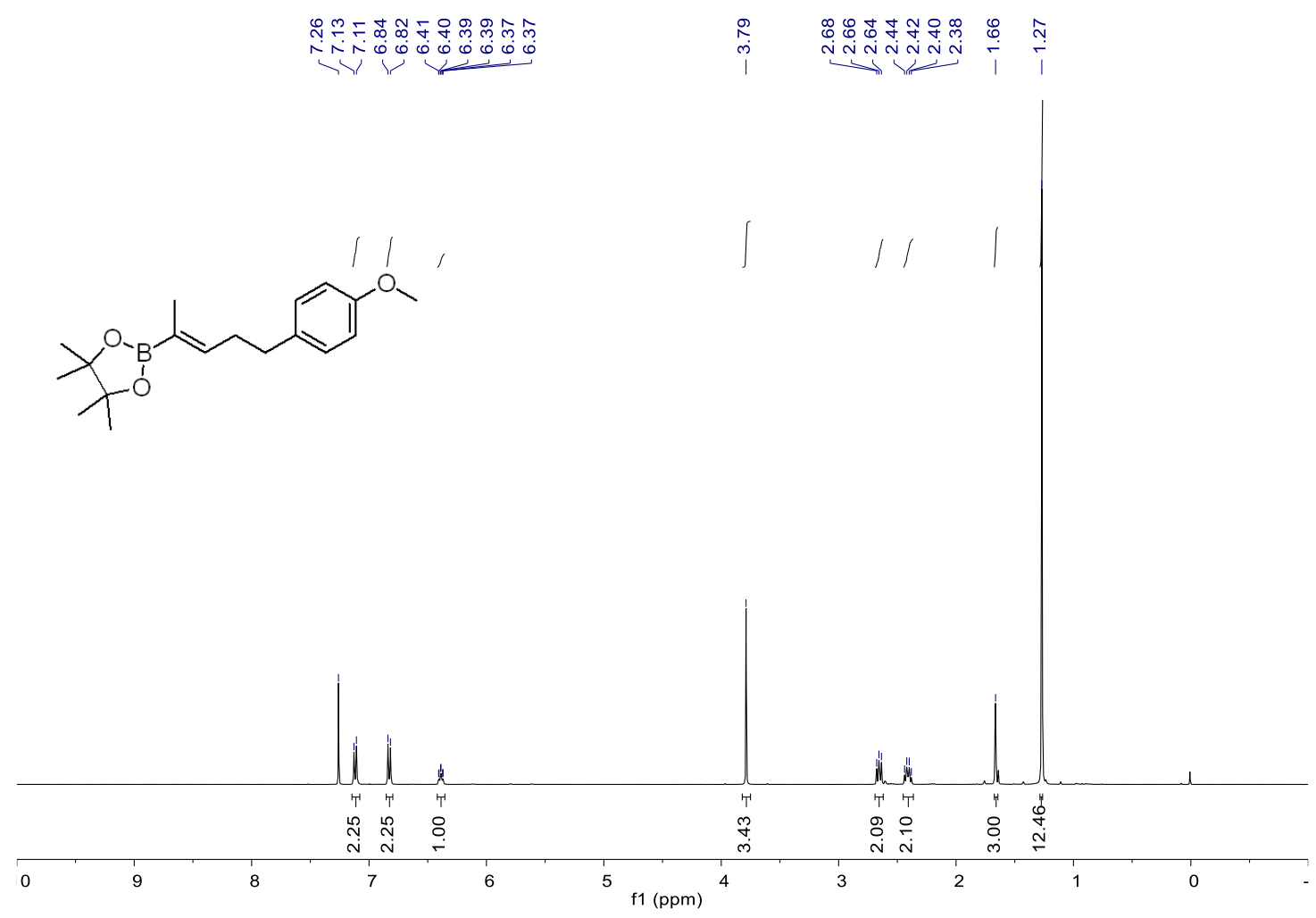

${ }^{1} \mathrm{H}$ NMR (400M, $\mathrm{CDCl}_{3}$ ) spectrum of $\mathbf{2 u}$

\begin{tabular}{|c|c|c|c|c|c|}
\hline 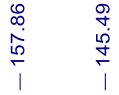 & 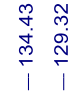 & 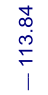 & 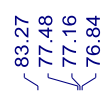 & 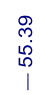 & 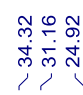 \\
\hline
\end{tabular}<smiles>COc1ccc(CC/C=C(\C)B2OC(C)(C)C(C)(C)O2)cc1</smiles>
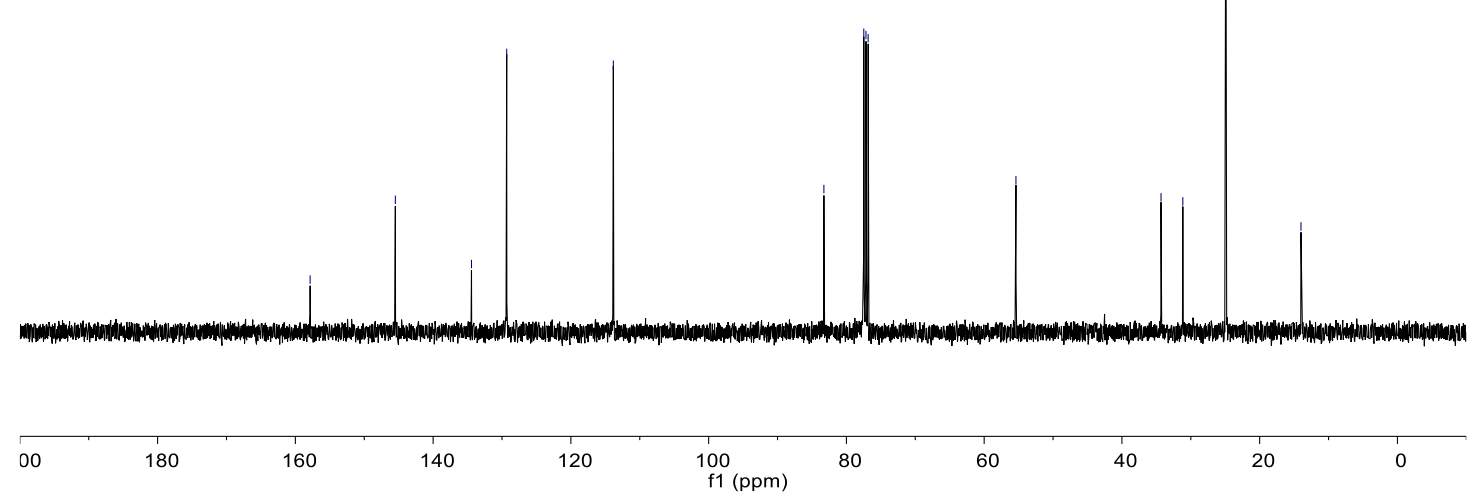

${ }^{13} \mathrm{C} \mathrm{NMR}\left(101 \mathrm{M}, \mathrm{CDCl}_{3}\right)$ spectrum of $\mathbf{2 u}$ 
<smiles>COc1ccc(CC/C=C(\C)B2OC(C)(C)C(C)(C)O2)cc1</smiles>

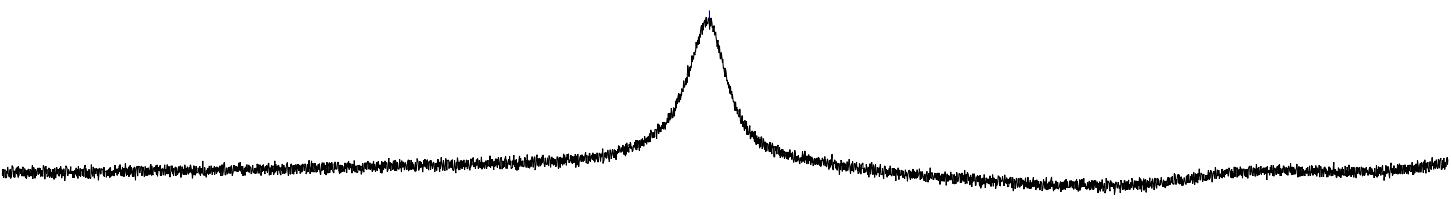

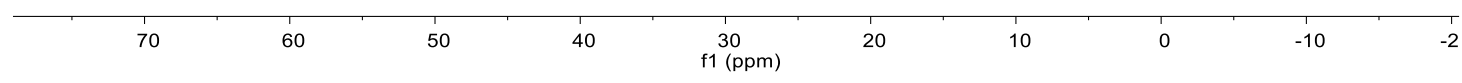

${ }^{11} \mathrm{~B}$ NMR $\left(128 \mathrm{M}, \mathrm{CDCl}_{3}\right)$ spectrum of $\mathbf{2 u}$

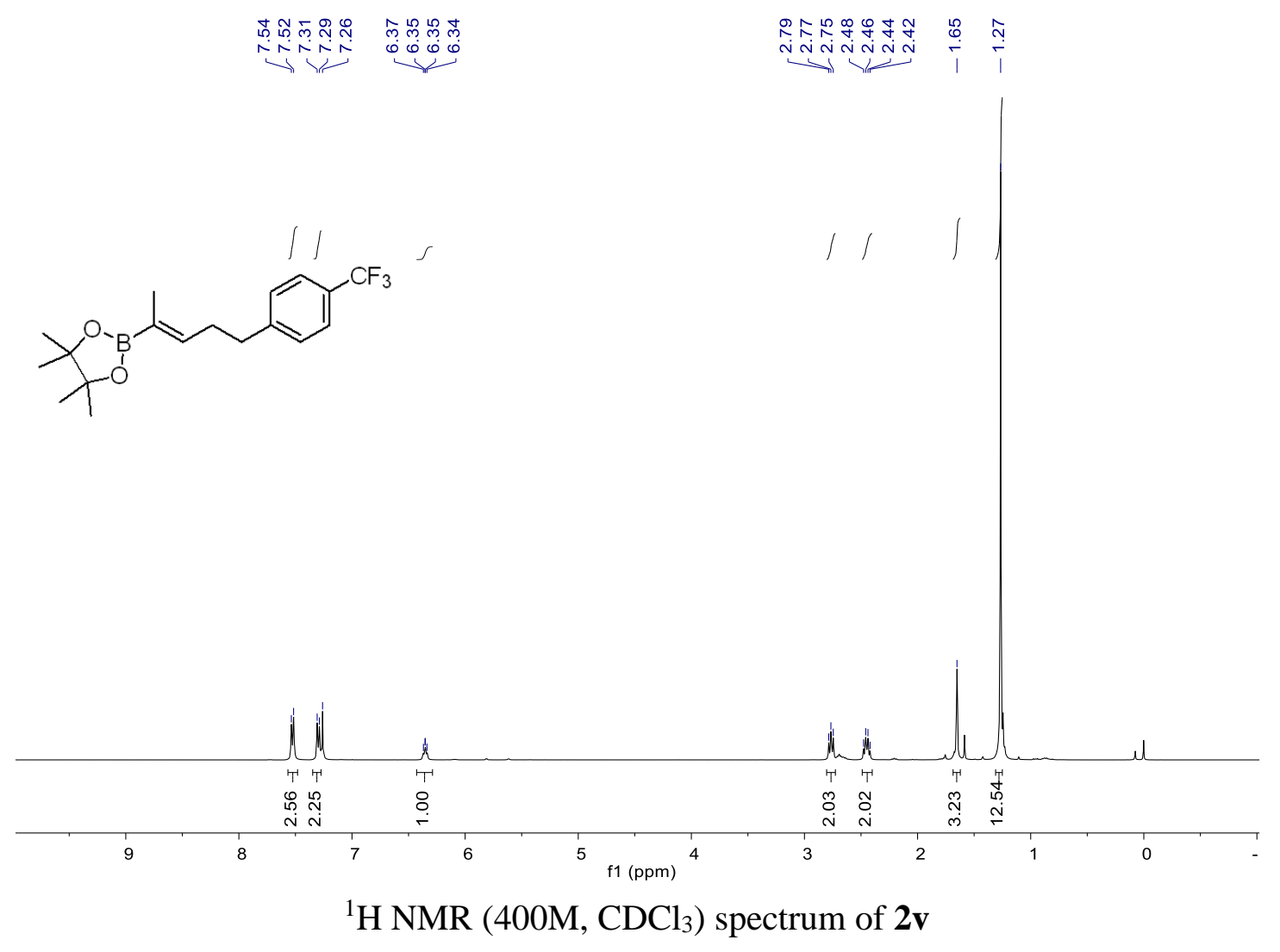



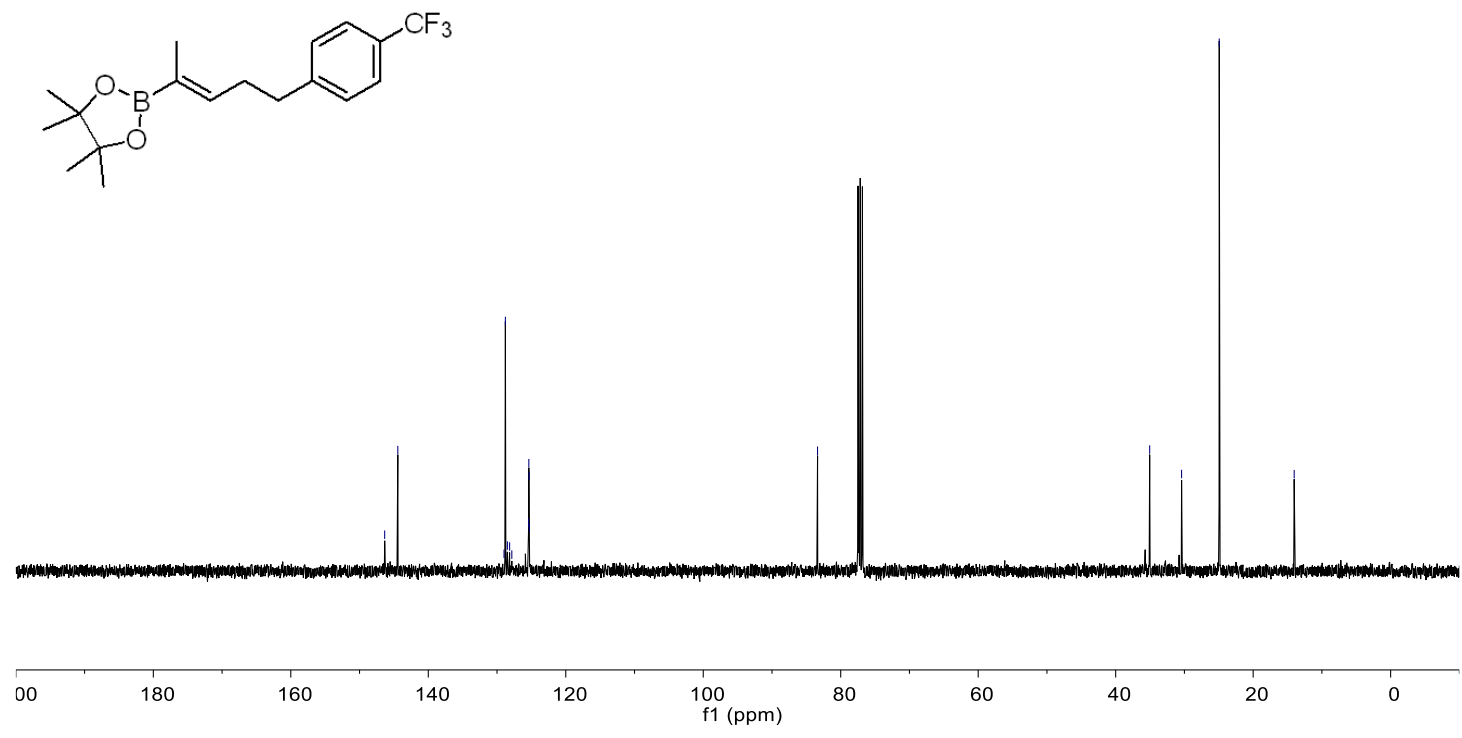

${ }^{13} \mathrm{C} \mathrm{NMR}\left(101 \mathrm{M}, \mathrm{CDCl}_{3}\right)$ spectrum of $\mathbf{2 v}$<smiles>C/C(=C\CCc1ccc(C(F)(F)F)cc1)B1OC(C)(C)C(C)(C)O1</smiles>

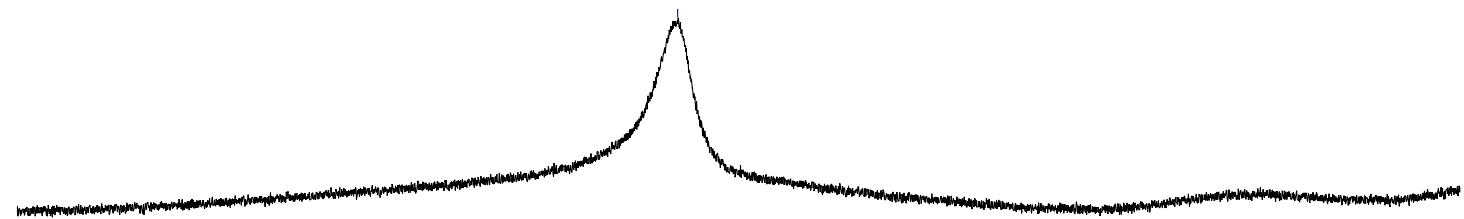

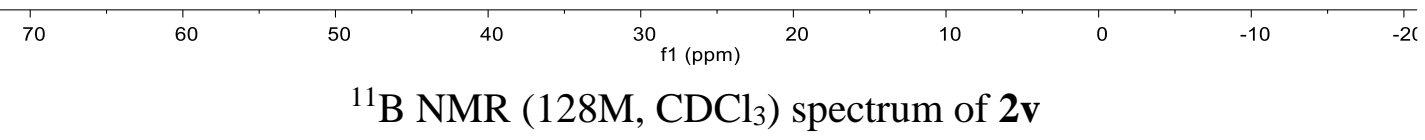




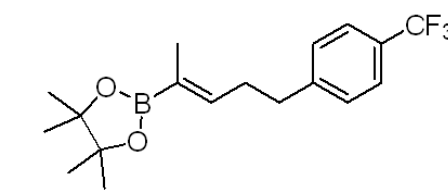

\begin{tabular}{|c|c|c|c|c|c|c|c|c|c|c|}
\hline 40 & 20 & 0 & -20 & -40 & $\begin{array}{c}-60 \\
\mathrm{f} 1(\mathrm{ppm})\end{array}$ & -80 & -100 & -120 & -140 & -160 \\
\hline
\end{tabular}

${ }^{19} \mathrm{~F} \mathrm{NMR}\left(377 \mathrm{M}, \mathrm{CDCl}_{3}\right)$ spectrum of $\mathbf{2 v}$

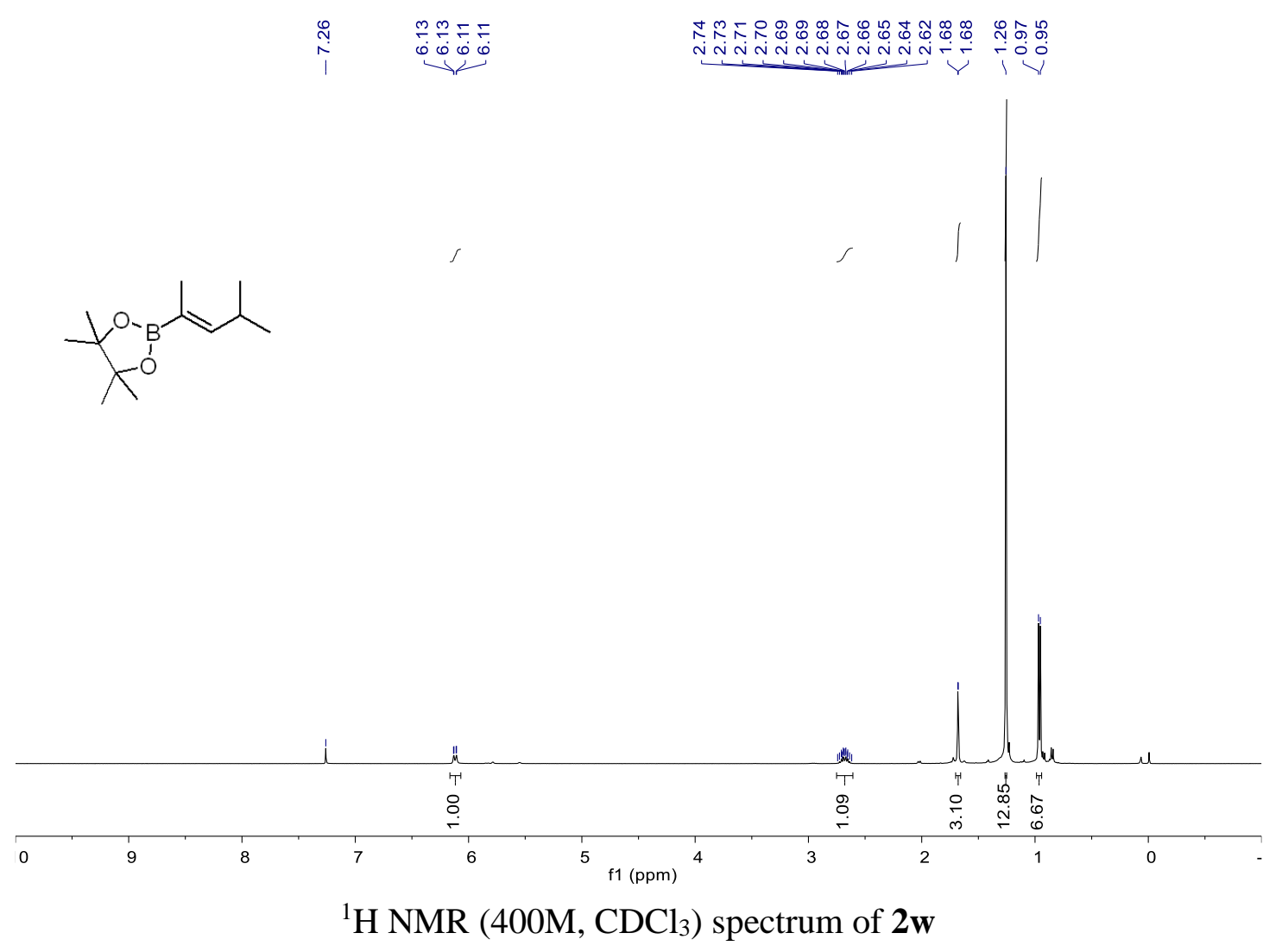



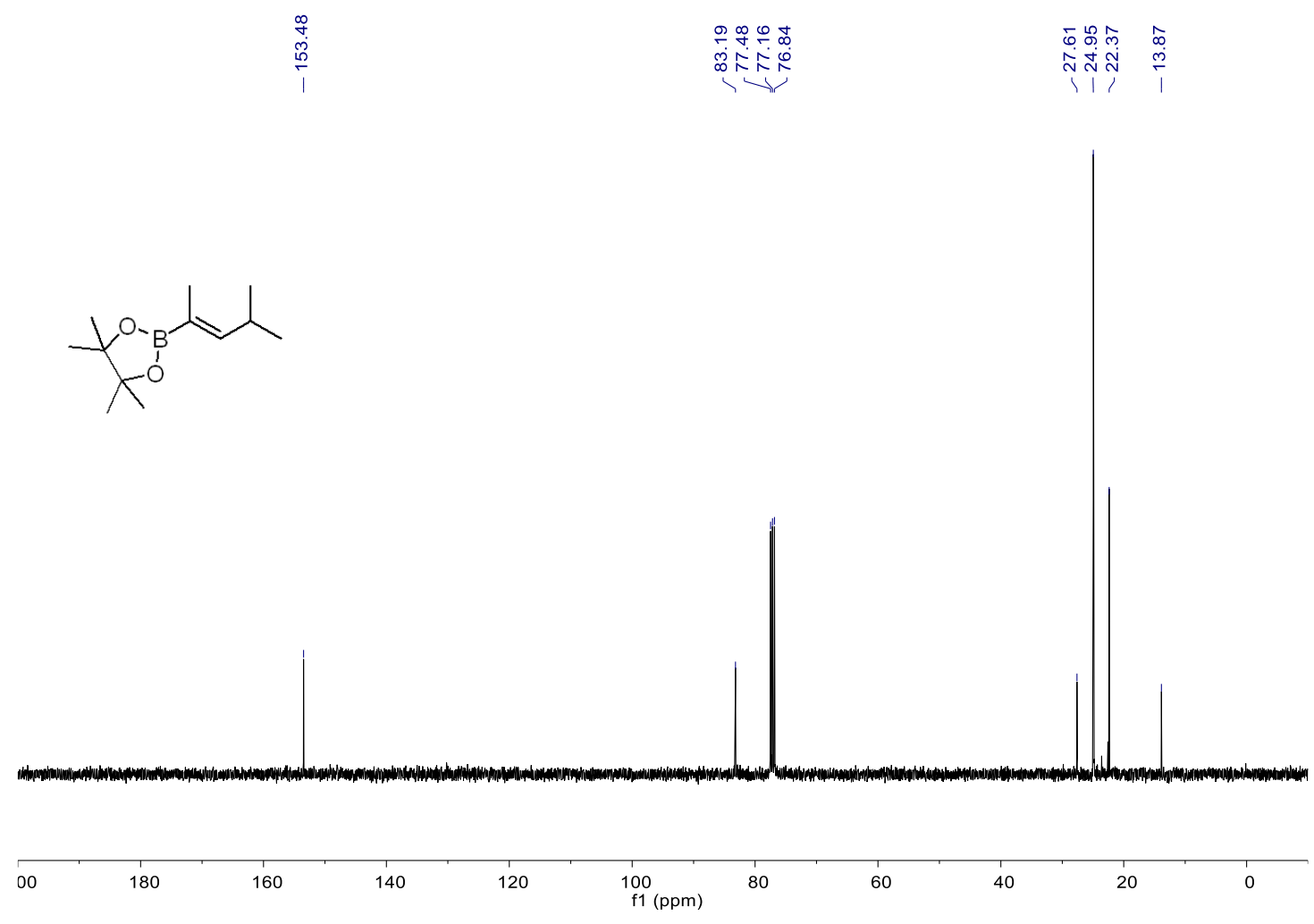

${ }^{13} \mathrm{C}$ NMR $\left(101 \mathrm{M}, \mathrm{CDCl}_{3}\right)$ spectrum of $\mathbf{2} \mathbf{w}$

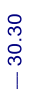
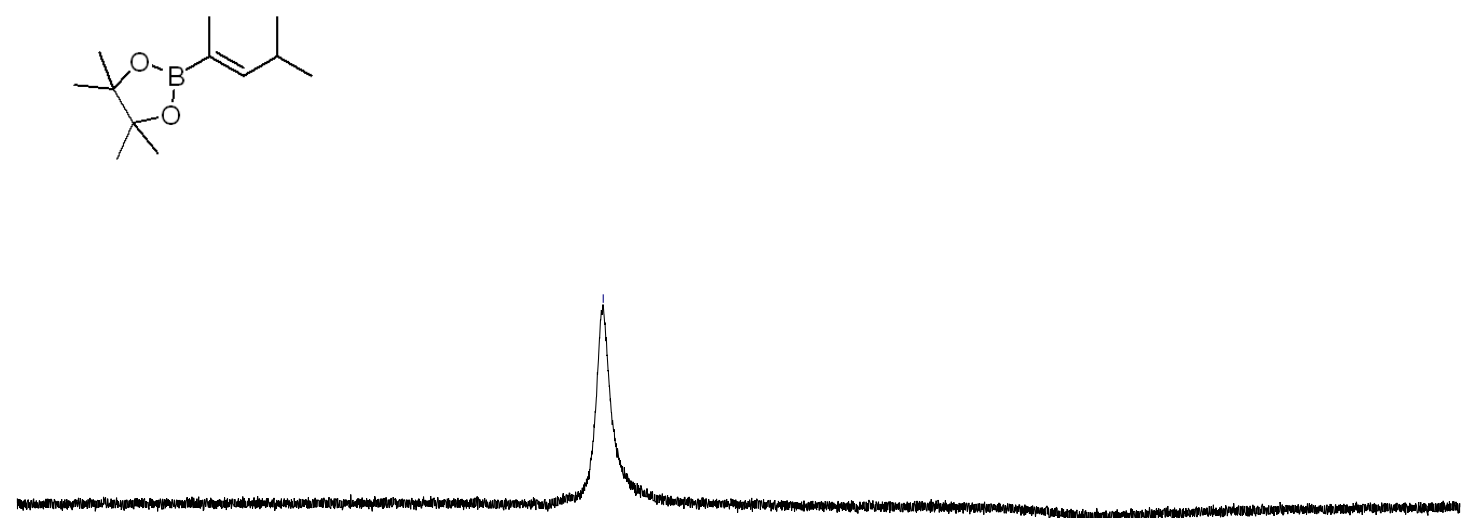

80

60

40

20 f1 (ppm)

$-20$

$-40$

$-60$

${ }^{11} \mathrm{~B}$ NMR $\left(128 \mathrm{M}, \mathrm{CDCl}_{3}\right)$ spectrum of $\mathbf{2 w}$ 


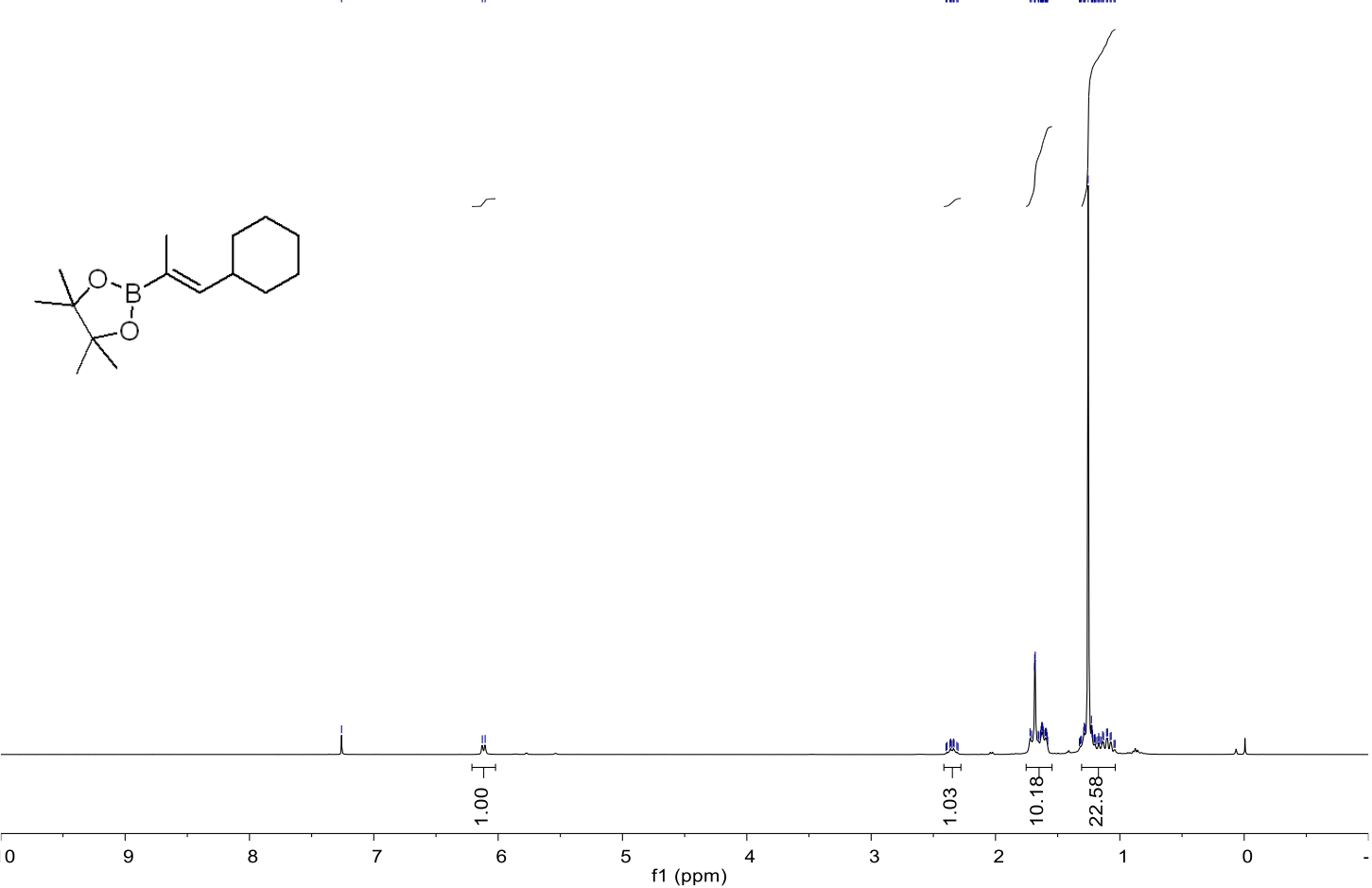

${ }^{1} \mathrm{H}$ NMR (400M, $\mathrm{CDCl}_{3}$ ) spectrum of $\mathbf{2 x}$
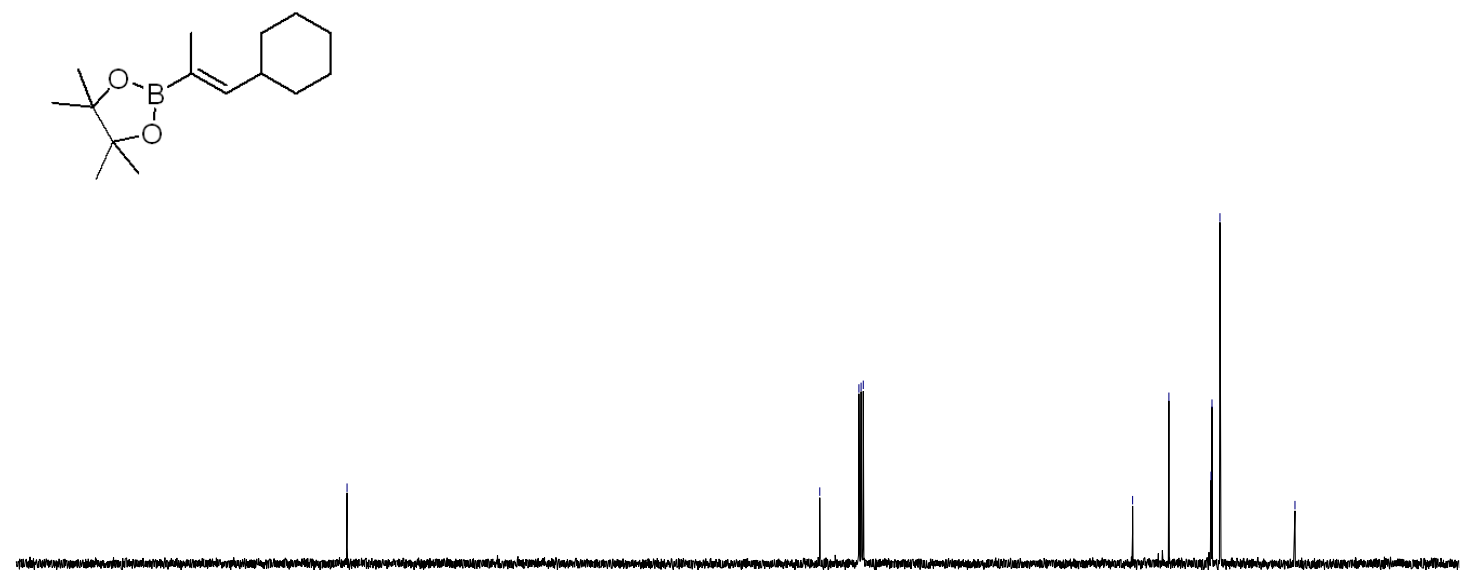

00

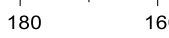

$160 \quad 140$

120

100
$\mathrm{f} 1(\mathrm{ppm})$

80

60

40

20

${ }^{13} \mathrm{C} \mathrm{NMR}\left(101 \mathrm{M}, \mathrm{CDCl}_{3}\right)$ spectrum of $\mathbf{2 x}$ 
<smiles>CC(=CC1CCCCC1)B1OC(C)(C)C(C)(C)O1</smiles>

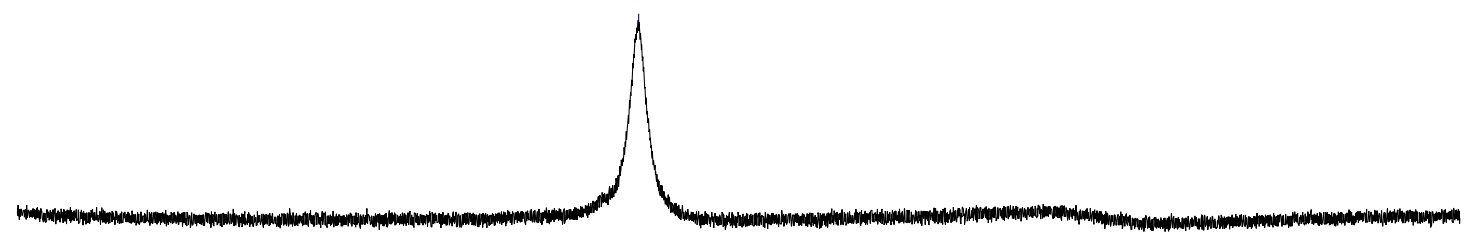

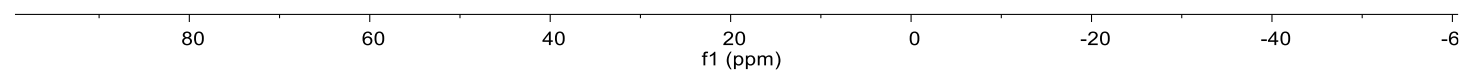

${ }^{11} \mathrm{~B}$ NMR $\left(128 \mathrm{M}, \mathrm{CDCl}_{3}\right)$ spectrum of $\mathbf{2 x}$

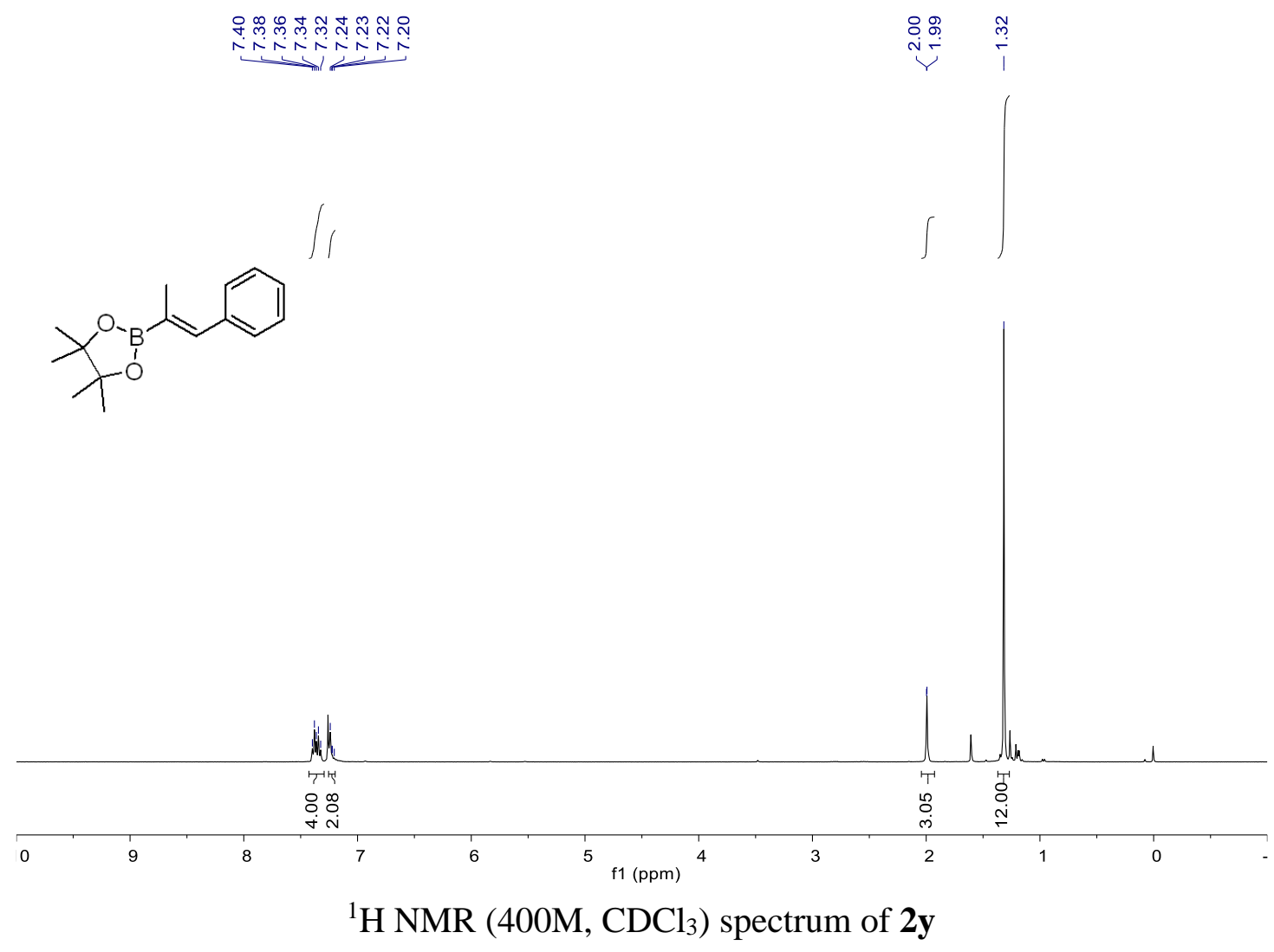



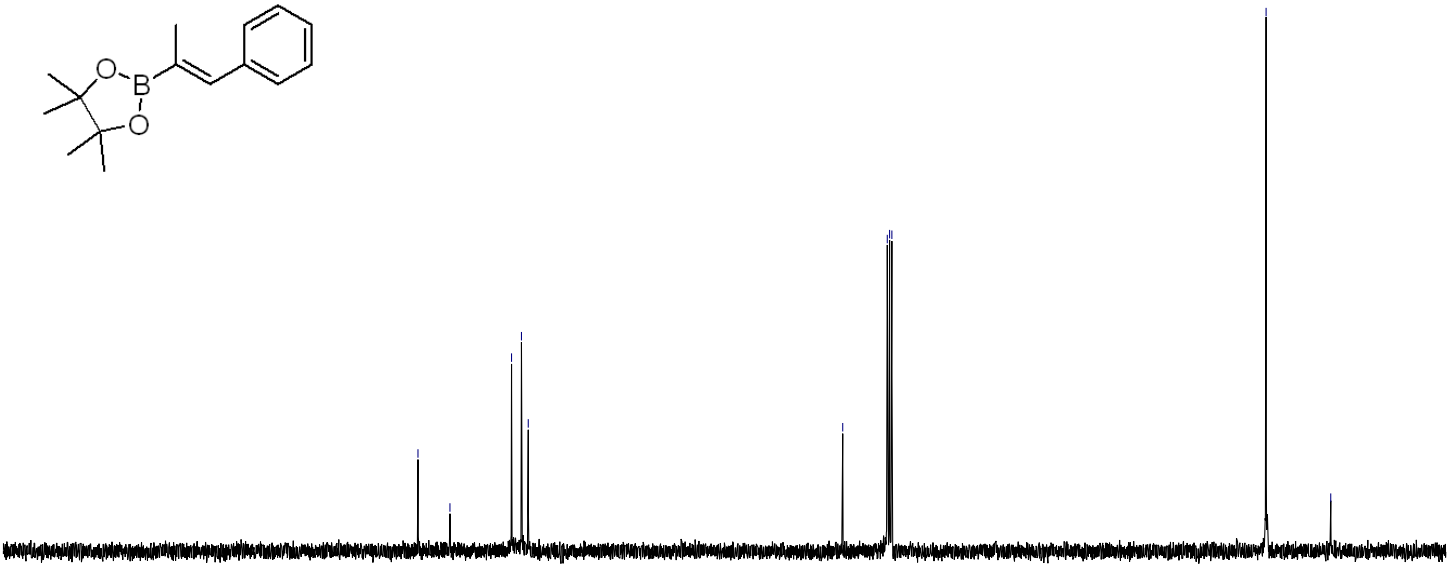

00 180 160 140 120 100
$\mathrm{f} 1(\mathrm{ppm})$ $80 \quad 60$

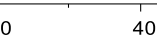

40 20

${ }^{13} \mathrm{C}$ NMR $\left(101 \mathrm{M}, \mathrm{CDCl}_{3}\right)$ spectrum of $\mathbf{2 y}$

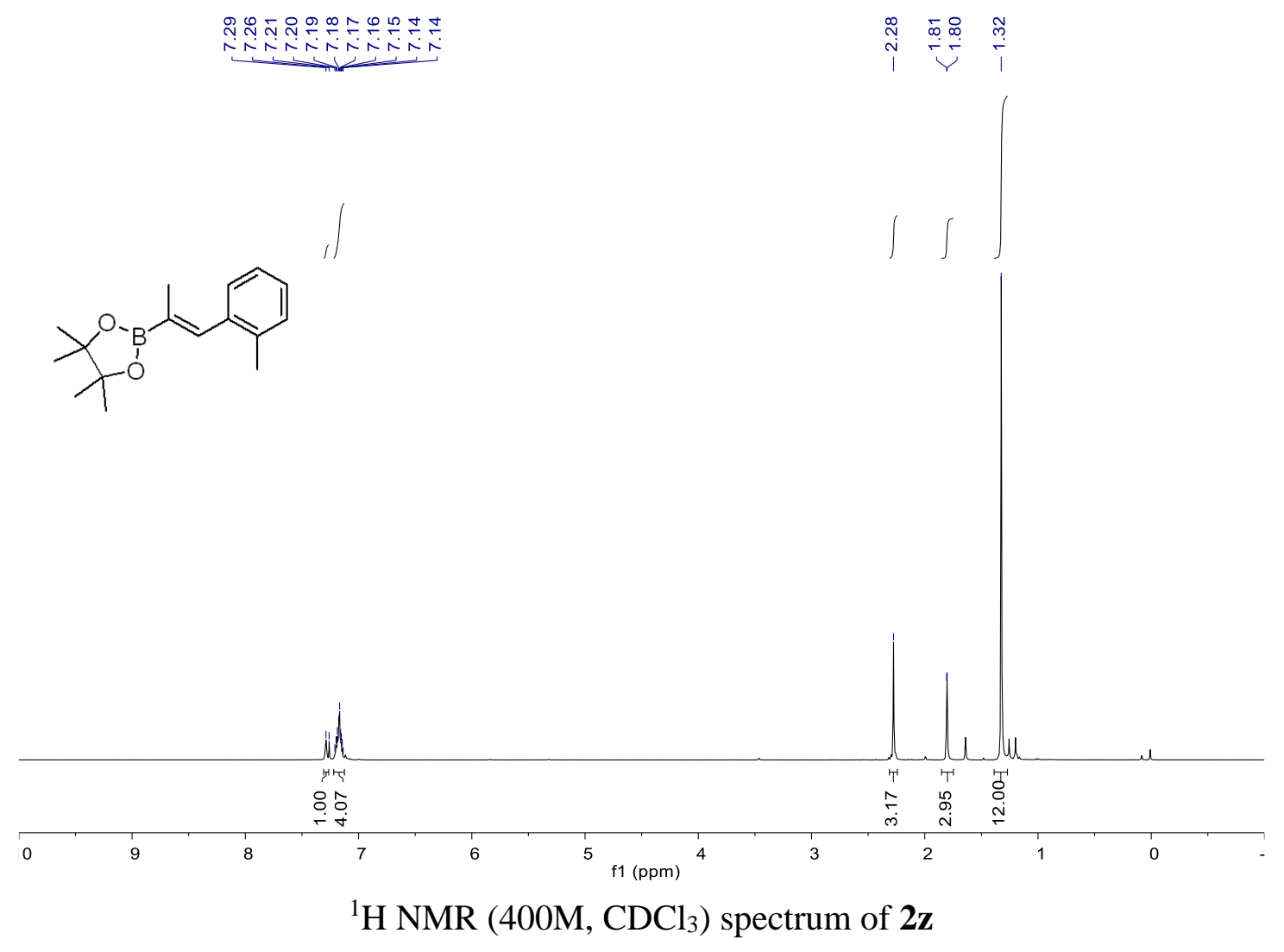



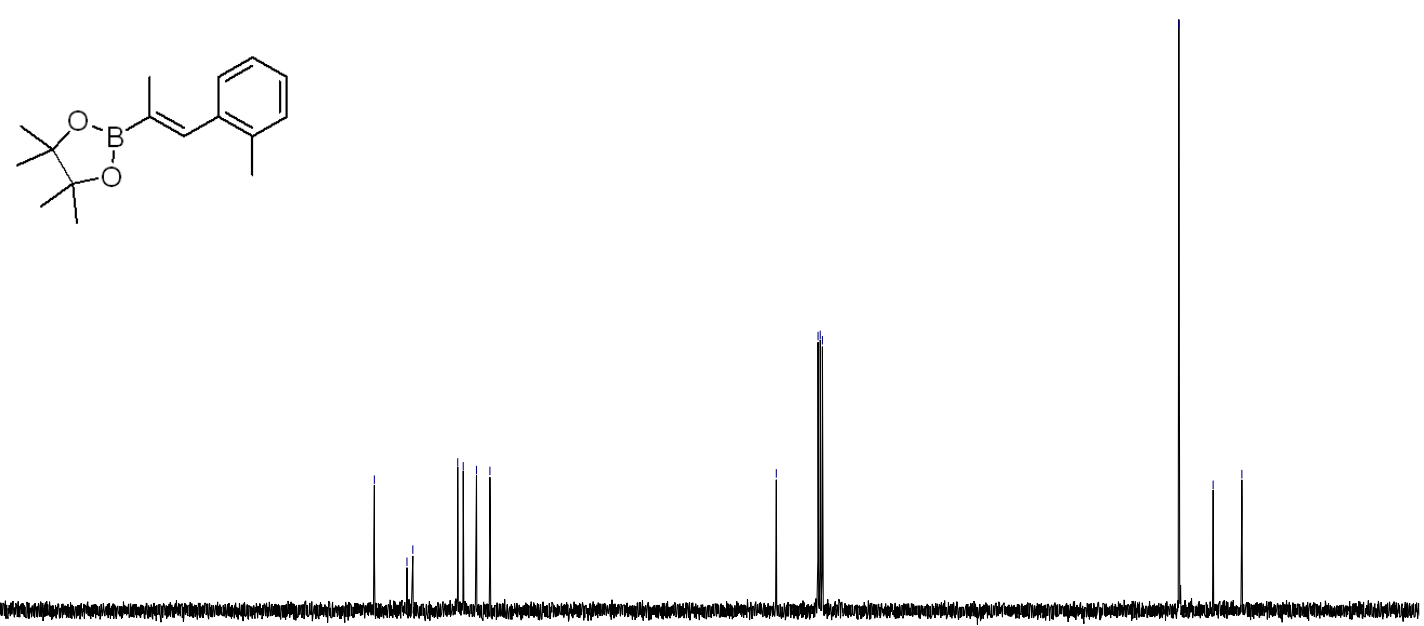

${ }^{13} \mathrm{C} \mathrm{NMR}\left(101 \mathrm{M}, \mathrm{CDCl}_{3}\right)$ spectrum of $\mathbf{2 z}$

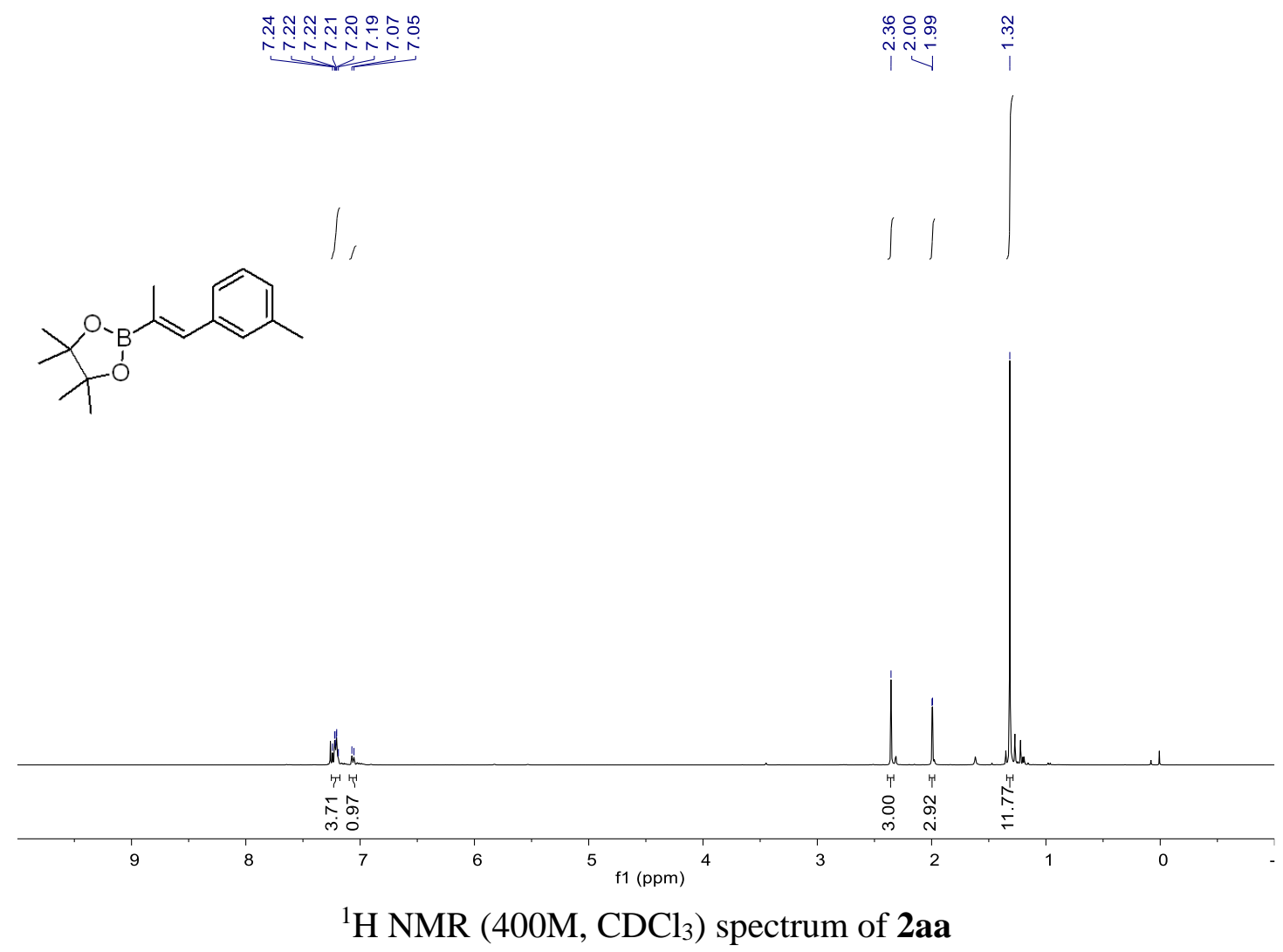


<smiles>C/C(=C\c1cccc(C)c1)B1OC(C)(C)C(C)(C)O1</smiles>

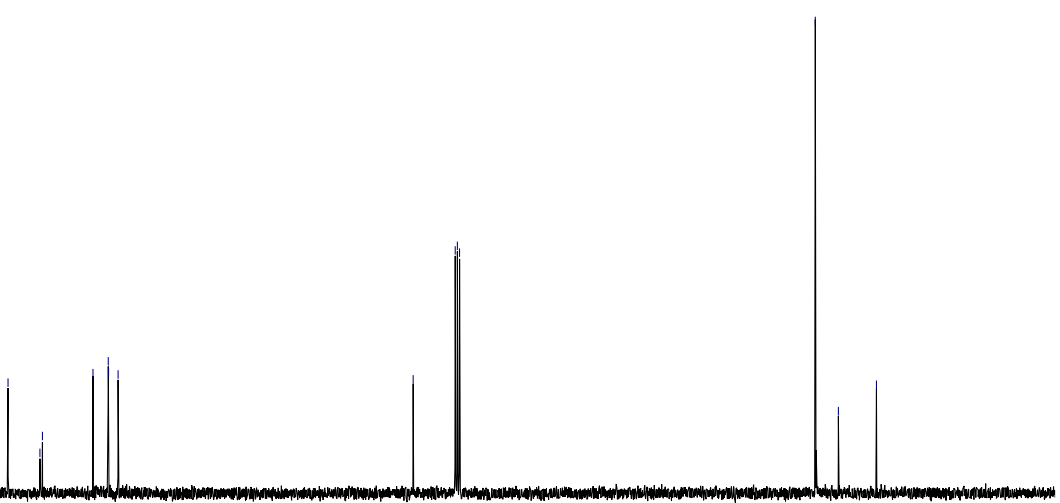

oo

180

160

140

120

100

$80 \quad 60$

40

20

${ }^{13} \mathrm{C}$ NMR (101M, $\mathrm{CDCl}_{3}$ ) spectrum of 2aa

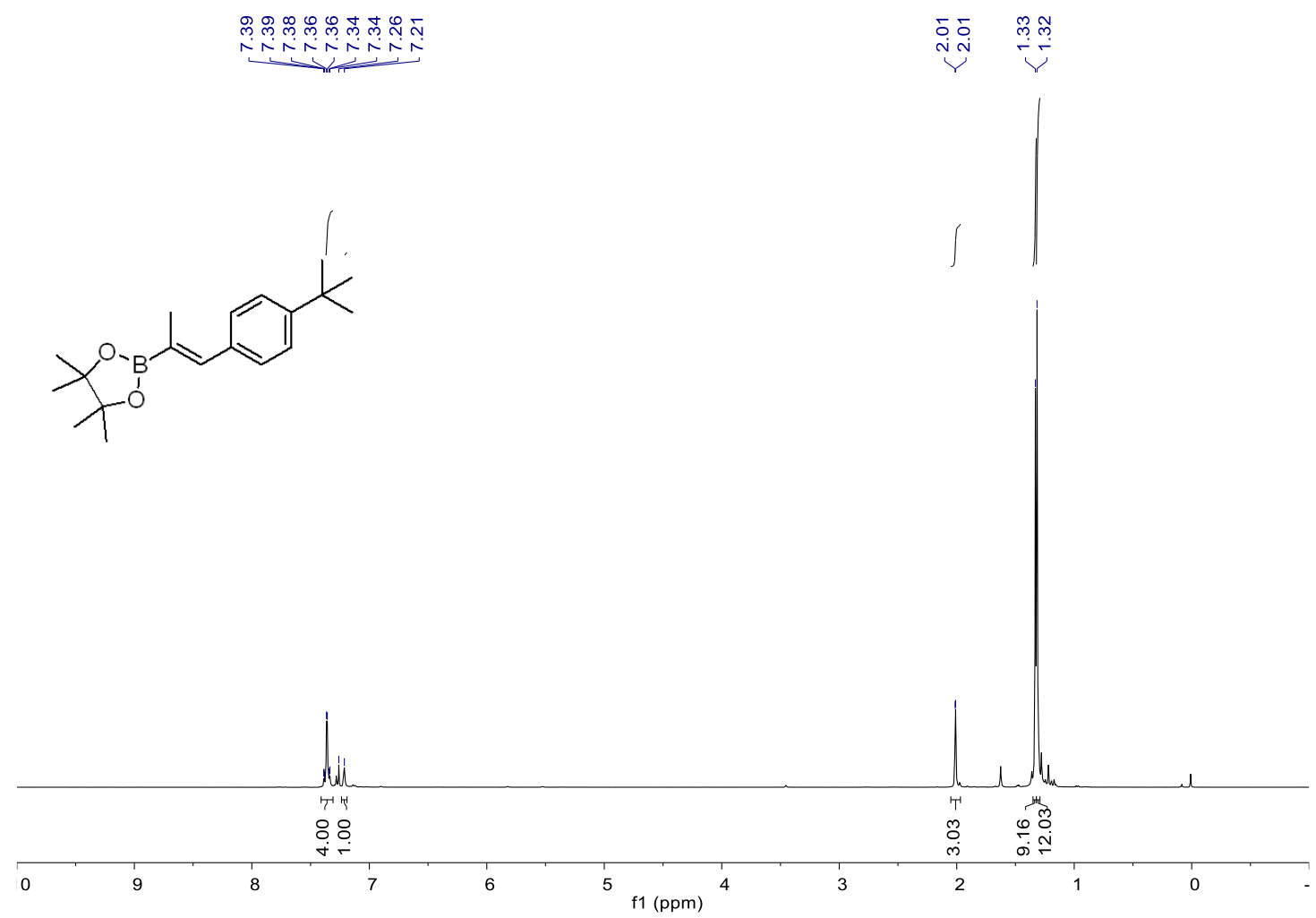

${ }^{1} \mathrm{H}$ NMR (400M, $\mathrm{CDCl}_{3}$ ) spectrum of $\mathbf{2 a b}$ 

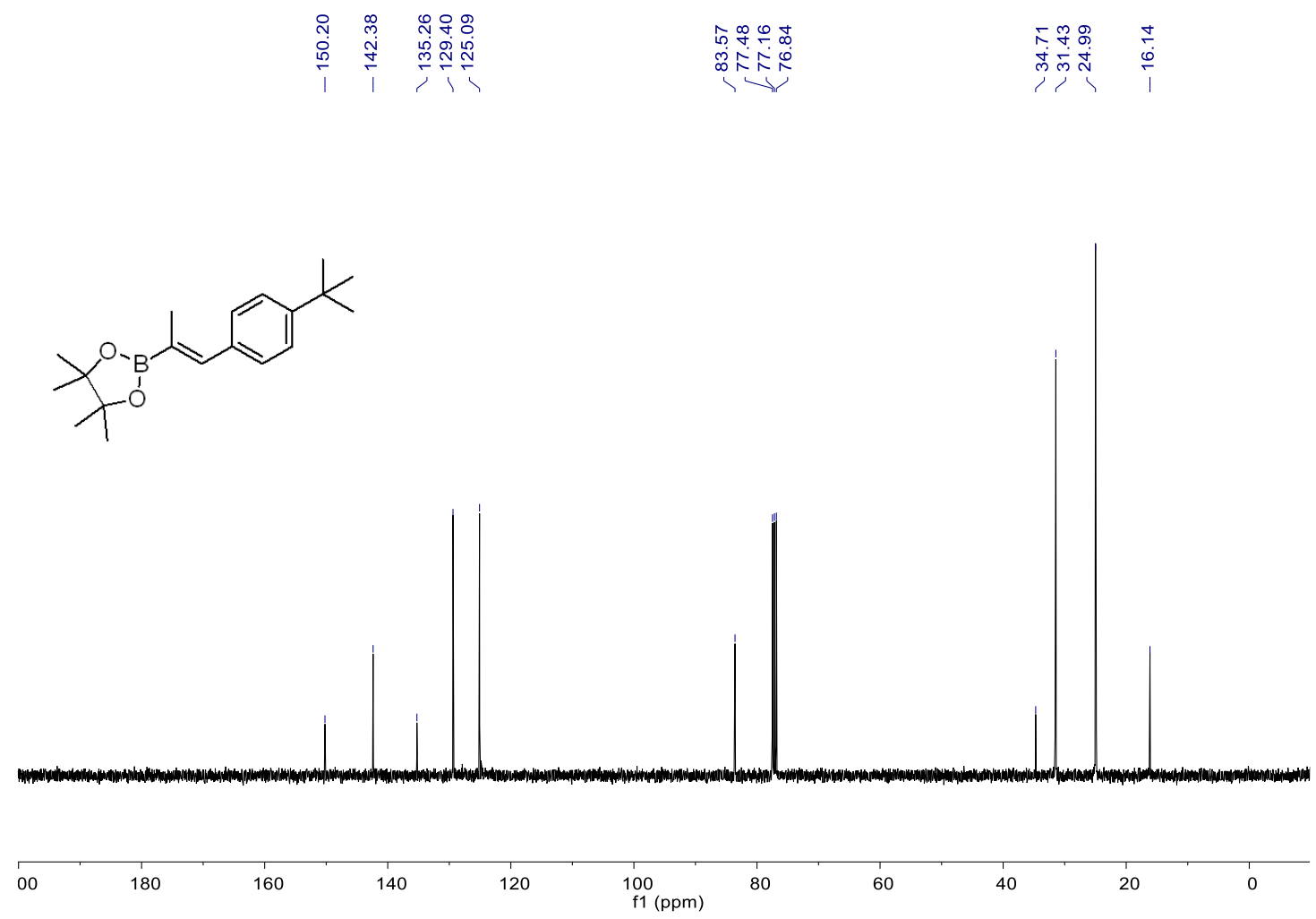

${ }^{13} \mathrm{C}$ NMR $\left(101 \mathrm{M}, \mathrm{CDCl}_{3}\right)$ spectrum of $\mathbf{2 a b}$

$\frac{5}{\check{m}}$<smiles>C/C(=C\c1ccc(C(C)(C)C)cc1)B1OC(C)(C)C(C)(C)O1</smiles>

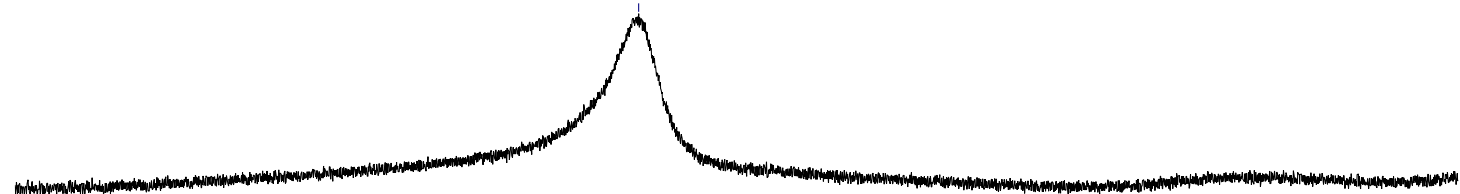

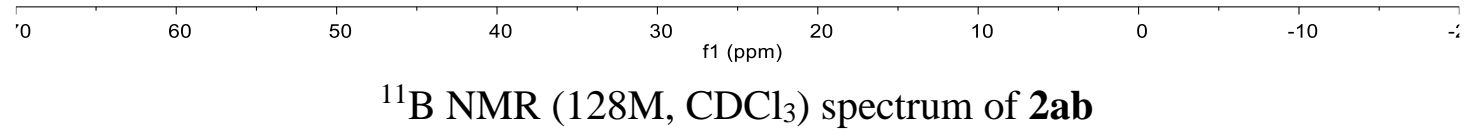




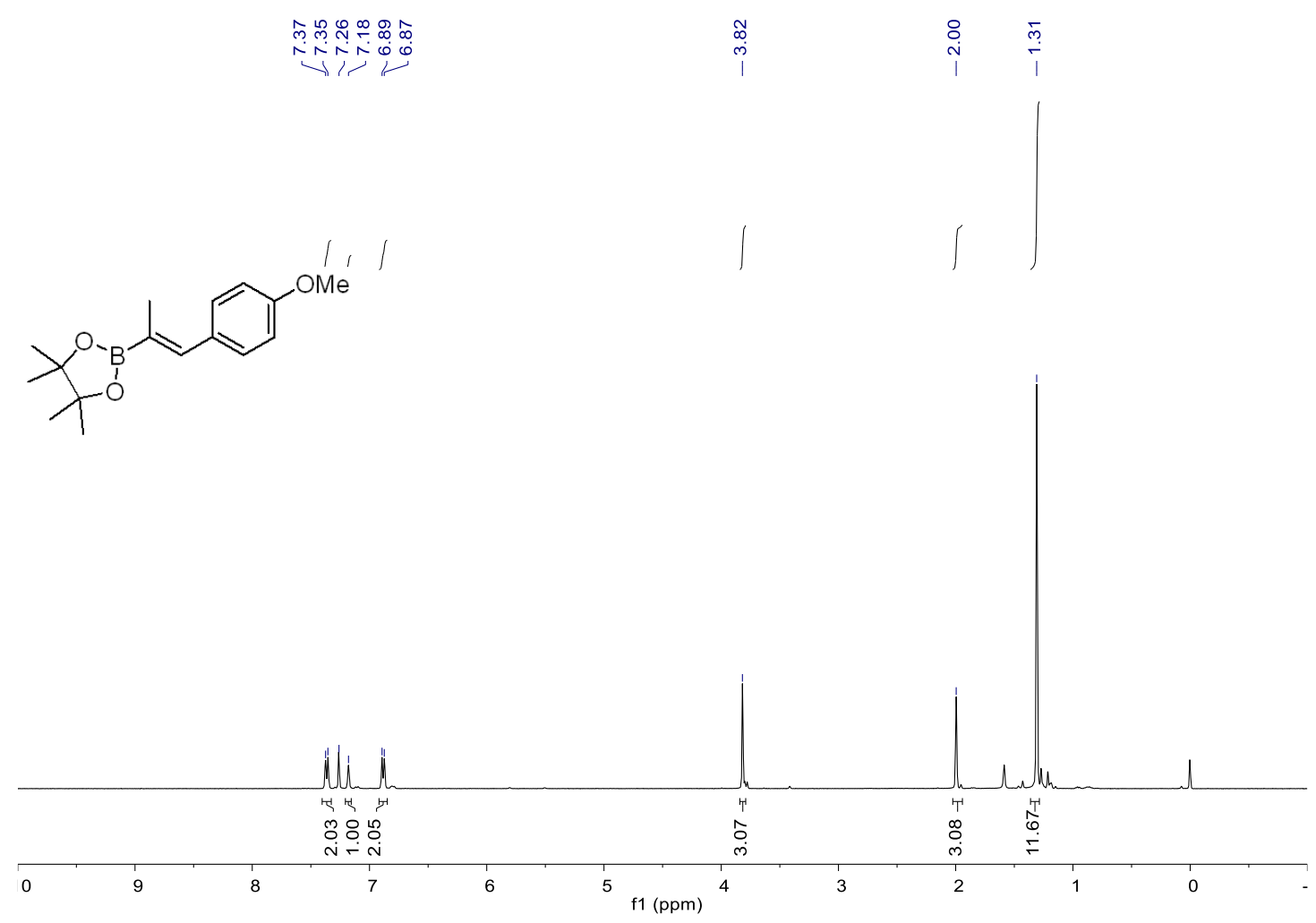

${ }^{1} \mathrm{H}$ NMR $\left(400 \mathrm{M}, \mathrm{CDCl}_{3}\right)$ spectrum of $\mathbf{2 a c}$

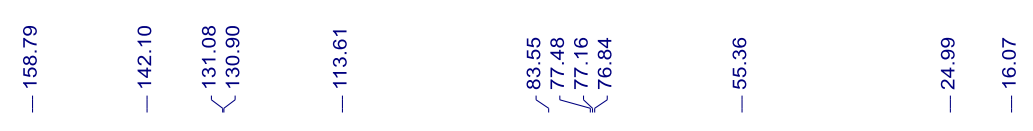<smiles>COc1ccc(/C=C(\C)B2OC(C)(C)C(C)(C)O2)cc1</smiles>

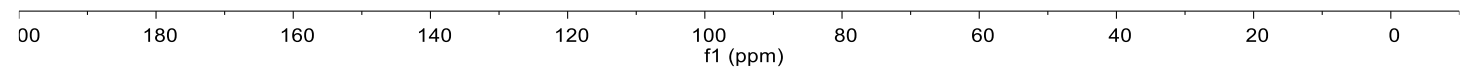

${ }^{13} \mathrm{C} \mathrm{NMR}\left(101 \mathrm{M}, \mathrm{CDCl}_{3}\right)$ spectrum of $\mathbf{2 a c}$ 

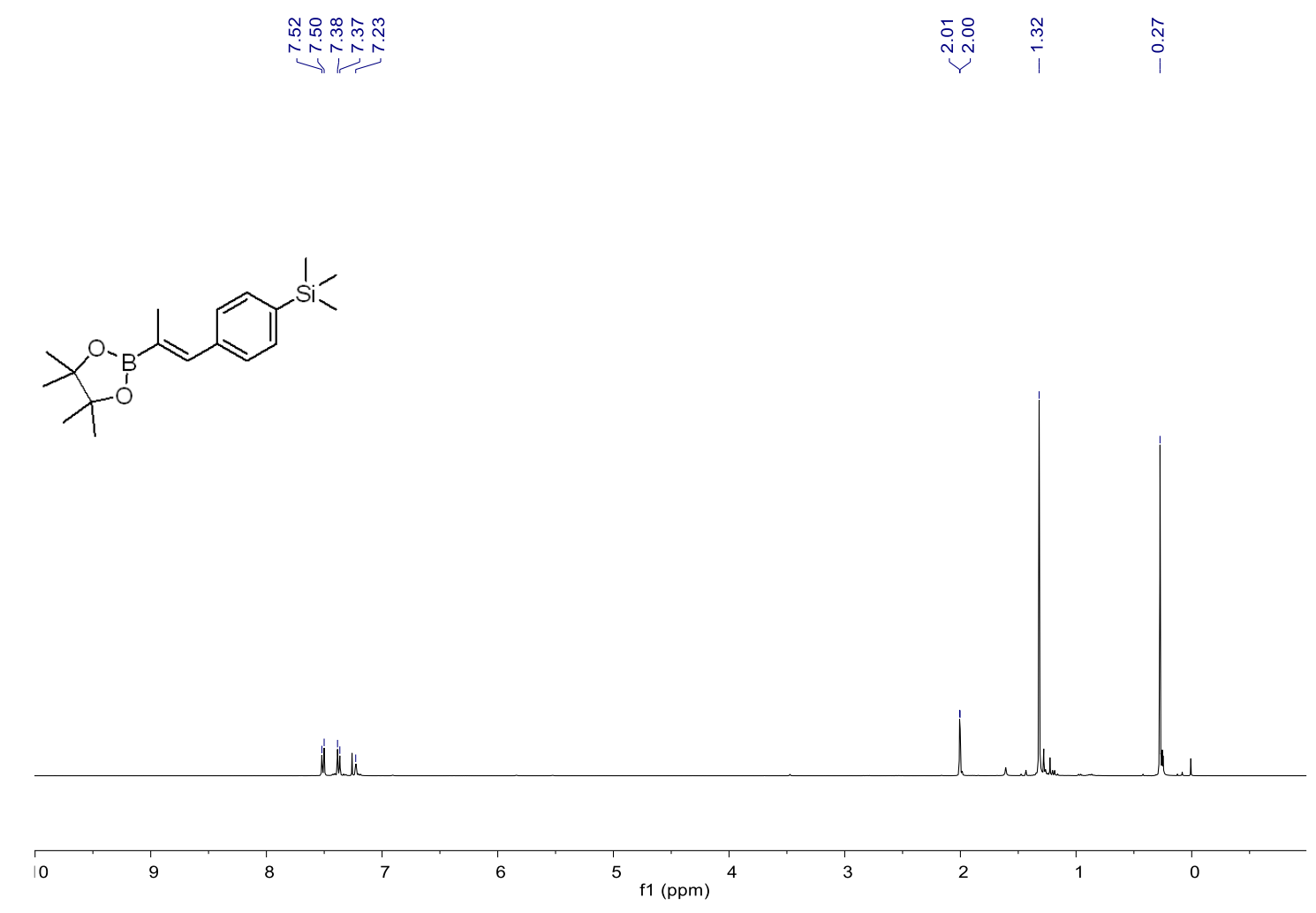

${ }^{1} \mathrm{H}$ NMR (400M, $\mathrm{CDCl}_{3}$ ) spectrum of $\mathbf{2 a d}$
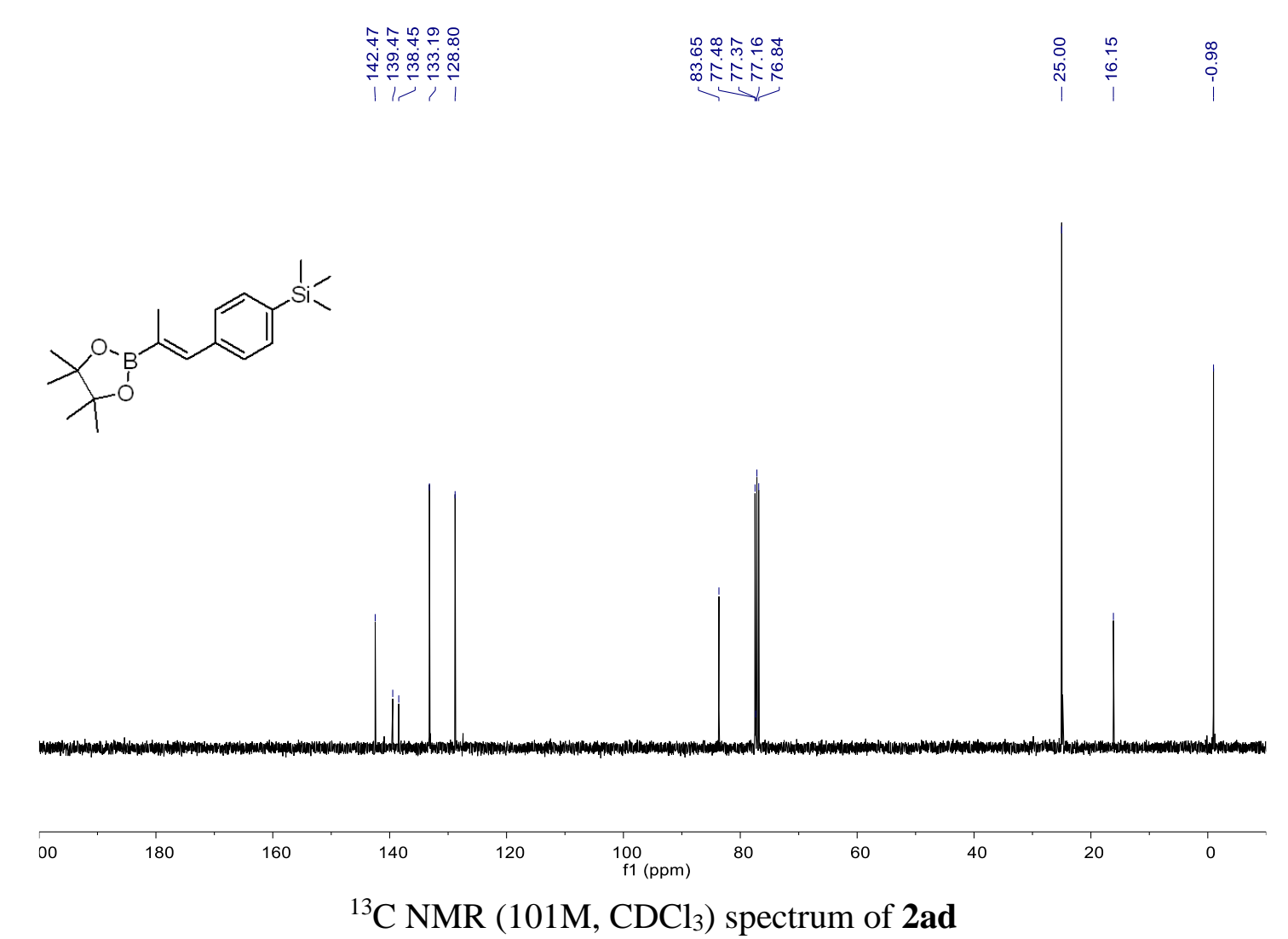
<smiles>C/C(=C\c1ccc([Si](C)(C)C)cc1)B1OC(C)(C)C(C)(C)O1</smiles>
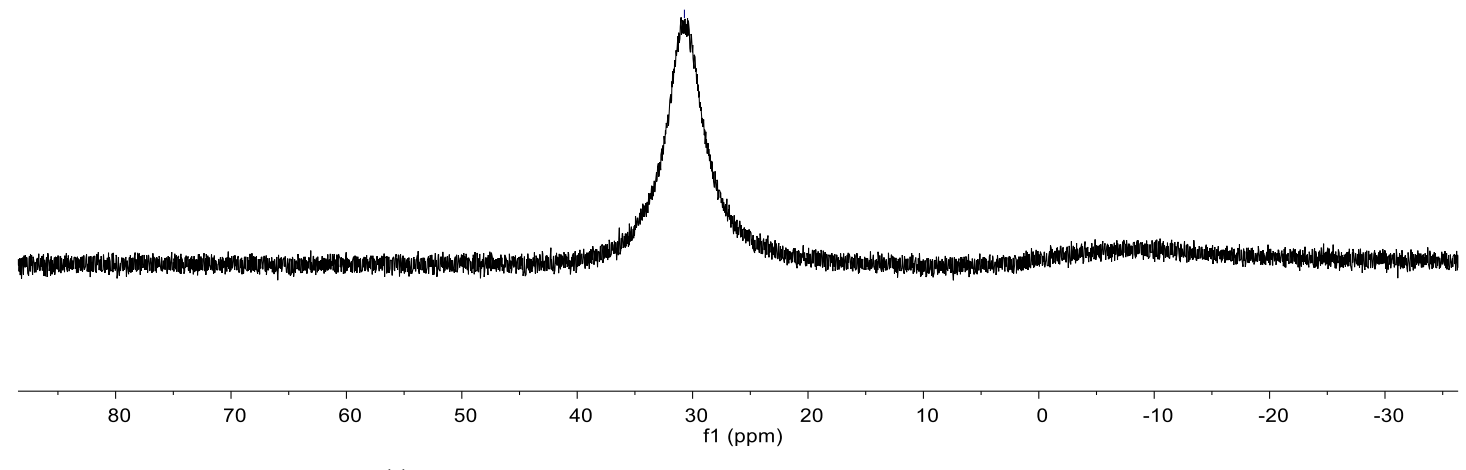

${ }^{11}$ B NMR (128M, $\left.\mathrm{CDCl}_{3}\right)$ spectrum of $\mathbf{2 a d}$

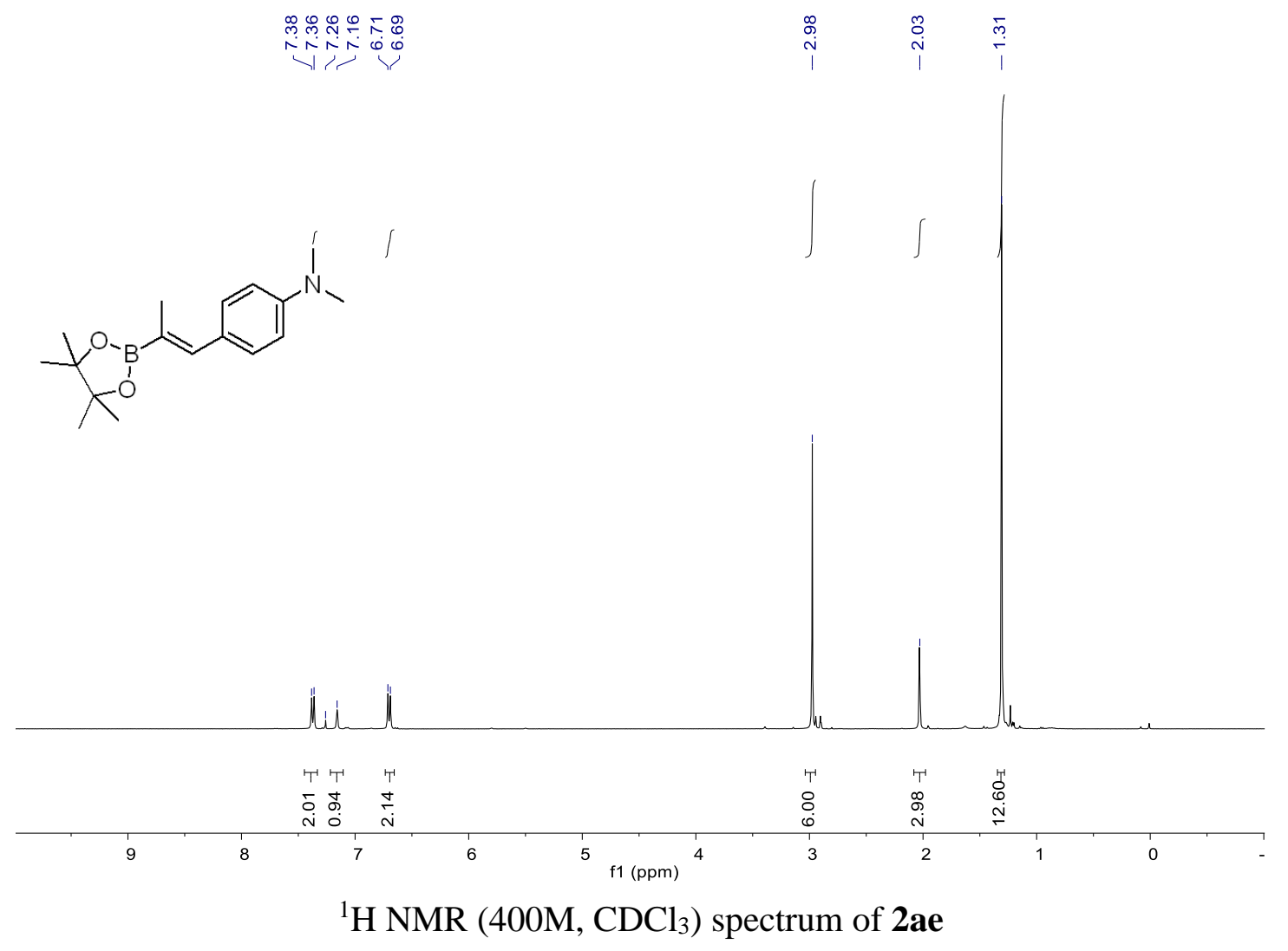


<smiles>C/C(=C\c1ccc(N(C)C)cc1)B1OC(C)(C)C(C)(C)O1</smiles>
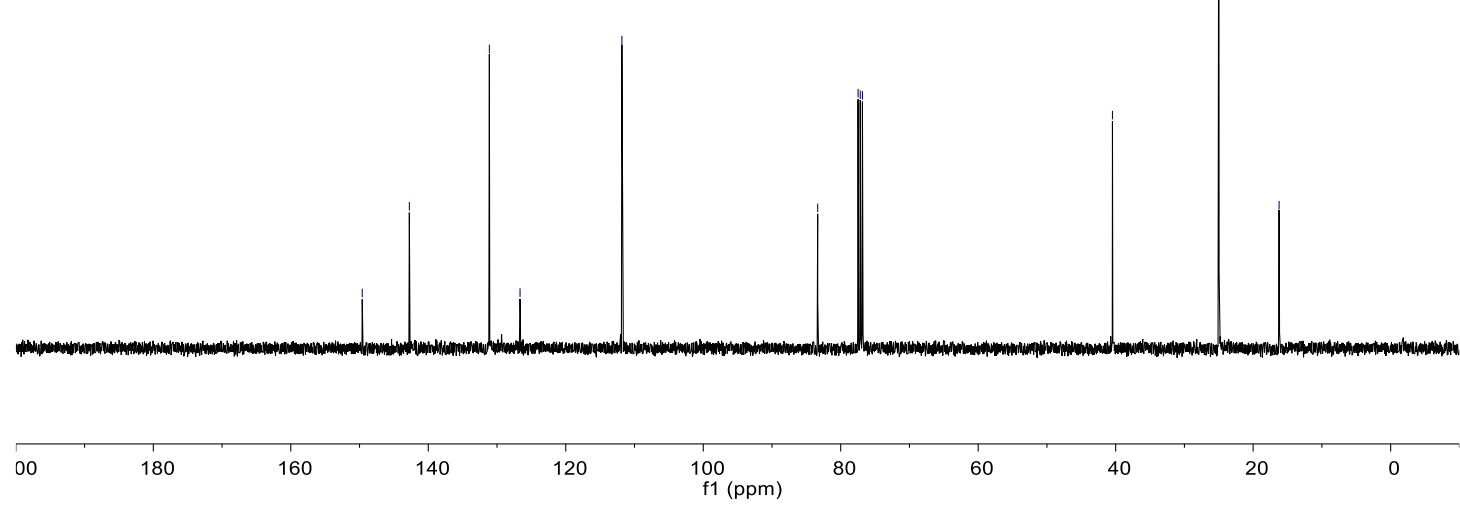

${ }^{13} \mathrm{C} \mathrm{NMR}\left(101 \mathrm{M}, \mathrm{CDCl}_{3}\right)$ spectrum of $\mathbf{2 a e}$<smiles>C/C(=C\c1ccc(N(C)C)cc1)B1OC(C)(C)C(C)(C)O1</smiles>

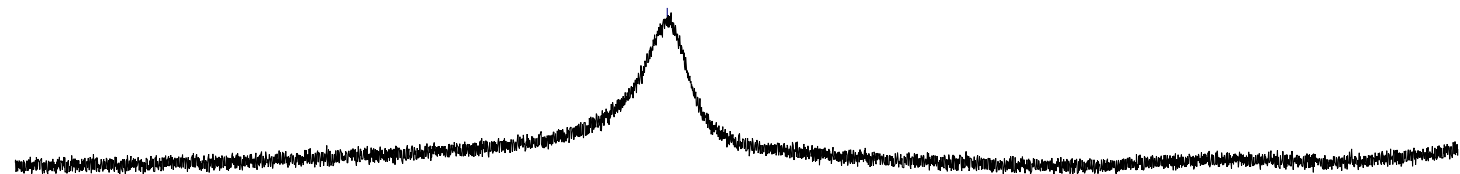

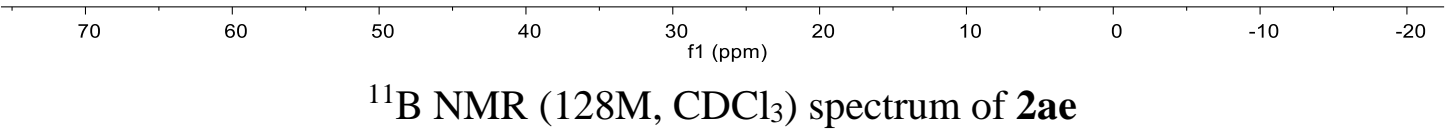




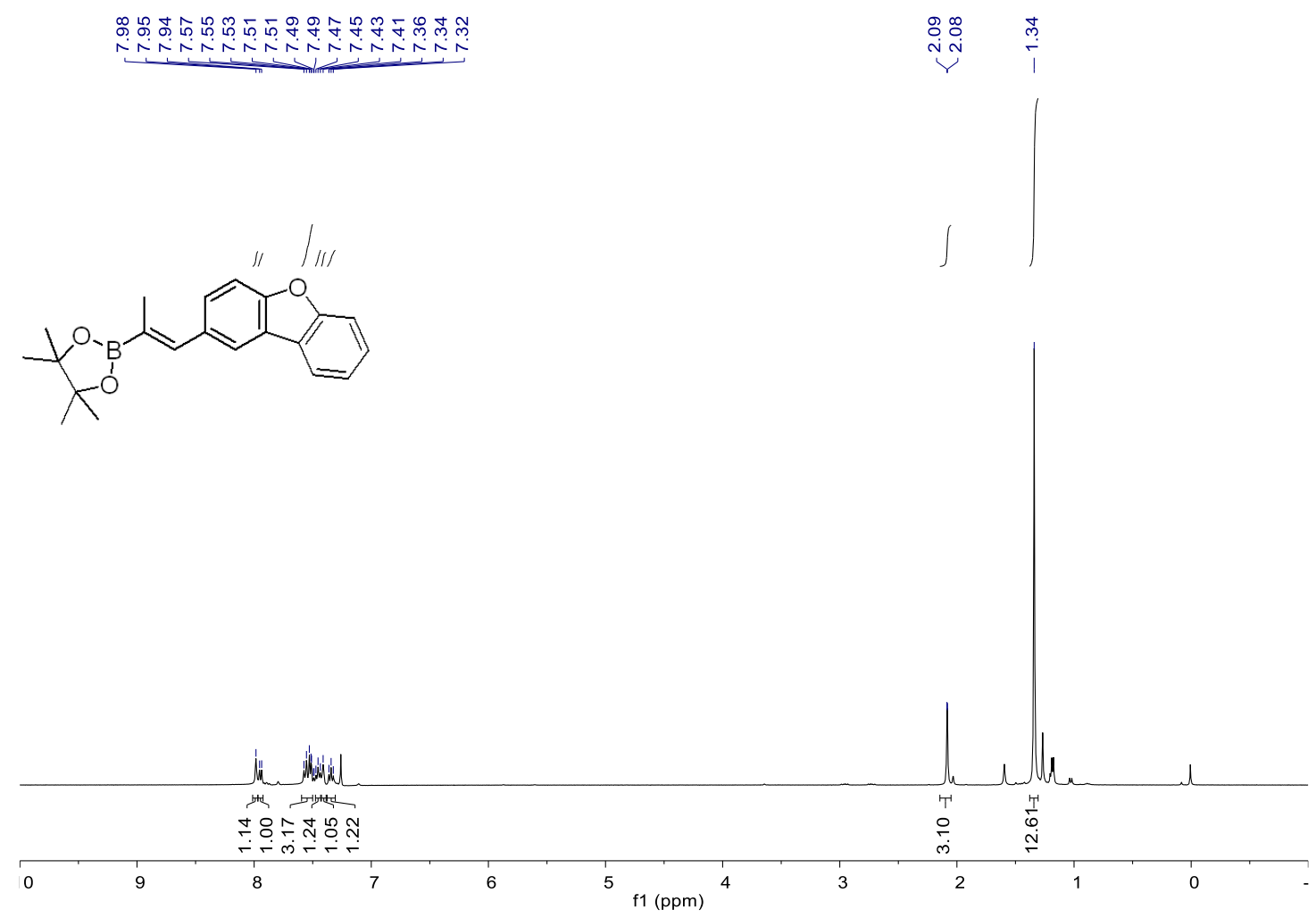

${ }^{1} \mathrm{H}$ NMR (400M, $\mathrm{CDCl}_{3}$ ) spectrum of $\mathbf{2 a f}$
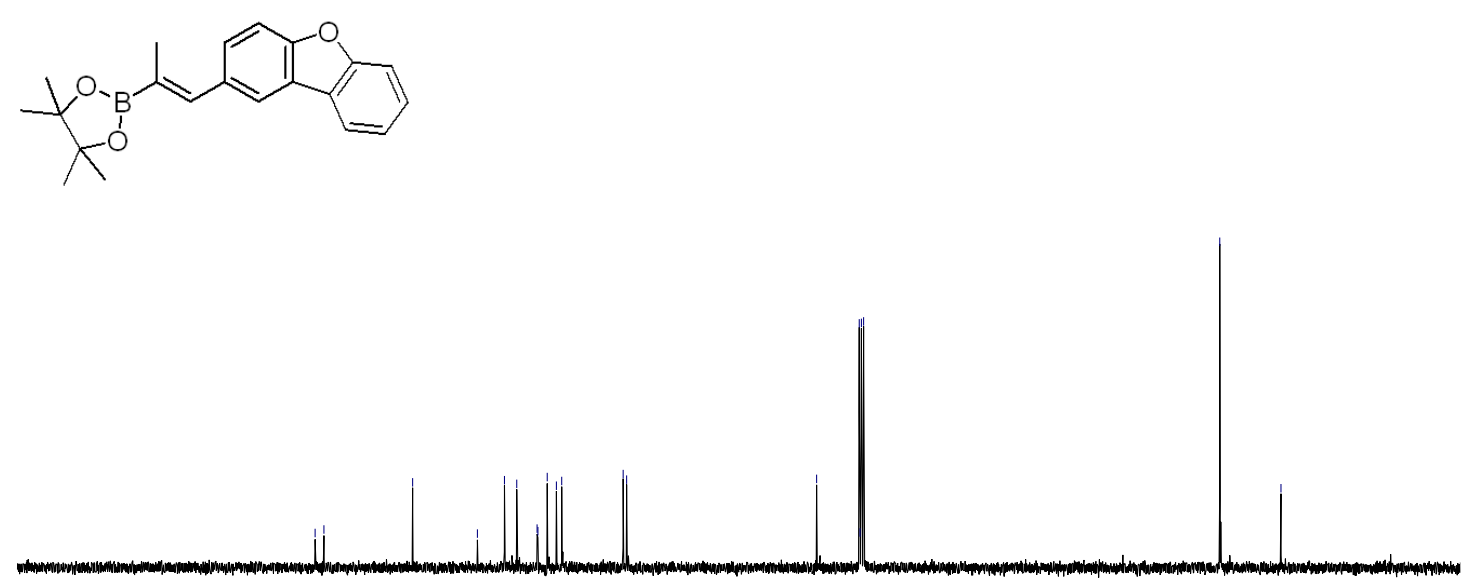

\begin{tabular}{|c|c|c|c|c|c|c|c|c|c|c|}
\hline 00 & 180 & 160 & 140 & 120 & $\begin{array}{l}100 \\
\mathrm{f} 1(\mathrm{ppm})\end{array}$ & 80 & 60 & 40 & 20 & 0 \\
\hline
\end{tabular}



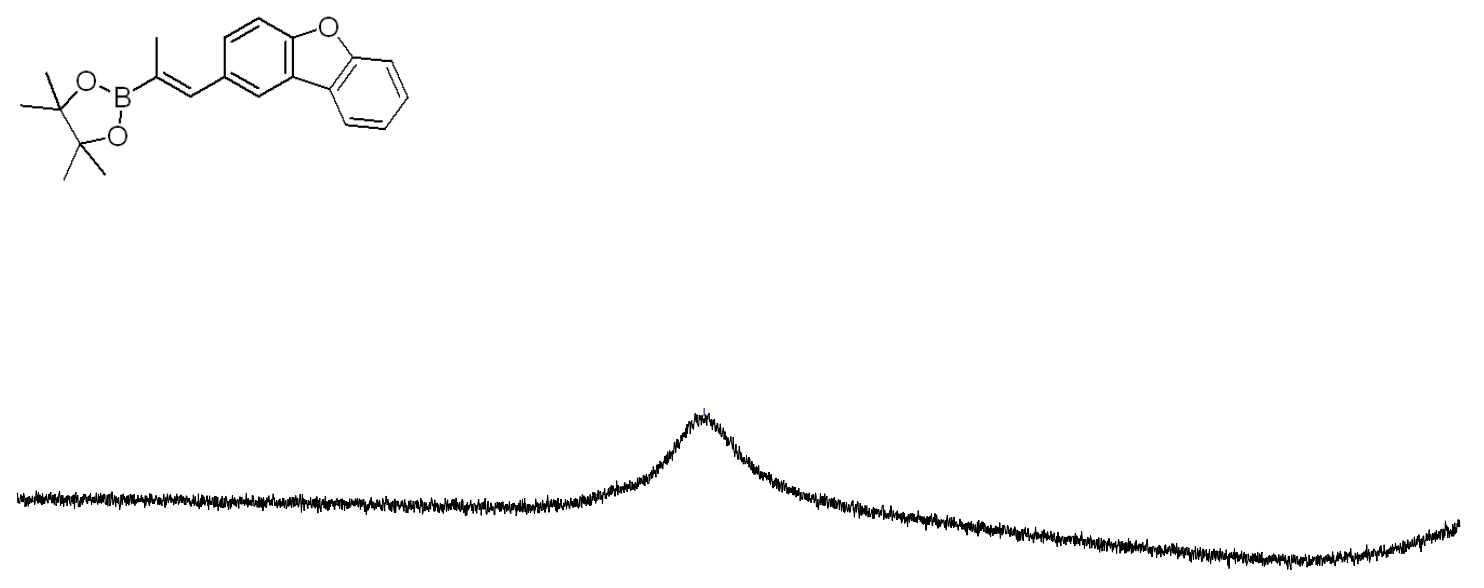

\begin{tabular}{rrrrr}
\hline 50 & 40 & 30 & 1 & 10 \\
\end{tabular}

${ }^{11} \mathrm{~B}$ NMR $\left(128 \mathrm{M}, \mathrm{CDCl}_{3}\right)$ spectrum of $\mathbf{2} \mathbf{a f}$

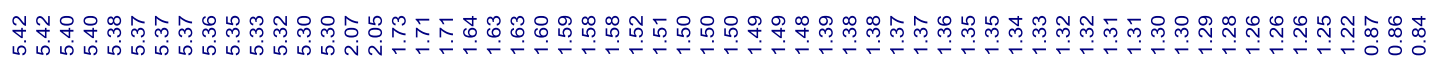<smiles>CC=CC(CCCCC)B1OC(C)(C)C(C)(C)O1</smiles>

$E / Z=1.3: 1$
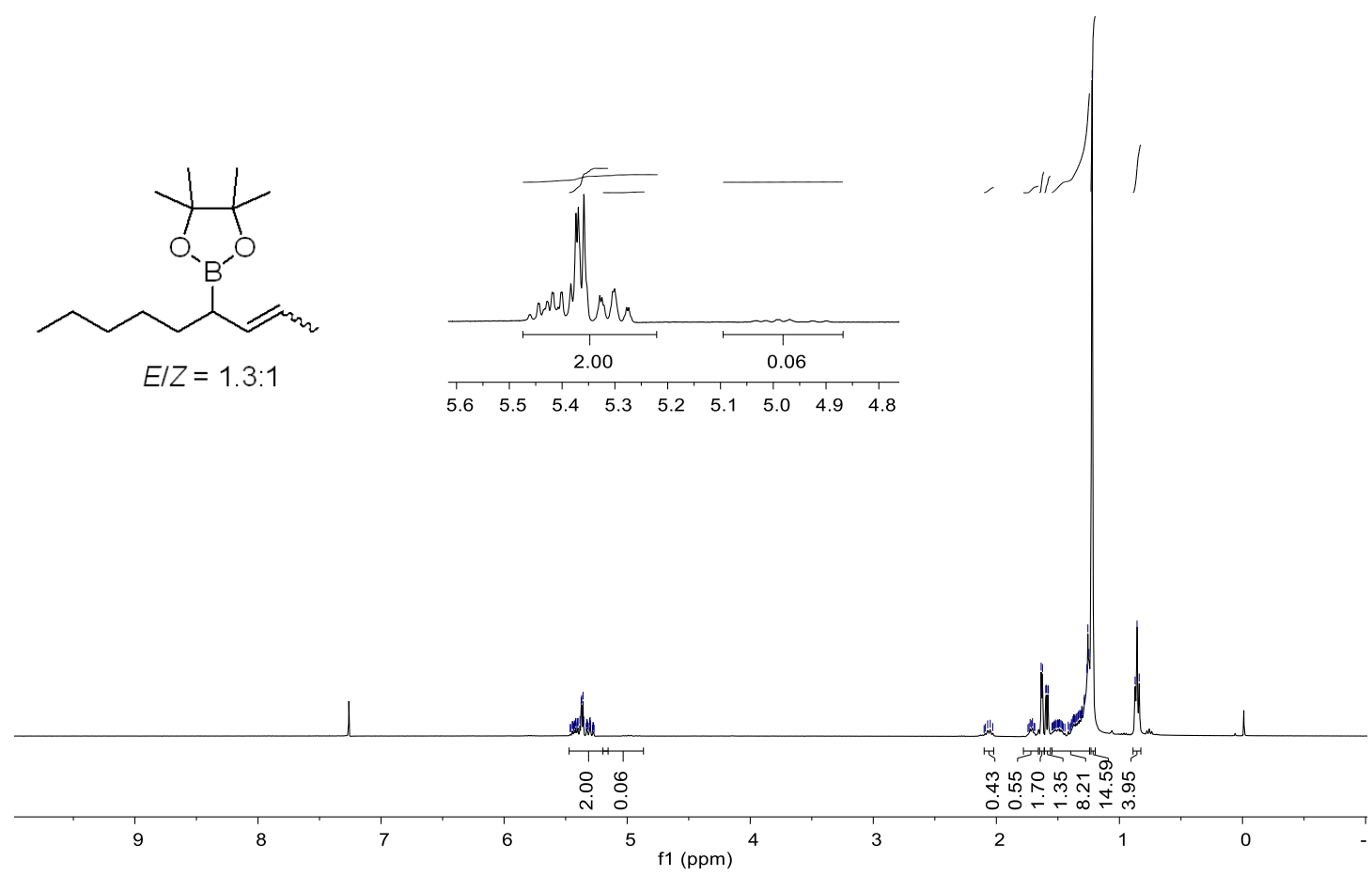

${ }^{1} \mathrm{H}$ NMR (400M, $\left.\mathrm{CDCl}_{3}\right)$ spectrum of $\mathbf{2 a j}$ 


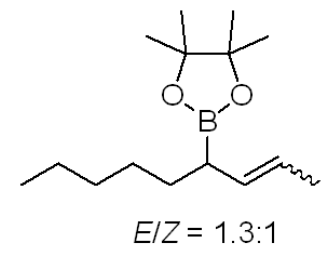

$E / Z=1.3: 1$
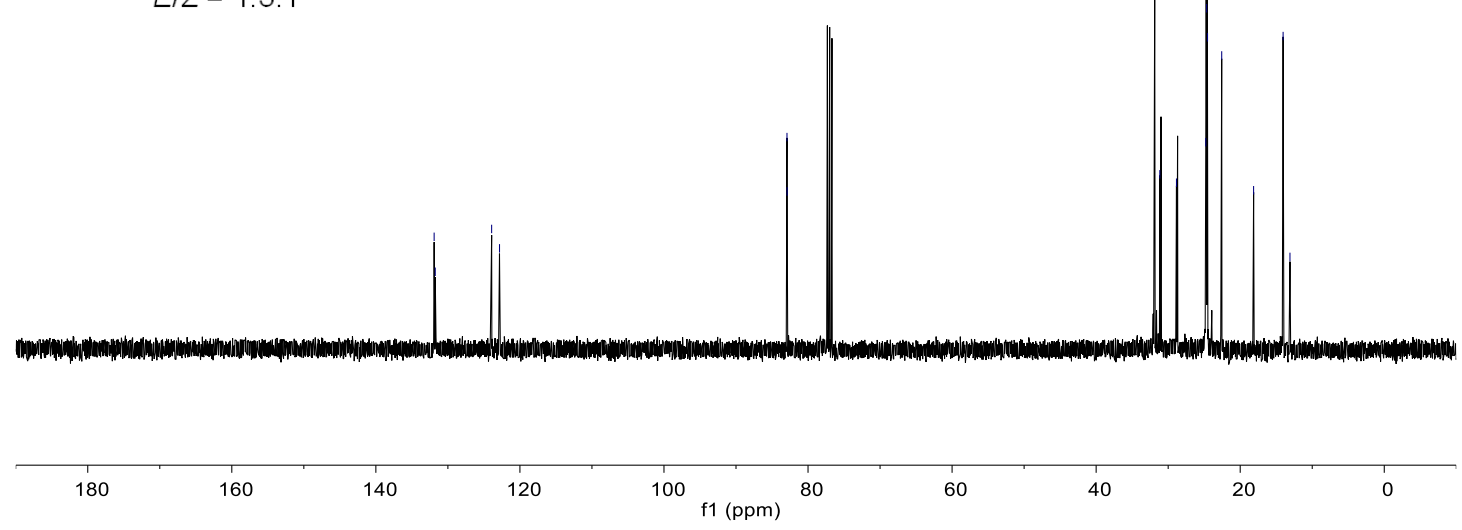

${ }^{13} \mathrm{C} \mathrm{NMR}\left(101 \mathrm{M}, \mathrm{CDCl}_{3}\right)$ spectrum of $\mathbf{2 a j}$

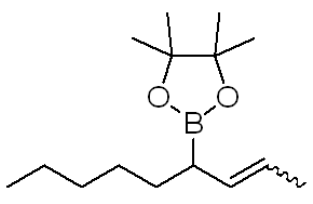

$E / Z=1.3: 1$

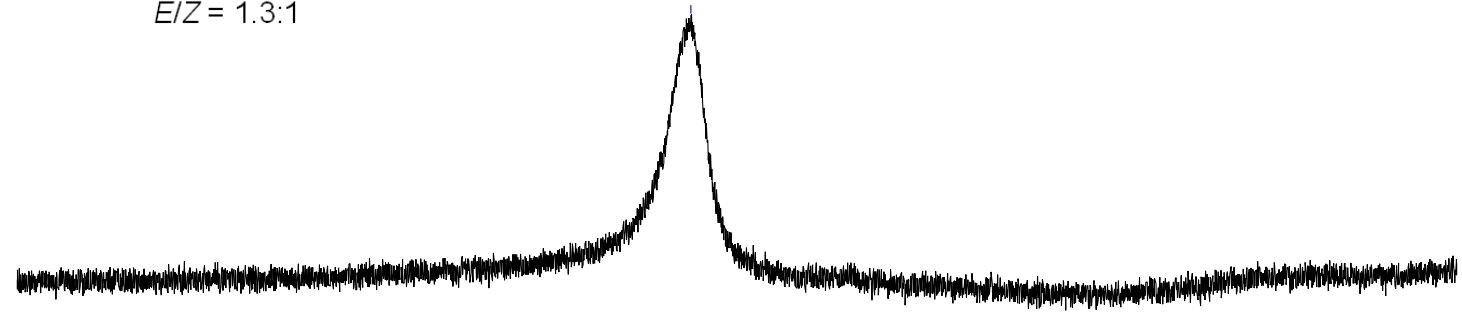




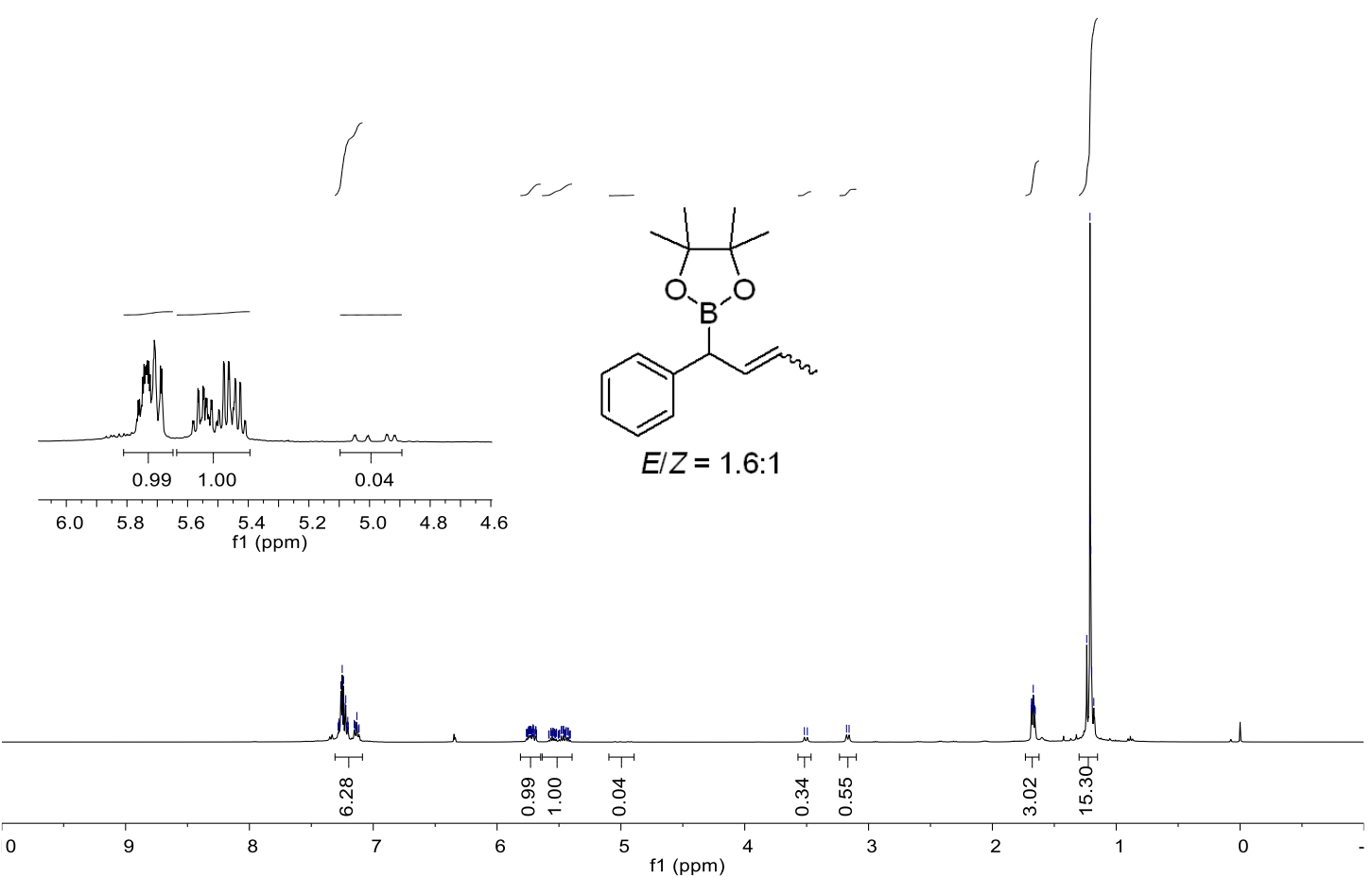

${ }^{1} \mathrm{H} \mathrm{NMR}\left(400 \mathrm{M}, \mathrm{CDCl}_{3}\right)$ spectrum of $\mathbf{2 a k}$

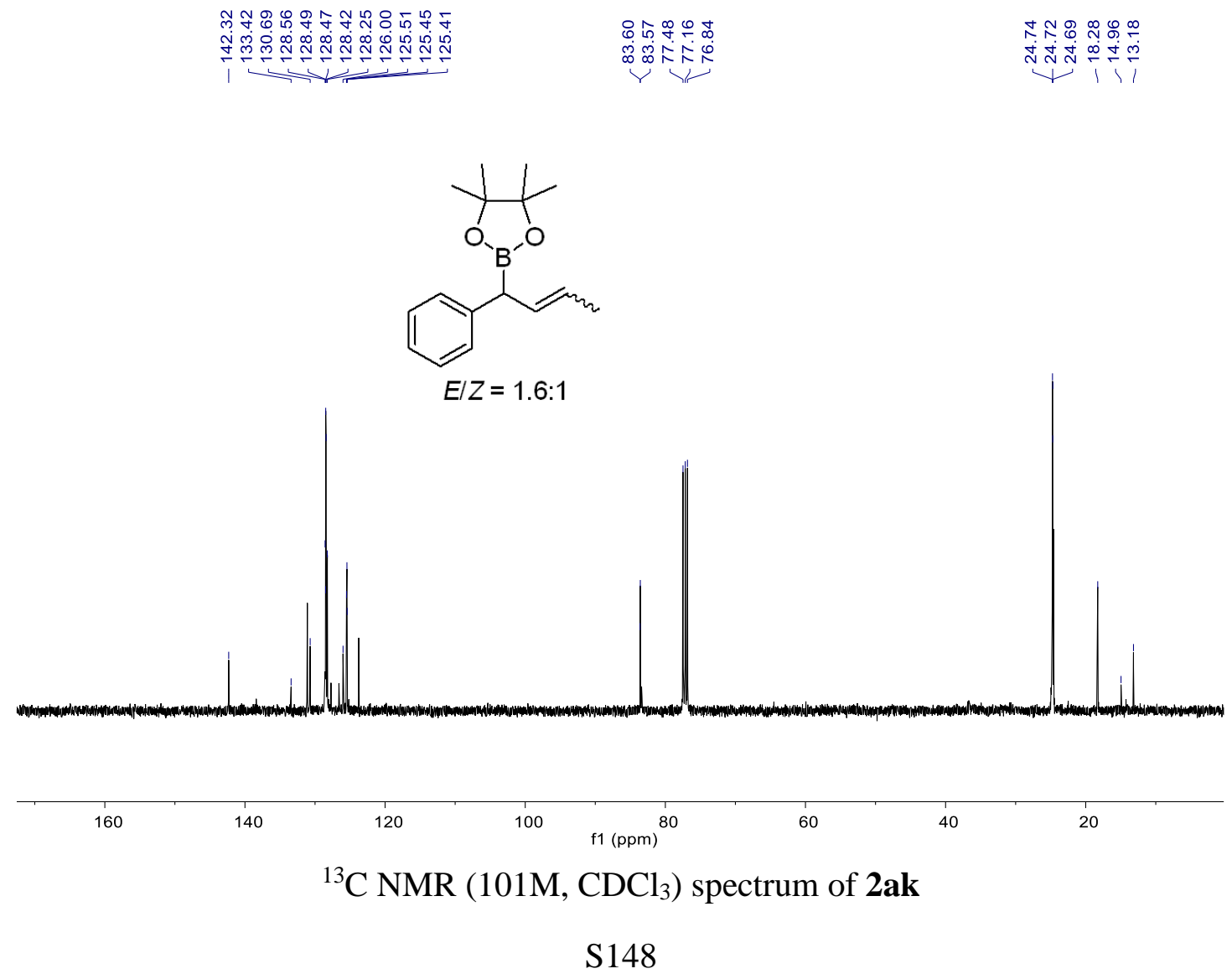




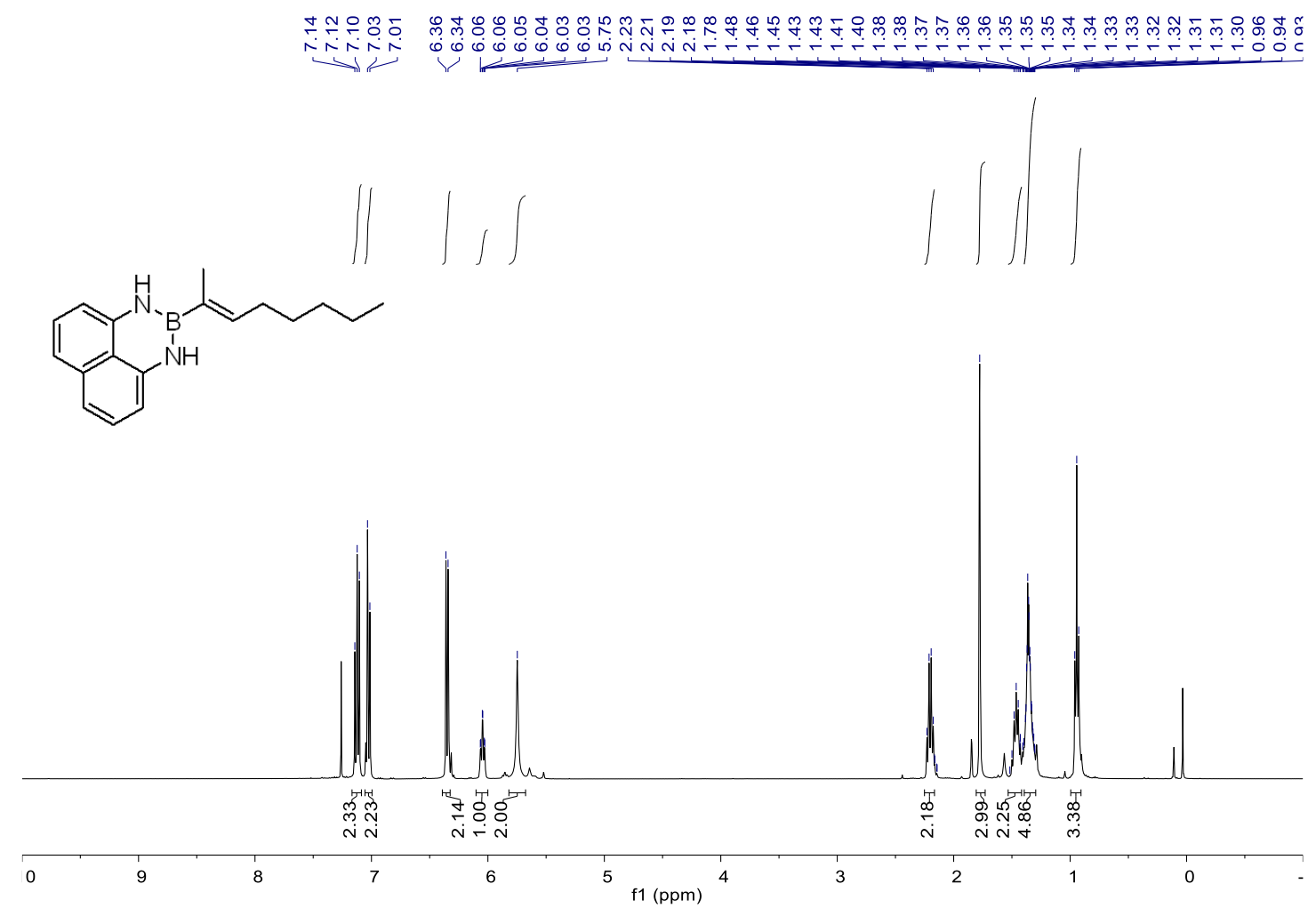

${ }^{1} \mathrm{H}$ NMR (400M, $\mathrm{CDCl}_{3}$ ) spectrum of $\mathbf{2 a m}$

\begin{tabular}{|c|c|c|c|c|c|}
\hline 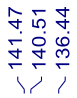 & $\underset{\substack{0 \\
\stackrel{0}{N}}}{i}$ & 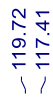 & $\begin{array}{l}\frac{\pi}{i} \\
\stackrel{0}{0}\end{array}$ & 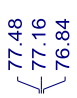 & 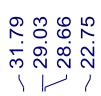 \\
\hline
\end{tabular}
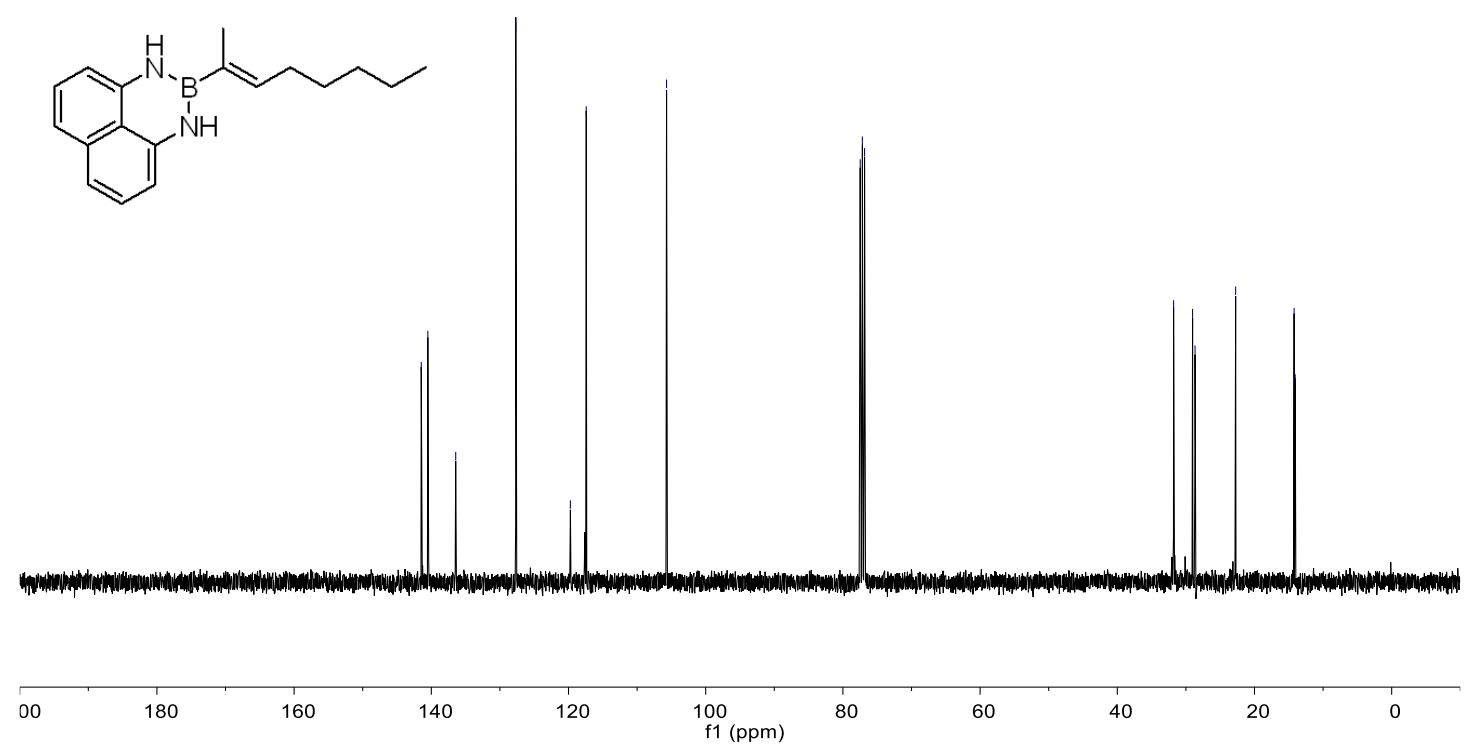

${ }^{13} \mathrm{C}$ NMR $\left(101 \mathrm{M}, \mathrm{CDCl}_{3}\right)$ spectrum of $\mathbf{2 a m}$ 

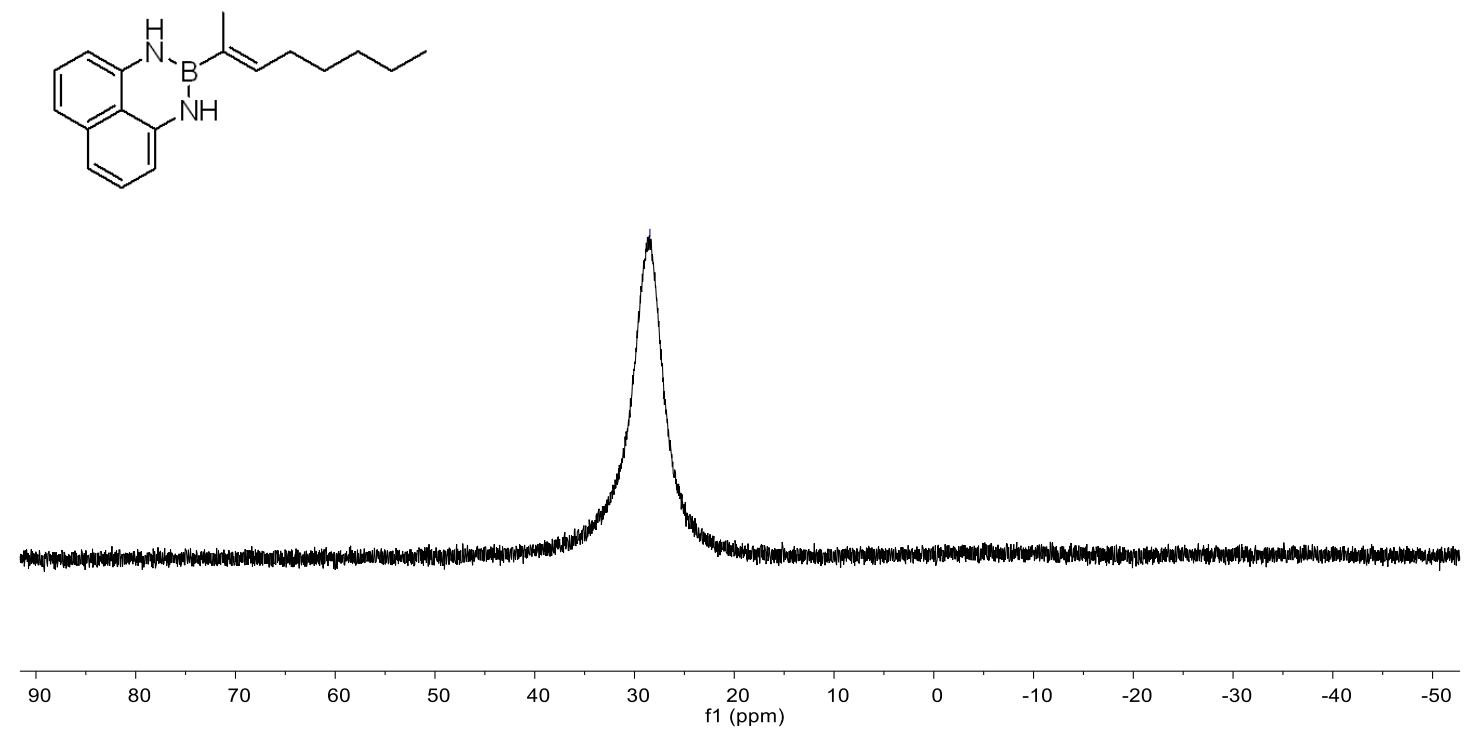

${ }^{11} \mathrm{~B}$ NMR $\left(128 \mathrm{M}, \mathrm{CDCl}_{3}\right)$ spectrum of $\mathbf{2 a m}$

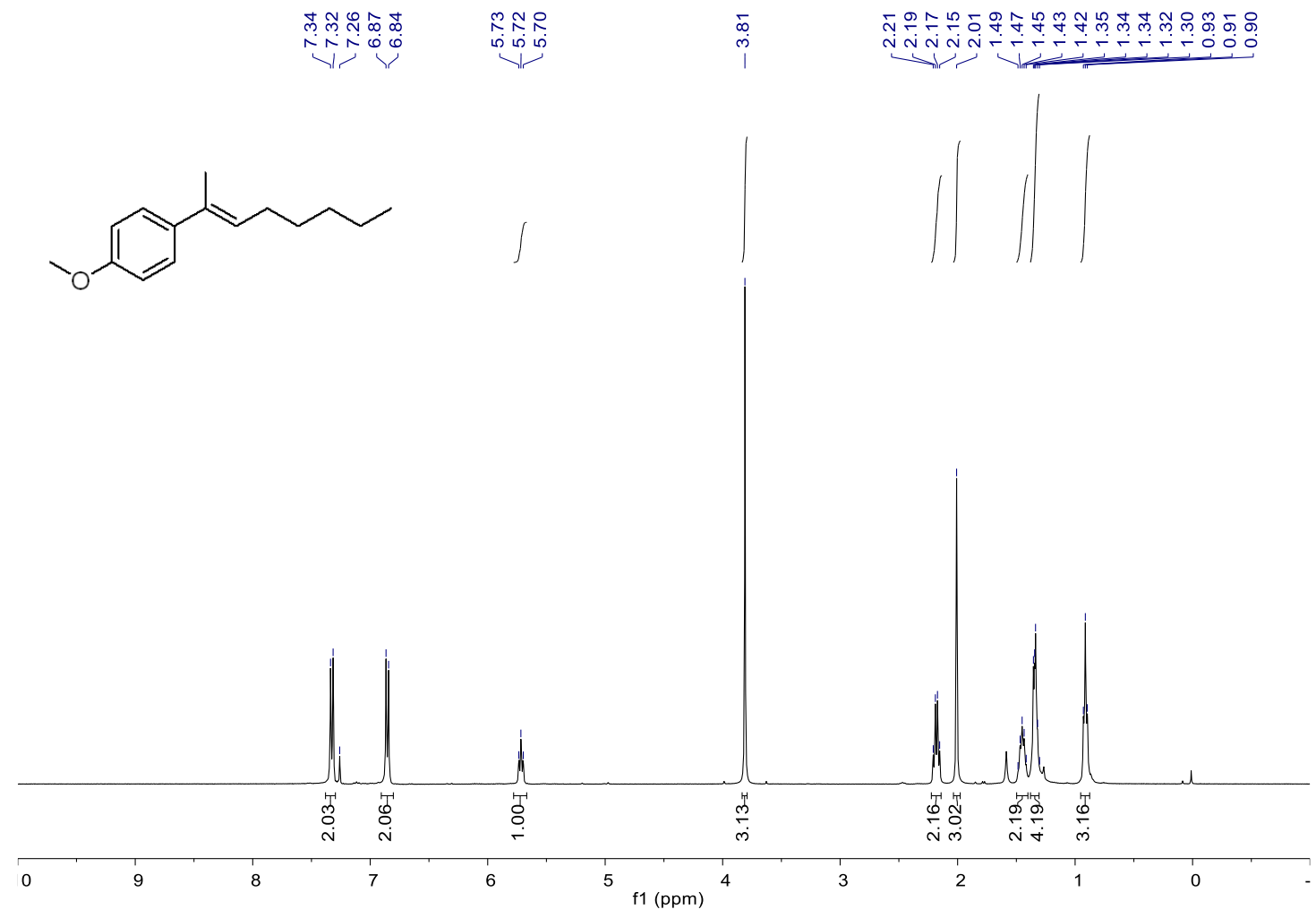

${ }^{1} \mathrm{H}$ NMR (400M, $\mathrm{CDCl}_{3}$ ) spectrum of $\mathbf{6 a}$ 

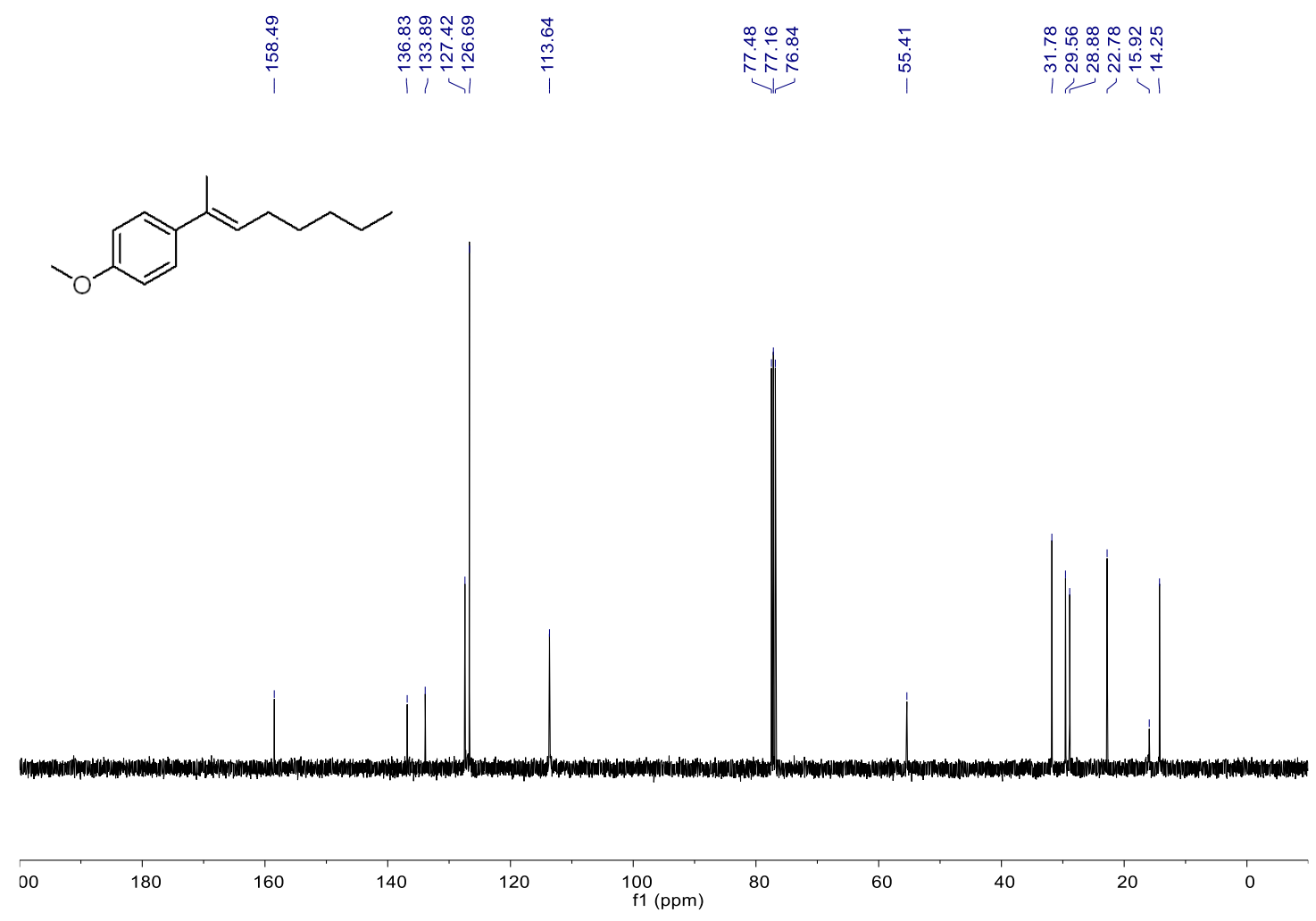

${ }^{13} \mathrm{C}$ NMR $\left(101 \mathrm{M}, \mathrm{CDCl}_{3}\right)$ spectrum of $\mathbf{6 a}$

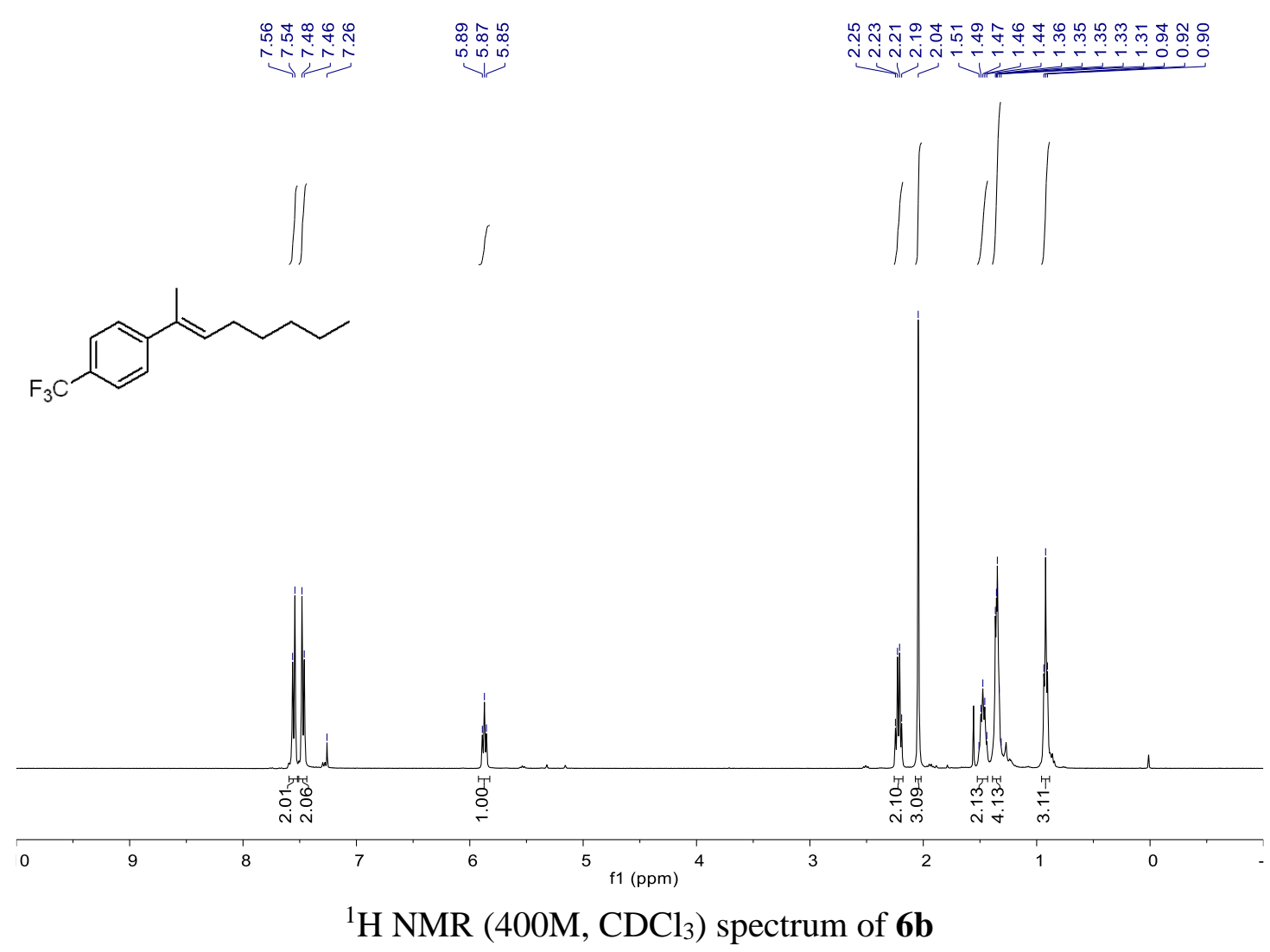




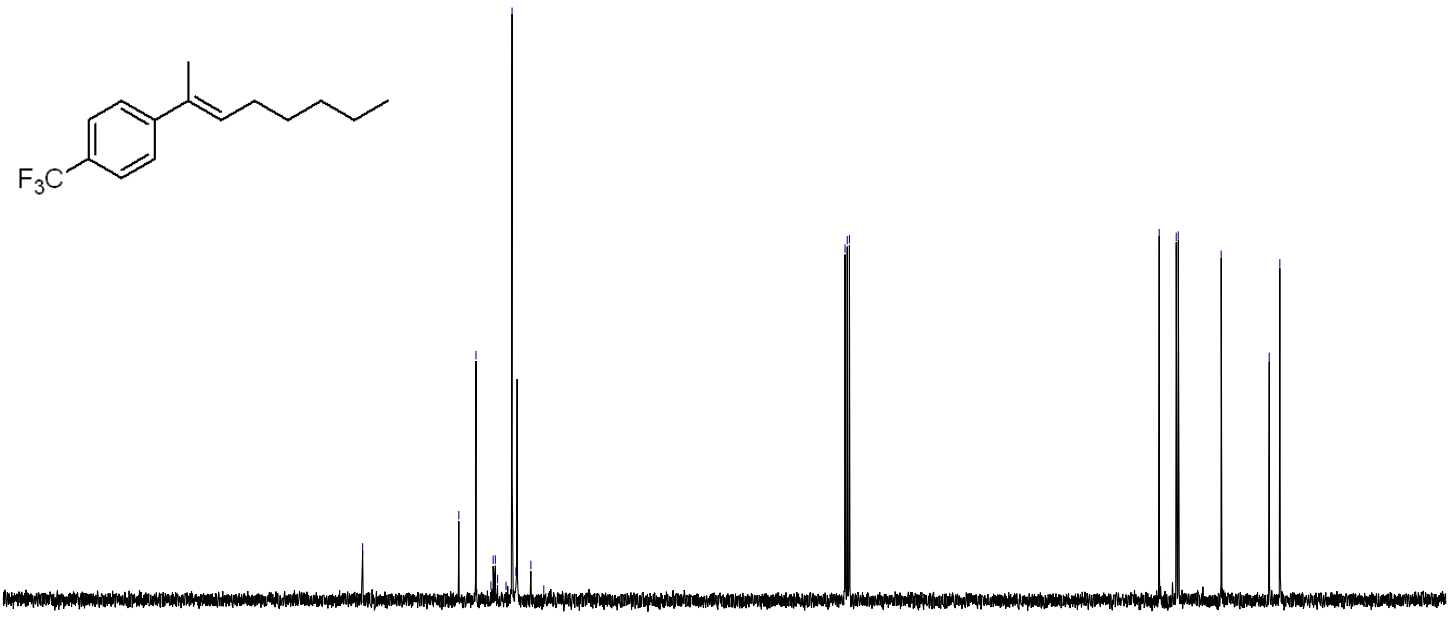

00

180

160

140

100
$\mathrm{f} 1(\mathrm{ppm})$

80

60

40

20

${ }^{13} \mathrm{C}$ NMR (101M, $\left.\mathrm{CDCl}_{3}\right)$ spectrum of $\mathbf{6} \mathbf{b}$<smiles>CCCCC=C(C)c1ccc(C(F)(F)F)cc1</smiles>

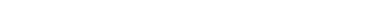

50

0

$-50$ $\mathrm{f}^{-100}(\mathrm{ppm})$

$-150$

$-200$

$-250$

${ }^{19} \mathrm{~F}$ NMR (377M, $\left.\mathrm{CDCl}_{3}\right)$ spectrum of $\mathbf{6 b}$ 


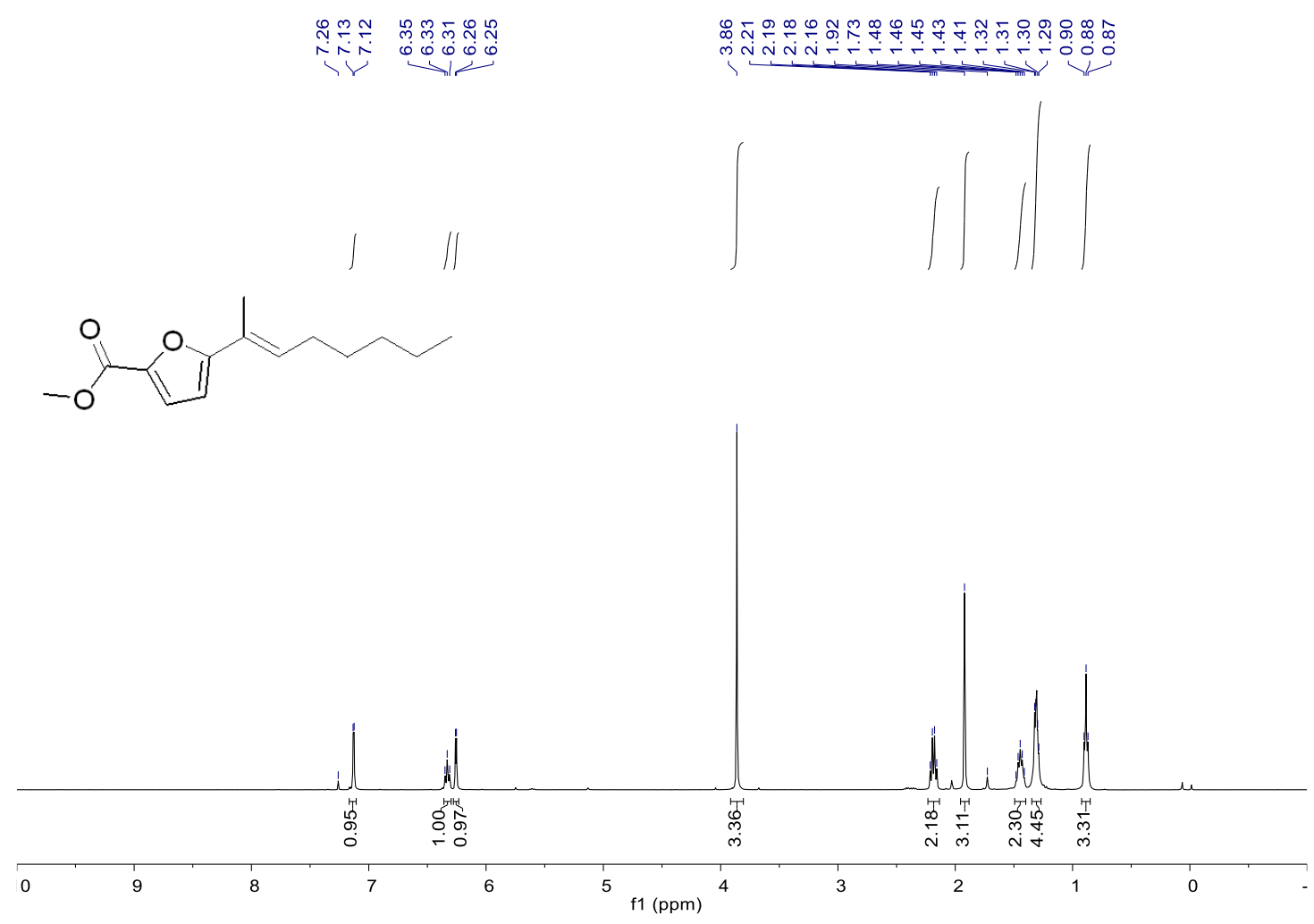

${ }^{1} \mathrm{H}$ NMR (400M, $\mathrm{CDCl}_{3}$ ) spectrum of $\mathbf{6 c}$

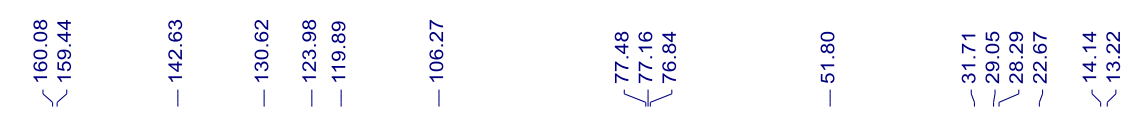
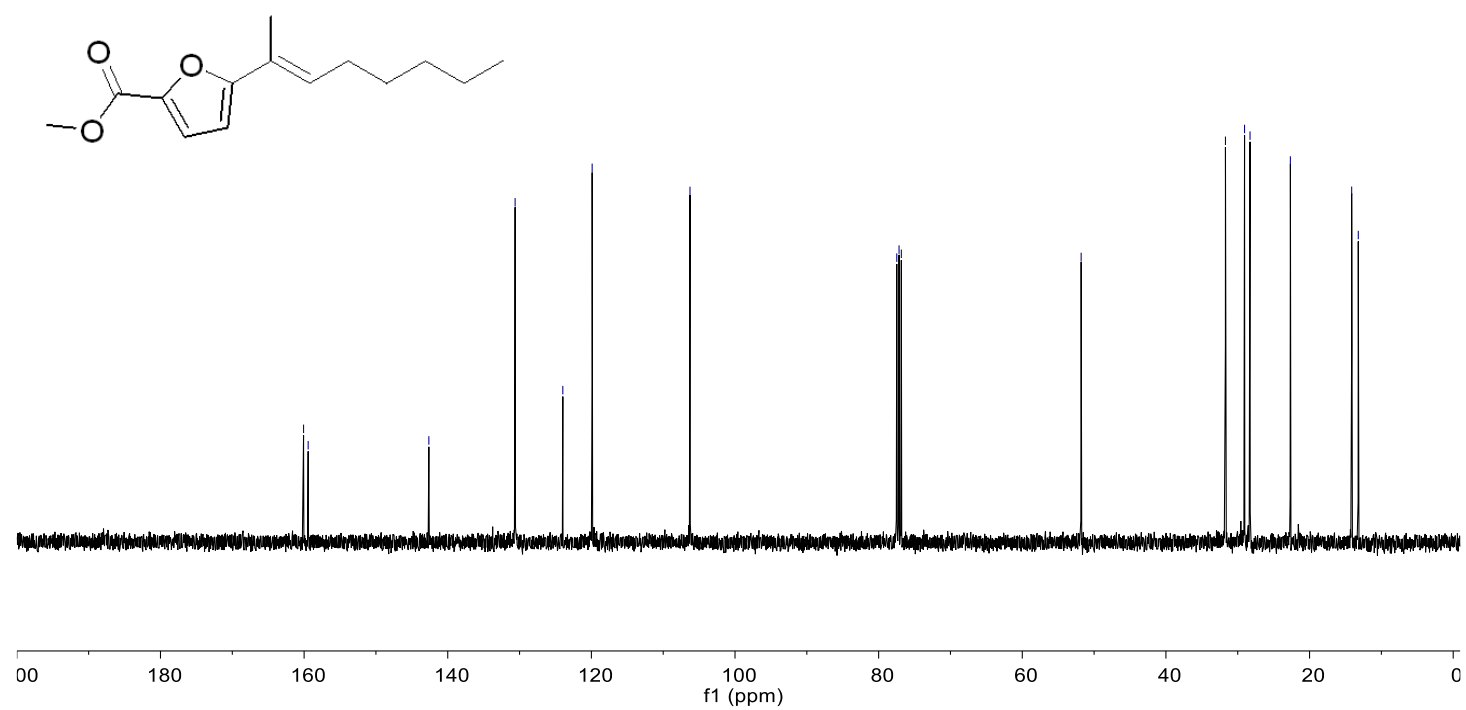

${ }^{13} \mathrm{C}$ NMR $\left(101 \mathrm{M}, \mathrm{CDCl}_{3}\right)$ spectrum of $\mathbf{6 c}$ 


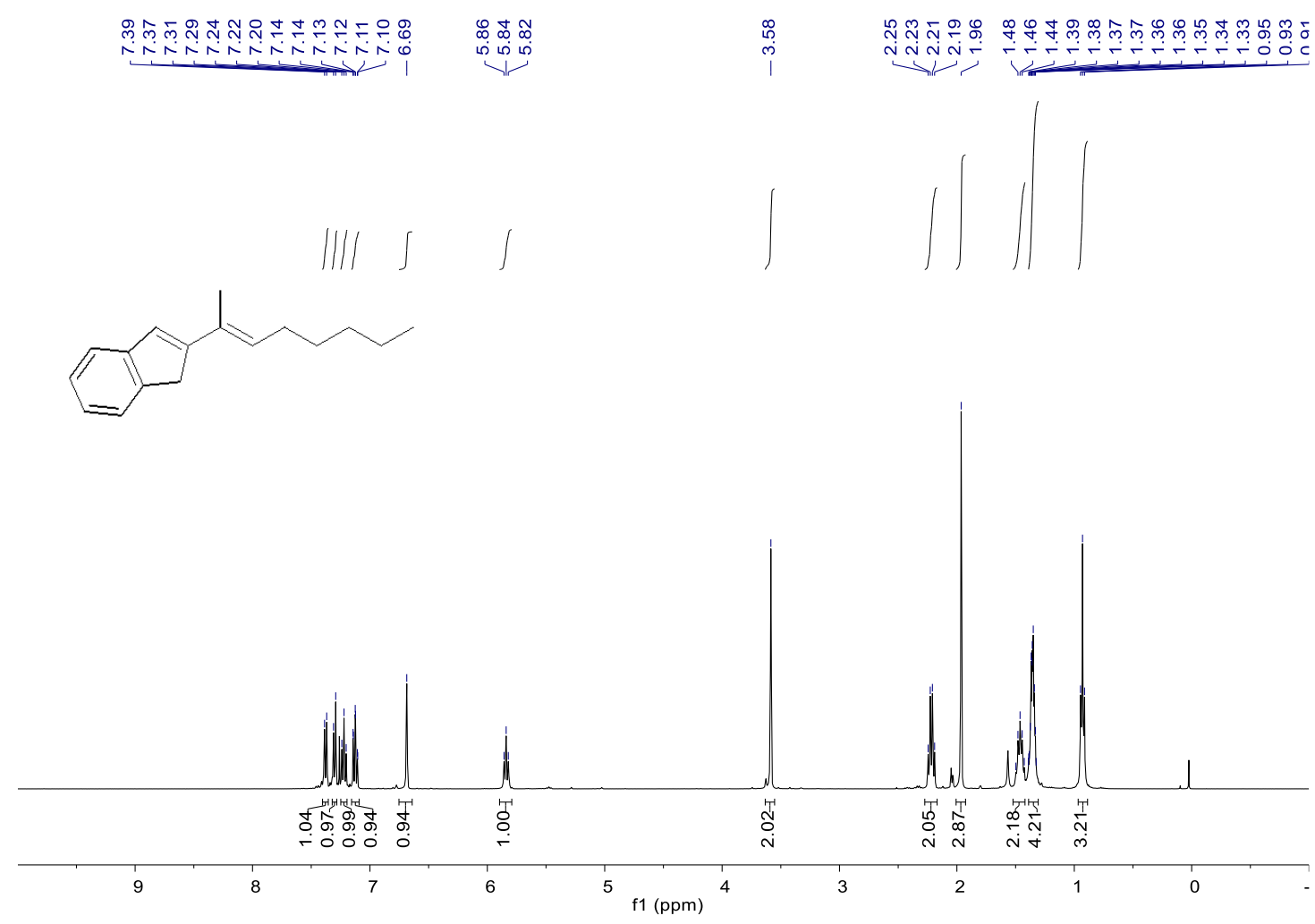

${ }^{1} \mathrm{H}$ NMR (400M, $\mathrm{CDCl}_{3}$ ) spectrum of $\mathbf{6 d}$

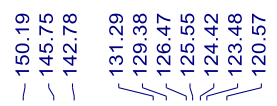

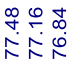

证

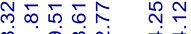

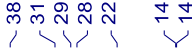
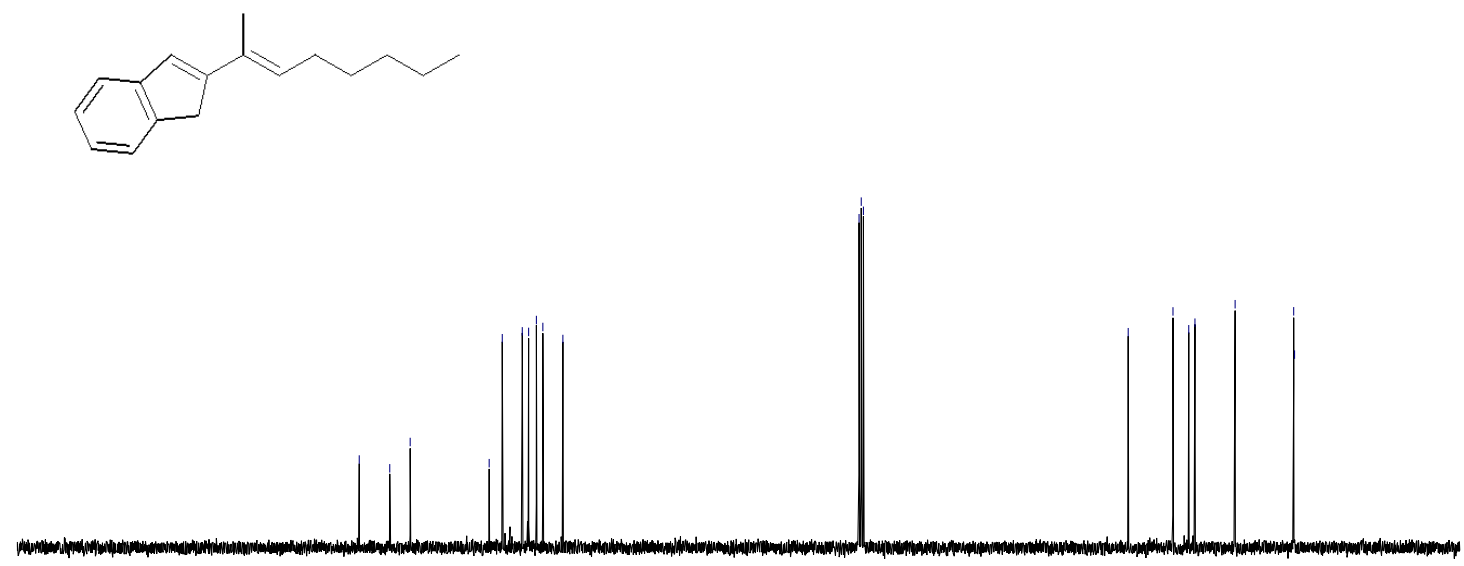

00

180

160

${ }_{\mathrm{f} 1}^{100}(\mathrm{ppm})$

$80 \quad 60$

40

20

${ }^{13} \mathrm{C}$ NMR (101M, $\left.\mathrm{CDCl}_{3}\right)$ spectrum of $\mathbf{6 d}$ 


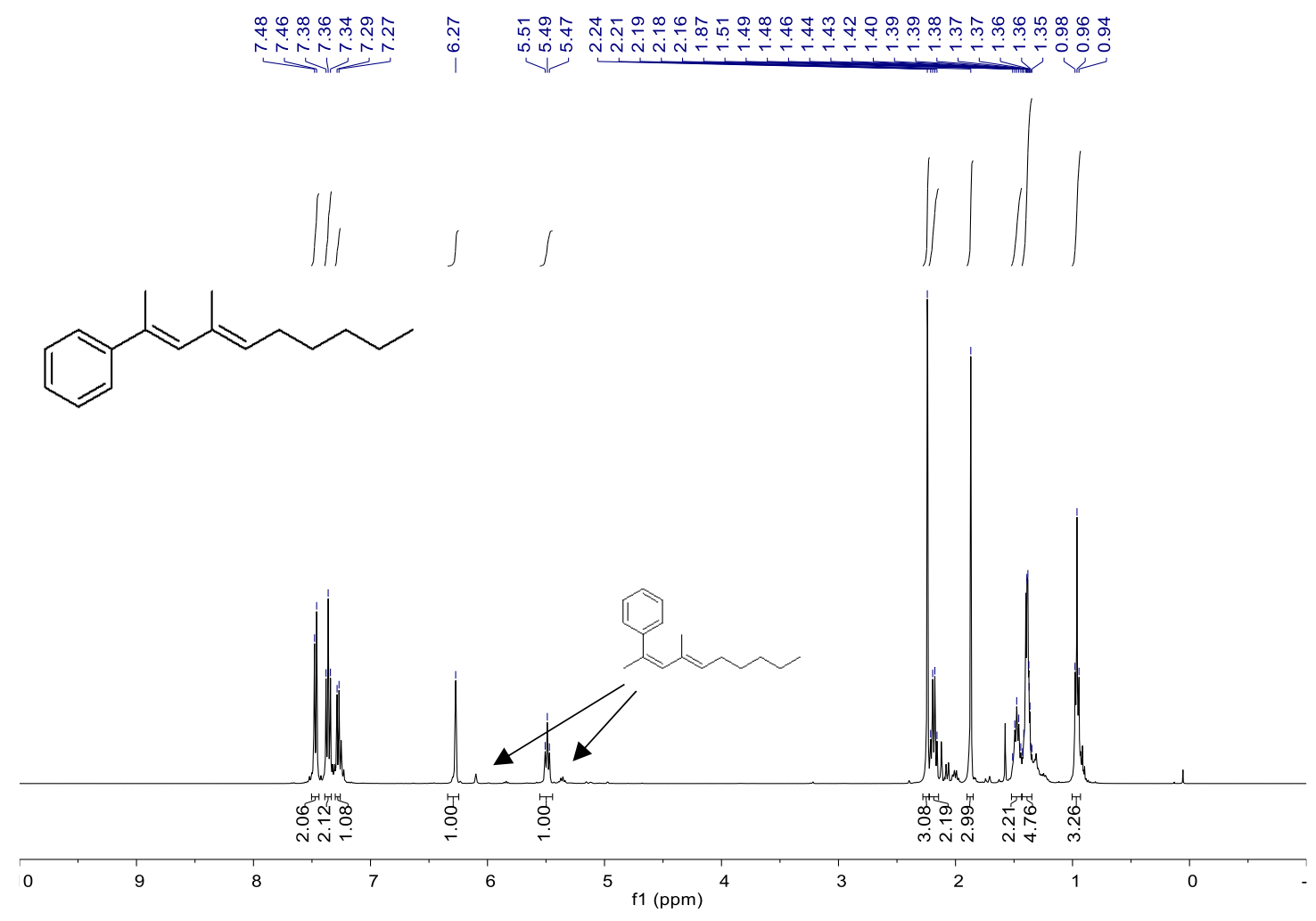

${ }^{1} \mathrm{H}$ NMR (400M, $\mathrm{CDCl}_{3}$ ) spectrum of $\mathbf{6 e}$

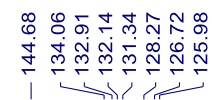

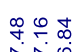

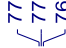

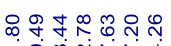

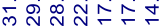

(
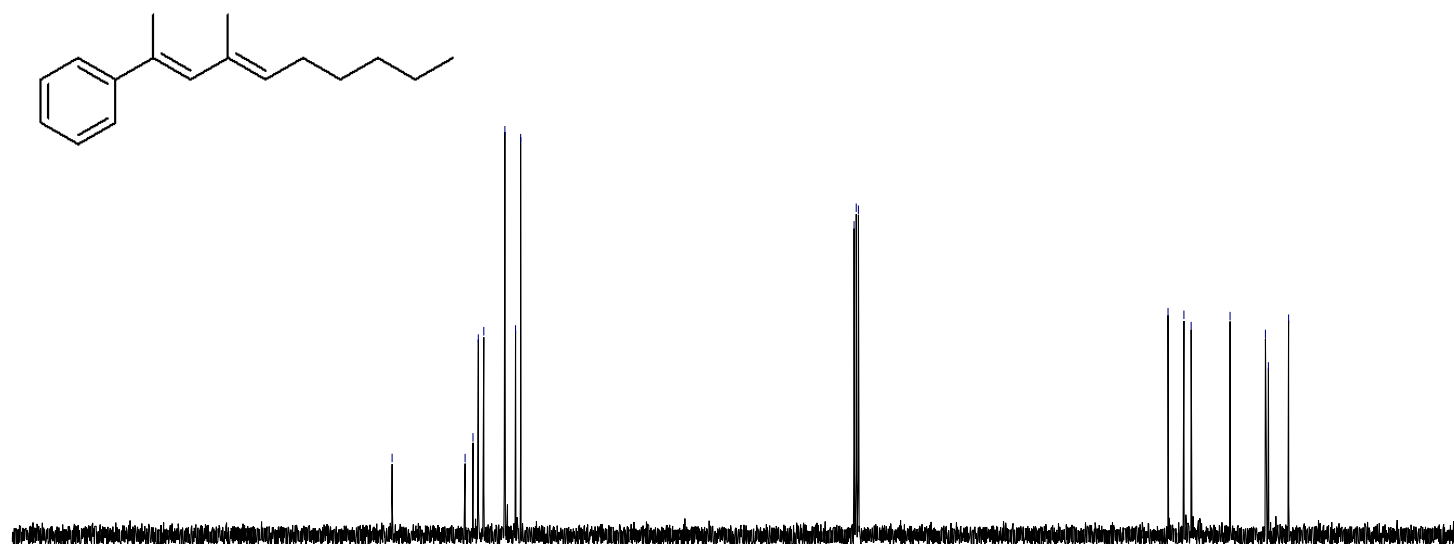

00

$180 \quad 160$

140

120

100
$\mathrm{f} 1(\mathrm{ppm})$

80

60

40

20

${ }^{13} \mathrm{C}$ NMR $\left(101 \mathrm{M}, \mathrm{CDCl}_{3}\right)$ spectrum of $\mathbf{6 e}$ 

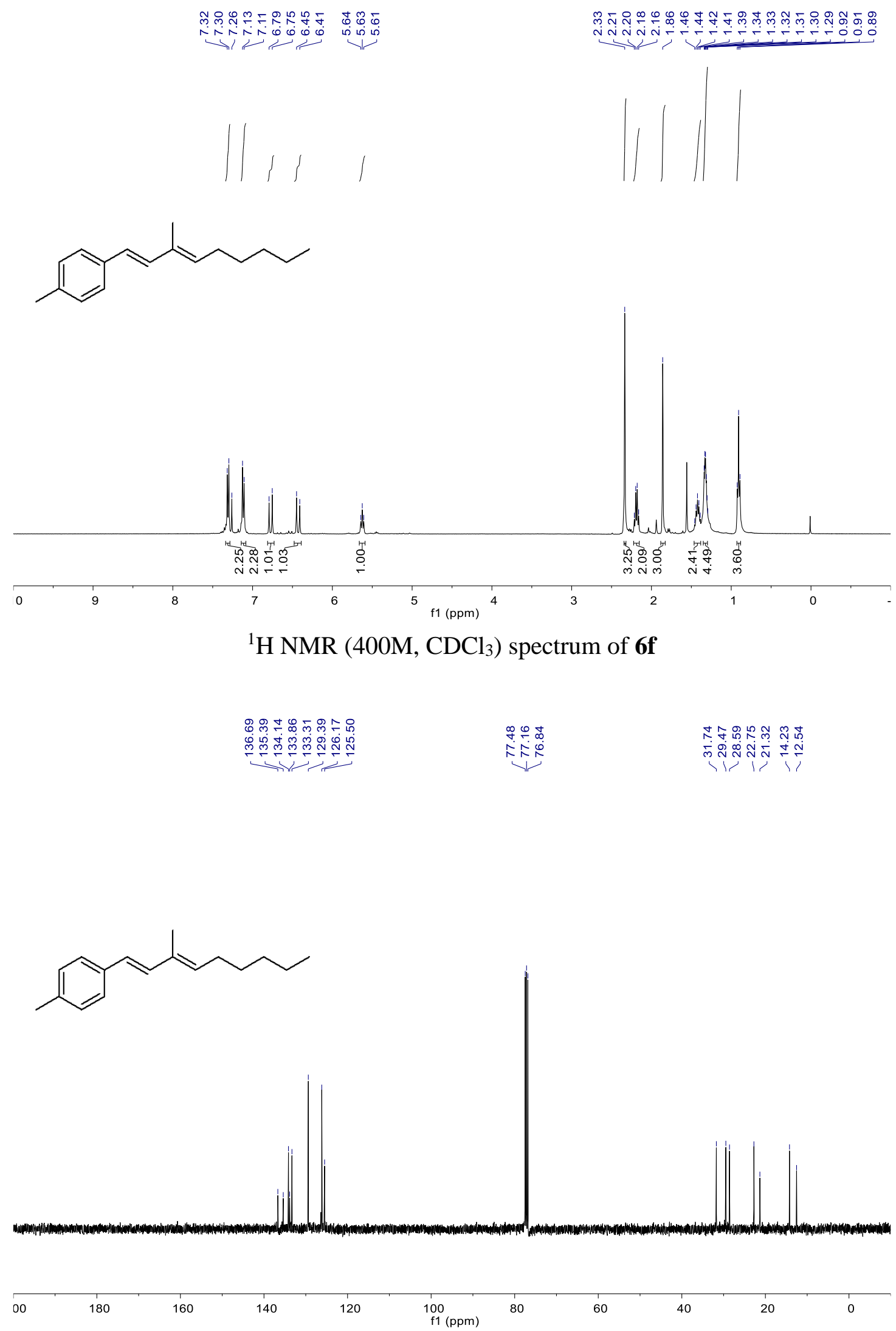

${ }^{13} \mathrm{C}$ NMR $\left(101 \mathrm{M}, \mathrm{CDCl}_{3}\right)$ spectrum of $\mathbf{6 f}$ 


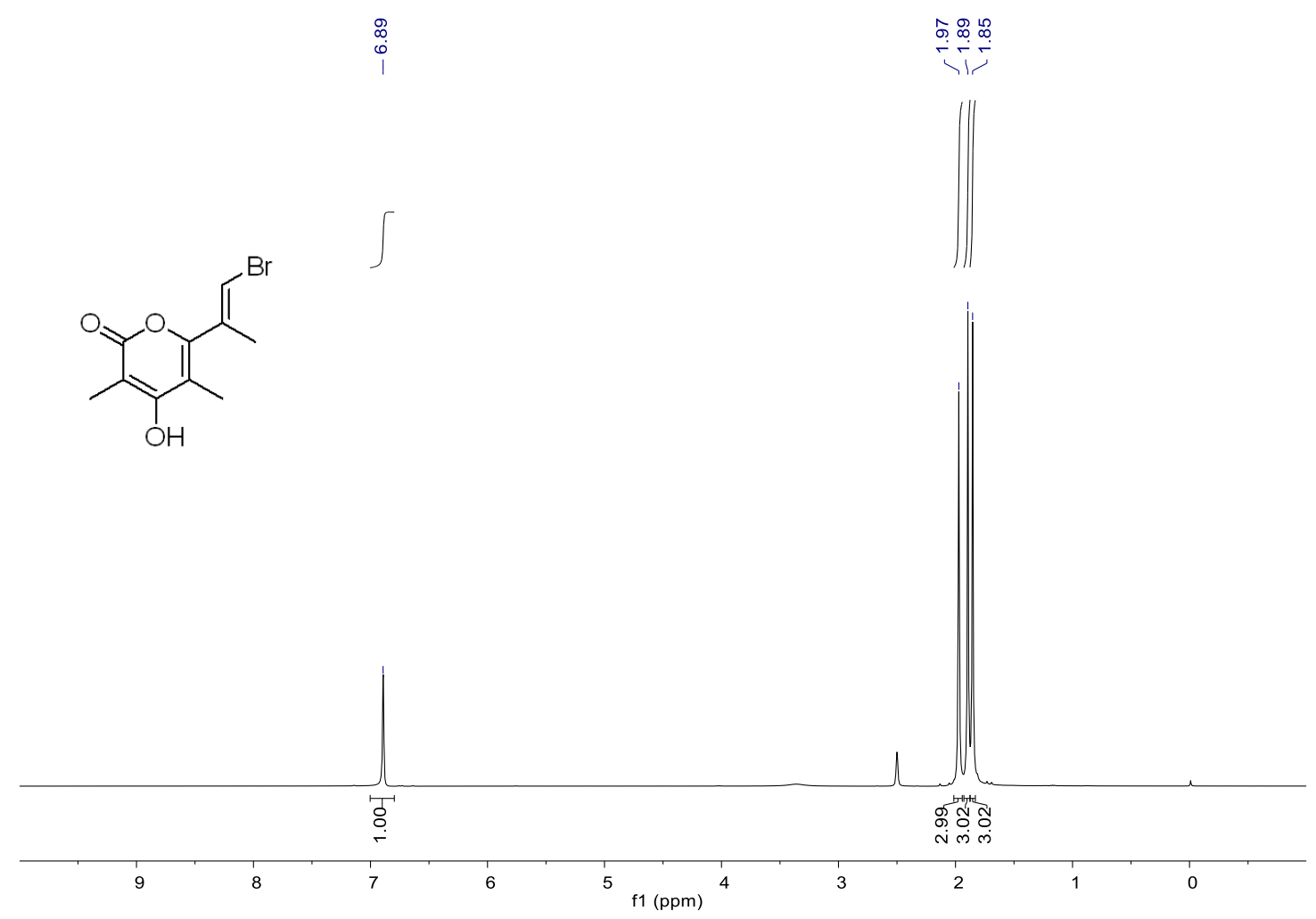

${ }^{1} \mathrm{H}$ NMR (400M, DMSO- $d_{6}$ ) spectrum of $\mathbf{1 1}$

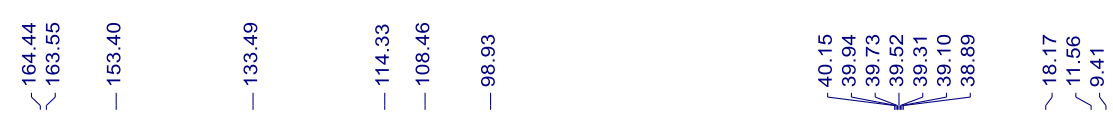<smiles>CC(=CBr)c1oc(=O)c(C)c(O)c1C</smiles>

00 100 f1 (ppm) 80 60 40 20 0

${ }^{13} \mathrm{C}$ NMR (101M, DMSO- $d_{6}$ ) spectrum of $\mathbf{1 1}$ 


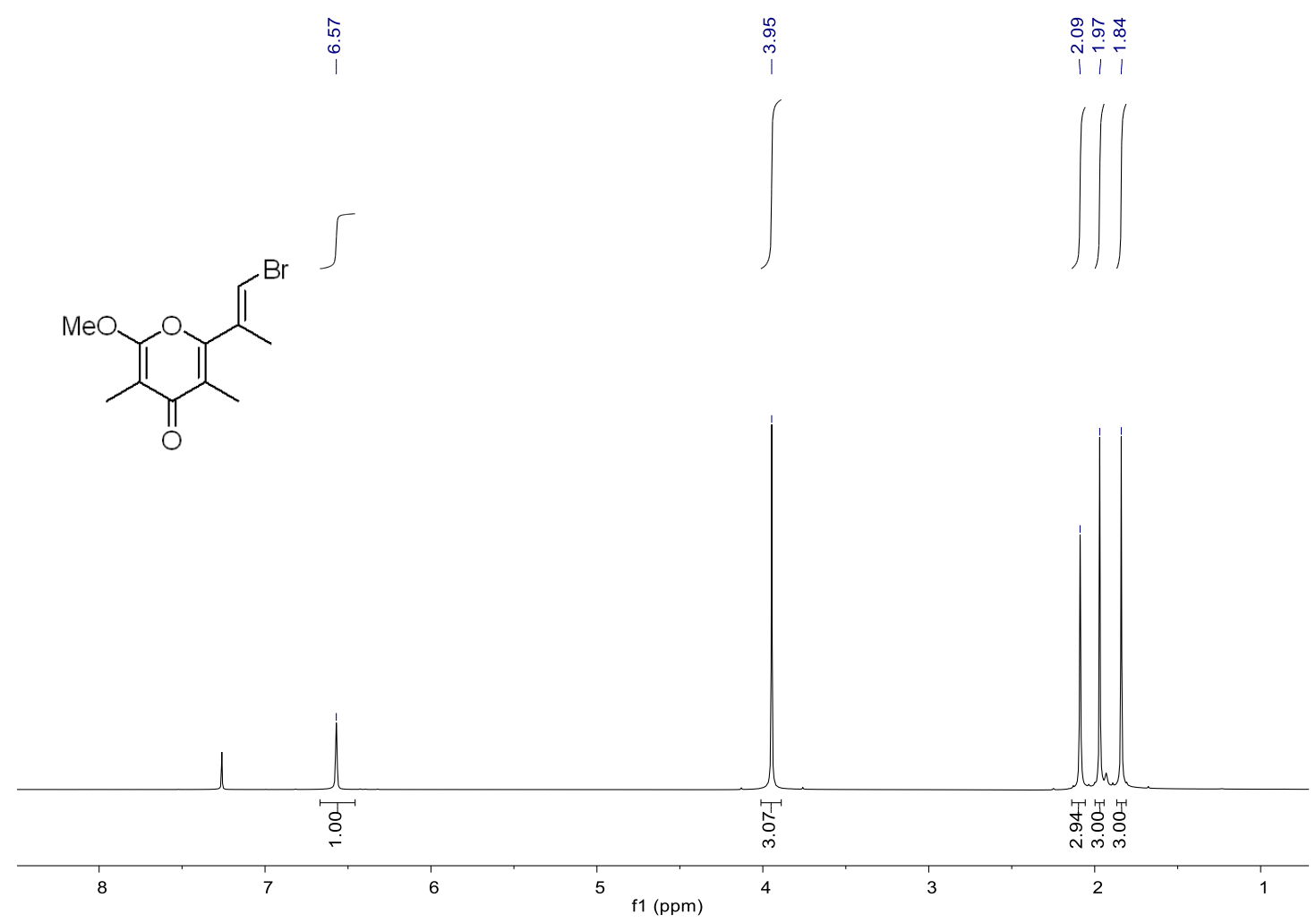

${ }^{1} \mathrm{H}$ NMR (400M, $\mathrm{CDCl}_{3}$ ) spectrum of 7

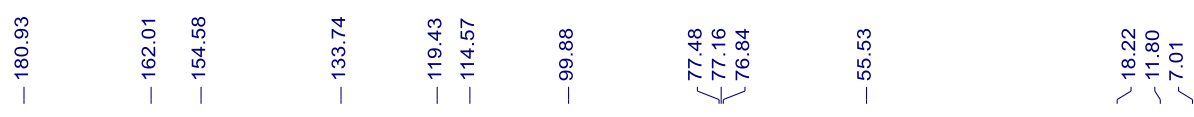<smiles>COc1oc(/C(C)=C/Br)c(C)c(=O)c1C</smiles>

200 180 160 140 120 ${ }_{\mathrm{f} 1}^{100}(\mathrm{ppm})$ 80 60 $40 \quad 20$ $20 \quad 0$

${ }^{13} \mathrm{C}$ NMR $\left(101 \mathrm{M}, \mathrm{CDCl}_{3}\right)$ spectrum of 7 


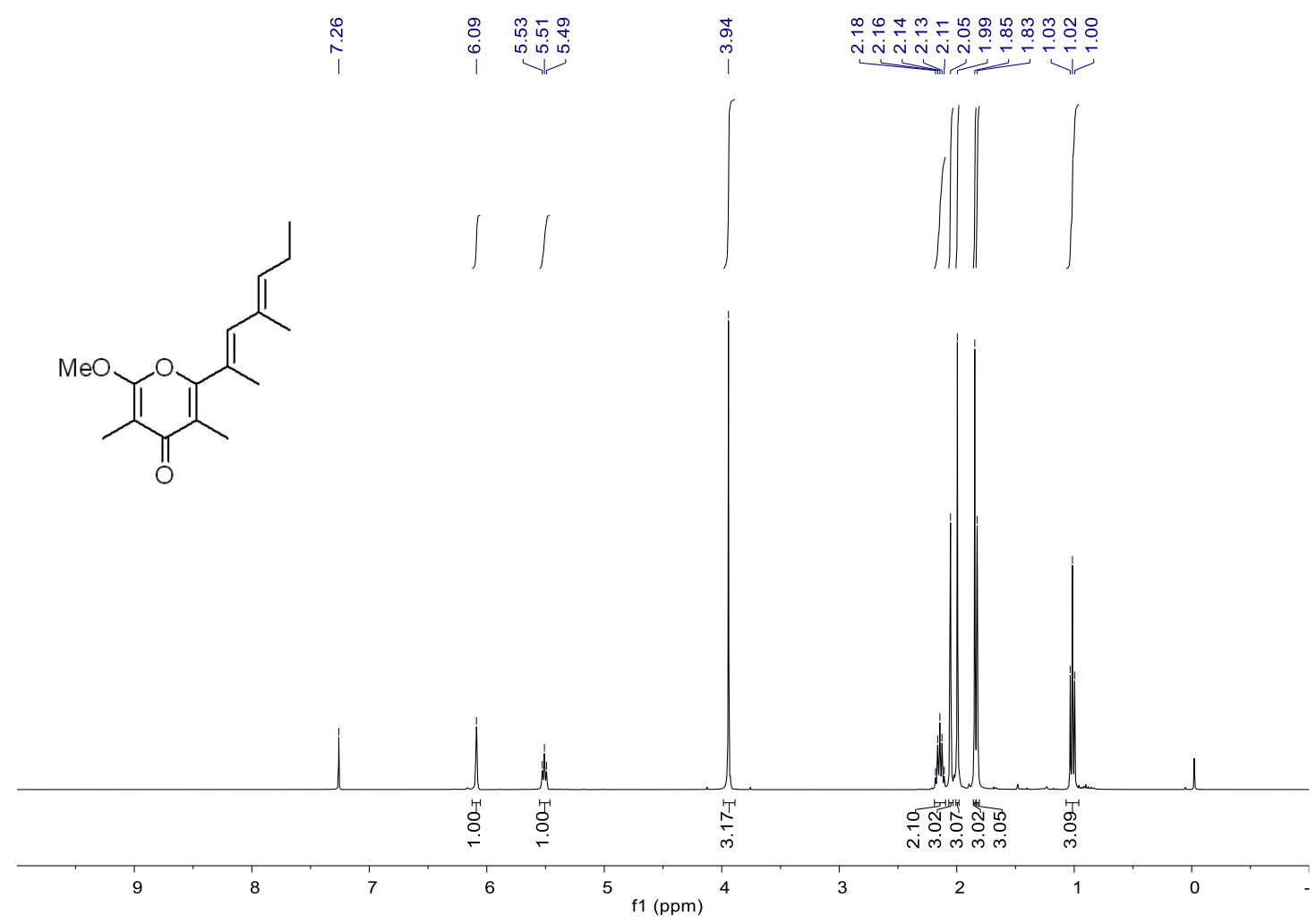

${ }^{1} \mathrm{H}$ NMR (400M, $\mathrm{CDCl}_{3}$ ) spectrum of $\mathbf{8}$
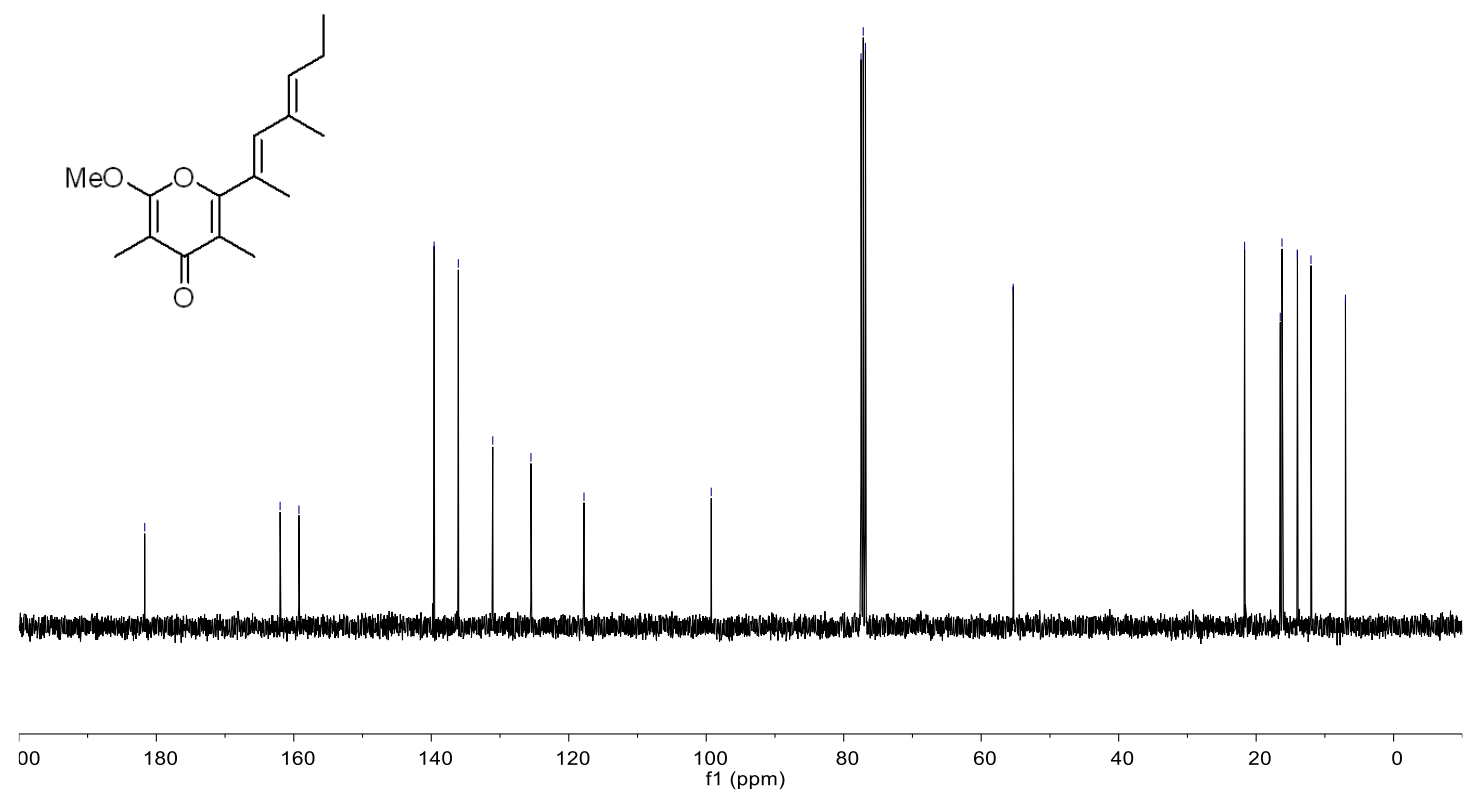

${ }^{13} \mathrm{C}$ NMR $\left(101 \mathrm{M}, \mathrm{CDCl}_{3}\right)$ spectrum of 8 